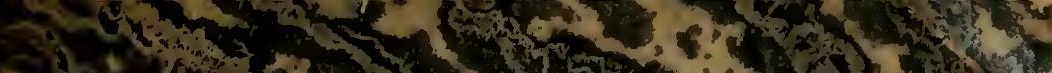

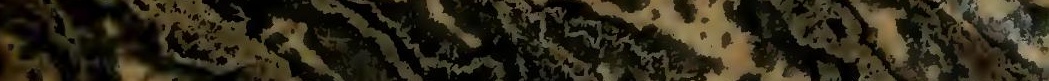

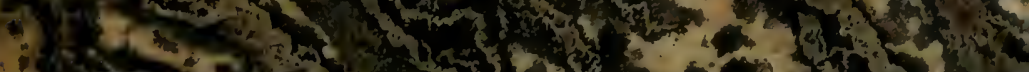

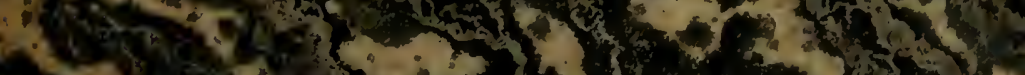

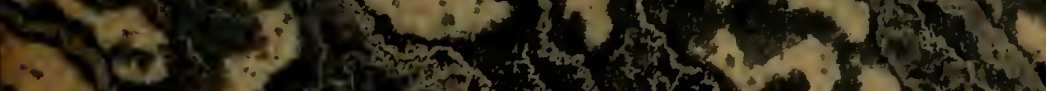

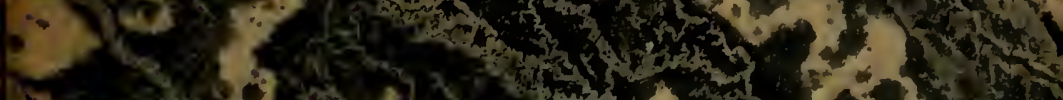

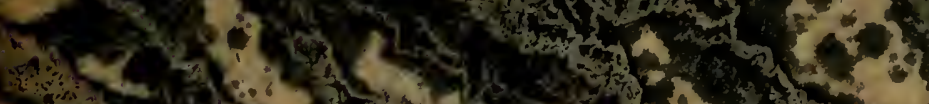

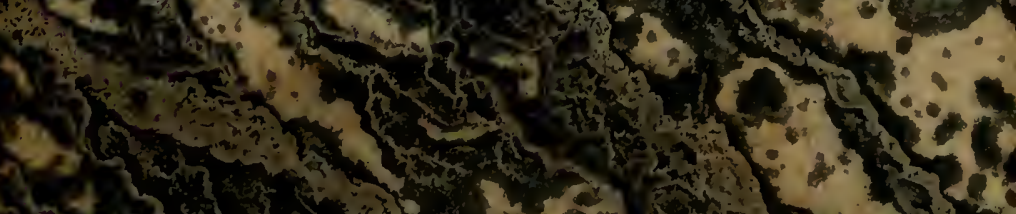
402 s.

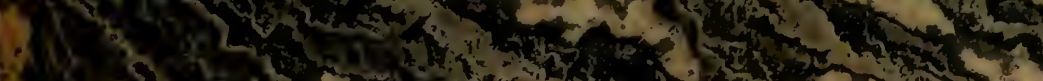

7.

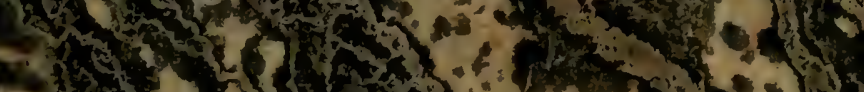

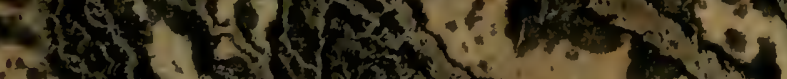

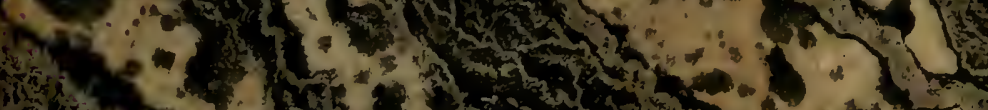

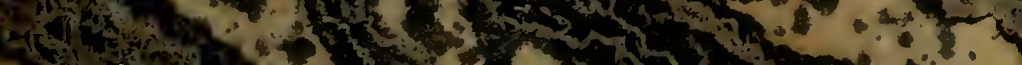

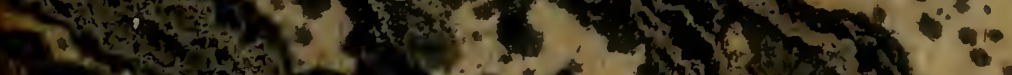

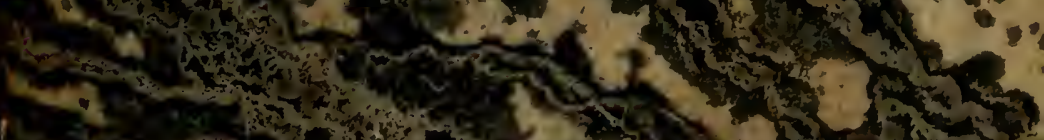
(3)

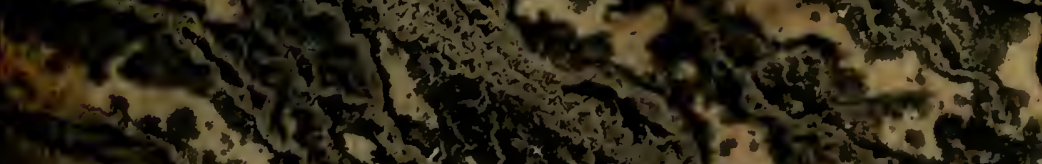

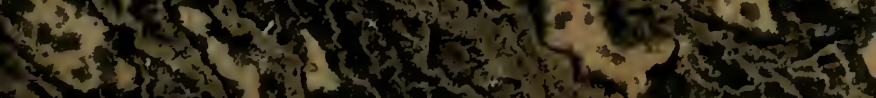

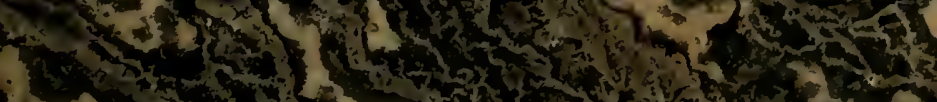

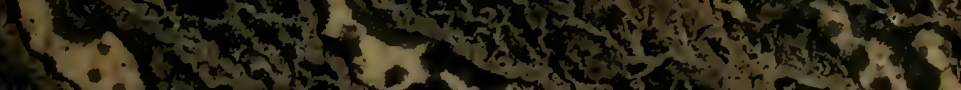

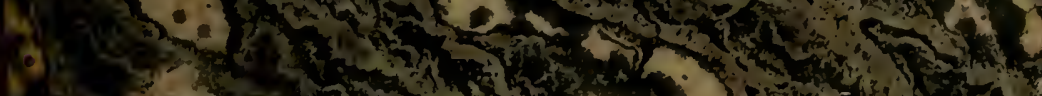
-25

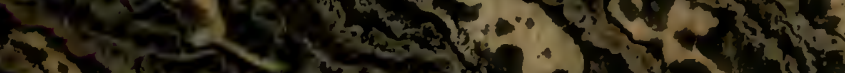

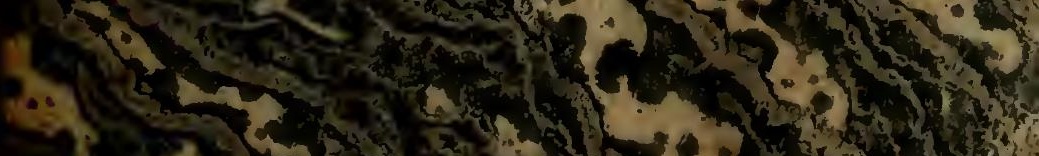

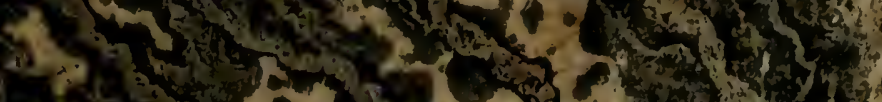

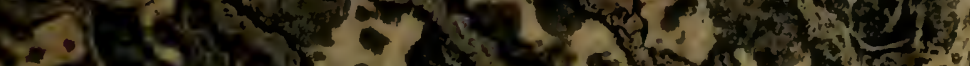




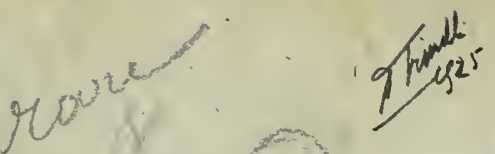


, 
Digitized by the Internet Archive in 2010 with funding from University of Ottawa 


\section{TRAITÉ}

ZOOLOGIQUE ET PHYSIOLOGIQUE

STjR

\section{LES VERS INTESTINAUX}

DE L'HOMME. 
PARIS. - IMPRIMERIE DE C.-L.-F. PANCKOUCKE, RUE DES POITEVINS, $\mathbb{N}^{\circ} 14$. 


\section{TRAITÉ}

ZOOLOGIQUE ET PHYSIOLOGIQUE

SUP

\section{LES VERS INTESTINAUX}

\section{DE LHOMME}

\section{PAR M. BREMSER D. M.}

TRADUIT DE L'ALLEMATD

PAR M. GRUNDLER D. M. P.

REVU ET AUGMENTÉ DE NOTES

PAR M. DE BLAINVILLE

D. 21. DE LA FACULTÉ DE PARIS ET PROFESSEUR D'ANATOMIE COMIPARÉE ET DE ZOOLOGIE A LA FACULTÉ DES SCIENCES, ETC. , ETC.

AVEC UN ATLAS

COMPOSE DE DOUTE PLATCEES IN-QCARTO.

\section{PARIS}

C. L. F. PANCKOUCK E EDITEUR

rue des Poitevins $\mathrm{n}^{9} .14$ 

Lettre de M. de Itumboidt à M. Panckoucke.

Monseur $_{\text {, }}$

Veuillez bieu m'excuser si, m'adressant à votre bienveillance souvent éprouvée, je vous parle d'une affaire à laquelle je prends le plus vif intérêt. L'excellent Traité sur les vers intestinaux de l'homme, du docteur liremser, est un ouvrage justement célèbre en Allemagne, en Italie et en Angleterre; il n'a point encore été traduit de l'allemand en français. Un de mes compatriotes, le docteur Grundler, qui habite depuis long-temps cette capitale, et qui connaît à la fois les langues et l'état de la science médicale, a entrepris cette traduction. J'ose espérer que, si quelque naturaliste français au courant de la matière, par exemple MI. de Blainville, voulait se charger de revoir la traduction, et d'y ajouter quelques notes instructives, MI. Grundler pourrait être assez henreux pour trouver un éditeur. L'ouvrage est également intéressant pour la médecine pratique et pour l'histoire naturelle descriptive.

Il existe au Muséum du Jardin du roi une belle collection des vers intestinaux de M. Bremser; elle prouve l'étendue du travail auquel ce savant s'est livré avec tant de succès. Je serais bien heureux d'apprendre que vous voulussiez vous intéresser, Monsieur, à cette utile entreprise : ce serait un nouveau service que vous rendriez aux sciences.

Agréez l'expression de la haute consideration avec laquelle j'ai l'honneur d'être,

Nonsieur,

Votre très-humble et très-obẻissant serviteur,

ALEXAXDRE DE HUNISOLDT. 

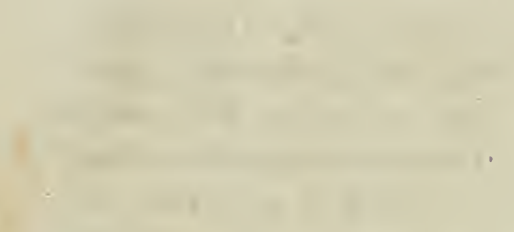

$+1$

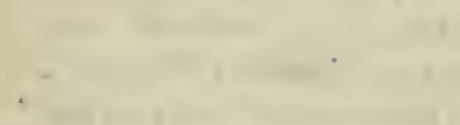

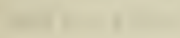

,

$+2$

7

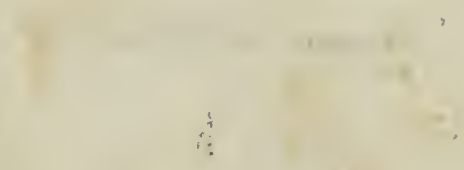




\section{AVERTISSEMENT.}

Dès que cet ouvrage nous fut connu, il y a plusieurs années, par l'entremise d'un jeune Allemand alors à Paris, qui venait de le recevoir de Vienne, la réputation de $\mathbf{M}$. Bremser, regardé avec juste raison comme le premier helminthologue praticien de l'Allemagne et de l'Europe entière, le besoin urgent que la médecine avait d'un traité qui contînt à la fois la description avec figures des vers qui séjournent dans l'es. pèce humaine, et des considérations générales et particulières sur les moyens bygiéniques et thérapeutiques propres a les combattre, nous firent vivement désirer que quelque médecin zoologiste en entreprît la traduction. Nous nous en serions peut-être même chargé volontiers, si nous n'eussions appris, dans une lettre de $M$. Bremser lui-même, qu'un médecin de Genève, alors à Vienne, était occupé à traduire son ouvrage en français sous ses yeux, et qu'il ne tarderait guère à paraître. Cependant une année et plus s'était déjà écoulée sans que nous eussions entendu parler le moins du monde de cette traduction, lorsque M. le docteur Grundler, médecin allemand, établi depuis un assez grand nombre d'années à Paris, et que nous avions l'avantage de connaître depuis long-temps, vint nous consulter sur la traduction qu'il avait commencée du 'Traité de M. Bremser, et nous demander de vouloir bien l'aider dans cette entreprise. Nous y fûmes aisément déterminé par l'intérêt mérité que nous lui portons et par l'utilité que nous voyions dans un ouvrage qui offre une des applica- 
tions les plus immédiates de la zoologie à la pratique mé. dicale. D'après nos conseils, il commença par avertir de son projet M. Bremser, en lui demandant s'il avait continué à faire traduire son ouvrage à Vienne, et, dans le cas contraire, s'il vovidrait y faire quelques changemens et additions. M. Bremser nous apprit que le médecin génevois ayant quitté Vienne avant la terminaison de sa traduction, il avait été obligé d'abandonner son projet, et qu'il nous fournirait plusieurs additions importantes. Il nous envoya en effet les matériaux de quelques changemens ou de rectifications de diverse nature; il y joignit des réponses aux observations critiques fondées, le plus souvent justes, mais aussi quelquefois passionnées qui ont été faites de son ouvrage depuis sa publication, et entre autres à celle qui a été insérée dans le journal itaiien intitulé Nuovi commentari di medicinae di chirurgia pour l'année I 820 ; critique extrêmenent longue, détaillée, qui a été tirée à part et répandue avec profusion en Allemagne, en Angleterre et en France, où elle a été traduite dans un de nos journaux de médecine. M. Bremser nous a aussi fourni quelques dissertations publiées en Allemagne par ses élèves sur plusieurs vers intestinaux propres à l'homme, que cependant il ne connaissait qu'incomplétement auparavant. Ces matériaux, auxquels nous avons cru devoir quelquefois ajouter des notes ampliatives ou explicatives étaient assez étendus pour qu'il fût difficile de les intercaler dans le corps de l'ouvrage, ou même en note au bas des pages, sans nuire à sa constitution primitive. Nous avons donc cru préférable de les comprendre dans une partie à part, que nous placerons à la fin du volume; et, comme elle sera composée d'autant de chapitres que l'ouvrage luimême, il sera aisé d'y avoir recours successivement après qu'on aura lu chaeun de cerix-ci. 
Voilà en quoi consiste le premier changement que nous ferons à l'ouvrage de M. Bremser, ce qui donnera à la traduction de M. Grundler une valeur évidemment supérieure à celle de l'ouvrage original.

Un autre changement moins important, mais qui nous a paru cependant nécessaire, est celui que nous avons fait dans la disposition de l'ouvrage. Nous n'avons pas suivi rigoureusement l'ordre de l'original, qui traite dans autant de chapitres, $x^{\circ}$ de la formation des vers intestinalux en général; $2^{\circ}$ de leur distribution systématique générale; $3^{\circ}$ de la description de ceux qui vicent dans le canal intestinal de l'homme; $4^{\circ}$ des causes de ia formation de ces vers; $5^{\circ}$ du diagnostic; $6^{\circ}$ des remèdes généraux; $7^{\circ}$ des remèdes spéciaux; $8^{\circ}$ de la description des vers nématoïdes qui séjournent hors du canal intestinal; $9^{\circ}$ des vers trématodes; $10^{\circ}$ des vers vésiculaires; $11^{\circ}$ des formules de médicamens; $12^{\circ}$ enfin des pseudohelminthes ou prétendus vers intestinaux. Il nous a semblé que ce mélange de la partie zoologique et médicale pouvait nuiré à la régularité de l'ouvrage, et par conséquent à sa conception, et nous l'avons nettement divisé en deux parties, la première physiologique et zoologique, la seconde médicale ou thérapeutique. Chacune est ensuite subdivisée en trois chapitres qui sont consacrés successivement, le pre. mier, aux considérations générales sur la formation des vers intestinaux; le deuxième, à la division systématique de tout ce groupe d'animaux; le troisième, à la description des espèces qui vivent dans le corps de l'homme. Ce chapitre est partagé en trois sections, vers intérieurs au canal intestinal, vers extérieurs et pseudoleelminthes. Les trois autres chapitres constituent la seconde partie de l'ourrage; le premier ou le quatrième de tout l'ouvrage traite des causes qui déterminent la formation:des vers chez l'honme; le deuxième ou cinquième 
donne le diagnostique, et enfin le troisième ou sixième et dernier, les moyens thérapeutiques propres à combattre les causes productrices des vers, et les vers eux-mêmes.

On trouvera aussi que le systeme de classification des vers intestinaux en général n'est pas rigoureusement semblable à ce qu'il est dans l'ourrage allemand; mais ici nous avons suivi les désirs de M. Bremser lui-même, qui nous a priés de donner ce système tel qu'il est dans le Synopsis publié par M. Rudolphi depuis son grand Traité sur les entozoaires. Nous avons même cru fairc encore mieux, en extrayant cetle partie de la dissertation de M. Fischer, publiée l'année dernière sous les yeux mêmes de M. Bremser à Vienne, et qui est sans doute ce qu'il y a de plus complet.

Les planches ne sont pas non plus rigoureusement semblables à celles de l'ouvrage allemand. D'abord nous avous été obligés de les réduire au format in-8 $8^{\circ}$, parce que le texte a ce format, an lieu de celui in $-4^{\circ}$ de l'ourrage original; ensuite, comme il y a une sorte de luxe dans les figures de M. Bremser, nous en avons un peu diminué le nombre, en conservant cependant toutes celles qui étaient évidemment nécessaires, même à des degrés différens. Nous avons composé la neuvième planche avec la figure des pseudohelminthes, représentés d'une manière pittoresque sur le titre même de l'ouvrage allemand. En général nous avons attaché une grande importance à ce que nos figures, quoique lithographiées, rendissent exactement celles de M. Bremser, qui sont véritablement excellentes.

Quant au texte même de l'ouvrage, nous n'avons dû, comme on le pense bien, rien changer à tout ce qui est de pure Gescription ou de pure thérapeutique; nous aurions pu ne pas faire de même de la partie étiologique qui traite de la formation des organisations vivantes dans les corps organisés, 


\section{AVERTISSEMENT.}

et nous aurions dû être d'autant plus naturellement portés à l'abréger, que c'est cette partie qui nous a donné le plus de mal; mais cornme les idées de l'auteur à ce sujet sont en rapport avec sa méthode thérapeutique, et que d'ailleurs il pourra être assez agréable aux lecteurs de connaître queiquesunes des idées de la philosophie allemande actuellement en vigueur, nous nous sommes décidés à ne rien changer à ce chapitre évidemment un peu long, sauf à donner quelques observations à ce sujetdans l'appendice.

Nous ne parlerons pas du style d'un ouvrage de cette nature; il nous suffira de dire que nous avons tâché de faire entendre les idées de $M$. Bremser, et que pour cela même nous n'aṛons pas toujours voulı luị ôter la physionomie un peu allemande conservée par le tradıcteur. Ce que nous savons, c'est que M. Grundler parait avoir eu beaucoup de peine, quelquefois à entendre, et souvent à rendre son original, comme au reste il le déçlare dans la note ci-jointe.

Nous devons avertir, en terminant, que les notes conservées an bas des pages appartiennent toutes à M. Bremser ou à M. Grundler; celles du premier étaient dans l'ouvrage original, ou ont été envoyées par lui au traducteur; celles du dernier n'ont trait qu’a la thérapeutique; les nôtres ne se trouveront que dans la troisiène partie ou dans l'appendice.

I’aris, Io anût 1823 .

H. D. DE BLAINVILLE. 


\section{Note dis traducteur.}

Les personnes versées dans la connaissance des deux langues, et qui liront l'ouvrage original, pourront seules juger de la difficulté que j’ai éprouvée à rendre les idées philosophiques de M. Brenser de la manière claire que l'on demande en France, ce qui a pu dépendre de plusieurs causes; la principale sans doute vient de moi, qui ne me suis pas essentiellement occupé de ces matières; mais n'est-il pas possible que M. Bremser lui-même ne se soit pas toujours exprimé d'une manière facile à saisir dans un sujet (la théorie de la formation du globe et des êtres vivans) encore si obscur? Aussi je ne veux pas assurer que j'y ai complétement réussi : je dois même déclarer que je n'aurais peut-être pas persisté dans mon entreprise, si d'après mon jugement, d'accord en cela avec un grand nombre de médecins allemands, anglais et mème français, et malgré la critique aussi amère qu'injuste d'un Italien, qui paraît être M. Brera, je n'eusse regardé l'ouvrage de M. Bremser comme très-important pour la pratique, et surtout si M. de Blainville, auquel j'en fais ici mes remercimens, n'avait bien voulı m'aider de ses conseils, et enfin si M. de Humboldt, qui exerce constamment un patronage si noble en faveur de ses compatriotes, ne s'était intéressé pour la publication de ma traduction, comme le prouve la lettre ci-jointe.

N. B. Les notes signées $B r$. m'ont été communiquées par M. Bremsez dans une lettre qu'il a bien voulu m'adresser. 


\section{PREFACE.}

$E_{\text {i }}$

publiant un nouvel ouvrage sur un sujet qui a déjà été traité par beaucoup d'autres auteurs, on contracte jusqu’à un certain point l'obligation de faire connaître les raisons pour lesquelles on s'y est déterminé. Je puis heureusement me dispenser de recourir à de longues explications, comme cela résultera tout à l'heure de la revue des ouvrages qui ont quelque ressemblance avec le mien. 11 est naturel, ce me semble, de ne pas parler des écrits dans lesquels il n'a été fait mention des vers intestinaux qu'en passant, parce qu'ils n'ont rien de commun avec mon livre. Mais il n'en est pas de nême des ouvrages d'Andry et de Leclerc, publiés il y a une centaine d'années, et de celui de Van Dovern, connu depuis cinquante ans; ils ont en effet quelque analogie avec le mien; car tous les trois ont traitć de l'helminthologie, comme moi, sous le double rapport de l'histoire naturelle et de la médecine pratique; mais, lorsque ces médecins écrivaient, l'lelmiuthologie était encore an herceau. 
Les ouvragés couronués de Bloch et de Goëze, noms connus sans doute de tous mes lecteurs, ne ressemblent que très-peu au micu. Les écrits de ces deux auteurs ont un intérêt plus réel pour les naturalistes, et particulièrement pour les helmiuthologues, que pour les médecins praticiens.

Mais, abstraction faite de la ressemblance que ces différens ouvrages peuvent avoir avec le mien, il fatt encore les regarder presque comme s'ils n'existaient pas; car on ne les trouve plus chez les libraires.

Zeder et Rudolphi ont enrichi l'helminthologie de beaucoup d'observations nouvelles : leurs ouvrages, quoique classiques, n'ont cependant qu'un rapport très-éloigné-avec le mien. M. Rudolphi, qui mérite à juste titre qu'on l'appelle le premier des helminthologues (car depuis long-temps aucune branche de la zoologie n'avait été développée aussi complétement que celle qui regarde les vers intestinaux, dans son Traité d'entozoologie); M. Rudolphi, dis-je, a consacré quelques chapitres aux médecins dans le premier volume de son incomparable ouvrage : cependant on n'y trouve pas ce qui intéresserait justement le plus les médecins praticiens, qui ne font pas ordinairement une étude particulière de l'histoire naturelle, savoir les figures des vers intes- 
linaux de l'homme et les règles de thérapeutique propres à combattre chaque espèce en particulier, choses auxquelles cet auteur, d'après son plan, nc pouvait pas donner un assez grand développement.

Les ouvrages encore recherchés aujourd'hui avec lesquels le mien peut encore être mis en comparaison se bornent (à l'exception peut-être de quelques-uns que je n'ai pas vus, et de quelques dissertations francaises qui ne se trouvent pas ordinairement chez les libraires ) à ceux de Joerdens, Brera et Bradley; je craindrais d'offenser ces trois médecins, si je mettais dans la même catégoric le livre insignifiant sur le même sujet de M. le docteur Albrecht, et plusieurs autres semblables. Je ne puis cependant pas regarder ces trois auteurs comme ayant répandu beaucoup de lumière sur la connaissance des vers intestinaux propres à l'espèce humaine, ces auteurs n'étant pas des helminthologues praticiens.

Joerdens s'est contenté de donner des descriptions et dẹs dessins des vers intestinaux de l'homme, mais il y a entremêlé une si grande quantité d'autres animaux qui ne sont pas des vers intestinaux, que ceux qui ne sont pas bien au courant de l'helminthologie ne savent trop à quoi s'en tenir. Du reste, cet autcur 
11 a pas envisagé les vers intestinaux sous le rapport de la pathologie, ni sous celui de la thérapeutique.

M. Brera a rédigé fidèlement ses leçons conformément au titre qu'il leur a donné, c'est-à-dire, que ce ne sont que de véritables leçons sur les principaux vers intestinaux propres à l'espèce humaine. Ces leçous ont été faites dans le geure de celles que l'on trouve ordinairement dans les thérapeutiques spéciales, dans le chapitre des vers intestinaux. Mais M. Brera a développé ce sujet beaucoup plus complétement que l'on n'a coutume de faire, et il a eu soin de citer les oúvrages qui s'y rapportent. Malgré quelques inexactitudes que l'on rencontre dans ce travail, et que je passe sous silence, il est toujours très-flatteur pour l'auteur que son petit livre ait été traduit en peu de temps, une fois en allemand et deux fois en français. Brera aurait dù se borner à ce travail sur l'helminthologie; les supplémens, quoique volumineux, qu'il y a ajoutés depuis, ne peuvent être regrardés, selon moi, que comme un amas d'erreurs et d'idées hasardées, et j’ai peine à croire que l'on puisse en prendre une autre opinion. Je pric mes lecteurs de veuloir bien regarder tout ce que j'en dis dans plusieurs endroits de mon onvrage, 
sous le seul point de vue scientifique; car sans cela ils pourraient croirc que jen veux à la personne de M. Brera, ce dont je suis cependant bien éloigné, n'ayaut jamais eu aucune espèce de relation avec lui, Au reste, mes observations critiques sur son livre sont toujours accompagnées de la citation des endroits qui s'y rapportent.

Bradley n'a également fait mention dans son ouvrage que des vers qui séjournent dans le canal intestinal de l'homme. 11 ne dit rien de noureau, et son livre n'a pas été accueilli avantageusement, même par ses compatriotes. Ses dessins sont en outre en -grande partie inexacts.

Par cette revue, un peu rapide à la vérité, mais complète, on voit donc, ce mé semble, qu'il n'existe pas encore d'ouvrage à l'aide duquel les médecins praticiens puissent connaître tous les vers observés jusqu'a présent dans l'homme, et tous les moyens thérapeutiques propres à combattre chaque espéce de vers en particulier. C'est pour remplir cette lacune, que j’ai travaillé depuis un grand nombre d'années, comme cela pourra malheureusement se voir assez aisément dans plusieurs répétitions qui se sont glissées dans cet ouvrage, et que l'on voudra Jien sans donte me pardonner. 
Il y a à présent. plus de douze ans que $M$. de Schreiber's fut mis à la tête de la dircction du cabinet impérial d'histoire naturelle de Vienne. Il s'était déjà livré depuis long-temps à l'étude de l'helninthologie. Plusieurs objets scientifiques furent cause que je fis sa connaissance particulière, et c'est depuis cette épơque, c'est-à-dire, depuis douze aus, que je m'occupe exclusivement d'helminthologie. La collection de vers intestinaux qui se trouve dans le cabinet impérial de Vienne, et qui n'a pas de pareille dans le monde, fera voir si j’ai travaillé avec quelque succès. Mon collègue, M. Joseph Nattere. et M. son frère Jeau Natterer mont aidé d'abord dans mes recherches sur les vers dans les animaux et dans la formation de cette collection. Le dernier de ces deux savans l'a enrichie considérablement, surtout de vers de poissons de mer trouvés dans les voyages qu'il a faits en Hongrie et le long des côtcs de la mer Adriatique et de la Méditerranée. Cependant j'étais seul chargé de la description, de la classification et de la conservation des vers recueillis. Je puis dire sans exagération quej’ai examinẻ moi-même au moins vingt-cinq mille animaux sous ce point de vue, et cela m'a valu, comme cela peut se concevoir aisément, une grande quantité de découvertes, tant 
sous le rapport de l'histoire vaturelle que sous celui de la médecine pratique. J'aurai bcaucoup d'obligations à ceux qui voudront bien soumettre mon ouvrage à une critique sévère, et je promets d'en profiter à la première occasion.

Je ue parle dans cet ouvrage que des vers qui ont été réellement trouvés dans le corps de l'homme, et que les helminthologues reconnaisseut positivement comme y ayant pris naissance. C'est pour cette raison que j'en compte moitié moins que M. Brera; mais la suite de l'ouvrage prouvera, je l'espère, que j'ai eu raison de n'en pas admettre davantage.

Je suis loin de regarder mes descriptions comme parfaites, mais je puis garantir l'exactitude des dessins; ils ont été faits sous mes yeux, et d'après nature, à l'exception de deux ou trois, qui ne sont que des copies; de ce nombre sont le filaire, copié d'après un dessin de M. Rudolphi, sur la troisième plauche, l'hamulaire lymphatique et le polystome pinguicole, dessinés d'après les figures de Treutler. L'extıémité de la queue du strongle, représentée figure $3 c \mathrm{pl}$. III, n'est aussi qu'une copie. Toutes les figures qui se trouvent neuvième planche, dans le groupe des pseudohelminthes, ne sont que des copies, à l'exception de la fig. $f$, qui est $u n$ dessin original. 
J'ai cité un très-grand nombre d'ouvrages; mais', pour ne pas être obligé de revenir un grand nombre de fois à la citation du même, j’ai cru devoir réunir les titres de ces ouvrages par ordre alphabétique, dans une table particủlière à la fin du traité. A fin que mes citations portent le cachet de la vérité, je me suis fait un devoir de lire mui-même tous les ouvrages mentionnés, à l'exception de quelques-uns marqués par un astérisque, que je n'ai pas pu me procurer.

Tienne, août 1818, 


\section{TABLE}

\section{DES MATIERES.}

\section{PREMIERE PARTIE. - CHAPITRE PREHLER.}

Sur la formation des organisalions vivantes dans les corps organisés.

Diverses opinions sur la formation des vers iutestinaux.........

On ne les trouve ni dans la terre, ni dans l'eau............

Des vers de terre et d'cau ne prennent pas une autre torme dans le corps animal..................................

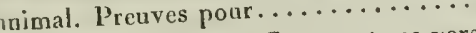

Examen de la question : Comment ees vers peuvent-ils arriver dais le eorps d'autres animaux ? La génération des vers ou deleurs oufs s'opère-t-elle par l'intermédiaire des alimeus ?........... 28

Les vers peuvent bien vivre comme parasites pendant quelque temps

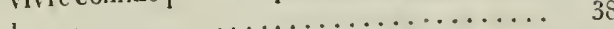

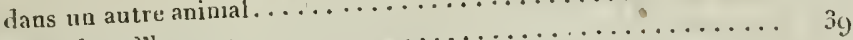

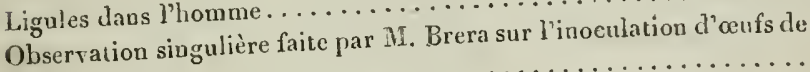
vers ......................

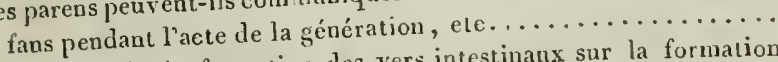
Digression de la formation des vers intestinanx sur la formation

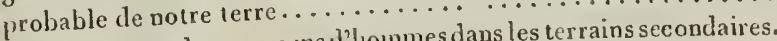
On ne tronve pas des ossemens d'hommes daus les ter rains secondaires. Nous ponvons distinguet trois genres de eorps sur notre tcre.....

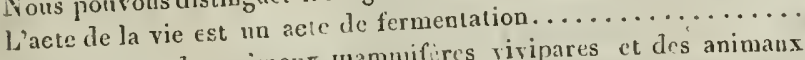
La différence des animanx mammifires vixipares et des animanx ovipares est pius grande qu'ou ne l'a cru ordinairement...... 3

Il parait qu'il a lieu, pou' ainsi clire, chez les vers intestinaux, une répélition de tous les modes de générations i'organisations animales. 109 


\title{
CHAPITIE DEUXIEME.
}

Division systématique des vers iutestinaux en général.

\section{CIIAPITRE TROISIEME.}

\author{
SECTION 1 .
}

Description desvers qui séjournent dans le canal intestinal da l'homıne $\quad 143$

I. Le tricocéphale. ....................... ib.

II. L'oxyure vermiculaire. ................... I4́

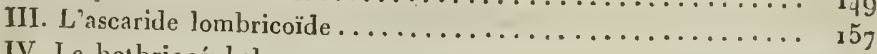

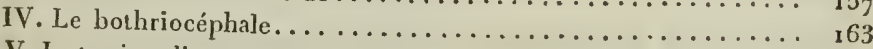

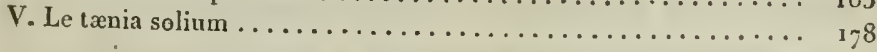

- SECTION Tr.

Description des rers qui séjournent hors du camal intestiual de

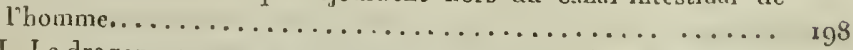

VI. Le dragonnean. ..................... ib.

Nom et histoire de ce ver, et diverses opinious sur sa nature... 199

Description de ce ver........................ 217

Du diagnostic de l'existence du dragonneau dans l'homme ....... 223

Des accidens qui arrirent pendant la durée de la maladie occasionée par la présence du dragonneau, et de son traitement....... 230

VII. Du hamulaire ......................... ${ }_{2} 50$

VIII. Du strongle géant. . . . . . . . . . . . . . . . . ${ }_{2} 53$

Des trématodes. . . . . . . . . . . . . . . . . . . . . . . . ${ }^{253}$

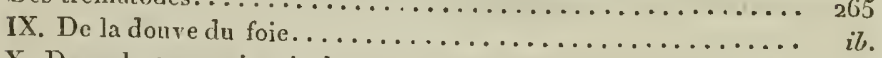

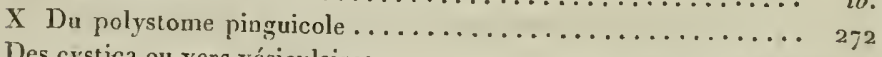

Des cystica ou vers vésiculaires......................... ${ }_{272}^{272}$

XI Dn cysticerque................................ ${ }_{28}^{2} 8$

XII. De I'échinoccoque . . . . . . . . . . . . . . . . . ${ }^{28} 3$

SECTYON III.

Sur les pseudohelminthes $\ldots \ldots \ldots \ldots \ldots \ldots \ldots \ldots \ldots \ldots \ldots \ldots \ldots$

I. Ditrachycère rude de Sulzer...................... ${ }^{319}$

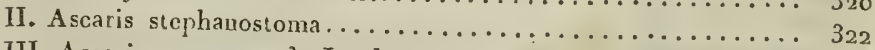

III. Ascaris conosoma de Jordens. .................. 322

IV. Cercosoma de Brera......................

V. Hexaı hyridium venarum de Tentler................ 325

VI. Diacau thos polycephaius de Stiebel................. 330

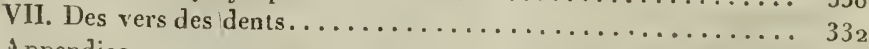

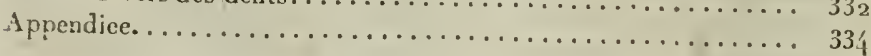




\section{TABLE DES MATIËRES.}

\section{SECONDE PARTIE - CHAPITRE QUATRIEME.}

Des causes de la formation des vers dans le canal intestinal de

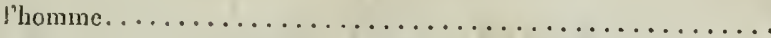

\section{CHAPITRE CINQUIEME.}

Un diagnostic de ja présence des vers dans le canal intestiual, et dies dérangemens qu'ils peuvent occasioner........... 35 f́ Symptòmés gétéraux....................... $i b$.

Quelques cas où on avait présumé que les rers avaient été la cause

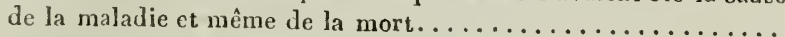
Ils causent divers embarras dans l'cstomac............ 384

Ou a tort de croire qu' ils perforent les intestins........... 385

On les accuse d'être une des causes de l'étranglement d'hernics... 394

\section{CIIAPIPE SIXIEMIE.}

Du traitement hygiévique et thérapeutique contre les vers intestinaux............................. 400

\section{SECTION I.}

Des remèdes en général...................... ib

Paison probable pourquoi nous en arons tant............ ib.

Expériences faites avec des remides hors du canal intestinal. ... . 404

I. Des remèdes qui agissent sur les vers d'une manière mécanique.. 407

II. Des remèdes qui agissent d'une manière spécifique contre les vers intestinaux......................... 4 ro

Remèdes à employer extérieurement. .............. 43 r

III. Des remèdes purgatifs..................... $\ldots \ldots \ldots 433$

\section{SECTION II.}

Du traitement particulier qu'il faut cmployer contre chaque espèce de vers............................ 444

Traitement particulier contre le tricocéphale............ ib.

- contre l'oxyure vermiculaire. ................ 445

- contre les ascarides lombricoïles............... $45 x$

- contre les cestoïdes. ....................... 454

SECTION III.

Des différentes méthodes de traitement contre le tæuia...... 455 SECTION IV.

Méthode de traitement de l'auteur................ 486

A ppendice par M. de Blainville..................

Table d'auteurs par ordre alphabétique............... 55 . 


\section{TRAITE}

S UR

\section{LES VERS INTESTINAUX \\ DE L'HOMME.}

\section{PREMIÈRE P A RTIE.}

\section{CHAPITRE PREMIER.}

Sur la formation des organisations vivantes dans les corps organisés.

Dans les infusions de substances tant végétales qu'animales, il se forme, après un certain temps, des animaux vivans, mais qui ne peuvent être aperçus qu'au moyen du microscope. Personne ne doute de ce fait, seulement on n'est pas d'accord sur le mode de form ationde ces êtres; en effet on ne sait pas cncore si ces animaux sont sortis d'oufs, qui auraient pu se trouver dans la substance soumise à l'infusion, dans l'eau ou bien dans l'air environnant, ou enfin s'ils sout un produit de la décomposition et de la fermentation de la substance infusée. On n'est pas non plus 
d'accord si chaque corps organisé vivant doit être nécessairement produit par d'autres corps organisés semblables à lui sous tous les rapports, ou bien si quelquesuns, dans des circonstances favorables, ne peuvent pas se former spontanément. Les naturalistes appellent cette dernière formation generatio spontanea ou aquivoca; je crois que l'on devrait l'appeler plutôt formatio primitiva; aussi je me servirai toujours de cette dernière dénomination.

Beaucoup de nos naturalistes modernes n'ont pas seulement regardé la matière verte de Priestley, les moisissures, les tremelles, les champignons, les nostocs, les infusoires, les animalcules spernatiques, etc., comme des organisations primitives, mais ils ont anssi considéré comme telles, les poux, les cirons et les vers intestinaux.

Comme la plupart de ces derniers se distinguent trop claircment par la grosseur, par un canal intestinal souvent complet, par des organes sexuels séparés, nême sur des individus différens, par une structure musculaire, et même par des nerfs, comme M. Otto l'a dernièrement démontré, de tous les animalcules microscopiques et de ces corps organisés encore si douteux, que quelques auteurs les rangent parmi les végétaux, il ne sera pas saus intérêt de s'occuper de l'origine des vers intestinaux, et d'examiner les différentes opinions émises sur ce sujet.

En examinant la question rigoureusement, il est évident qu'il ne peut y avoir que deux origines pour les vers daus le corps de l'homme et dans 
celui des animaux. Dans l'une ils proviennent du dehors, et dans l'autre leur source est dans le corps animal lui-nême, où ils se forment spontanémeut. Dans le premier cas, ils parviennent daus le corps animal ou à l'état de vers, ou à l'état d'œufs, n'inporte que la mère qui leur a donné naissance soit androgyne, ou qu'elle ait cu besoin, pour être fécondée, de l'action d'un individu mâle, et encore plus d'où elle provient elle-même.

Dans le second cas, ces ver's, ou au moins ceux qui se trouvent pour la prenière fois dans un individu, doivent être nécessairement regardés comme des animaux sans parens, comme une production spontanée de la substance vivante (de la matière organique) qui, partout et de toute éternité, tend à former des êtres distincts et complets en eux-mêmes, ou, en d'autres termes, les vers doivent leur existence à une formation primitive. Cependant, comme cette dernière supposition déplaî singulièrement à quelques médecins et natuialistes, qui la regardent presque comme un blasphème, l'ou s'est efforcé, pour n'être pas obligé d'adopter cette opinion, de découvrir d'autres manières par le moyen desquelles les vers pourraient arriver dans le corps animal. Nous allons examiner successivement les diverses opinions à ce sujet.

Les auteurs qui veulent rendre la chose plus facile à concevoir, à ce qu'il leur semble, supposent que les vers intestinaux proviennent des vers de terre ou d'cau, et qu'ils ont pu s'introduire dans le corps ani- 
mal avec les alimens, soit à l'état de vers, soit à celui de germe ou d'œuf.

Les défenseurs de cette hypothèse s'appuient sur la prétendue observation, que les vers iutestinaux de l'homme et ceux des animaux se trouvent également dans l'eau ou dans la terre; mais nous allons voï que cette hypothèse est basée sur des faits mal observés.

Linné a cru à tort, avoir trouvé la douve du foie (distoma hepaticum), le tæuia large (tania lata) et l'ascaride vermiculaire (ascaris vermicularis) dans des marais ou dans des racines de plantes pourries; car Otto-Frédéric Müller ${ }^{x}$ a prouvé, jusqu’à l'évidence, que cet auteur s'était trompé, qu'il a encore confondu le bothriocephalus solidus (Rud.) tantôt avec la douve du foie, tantôt arec le tænia large (tania lata), et qu'il confond également ce dernier avec le tænia du cheval. Il parât également qu'il a regardé à tort un ver qui vit dans les marais, comme un ascaride vermiculaire (ascaris vermicularis)'. Linné, homme du plus grand mérite, ne peut cependant être considéré en aucune manière comme juge compétent dans les discussions sur l'helminthologie. 11 a vu et examiné trop peu de vers intestinaux, car sans cela il n'aurait pas nié dans les tænias la présence de la tête, que l'on peut voir avec tant de facilité sur la grande espèce du cheval.

Gadd a commis à peu près la même erreur que

N Naturforscher, st. 18, s. $2 \mathrm{I}-33_{7}$.

- Verm. terr., vol. I, part. 2, p. 36. 
Linné; voici comme il s'exprime : "Le même tænia que Linué a rencontré pendant son voyage en Laponie (1734), dans une source ferrugineuse, a été trouvé par moi, en 1747 , dans un endroit semblable. Ce tænia était articulé, plane, et pourvu de deux ouvertures latérales (tonia articulata, plana, osculis lateralibus geminis), et par conséquent doit être regardé comme le même qui séjourne dans l'homme. " L'cn voit, par la définition que Gadd a donnée de ee ver, et par la conclusion qu'il en a tirée, avec combien peu d'attention il l'a examiné. Du reste, Gadd lui-même n'a pas la prétention d'être helminthologue.

Unzer regarde comme une chose prouvée, que les lombrics ou vers de terre, et les asearides sont les mèmes animaux, et que la couleur différente de ces derniers (comme si toute leur différence ne consistait qu'en cela) provient du lait qu'ils rencontrent et mangent souvent daus le canal intestinal de l'homme. Cet auteur a également sontenu avoir recueilli dans une fontaine des moreeaux de tænia; mais Outo-Frédéric Müller lui ayant demandé quelques délails plus circonstanciés sur ce fait, Unzer répondit: "Il se pourrait bien que ce seul échantillon eût été déposé ou par un poisson ou par un hormexe."

\section{Transeat arm ceteris.}

Tissol communique, dans une lettre à Zimmermann, l'histoire de la maladie d'un jeune garçon,

${ }^{2}$ Gexe. A. G. D. Eingers, p. 15. 
dans laquelle il est encorc question d'un ver intestinal analogue à un ver extérieur trouvé daus une fontaine. Voici ses propres paroles: "Mane in lecto cum levi ani pruritu dejicit simul et teretem et nascentem toniam, filum nimirum crassum, album aequabile, viginti quinque circiter pollices longum, quatuor ant quinque circumvolutum giris, iisque omniǹ similem, quas in fontibus Suecia invenit Ill. Limnceus et in fonte Helvetico amicus medicus. "Par ces détails, on ne peut pas savoir au juste quel ver Tissot a vu, encore moins à quelle espèce de ver trouvéc par Linné il ressemble, mais encore beaucoup moins quelle forme avaient les vers que son ami a observés, et comme les connaissances de ce dernier sous le rapport de l'helminthologie nous sont tout à fait inconnues, son observation ne peut pas trouver place parmi les preuves qu'il existe daus l'eau des ver's analogues aux véritables entozoaires.

Beireis racontc aussi qu'il a découvert, daus la fontaine de Eudger, près Helmstædt, et dans une fontaine près Ballenstædt, l'ascaride lombricoïde de l'homme. Cependant il résulte de l'ensemble de sa description, que ce ver, malgré les trois petits bontons et le suçoir de la tćte, u'était autre chose que le même animal qu'Otto-Frédéric Müller ${ }^{x}$ a décrit, et que l'ou voil souvent daus l'ean. Le ver décrit par Beireis était blanc comme de la neige, il n'avait que trois ou quatre lignes de longueur, et de-

IVerm. terr. hist., rol. I, part. I, p. 36. 
vait parvenir daus les intestins de l'homme (lieu, sclon cet auteur, le plus favorable pour la nourriture et pour la propagation des vers), à une grosseur pareille à celle de l'ascaride lombricoïde. Beireis ne dit rien de la structure interne du ver, non plus que de la conformation de la fin de la queue, etc. : c'est ce que Gœze a remarqué comme nous. L'expérience nous démontre cependant, que chaque animal se trouve le plus convenablement dans les endroits que la nature lui a assignés. Les animaux du sud ne supportent pas le climat froid du nord; les rennes, par exemple, bés en Laponie, ne peuvent vivre en Allemague, pas même dans la partie septeutrionale, et encore moins dans l'Italie. L'on ne conçoit pas, par celte raison, comment unver, destiné d'ailleurs à vivre dans l'eau froide (Beireis en a trouvé une fois un au mois de décembre), pourrait supporter aussi bien, en arrivant dans le corps animal, des changemens si subits et si brusques sous le rapport de la température du milieu et de la nourriture, de manière que ces changemens opéreraient encore en lui un développement assez considérable pour le faire parvenir à la grandeur de l'ascaride lombricoïde. Cepeudaut nous observons que le contraire arrive chez tous les autres animaux, quand ils se trouvent soumis à des circonstances à peu près semblables. Beireis a avancé par conséquent une pure hypothèse, en disant que les lombricö̈des et lcs vers 'qu'il a rencontrés dans l'eau sont les mèmes, excepté que les prèmiers ve sont qu'un peu plus développés. 
Gmelin ' donne la description de tænias qu’il a observés dans une eau stagnante, et il les désigne avec raison sous le nom de tania dubia; car il est certain que ces txnias douteux n'étaient autre chose que du frai de crapauds et non pas de grenouilles, comme Pallas ${ }^{2}$ l'a soutenu. Cela résulte, non-seulement de la description, mais se confirme encore davantage par la figure, si on la compare avec la planche $\mathrm{xx}$ de Røesel.

L'on compte également Leeuwenhoeck parmi les personnes qui ont trouvé, dit-on, des vers intestinaux. hors du corps animal. Cependant son mémoire, qui a donné lieu à cette assertion, nous apprend uniquement que l'on observe des vers dans le foie des moutons et des bœufs, et qu'il y a aussi de très-petits ver's dans la terre, deux choses dont persoune, jusqu'à présent, n'a douté. Du réste, il n'est pas même question, dans ce mémoire, d'une comparaison entre ces deux espèces de vers, et encore moins que cet auteur ait regardé les vers de terre comme identiques avec les douves du foie.

Schæffer ${ }^{3}$ a également prétendu avoir vu des douves du foic dans l'eau; cependant il est très-probable qu'il s'est trompé, car Otto-Frédéric Müller ni aucun autre naturaliste n'ont pu y en découvrir. Supposé même que Sclıæffer ait-trouvẻ des douves

$=$ Reisen 3ter Theil., s. 3o2, tab. 3 o.

- Nordische beitr., 1, s. 42 .

${ }^{3}$ Die Egelschnecken, s. 29. 
du foie dans l'eau, il nous reste toujours à soupçonner que ces vers ont pu y avoir été déposés par des brebis qui paissaient près de là.

Hahn, dans une lettre à Pallas ${ }^{2}$ sur une épizootic qui avait régné tout le long de la rivière $\mathrm{Ob}$, en Russie, croit qu'elle pouvait être attribuée à ce que les petites rivières et eaux stagnantes de ce pays étaient, à cette époque, remplies d'une quantité considérable de filaires (gordius aquaticus). 11 pense que les bœufs et les chevaux- qui furent atteints de cette maladie les avaient avalés en buvant, et que ces vers s'étaient frayé une route à travers les parois de l'estomac, pour arriver dans les poumons et dans le foie, où on les a trouvés. Il fait observer que ceux de ces animaux auxquels on a donné à temps des sels et des vermifugès furent sauvés.

A juger d'après ce récit, il paraît que l'on n'a pas du tout rencontré de vers dans l'estomac des animaux atteints de l'épizootie. Quant à moi, il me semble plus probable que ces vers se sont engendlés dans leurs poumons, comme cela a lieu très-souvent chez les moutons, et qu'ensuite ils les ont communiqués aux eaux dans lesquelles ils s'abreuvaient, par l'expectoration; car Pallas dit lui-même, comme nous le verrons plus tard, quand il sera question du filaire de Médine (filaria Medinensis), qu'il a observé très-fréquemment le gordius aquaticus dans l'eau de quelques contrées, sans avoir entendu dire

Nord. bcitr., st. I, p. 160. 
qu'on l'aic jamais rencontré ni dans l'homme ni dans les animaux ${ }^{x}$.

Voilà, à ce que je crois, tous les exemples de vers intestinaux regardés comme ayant pris naissance dans la terre et dans l'eau, séparés du corps animal, ou, en d'autres termes, ce sont tous les faits venus à ma connaissance qui sont adoptés comme preuves, que les vers intestinaux doivent être consićérés comme semblables, sous tous les rapports, aux vers que l'ou trouve daans la terre et dans l'eau. L'on voit aisément quel peu de cas on doit faire de pareilles preuves; car on peut supposer que les vers trouvés dans ces circonstances n'étaient'pas les mêmes que cenx qu'on observe dans l'homme et dans les animaux; ou bien, cela fùt-il vrai, il resterait encore à supposer que ce pouvait être ou des hommes ou des animaux qui les avaient rendus. En dernière analyse, si l'on réfléchit que chaque espèce d'animal se rencontre plus fréquemment dans le lieu que la nature lui a assigné que partout ailleurs, cela doit faire naître en nous encore plus de doute sur les observations mentionnées ci-dessus; car si les vers que l'on découvre assez souveut dans l'homme et dans les animaux séjournaient originairement dans l'eau ou dans la terre, l'on devrait lcs reneontrer encore beaucoup plus

- Le docteur Karl a également tronvé en Moravie, dans les poumons du beeuf, une espèce de strongle (stronzylus filaria), que je n'ai rencontrée que dans les moutons, les antilopes el les chamois. (Br.) 
fréquemment dans ces derniers lieux, chose qui n'est certainement pas.

Quoique l'on ne puisse pas aisément combattre l'exactitude de cette conclusion, et que l'on ve prisse pas non plus prouver la présence de vers intestinaux hors du corps animal, les défenseurs de cette théoric, pour n'être pas obligés de l'ahandonner tout à fait, ont cherché à la défendre par une autre hypothèse; ils prétendent que les vers de terre et d'eau ne prennent la forme spécifique des vers intestinaux qu'en arrivant dans le corps animal. D'après cette assertion, le changement de séjour peut próduire un changement de conformation. M. Brera est surtout très-porté pour cetie opinion; et, pour lui donner plus de poids, il dit que plusieurs plantes changent peu à peu leur forme originelle, quand elles se trouvent soumises à l'influence d'une culture différente et d'un climat étranger. Il cite, entre autres choses, un exemple ${ }^{x}$ de grains d'avoine, qu'un soldat avait avalés pendant l'hiver, et qui, pendant l'été suivant, commencèreut à germer dans son estomac, à pousser des racines et même des tiges, non pas cependant de la même manière que cela a lieu Jorsque ces semences sont mises dans la terre; observation qui me paraît la seule vérité de toute cette citation.

Cependant M. Brera ne semble pas avoir réfléchi que le changement de forme parmi les plantes n'a pas lieu subitenent, et que cela n'arrive que très-lente-

- Memorie, p. 420. 
nient et après plusieurs générations. Si l'on voulait même supposer que les vers eux-mêmes ne s'introduisent pas daus le corps animal, et que ce ne sont que leurs germes, ou plutôt leurs ocufs fécondés qui y arrivent, l'analogie seule ne nous met cependant pas en droit de croire que les petits qui écloraient des œufs prendraient une forme différente de celle de lcurs parens, car la forme spécifique leur a élé donnée par l'acte de la génératiou, et elle ne se laisse plus changer par les circonstances au moyen desquelles ecs jeunes vers reçoivent leur développement complet. D'une semence placée dans une terre ou sous une zone quelconque, il sortira (supposéqu'elle ne périsse pas) la même plante dont elle provient, n'importe que cette plante croisse originairement au Cap de Bonne-Espérance ou bien au pôle du nord, seulement avec la différence qu'elle se développera mieux daus sa mère-patrie. Un œuf de coucou, par exemple, produira un animal semblable, n'importe par quel oiseau il aura été couvé. A riuste dit, par cette raison, très-à-propos :

Da vacca nas:er cerva non vedesti,

No nuai colomba d'aquilas.

Cependan les naturalistes au-delà des Alpes ne paraissent pas vouloir ajouter foi aux vers de leur compatriote; car Gautieri ${ }^{2}$ par exemple, ne doute

- Ces vers sont également cités dans l'ouvagge de Vallisnieri.

= Ouvrage cité , p. 81. 
nullement que les cestoïdes, les échinorhynques, les nématoïdes et les hydatides puissent se développer des mêmes germes.

On me fera peut-être l'objection suivante : quelques animaux subissent, avec le temps, une métamorphose, quoique incomplète, dans laquelle ils passent à l'état de chrysalide, comme cela arrive chez les insectes. Ils éprouvent alors un changement si considérable dans leur structure extérieure, que l'on ne reconnait plus leur forme primitive dans l'animal complétement développé. Cela se voit, par exemple, cliez les crapauds et les grenouilles. Ne pourrait-on pas éça'ement supposer qu'un semblable changement lent dans la structure extérieure et intérieure pût être accéléré ou retardé par quelque différence dans le séjour, la température et la nourriture? Ne pourrait-on pas encore admettre que ces mênes circonstances pussent donner à la structure un tout autre mode de conformation, de manière qu'il en résultât un changement tel, qu'un ver, par exemple, qui aurait pris naissance dans son élément naturel, c'est-à-dire dans l'eau ou la terre, et qui se serait développé par la suite dans le corps animal, ne se ressemblerait aucunement dans ces deux états.

Je répouds à ceła : les vers en général, et les vers intestinaux en particulier n'éprouvent jamais un pareil changement de forme. Il y a cependant quelques exceptions, dont je parlcrai plus tard; mais dans ce cas nous pouvons clairement voir la transition d'une forme à une autre. On a examiné dans le cabinet 
d'histoire naturelle de Vienne cinquante mille animaux au moins, dans le but de découvrir des vers intestinaux. Cenx que l'on a trouvés, et dont le nombre n'est pas peu cousidérable, ont tous passé plusieurs fois par mes mains; mais je n'ai jamais été embarrassé, en voyant un ver vivant, pour dire surle-champ, si c'était un ver intestinal, ou bien un ver de terre ou d'eau.

Tous les vers vivans que l'ou a trouvés dans les animaux examinés portaient les caractères spécifiques des vers intestinaux. On a bien remarqué quelquefois d'autres vers dans l'estomac de ceux qui s'en nourrissen, mais ces vers étaient morts et presque toujours à moitié digrérés, cornme par exemple des larves d'insectes. Dans un nombre de recherches aussi considérable, il aurait dû arriver quelquefois que l'on eût rencontré des vers vivans provenant de la terre ou de l'eau, et que l'on eût observé la transition graduelle de leur forme en celle des vers intestinaux; mais pareille chose n'a jamais eu licu.

Je suppose, sans vouloir cependant l'affirmer, que la structure des nématoïdes et des trématodes ait pu donner lieu à une illusion, à cause de la légère ressemblance qui existe peut-être entre ceux-ci et certains vers de terre et d'ean, de manièré que l'on se serait cru en droit de regarder de véritables descendans de ces derniers comme, nés dans le corps animal. Mais, je le demande, où trouvons-nous hors de lui des ver's que nous puissions regarder comme parens primitifs des échinorhyuques, par excmple. Renier a cepen- 
dant compris parmi les vers intestinaux (Vermi, Tab. vı) un échinorhynque (echinorinco scudato) provenant de la mer Adriatique; mais sitôt que cet auteur eut vu chez moi de véritables échinorhynques, il fit sur-le-champ, du ver qu'il avait observé, un genre particulier. Je demande ensuite que l'on me désigne, parmi les vers de terre on d'eau, ceux que l'on peut regarder comme la souche de l'ordre, si riche en espèces et en genres, des cestoïdes; que l'on m'indique enfin un ver hors du corps animal, ou bien un être quelconque vivant, duquel on pourrait dériver l'origine des hydatides, qui ne se tronvent pas même, à ce que l'on peut juger jusqu’à présent, dans tous les genres d'animaux, et qui vivent seulement dans le corps des mammifères, et principalement dans les animaux rongeurs et ruminans, animaux qui broient leur nourriture très-soigneusement. Si l'on voulait par hasard regarder comme tel quelque animal du genre medusa de Linnæus, je prie que l'on m'indique par quel chemin il aurait pu arriver de la mer Adriatique dans le foie d'une souris domestique de Vienne, ou dans le mésentère d'un chamois qui vit dans les Alpes de la Styrie.

Je crois avoir démontré par cête discussion d'une manière suffisante que les vers intestinaux ne peuvent pas être des descendans de vers, qui vivent originairement dans l'eau ou dans la terre, d'où il résulte par conséquent waturellenent, que l'on doit en former un ordre particulier, comme d'êtres qui peuvent seulement exister dans le corps de l'hom me et 
dans celui des animaux. Cependant cette couclusion ne s'appuie pas seulement sur la preuve négative rapportée ci-dessus, mais elle est encore confirmée davantage, je pourrais même dire qu'elle se trouve élevée jusqu'à la certitude mathématique par les considérations suivantes.

$x^{\circ}$. Les vers qui vivent dans le corps de l'homme et dans celui des animaux en général, ont une structure toute particulière par laquelle ils se distinguent clairement de ceux qui séjournent dans l'eau ou bien dans la terre. Aussi, de même qu'un botaniste habile distinguera sur-le-champ, par la conformation extérieure, une plante aquatique de celle qui croît sur les Alpes, aussi facilement l'helminthologue exercé décidera si tel ou tel ver doit être compté parmi les vers intestinaux ou non.

Il y a quelques années que l'on m'apporta de trèspetits vers que l'on prétendait provenir d'un animal dont je ne me rappelle plus le nom. Je doutai sur.lechamp qu'ils fusseni des vers intestinaux, et je me proposai de les examiner pendant qu'ils vivaient encore. Le leudemain on m'en apporta de pareils, que l'on avait encore trouvés dans les intestins de deux différens animaux. Il résulta, de l'examen particulier que j'en fis, que c'étaient de petites sangsues (7hirudo) que l'on avait recueillies dans l'eau dans laquelle on avait lavé ces intestins.

Une autre fois, on me remit deux petits vers contenus dans un vase rempli d'eau, sans me dire d'où ils provenaient; je déclarai également, sáns hésita- 
tion, que ce n'étaient pas des vers intestinaux, en effet c'étaient des planaires que l'on avait recueillies dans l'eau, et ces dernières ont cependant quelque ressemblance avec les trématodes, qui séjournent dans le ccrps des animaux. Nous avons déjà remarqué que l'on ne trouve aucun animal ni dans l'eau, ni dans la terre, qui ressemble, quant à la conformation, aux échinorhynques, aux cestoïdes ni aux hydatides. Chaque famille de vers intestinaux a même tant de signes caractéristiques qui lui sont particuliers, que le naturaliste exercé n'hésite pas long-temps dans laquelle il doitranger tel ou tel ver qui lui est présenté.

$2^{\circ}$. Quelques animaux ont des vers intestinaux qui leur sont propres, et que l'on ne rencontre pas dans d'autres. Je ne veux cependant pas admettre pour cela, comme cela a été fait à tort par quelques personnes, que ehaque espèce d'animaux ait des espèces de vers particulières, et que l'on ne puisse découvrir dans d'autres, car l'expérience nous fournit plusieurs exemples qui prouvent le contraire. L'ascaride (ascaris lombricoïdes), par exemple, qui séjourne dans les intestins grêles de l'homme, ne se distingue en rien des ascarides que l'on voit souvent dans le canal intestinal des cochons, des bœufs et des chevaux. Les douves du foie, que l'on a trouvées jusqu'à présent dans le corps de plusieurs mammifères, c'est-à-dire dans celui de l'homme, des lièvres, des bœufs, des chameaux, des cerfs, des chevaux el des cochons, appartiennent toutes à la même espèce. M. Rudolphi a vu dans le corps d'un lion né à Lon- 
dres, une quantité extraordinaire d'ascarides, et moi-même j’ai rencontré dans un individu de cette espèce né à Tunis, de pareils vers. Mais le bothriocépliale et le tania propres à l'homme se distinguent clairement de tous lesautres bothriocéphales et rænias propres aux animaux; il en est de même de plusieurs autres vers qui séjournent dans le corps des différens aninsaux.

3॰. On trouve des vers dans toutes les parties du corps animal : on en rencontre dans le canal intestinal d'un bout à l'autre, dans le tissu cellulaire, entre la peau et les muscles, dans le foie, dans la vésicule du fiel, dans la trachée-artère, dans les poumons, dans le cerveau, dans les reins, dans le cour, dans la rate, dans la vésicule aérienne des poissons, dans la vessie abdominale des reptiles et même dans des parties désorganisées du corps animal. Treuller a observé le poly stome pinguicole dans un steatôme des ovaires d'une femme, et le strongle armé dans des anévrýsmes des artères du mésentère des chevaux.

Hopkinson et Morgan ont rencontré des filaires vivans (filaria papillosa) dans la cliambre antérieure de l'oil des chevaux.

Plusieurs médecias et moi, nous avons vu également de pareils vers se nouvoir dans la chambre antérieure de l'œil d'un cheval qui se trouvait en 1813 à l'école vétérinaire de Vienne.

M. Jean Natterer a observé en Italie, eu 1815, deux fois chez la mouelte brune (larus fuscus) et trois fois chez la mouette glauque (larus glaucus) des dis- 
tomes de la longueur de trois ou quatre lignes et de la largeur de plus d'une ligue sous la troisième paupière. Il y avait trente-un de ces vers dans les yeux d'un de ces oiseaux. Je possède plusieurs nématoïdes de la longueur d'un pouce, qui avaient séjourné sous la troisième paupière et dans le conduit auditif du faucon bleu (falco naevius), d'une part, et de l'autre dans le sinus maxillaire (antrum Highmori) du faucon noir (falco ater) et de l'échasse (charadrius himantopus). On observe souvent, dans la cavité du tympan du marsouin (delphinus phocana), des strongles. J'ai découvert deux fois des cysticerques ténuicolles (cysticercus tenuicollis, Rud.) dans le cœur de deux bœufs, et j’ai conservé un morceau de l'un de ces cours, sur lequel on peut voir la capsule dans laquelle a séjourné un de cés vers. La rate n'est pas non plus exempte de vers; exemple les amphistomes ( amphistoma) que Caldani, en premier lieu, et moi, plus tard, avons observés dans cet organe. Ces derniers animaux, enfermés dans des capsules particulières, se trouvent presque toujours dans toutes les parties internes de la grenouille verte (rana esculenta, L.), et sont implantés à la superficie de la rate, ainsi que sur celle des autres viscères. M. Lüdersen a également découvert des hydatides dans la substance de la rate. M. Rudolphi croyait que ces dernières ne devaient pas être regardées comme de véritables hydatides; cependant je démontrerai, quand il sera question de ce genre de vers, qu'elles doivent l'être comme telles. 
$4^{\circ}$. Certains genres et espèces de vers ne se trouvent jamais que dans les mêmes parties et dans les mêmes organes du corps animal. Les douves du foie, par exemple, ne s'observent que dans le foie et la vésicule du fiel des mammifères. Si elles provenaient de vers qui vivent dans l'eau, et qu'elles eussent été introduites dans le corps de ces animaux avec les boissons, on devrait plutôt les rencontrer dans l'estomac ou dans le reste du canal intestinal qu'ailleurs, par la raison que ces vers trouveraient dans ces endroits de l'eau, c'est-à-dire leur nourriture accoutumée, tandis qu'ils ne rencontrent rien d'analogue dans la vésicule du fiel.

Le polystome (polystoma integerrimum) séjourne uniquement dans la vessie abdominale des crapauds et des grenouilles, et l'ascaride à veines noires (ascaris nigrovenosa) dans les poumons de ces animaux.

Le conure cérébral (coenurus cerebralis) s'observe seulement dans le cerveau des moutons atteints du tournis, et jamais dans le foie, où séjournent cependant bien souvent des hydatides, mais qui appartiennent à un autre genre. On voit souvent dans la vessie aérienne des truites (salmo fario) des milliers de vers de la même espèce, et dont on ne rencontre aucun individu ni dans le canal intestinal ni dans aucun autre viscère de ces poissons : j'en ai cependant examiné huit cent cinquante-huit.

Le canal intestinal, qui n'offre aux vers intestinaux aucun obstacle mécanique à un changement de demeure, a néanmoins des endroits déterminés où nous 
trouvons seulement tel ou tel ver. Le strongle (strongylus horridus ou papillosus, Rud.) se trouve seulement dans l'œsophage ou l'estomac de plusieurs oiseaux aquatiques; l'ascaride obtus (ascaris obtusa, R.) uniquement dans l'estomac des souris ; le distome ténuicolle (distoma tenuicolle, Z.), dans celui de quelques poissons voraces; dans tout le reste du canal alimentaire de ces derniers, on ne rencontre pas un seul de ces vers. Les ascarides séjournent presqu'exclusivement dans l'estomac et les intestins grêles, ę non dans d'autres organes, comme quelques naturalistes l'ont prétendu. Les trichocéphales et les oxyures se trouvent dans les gros intestins, les premiers bornés même à l'intestin cœcum, et l'amphistome en massuc (amphistoma subclavatum) n'existe que dans le rectum.

Si ces vers séjournaient originellement dans la terre ou dans l'eau, et s'ils pouvaient quitter leur demeure et leur nourriture ordinaires sans préjudice de leur bien-être, pour se rendre dans un lieu tout à fait étranger, l'on ne conçoit pas pourquoi un ver, que l'on ne rencontre que daus le rectum, supposé que ce ver se füt introduit dans le corps animal avec les alimens, n'aurait pas choisi aussi bien un autre endroit que le rectum, dans lequel il aurait vécu; on ne conçoit pas non plus qu'est-ce qui aurait pu l'empêcher de prendre quelquefois une direction latérale, conme le fait la douve du foie dans les canaux hépatiques; on comprend encore moins pourquoi on ne l'aurait jamais rencontré, pendant qu'il voyagerait 
de la bouche à l'anus, que justement dans un seul endroit, c'est-à-dire dans le rectum.

Dans le cas où des vers s'introduiraient réellement du dehors dans le canal alimentaire, et qu'ils y vivraient comme parasites ", ils seraient arrêtés par la suite dans leur trajet, comme nous aurons occasion plus tard d'en douner un exemple.

$5^{\circ}$. Tous les vers intestinaux ne se conservent pas seulement dans le corps animal, mais ils y multiplient; ils meurent au contraire très-vite, quand ils sont forcés de le quitter. C'est là une des plus fortes preuves en faveur de l'opinion que les vers intestinaux sont propres au corps animal, et qu'ils lui appartiennent exclusivement. Si ces vers n'étaient pas une production particulière du corps animal, si ce dernier n'était pas leur demeure naturelle, ils y mourraient aussi bien que les vers de terre et d'eau, au moins ils ne s'y multiplieraient pas. 11 y a cependant quelques larves d'insectes qui se conservent dans le corps animal, et on les y trouve sous la peau, dans les fosses nasales, dans l'estomac, dans le rectum, elc. Mais nous savons très-bien que les œuls dont elles proviennent ont été déposés dans ces endroits par des insectes, afin que la larve aussitôt sortie de l'œuf, y trouvât une nourriture convenable.

Rigoureusement parlant, on devrait regarder tous les vers intestinaux comme parasites. Je me sers ici seulement du mot parasite pour indiquer les vers qu'un animal a gagnés en en mangeant un autre. 
Du reste, le séjour de pareilles larves d'insectes dans le corps animal ne se prolonge que pendant un temps déterminé, c'est-à-dire jusqu'à l'époque où elles doivent, bientôt après, se changer en chrysalide; sitôt que cette époque est arrivée, elles quittent l'animal aux dépens duquel elles s'écaient nourries pendant tout ce temps, et parviennent au dehors à l'état d'insecte parfait ${ }^{3}$. Ce dernier, par conséquent, continue à vivre, quoique sous une forme différente, hors du corps animal, ce qui causerait, au contraire, la mort d'un ver intestinal.

Schæffer, qui a prétendu, comme nous l'avons remarqué plus haut, avoir rencontré des douves du foie dans l'eau, n'a pas manqué d'observer que l'on pourrait, à l'aide de raisonnemens semblables à ceux que nous venons de faire tout à l'heure, mettre en doute l'identité des vers trouvés dans l'eau et de ceux qui séjournent dans le foie des mammifères. Cet auteur a tàché d'expliquer la mort subite de ces douves quand on les ôte de leur séjour naturel, en disant que ces animaux étaient accoutumés, depuis trois ou quatre générations, à la température élevéc du corps animal, de manière qu'ils ne peuvent descendre à celle de l'eau froide sans perdre la vic. Mais, à juger d'après une analogie raisonnable, on devrait admettre également que des vers qui auraient vécu auparavant pendant ccnt ou mille générations dans une eau froide pourraient supporter encore bien moins un clianges

- Voyez l'ouvrage do Clark sur les oestres. 
ment aussi subit que celui qu'ils éprouveraient en passant dans une température aussi élevée que celle $\mathrm{du}$ corps des mammifères. Les vers intestinaux meurent riéanmoins quand on les retire de leur séjour habituel, même en les exposant à une température semblable à celle du corps animal dans lequel ils avaient vécu. La mort est seulement retardée, parce qu'une influence nuisible, c'est-à-dire le changement de température, agit de moins sur eux.

Nous devons en outre considérer que chaque animal revient beaucoup plus aisément à son état primitif et naturel, qu'il ne s'accoutume, après en avoir été tiré, à un autre état d'existence qui lui est étranger. Nous serions donc obligés de regarder les vers intestinaux, supposé même qu'ils séjournent origivairement dans l'eau ou dans la terre, comme des animaux qui se trouvent, dans le corps animal, dans un état d'existence contraire à leur nature. Ne devrait-on pas conclure, par analogie, que les vers intestinaux rendus à la terre ou à l'eau, desquelles ils sont provenus, dans la supposition que nous combattons, pourraient revenir ou se réaccoutumer à leur ancien état primitif d'existence? L'expérience prouve, comme nous l'avons démontré, justement le contraire. Voilà par conséquent une nouvelle preuve contre l'idée que les vers intestinaux tirent leur origine des vers de terre et d'eau, ce qui confirme en même temps leur nature particulière.

$6^{\circ}$. Il existe souvent une grande quantité de vers intestinaux dans le corps de l'homme ou dans celui 
d'autres apimaux, sans qu'il en résulte la moindre gêne ou le plus petit changement dans leur santé. J'ai connu plusieurs personues dans le corps desquelles séjournaient des tænias, vers regardés par beaucoup de médecins comme extrêmement dangereux pour la santé, sans qu'elles en fussent incommodées le moins du monde. Ces mêmes personnes ne se seraient jamais doutées d'avoir le tænia, si elles n'en avaient pas remarqué par hasard quelques morceaux dans leurs selles.

Le cabinet d'histoire naturelle de Vienne possède plusieurs morceaux d'intestins sur lesquels se trouvent implantés une grande quantité de vers intestinaux. Il y a, entre autres échantillons, un morceau d'intestin de la longueur de deux pouces et demi, provenant d'un edicnème ou pluvier de terre (charadrius œedicnemus, L.), sur lequel soixante-cinq échinorhynques de la longueur de cinq lignes sont implantés, sans compter les individus qui s'en sont détachés pendant la préparation de ce morceau. Notre cabinet possède également une portion d'iutestiu de la longueur de trois pouces à peu près, provenant d'un hibou (strix otus, L.), sur lequel on voit fixés au moins deux cents amphistomes macrocéphales (amphistoma macrocephalum), et malgré cela ces deux oiseaux n'étaient pas maigres, et n'avaient pas l'air malade.

Il y a cependant des cas où les vers intestinaux peuvent être extrêmement nuisibles à la sauté, et nous en citerons des exemples par la suite, mais cela 
n'arrive pas constamment. D'autres vers, aucontraire, ou bien des larves d'insectes transmis de dehors dans le corps animal, ne peuvent pas y vivre sans causer beaucoup de gêne, et ceux de ces animaux qui ont été introduits avec les alimens cu boissous dans le canal intestinal sont ordinairement soumis, comme nous l'avons déjà remarqué, aux lois de la digestion. $7^{\circ}$. On a découvert des vers intestinaux dans des fœtus nouvellement nés. Tous les exemples de ce genre cités par les auteurs ne méritent peut-être pas que l'on y ajoute foi, mais néanmoins ce fait a été constaté par un certain nombre d'hommes dignes de confiance, de manière que l'on ne peut pas douter de la vérité de leurs observations.

Fromann a trouvé des douves du foie, non-seulement chez les moutons, mais encore chez des agneaux nouveau-nés qui avaient succombé à une épizootie pendant laquelle des troupeaux entiers de ces animaux ont péri.

Kerkring a vu un fœtus dont le canal intestinal était presqu'entièrement rempli de petits vers; le même auteur a découvert une autre fois des ascarides d'un volume trois fois plus considérable qu'à l'ordinaire, dans l'estomac d'un foetus de six mois et demi.

Pallas ${ }^{*}$ et Bloch assurent que Brendel ${ }^{2}$ et Heim ont trouvé des tænias dans des foctus.

Blumenbach a reucontré, d'après le témoignage de

- N. n., beitr., I, s. 43.

Ouvrage cité, p. 38. 
M. Rudolphi ', plusieurs tænias dans le canal intestinal d'un chien nouveau-né.

M. Hirsch, médecin à Beyreuth, a vu dans le jéjunum d'un enfant, qui avait été probablement étrauglé en naissant, un ascaride de deux pouces et demi de longueur.

Gæze $^{2}$, Bloch ${ }^{3}$ et Rudolphi ont souvent observé des tænias d'une très-grande dimension chez des agneaux qui têtaient encore.

Ce dernier assure avoir vu des douves du foie chez plusieurs jeunes oiseaux qui étaient encore presque sans plumes.

J'ai découvert moi-même, il y a quelques années, chez un freux (corvus frugilegus, L ) très-jeune et sans plumes, quarante-cinq tænias de plusieurs pouces de long.

11 me serait facile de doubler, et même de tripler le nombre de ces observations : les sources dans lesquelles elles ont été puisées sonı d'une nature à pouvoir aisément contrebalancer celles que nous avons rapportées plus haut, et d'après lesquelles des vers intestinaux auraient été trouvés hors du corps animal.

11 reste prouvé, à ce qu'il nons semble, d'une manière suffisante, que les vers intestinaux ne peurent pas tirer leur origine de vers qui, d'après les lois de la nature, vivent hors du corps animal, et qu'il faut

- Je n'ai pas encorc pu me procurer l'édition dans laquetle il est mention de ce fait.

' N. G.d. Eingew., p. 37 1.

${ }^{3}$ Ouvrage cité, p. 38. 
les regarder comme des animaux d'un genre particulier.

Il reste cependant encore la question suivante à résoudre : de quelle manière les vers, qui forment ainsi un ordre particulier d'êtres dans le règne animal, arrivent-ils dans le corps d'autres animaux?

L'on peut concevoir la chose de deux manières:

Les vers ou leurs oufs propres à l'espèce humaine et aux animaux, et évacués par eux, peuvent être communiqués à d'autres hommes et à d'autres animaux par les alimens, par les boissons, et peutêtre même par l'air.

C'est surtout Pallas "'qui.a le plus soutenu cette opinion : elle a eu également pour partisans M. Reinlein et Brera. Il n'y a cependant que Brera qui ait cité une observation à l'appui de cette opinion, observation qui sera examinée à la fin de ce paragraphe.

Les preuves que Pallas a apportées pour soutenir son assertion, sont les suivantes :

1․ "La maladie vermineuse est très-répandue parmi les hommes et les animaux qui vivent dans les grandes villes ou dans des endroits très-populeux, surtout où les hommes se tiennent d'une manière malpropre, où l'humidité de l'air et de la contrée est propice à la conservation des œufs horś de leur séjour - naturel, et où l'on se sert, pour boisson ordinaire, l'eau de réservoirs, de sources ouvertes et de rivières qui reçoivent toutes les immondices. On rencontre au

${ }^{3}$ N. n. beitr., s. 43. 
contraire très-rarement ces différentes espèces de vers intestinaux dans les contrées peu peuplées de la Russie et de la Sibéric. On en voit également peu souvent chez les peuplades errantes, qui changent leurs demeures très-fréquemment; à peine ai-je trouvé dans les animaux sauvages de ces pays la centième partie de ce que j’ai rencontré de vers intestinaux dans les animaux d'Europe.

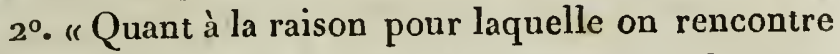
constamment telles ou telles espèces de vers, les unes dans les animaux à sang chaud, les autres dans les oiseaux et dans les poissons, il me semble que l'on peut la trouver, en disant que les œufs dont sortent les vers ne trouvent que dans tel ou tel animal les circonstances, la chaleur et la nourriture nécessaires à leur développement, et sans lesquelles ils périraient.

"J'ajoute à cela $3^{\circ}$., l'observation déjà faite par Hippocrate, que l'on rencontre des tænias dans les enfans nouveau-nés, et, d'après Brendel, même dans les fotus.

“ On a également remarqué que plusieurs personnes appartenantes à la même famille sont souvent tourmentées par le tænia comme d'un mal endémique.

" Il a été également pour moi très-remarquable et concluant de voir que les animaux de proie, les oiseaux carnivores, surtout ceux qui vivent dans le voisinage des hommes, et les poissons voraces (voyageant en troupes et jouissant d'une vie plus longue que les autres poissons) sont ordinairement sujets aux vers intestinaux; le contraire a lieu chez les animaux 
rongeurs, qui se nourrissent avec beaucoup de précaution, et cliez les animaux ruminans, qui broyent leur nouriture avec soin. 》

J'oppose à la première assertion de Pallas, que s'il arrive réellement (ce dout je doute encore) que la présence des vers intestinaux soic plus fréquente parmi les honımes qui habitent les grandes villes, on pourrait assez bien en trouver la raison dans ce que leur nourriture est très-mélangée, et qu'ils ne jouissent ordivairement pas d'une boune digestion. Les habitans de la Russie et des pays encore plus éloignés de nous sont au contraire très-robustes;leur digestion par conséquent se fait bien; ces hommes font surtout un grand usage d'eau-de-vie, et se nourrissent de choses très-simples. Cela est peut-être aussi la raison pourquoi les vers intestinaux se trouvent plus rarement chez eux.

Il y a en géuéral sur ce sujet encore beaucoup de choses dont nous ne pouvons nous rendre compte. J'ai trouvé, par exemple, chez plusieurs animaux très-communs, des vers qui n'ont pas encore été découverts par d'autres naturalistes habitant d'autres pays, landis que j'ai cherché vainement quelques vers qı:e ces naturalistes avaient déjà observés depuis long-temps.

Les entomologistes ont remarqué que les insectes et leurs larves sont plus sujets aux filaires dans une année que dans une autre.

Abildgard prétend avoir observé que les chevaux sont très-incommodés pardes vers à certaines époques 
de l'année. Sloane dit quelque chose de semblable concernant le ver de Médine.

Pendant les cinq premières années de nos recherches sur les vers, nous avons examiné quinze cent soixante-trois campagnols (mus arvalis, $\mathrm{L}_{\text {. }}$ ) et nous n'avons trouvé que trois fois des échinorhynques bien développés dans des individus qui en avaient trois ou quatre en même temps. En 1812 nous avons encore rencontré quatre de ces vers parmi quatre cent trentedeux individus de la même espèce. Deux échinorhynques (parasites) furent également découverts à cette époque, une fois dans un putois, et l'autre fois dans un faucon cendré (falco cinereus, L.). Tous ces campagnols, au nombre de dix-neuf cent quatrevingt-quinze, ne nous ont fourni dans les prenières années de nos travaux que deux fois l'occasion d'observer des cysticerques qui nageaient en grande quantité, librement, dans la cavité thorachique de deux individus, et depuis nous n'en avons jamais rencontré d'autres. Au commencement de l'année 1807 j'ai trouvé entre les tégumens et les muscles d'une grenonille verte (rana esculenta, L.) un filaire que j'ai cherché vainement par la suite parmi plus de douze cents individus de cette espèce, et ce n'est que dans l'année 1813 que j'ai revu ce ver trois ou quatre fois dans la même espèce, sur un petit nombre d'individus.

Dans quelques pays on peut regarder les vers intestinaux comme un mal endémique; dans d'autres on ne les voit que d'une manière sporadique. Les ha- 
bitans de la Russic, de la Pologne, de la Suisse et de quelques contrées de la France sont sujets au bothriocéphale; le reste des Européens est incommodé par le tænia. Qui peut nous indiquer la raison de tout cela? Ce n'est pas moi, assurément.

Le second argument de Pallas prouve plutôt contre lui qu'en sa faveur, car cette constance avec laquelle on ne rencontre que telle espèce de ver dans telle espèce d'animal nous fait présumer d'autant moins que les vers puissent arriver du dehors dans le corps animal. Si nous voulions poser en fait, pendant un moment, que cela fût possible, nous serious alors obligés d'admettre : d'abord que le ver transmis dans le corps d'un animal prendrait la forme particulière de l'espèce de ver qui est propre à cet animal; ou, en d'autres termes, la forme et la structure du ver seraient déterminées par l'animal dans lequel il devrait séjourner; et ensuite qu'il ne peut se développer dans un animal que des oufs ou des germes de vers qui soient propres à son espèce, ou, pour le moins, à son genre.

Nous avons déjà démontré plus haut, lorsqu'il était question de la prétendue métamorphose des vers de terre et d'eau en vers intestinaux, que le premier cas ne peut pas avoir lieu; et, en effet, quel changement tout à fait singulier ne devrait-il pas s'opérer dans plusieurs espèces de vers, si cela arrivait ainsi? Supposons qu'une souris eut avalé les oufs d'une espèce d'ascaride particulière à l'homme, et qu'un autre cut mangé les oufs d'un ascaride propre aux chats. Ces 


\section{DE L'HOMME.}

deux ascarides ne diffèrent pas seulement entre enxmêmes, mais ils diffèrent encore de l'ascaride particulier à la souris. Fût-il par conséquent possible que l'ascaride trouvé dans le corps d'un auimal ait pu tirer son origine d'un ascaride d'un autre animal d'un genre différent, nous devrions alors présumer dans le cas supposé (c'est-à-dire où les œufs de ces deux différens ascarides auraient pu se développer sous la forme d'ascarides propres aux souris) que les changemens suivans auraient dù s'opérer. $1^{\circ}$. Les rainures latérales propres aux ascarides de l'homme auraient dù se remplir et s'aplanir sur les individus qui se seraient trouvés dans le corps de la première souris; et $2^{\circ}$. les nembranes latérales en forme d'ailes, qui caractérisent l'ascaride du chat, auraient dú se rétracter chez les individus qui se seraient trouvés dans le corps de l'autre souris; par conséquent celte métamorphose aurait nécessité deux opérations tout à fait opposées, sans parler de beaucoup d'autres qui auraient dù encore s'effectuer avant que la forme des ascarides propres à l'homme et au chat, et transférés dans le corps de ces deux souris, fùt devenue identique à celle qui caractérise l'ascaride de la souris. Ces vers nouvellement transformés auraient subi encore une autre métamorphose, si ces deux souris eussent été avalées par un chat.

Quand même on roudrait admettre que les œufs de tel ou tel ver pourraient se développer dans un animal, pourvu qu'ils provinssent d'un individu de la même espèce que celui-ci, on rencontrerait en- 
core une nouvelle difficulté, en ce que l'on ne pourrait nullement concevoir par quel moyen, chez certains animaux, cette communication des oufs aurait p'l s'effectuer. Aiusi hi fiéquence des vers chez les homanes extrêmement propres, comme les Hollandais, ne pourrait être expliquée; en effet, quoique l'on ail raison de dire, homo homini lupus, on voit, all moins en Europe, que les hommes ne se mangent entre eux que dans un sens figuré; ce ne serait alors que par les matières fécales qu'une pareille communication pourrait s'opérer; cependant cela ne peut être supposé chez les hommes, qui ont tonjours soin d'éloigner ces matières des choses qui leur servent de nourriture. La possibilité d'une pareille communication se concoit cependant par l'eau, en ce que nos latrines s'écoulent ordinairement dans les rivières, qui sunt souvent en communication directe avec vos puits. Mais quel chemin l'ouf du ver n'aurait-il pas à faire? Pendant combien de temps ne serait-il pas obligé de conserver la vie dans des circonstances défavorables, et dans lesquelles les vers iutestinaux meurent si vite? Pallas dit lui-même que les oufs des vers périssent, s'ils ne rencontrent des circonstances favorables à leur conservation, c'est-à-dire la nourriture el la chaleur.

Nous rencontrons encore plus de difficultés pour expliquer la communication des oufs de vers intestimanx entre les animaux, qui we boivent pas en général, comme cela a lieu pour les oiseaux de proie. Dans l'état libre ces animaux ne se mangent 
pas entre eux, et ils mangent encore moins leurs ex-crémens; les animaux qui leur servent de nourriture n'avalent pas non plus les matières fécales que ces oiseaux rejettent; car dans ce cas on pourrait supposer que les oufs des vers provenant d'un de ces oiseaux auraient pu être communiqués à un autre individu de la même espèce par cet intermédiaire.

Il nous reste enfin encore à supposer que c'est peut-être par le moyen de l'air que celte communication des œufs de vers intestinaux peul s'effectuer; mais cette supposition? En effet un corps qui, à cause de sa pesanteur spécifique, ne se soutient pas nême sur la surface de l'eau, comme cela arrive pour les oufs de vers intestinaux, peut encore moins s'élever et flotter dans l'air. Pour que cela pût avoir lieu, il faudrail nécessairement supposer qu'ils se seraient préalablemeut desséchés de telle sorte, qu'ils ne formeraient pour ainsi dire qu'une espèce de poussière. Mais est-il probable que ces œufs, ainsi desséchés, pussent encore conserver la faculté de se développer?

Ce que Pallas a envisagé comme un troisième argument pour soutenir son hypothèse, sert justement à la réfuter; car on doit plutôt regarder comme une contre-preuve la circonstance que l'on a rencontré des vers dans des focus, qui assurément n'ont pas pu manger des substances chargées d'oufs de vers. Du reste, plus loin nous aurons également occasion de démontrer qu'il est tout à fait impossible qu'une mère puisse transmettre des œufs de vers au foetus qu'elle 
porte dans son sein. S'il arrive quelquefois que plusieurs personnes de la même famille soient sujettes aux vers intestinaux, cela s'explique aussi facilement que la raison pour laquelle plusieurs personnes appartenantes à la mêtme famille ont de mauvaises dents, on qu'elles sont sujettes à la goutte.

L'assertion du même auteur, que les animaux carnassiers ont beaucoup plus souvent des vers que les animaux rongeurs et ruminans, ne se coufirme nullement par les observations que j'ai faites à ce sujet : je vais seulement citer quẹlques exemples. Parmi vingt-une loutres, il n'y en avait pas une seule dans le corps de laquelle il se soit trouvé un ver. Parmi cinquante-quatre lapins sauvages, il n'y en avait an contraire que cinq qui en fussent dépourvus. Nous avons trouvé dans plusieurs de ces animaux en même temps des nématöides, des cestoïdes et des cystoïdes. La loutre est cependant uu animal carwassier qui mange presqu'exclusivement des animaux chez lesquels on rencontre beaucoup de vers. Le lapiu, au contraire, ne vit que de végétaux; c'est un animal rongeur et qui rumine également.

J'ai disséqué en 1816 , dans le unois de septembre, dix-sept chamois qui ont été tués à une chasse sur les Alpes de la Styrie. Parmi ces animaux il n'y en avait qu'un seul qui n'eût pas de vers; quelques-mns avaient des trichocéphales ou des strongles, et huit des tænias. Les chamois vivent cependant bien éloignés des grandes villes.

Sans doute on pourrail encore dire que les ani- 
maux qui ne mangent pas de chair penvent se communiquer leurs vers, en admettant que, rejetés avec les excrémens, ceux-ci se trouveraient mêlés avec les alimens ou avec les breuvages; mais comment expliquer la communication d'hydatides, chez lesquelles nous ne connaissons point d'œufs, et qui, enfermées dans des capsules particulières, séjournent dans des viscères qui n'out aucune communication directe avec le canal intestinal, ce qui empêche par conséquent de supposer que les vers ou leurs oufs pourraient être transmis à ces viscères par celte voic. Alors, se demande-t-on tonjours, par quel chemin les hydatides sont-elles arrivées dans les organes dans lesquels nous les trouvons? Les animaux rongeurs et ruminans sout réellenent, au moins dans beaucoup de cas, presque remplis d'inydatides; nous en observons au contraire très-rarement chez les animaux carnassiers : on en a tout au plus deux ou trois exemples.

Le fait suivant est très-concluant et vicat à l'appui de ma théorie. M. Schreiber a nourri en 1806 un putois (mustela putorius, L.) pendant six mois, uniquement de lait, de vers intestivaux de toute espèce et de leurs oufs; au lieu de cette nourriture, on na substitué que très-rarement un peu de nie de pain. Cet animal fut tué ensuite et examiné; matis, au grand étonnement de tout le monde, on n'y trouva pas la trace d'an ver quelconque. Il est à regretter que le temps ell'occasion nous aient manqué pour répétcr cette expérience sur d'autres animaux el dans deș circonstances différentes. 
Quoique j’aie posé en fait que la communication des vers ne pouvait pas avoir lieu par l'intermèdiaire des alimens et des boissons, je ne veux pas cependant nier la possibilité que les vers qui se sont introduits par ce moyen daus le canal intestinal d'un animal, ne puissent y vivre, dans quelques cas, pendant un certain temps. J'ai rapporté moi-même plus haut que l'échinorhynque, que l'on voit très-rarement dans le campagnol, fut rencontré comme parasite dans un putois, et une autre fois dans un faucon cendré (falco cineraceus); mais on a trouvé en même temps dans les deux cas les restes des campagnols dans l'estumac de ces animaux.

On a observé également l'échinorhynque nommé echinorhynchus hoeruca, qui ne se rencontre que dans 1a grenouille verte, dans l'estomac dù crapaud gris (bufo cinereus, Rec.); mais on y veyait encore les restes d'une jeune grenouille.

J'ai observé dans l'estomac d'une couleuvre à collier (coluber natrix, L.) implantée dans les parois de cet organe une espèce de nématoïde, qui ne séjourne ordinairement que dans les crapauds couleur de feu (rana bombyna), dans les salamandres aquatiques et dans les protées de la Carniole (proteus anguinus). Mais cette conleuvre avait vomi, dans les vingt-quatre heures qu'elle avait élé enfermée, un crapand couleur de feu.

On découvre souvent des ligules (qui se trouvent originairement dans la cavité abdominale des poissons du genre cyprinus) comme parasites dans le ca- 
nal alimentaire d'oiseaux aquatiques, et dans celui de poissons voraces. Mais ces vers, qui ont la vie très-dure, nous fournissent un exemple de l'influence des fonctions digestives : on en remarque quelquefois tout le long du canal intestinal de ces oisenux, mais ces vers, quoique vivans, sont dans un état trèsdifférent; ils n'éprouvent en effet aucune altération dans l'estomac, on les y voit tels qu'ils se trouvent dans la car iıéabdominale des poissons; mais ceux qui se sont glissés plus loin que l'estomac on céprouvé une altération, et elle cst d'autant plus visible, qu'ils se trouvent plus éloignés de ce viscère. On voit alors que la couleur blanclie de ces vers s'est changée en un jaune sale; leur corps est allongé, aminei, ou au moins lésé ou comme macéré à l'une de sess extrémités, de manière que l'on aperçoit clairemeut qu'ịs ont été en partie soumis aux lois de la digestion.

Je n'ai observédes ligules chez les poissons voraces que dans l'estomac, mais il y avait toujours en même temps des restes de poissons nou digérés. Je n'ai jamais rencontré ces vers dans les intestins; il me paraît probable qu'ils sont entièrement dissous dans l'estomac.

Montin prétend avoir observé des morceaux de ligules dans les déjections d'une jeune dame de vingreinq ans; cependant on peut bien présnmer qu'elle n'avait pas mangé de poissons crus pourvus de leurs intestins. Cet anteur ajoute qu'elle a rench en même temps des ascarides et des tænias. Il est très-probable que Montin a pris un morceau de ces derniers très- 
allongé, ou peut-être très-contracté, pour celui d'une ligule. D'après les expériences de Bloch ${ }^{x}$, les ligules meurent dans l'eau bouillante dans l'espace de deux minutes, et je crois que l'on fait bouillir les poissons au moins autant de temps en Suède pour les manger. L'observation de Montin ne met donc uullement en droit de placer les ligules parmi les vers intestinaux propres ì l'homme, comme l'a fait Brera.

Rosenstein a prétendu, indubitablement à tort, avoir tronvé des ligules vivantes dans des poissons bouillis. Quand on transporte un de ces vers d'une assiette très-chaude sur une très-froide, il s'opère alors par le changentent de température des contractions inégales des fibres, que l'on peut aisément prendre, pour des mouvemens volontaires.

De potits vers retirés de l'esprit-de-vin, où ils ont été depuis très-long-temps, et placés dans de l'eau, semblent se mouvoir, quoique morts, d'une manière extraordinaire, et ces mouvemens durent jusqu'à ce que l'esprit-de-vin contenu dans ces vers se soit entièrement mêlé avec l'eau.

La prétendue expérience de Rolandson Martin sur l'introduction de vers de poissons dans le canal inteśtinal de l'homme mérite encore moins notre attention. Cet auteur raconte qu'il a rendu souvent, pendant qu'il demeurait dans le voisinage de la mer, des petits vers qu'il croyait être des oxyures, quoique son àge aurait dû, d'après son opinion, l'en exempter.

* Ourrage citć. 


\section{DE L'HOMME.}

J'ai connu moi-même un vieillard de quatre-vingts ans et plus, qui en rendait très-souvent, preuve que l'âge avancé ne met pas à l'abri de ces vers.

Rolasdson examinant un jour la vessie d'un éperIan (salmo eperlanus, L.), poisson dont il mangeait très-fréquemment, y trouva une grande quantité de petits vers, qu'il décrit avec beaucoup de soin; il croit que les petits vers qu'il a rendus souvent provenaient de ce qu'il s'était nourri de ces poissons.

La description que Rolandson donne des vers trouvés dans cel éperlan, prouve clairement la grande différence qui existe entre ces vers et les oxyures propres à l'homme. Ceux-là sont probablement les mêmes que Fischer a observés le premiel dans la vessie aćrienue d'une truite (salmo fario, L.), et que cet auteur a décrits sous le nom de cystidicola. Tout ce que Rolandson dit des vers rendus par lui se rapporte parfaitement à la conformation des oxyures. On voit dans chaque ligne de ce récit, qu'il est sorti de la têtc d'un hypocondriaque.

Pallas ${ }^{1}$ a prouvé par une expérience, que des vers peuvent se développer par l'insertion de leurs oufs daus un animal. Voici ses propres paroles : “J'ai essayé, par le moyen d'nne petite incision, à introduire les œufs rouges du tænia du chieu dans la cavité abdominale d'un jeune animal de cette espèce. J'ai trouvé en eflet, après l'espace d'un mois, des petits tænias renfermés dans cette carité. Ces vers avaient tout au plus un pouce de long, et leurs articu'N.n. Beilr, p. 58. 
lations étaient encore plus courtes que celles représentées fiğ. xir. Je suis fâché quac le temps et l'occasion m'aient manqués pour répéter cette expérience de différentes manières, et même avec des vers cucurbitains. " Pallas est un observateur fidèle, et on ne doit pas douter de la vérité de son observation, qui ne prouve cependant rien contre ma théorie; car ces œufs avaient été pris fraîchement d'un tænia de chien, par conséquent ils étaient doués de vie, et ils ont trouvé dans la cavité abdominale d'un antre chieu tout ce qui était nécessaire à leur développenent, c'est-à-dire la chaleur et l'humidité, et dans celle-ci il y avait même quelque chose qui a pu leur servir de nourriture. Le développement peu considérable opéré dans l'espace d'un mois sur ces tænias, qui grandissent en général très-vite, doit être attribué à la petite quantité de nourriture que ces vers ont rencontréc dans la cavité abdominale, c'est-à-dire hors du tubc alimentairc. Cette expérience ne prouve cependant nullement que ces vers introduits par la bouche n'auraient pas été soumis aux lois de la digestion, comme nous l'ivons vu arriver chez le putois.

Brera ' s'est flatté d'avoir fait une experience semblable à celle de Pallas; cependant il n'en est pas ainsi, comme nous allons le voir. Le fait suivant, communiqué par Rubini à Brera, a fourni matière à cette expérience : une petite fille de deux ans, jouissant d'une bonne santé, rendit, avec les matières fécales, des petits corps arrondis et jaunâtres. En les

Memorie, p. 186. 
pressant entre les ongles, il en sortit une humeur blanchâtre; leur enveloppe semblait composée de deux membranes, une interne blanchâtre, et une externe jaunâtre. Plusicurs de ces corps furent placés dans une boîte, et le lendemain on remarqua que quelques-uos s'etaient ouverts, et que plusieurs petits oxyures très-vifs en sortirent; ccpendant ces vers moururent promplement. Le frère de cette petite fille évacua de pareils corps l'année suivante. Rubini en envoya à Brera, en $\mathbf{1} 805$, a u mois de février, plusieurs dans une petite boîte. Il est à remarquer que le thermomètre était à deux degrés audessous de zéro.

Ces corps étaient petits, durs, tout à fait desséchés, et ressemblaient à des grains de sable. A une loupe, qui grossissait dix fois, ils paraissaient cordiformes; sous un microscope de Dollond, leur superficie était velue. En les fendant dans tonte leur longueur, on remarqua que ces prétendus œufs n'étaient qu'un agrégat ou plutôt un réceptacle de plusieurs œufs de différentes grosseurs, qui contenaient encore, sans doute, comme Brera le présume, d'autres œufs trèspetits et imperceptibles. Brera, persuadé que ces corps contenaient plusieurs germes de vers, en introduisit dix (le 4 février, c'est-à-dire un mois après qu'ils avaient été évacués) dans la cavité abdominale d'un jeune chien, noyennant une petite incision pratiquée à l'extérieur. La plaie se cicatrisa dans l'es. pace de quatre jours, et l'animal fut tué vingt-un jours après. On trouva la cavité abdonivale remplie de petits vers qui portaient rous les caractères des 
oxyures. Leur conleur était jaunâtre, leur longueur de quatre millimètres à peu près, ct la grosseur la plus considérable du corps d'un millimètre et demi. Ces vers étaient très-vifs, et sautaient à la lucur d'une chandelle. Leur tête était grosse, obtuse, et leur queue se terminait en un cône obtus, au lieur d'être pointue.

Voilà l'essentiel du contenu de l'observation de Brera, et si elle est exacte, elle servirait de preuve que des vers provenant d'œufs tout à fait desséchés peuvent se développer dans des circonstances favorables; pourquoi n'en serait-il pas de même de ceux qui flotteraient dans l'eau?

Mais je me permets de faire observer à Brera que les petits corps que Rubini lui envoya n'étaient pas des œufs de vers.

Si Brera était aussi bon observateur qu'il s'annonce lui-même, il aurait dû d'abord douter de la pature d'œufs qu'il attribue à ces corps, et s'étonner ensuite que ces deux enfans n'eussent jamais rendu de vers, cc que leur mère, du reste, aurait dû facilement remarquer, quoique ces vers ve se fussent pas fait sentir par des démaugeaisons à l'anus. Mais il est dit expressément, en parlant de cette fille, qu'elle n'a éprouvé aucun symptôme morbide (sensa essere a)Setta da verun sintomo morboso); cependant il parait que Brera ue s'en souvenait pas à la page 374 , où il cite ce cas et le désigne comme une maladie vernineuse très-grave, excitée par la présenee d'oxyures : (caso di verminatione incommodissima cccitata della pre- 
senza delle ascaridi vermicolari). En lisant l'ouvrage de Brera avec attention, on trouvera souvent de pareilles erreurs et contradictions.

Cet auteur aurait dû, en outre, trouver étrange que ces vers u'eussent besoin, la prentière fois, pour se développer, que d'une seule nuit, tandis que dans la seconde ils restèrent un mois šans le faire. Si Brera les cût bien examinés, il aurait vu sur-le-champ que c'était contre toute analogie que ce fussent des capsules qui en auraient contenu d'autres, daus lesquelles à la fin les véritables ocufs seraient enfermés. Il y a bien des infusoires, par exemple le volvox globuleux (volvox globator), où un animal se trouve enfermé dans l'autre, et où les jeunes en contiennent encore d'autres plus petits, mais je ne connais aucun cxemple où des oufs séparés de la mère et provenant surtout d'animaux qui ont des parties sexuelles séparées, et qui s'accouplent complétement, comme toutes les espèces de nématö̈des, puissent être réunis dans une seule capsule. Cela peut arriver tant que les œufs sont encore contenus dans le sein de leur mère, soit dans une membrane, soit dans une sorte de matrice, mais non pas quand ils en sont sortis. Brera aurait dû encore être surpris qu'il ne sortît la première fois de chaque corps qu'uu senl ver, tandis que des milliers se développèrent lors de son expérience de dix introduits dans l'abdomen du chien. Il aurait pu se convaincre aisément que ce n'était pas des œufs d'oxyures qu'il observait, s'il s'était donné la peine de les comparer avec des œufs sortis récenıment d'une 
femelle de cette espèce; il anrait alors vu que ces oufs sont tellement petits, qu'il est impossible de les apercevoir à l'oeil nu, de les compter lorsqu'ils sont desséchés, et encore bien moins de les disséquer. Les œufs des oxyures desséchés sur une plaque de verre, ne peavent pas même être sentis par le toucher; ceux de Brera, d'après sa description, avaient une enveloppe dure, composée de deux membranes, et ils étaient aussi gros, et peut-être plus que certains oxyures. Il nous dit aussi que ces corps étaient cordiformes; cela se peut bien, mais les oufs d'oxyures sont ovalaires, justement comme il les a représentés, tab. Iv, fig. 10, quoique un peu inexactement, d'après une figure de Goze. Ces œufs ont, même en les grossissant sous les plus fortes loupes, une superficie tout à fait lisse, et non pas velue, comme Brera le prétend des corps qu'il a observés. Ainsi donc, si Brera n'avait pas été trop prompt dans ses conclusions et assertions, l'idée ne lii serait pas venue de regarder les oeufs d'oxyures comme identiques avec ces petits corps.

Le lecteur demandera enfin, qu'était-ce donc que ces corps? Ne les connaissant que par une descriplion et une figure qui se trouve copiéc pl. Ix, fig. I, je ne puis rien avancer là-dessus; mais qu'il me soit permis de faire une observation. Au premier aspect je les ai pris pour les graines d'une plante, mais je ne savais pas à laquelle elles appartenaient. Pour m'éclairer là-dessus, je demandai à $\mathbf{M}$. le baron de $\mathbf{J a -}$ quin quelles graines de cette forme ct grosseur avaient 
pu être mangées par un enfant : cet auteur me répondit que c'était probablement celles des fraises, qui se trouvent au dehor's du fruit, et qui, introduites dans le canal intestinal, s’y grossissent considérableinent. Nous en examinâmes sur-le-champ qui avaient été recueillies fraîcliement de ce fruit, et nous vîmes qu'elles ressemblaient parfaitement aux corps représcutés par Brera. J'en donnai ensuitc à un enfant à manger, et quarante-lauit heures après je retrouvai dans ses matières fécales les graines considérablement gonflées : il sortait de ces derniéres, écrasées entre les ongles, non pas une humeur blanchâtre, mais bien un germe déjà développé. C'était probablement de fraises fraîchement cueillies, que provenaient les premiers corps observés par Rubini au mois de juin, tandis que les autres observées par lui en hiver provenaient sans doute de fraises confites. Les mouvemens vifs dont parle cet auteur se laissent eyalement expliquer. Ces graines furent probablement mises dans l'cau, où il s'opéra une absorption inégale de ce liquide, qui, causant une différente tension des fibres, produisit des sautillemens en différens sens, qu'un homme peu exercé a pu regarder comme des mouvemens volontaires.

La villosité apparente, qui n'est pas propre aux graines de fraises, pouvait provenir de la poussière adhérente, ou d'une macération de l'épiderme, ou peut-être même était-elle due à de la moisissure.

Les corps ronds que Brera prétcnd avoir observés dans ces apsules n'étaient très-probablement dus 
qu'à une illusion d'optique. Celui qui ne sait pas bien manier le microscope composé ne doit pas trop se fier à sés yeux, car rien n'est plus facile que de se tromper dans l'emploi de cet instrument.

L'origine des milliers de vers rencontrés ensuite par Brera dans la cavité abdominale du chien ne peut être aussi facilement expliquée : j’ai cependant la certitude que ce n'étaient pas des oxyures. Ces vers étaient d'un jaune foncé : les oxyures sonı blancs comme du lait; la longueur de chacun était de quatre millimètres à peu près, et la grosseur la pius considérable d'un millimètre et demi, par conséquent une proportion de la longueur à la grosseur, comme trois à huit. Chez les oxyures, cette proportion est d'un à vingt, sans compter la partia la plus pointue de la queue. Ils avaient la tête grosse et obtuse. Les oxyures sont très-fortement amincis vers l'extrémité antérieure, qui est pourvue d'une membrane latérale; la queue formait un cône obtus, tandis que la queuc des oxyures est très-pointue et est tellement amincie vers son extrémité, qu'on ne peut guère la voir qu'avec l'œil armé du microscope. Peut-on trouver des différences plus grandes, je le demande, chez des aninaux, comme les nématoïdes, dout les geures et les espèces sont seulement déterminés par la proportion de la grosseur avec la longuenr, et par la conformation de la tête et de la queue? Et Brera dit cependant qu'ils offraient tous lescaractères des oxyures (offrivano tutti $i$ caratleri dell' ascaride vermicolare). Les différences, selon lui, auraient pu provenir de ce 
qu'ils ne s'étaient pas développés daus le corps de l'homme. En général Brera a supposé bien des choses qui ne sont nullement fondées : je vais en donner quelques exemples. Il croit que le polystome tæniö̈de (polystoma tcenioides, Rud.), dentelé sur les bords, et non pas articulé, provenant des sinus frontaux du chien, est identique avec le véritable tænia articulé (tania lanceolata), qui séjourne dans les intestins de l'oie, d'où il tire diverses conclusions. 11 a décrit une larve de mouche trouvée dans le pot de chambre d'une femme, pour un nouveau ver intes-

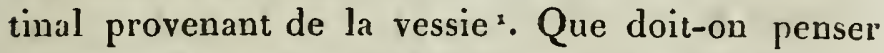
d'un pareil observateur? Comment peut-on ajouteı' foi à ce qu'il dit, quand on sait qu'il rapporte infidèlement, et qu'il arrange, comme bon lui semble, les expériences faites par d'autres, comme nous le verrons quand il sera question de l'hamulaire lymphatique de Treutler et du polystome des veines (polystoma venarum)? Qui s'étonnera alors, si je soupçonne que Brera n'a trouvé ses prétendus oxyures que plusieurs jours après l'ouverture du chien, ce qu'il lui aura paru convenable de nous laisser ignorer, ou que pendant cet espace de temps, les mouches ont pu y déposer leurs oufs, et qu'il a pris alors leurs larves pour des vers? Toute sa description rend cette supposition très-probable.

Je prie ceux de mes lecteurs qui pourraient croire que je me suis occupé trop long-temps de réfuter

?. Voyez Circosoma, parmi les pseudohelminthes. 
Brera, de considérer que plusieurs raisons ont dû m'y déterminer. Brera est, pour ainsi dire, le seul (Joerdens et plusieurs auteurs de dissertations exceptés) qui ait publié dans les temps modernes un ouvrage même très-volumineux 'sur les vers intestinaux de l'homme. Cet auteur s'annonce lui-même comme un homme qui a approfondi et examiné avec le plus grand soin tout ce qui a rapport à ce sujet. Quel médecin n'étant pas lui-même helminthologue, ne croirait pas Brera sur parole? Alors, entraîné par les raisonnemens de cet, auteur, il n'hésitera pas d'admettre ces trois choses : $1^{\circ}$. que des oufs de vers tout à fait desséchés peuvent éclore si on les. soumet à l'influence de la chaleur animale et de l'humidité; $2^{\circ}$. que les oeufs de vers peuvent être transférés d'un animal dans un autre, et $y$ atteindre leur développement; $3^{\circ}$. que la structure des vers prend une toute autre forme quand ceux-ci sont éclos dans un corps organisé autre que celui dans lequel ils séjournent originairement. Réfuter ces trois points, prouver leur peu de vraisemblance et même leur impossibilité, était le but de toute cette discussion. J'étais surtout obligé de réfuter avec beaucoup de détails la prétendue observation concernant le développement de vers tout à fait desséchés, et par laquelle ma théorie aurait complétement échoué, afin de mettre mes lecteurs en état d'en pouvoir juger euxmêmes. Ceux qui ne veulent pas ajouter foi à mes assertions peuvent facilement, par des expériences, se convaincre de leur vérité. 
Après avoir prouvé par des raisonnemens l'impossibilité de la communication des vers intestinaux par l'intermédiaire des alimens, des boissons et même de l'air, il ne reste d'autre voie, dans la supposition que les vers intestinaux seraient gagnés par communication, que celle de la succession par les parens, c'està-dire que ceux-ci les transmettraient à leurs enfans, soit par l'acte de la génération, soit par la nutrition, dans le sein de la mère, soit enfin par l'allaitement.

Les défenseurs de cette hypothèse sont obligés de convenir, ou plutôt ils sont forcés d'admettre que les parens primitifs de l'homme et de tous les autres animaux ont porté en eux toutes les espèces de vers particulières à chaque espèce d'animal. Si l'on considère combien d'espèces de vers on rencontre chez quelques animaux, chez l'homme, par exemple, douze', sans compter le tænia vulgaire comme une espèce particulière, ct sans y comprendre le polystome des veines (polystoma venarum), et plusieurs autres dont nous parlerons par la suite; chez le chien huit; chez le renard, neuf; chez le putois, également neuf; chez le hérisson, dix à onze; chez le campagnol, sept; chez le lièvre, huit; chez le mouton, neuf; chez le bouf, dix à onze; chez le cochon, huit à neuf; chez le cheval, neuf; chez le faucon cendré, huit; chez la pie, huit ; chez la corncille bleue, sept; chez le cormoran, huit; chez la cicogne, sept; chez le vanneau, dix; chez le pluvier, sept; chez la grenouille temporaire, huit; chez la grenouille verte, dix ; chez le silure, sept; chez la perche, onze; chez le sandre (perca 
lucio, L.) sept; chez la truite, dix; chez la truite saumonnée, neuf; chez le saumon, huit; chez le brochet , dix, etc., sans parler de ceux que nous ne connaissons pas encore, et de ceux dont la race s'est peut-être éteinte; il faudrait alors admettre que ces parens primitifs auraient été, pour ainsi dire, de véritables magasins de vers, auxquels la génération de ces parasites a dî canser plus d'obstacles que la conservation de leur propre race.

On pourra sans doute objecter contre cette assertion, que les parens primitifs ont dù avoir nourri dans leur corps toutes les espèces de vers que l'on rencontre dans leur's descendans; que cela n'a pas été absolument nécessaire, par la raison que les vers appartenant originairement à la même espèce, auraient pu recevoir, par le croisement des espèces, par liufluence du climat, de la nourriture, du chyle, une structure tout à fait différente de leur première forme, de mauière à ne plus être reconnus, ce qui fait que nos naturalistes d'aujourd'hui les regardent comme autant d'espèces différentes, ce que nous avons également raison d'admettre pour les animaux d'une conformation parfaite, savoir que ceux que nous regardons aujourd'hui comme formant des races particulières, ne furent primitivement que des variétés.

Quand même on voudrait admettre que cela puisse arriver dans quelques cas pour les vers appartenant à la ueème famille, ou pour ceux qui séjournent dans le mème organe ou viscère, on concevrait difficilement que cela eût pu avoir lieu pour des vers, qui dif- 
fèrent entre eux, non-seulement d'espèce et de genre, mais encore d'ordre, et qui séjournent dans des or.ganes différens. Si l'on voulait supposer, par excmple, que l'hydatide qui se trouve dans le cerveau du mouton, le nématoïde dans la trachée-artère du nêême animal, le tænia dans ses intestins grêles, et le ver (de l'ordre des trématodes) dans son foie, aient eu le mênie père primitivement, on ne pourrait pas, par conséquent, regarder comme un homme dépourvu de bon sens celui qui croirait qu'un animal quelconque, l'éléphant, par exemple, serait à la fois le père des baleines, des bouquetins, des lions, des kanguroos, etc.; et cependant dans ce cas la différence rie serait pas même aussi grande, car ces animaux appartiennent tous à la classe des mammifères.

Quoiqu'il'ne soit pas probable que chaque individu des parens primitifs de l'homme ou des animaux ait porté autant de vers différens dans son corps, il n'est cependant pas encore clairement démontré que cela ne soit pas possible. Je ne veux pas, pour cette raison, que l'on regarde ce que j'ai avancé jusqu'à présent à ce sujet, comme une preuve admissible contre cette hypothèse. Supposé que les parens puissent communiquer les vers à leurs enfans, on devrait nécessairement admettre en même temps que les parens portaient en eux-mêmes les vers qu'ils devaient transmettre à leurs descendans; car on ne pent pas communiquer quelque chose que l'ou ne possède pas soi-même. Mais c'est justement ici que cette hypothèse n'est pas confirmée par l'expéricuce. Nous 
voyons beaucoup de personnes très-sujettes aux vers, sans que l'on en ait vu une trace chez leurs parens; comment ceux-ci, par conséquent, auraient-ils pu les leur transmettre? Alors, par quel moyen cette communication s'est-elle opérée? Brera ${ }^{\mathrm{x}}$, défenseur de cette hypothèse, a toujours un refuge quand il se trouve embarrassé dans ses assertions, et c'est en effet par un moyen de cette sorte, qu'il a cherché à la soutenir.

Cet auteur, s'en rapportant à ses expériences, dit que quelques hommes sont quelquefois très-sujets aux vers, tandis que leurs enfans n'en ont pas, et que les mêmes vers dont leurs pères ont été affectés ve se montrent que chez leurs petits-fils. On ne peut rien objecter contre cette expérience, et il peut même arriver que les arrière-petits-fils aient les mêmes vers que leurs bisaïeux avaient jadis, tandis que tous les autres membres de cette famille n'en avaient pas. Mais je doute de la conclusion que Brera en tire, savoir que les œufs des vers du grand-père ont traversé le corps du fils, dans lequel ils n'auraient pas trouvé une occasion favorable à leur développement, pour se rendre dans celui du petit-fils.

Le lecteur doit facilement voir tout le ridicule que contient l'idée d'une pareille succession de vers du grand-père an petit-fils, en omettant son propre fils. Je crois cependant encore nécessaire de faire remarquer les points suivans ؛

1 Memorie, p. 4 or. 
1. Nous pouvons supposer que les deux sexes de l'espèce humaine, arrivés à l'époque à laquelle ils peuvent se reproduire, ne conservent pas, à cause du changement continuel de la matière un seul grain de la substance osseuse primitive qu'ils avaient en quittant le sein de leur mère; encore moins les corps étrangers, parmi lesquels je compte les vers, pour-a raient-ils se conserver aussi long-temps dans l'iutérieur du corps, où des évacuations continuelles ont licu par le moyen des vaisseaux excréteurs. Brera a. dit lui-même que, lorsque les oufs de vers ne trouvent pas dans un corps les conditions nécessaires à leur développement, ils sont évacués intacts (quoique cette assertion ne soit appuyée d'aucune preuve) comme d'autres substances destinées à être excrétces. Pourquoi les oufs de vers communiqués par la génération feraient-ils une exception à cette règle? Cas ou l'œuf comme tel est détruit, comme cela a lieu quand il arrive dans l'estomac, ce que du reste l'expérience rapportée sur le putois a prouvé, ou bien l'œuf rencontre les conditions requises ì son développement, et le ver en sort. En effet, à juger d'après toute analogie, l'œuf d'un ver vivant et fécondé, par: exemple celui d'un mammifère, n'a pas besoin d'autre condition pour son développement que la chaleup animale et l'humidité. Au moins on ne voit point la raison pour laquelle un ouf qui se trouverais dans de pareilles circonstances. ne pourrait pas se développer aussi bien qu'un ouf de poule, qui n'a besoin pour cela que d'une chaleur sèche dans un four. 
Le ver par conséquent se développera toujours et à un endroit quelconque du corps, pourvu qu'il ne rencontre pas quelque chose, comme par exemple dans l'estonac, qui agisse sur lui d'une manière destructive; mais autre chose est sa croissance et sa conservation future. L'expérience précitée de Pallas faite sur les œufs du tænia du chien paraît non-seulement prouver qu'il peut s'accroître, mais encore donner des éclaircissemens sur sou développement ultérieur.

Si des œufs de vers pouvaient être introduits dans la matrice avec le sperme de l'homme, il serait sans doute à craindre, comme ils y trouvent de la chaleur animale et de l'humidité, que ces vers ne se développassent plus tôt que le foetus dans lequel ils devaient séjourner, qu'ils ne le.mangeassent à l'état d'embryon, et que la femme, à la fin, n'accouchât, au lieu d'un enfant, d'un amas de tæuias ou d'ascarides; mais un faiı semblable n'a jamais été observé.

$2^{\circ}$. La chose ne devient pas encore claire, en admettant même que des vers puissent être communiqués an foetus pendant l'acte de la génération (je démontrerai cependant par la suite que cela ne peut pas àvoir lieu ); qu'ils puissent être seulement déposés dans les organes générateurs par une loi d'attraction, bien difficile à la vérité à expliquer, et qu'ils puissent y séjourner tranquillement jusqu'à ce que ces organes soient arrivés à leur développement complet; caril est impossible qu'un père communique à son fils des millions d'œufs, surtout quand ils sont de la grosseur de ceux dont Brera a parlé, et les œufs, 
comme tels, ne peuvent pas non plus se multiplier. Quand on rélléchit combien de sperme l'homme perd ou seulement combien il s'en forme dans ses testicules, et quelle quantité est de nouveau absorbée, avant qu'il ne cohabite avec une femme; quand on considère qu'il ne faut qu'un seul acte de génération pour la fécondation, on serait forcé, si on voulait défendre l'hypothèse de Brera, d'admettre qu'une puissance surnaturelle surveille ces œufs de vers, et que par sa prévoyance il ne s'en détacherait que quand le coït serait fécond, et chaque fois seulement une certaine quantité.

$3^{\circ}$. Quand on pense combien peu de fois nous rencontrons certaines espèces de vers chez l'homme et les animaux ( par exemple, chez le premier, les hydatides, les douves du foie, et les strongles dans les reins, l'hamulaire lymphatique et le polystome pinguicole, deux vers qui n'ont été trouvés qu'une seule fois), on est, pour ainsi dire, forcé de croire que quelques oufs de vers auraient été obligés de parcourir, à cet état, trente à quaranté générations, jusqu'à ce qu'enfin un ver, peut.être après mille ans, eut réussi à sortir de l'œuf. Qui pourrait admettre, je le demande, une pareille supposition?

$4^{\circ}$. Toute cette hypothèse est réfutée par un seul ver, c'est-à-dire le cœnure cérébral dans le cerveau des moutons atteints du tournis. Ce ne sont ordinairement que les agncaux qui en sont incommodés la première année de leur vie; cependant la même chose s'observe quelquefois aussi chez les béliers et 
les brebis. Cette maladie devient toujours mortelle, à moins qu'on ne parvienne à détruire le ver par le moyen de la trépanation. Si le premicr ver de cette espèce avait été créé au même moment que le premier mouton, celui-ci aurait dû être nécessairement détruit par la présence de ce ver, comme cela arrive encore aujourd'hui, et par conséquent la propagation des moutons n'aurait pu avoir lieu. L'expérience cependant nous démontre le contraire.

$5^{\circ}$. Quand même nous trouverions chez les parens les mêmes vers que nous observons chez leurs enfans, il ne serait pas prouvé pour ccla que les derniers les anraient gagnés des premiers. Une telle communication du côté du père n'est pas même imaginable, car quand mêne un mélange réel de l'humeur spermatique des deux sexes aurait lieu pendant l'acte de la génération, chez l'homme et les mammifères, la même chose n'arrive cependant pas dans la plus grande partie des animaux des autres classes du régne animal. J'aurai occasion, par la suite, de démontrer limpossibilité d'un parcil mélange dans unc grande partic des oiseaux.

Spallanzani nous a démontré, par des expériences faites à ce sujet, combicn peu de sperme il faut pour rendre fécond : trois grains de celui d'une grenouille mâlc étendus dans une livre d'cau, furent suffisans pour fécondér une grande quantité de frai de grenouille. L'attouchement seul de la pointe d'une épingle trempée dans le sperme rẹdit un œuf cornplétement f́cond. Qui pourrait donc croire que 
cette petite portion d'humeur spermatique adhérente à la pointe de l'épingle ait encore contenu les oufs de dix espèces différentes de vers, et dont on rencontre souvent six à sept dans une grenouille verte, sans parler des individus dont il y a souvent une centaine à la fois; car on trouve fréquemment les vers suivans dans le corps de cet animal, savoir, dans ses intestins grêles, des strongles, des échinorhynques, des distomes (distoma, Rud. Zed.); dans le rectum, une espèce de nématoïde et des amphistomes; dans les poumons, une espèce de nématoïde et des distomes; dans la vessie abdominale des distomes (tous ces trois distomes diffèrent entre eux spécifiquement); sous les tégumens, des filaires; outre cela ilịy a encore souvent dans lo tissu cellulaire de tous les viscères et muscles des amphistomes enfermés dans des capsules. Comment les oufs des vers de tous ces différens viscères pourraient-ils arriver dans les testicules pour être excrétés de là avec le sperme? Par quel moyen pourraient-ils s'introduire dans l'œuf de la grenouille, et y rester jusqu'à ce que cet animal eut atteint sa maturité? Et comment enfin, claque espèce d'œufs de vers pourrait-elle justement arriver dans l'organe déterminé pour s'y développer. J'ajoute encore que trois espèces de ces vers sont vivipares, savoir l'ascaride (de l'ordre des vématoïdes), provenant des poumous, une autre ascaride du même ordre que le précédent, provenant du rectum, et l'amphistome, du même orgaue.

Si par conséquenı une communication de ces vers 
avait eu lieu par le moyen du père, on devrait voir nager ces jeunes vers dans l'humeur spermatique, à l'aide d'un microscope ordinaire, cependant il n'en est pas ainsi.

Des difficultés insurmontables s'opposent également à ce que la communication se fasse par la mère; car pour qu'elle pût avoir lieu, il faudrait nécessairement admettre que les oufs des vers qui séjournent dans les différens viscères de la mère seraient absorbés par les vaisseaux lymphatiques, et conduits de là dans la masse du sang, qui les déposerait ensuite, par le moyen des vaisseaux exhalans, dans la matrice, d'où le foetus devrait les absorber de nouveau par ses vaisseaux lymphatiques, qui eux-mêmes les porteraient dans le torrent de la circulation, par l'intermède de laquelle ils seraient enfin transmis à des organes propres à leur développement, où ils seraient déposés par les vaisseaux exhalans. C'est, à la vérité, un chemin à la fois long et dangereux que l'œuf aurait à parcourir, car il courrait continuellement le risque d'être transporté dans un autre organe excréteur, où il serait perdu à jamais. Supposous même que dix mille se perdissent a vant que l'un ou l'autre pût atteindre sa destination, on devrait alors retrouver ces œufs, non-seulement dans le sang de la mère, mais encore dans celui du fotus; cependant on n'y en trouve point, et même il est impossible d'en rencontrer, quoique le volume des œufs provenant des plus petits vers, soit, d'après un calcul approximatif de M. Rudolphi, dix mille fois plus gros 
que celui des globules du sang, estimation qui ne paraît pas exagérée. Or, nous savons que les dernières ramifications des vaisseaux que les œufs des vers devraient parcourir à plusieurs reprises, s'ils suivaient le chemin mentionué, ne laissent pas même passer une globule rouge du sang, encore moins un ouf de ver pourrait-il y circuler. Chez les animaux ovipares, la grenouille, par exemple, le commencement primitif de l'œuf, entourć d'une membrane particulière, forme un tout par lui-même, qui dans son origine n'est pas probablement plus grand qu'un œuf de ver. La membrane qui couvre l'ouf de la grenouille est cause que cet œuf ne peut recevoir sa nourriture que sous forme de vapeur. Comment un œuf de ver, visible à l'aide d'une simple loupe, pourrait-il s'y introduire, n'ayant pas d'organes par le moyen desquels il se frayerait un chemin? Ces raisonnemens suffiront, ce me semble, pour se convainere de l'impossibilité de la communication des oufs de vers de la mère à son fotus.

Brera n'est pas de l'opinion que l'on doive regarder ces petits corps ovales, elliptiques et sphériques que nous trouvons dans le corps des vers intestinaux comme des œufs simples, et il prétend que ce ne sont que des capsules dans lesquelles les œufs sont enfermés. Mais comme cette hypothèse n'est soutenue ni par l'analogie ni par l'expérience, on me pardonnera, ce ne semble, si je passe outre.

Eufin l'expérience prouve encore contre l'hypothèse d'après laquelle les parens communiqueraient 
les vers à leurs enfans; je vais le montrer par quelques faits. L'Européen u'est jamais incommodé par le ver de Guinée ( filaria dracunculus), quelle que soit la durée du temps qu'il reste en Europe; cependant cela arrive très-facilement quand il a habité les pays où ce ver séjourne. Il est évident qu'il n'a pas pu en hériter de ses parens, et nous savons également que ses enfans, petits-fils et arrière-petits-fils n'en seront jarnais attaqués, pourvu qu'ils ne se rendent pas eux-mêmes dans ces contrées.

Les cochons domestiques, qui ont sans doute tiré leur origine du sanglier, ont cependant une espèce de vers (le cysticerque, cysticercus cellulosus, Rud.) que l'on ne rencontre jamais chez les sangliers; il résulte alors de là que les cochons n'ont pas pu gagner ces vers par voie de succession.

Les raisons qui nous ont servi à démontrer que les pareus ne peuvent pas communiquer les vers au fotus par l'acte de la génération, ni pendant que celui-ci était encore nourri dans le sein de sa mère, suffisent aussi pour prouver que le foctus ne peut pas les gagner par le lait de celle-ci, ce qui a été admis par Thomas. 11 est d'ailleurs de fait que beaucoup d'enfans, élevés sans avoir tetté, sont souvent trèssujets aux vers. Du reste, cette dernière sorte de communication ne pourrait avoir lieu que chez les mammiféres. On objecte à cela que les oiseaux pourraient communiquer les vers à leurs petits avec la nourriture qu'ils leur dégorgent; mais beaucoup d'oiscaux ne nourrissent pas leurs petits de cette 
manière, et cela n'empêche pas ces derniers d'avoir des vers.

Les amphibies et les poissons ne s'occupent pas du tout de leurs petits, qui habitent souvent d'autres endroits que leurs parens : la salamandre terrestre, par exemple, vit sur terre, tandis que ses petits vivent dans l'eau. Chez les insectes, la mère est ordinairement morte depuis long-temps avant que le petit ne sorte de l'œuf. Comment, dans ces cas-ci, pourrait-il y avoir 'une communication de vers, qui, comme nous l'avons démontré, est impossible dans le sein de la mère.

Les raisonnemens précédens ont, comme je l'es père, convaincu mes lecteurs que les vers intestinaux ne peuvent pas arriver du dehors dans le corps animal; j'ai posé, dans le commencement, pour principe, qu'il ne peut y avoir que deux origines pour les vers ou leurs oufs dans les animaux. Dans l'une ils proviennent du dehors, et dans l'autre leur origine est dans l'animal lui-même, où ils se forment spontanément.

Si j'ai réussi à prouver que la communication par la première voie ne peut s'effectuer, j'aurai alors, par cela même, fourni une preuve, négative il est vrai, en faveur de la réalité de la dernière; je vais essayer maintenant d'y ajouter quelque chose d'affirmatif. Pour atteindre ce but, je suis obligé, autant que cela peut se faire, de remonter à la formation de tous les corps organisés; mais avant d'entrer dans ce sujet, je suis forcé de m'occuper d'abord de la formation probable de notre globe. 
Les recherches sur la formation de notre planète nous ont appris les choses suivantes : la dernière couche jusqu'à laquelle nous avons pu pénétrer est composée de granit, c'est ce qui forme les terrains primitifs; an-dessus d'elle se trouvent les terrains de trausition, placés couche par couche, et au-desșus les terrains secondaires. Outre cela, nous distinguons les terrains d'alluvions et les terrains volcaniques. Dans les terrains primitifs, comme dans ceux de transition, nous ne trouvons pas de traces d'êtres qui aient en vie; ce n'est que dans la première couche des terrains secondaires que nous rencontrons des restes de formations organiques; ce sont en grande partic des crustacés ou des animaux aquatiques du premier degré d'organisation. Dans les couches déposées plus tard, et par conséquent plus haut, nous observons des restes d'animaux qui ont jadis vécu sur la terre; mais dans celles situées un peu plus profondément, nous ne découvrons que des restes d'auimaux dont la terre, dans son état actuel, ne possède plus d'analogues, au moins en espèce. Ce ne sont que les couches les plus récentes des montagues secondaires qui nous fournissent des restes d'animaux semblables aux nôtres, ou ayant au moins quelque affinité avec ceux qui existeut encore. On ne découvre dans aucun terrain secondaire des ossemens d'hommes; il y en a.cependant quelquefois dans des profondeurs considérables; mais ces dernières n'étaient formées originairement que par des crevasses qui ont été probablement remplies plus tard par les éboulemens des parties supé- 
ricures. Nous ne connaissons donc pas encore de véritables anthropolithes. Les vertèbres pétrifiées trouvées par Scheuchzer, près d'Altorf, ont appartenu, comme M. Cuvier ${ }^{x}$ l'a démontré, à une espèce de crocodile, et son témoin du déluge (homo diluvii testis) n'a jamais été regardé par un autre naturaliste que lui comme un véritable squelette d'homme, ce qui résulte des recherches faites à ce sujet par Kargs et M. Cuvier. Le squeletie humain fossile apporté par Cochrane, de la Guadeloupe à Londres, n'est pas non plus un véritable anthropolithe. - Voy. Mémoire sur un squelette lumain fossile de la Guadeloupe, par Charles Konig, écuyer, extrait d'une lettre à l'honorable sir Joseph Banks, dans les Transactions philosophiques, Londres, 1814; dans le Journal de physique et d'histoire naturelle, septembre 1814, p. 196, ainsi que dans lessuivans: Allgemeine geographische ephemeriden, herausgegeben von Bertuch; Julius, 1814, p. 536; et le Bulletin des sciences par la Société philomatique de Paris, livraison de novembre 1814, p. 149.

Cette structure de la terre me paraît devoir conduire à penser que primitivement, au moment où elle formait un tout existant en lui-même, ce n'était en quel que sorte qu'une masse liquide (tropfen) sans forme, mais vivifiée, 'c'est-à-dire douée de l'esprit vivant, ou bien simplement del'esprit universel. On ne doit pas cependant confoudre ce dernier, appelé par moi esprit vivant, avec l'ame du monde de nos philo-

Ossemens fossiles, t. 4. 
sophes, ame qui a créé non-seulement le monde d'après leur opinion, mais qui s'est encore créée ellemême; car je n'entends autre chose par cet esprit que ce qu'on pourrait appeler également la vie, force vitale, en uu mot la cause primitive de la vie en général, de laquelle tous les mondes, lors de leur création, ont été doués, ou plutôt animalisés par l'être des êtres, par Dieu le créateur ${ }^{x}$.

Cette masse s'était probablement détachée du soleil, ainsi qu'il y a lieu de croire que la lune s'est séparée plus tard de nolre terre, l'une et l'autre pour former un tout clos, un système, et pour jouir d'une vie particulière, chacune séparément. Buffon était déjà de celle opinion. (Voy. Epoques de la nature; première époque.) Quoique celte opinion appartienne à un naturaliste déjà un peu ancien, et non pas à un de nos modernes, je ne vois pas de raison suffisante pour ne pas l'adopter. Du reste, dans la nature de mes recherches, il est tout à fait indifférent que notre terre ait formé primitivement un tout en elle-même, ou bien qu'elle ne soit effectivement qu'un morceau séparé du soleil. Je ne disputerai pour cela avec personne, car il est à peu près impossible de savoir quelque chose de certaiu là-dessus.

I Le conseiller Voigt a également désigné, dans ses Elémens d'histoire naturelle publiés en ${ }^{8} 8 r_{7}$, sous le nom d'esprit la cause primitive de la vie; cependant je n'ai pas pu lui emprunter cette expression, par la raison que mon ouvrage avait déjà reçu l'imprimatur au mois de juin 18,5 . 
Peu à peu il se développa dans cette masse liquide sans forme un noyau, c'est-à-dire nos terrains primitifs. La cause principale de cette formation du noyau, ainsi que celle des précipitations successives sur ce noyau, c'est-à-dire des terrains de transition et des montagnes secondaires, peutêtre cherchéc ou dans une force particulière appclée morte et inhérente à la matière comme telle, ou bien dans l'esprit, qui vivifie la substance et qui la conserve comme un tout clos par lui-même. La force que l'on prétend inhérente à la matière a été désignée par nos naturalistes sous le nom de gravitation (gravitatio), que l'on veut expliquer par une tendance vers le centre. Si cette force était la seule agissante sur notre glole, il serait déjà depuis long-temps contracté en une masse morte. On en a par conséquent imaginé une autre, directement opposée à la force attractive vers le centre, ou à la contraction, et on l'a appelée force expansive (expansio). Mais quoique je ne sois pas porté à chercher dans la gravitation, pour me servir d'un terme plus court, la cause de la formation de notre terre et de la conservation de son existence, je ne crois pas cependant que l'on ait besoin, pour expliquer les phénomènes qu'elle présente, d'avoir recours à une nouvelle force expansive particulière. Nous savons que le grand corps attire le petit. Le soleil est en effet un corps beaucoup plus grand que notre terre : la force attractive vers lui-même doit nécessairement affaiblir celle de notre terre vers son centre, car celle-ci est encorc située dans la sphère du soleil, 
dont elle forme une partie intégrante. La force attractive du soleil est affaiblie nécessairement par la graude distance; car sans cela elle aurait depuis long-tcmps englouti la terre; par conséquent elle contrebalance la force attractive de la terre, et empêche celle-ci de se contracter (erstarren ) complétement. D'après cette supposition', la cause des phénomènes doit être attribuée à la force attractive du soleil, et non pas à une force expansive particulière, comme ou l'a admis à tort.

On peut ainsi se rendre raison de la conservation de la terre comme telle, mais nullenıent de l'origine et de la formation des corps isolés, qui formeraient en eux-mêmes un tont, on bien un monde particulier. Si je me représente la force attractive aje la terre vers son centre, comme agịssant de $a$ à $m$, et la force attractive du soleil comme de $z$ à $m$, je conçois que ces forces puissent agil avec une intensité égale ou inégale. Nous avons déjà démontré que ce dernier cas, à ce qu'il parât au moins, ne pouvait pas avoir lieu, par la raison que la terre se serait depuis longtemps contractée en une masse morte, ou bien qu'elle aurait été engloutie par le soleil. Si au contraire le premier cas avait lieu, on ne conçoit pas pourquoi la terre n'aurait pas continué de rester dans le même état dans lequel elle s'est trouvée lorsqu'elle s'est détachée du soleil, et pourquoi elle n'y aurait pas resté jusqu'à sa destruction.

On pourrait cependant objecter que par le mouvemeat de rotation de la terre, un de ses hémisphères 
se trouve toujours moins exposé à l'action de la force attractive du soleil que l'autre, et que la force attractive de la terre devrait alors agir avec plus d'intensité sur l'hémisjuhère où il fait nuit, que sur l'autre où il fait jour, et où la force attractive du soleil devrait avoir la prépondérance.

Quand même nous concéderions cela, et que nous admettrions par conséquent que les deux forces aient continué leur action en ligne directe, c'est-à-dire la force active de la terre de $a$ à $b, c$, etc., et la force réactive du soleil de $z$ à $y, x$, etc., il n'aurait pu résulter de ces actions autre chose qu'une masse norte d'un côté, et d'éther de l'autre '. Il reste cependant inexplicable comment un corps organisé vivant, qu'il soit animal ou bien plante, aurait pu se développer par ces simples réactions.

L'explication de la formation de la terre et de celle des corps organisés offre moins de difficulı́s, si nous cherchons la cause principale dans quelque chose de plus élevé, c'est-à-dire dans l'esprit même, dans là tendance à dominer la matière et à former continucllement, par sa liaison intime avec elle, des tous clos existans par eux-mêmes, comme nous le voyons journellement dans la formation de chaque corps organisé. Dans cctte idée l'esprit sépara d’abord Ja matière

- Ou rigoureusement et métaphysiquement parlant, la terre serait réduite, d'un côté, à un point mathématique égal à rien, et, de l'autre, son expansion aurait ćlć cgalement poussće jusqu'à un rien absolu. 
brute, la rejeta au centre de la terre, et c'est ainsi que les terrains primitifs se formèrent. Peut-être a-t-il fallu des milliers d'anuées pour arriver à ce résultat; car la formation de ces terrains paraît s'être opérée peu à peu par cristalisation. Aprés que la plus grande partie de la matière qui était la moins propice à la vic, c’est-ì-dire à celle des corps isolés, se fut cristallisée, I'esprit put agir déjà plus librement; il s'effectua alors une révolution ou bien une fermentation dans la totalité de la masse, et les terraius de transicion se précipitérent probablement d'une manière subite. Cependant on peut présumer par la disposition stratifiée de ces terraius, que plusieurs fermentations semblables ont dû coutribuer à leur formation. Jusqu'à celte époque, c'est-àd dire jusqu'aı complétentent des terrains de transition, la terre continua encore une vie universelle, c'est-à-dire une vie qui n'élait pas encore divisée, ou bien qui n'était pas encore communiquée à des corps isolés; car nous ne trouvons nulle part, ni daus les formations primitives, ni dans celles de transition, aucune trace d'êtres jadis vivans, et encure bien moins d'organisations aninales.

Ce n'est qu'après la précipitation de ces terrains, que l'espric fut ì même de s'enparer de telles ou telles parties de la matière, et d'en former des corps isolés doués d'uue vie individuelle. Nous trouvous les restes de corps jadis vivans dans les conches inférieures des terraius secondaires, qui, selon toutes les apparences, se sont formós, comme les terrains de tran- 
sition, après des fermentations semblables et partielles. Les corps anciennement vivans que nous découvrons dans les couches inférieures des terrains secoudaires appartiennent tous à des animaux aquatiques; on n'y trouve pas de plautes. A près la formation des terrains de transition et avant la précipitation des premiers terrains secoudaires, il est à présumer qu'il n'y avait point de terrain à découvert, non plus peut-être que d'atmosphère, de mêne que la lune, conme partie détachée plus tard de la terre, en est encore actuellement privée.

Par la suite il s'opéra une nouvelle révolution ow fermentation. La première création fut détruite par la précipitation suivante, el la terre ful de nouveat peuplée d'aninaux qui étaieat cependant d'une autre espèce que les premiers. On ne peut déterminer au juste combien il y a eu de pareilles révolutions suivies de précipitations, qui avaient lieu, chaque fois at moius, sur de grandes étendues de la terre. Il est seulement certain que chaque précipitation fut suivie d'une nouvelle création, et que l'homme est un produit de la dernière ${ }^{3}$; car on n'observe, comme il a été remarqué, aucun ossement d'homme, pas même dans les couches supérieures des terrains secondaires; et, qui plus est, on ne commence à voir des ossemens de mammifères que dans ces couches.

${ }^{x}$ Cela se rapporte parfaitement avec le premier chapitre de la Genèse. On n’a qu' à s'imaginer, comme buffon l'a déja observé, au licu des jours, de grantes éporgues. 
supérieures, et. M. Cuvier ${ }^{3}$ présume par cette raison, qu'ils sont un produit de l'avant-dernière révolution de notre terre.

Comme après chaque précipitation il se formait toujours des êtres plús parfaits, et enfin celui qui jusqu'à présent est le plus parfait de tous, c'est-à-dire l'homme, mon opinion, de voir la canse principale d'action dans l'esprit, ct dans sa tendance à dominer la matière, gagne, par celte raison, toujours plus de probabilité. C'est bien un esprit qui vivifie l'huître et qui anime l'homme; mais l'esprit est, dans les deux cas, pour me servir d'une expression de l'électricité, sous des degrés très-différens de tension; dans l'homme il est monté jusqu'à l'intelligence, et dans l'huître nous trouvons à peine des traces de sentiment. Les animaux de la première création ne pouvaient pas être aussi parfaits que ceux de la dernière; dans la première, l'esprit était encore trop enchainé à la matière, et ce n'est qu'après s'être débarrassé de cette dernière, non propice à l'animalisation, qu'il pouvait agir plus librement, et parvenir à la fin à gouverner l'existence corporelle de l'organisation, à laquelle il esı inhérent; car l'homme animé par l'esprit veut, et sa volonté est une loi pour la nuatière. Cette assertion souffe cependant quelquefois des exceptions dans certains cas; mais alors l'esprit demande plus que la matière ne peut faire, eı nous devons également considérer que l'homme n'est pas un

3 Ossemens fossites, discours préliminaire, p. $7^{0}$. 
pur esprit, mais senlement un esprit borné par la matière de différentes manières. En un mot, l'homme n'est pas un dieu, mais malgré la captivité de l'esprit dans sa corporéité, celui-ci est déjà devenu assez libre en lui pour qu'il s'aperçoive qu'il est gouverné par un esprit plus élevé que le sien, c'est-à-dire par un dieu. Pouvoir ou plutôt devoir comprendre cela est ce qui forme la différence entre l'homme et les animaux, différence que l'on a voulu chercher dans l'absence du ligament cervical et de l'os inter-maxillaire, dans la coïncidence des dents canines, dans la réunion du pouce aux autres doigts dans les extrémités inférieures, dans sa station bipède, etc. Schrank', qui a rendu tant de services à l'histoire naturelle, a placé avec raison l'homme dans une classe particulière du règne animal.

ll est encore à présumer, dans la supposition qu'il y aurait une nouvelle précipitation, que des êtres beaucoup plus parfaits que ceux qui ont été le résultat des précédentes seraịent créés. L'esprit dans l'homme est à la matière dans la proportion de 50 à 50 , avec de légères différences en plus on en moins, car c'est tantôt l'esprit et tantôt la matière qui domine. Dans une création subséquente, si celle qui a formél'homme n'est pas la dernière, il y aurait apparemment des or-

'Briffe an Nau, p. 247. Il a cependant oublié un signe caractéristique, c'est-ì-dire que l'homme peut devenir fon : bonne occasion pour certains critiques de mettre au jour une idée spirituclle. 
ganisations où l'esprit agirait plus librement, et où il serait dans la proportion de 75 à 25 . Il résulte de cette considération, que l'homme a été formé comme tel à l'époque la plus passive de l'existence de notre terre. L'homme est un triste moyen terme entre l'animal et l'ange ${ }^{x}$; il tend aux connaissances élevées et ne peut pas y atteindre; quoique nos philosophes modernes le croyent quelquefois, cela n'est réellement pas. L'homme veut approfondir la cause première de tout ce qui est, mais il ne peut pas y parvenir : avec moins de facultés intellectuelles, il n'aurait pas la présomption de vouloir connaître ces causes, qui seraient au contraire claires pour lui, s'il était doué d'un esprit plus étendu. L'homme se fait une idée incomplète ou fausse du temps et de l'espace, quoiqu'il sache, ou plutôt qu'il doive savoir qu'il n'y a pas de temps pour l'éternité, ni d'espace pour l'infinité ou pour limmensité. Les idées d'espace et de temps lui sont en effet innées, ou bien elles sont jointes nécessairement à son existence conıme homme,

- Je ne veux nullement dire par cela que l'homme soit quelque chose de vil ou de misérable, car il est, au moins sur notre globe, l'êire le plus parfait, le chcf-d'œurre de la création; j’ai voulu seulement indiquer que l'homme n'est ni un ange, ni un dieu, qu'il doit ètre très-pénible pour lui de n'avoir jusłement qu'autaut d'esprit qu'il cn faut pour concevoir qu'il n'en a pas assez pour approfondir les choses qu'il désire, par use tendance innée, le plus ardemment de connaître; cependant il n'a pas le droit de s'en plaindre. Ic prophèic Isaïc s'exprime là-dessus d'une manière trc̀s-justc. Voy. chap. 45 , vers. 9 . 


\section{DE L'HOMME.}

mais elles ne sont pas placées dans l'esprit, qui est infini, sans bornes et éternel, et elles lui sont pour ainsi dire imposées par sa corporéité, par la matière, qui gêne l'action libre de l'esprit, comme esprit dans toute sá pureté. L'hornme, tel qu'il est dans sa corporéité, ne parvient pas même autrement à la connaissance de lui-même que par la réflexion de l'esprit sur la matière. Mais ces considérations n'appartiennent pas à mes recherches, et j'en reprends par conséquent la continuation.

De même qu'il est probable que chacune des précipitations qui formèrent notre globe eut lien subitement, les corps des animaux et des plantes dûrent se former jadis aussi d'une manière subite ou d'un seul jet. Dieu voulut et sa volonté fut faite; car je crois aussi peu que le cèdre du Liban fut originellement un lichen, que l'éléphant doive son origine à une huître ou à un zoophite, en̂t-il passé même par mille gradations ; jadmets encore moins que l'homme ait été originellement un poisson ou un animal couvert d'écailles, comme quelques naturalistes modernes s'efforcent de nous l'expliquer. Si les choses se fussent passées ainsi, alors de pareilles métamorphoses progressives, ou bien des formations graduelles d'êtres, en d'autres de plus en plus parfaits, soit chez les plantes soit chez les animaux, devraient avoir lieu journellement sous nos yeux. Mais, pour parler seulement de l'homme, aucun fait ne nous prouve qu'il y ait dans son organisation physique et morale, aucun progrès qui indiquerait un développement ultérieur; il est toujours le 
même, tel qu'il fut il y a des millièrs d'années. La manière dont les gouvernemens, l'éducation et le sol ont influé sur quelques peuples, ne peut pas être prise en considération; il existait dans les temps les plus reculés des hommes doués d'un esprit élevé et des hommes bornés, ainsi que nous l'observons encore actuellement.

Les vers intestinaux mêmes, qui s'engendrent journellement sous nos yeux, prouvent contre une pareille transformation progressive d'animaux de degrés inférieurs en des animaux de classes plus élevées. En effet, si cela avait lieu, les vers les moins parfaits devraicnt toujours se former les premiers, et les plus parfaits se développer par la suite; mais aucune observation ne nous met en droit de croire qu'une ascaride, par exemple, tire son origine d'une hydatide ou d'un tænia. Dans cette hypothèse on présume, comme cela se voit, que la plus grande perfection consisterait dans une composition plus grande et plus variée, et que l'imperfection serait en rapport direct avec la simplicité; ce que je viens de dire arriverait cependant, quand même l'opposé aurait lieu.

Je ne puis pas décider si les premières plantes et les premiers animaux se sont détachés de la terre comme totalités sans forme, mais ayant une existence propre, c'est-à-dire comme des embryons qui n'auraient reçu leur développenent complet que peu à peu, ou bien s’ils se sont présentés, dès leur origine, entièrement formés et à l'état adulte. Si le premier cas avaiı eu lieu, le développement aurait dî s'opérer 
plus vîte que dans la suite par la voie de la genération. Je crois cependant que le têtard et la chenille existaient avanı la grenouille et le papillon; mais comme tout cela est indifférent par rapport à l'examen du sujet dont je m'occupe actuellement, je passe d'autres recherches de cette nature sous silence.

J'ai voulu uniquement démontrer par la précédente digression, que notre terre dans son état primitif et sans forme, jouissait seulement d'une vic universelle, el que ce n'est qu'après la séparation des substances, qui étaient plus propres à former le squelette du corps de la terre, qu'à jouir d'une vie particulière et individuelle, que la vie se présenta sur notre terre dans des organisations individuelles innombrables.

Si nous considérons l'état de notre terre dans le moment actuel, et les substances desquelles elle est composée, nous pouvons distinguer clairement trois genres de corps.

$1^{\circ}$. Des corps morts inorganiques. -. Minéraux.

$2^{\circ}$. Des corps vivans ou organisés. - Plantes, animaux.

$3^{\circ}$. Des corps sans forme tenant le milieu des deux précédentes espèces. -- Air, éau.

\section{$1^{\circ}$. Des corps morts inorganiques.}

Nous appelons peut-être à tort les minéraux des corps morts, car nous ne savons pas d'abord quelle part ils peuvent avoir à la vie universelle de la terre, 
et ensuite nous trouvons dans le sang chaud et rouge le fer à l'état liquide et par conséquent vivant ; ce métal peut de nouveau reparaître sous forme solide par des procédés chimiques. Nous voyons aussi quelquefois que des parcelles provenant d'un corps organisé se décomposent sur le point le plus élevé d'une roche nue, quelques gouttes de pluie les arrosent, et un lichen vivant se produit. Il suit de là que le mort, le solide, peut devenir partie intégrante de la vie, et qu'il peut être vivifié lui-même.

Il résulte encore de là que les minéraux ne doivent pas être considérés comme absolument privés de vie, cependant ils le sont quand on les compare avec de véritables corps organisés, à cause de leur tension vitale, si peu perceptible. Du reste, dans la nature inorganique, tout se forme par lignes droites, par angles et par cristaux.

\section{$2^{\circ}$. Des corps vivans ou organisés.}

Ceux-ci se forment par lignes courbes : j'y range tous les animaux, les végétaux ou bien leurs parties, n'importe que ces corps aient une vie propre perceptible à nos sens, ou qu'elle se trouve chez eux dans un état latent. Le dernier cas a lieu chez tous les corps privés de la vie dont ils ont joui, que l'on ne doit nullement confondre avec les corps morts ou minéraux; car les corps qui ont cessé de vivre peuvent non-seulement être employés à la conservation de la vie active, c'est-à-dire à la nourriture d'autres corps vivans, 
mais encore ils peuvent être rappelés de nouveau à une vie active propre sous une forme, il est vrai, différente de celle qu'ils avaient précédemment.

On s'étonnerait à tort que j'admette une vie à l'état latent; car nous avons beaucoup d'exemples où la vie propre et individuelle reste pendant un très-long espace de temps dans un pareil état. Nous amassons ordinairement les œufs des poules pendant plusieurs semaines avant que de les donner à couver; les œufs du ver à soie (bombix mori) se conservent d'une année à l'autre, et les graines des plantes peuvent être gardées pendant plusieurs années sans perdre leur vie individuelle. Dans ces différens cas, la vie est assurément dans un état latent; cependant la durée de cette vie propre et latente n'est pas toujours égale, elle est plus courte chez les animaux, et beaucoup plus longue chez les plantes : chez celles-ci elle est même quelquefois tellement longue, que l'on ne peut déterminer au juste le temps de sa durée. Van Swiéten = raconte que des haricots qui avaient été renfermés pendant deux cents ans, ont germé, et sont parvenusà une hauteur très-considérable. Cet auteur a vu lui-même germer des graines provenant de la sensitive (mimosa sensitiva), que l'on avait conservées depuis quatrevingts ans. Des faits semblables de germination de graines de soixante ou soixante-dix ans sont connus de tous les jardiniers. Toutes les graines de plantes ne se ressemblent cependant pas, quant à la durée de leur

${ }^{2}$ Comment. v1, ad $\S 125$, de podagrá , p. 260 . 
conservation; chez les unes le principe vital s'éteint plus tôt que chez les autres. Nous ne sommes pas encore parvenusà découvrir à l'extérieur ouà l'intérieur une différence entre une graine véritablement privée de vic et celle qui en est encore douée. En les mettant toutes deux dans la terre, il ne se développera un germe que de la dernière. Mais le fait qu'il peut se développer de la première de la moisissure et des infusoires, sert de preuve qu'elle n'avait pas perdu la vic universelle en perdant la vie individuelle.

$3^{\circ}$. Des corps sans forme tenant le milieu des deux précédentes espèces : air, eau.

J'ai dit plus haut que les corps sans forme, l'air et l'eau, tienuent le milieu entre les deux autres genres de corps organiques et inorganiques, par la raison qu'ils appartiennent aussi bien à l'un ou à l'autre de ces règnes. D'après les expériences d'Ingenhouss, il se forme de la matière verte ${ }^{\prime}$ dans de l'eau pure mise sous un globe de verre. D'un autre côté, de l'eau mêlée avec une certaine quantité de plâtre calciné se so-

x Cependant il n'a jamais vu se reproduire de matic̀re verte dans l'eau bouillie pendant deux ou trois heures, et qu'il avait empêché de communiquer avec l'air, par le moyen du mercure, quoique le vase qui la contenait eût resté exposé aux rayons solaires pendant dix-huit mois. Cette matière se reproduit cependant, si l'on introduit dans cette eau une substance organisée quelconque, par exemple de la viande toute fraîche, et pourvue encore de sa contractilité. 
lidifie en une masse morte. La partie de l'air atmosphérique que nous appelons oxygène est une condition néccssaire pour la conservation de chaque être organisé doué d'une vie propre et active; ce même oxygène cesse d'être fluide élastique, et devient solide par sa combinaison avec le mercure coulant, et il se forme alors un oxyde solide. L'animal meurt presque subitement dans l'autre partie de l'air atmosphérique, c'est-à-dire dans l'azote, dont le corps animal est en grande partie composé. Il résulte de là que l'on ne doit pas compter ces deux corps sans forme (l'oxygène et l'azote) parmi les corps morts, mais que l'on ne doit pas non plus chercher en eux l'esprit qui vivifie la matière. Cependant ils sont certainement des conditions nécessaires, non-seulement à la formation de chaque être doué de la vie active, mais encore à la conservation des êtres déjà développés; car, pour qu'il puisse se former une nouvelle vie de la matière convenable au développement et à la conservation des corps organisés, il est nécessaire qu'elle passe préalablement à l'état fluide ou sans forme. Cela ne peut s'effectuer autrement que par le mélange de l'air et de l'eau. Chaque corps vivant ou chaque organisation, n'importe qu'elle s'appelle animal ou plante, commence sa vie dans l'état amorphe. La graine mise dans la terre se liquéfie (loest sich auf) avant que le germe ne paraisse; mais la graine ellemême, avant qu'elle se produise comme telle dans la mère plante, n'est qu'une goutte sans forme. Le pre- 
mier développement de chaque animal n'est absolument rien autre chose; car autrement, comment aurait-il pu se faire qu'un foetus se trouvât enfermé dans un autre foetus, et ce dont nous avons plusieurs exemples ? Les plantes nc peuvent prendre leur nourriture autrement qu'à l'état informe ou liquide. Les alimens mangés par les animaux ne peuvent pas non plus être employés à la nutrition avant que ces alimens ne soient passés à l'élat informe; car les animanx reçoivent, également leur nourriture par des espéces de racines qui se tronvent placées dans les intestins. Cette observation a été faite, si je ne me trompe, par Boerhaave.

Mais que deviennent les substances pendant qu'elles. se trouvent à l'état amorphe? Celles qui étaient auparavant unies se séparent et entrent dans de nouvelles combinaisons avec d'autres. Ces séparations, ces décompositions et nouvelles combinaisons n'ont pas seulement lieu à la première formation de chaque corps organisé, mais elles se continuent pendant toute la durée de sa vie individuelle, et ne cessent qu'avec elle. Nous désignous une pareillc décomposition et nouvelle composition de substauces sous le nom de fermentation; par conséquent l'acte de la vie est scmblable à eclui de la fermentation.

Je prie mes lecteurs de ne pas s'impatienter, et de

- Voy. Med. Jahrb. des oesterr. staates II Bd.4tes st.WFien., 1814, 8. s.67.ff. 
vouloir bien me suivre encore quelques momens; car je m’imagine déjà voir quelques personnes sourire de pitié à l'emploi de cette ancienue théorie rebattue de la fermentation; je les entends également demander, avec un ton moqueur, si l'auteur enfin ne va pas distiller des hommes dans sa cornue? Patience, messieurs, cette théorie n'est pas aussi absurde qu'elle paraît l'être. J'envisage la fermentation sous un tout autre point de vue que nos chimistes; ils nous indiquent, par exemple, dans la fermentation du suc exprimé d'une plante avec l'exactitude la plus consciencieuse sur des tables de plusicurs pages, chaque millième partie de tel ou tel gaz dégagé pendaut celte fermentation, ou dans quelle nouvelle combinaison il est entré; ils décomposent encore tout le résidu de la manière la plus exacte, ponr nous démontrer la suite de l'opération, jusqu'à ce que ce suc soit converti en vinaigre; mais ils passent totalement sous silence le dernier produit de la fermentation, c'est-à-dire la formation de la moisissure et des infusoircs; ils font. cependant quelçuefois mention de la fermentation putride qui suit la fermentation acétique, et des substances qui se forment pendant sa durée; mais ils ne disent pas un mot de l'acte particulier de la vie, qui se développe justement à cette époque de la fel\% mentation. Cela provient de ce que je chimiste regarde tous les corps qu'il soumet à son examen comme morts, et qu'il ne fait aucune différence entre un corps mort et celui qui a été privé de vie, entre lesquels il en existe cependant une considérable. 
$1^{\circ}$. Il ne peut jamais se développer du mort ${ }^{x}$ un être vivant, et le mort ne peut jamais non plus servir a la conservation d'une vie déjà existante. On a beau arroser de la terre pure ou de la limaille d'un métalavec de l'eau, et les conserver pendant des années, jamais il ne s'en développera un infusoire. En exposant ces substances au soleil, il pourra peut-être se former de la natière verte, mais c'est un produit de l'eau auquel ces substances mortes mélangées n'ont aucune part. M. Alexandre de Humboldt nous a bien rapporté (Rel.hist.) que les Otomaques, près de l'Orénoque, se nourrissent d'une espèce de terre glaise pendant la saison pluvieuse, qui dure à peu près deux à trois mois. M. Vauquelin a examiné cette terre chimiquement, et l'a trouvée toute pure, sans être mélangée d'aucuue trace de substance organisée; mais, plus loiu M. de Humboldı dit : “ Ces hommes mangent de temps à autre (s'ils peuvent se le procurer) un lézard, un petit poisson ou une racine de fougère. "Ce sont par conséquent ces substances qui servent de nourriture, et non pas la terre pure, qui cependant, malgré toutes les recherches"chimiques, pourrait encore contenir des substances propres à la nutrition, substances qui, pendant l'analyse, ont pu s'évaporer.

Les ours, les marmottes, les loirs et d'autres animaux ne prennent non plus aucune nourriture pendant l'hiver, pas même de l'eau avec laquelle les

"Il faut se rappeler que l'auteur emploie le terme mort pour celui de brute ou inorganique. ( $G$.) 
sauvages de l'Orénoque arrosent la terre qu'ils mangent avant de l'avaler. Si cette cau se trouve chargée d'insectes ou de reptiles pourris, elle peut alors remplacer en quelque sorte un potage d'Oglio. Le passage suivant, tiré de l'ouvragge du même auteur, p. 609, prouve que l'Otomaque ne doit pas être très-difficile dans le choix de sa boisson. "Quand les nations éloignées de l'Orénoque veulent parler de quelque chose très-malpropre, ils se servent d'un proverbe qui dit : que rien n'est si dégoûtant qu'un Ocomaque ne le mange. "Du reste, il est probable que la manière de vivre d'un Otomaque pendant le temps pluvieux, ne doit pas beaucoup différer du sommeil d'une marmotte pendani l'hiver.

$2^{\circ}$. Le corps mort est composé de substauces toutes. différentes que celui qui est organisé, et que les parties qui en proviennent après sa mort. Les corps. morts se laissent aussi bien dissoudre et décomposer que les corps privés de vie. Quelques corps morts, comme par exemple les métaux, se laissent même transformer dans des états sous lesquels on peut à peine deviner leur état primitif ; mais par des procédés chimiques on peut de nouveau les réduire et les ramener à cet état primitif. Par exemple, le fer, dis-o sous dans l'eau sans en altérer la limpidité, peut être de nouveau réduit à son état métallique. Des corps organisés privés de vie se laissent également décomposer par l'art dans leurs substances originelles, mais jamais un chimiste n'a réussi à rendre ensuite à un. corps organisé et décomposé sa structure véritable: 
et primitive. Le chimiste peut décomposer le cinabre en soufre et en mercure; il peut préparer avec le premier de lacide sulfurrique, du sublimé corrosif avec l'autre, et dissoudre ce dernier dans l'eau. II résulte de là deux liquides aussi limpides qu'elle, et daus lesquels on n'aperçoit pas une trace de l'état solide des substances employées primrtivement. Le chiniste peut eependant tirer de ces deux liquides, par des nouvelles opérations, du cinabre solide tel qu'il était originairement, et qui aura toutes les qualités de celui avec lequel on avait préparé ces liquides. Cela ne se passe pas de la même manière dans les corps organisés. En versant de l'eau bouillante sur de l'amidon, il se forme une sorte de bouillie, mais le chimiste emploiera inutilement tout son art pour former de l'amidon tel qu'il était. Sitôt que le sang sorti de la veine s'est divisé en partic fibreuse et en partie aqueuse, jamais on ne parviendra à lui rendre son ancienne liquidité.

$3^{\circ}$. Tous les corps morts, c'est-à-dire les minéraux autant que nous en connaissons, diffèrent les uns des autres par la nature de leurs parties constituantes; il en est même qui ne consistent que dans une seule substance. Tous les corps organisés sont composés de plusieurs substauces, mais tous des mêmes : la différence ne consiste que dans la diverse proportion de ces substances entre elles.

Cependaut on ne doit pas croire que la cause principale de la vie repose dans le mélange de ces substances; l'admettre serait du matérialisme tout pur. 
La cause principale de la vie est placée dans ce quc j'ai nommé l'esprit, et que l'ou pourra appeler $x$ si l'ou veut; mais qui est tout à fait différent du mélange des substances, et par lequel ce mélange devient vivant ${ }^{3}$. Si la vie n'était que le produit d'un certain mélange proportionné des substances, le chimiste, après avoir décomposé un corps organisé, pourrait redonner à ce dernier sa structure primitive; mais c'est ce que ne peut faire le chimiste, par la raison qu'il n'est pas maître de l'esprit. Celui-ci cependant

' On m'objectera que je cherche la cause principale de la vie dans une force cachée inexplicable, et que l'esprit est une faculté occulte (facultas occulta). Mais sommes-nous plus avancés dans l'explication des autres forces? Nous apercevons seulement les phénomènes dans ce monde corporel, et nous concluons de là une cause principale occasionelle, que nous appelons force, sans avoir expliqué quelque chose avec cela : qu'est-ce autre chose que la force vitale, si en faveur aujourd'hui, qu'une faculté occulte, qu'une cause à nous inconnue de certains phénomènes que nons appelons phénomènes de la vie, ou simplement la vie? Que nous a expliqué Newton, en disant : la gravitation est l'attraction vers le centre de la terre? Quelle est la cause de cette allraction? peut-être que les grands corps attirent les petits; bien : mais quelle est la cause que le plus grand attire le plus petit? Personne ne peut l'expliquer, et personne ne le comprendra aussi longtemps que notre esprit sera captivé par notre corporéité; mais nous le saurons quand cette tension élevée de l'esprit, ce que nous appelons dans le sens strict esprit on intellt toence, aura quitté la matière ; car cel esprit arrivé dans mon moi jusqu'à l'intelligence existera assurément toujours, et ne sera janais cimplojé à la vivification d'un limaçon ou d'une graine quelconque. 
peut faire beaucoup plus, car il peut changer même des substances dans d'autres, et en créer de nouvelles qlii n'existaient pas auparavant. Nous ne trouvons dans l'eau et le pain ni ammoniaque, ni phosphore, ni urée, etc.; mais nous pouvons tirer ces substances du corps des hommes et des animaux qui se sout uniquement nouris d'eau et de pain. M. Vauquelin a fait des recherches sur la formation de la chaux dans le corps des poules, et a trouvé que les alimens qui avaient servi à la nourriture d'un de ces oiseaux, ne contenaient pas autant de chaux, à beaucoup près, qu'il y en avait dans les coques de l'œuf et les matières fécales. Cet auteur a trouvé au contraire une diminution de la terre siliceuse contenue dans ces alimens. Une autre particularité digne d'être remarquée, c'est que le sang des animaux à sang chaud reste à peu près toujours dans le même degrré de température, sous des degrés très-différens en plus ou en moins de température extérieure; la vie par conséquent n'est pas un produit du mélange de certaines substances, mais bien un produit de l'esprit.

Quand l'esprit doit former, par le mélange de ces substances une nouvelle vie individuelle, ou quand il doit conserver celle qui existait déjà, il est absolument nécessaire, comme nous l'avons déjà démontré plus haut, que les substances se trouvent à l'état amorplı.' Si, d'après la supposition que nous avons établie daus le commencement, la totalité de la terre se trouvait à l'état sans forme avani l'existence des corps organisés vivans; $c t$ si, également d'après notre 
supposition, ces corps se développèrent seulement de la matière amorplie, wous ne devons pas assurénent nous étonner si la même chose se produit encore aujourd'hui, e'est-à-dire, si, partout où il se trouve de la matière animalisée à l'état sans forme, il se développe encore de nouvelles vies individuelles ou bien de nouvelles organisations. Que nos infusions ne produisent pas des éléphans ou des baleiues, et qu'il ne se forme dans nos fumiers que des champignons et non pas des chênes et des pins, cela est facile à concevoir, quand on compare les masses en fermentation entre elles; car celles qui fermentent sous nos yeux, et qui produisent de nouvelles vies individuelles, ne sont pour ainsi dire que des points mathématiques en comparaison avec la totalité de la masse de notre torre jadisen fermentation. Qui sait quel serait le produit, si des millions de grands corps organisés étaient soumis à la fois à une pareille fermentation prolongée et entretenue avec soin?

Ce que j'ai dit jusqu'à présent sert à prouver qu'il ne peut pas être question de mort dans toute la nature organisée. La mort n'est qu'une transition à une nouvelle vie ou bien it une autre forme de vie ${ }^{3}$ : c'est une vérité que nous trouvons constatée dans toute la na=

- Terra nostra telluris putredinis producta absurbcndo nigra et fortitissima cradit, hinc plantis prostantissimum probet pabulum. IIinc elucescit morte, et putrefuctione hominis corpus non pcrive, sed duntaxat ejusdem structuram organicam deleri, et perenni circulo elementurum unius destructionem alterius esse generationem. Plenk, IIysrogologia. 
ture organique. Les corps privés de vie ne servent pas seulement, comme nous l'avons déjà souvent remarqué, à la conservation de la vie déjà existante, mais encore il peut même s'en développer dẹ nouvelles vies individuelles, comme la moisissure, les nostocs, les champignons, la matière verte de Priestley, les infusoires, etc., nous le prouvent jusqu’à l'évidence.

Mais jentends ine faire cette question : est-ce une chose bien prouvée et constatée, que ces organisations doivent leur existence à une formation spontanée ou primitive, et qu'elles ne se sont pas développées, comme toutes les autres, par le moyen de graines et d'œufs? Un critique ${ }^{x}$, après s'être appuyé dans son texte sur des argumens bien faibles contre la formation spontanée des vers intestiuaux, s'exprime encore dans une note de la manière suivante : "Les infusoires ne nous mettent pas tout à fait en droit de détruire l'axiome omne vivum ex ovo ";

- Annalen der litteratur und kunst in dem oesterr kaiserthum gtes IIeft. 1812 in der Recension des Buchs : de tæuniâ latâ vom Hrn, Prof : Reinlein, s. $3 \mathbf{1} 7$.

${ }^{2}$ L'axiome omne vioum ex ovo a été attribué à tort par ce critique à Linné, tandis qu'il provient de Harvey. Si ce critique aime à s'appuyer sur des autorités, je peux cependant lui en opposer une autre, qui dit justement le contraire ; il la trouvera dans l'ouvrage de M. Oken, ayant pour titre : Die Zeugung. Voyez la dernière page, où il est dit: nullun vivum ex ovo, omne vivum è vivo. Un grand partisan de M. Oken, M. Goldfuss, a cependant rétabli l'axione omne vivum ex orn; mais ce dernier auleur ne veut pas 
car il est plus facile de concevoir que ces animaux se formeuı de germes existans, mais développés depuis peu, que d'admettre qu'ils tirent leur origine d'un mélange produit par la décomposition des végétaux infusés. ")

Il est vrai que ce mode de formation ne saute pas immédiatement aux yeux, c'est probablement pourquoi ce critique ne l'a pas pu comprendre.

Quand certains auteurs se trouvent embarrassés, et qu'ils ne savent trop que dire, ils cherchent alors prudenıment à cacher leur ignorance sous des expressions douteuses et hasardées : cela a au moins un air savant, mais il s'en faut beaucoup que ce le soit réellement; car on ne sait trop quelle conséquence on pourrait tirer d'une manière de voir qui, du reste, n'apprend rien. Le vulgaire des lecteurs s'imagine cepeudant assez souvent à tort qu'un parèil langage renferme quelque chose d'une certaine importance.

Dans toutes les organisations je ne connais que les modes suivans de génération : ou elle s'opère par la naissance de petits vivans ${ }^{3}$, par des oufs et par des graines $^{2}$, ou bien par marcottes ${ }^{3}$, en y comprenant la reproduction des greffes et bourgeons. On appelle germe, dans le langage usité, la première formation perceptible d'une plante ou d'une de ses parties, ou,

désigner par là un ouf pondu par un animal, mais bien un œuf inaginé, et appelé par cet auteur zoographique polarisé.

" Génération vivipare.

* Génération ovipare.

${ }^{3}$ Génération scissipare. 
en un mot, son premier développement visible; mais je ne puis pas me former une idée de la sorte de germes dont les infusoires pourraient se développer. Ce critique n'a pas osé parler d'œufs, par la raison que l'on n'en aperçoit point chez ces animaux. Il a employé par conséquent le mot germe, par lequel il s'est imaginé produirc le même effet que celui que quelques auteurs se promettent en ajoutant une barre à la fiu d'une phrase, c'est-à-dire d'inviter les lecteurs à pousser encore plụs loin la pensée de l'auteur, si cela leur est possible. Cependant ce critique exige encore davantage de ses lecteurs, comme cela résulte du passage suivant, p. 316 : "J'ai trouvé les reins d'une femme couverts de grandes hydatides (an strongylus hydatis gigas?'), mais à cette époque j'étais trop peu naturaliste pour examiner ces vers. "Il paraît que ce critique s'est imaginé qu'il était plus savant lorsqu'il avança une erreur aussi grave. Pour mettre mes lecteur's à même de concevoir toute l'étendue de l'erreur contenue daus ces trois mots, je suis obligé de leur expliquer les deux premiers l'un après l'autre. Les helminthologues désignent par le mot strongle un ver qui a le corps allongé, cylindrique, élastique, aminci vers les deux extrémités, pourvu de fibres musculaires, d'une.ouverture buccale, d'un canal alimentaire très-visible et d'un appareil générateur de l'un ou de l'autre sexe. Le strongle géant ( strongylus gigas), chez lequel M. Otto croit même avoir découvert un système nerveux, a été très-souvent confondu par les médecins avec l'as- 
caricle lombricoïde (ascaris lombricoides) Le strongle se trouve représenté pl. III , fig. 3. On comprend sous le nom d'hydatide (hydatis) une espèce de boule d'eau, c'est-à-dire un sac formé par une membrane trèsnince, presque toujours d'une figure sphérique, contenant une liqueur transparente, limpide comme de l'eau, ou bien trouble, et sur lequel on n'aperçoit aucun organe ni à l'extérieur ni à l'intérieur.

Quel homme, je le demande, peut réunir ces deux idées disparates dans une seule? On ne devrait pas au moins charger une personne capable d'avancer des choses aussi incohérentes, de juger les écrits scientifiques des autres .

Je conseille, au reste, à ce critique, de lire un passage du second livre de Samuel " et de bien étudier la Biologie de Tréviranus. Je recommande également ce dernier ouvrage, vraiment classique, à tous mes lecteurs qui auraient encore quelque doute sur la formation primitive de la matic̀re verte de Priestley, des infusoires et de la moisissure.

Tréviranus n'a pas seulement examiné avec une attention particulière les expériences de Needham, Wrisberg, Otto-Frédéric Müller, Ingenhouss, etc., auteurs qui croient à cette formation; mais il a encore écudié avec beaucoup de sagacité les recherches de Spallanzani et Therechowsky, auteurs qui ont cru réfuter, par leurs expériences, celles des naturalistes que nous venons de mentionner. Treviranus, du

- Livre de Samuel, chap. ıo, vers. 5. 
reste, a fait lui-même encore quelques expériences à ce sujet; en un mot cet auteur a, pour ainsi dire, épuisé cette matière, et ce serait une vaine entreprise de ma part de vonloir encore y ajouter quelque chose ; car celui qui n'a pas été convaincu par 'Tréviranus de la formation primitive des infusoires, ne le sera pas nou plus par moi, et ne le sera probablement jamais.

Quant à moi, je regarde comme une chose prouvée la formation primitive de la moisissure et des infusoires, par le moyen des corps organisés privés de vie.

Mais s'il se développe réellement de ces derniers des organisations vivantes et existantès par elles-mêmes, une semblable formation, ce me semble, doit d'autant plus facilement avoir lieu dans les organisations vivantes clles-mêmes. Il nous est encore permis d'admettre que les organisations qui se développent dans le vivant, n'importe que ce soit dans un homme, dans un animal ou dans une plante, doivent être beancoup plus parfaites que celles formées d'un corps privé de vie, par la raison que le principe de la vie, dans le premier cas, se trouve monté plus haut, et agit d'une manière plus intense. L'expérience même nous le démontre aussi réellement. Il se forme des plantes ou des organisations privées de vie, selon les circonstances, tantôt de la moisissure et tantôt des infusoires. Nous voyons, par exemple, que des infusoires se développent dans la colle de farine fortement délayée, et que de la moisissure croît sur un morceau 
de viande rotie mouillé; mais le contraire a lieu si la chair trempe dans beaucoup d'eau, et si on laisse fermenter la colle telle qu'elle est'. Dans les organisations vivantes, le nouveau produit est toujours déterminé par la nature de l'organisation dans laquelle il a été formé. Des lichens et des mousses croissent sur des plantes, et il se forme des vers intestinaux, des pous et des cirons dans le corps animal.

Je m'attends que l'on me dira : comment, vous croyez aussi que les pous doivent leur existence, dans certains cas, à une formation primitive? Ces animaux pondent des œufs; la manière et le mode de leur développement et de leur génération sont par conséquent tout à fait évidens. Je sais très-bien que ces animaux pondent des œufs, et qu'il en est de même d'une grande partie des vers intestinaux, et que d'autres parmi ceux-ci sont même vivipares; cependant je prétends que les vers intestinaux, c'est-à-dire ceux qui se trouvent pour la première fois dans le corps animal, sont le produit d'une formation spontanée, et qu'il ev est de même des pous dans certains cas. L'on voit quelquefuis sur la tête d'un enfant en bas âge une quantité innombrable de ces animaux, sans cependant observer d'œufs, et on remarque encore que la mère et la nourrice de cet enfant n'en avaient point. D'où provienuent alors les pous dans un cas senblable? On me dira sans doute : il est possible quic

- Voyez Rheinische Jahy bucher fiir med. und chir. v. Harles Bon! 1820. $(B r$. 
cette communication ait été opérée par une troisième personne. Supposé même que les choses se fussent passées ainsi, nous pouvons cependant présumer avec raison que cette personne n'aurait pas pu transmettre, pour ainsi dire, invisiblement, une si grande quantité de pous; en admettant même que cette personne eût communiqué à l'enfant deux ou trois individus, ceux-ci, avant de se multiplier de cette sorte, auraient dû d'abord pondre des œufs, que l'on aurait infailliblement remarqués dans les cheveux de cet enfant; cependant cela n'a pas eu lieu, car de pareils petits pous se développent dans quelques cas, pour ainsi dire dans une seule nuit, et souvent on ne peut pas même parvenir à les détruire. D'où viennent encore les pous dans cette affreuse maladie nommée ph thiriasis, de laquelle nous avons des exemples même récens, qui ont été rapportés par M. Hufeland ${ }^{*}$ ?

M. le professeur Rust a traité en Pologne un employé atteiut de cette maladie, et il a fallu neuf mois à ce médecin avant de le guérir radicalement, malgré l'emploi des remèdes les plus efficaces. 11 n'est pas aussi difficile de détruire les pous qui ont eté gagnés par une communication directe. Il y a neuf à dix ans que beaucoup de marchands allemands se rendirent par la Bosnie et l'Albanie à Salonique. Le troisième ou quatrième jour de leur voyage, tous ces hommes furent couverts de pous qu'ils avaient gagnés de

I Jahrăgang 18 × 3. 3tes Heft page $122, f$. 
Turcs, qui voyageaient dans leur société. Après le départ de ces derniers, et après s'être bien nétoyés, ils en furent entièrement débarrassés.

Ce fail démontre la grande différence qui existe entre les pous gagnés par communication directe et ceux qui se sont formés spontanément. M. le docteur Fechner croit avoir observé que quelques personnes atteintes de maladies chroniques, et qui n'avaient pas élé auparavant incommodées par les pous, commencèrent cependant à en être remplies peu de temps avant de mourir.

M. Rust m'a communiqué l'observation suivante, digne d'être citée. Voici ses propres expressions : "Je fus engagé, en ISo8, par M. le docteur Müller, pendant que je séjournais à Zaslaw, en Volnie, à la cour du prince Sangusko, à assister à une consultation devant se faire chez un enfant juif du sexe masculin, âgé de treize ans, qui avait une très-grosse tumeur sur la têtc, et pour laquelle on avait déjà employé inutilement heaucoup de remédes sans pouvoir la dis. soudre. Je m'y rendis et je vis que la plus grande partie du crâne était occupée par une tumeur très-élevée, mollasse, mais sans la moindre fluctuation. Je ne pus non plus trouver ni trace d'inflammation actuelle ou passée, ni lésion ou anomalie dans les tégumens du crâne. Le malade avait un air cachectique et se plaignait seulement d'une démangeaison insupportable dans l'intérieur de la tumeur, qui semblait avoir éte formée par métastase à la suite d'une fièvre nerveuse. Cette tumeur s'était grossie dans l'espace de 
huit jours jusqu'à un volume considérable. Pour nous faire une idée exacte de la nature du contenu, nous convinmes de faire une incision dans la tumeur, et cela fut pratiqué sur-le-champ; alors une quantité énorme de petits pous blancs en sortit, lesquels, réunis et mesurés ensemble, remplissaient une pinte de Pologne; ce fut tout ce que contenait la tumeur. Des frictions faites avec l'onguent mercuriel sur les tégumens du crâne, et des injections de même nature dans la cavité de la tumeur, jointes à l'usage de médicamens intérieurs, rétablirent bientôt le malade sans que nous ayons pu connaître la nature de celte maladie singulière; il est encore digne de remarque quece malade, d'après les renseignemens donnés par les parens, n'avait jamais eu auparavant de croûtes laiteuses sur la tête, et qu'il avait eu toujours moins de pous dans son enfance que les autres enfans n'out coutume d'en avoir."

Le ciron (acarus exulcerans, L.), dans mon opinion, n'est pas la cause, mais une production de la gale, ou du pus qui est contenu dans les boutons des galeux; c'est par cet e ràison que l'on ne rencontre pas cet insecte dans toutes les éruptions de cette natare, et sa production paraît dépendre de causes particulic̀res à nous inconnues. D'Azara raconte : «Quelques habitans du Paraguay sont sujetsà une espèce de gale qui diffère de la gale ordinaire; il se forme dans chaque bouton un petit insecte blanc de la grandeur d'une puce. Ce sont ordinairement des femmes qui les extraient avec la pointe d'une épingle, et la guérison 
de ces malades en résultc. J'ai vu retirer plus de soixante de ces insectes des fesses d'une femme. Il paraît qu'ils ne se produisent pas par l'acte de la génération, mais bien par une disposition particulière des humeurs du malade. Les vers ${ }^{x}$ que l'on rencontre dans les reins de l'aguara-guaza (espèce de chien sauvage) semblent avoir la même origine. ")

Mais j'entends dire : nous ne pouvons nullement concevoir comment un corps organisé vivanı pourrait se développer sans l'intermède d'un autre corps organisé de même nature, ou semblable à lui sous tous les rapports. Je conviens qu'une pareille formation n'est pas facile à comprendre, mais j'ajoute que la plus grande quantité des phénomènes de la nature en général, sont encore des problèmes pour nous; car, je le demande, quel est l'homme qui sache au jusle comment une nouvelle vie individielle se produit par la voie de la génération? Celle des mammiféres est pentêtre la moins difficile à concevoir; mais celle de la plus grande partic des autres animaux est, pour nous, aussi incompréhensible que la formation primitive. On ne doit pas s'imaginer que la différence entre les mammifères vivipares et les animaux ovipares soit aussi peu considérable que plusieurs physiologistes, et entr'autres Gautieri, se plaisent à croire. Ce dernier, par exemple, place les vivipares et les ovipares sous

I Il résulte de ce que cet auteur dit dans son Essai sur l'histoire naturelle des quadrupèdes de la province du Paraguay, t. 1 , p. 3. 3 , que ce sont des strongles géans. 
le rapport de la générątion dans la même classe, et il admet pour toute différence entre ces deux classes d'animaux, que l'œuf, chez les premiers, se développe dans la matrice elle-même, et dans les autres au dehors. Il est vrai que le mode de génération des salamandres et des orvets vivipares, et celui des lésards et couleurres ovipares, n'est pas très-différent ; bien plus, c'est presque le même; car chez les lésards el les couleuvres l'œuf parfait se sépare de la mère avant que le jeune animal ne soit formé complétement. Chez les salamandres et les orvets l'ouf séjourne dans le sein de la mère jusqu'au développement complet du petit, cependant, sans que cet œuf semble avoir plus de rapports directs avec sa mère, que celui qui est déposé immédiatement sur la terre par la sienne.

Les différences sont plus considérables et plus essentielles entre les mammifères vivipares et tous les autres animaux ovipares ou vivipares; les voici :

$1^{\circ}$. Chez les nammifères un accouplement complet a lieu. Les humeurs spernatiques du mâle et de la femelle se mêlent ensemble, au moins cela paraît se passer ainsi.

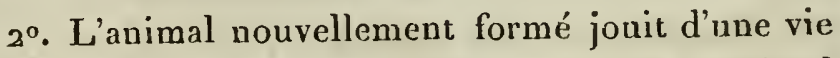
propre, dès le moment de la fécondation. Cet animal semble, à la vérité, ne consister, au cómmencement, que dans un point sautillant (punctum saliens); mais c'est néanmoins déjà un véritable animal; il grandit, il se développe peu à peu, et il se sépare de sa mère après un temps exactement déterminé; mais il meurt 
si le développement progress:f de sa formation est interrompu par une cause quelconque. Les choses se passent autrement si la conception a eu lieu hors de la matrice, et chez le fotus enfermé dans un autre.

$3^{\circ}$. Ce qu'on nonme à tort l'œuf ' (car on ne peut pas avec juste raison lui donner ce nom, en le comparaut avec les véritables œufs) ou bien les membranes qui entourent le nouvel animal, se forment plus tard:

$4^{\circ}$. Le placenta, quelque forme qu'il ait, par lequel le jeune mammifère reçoit sa nourriture, est placé hors de ce prétendu ouf, ou bien hors de ses membranes, et il est au moins dans une communication médiate avec la mère; car, quoique-les vaisseaux du placenta ne s'anastomosent pas avec ceux de la matrice, cette dernière acquiert cependant un volume beaucoup pluś considérable pendant la grossesse, c'est-ì-dire pendant le développenen $\iota$ du jeune animal; les vaisseaux de cet organe se dilatent considérablement, et il en suinte des substances nutri-

- Les vésicules de Graafne sont pas de véritables œufs, et elles ne doivent nullement être comparées avec des œufs provenant d'animaux ovipares; car le jeune animal ne se forme pas dans des vésicnles; elles ne grossissent pas comme d'autres cufs, et elles ne descendent pas non plus dans la matrice. Ossiander s'exprime làdessus ainsi : "Les œufs de Graaf ne sont pas de véritables œufs, mais bien des bourses gélatineuses de forme irrégulière et de grosseur très-variable. Les corps, de couleur jaune, ne sont également rien autre chose que des bourses semblables remplies d'une matière (smegrma) colorée. Voyez Gocttingsche gelehirte anzeigen 1814.163 tes stüsts. 
tives selon le besoin du foetus, qui se développe toujours de plus en plus. Ces substances sont absorbées par les vaisseaux du placenta et communiquées ensuite au jeune animal. En un mot celui-ci se nourrit aux dépens de sa mère aussi long-temps qu'il reste enfermé dans son sein.

Examinons à présent ce qui a lieu dans les animaux ovipares, et surtout chez les animaux á sang chaud, les oiseaux, qui ont le plus d'affinité avec les mammifères; car, si nous voulions aller plus loin, nous pourrions facilement rencontrer des animaux chez lesquels nous ne trouverions plus de points de comparaison.

$1^{\circ} .11$ se détache de la totalité de la masse de l'ovaire de la poule une goutte d'une substance amorphe, et il s'en forme un tout clos en lui-même, le commencement de l'œuf. Ce développement primitif de l'œuf perceptible à nos yeux est bien renfermé dans une poche, qui est fixée à l'ovaire par un pédoncule, mais l'ouf forme néanmoins par lui-même un tout séparé. $2^{\circ}$. L'œuf qui s'est formé spontanément dans le corps de la poule, c'est-à-dire sans l'intermédiaire du coq, se développe néanmoins et jouit d'une vie individuelle. Il grandit, et non pas comme un cristal, par couches de substances semblables (per juxtapositionem), mais par sa propre force vitale interne (per intussusceptionem ). 11 faut cependant que l'œuf recoive du dehors, c'est-à-dire de sa mère, les substances nécessaires à son propre accroissement; car sans cela il ne pourrait pas atteindre son développement com- 
plet. Il paraît aussi qu'elles doivenı être extrêmement simples et ténues pour pénétrer à travers des nembranes qui peuvent retenir l'air dont on les a remplies. L'œuf forme, de ces substances qui lui ont été communiquées par sa mère, le jaune et le blanc; il les prépare lui-même, car il ne les reçoit pas tout formés de la poule. L'œuf, par conséquent, a sa vie propre parfaitement semblable à celle des autres corps organisés. Après avoir altein son développement complet, il se couvre, daus le rectum, de la coque calcaire, et il ne diflère à l'extérieur en rien d'un ouf fécondé par l'intermédiaire du coq. La différence se trouve donc dans son intérieur, en ce qu'il ne peut jamais s'en développer un petit. L'ouf non fécondé et formé spontanément dans la poule, nous fuurnit ainsi un exemple remarquable d'une vie individuelle dans une subsiance saus forme, car le jaune et le blanc sont liquides. Comme l'ouf se forme, se conserve et se développe tout à fait à la manière de tous les corps organisés vivans, nous sommes par celle raison obligés d'admettre qu'il viı réellement.

$3^{\circ}$. L'ouf, qui s'était déjìt formé avant que le cor n'eût agi sur la poule, est bien rendu fécond par cet acte de la génération; mais ici il ne s'opère pas, comme dans l'accouplement des mammifères, un mélange des humeurs spermatiques; il est même impossible qu'un peu de sperne du coq puisse arriver jusque dans le voisinage de l'ouf. Chez les coqs et la plus grande partic des oiseaux, les canaux déférens partent des testicules, et ils se terminent dans le 
rectum par deux petits mamelons; mais ceux-ci sont beaucoup trop petits pour atteindre le rectum de la poule, et encore moins pour pouvoir s'y introduire pendant l'accouplenıent; les plumes ınêmes offrent un obstacle à l'éjaculation du sperme jusqu'à cet endroit. Par conséquent, dansce cas-ci rien de corporel ou de matériel ne peut contribuer à la fécondation, et ce qui rend l'œuf fécond doit être placé dans une force particulière, qui a nćcessairement le sperme pour conducteur; cependani cette force se communique à l'œuf d'une manière inconcevable pour nous, et lui donne, dans quelque degré de développement qu'il soit (car plusicurs neufs sont fécondés à la fois, et chacun se trouve dans un état particulier de développement) la faculté de produire un petit animal sem. blable à ses parens.

$4^{\circ}$. Mais le commencement de la vie individuelle du jeune oiseau ne résulte pas encore de cet acte de la gévération, et de cette fécondation, la possibilité de son développement est seule déterninée par là. Clıez les manmifères, la nouvelle vie commence an moment même d'un accouplement fécond, et après un espace de temps exactement déterminé pour chaque espèce, le jeune animal se sépare de sa mère. Cela ne se passe pas ainsi chez l'oiseau. Nous remarquons bien le germe dans un œuf fécondé; mais le punctum saliens, c'est-à-dire le commencement de la vie individuelle, ne se dévcloppe que pendant l'incubation, et à dater de cette époque seulement, on peut déterminer le moment de la sortie du petit. Il est du reste 
indifférent que l'œuf ait été fécondé à un degré quelconque du développement qui lui est propre; mais ce qui est seulement nécessaire, et ce qui détermine la principale différence entre le développement d'un mammifère eı celui d'un oiseau, est que, chez le dernier, l'œuf doit être complétement développé avant que la nouvelle vie individuelle puisse commencer, au contraire de ce qui a lieu dans le premier; d'où résulte la cinquième différence entre les mammifères et les oiseaux, c'est-à-dire que le placenta ne se trouve pas au dehors, mais bien dans l'intérieur de l'œuf, dans lequel il a déjà été amassé par avance autant de substance qu'il en est besoin pour nourrir et conserver le jeune oiseau jusqu'au moment de sa sorlie, tandis que cela se passe tout différemment chez les mammifères, comme nous l'avons déjà vu.

Ces réflexions peuvent suffire pour démontrer la grande différence qui existe réellement entre le mode de génération des mammifères vivipares et celui de toutes les autres classes d'animaux. Nous avons également montré dans nos réflexions qu'une nouvelle vie individuelle peut réellement se développer spontauément daus un corps organisé: l'œuf non fécondé de l'oi. seau nous en fournit un exemple remarquable. Lion m'objectera peut-ĉtre, quel'appareil générateur de la poule est conformé de manière à ce qu'un ouf puisse ou plutôt doive s'y développer spontanément, et que la productiou des oufs est inhérente aux fonctions, naturelles des organes sexuels de cet anima]. Mais je pourrais alors aussi bien admettre que la production 
des ascarides et des cestö̈des est inhérente aux fonctions du canal intestinal, et que la formation des douves et des hydatides appartient aux fonctions du foie. Si on voulait me dire que ces vers ne se trouvent ni dans tous les intestins ni dans tous les foies, je répondrais qu'il y a également des animaux qui ne sont pas non plus susceptibles d'être fécondés, et qui ne pondent jamais d'œufs. Les personnes qui regardent la production des vers intestinaux comme le résultat ou plutôt comme la suite d'une lésion des fonctions des organes en général, peuvent encore avoir raison. Il reste cependant certain que telle ou telle espèce de vers se développe seulement dans des organes déterminés; car les douves du foic, par exemple, se produisent seulement dans le foie, etc. Nous reviendrons plus tard sur ce sujet.

J'ai tâché jusqu'à présent de démontrer que le développement d'un jeune animal, surtout quand il n'appartient pas à la classe des mammifères, opéré à la suite d'un accouplement fécond, est aussi incompréhensible que le développement d'un animal sans l'intermédiaire de parens, .c'est-à-dire par formation primitive ou spontanée. Nous sommes donc obligés de recourir à la formation probable des premiers corps organisés; ce n'est pas dans l'espoir de comprendre par là la génération ou une formation quelconque, n'importe de quelle manière elle s'opère, mais bien pour qu'il nous soit possible de déduire les mênes effets des mêmes causes. Eu suivant celte route, nous devons nécessairement arriver à des corpss organisés 
qui n'ont pas été produits par des parens, mais bien qui se sont développés spontanément par la matière sans forme; car j'ai tâché de démontrer dans le commencement de cette digression, que notre terre existait long-temps avant la formatiou des corps organisés isolés, et que les matières brutes ont dû être séparées d'abord comme masses mortes, avant que l'esprit ait pu transformer les parties intégrantes de la matière épurée en corps isolés doués d'une vie individuelle. Nous avons également démontré que plusieurs créations semblables ont dû avoir lieu sucessivement, et qu'elles ont été la suite des fermentations générales de la terre. Chaque corps organisé vivant doit par conséquent être considéré comme une partie intégrante de toute la terre vivante; et il forme en lui-même un monde, qui est à la terre comme celle-ci est au soleil. Il s'opère en lui en petit ce qui s'opère cn grand, ou plutôt ce qui s'est déjà opéré dans la terre; car depuis que la vie individuelle de cette dernière a été divisée et communiquée à tant d'êtres isolés, elle jouit d'une vie plus tranquille; sa vie actuelle esı à comparer, pour ainsi dire, au sommeil ${ }^{x}$ La vie in-

${ }^{3}$ Ce sommeil est cependant quelquefois interrompu par des rếves violens, en considérant comme tels les éruptions volcaniques, les tremblemens de terre, les écroulemens de montagnes, elc. Du reste, nous ne pouvons pas démontrer avec certitude qu'il n'y aura pas un jour encore une fermentation générale de la terre; dans ce cas on peut trìs-bien s'imaginer que d'antres substances pourraient être encore précipitées comme brutes au-dessus des 
dividuelle, principalement celle des organisations animales, est plutôt à comparer à la vie de la terre lors de ses époques tumultueuses, où les grandes fermentations suivies de nouvelles créations avaient lieu. La vie universelle, ou bien l'acte éternel de fermentation, a été en quelque sorte transférée à des corps isolés doués de vie. Dans chaque organisation animale existe une fermentation contiuuelle, pendant laquelle de nouvelles substances sont admises, précipitées , appropriées, dissoutes, décomposées et excrétées; en un mot, la vie consiste dans une décomposition continuelle et dans une combinaison nouvelle de substances. Si nous réfléchissons à la grande quantité de substances animalisées sans forme qui se trouvent dans chaque corps animal, est-il alors étonnant qu'il puisse se former de celle dont le corps n'a pas besoin pour sa nutrition, ou qui n'y est pas même propre à cause de sa composition particulière, une petite masse, un tout existant par lui-même, ou bien qu'il se produise un ver intestinal dans ce petit monde ( mitcrocosmus), à l'exemple du grand, dans lequel se dévcloppa jadis un ver de terre. Ce nouveau ver unc fois

autres masses mortes, et qu'il se produirait alors des êtres dans lesquels l'esprit agirait encore plus librement que dans l'homme; car quoique la création actuelle ne puisse pas exister sans eau liquide et sans une atmosphère qui fait monter le mercure à vinglhuit degrés dans le baromètre, il ne suit nullement de là que la même chose devrait avoir nécessairement lieu dans une création subséq̧uente. 
formé, pourra ensuite multiplier son espèce en produisant des animaux semblables à lui. Nous sommes obligés de nous imagiver, cómme il a été déjà remarqué, les parens primitifs de tous les animaux à nous connus, comme des animaux originairement sans parens, et cependant comme doués de la faculté de régénérer leur espèce de manière différente; et justement les différens modes de génération que l'on ol)serve chez les vers intestinaux, prouvent que la marche de la nature créatrice eu petit est tout à fait semblable à sa marche en grand, et qu'il ne s'opère actuellement rien autre chose dans le corps animal vivant, que ce qui s'opèra de même anciennement dans notre globe vivant. En effet, chez les vers intestinaux, on trouve, pour ainsi dire, une répétition de tous les modes de génération qui existent dans la série des organisations animales.

L'échinocoque se trouve au plus bas degré de formation organique. J'ai eu occasion de faire les observations suivantes sur son mode de génération. Un foie de bœuf pesant cinquante-deux livres, était rempli d'hydatides, parmi lesquelles il y en avait quelques unes de la grosseur du poignet d'un homme robuste. Je tâchai de les disséquer à la manière accoutumée, mais je ne pus atteindre mon but, parce que la membrane interne ou bien la membrane particulière du ver était fortement adhérente aux parties environnautes, parties par le moyen desquelles la capsule s'était formée. En incisant une de ces vessies d'une extrémité à l'autre, il en sortit une quantité 
considérable de vésicules formées par une membrane très-mince remplie d'eau, et de différentes grosseurs, les plus petites noins grosses qu'un pois, et les plus fortes de la grosseur d'une noix. En ouvrant une des dernières, je trouvai qu'elle en contenait encore plusieurs plus petites, et ce ne fut que dans celles-ci que je remarquai la matière appelée granuleuse ( $m a-$ teries granulosa), c'est-à-dire les véritables échinocoques. J'ai trouvé également des emboîtemens semblables dans une liydatide énorme provenant du foie

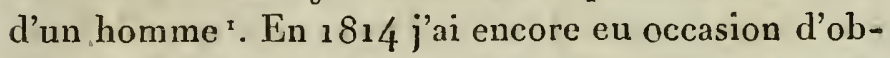
server sur les poumons et le foie d'un chameau (camelus dromedarius) la génération des échinocoques, ce qui m'a permis de confirmer ce que j’avais vu précédemment. Ces animalcules, placés au plus bas degré de la série animale, se multiplient par conséquent de la rnanière la plus simple. L'animal qui reproduit ses descendans cesse lui-même d'être un animal et se transforme en une membrane dans laquelle ses petits se trouvent enfermés, comme la graine cesse d'exister comme telle, sitôt que le germe de la nouvelle plante s'est développé en elle. On observe un semblable mode de génération chez le kolpode capuchon (kolpoda cucullus) ${ }^{2}$ et chez le volvoce globuleux (volvox globator $)^{3}$.

- Voyez, plus loin, le chapitre sur l'échinocoque.

o. Fr. Müller, Verm. terrestr., vol. I, part. 1, p. 58.

${ }^{3}$ Resel Insecten belustigungen, 3 Th. s. 6r 7. Tab. 101, fig. 1, 2, 3. De geer in den schwed. Abhandl. aif das Jahr 176r. Bd. 23 . s. 112, tab. IIx, fig. $1-5$. 
On n’a pas encore pu découvrir les organes destinés à la génération chez le cysticerque, animal également d'une structure très-sinple : je crois cependant avoir observé quelque chose à ce sujet. J'ai trouvé dans la cavité thoracique de deux campagnols (mus arvalis, L.) des cysticerques en assez grand nombre, et qui y nageaient librement ${ }^{x}$. Ces vers élaient ì peine un peu plus gros qu'un grain de millet. A la vessie terminale de plusieurs de ces individus, on voyait pendre an dehors un, plus souvent deux, mais plus rarement trois jeunes cysticerques. On ne doit nullement croire que j’aie peut-être pris des aspérités de la vessie caudale pour des vers, car on voyait clairement leur cou (leur tête, à cause de sa petitesse, ne put pas être vue ni séparée ) à l'aide duquel ils étaient implantés comme par un pédoncule sur la mère. Il paraît que Goeze a observé quelque chose de semblable. Par conséquent la génération de ces vers s'opère en quelque sorte par marcottes, comme cela a lieu chez les polypes et chez les animaux du corail.

Le polycéphale (polycephalus, Zed., conürus, Rud.) fait peut-être la transition du premier mode de génération au second. Ici plusieurs têtes sont implantées sur nne vessie commune. Peut-être ces têtes se forment-elles peu à peu : mais nous ne savous encore rien de positif là-dessus²

- C'était, en effet, une rencontre très-rare, car ces vers, ainsi que les hydatides en général, sont ordinairement enfermés dans des capsules particulières.

${ }^{2}$ Voyez Fischer dissert. ; il y est question d'un polycéphale de la 
Les cestoïdes sont de véritables liermaphrodites, et les articulations du même ver peuvent s'accoupler mutuellement.

Les trématodes sont androgynes. L’apparcil générateur des deux sexes se trouve réuni dans chaque individu; mais celui-ci ne peut pas se féconder luimême; il a besoin pour cela d'un autre individu de son espèce, qui est fécondé à son tour par le premier. Les trématodes sont en général ovipares, mais d'après le témoignage de Zéder, l'amphistome subelaviforme (amphistoma subclavatum), provenant du rectum des grenouilles, est vivipare. 11 y a par conséquent ici un rapprochement daus le mode de génération avec les animaux d'une organisation plus parfaite, c'est-à-dire avec ceux de la classe des reptiles, qqui sont également en général ovipares; mais dont quelques-uns sont aussi vivipares, comme les orvets, les vipères, les salamandres, elc.

Les acantocéphales ont bien des parties sexuelles séparées, et le mâle se distingue par une vessie qui se trouve placée à la partie postérieure, mais il n'y a pas d'accouplement, et la fécondation s'opère d'après la supposition très-probable de M. Rudolphi, comme chez les poissons, les crapauds, les grenonilles, etc., par aspersion des oufs avec le sperme du mâle au dehors de la mère.

Nous arrivons enfin aux vers intestinaux du plus grosseur d'un pois, que l'on a trouvé dans les ventricules d'un mouton d'Espagne. ( $B r_{\text {. }}$ ) 
haut degré d'órganisation, c'est-à-dire aux nématoïdes. Ceux-ci ont des organes sexuels séparés. Les femelles sont pourvues d'un vagin, et les máles ont un péuis (qui est peut-être double et bifurqué) très-facile à apercevoir. On trouve dans le corps des premières une espèce de matrice et des oviductes, et dans celui des derniers des vaisseaux spermatiques. La plus grande partie de ces vers est ovipare; mais il y en a quelques-uns qui sont vivipares, comme, par exemple, le genre entier des cucullaus, et les nématoïdes provenant du poumon et du rectum des crapauds, des grenouilles et de quelques autres reptiles.

Quoique les vers intestinaux aient la faculté de se reproduire de tant de manières différentes, cepen-dant, comme ils ne peuvent pas être transmis d'un animal dans un autre, ce qui a été déjà prouvé, il faut nécessairement que les premiers de ces individus; c'est-à-dire ceux qui se trouvent pour la première fois dans un corps animal, n'importe lequel, se forment spontanément. Mais ici il ne s'opère rien autre chose que ce qui s'était déjà souvent opéré en grand sur la terre: chaque fois que la terre changea la forme de son existence ${ }^{1}$, la création existante fut aussi chaque fois détruite. La même chose arrive quand l'animal,

- Je ne veux pas parler ici de la forme extérieure, car elle a été probablement toujours sphérique; mais.j'entends les différens modes d'être, les différens états sous lesquels elle s'est trouvée, depuis qu'elle a formé un tout existant par lui-même. 
dans lequel les vers s'étaient produits et multiplićs, meurt. L'animal, après sa mort, passe à une autre forme d'existence, ou bien à une autre forme de vie; le ver, qui est le produit de l'animal daus lequel il séjourne, doit, par conséquent, nécessairement changer avec lui, en même temps, sa forme d'existence ou bien sa forme de vie. Le ver est soumis, comme l'animal dans lequel il s'est produit, aux lois de la putréfaction, à moins qu'un helminthologue ne s'en empare à temps, et ne sauve la forme primitive du ver.

Si quelqu'un voulait m'objecter que dans ma manière de voir, la terre a toujours produit à chacune de ses métamorphoses des êtres qui ne ressemblaient pas à ceux des précédentes, mais que ce n'est pas le cas chez les vers intestinaux, puisque de temps immémorial nous rencoutrons toujours les mêmes vers chez l'homme, et que, par consequent, ma comparaison ne peut se soutenir ; je répondrais à cela que notre terre, à l'exception de la lune, n'a pas produit úc globes ou de petits semblables à elle. Du reste, nous ne savons pas ce qui se passe dans la lune; nous ignorons également si elle n'a pas déjà subi les mêmes métamorphoses que notre terre, ou bien si elle est encore à les éprouver. Chez les hommes et les animaux, le semblable reproduii le semblable; par conséquent, les animaux qui se sont développés d'eux el dans cux, doivent nécessairement se ressembler. Nंous observons cependant dans le microcosmus, dans l'homme comme animal, sonmis à une métamorphose moins 
lente, une différence ou une tendance prédominante à la formation spontanée de tel ou tel parasite, selon que ce microcosme se trouve à tel ou tel degré de développement. Ainsi, pour ne parler que de nos vers, il ne se produit chez les enfans en général, car il n'y a rien sans exception dans ce monde, que des ascarides et des oxyures, ct chez les adultes, nous observons plus souvent des cestoïdes, des strongles, etc. Voilà donc encore ici un rapport avec ce qui a eu lieu.

La certitude de la formation primitive des vers intestinaux est donc clairement établie, non-seulement par des preuves négatives, puisque les vers intestinaux ne peuvent pas arriver du dehors dans le corps animal, mais elle est encore déduite de l'analogie de la formation primitive des infusoires, de la moisissure, etc. , et enfin elle cst démontrée par l'induction tirée de la formation première probable de tous les corps vivans; mais, pour un observateur exact, il existe encore des preuves directes.

M. Rudolphi x croit avoir observé le développement du tænia dans un chien. On rencontre assez fréquemment le géroflé (caryophyllocus mutabilis, Rud.) daus les poissons du genre cyprinus. Ce ver a reçu son premier nom de sá ressemblance avec un clou de girofle, et la grande variabilité de la partie antérieure de la tête lui a valu le second. J'ai remarqué très-souvent le premier développement de ces vers; j'ai trouvé notamment bien souvent dans la

Entoz., vol. I, p. 41 . 
mucosité, dont les parois internes des intestins de ces poissons sont enduites, de petits corps de la longueur d'une ou deux lignes, et même quelquefois un peu plus, qui remuaient et qui donnaient clairement des sigues de vie. Ces corps ressemblaient, quant à leur couleur et à la nature de leur substance, aux géroflés; mais ils étaient dépourvus de tête. Je puis assurer que la tête n'était ni déchirée ni rétractée, car je les ai examinés long-temps avee la plus grande exactitude, et jamais je n'ai vu quelque chose qui ressemblât à la tête. A près les avoir nétoyés, à l'aide d'un pinceau, de la mucosité dont ils étaient entourés, on voyait qu'ils formaient un tout clos, et on ne remarquait, à l'une ou l'autre de leurs extrémités, aucun filament, aucune villosité, comme cela aurait eu lieu si la tête avait éié déchirée. La formation primitive de ces vers s'opère, suivant moi, de la manière suivante : une partie de la mucosité intestinale, ou plutôt de la mucosité vivante et sans forme, se coagule en une masse un peu conipacte; elle se couvre d'un épiderme, et jouit, dès ce moment, d'une vie propre; la tête se forme par la suite, et à la fin les organes de la génération paraissent. Cette supposition a gagné chez moi encore plus de probabilité, par la raison que j'ai rencontré de pareils géroflés beaucoup plus petits, pourvus d'une tête complétement développée. 11 paraît, par conséquent, que tantôt une plus grande quantité, et tantôt une plus petite quantité de cette mucosité, se coagule en une totalité, et forme le commencement du ver. Ces 
vers, incomplétement développés, sont cependant tous amiocis vers une de leurs extrémités, absolument conme le clou de girofle. J'ai trouvé également quelques individus qui comnençaient déjà à devenir plus larges et plus transparens à l'autre extrémité; c'était, par conséquent, le premier développement de la tête.

Pourrait-il rester encore quelque doute sur la formation primitive des vers intestinaux, après tant de preuves, tant négatives qu'affirmatives?

Celui qui douterait de la formation primitive par la seule raison qu'il ne la comprend pas, et que les animaux ont, en général, la faculté de se multiplier par l'acte de la génération, serait à comparer à un homme qui voudrait nier l'inflammation spontanéc de la matière électrique, la foudre, par la raison qu'il ne peut produire lui-même des étincelles électriques que par le frottenient des corps solides.

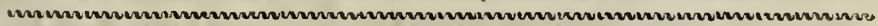

\section{CHAPITRE DEUXIEME.}

Division systématique des vers intestinaux en général.

On sai que les médecins et les naturalistes anciens. n'étaient pas très-avancés dans l'helminthologie : leur science se bornait à connaître quelques-unes des espèces de vers intestinaux que l'on rencontre dans le corps humain et que l'on trouve désignés, dans leurs. ouvrages, sous les noms suivans : ascarides, lumbrici 
teretes, lumbrici lati, tcenia lata, tania solium, vermes cucurbitini. Ces derniers ont été regardés à tort comme formant un genre particulier, puisque ce ne sontque des articulations détaclıées du tænia. Le dragonneau (filaria dracunculus) était également connu par beaucoup de persomes; mais on ne le plaçait pas parmi les vers intestinaux, parce $q u^{2}$ on le confondait avec lc filaire aquatique (gordius aquaticus), et que l'on croyait que ces deux vers n'en formaient qu'un.

Avant Redi, médecin du grand-duc Cosme III de Médicis, dans le dix-septième siècle, il n'était venu dans l'idée de personne d'examiner les animaux. sous le rapport helmintlologique : il fut le fondateur le cette science; mais il se contenta de décrire et de dessiner les vers intestinaux à mesure qu'il en trouvait, et cela ne pouvait pas se faire autrement à cette époque. Après lui, cette branche de l'histoire naturelle resta long-temps inculte; personne ne s'en occupa d'une manière spéciale, à l'exception de Léonard Frisch, qui a fait insérer quelques mémoires sur les vers intestinaux, dans les Mélariges de Berlin.

Daus la deruière moitié du siècle précédent, Pallas, Otto-Fréd. Müller et Otto Fabricius commencèrent à regarder ces animaux comme dignes de quelqu'attention. Linné leur assigna bien une place dans son Système de la nature, mais il les rangea parmi les autres vers, et plaça les filaires (gordius filaria), les ascarides (ascaris) ct les douves ou fascioles (distoma), parmi les vers intestinaux; l'hydatide et le tænia, parmi les zoophytes. 
La société des sciences de Copenhague paraît avoir éveillé le goût pour cette science, principalement parmi les naturalistes allemands, en proposant pour prix, en 1780 , de chercher, par l'expérience et le raisonnement, si les œufs des vers intestinaux, comme, par exemple, ceux du tænia, de la douve-fásciole, sont innés dans les animaux, ous'ils y arrivent du dehors, et, dans ce cas, d'exposer les moyens propres à s'opposer à leur introduction. La grandequantité de vers intestinaux que l'on découvrit alors journellement, et qui sont si différens. entre eux, tani par leur forme extérieure que par leur structure intérieure, néccssita une classification.

Bloch, qui remporta le prix, les a divisés dans son ouvrage en deux ordres : le premier comprend les vers plats ou larges; le second, les vers ronds. 11 compte, parmi les premiers, les ligules (ligula), les douves (distoma) et le ver solitaire; et parmi les seconds, les hydatides et les caryophillées.

Goeze se contenta d'établir seulement des genres.

Otto-Fréd. Müller ${ }^{x}$ et François de Paula Sclıranck suivirent son exemple; ces deux derniers ont publié presqu'en même temps, c'est-à-dire en ${ }^{7} 787$ et en 1788 , des tableaux de tous les vers intestinaux découverts jusqu'alors; mais il manque, dans tous ces travaux, un bon principe de classification, et les différens genres de vers intestinaux sont souvent rapprocliés d'une manière peu naturelle.

. Naturforscher, st. 22. s. 33 et 86. 
Nous devons à Zeder, homme du plus grand mérite, les prenières bases d'une bonne division systématique; elle fut publiée en 1800 , dans son premier supplément pour l'histoire naturelle des verş intestinaux, par Goëze : il est nalheureux qu'il ait renoncé depuis à l'étude de cette science. Il partage tous les vers intestinaux en cinq elasses, auxquelles il a substitué plus tard le nom de familles, d'après le - conseil de M. Rudolphi '. Celles - ci sont subdivisées en genres, qui quelquefois sont subdivisés de nouveau en sections, et les genres en espèces. Cette division systématique a été adoptée par M. Rudolphi, dans son grand ouvrage; cependant il a trouvé convenable de faire quelques changemens dans la circonscription des genres et dans leur disposition. Il a aussi remarqué que les deux derniers ordres ne sont pas naturels. Je crois, en outre, que le troisième et le quatrième auront besoin de quelques modifications, et je pense que M. Rudolphi sera obligé de réunir dans son ouvrage supplémentaire les monostomes hypostomes (monostomata hypostomata) et les polystomes pentastomes (polystomata pentaslomata), qui formeront, avec le caryophillée, un sixième ordre, que l'on sera peut-être obligé de placer entre le troisième et le quatrième.

Wilbrand a imité, comme M. Rudolphi, la classification de Zeder. M. Olfurss s'en est écarté de même que M. Cuvier, et ce dernier a réuni des

"IVicdemanns archiv. II. s. 4t. 
vers extérieurs avec les vers intestinaux; enfin, M. Brera a classé les vers intestinaux de I'homme à sa manière : il en forme cinq ordres, qui sont encore subdivisés en douze genres et en vingt-six espèces, ce qui fait justement une fois plus que je n'en connais; il est vrai qu'il y en a quelques-unes parni elles qui ne sont pas même des vers. Je vais me bornerà exposer la classification de M. Ruidolphi, telle qu'il l'a établie dans son Traité sur les entozoaires ${ }^{\text {. }}$.

\section{ORD. I. NÉMATOIDES (Nematoidea).}

Corps arrondi, élastique ; canal intestinal complel, pourvu d'une bouche et॰d'un anus. Les deux sexés séparés sur deux individus différens.

Cet ordre comprend des vers allongés, cyliudiriques dans presque toute leur longueur, et plus ou moins atténués aux deux extrémités. La tête, non distincte, est obtuse ou tronquée, quelquefois accompagnée de membranes latérales. L'ouverture de la

M. Bremser, dans son ouvraģe, do nne ici la méthode suivie par M. Rudolphi dans son grand Traité sur les entozoaires; mais il nous a invités à y substituer celle que ce dernier a adoptée dans son Synopsis, publié en r818. Nous avons cru faire encore mieux eu traduisant presque mot à moí l'extrait qu'en a publié à Vienne, en 1822, M. le docteur Fischer, aide-naturaliste dans l'université impériale de Vieune, sous les yeux de M. Bremser lui-même, sous le titre suivant : Brevis entozoorum seu vermium intestinalium expositio et methodus cosdem investigandi ct consercandi, cum icone; nous en reparlerous dans notre supplément. 
bouche offre de bons caractères génériques dans sa forme, et surtout dans l'existence des lèvres, des tubercules ou des valvules dont elle peut être pourvue. La queue continue avec le corps, a son extrémité obtuse ou aiguë, droite ou oblique, fléchic ou non. Les individus mâles ont presque toujours le corps plus cour't, plus grêle que les femelles; l'extrémité de leur queue infléchie offre souvent des aiguillons ou des filamens qui appartiennent à l'appareil de la génération. Dans les deux sexes, les organes internes de la génération, les ovaires dans les femelles, les testicules daus les mâles, ont la forme de longs filamens très-fins qui s'entortillent autour du canal intestinal, souvent plus coloré que le reste, et leur communication extérieure se fait par un orifice médian situé vers le tiers antérieur du corps.

Les différentes espèces de cet ordre se trouvent dans toutes les classes d'animaux beaucoup plus souvent dans le canal intestinal que dans le tissu des autres parties.

On les partage en onze genres, qui sont les suivans:

\section{G. i. Filaire, Filaria.}

Corps allongé, cylindrique, presque d'un égal diamètre dans toute son étendue; bouche orbiculaire; l'orgıne excitateur mâle formé par un seul ou par un double aiguillon.

Les filaires se trouvent non-seulement dans les animaux vertébrés, mais encore dans les insectes et dans 
leur's larves; savoir dans le tissu cellulaire sous-cutané, autour des yeux, des oreilles, des joues, du cou, sous la peau des narines, entre les muscles du. cou, dans l'œsophage, dans la cavité de la poitrine, daus les bronches, dans les cavités de la plèvre, adhérens au cœur, dans l'estomac, à la superficie externe des intestins, dans le foie, le mésentère, l'épiploon, dans la région lombaire ou coxale, et même dans les parties génitales; libres, adhérens aux organes ou comme mêlés avec le tissu cellulaire.

\section{A. Espèces à bouche simple.}

Le F. de MÉdine , F. medinensis, dans le tissu cellulaire de l'homme.

Le F. GRÈLE, $F$. gracilis, dans la cavité abdominale des sapajous.

Le F. A TTÉn UÉ, F. attenuata, dans la cavité abdoninale du geai, C. frugilegus.

B. Espèces à bouche papilleuse ou labiée.

Le F. papilleux , $F$. papillosa, dans la cavité thoracique du cheval.

\section{G. II. TRIChоsome, Trichosoma.}

Corps arrondi, élastique, très-fin en avant et s'accroissant peu à peu en arrière; bouche ponctiforme à l'extrémité amincie; l'organe excitateur mâle formé par un fil simple contenu dans une gaine.

Les espèces de ce genre existent chez les mammi- 


\section{SUR LES VERS INTESTINAUX}

fères, les oiseaúx et les amphibies, entre les membranes de l'estomac, dans les intestins grêles ou gros, et surtout dans le cœeum, dans la vessie urinaire.

lix. Le T. InfLÉCH , $T$. inflexum, des intestin's du merle bleu, et très-communément de ceux des pigeons et de beaucoup d'autres oiseaux.

\section{G. III. Trichocéphale, Trichocephalus.}

Corps arrondi, élastique, capillaire en avant et se renflant subitement en arrière; bouche orbiculaire; l'organe excitateur mâle simple, et contenu dans une gaîne.

Dans l'intestin coecum des mammifères.

\section{A. Espèces inermes.}

Le T. de L'номme, $T$. dispar, dans l'intestin cœcum de l'espèce humaine.

Le T. DÉprimé, $\boldsymbol{T}$. depressiusculus, daus les intestins du chien.

\section{B. Espèce armée.}

Le T. HÉrissé, $\boldsymbol{T}$. echinatus, dans l'estomac du lézard apode ( Lac. apoda, L.).

\section{G. IV. OxyuRE, Oxyuris.}

Corps arrondi, élastique, subulé à sa partie postérieure; bouche orbiculaire; organe excitateur dans une gaîne.

- LLes espèces de ce genre n'ont été jusqu'ici trou- 
vées que dans les gros intestins des mammifères. L'une d'elles, la plus commune, l'O. vermiculaire, O. vermicularis, placée par Rudolphi parmi les ascarides, se trouve communément dans le rectum del'espèce humaine; elle est figurée pl, I et II, fig. 3 ct 1-2.

I'O. Douteux, O. ambigua, vient des intestins du apin sauvage.

\section{G. V. Cucullan. Cucullanus.}

Corps cylindrique, élastique, aminci postérieurement; la tête pourvue d'une bouche orbiculaire et d'une espèce de capuchon strié; organe excitateur mâle formé par un aiguillon sinjple.

Dans les intestins et l'abdomen des reptiles et des poissons.

Le C. ÉLÉcant, C. elegans, dans les appendiccs. pyloriques de la sandre ( perca lucio-perca, L.).

G. VI. Spiroptère, Spiroptera.

Corps arrondi, élastique, aminci anx deux extrémités; la bouche orbiculaire ; l'organe excitateur sortant entre les ailes latérales d'une queue roulée en spirale.

Dans les animaux vertébrés, entre la troisième paupière et le bulbe de l'œil, dans l'œsophnge, l'estomac; très-fréquemment entre les membranes et dans des tubercules de cet organe chez'les poissons, 
dans les intestins et la vessie natatoire de ces mêmes animaux.

\section{A. Espèce à bouche nue.}

Le Sp. strongloïde, $S$. strongylina, dans l'estomac du sanglier.

B. Espèce à bouche papilleuse.

Le Sp. овтus, S. obtusa, dans l'estomac de la souris.

On a aussi trouvé des spiroptères dans la vessie urinaire de l'espèce humaine.

\section{G. VII. Pirysaloptère, Physaloptera.}

Corps arrondi, élastique, aminci aux deux extrémités; la bouche orbiculaire; la queue du mâle défléchie, ailée de chaque côté, ct pourvue inférieurement d'une sorte de vessie; l'organe excitateur sortant d'un tubercule.

Dans l'estomac des mammifères, des oiseaux et des reptiles.

Le P. enfermé, $P$. clausa, dans l'estomac du hérisson.

\section{G. VIII. Strongle, Strongylus.}

Corps arrondi, élastique, aminci aux deux extrémités; la bouche orbiculaire ou anguleuse; la pointe de la queue du rnâle terminée par une bourse d'où sort l'organe excitateur.

Les espèces assez nombreuses de ce genre ont été trouvées dans les trois premières classes d'animaux 
vertébrés, dans la caisse du tympan, la trachéeartère, les bronches, l'osophage, les poumons, le cour, les intestins, le foie, les reins, et dans des tumeurs anévrismales des artẹres mésaraïques.

A. Espèce à bouche orbiculaire ou aiguillonnée (sclerostomata).

Le S. ARMÉ, S. armatus, dans l'intestin cœcum du cheval.

B. Espèce à bouche orbiculaire et papilleuse.

Le S. Gx́ant, S. gigas, dans les reins de l'homme; du chien, de la loutre, du phoque, du cheval, du bœuf, etc.

C. Espèce à bouche nue.

Le S. FILAIre, S. flaria, dans la trachée-artère et les bronches des moutons, où il est souvent en grand nombre.

\section{G. IX. Ascaride, Ascaris.}

Corps arrondi, élastique, atténué aux deux extrémités; la bouche à trois valvules; l'organe excitateur. mâle formé par un double aiguillon.

Dans presque toutes les parties du corps des animaux vertébrés; une seule espèce a été découverte dans un animal du type des mollusques (lepas fascicularis ), par M. Sœmmering. On trouve des ascarides dans la gorge, l'œsophage, entre les bronches, dans les poumons, l'estomac et surtout dans les intestins, leurs membranes et leurs tubercules, dans l'abdomen, le foie, la rate, le mésentère, le périnée, le plus souvent libres, mais aussi quelquefois adhérens. 
Ce genre, le plus nombreux parmi les vers intestinaux, contient un très-grand nombre'd'especes, dont quatre-vingt seulement sont bien déterminées.

A. Espèces atténuées également aux deux extrémités.

\section{a. La tête nue.}

L'A. nombricoïde, A. lombricoides, dans les intestins de l'homme et de plusieurs autres animaux mammifères.

\section{b. La tête ailée.}

L'A. Du CHAT, A. mystax, dans les intestins du chat domestique.

B. Espèces dont l'extrémité antérieure est la plus grosse.

\section{a. La tête ailée.}

l'A. vermiculatre, A. vermicularis (oxyuris vermicularis, Br.), dans le canal intestinal de l'espèce humaine.

L'A. tacheté, $A$.maculosa, dans le pigeon domestique.
,
b. La tếle nue.

L'A. DENTÉ, $A$. dentata, dans le canal intestinal du barbeau (cyprinus barbus).

C. Espèces dont l'extrémitế postérieure est la plus grosse.

a. La têté nue.

L'A. spiculigìre, $A$. spiculigera, dans l'estomac du cormoran (pelecanus carbo). 
b. La tếle ailée.

L'A. serpentule, $A$. serpentulus, dans le héron cendré.

G. X. Oрниолтоме, Ophiostoma.

Corps rond, élastique, atténué aux deux extrémités; la bouche pourvue de deux lèvres; l'une supérieure et l'autre inférieure.

Dans les intestins grêles et gros des mammifères et des poissons.

L'O. SPHÉROCÉPHALE, O. sphcerocephalus, des gros intestins de l'esturgeon ( $A$. luuso).

\section{G. XI. LiorhynQue, Liorhynchus.}

Corps rond, élastique; bouche à l'extrémité d'une sorte de trompe érectile et lisse.

Dans l'estomac et les intestins de quelques mammifères et de plusieurs poissons.

Le L. DE L'ANG UILLE, $L$. denticulatus, dans l'anguille.

\section{ORD. II. ACANTHOCÉPIALES (Acanthocephala).}

Corps subarrondi, utriculaire, élastique; l'extrémité antéricure prolongée en une sorte de trompe rétractile et garnie de crochets disposés par séries. Les deux sexes distincts sur des individus différens.

Cet ordre ne contient encore qu'un seul genre. 
G. XII. LchinonirneUe, Echynorhynchus.

Corps subarrondi, utriculaire, élastique; une trompe rétractile pourvue de crocliets, les sexes distincts sur des individus différens.

Les espèces de ce genre, qui sont assez nombreuses, existent dans toutes les classes d'animaux vertébrés; le plus souvent fixées dans le canal intestinal, entre ses membranes et même à l'extérieur dans l'épiploon et le péritoine; on en a aussi trouvé quelquefois au cou, sous la peau.

La forme de la trompe, l'existence ou l'absence d'un rétrécissement ou d'une espèce de cou entre elle et le corps, l'existence ou l'absence de crochets sur l'une ou l'autre de ces trois parties fournissent les bases de la subdivision des espèces.

A. Espèces dont le cou et le corps sont sans crochets.

a. Le cou très-court ou long.

$1^{\circ}$. La trompe subglobuleuse.

L'E. GÉanT, $E$. gigas, des intestins du cochon.

$2^{\circ}$. La trompe ovale.

L'E. clobuleux, E. globulosus, dans la sciène ombre et plusieurs autres espèces de poissons.

3•. La trompe oblongue, plus grosse au milieu.

L'E. ceint, E. cinctus, de la couleuvre noir-verdâtre (col. atrovirescens).

$4^{\circ}$. La trompe claviforme, ou plus grosse à l'extrémité. 
L'E. AGILE, $E$. agilis, desintestins du muge (mug. cephalus).

$5^{\circ}$. La trompe conique ou plus épaisse à la base.

L'E. chenille, $E$. haruca, de la grenouille ordinaire ( $r$. esculenta).

$6^{\circ}$. La trompe cylindrique ou linéaire.

L'E. A QUEUE, E. caudatus, dans les faucons.

\section{b. Le cou long.}

L'E. noduleux , E. nodulosus, dans le barbeau (cyp. barbus).

B. Le cou ou le corps armé.

L'E. SPHÉrocéphaLE, $E$. sphcerocephalus, dans l'huîtrier (himant. ostralegus).

\section{ORD. III. TRÉMATODES (tremaloda).}

Corps déprimé ou subarrondi, mou; des pores ou suçoirs; tous les individus androgynes.

Les vers intestinaux compris dans cet ordre ont une forme très-variée; la tête est très-rarement distincte, et par conséquent le cou, sous lequel sort un filament cirreux servant à la génération. Le corps, ordinairement déprimé, ovale, elliptique, linéaire ou subarrondi, est souvent nu, mou, rarement denticulé sur ses bords; on y remarque au-dessous un, deux ou plusienrs pores plus ou moins antérieurs, disposés de manière différente, outre un autre qui se trouve quelquefois en arrière. Ces pores, le plus 
souvent simples, sont aussi quelquefois pourvus de nodules on d'aiguillons. Il n'y a pas de canal intestinal proprement dit; tous les individus ont à la fois les organes génitaux des deux sexes.

On trouveles trématodes dans les animaux vertébrés.

G. XIII. Monostome, Monostoma.

Corps mou, subarrondi ou déprimé; un seul pore antéricur.

Dans les mammifères, les oiseaux, les reptiles et les poissons, entre les muscles, dans le thorax, les poumons, les intestins et l'abdomen.

A. L'ouverture du pore inférieure.

Le M. caryophyluin, M. caryophyllinus, dans les intestins de l'épinoche (gast. aculeatus).

B. L'ouverture du pore antérieure.

Le M. Foliacé, $M$. foliaceum, dans l'esturgeon ( $a$. sturio ).

G. XIV. Ampirstome, Amphistoma.

Corps mou, subarrondi; deux pores, l'un antérieur et l'autre postérieur.

Dans l'estomac, les intestins, l'abdomen et les hydatides des viscères des mammifères, des oiseaux et des reptiles.

A. La tête distincte.

L'A. a long cou, $A$. longicolle, dans les hérons. 
B. La tête non distincte.

L'A. subclaviforme, $A$. subclavatum, dans les grenouilles, crapauds et rainettes.

\section{G. XV. Distome, Distoma.}

Corps mou, déprimé ou subarrondi ; deux pores, dont l'un antéricur et l'autre ventral.

Les espèces de ce genre, qui montent aujourd'hui à plus de cent quarante, peuvent être divisées ainsi :

\section{A. Espèces non armées.}

a. Planes ou déprimées.

$1^{\circ}$. Le pore ventral le plus grand.

Le D. ou fole, $D$. hepaticum, dans l'homme, plusieurs rongeurs, le cheval, le cochon et presque tous les animaux ruminans.

$2^{\circ}$. Le pore antérieur le plus grand.

Le D. MÉgastone, D. megastomum, dans l'estomac du squale milandre ( $s q$. galeus $)$.

$3^{\circ}$. Les pores égaux.

Le D. macrostome, D. macrostomum, dans les espèces du genre fauvette (motacilla).

\section{b. Subcylindriques.}

$I^{\circ}$. Le pore ventral le plus grand.

Le D. Fourciu , D. furcalum, dans les intestins du surnulet (mullus surmulctus). 
$2^{\circ}$. Le pore antérieur plus grand.

Le D. coupé, $D$. excisum, dans l'estomac du maquereau ( $S$. scomber).

$3^{\circ}$. Les pores égaux.

Le D. point, D. punctum, dans le barbeau (cyp. barbus).

3. Espèces armées.

\section{a. Noduleruses ou papilleuses.}

Le D. Noduleux, D. nodulosum, dans les perches.

\section{b. Aiguillonnées.}

LeD. AIguillon Né, D. echinatum, dans les hérons, les canards, etc.

\section{G. XVI. Tristome, Tristoma}

Corps dẻprimé; trois pores, dont deux antérieurs simples, et le troisième postérieur rayonné.

Le T. orangé, $T$. coccineum. La seule espèce de ce genre a été trouvée fixée aux branchies et au corps du poisson lune (ortliragoriscus mola).

\section{G. XVII. Pentastone, Pentastoma.}

Corps un peu arrondi ou déprimé; la bouche entre deux pores de chaque côté, d'où sort un aiguillon.

Dans les sinus frontaux, les poumons, à la surface du foie des mammifères et des reptiles.

Le P. т®niö̈de, $P$. tonioides, dans le chien, le loup, le cheval. 


\section{G. XVIII. Polystome, Polystoma.}

Corps subarrondi ou déprimé; six pores antérieurs, outre un ventral et un postérieur.

Dans la gorge, les branchies des poissons, la vessie urinaire des grenonilles, dans l'oraire de l'espèce htimaine.

Le P. pinguicole, $P$. pinguicola, dans l'homme.

Ord. IV. CESTOIDES (Cestoidea).

Corps allongé, déprimé, continu ou articulé; la tête très-rarement pourvue de lèvres simples, et le plus souvent de deux ou quatre fossettes ou suçoirs; tous les individus androgynes.

Les vers rangés dans cet ordre ne forment pas une famille véritablement naturelle, tant la tête diffère de structure dans chaque genre. Dans quelques-uns, en effet, elle est polymorphe, tétragone, pyramidale, comprimée, avec la bouche pourvue dejèvres ou suçoirs, tandis que dans les autres elle est tronquée, hémisphérique ou globuleuse, lisse en avant et pourvue d'un tubercule ou d'un rostre, inerme ou armée d'une couronne simple ou double de crochets; quelquefois même on y remarque quatre espèces de trompes armées de crochets rétractiles. Le cou est souvent nul. Le corps est allongé, déprimé, mou, continu ou formé d'un grand nombre d'articulations 
percées de pores latéraux ou marginaux, et pourvues de papilles ou de filamens érectiles. La queue est obtuse ou articulée. Le canal intestinal n'est pas visible ; il est remplacé par des espèces de vaisseaux qui provienuent des suçoirs; il en est de même des organes de la génération; quelquefois cependant on voit une série d'ovaires simples en forme de taches dans toute la ligne médiane, et des orifices des articulations sortent des filamens que l'on regarde comme des organes mâles de la génération.

On les trouve dans le canal intestinal.

\section{G. XIX. Géroflé, Caryophyllauus.}

Corps déprimé, continu; la tête dilatée, divisée en lanières et pourvue de lèvres, l'une supérieure et l'autre inférieure.

Daus les intestius des poissons.

Le G. changeant, C. mutabilis, dans les carpes, les cobites.

G. XX. Massìte, Scolex.

Corps déprimé, continu; la tête pourvue de quatre fossettes.

Dans les intestins et la cavité abdominale des poissons et des sèches (sepia).

La M. POLYMORPHE, S. polymorplues.

G. XXl. Gymnor hy yque, Gymnorhynchus.

Corps déprimé, continu, très-long, avec un récep- 
tacle globuleux au cou; la tête pourvue de deux fossettes bipartites et de quatre trompes nues, rétractiles.

Dans la chair de plusieurs poissons.

Le G. rampant, G. reptans, dans la chair du spare de Ray ( $s p$. Raii).

G. XXII. Tétrariynque, Tetrarhynchus.

Corps déprimé, continu; la tête pourvue de deux fossettes bipartites et de quatre trompes rétractiles garnies a'aiguillons recourbés.

Dans les reptiles, les poissons et les mollusques, dans les chairs, les branchies, l'estomac et ses membranes, le foie, le péritoine.

Le T. DIscophore, T. discophorus, dans les branchies et entre les membranes de l'estomac du spare de Ray (sp. Raii).

\section{G. XXIII. Lrgule, Ligula.}

Dans son premier degré de développement : corps déprimé, continu, très-long, avec un sillon longitudinal, sans apparence de tête ni d'organes de la ggénération.

Dans son état complet : corps déprimé, continu, très-long; la tête pourvue de chaque côté d'une fossette très-simple; des ovaires avec des lemnisques formant une série simple ou double dans la ligne médiane.

Les animaux de ce genre, rès-communs daus les 
oiseaux et les poissons, sont au contraire très-rares dans les mammifères.

A. Espèces à ovaires distincts.

La L. unisériale, $L$. uniserialis, dans les oiseaux du genre falco.

B. Espèce à ovaires évidens.

Le L. TRÈs-SIæPLE, L. simplicissima, dans beaucoup de poissons.

\section{G. XXIV. Tricuspidaire, Trionophorus}

Corps allongé, déprimé, subarticulé; la bouche bilabiće et armée de chaque côté de deux aiguillons tricuspides.

Dans les intestins des poissons, dans des kystes du mésentère et du foie.

Le T. Noduleux, T.nodulosus, dans la perche, le brochet, le saumon, etc.

\section{G. XXV. Bothriocéphale, Bothriocephalus.}

Corps allongé, déprimé, articulé; la tête subtétragone et pourvue de deux ou quatre fossettes opposées.

Les espèces de ce gevre se trouvent très-fréquemment dans les poissons et les oiseaux, et plus rarement dans les mammifères et dans l'espèce humaine, dans les branchies, l'œesophage, les appendices pyloriques, les intestins, la cavité abdominale. 
A. Espèces non armées (gymnobothrii).

a. $A$ deux fossettes (dibothrii).

Le B. Large, B. latus (Br.) (Tam. lata, Aucth.), dans les intestins de l'espèce humaine en France, en Suisse, en Russie.

\section{b. $A$ quatre fossettes (tetrabothrii).}

I,e B. Renflé, B. tumidulus, dans la raie pasténaque.

B. Espèces armécs. Toutes sont à quatre fossettes (tetrabothriii.)

a. Aiguillonnées (onchobothrii)

Le B. couronné, B. coronatus, dans les raies et les squales.

b. Pourvies d'une trompe.

Le B. A suçoirs hérissés, $B$. corollatus, dans le même groupe de poissons.

\section{G. XXVI. TEN1A.}

Corps déprimé, allongé, articulé; quatre sụçoirs à la tête.

Dans les intestins, les conduits biliaires, la vésicule du fiel, le foie des animaux vertébrés; une seule fois dans la cavité abdominale.

A. Espèces non armées.

a. La tête simple ou sans rostre.

Le T. pectiné, $\boldsymbol{T}$. pectinata, dans les lapins, la marmotte. 


\section{b. La tếle avec un rostre rétractile.}

Le T. villeux, $T$. villosa, dans l'outarde.

B. Espèces armées.

Le T. cucurbitain, $T$. solium, dans le canal intestinal de l'espèce humaine en Allemagne, en Argleterre, en Hollande et dans l'Orient.

\section{ORD. V. CYSTOIDES (Cystica).}

Corps déprimé ou un peu arrondi, se terminant en arrière par une vessie pour chaque individu, ou commune à plusieurs; la tête pourvue de deux ou quatre fossettes ou de quatre suçoirs avec une couronne de crochets ou enfin de quatre trompes. Les organes de la génération presque inconnus.

Cet ordre renferme les vers intestinaux dont l'organisation est la plus simple. Leur corps est formé par un sac membraneux, pellucide, plein d'une humeur aqueuse, et le plus souvent est renfermé dans un autre sac formé par l'organe dans lequel se trouve l'animal. La tête est pourvue de quatre onvertures et d'aiguillons ou de quatre trompes; elle est rétractile dans le corps. Dans un genre plusieurs têtes appartiennent à la même vessie; dans un autre, les vers sont extrêmement petits, et occupent la face interne de la vessie, ou nagent librement dans le fluide qu'elle contient. Les organes qui servent à la mutrition et à la 


\section{DE L'HOMME.}

génération de ces animaux n'ont pas encore été découverts.

G. XXVII. Anthocépinale, Anthocephalus.

Corps allongé, déprimé, tẹrminé en arrière par une vessie caudale, et en avant par. une tête pourvue de deux ou quatre fossettes et de quatre trompes garnies d'aiguillons.

Contenu solitairement dans une double vessie, dont l'une externe dure, élastique, et l'autre interne plus mince.

Dans le foie, le mésentère, le péritoine, les hydatides viscérales des poissons.

L'A. macroune, $A$. macrourus, du spare.

G. XXVili. Cysticerque, Cysticercus.

Corps subarrondi ou déprimé, terminé par une vessic caudale; la tête avec quatre suçoirs et un rostre garni de crochets recourbés.

Contenu solitairement dans une vessie externe simple.

Entre les muscles, dans la graisse, dans le cerveau, le thorax, la plèvre, le coeur, le foie, le mésentère, le péritoine des mammifêres.

Le C. du tissu cellulaire, C. cellulosus, dans l'honme, les singes, les cochons.

G. XXIX. Cenvee, Canurus.

Corps allongé, subdéprimé, rugueux, avec une 


\section{SUR LES VERS INTESTINAUX}

tête pourvue d'un rostre garni de crochets et de quatre suçoirs, adhérens en plus ou moins grand nombre à la face interne d'une vessie simple remplie de fluide.

Le C. cérébra L, C. cerebralis, dans le cerveau des moutons, des bœufs et des antilopes.

L'aperçu systématique que je viens d'exposer servira à mes lecteurs pour assigner à chaque ver qu'ils pourront rencontrer dans l'homme, sa place dans le système; je ve le suivrai cependant pas ponctuellement, et la division que j'établis conviendra mieux au médecin praticien. Je partage les vers en ceux qui séjournent dans le canal intestinal de l'homme, et en ceux qui se trouvent dans un autre organe. On peut, sur les premiers, donner beaucoup de généralités sous les rapports de l'étiologie, du diagnostic et de la thérapeutique, ce qui m'évitera plusieur's répétitions inutiles; quant aux seconds, au moins pour la plus grande partie, on ne peut presque en rien dire d'un peu certain, même de particulier, et à plus forte raison de général.

Je donnerai d'abord une courte descripition zoologique des vers qui séjournent dans le canal intestinal de l'homme, et j'aurai toujours soị de renvoyer aux figures, qui, comme je me plais à le croire, ont été exécutées d'une manière à rendre superflues des descriptions plus détaillées.

Puis je m'occuperai des causes les plus prochaines de leur production, des signes àl'aide desquels on pré- 
sume ordinairement leur présence, et enfin des remèdes propres à les expulser. A près cela je donnerai une description des vers qui séjournent hors du canal intestinal, et je ferai connaître de chacun en particulier tout ce que l'on sait jusqu'à présent d'intéressant pour le médecin praticien ${ }^{\text {. }}$.

\section{CHAPITRE TROISIEME.}

\section{SECTION PREMIÈRE.}

\section{Des vers qui séjournent dans le canal intestinal de l'homme.}

\section{TRICHOCÉPHALE.}

Trichocephalus dispar ( en allem. der Peitschenwirm on Ilaarkopf) Tab. I, fig. I et 2.

Trichocephalus : parte capillari longissima, capite acuto indistinclo, curpore maris spiraliter involuto, femince subrecto. Rud. Morgagni, Epist. anatomica XIV , art. 42. Roederer et Wagler, L. C. , trichuris.

' Nous avons jugé plus convenable d'établir une disposition de chapitres un pen différente de celle de l'auteur, pour ne pas rompre la partie descriptive par des considérations de sénéiotique et de thérapeutique, et pour rendre chaque partie indépendante des autres. 
Gœze, Eingeweidew., p. 112 , 116 , tab. VI, fig. I et 5, trichocephalus hominis.

Gmelin, Syst. nat., p. p. $5 \circ 37, \mathrm{n}^{0} . \mathbf{1}$, trichocephalus hominis.

Werner, I'erm. intest., p. 84, ascaris trichiura.

Joerdens, Helninth., p. 17 , tab. I, fig. 6 et 1o, trichocephalus hominis.

Brera, Vorlesung, p. I6, tabl. 1v, fig. I et 5 der Haarkopf; Voy. aussi Memorie du même, p. 17 I, tricocefalo.

Zeder, Anleit., p. 69, mastigodes hominis.

Rudolphi, Entoz., tom. II, p. 88, trichocephalus dispar.

Bradley, A treatise on worms, p. $7^{2}$, plate 111 , fig. I et 3 , the long thread worm.

Cuvier, Regne animal, t. $1 \mathrm{v}, \mathrm{p} .3 \mathrm{I}$, trichocéphale de l'homme.

De Lamarck, Anim. sans vert., tom. III, p. 212 , le trichiure de l'homme.

Cette espèce séjourne ordinairement dans les gros intestins, mais principalement dans l'intestiu cœecum; Werner prétend cependant l'avoir rencontrée dans la partie inférieure de l'iléon.

\section{DESCRIPTION.}

Ce ver est de la longueur d'un pouce et demi à deux pouces; la partie mince capillaire forme les deux tiers de toute sa longueur; il est ordinairement blanc, quelquefois cependant il est coloré par les alimens dont il est rempli. La partie capillaire ou antérieure pasșe assez brusquement à la partie renflée ou postérieure.

Le mâle, fig. $1,1 a$. et $1 b$., plus petit que la femelle, est tellement pointu vers le commencement de la tète, que l'on peut à peine voir l'ouverture de la 
bouche. Wrisberg ${ }^{x}$ croit avcir trouvé à cet endroit un petit tube; mais ni Müller, ni Rudolphi, ni moi n'avons pu le décou rrir. Le canal al mentaire traverse en ligne directe la partię antírieure capillaire qui est striée en travers; il s'étend̉ dans la partie postérieure plus épaisse, qui est un peu sp.riforme. Dans cette dérnière partie se trouvent aussi les vaisseaux spermatiques, repliés sur eux-mêmes, et qui se terminent dans la partie inférieure de la fin de la queue par un petit tube transparent ou espèce de vagin, par lequel sort le pénis; ce tube ou ce vagin n'a pas toujours la même forme, comme cela se voit par les fig. 1.a., et I $b .$, pl. I.

La femelle fig. 2., pl. r, se distingue du mâle, d'abord par une plus longue partie antérieure et capillaire, et parce que la partiepostérieure est presque droite ou à peine recourbée; c'est là que se trouvent les oviductes et les œufs d'une forme elliptique placés autour du tube intestinal; il y a à son extrémité une petite ouverture, qui peut servir en même temps d'anus et de vagin.

REMARQUES.

Il n'y a pas encore soixante ans que les naturalistes et les médecins ont quelque connaissance de cette espèce et même de ce genre de vers. Morgagni l'avait connu bien antérieurement comme Rudolphi l'a prouvé; mais cette découverte, comme beaucoup d'autres, était tombée dans l'oubli pour reparaître plus

: Ouvrage cité, p. 13. 
tard comme nouvelle. Un étudiant disséquait, dans l'hiver de 1760 à 1761 , à lamphithéâtre anatomique de Goetlingue, la vąlvule du colon d'un enfant de cinq ans, du sexe féminin. Il fit par hasard une petite ouverture dans le coecum, et il en sortit plusieurs des vers dont nous nous occupons. Wrisberg et plusieurs autres jeunes médecins les regardèrent comme une espèce de vers inconnue jusqu'alors; le prosecteur Wagler crut que ce n'était que des oxyures (ascarides vermiculaires) d'une taille extraordinaire; d'autres les prirent pour de jeunes lombricoïdes. Cette incertitude donna lieu à une dispute assez sérieuse, mais il ne vint dans l'idée de personne d'éclaircir la chose par des comparaisons, des examens et des recherches exactes. Roederer, informé de cette dispute, en fit l'examen lui-même; ce dernier et Büttner regardèrent ces̉ vers comme une espèce inconnue jusqu'alor's, et Büttner lui donna le nom de trichuris; dès ce moment les intestins de tous les cadavres furent examinés soigneusement. A cette époque il régna une épidémic dans un corps d'armée français stationné à Goettingue. Roderer ei Wagler ont décrit cette épidémie sous le nom de morbus mucosus, et l'on trouva souvent de ces vers dans les cadavres des soldats qui monrurent à la suite de cette épidémie, ce qui engagea Roderer à regarder ces animaux comme une production de cette maladie; mais Wrisberg remarqua quion les aurait assurément trouvés plus tôt si l'on y avait fait attention. On les rencontre en effet aujourd'hui daps presque tous les 
cadavres d'hommes, mais très-souvent il $\mathrm{n}_{\text {}} \mathrm{y}$ en a qu'un seul; M. Rudolphi en a cependant observé une fois plus de mille ensemble.

L'on regarda d'abord la partie mince ou capillaire comme la queue du vèr d'où vient le nom de trichuris, et l'on crut avoir trouvé une trompe dans le tube ou dans le vagin, qui entoure le pénis; et comme celui-ci ne se remarque pas dans la femelle, Roderer, Wagler et Wrisberg ont pris les deux sexes pour deux différentes espèces. Bloch n'a vu qu'une seule femelle de cette espèce de ver, qui provenait d'un homme, et il l'appela également trichuris. 11 paraît que Werner, qui le désigne sous le nom d'ascaris trichiura, n'a vu que des mâles, et il présuma que la trompe que l'on ne trouve pas chez les individus presque droits, c'est-à-dire chez les femelles, s'était détachée par suite de la putréfaction. Werner n'aurait pas fait cette supposition s'il avait vu la femelle elle-même; car il avait trop de connaissances en helminthologie pour ne pouvoir pas distinguer un ver entier de celui qui est à moitié pourri. Il ne faut pas s'étonver que Jœrdens, qui du reste n'iguorait pas ce que Pallas et Goeze ont avancé sur ce sujet, ait adopté les idées de Werner; car Joerdens n'a jamais observé ou exaniné un seul ver lui-même; le noin de tricocephalus hominis, que l'on trouve dans l'ouvrage de ce dernier est assez mal inventé.

Pallas, qui rencontra vers l'époque de cette découverte, dans une espèce de lésard (lacerta apus), 
un ver appartenant à ce genre, et auquel il donne assez inexactement le nom de tania spiralis; Goze, qui a reçu de Wagler plus de cent de ces vers, et qui les a comparés avec les vers du même genre, provenant des souris et des sangliers (car cclui du clieval n'appartient pas à ce genre ); et Müller "ont prouvé jusqu'à l'évidence que l'on ne doit chercher la tête de ces vers qu'a la partie la plus pointue. Ces auteurs ont également prouvé que les spiriformes sont les mâles, et les droits les femelles. Depuis l'on a rencontré dans diverses espèces de singes, dans les chiens, dans les renards, dans beaucoup d'animaux rongeurs, et dans un grand nombre d'animaux ruminans, comme les chamois, les cerfs, les gazelles et les moutons, beaucoup de vers de ce genre, et toujours les deux sexes dans chacun de ces animaux; et je crois qu'il n'y a plus de naturalistes qui n'aient pas connaissance aujourd'hui de leur véritable nature. Une chose qui vient à l'appui de ces découvertes, est que la partie la plus pointue est toujours assez fortement implantée dans les parois intestinales, tandis que l'extrémité la plus grosse, qu'elle soit droite ou spiriforme, se trouve libre dans les matières fécales; tout cela prouve que la partie la plus pointue est le côté où est la tête. On ne conçoit donc pas comment Brera" peut encore engager les naturalistes à éclaircirce sujet par des recherches plus exactes.

Naturforcher 12 Stück. s. 182 in der note.

Meinorie, p. 77 . 
Nous serions fort heureux d'être aussi avancés dans d'autres points d'helminthologie que nous le sommes sur celüi-ci.

\section{OXỴURE VERMICULAIRE.}

Oxyaris vernicularis (en allem. der pfriemenschwanz), pl. I, fig. 3 , le mâle, et pl. II, fig. I, la femelle.

OXYUris : capitis obtusi membrana laterali utrinque vesiculari, cauda maris spirali obtusa, femina subulata recta.

Bloch, Abhandl. s. 31. Ascaris vermicularis. Der aflerwurn.

Goeze, Engeweidew. s. 102-1 o6, tab. v, fig. $x$ et 5., der menschiiche pfriemenschwanz.

Werner, Vermes intest., p. 72 , fig. 133 et 157 , asc. vermicularis. Gnelin., Syst. nat., p. 3o29, r, asc. vermic.

Joerdens, Helminthol., page 19 , tab. II , fig. I et 5 , asc. vermic. Der afterwurm.

Zeder, Anteitnns, p. 107, n., fusaria vermic.

Brera, Vorlesung, p. 18, tab. Iv, fig. 7 et 11 ; Memorie du même, p. 178 , tab. III, fig. 14 et 15 , ascaride vermicolare.

Rudolphi, Entoz., t. II, part. I , pag. 152, $\mathrm{n}^{\circ} .31$, ascaris vermicularis.

Bralley, $A$ treatise, p. 56 , vol. II, fig. 1 et 3 , the ascaris vermiaularis, commonly called the max, or thread worm.

Cuvier, Règne animal, t. Iv, p. 33, l'ascaride vermiculaire.

De Lamarck, Anim. sans vert., t. III, p. 104 , l'oxyzure vermiculaire.

Ce ver a encore les dénominations suivantes : les Allemands l'appellent der kinderwurm, mustdarmwurm, madenwurm, die ascaride, die arschmade, darmschabe; les Hollandais, aarsmade; les Danois, smaa spolorme, boerneorm; les Suédois, barnmask; les Auglais, bots; les Français, ascarides.

L'oxyure vermiculaire séjourne dans les gros intestins, et principalenent dans le rectum. 
DESCRIPTION.

Le mâle, de la longueur d'une ligne ou d'une ligue eı demic, a le corps mince, très-élastique, et d'une couleur blanche; la partie antérieure, obtuse, est cntourće d'une membrane transparente (Voyez pl. 1, fig. 3); on apercoit à travers cette dernière, formant une espèce de vessie, un tube droit, qui est l'œsophage, et qui devient claviforne à l'endroit où il se perd dans un estomac globulenx. Le tube intestinal s'étend dans toute la longueur du corps, qui devient peu à peu plus gros, et se contourne en spirale vers la quene. L'on ne peut pas bien voir les vaisseaux spermatiques, qui entourent le canal alimentaire, chez les vers qui ont resté pendant quelque temps dans l'esprit-de-vin, et jusqu'à présent jè n'ai pas eucore cu occasion d'en examiner de vivans. Je n'ai pas encore observé non plus un pénis allongé chez celte espèce d'oxyure, mais bien chez les vers du même genre provenant des lapins sauvages.

La femellc, pl. 2, fig. $1 \mathrm{ct} 2$, est plus grande etacquiert une longueur de quatre à cinq lignes; la conformation de la partic antérieure ressemble, par sa structure intéricure et par l'extérieure, parfaitement à celle du mâle, jusquà l'endroit où sc tcrmine l'estomac; à partir de cet endroit, le canal alimentaire est entouré de tous côtés par les oviductes; le ver augmente toujours en grosseur depuis la tête jusque vers le premier liers de sa longueur; au-delà il 
devient plus mince, et la queue se termine en forme de poinçon, tellement fin à son extrémité que l'œil non armé du microscope a de la peine à l'apercevoir : la fig. $2 b$. pl. 2 , représente un morceau de ce ver considérablement grossi et dans lequel on peut voir les œufs.

\section{REMARQUES.}

Ce ver est connu des médecins de temps immémorial, et conme il est très-commun chez les enfaus, que sa conformation diffère de celle des autres vers de l'homme, on devrait croire qu'il aurait été impossible de le méconaaître. Néanmoins, nons trouvons beaucoup d'exemples chez les auteurs, où des larves de mouches et des articulations détachées du tænia ont été prises pour cette espèce de ver. L'on a préteudu également en avoir rencontré hors des gros intestins dans d'autres parties du corps; Bloch ${ }^{2}$ rapporte que Wulf en a trouvé une grande quantité dars un sac formé daus les parois de l'estomac.

Brera ${ }^{2}$ dit en avoir observé dans l'œsophage d'une fenme une quantité innombrable. Cette personne avait succombé ì la suite d'une fièvre lente verveuse. Le même auteur cite plus loin ${ }^{3}$ une observation faite par Bianchi, qui prétend en avoir vu dans les ventricules du cerveati.

Abhandl, s. 3 r.

"Vorlesung, p. I9.

3 Memorie, p. $18 \mathbf{r}$. 
Je passe sous silence d'autres observations semblables, et je doute très-fort qu'il y ait un naturaliste qui croye que c'étaient de véritables oxyures, avant qu'il n'ait été convaineu par ses propres observations.

Goeze a pensé à tort que les oxyures sont vivipares, probablement parce qu'ayant fait l'observation que les œufs de ces animaux sont dans un mouvement continuel d'avant en arrière et d'arrière en avant, il les a regardés comme des fotus. Il est bien vrai qu'il existe dans le rectum des grenouilles et des crapauds des petits vers qui sont vraimeat vivipares, mais ils appartieunent à un autre genre.

Les anciens médecins ont appelé ces vers ascarides, et ils voulaient les distinguer, par eette dénomination, des lombricoïdes, auxquels ils avaient donné le nom de lumbrici teretes. Linné employa plus tard le nom d'ascaris comme nom de genre, et alors le lumbricus teres a été appelé ascaris lombricoides, et le ver dont nous parlons ascaris vermicularis; mais dans les derniers temps, on a mieux approfondi la chose, et l'on s'èst convaincu que ces deux ver's ne diffèrent pas seulement entre eux comme espèces, mais même qu'ils ne sont, pas du même genre, comme nous le verrons par la suite.

M. Rudolphi, qui doutait depuis long-temps ' que ceux observés par Goeze dans les gros intestins du cheval (ces vers étaiẹnt renflés à une de leurs extrémités et très-pointus à l'autre) appartinssent au

- Wiedemanns archiv. 
genre trichocéphale, confirma plus tard sa supposition par un examen particulier; d'après cela il crut devoir former de ces vers un genre distinct sous le nom d'oxyure.

Je trouvai dans l'hiver de 1809 une grande quantité d'une espèce de vers dans les gros intestins de plusieurs lapins sauvages, et je n'hésitai pas un instant à.les ranger dans ce geure, quoiqu'alors je ne connusse les oxyures du cheval que par les dessins de Goeze et de Rudolphi ${ }^{x}$. Lorsque plus tardjjeffis dessiner ces vers sous une échelle beaucoup plus grande que nature, je fus frappé de leur ressemblance avec ceux qui proviennent du rectum de l'homme; je les comparai alors plus soigueusement, et je me convainquis que ces derniers (les oxyures) ne doivent plus être rangés avec les lombricoïdes; car ceux-ci sont toujour's amincis (attenuatce) vers les deux extrémités, et en outre se distinguent très-clairement de tous les autres nématoïdes par trois papilles ou boutons à l'extrémité antérieure ${ }^{2}$; les oxyures sont bien également amincis vers leur extrémité antéricure, mais ils se terminent à l'autre en forme de poinçon ( subulatce); cela a lieu au moins chez les femelles, et du reste il leur mauque les trois papilles au commencement de la tête; la structure intérieure de ces deux

"J'en ai reçu plus tard une grande quantité du docteur Brosché, de Dresde. ( $B r_{\text {. }}$ )

"Il faut comparer les signes caractéristiques de ces deux genres de vers rapportés plus haut. 
espèces de vers n'est pas moins différente. Je communiquai mes observations à $\mathbf{M}$. Rudolphi ; ce savant observateur partagea mon opinion. Son volume supplémentaire parlera de plusieurs nouvelles espèces de ce zenre; mais avec tout cela je n'étais cependant pas cncore sur ce sujet arrivé au degré de certitude que je désirais.

Goeze a bien dessiné un ver de ce genre (voyez sa pl. 5, fig. 5), qu'il regarde comme un mâle, probaHement par la raison qu'il ne put apercevoir d'œufs. On trouve un ver semblable dessiné sur ma première planche (voy. fig. 3 et $3 a$.). Les mâles de tous les nématoïdes sont en général plus petits d'un quart ou d'un tiers que les femclles, et la terminaisón de leur quene est tout à fait différente. Dans les figures de Goeze, copiécs par Jœrdens et Brera, on voit que la grandeur est la même, et que la terminaison de la queue est absolument semblable; mais on n'observe point d'oufs sur les individus représentés dans les figures 8 et 9 ; c'étaient peut-être des femelles qui avaient pondu leurs oufs, ou bien ces œufs n'élaient pas cncore complétement formés ou n'étaient pas encore fécondés. Enfin ces individus n'étaient-ils pas dépourvus d'organes sexuels, comme il s'eu trouve parmi les abeilles et les fourmis? je ne puis rien décider là-dessus. Les oxyures que M. Rudolphi a trouvés dans les chevaux avaieut tous des oeufs, les miens pareillement; tous étaient donc par conséquent des femelles. Parmi les vers provenant des lapins sauvages, j'en ai rencontré beaucoup qui 
avaient la fin de la quene obtuse et roulée en spirale; jai remarqué également sur plusieurs un petit dard (spiculum) allongé, et ceux-ci étaient en général plus petits que ne le sont ordinairement les femelles; je conclus de là que les mâles des oxyures provenant de l'homme devaient être conformés de la même manière; mais parmi tous les individus que j'eus à ma disposition, je ve pus en trouver un seul qui eût les mêmes signes caractéristiques; il en fut long-temps de même des oxyures provenant des lapins domestiques, et d'un grand nombre de souris. Cette circonstance m'aurait presqu'engagé à regarder la génération des oxyures comme analogue à celle des pucerons, qui sont en général vivipares, et qui ne produisent que des femelles en été, pendant la durée duquel ils ont de la nourriture en abondance, mais qui au contraire pondent en automne des oufs dont il se développe au printemps (fabula si vera) des femelles et des mâles, et ces derniers, comme on l'a prétendu, rendent alors féconde toute la génération pour l'année prochaine; mais en faisant l'observation que les lapins domestiques, l'homme et leç cheval reçoivent ordinairement de la nourriture en suffisante quantité, et que par conséquent les vers qu'ils portent en cux ne doivent pas en manquer, je fus porté à présumer que les femelles des oxyures étaient, peut-être à cause de cela, en état de multiplier leur espèce sans l'entremise du mâle. Il arrive au contraire bien sou vent que les lapins sauvages, et par conséquent leur vers, manquent de nourriture pendant l'hiver, et 
c'est justement dans cette saișon que je n'ai trouvé que des oxyures mâles. La faim n'influe pas avantageusemeut, comme nous le savons, sur la faculté prolifique; il me parut alors conforme aux sages dispositions de la nature, qui n'aime qu'à produire et conserver la vie, que la faculté générative eût été partagée entre deux individus, afin que chacun d'eux eût moins de peine à conserver son espèce, et que les vers provenant d'une formation primitive ne pussent pas être détruits entièrement. Pendant que je me livrais à ces conjectures et que javais une sorte de satisfaction d'avoir entrevu, à ce que je croyais, pourquoi on ne rencontre pas d'oxyures mâles chez de certains animaux, laissant à d'autres à juger la probabilité de ces conjectures, je communiquai mon opinion à M. Sœmmerring, et peu de temps après il cut la bonté de m'envoyer un petit vase rempli d'oxyures dans l'esprit-de-vin. Ces vers provenaient de son propre fils, qui les avait rendus après avoir pris un lavement d'huile d'olive. Sœmmerring m'observa que je trouverais peut-être parmi eux quelques individus qui offriraient les signes caractéristiques des mâles, ạ la recherche desquels j'étais, comme il le savait, depuis long-temps. Je les ai examinés, et j’ai trouvé en effet ces caractères (Voyez pl. 1, fig. 3). $J$ 'en ai reçu plus tard de pareils encore une fois du même médecin et de M. Hermann. M. Rudolphi n'a pas hésité un instant à les reconnaître pour des mâles, et il en aura probablement trouvé depuis luimême. 
Il résulte de ces observations, que les vers connus sous le nom d'ascarides vermiculaires, doivent être compris dorénavant dans le genre oxyuris, et non pas dans le genre ascaris, et que les deux sexes de ces oxyures se distinguent entre eux par les caractères que nous avons rapportés plus haut.

\section{Ascakide tombricoide.}

Ascaris lombricoides (en allem. der spulwurm), pl. II, fig. 2-3.

AsCaRIs : corpore utrinque sulcato, cauda obtusiaccula.

Bloch, Abhandlung, p. 29, tab. vil, fig. 4-6, asc. lumb.

Goeze, Eingeweidew, p. 65 et $7^{2}$, tab. I, fig. 1-3, asc. gigas.

Werner, Verm. intest., p. $7^{5}$ et $8 \%$, tab. vil, fig. 153 et 159 , asc. lumb.

Gmelin, Syst.nat., p. 3o29, $\mathrm{n}^{\mathrm{o}}{ }_{2}$, asc. lumb.

Zeder, Nachtrag, p. ${ }_{2} 5$ et $3 \mathrm{r}$, fusaria lumbricoides; le même, $\mathrm{An}$ leitung, p. $102, \mathrm{n}^{\mathrm{0}} . \mathrm{x}$, fus. lumb.

Joerdens, Helminth. , p. 22 , tab. 1I, fig. 6 et 15 , asc. lumbr. Brera, Vorlesung, p. 21 , tab. v, fig. I et $\mathbf{I}$; le même, Memorie, p. $19^{5}$, tab. In, fig. 18 et $20, l o m b r$.

Rudolphi, Wiedemanns archiv., $\mathrm{n}^{\circ} .2$, page. ${ }^{\circ}$, asc. lumbr.; le même, Entozcolog. 11 , part. 1, p. $124, n^{\circ} .1$, asc. lumbr.

Bradley, A treatise, p. 34 et 55, tab. I, the asc. lumbr.

Cuvier, Règne animal, tom. Iv, p. 33, l'ascaride lombrical.

De Lamarck, Anim. sans vert., t. III, p. 207, l'ascaride lombricoide.

Les Allemands l'appellent encore rundwurm, les Hollandais rondewurm, menschenworm, kinderenworm; les Danois menneskeorm, spolorm, skolorm; les Suédois mennisko-mask, spolmask; les Anglais the round worm, large round worm, round gut worm; Ifs Français lombric des intestins, strongle; les Italiens verme rondo, lombrico; les Espagnols lombriz; les Portugais lombriga. 
Cette espèce de vers ne séjourne pas seulement dans les intestins grêles de l'homme, mais elle se trouve également dans ceux des boufs, des chevaux et des cochons.

\section{DESCRIPTION.}

Ces vers ont le corps de la grosseur de deux ou trois lignes, sur une longueur de six, dix, jusqu'à quinze pouces. Les petits, de la longueur d'un pouce et demi, sont rares; on en trouverait sans doute beaucoup plus souvent, si l'on examinait les intestins des cadavres d'homme aussi souveut que cela a lieu pour ceux des autres animaux. Lá couleur de ces vers est ordinairement rouge brunâtre; mais elle varic cependant quelquefois ; elle est plus claire ou plus foncée, selon la couleur des alimens dont ils sont gorgés; elle est même quelquefois d'un rouge de sang; les organes de la génération sont très-souvent visibles à travers les tégumens; il en est de même du canal alimentaire, que l'on reconnaît à sa couleur brunâtre; la tête, pl.2, fig. $3 a$., se distingue du reste du corps par uu enfoncement ou dépression circulaire. Il se trouve au-dessus de cette dépression trois boutons ou plutôt trois valvules, qui peuvent s'ouvrir et se fermer; quand elles s'ouvrent, il se présente alors au milieu d'elles un petit tube, qui est l'ouverture de la bouche proprement dite. Le corps est cylindrique, et presque également aninci vers ses deux extrémités; il l'est cependant plus du côté de la tête; il y a dechaque côté et le long du corps une petite rainurc. Le canal 
intestinal, reconnaissable à sa coulcur brunâtre, se termine par une fente transversale ou anus, qui est située à la partie inférieure un peu avant l'extrémité postérieure du corps.

Le mâle se distingue de la femelle (qui est ordinairement plus grande) par la fin dé la queue, qui est recourbée, et par laquelle sort quelquefois un double pénis. Voy. pl. 2 , fig. $3 c$.

L'appareil générateur du mâle est d'un volume moins grand que celui de la femellc: cette dernière est représentée dans la fig. 3. Les or ğanes de la génération de la fenielle remplissent pour ainsi dire tout le corps, et la fin de la queue est droite. A l'endroit où les tégumens du ver avaient été déchirés, voy. fig. 3 , on remarque une très-petite ouverture, qui est l'entrée du vagin. Comme ce ver est connu de tout le monde; je n'ai faitreprésenter avcc dessein qu'une femelle, dont le ventre se trouvait accidentellement ouvert, afin quel'on puisse se faire une idée de son organisation intérieurc. L'intestin large et brunâtre, que l'on voit au dehors, est une partie du canal alimentaire, et les autres parties blanches sont les organes de la génération. Les plus volumineuses sont les oviductes, que Zeder appelle la matrice. Les plus minces sont les canaux excréteurs deś œufs, d'une forme cylindrique; car ces vers sont ovipares, et non pas vivipares, comme Wendelstadt l'a prétendu à tort; Werner croit cependant avoir observé des fotus déjà développés dans les oufs, et 'c'est ce que M. Rudolphi paraît vouloir confirmer également. Ceux qui 
voudiont counaître la disposition des organes sexuels du mâle et de la femelle de cette espèce de ver peuvent les étudier dans l'ouvrage de Werner. Ses figures ont été copiées par Jœrdens et Brera. Il n'entrait pas dans mon plan de donner à mes lectcurs l'anatomie des ver's intestinaux, mon but était seulement de leụr offrir la description et la figure de tous ceux qui ont été trouvés dans l'homme jusqu'ici, afin de les mettre en état de pouvoir reconnaitre, par des caractères certains, les différentes espèces de vers qui pourront se présenter à eux; j’ai regardé par conséquent comme superflu d'ajouter des dessins d'anatomie, ce qui du reste aurait augmenté de beancoup le nombre des planches et le prix de l'ouvrage; les médecius, qui auront envie de corinaitre la structure intérieure de ces vers, se donneront eux-mêmes volontiers la peiue de les examiner sous le rapport anatomique; ce qu'ils peuvent faire d'autant plus facilement, qu'ils sont toujours à même de s'en procurer, et ils n'auront pas à regretter le temps qu'ils y auront employé, quand même ces conuaissances ne leur serviraient seulement qu'ìse mettre àl'abri du rèproche d'être ignorans, quandil s'agira de donner leur avis sur ce sujet.

L'anecdote suivante publiée par Goeze ${ }^{i}$ peut servir à les engager davantage à ce geure de travail. "Quelques enfans firent un jour une incision sur un lombricoïde, et cela fit sortir ses intestins au dehors; le père, qui arriva dans ce noment, recueillit le ver

Ourrage cilé, p. jo. 
et le conserva dans de l'esprit-de-vin, pour demander à son médecin (du reste homme habile) ce qu'il croyait que cela pouvait être. Le médecin regarda les oviductes sortis au dehors, et les prit pour de jeunes ascarides, et le canal alimentaire pour un jeune tænia. Goeze rectifia cette erreur en présence du disciple d'Hippocrate qui avait porté ce jugement erroné, et qui par conséquent eut un peu à rougir de son ignorance.

La fig. 3 représente une petite femelle d'ascaride de grandeur naturelle; cet individu provenait du nez d'une vieille femme, qui l'avait rendu en se mouchant. Un de mes confrères m'a communiqué ce ver sans cependant pouvoir me donner beaucoup de détails sur ce fait; il ajouta, que cette femme était trop bornée, pour qu'il fùt possible de tirer d'elle quelques renseignemens détaillés; tout ce qu'il a pu apprendre se borna, à ce qu'elle avait éprouvé avant que de le rendre un grand mal de tête. Ce ver s'était sans doute glissé sur la voûte palatine par suite d'un vomissement qu'elle avait éprouvé précédemment, et était resté très-probablement depuis quelque temps dans la région supérieure des fosses nasales, jusqu'à ce qu'un effort pour se moucher l'ait enfin fait, sortir de cet endroil.

REMARQUES.

Le lombricoïde est connu des médecins depuis aussi long-temps que cẹlui dont nous vemons de nous 
occuper précédemment; on l'appela lumbricus; et, pour le distinguer des cestoïdes, il fut nommé lumbricus teres. On le regarda également comme identique avec le lumbricus terestris; mais nos meilleurs naturalistes ont découvert tant de caractères propres à distinguer ces deux animaux, qui n'avaient pas même échappé à Tyson ', qu'il est impossible de supposer qu'il puisse exister' aujourd'hui un médecin qui les confondrait.

Cependant Brera, pour montrer le genre d'exactitude qu'il met dans ses recherches et ses examens, a encore employé dervièrement six grandes pages in-quarto ${ }^{2}$ de son ouvrage pour montrer la ressemblance qu'il suppose entre ces deux vers, où il met toujours en avant son ancienne hypothèse (regardée par lui comme prouvée), que toutes les différences de conformation ne dépendent que $x^{\circ}$. des endroits dans lesquels le développement s'opère, $2^{\circ}$. de la nourriture, $3^{\circ}$. de la température. Si l'on voulait étendre autant que Brera, l'influence de la manière de vivre, de la nourriture, du climat, etc., sur les corps organisés, on pourrait alors facilement prouver, que l'homme, le singe, le maki, et beaucoup d'autres animaux proviennent tous de la même souche, et que la différence de leur structure, tant intérieure qu'extérieure, n'est résultée que des diverses influences dont nous venons de parler. Je ne suis pas du tout

- Philosophical transactions, I683, p. I53.

$\therefore$ Memorie, p. 201-206. 
disposé à entrer en dispute avec Brera, sur ce sujet, car Jacques-Théodore Klein, Goeze, Pallas, etc. ont prouvé jusqu'à l'évidence que ces deux animaux diffèrent tout à fait l'un de l'autre. J'engage mes lecteurs à décider eux-mêmes cette question. Ils n'ont qu'à comparer ces deux animaux ensemble, et ils verront aisément de quel côté est la vérité.

Le stomachide de Peereboom n'est rien autre chose qu'un lombricoïde mutilé ou défiguré, et il ne doit pas être considéré comme une nouvelle espèce. Il en est de même de celui trouvé par Treutler ${ }^{2}$ parmi beaucoup d'autres lombricoïdes bien conformés. Ce ver n'avait que deux valvules à la bouche, et ne doit être regardé que comme une difformité.

\section{BothriocépHaLe.}

Bothriocephalus latus (en allem. der bandwurm), pl. Iv et v.

Bothriocephalus : capite foveisque marginalibus oblongis, collo subnullo, articulis anterioribus ruga formibus, insequentibus plurimis brevibus subquadratis latioribus, ultimis longiusculis.

Bonnet; Mémoires présentés, t. I, p. 478, tab. I et II, tania à anneaux courts ou à mammelons ombilicaux; le même, Nouvelles recherches dans les observations de Rozier sur la physique, t. IX, p. $24^{3}-257$, tab. I, fig. I et 12 .

Pallas, Elenchus zoophyt., p. $408, \mathrm{n}^{0} .3$, t. grisea; p. $4 \mathrm{10}, \mathrm{n}^{0} .4$, t. lata.

Bloch, Abandlung, p. 17, tcenia lata, der breite bandwurm.

Goeze, Eingeweidew, s. $29^{\circ}$, fig. 8 , t. lata.

Batsch, Bandw., s. 1 07, fig. 33-5o, t. membranea; Der houtige bandwurm, s. 111, fig. $51-52$, t. lata.

× Ourrage cité, p. 17 , fig. 6 et 7 . 
Gmelin, Syst. nat., p. 3o65, t. vulgaris; p. $3 \circ 67$, no. 3, t. lata. Jœrdens, Helminth., s. 47 , t. IV , fig. 1-4, t. vulgaris; der kurzgliedrichte bandwiturm, s. 49 , t. Iv , fig. $5,8,9$, 10, der breite bandwurn.

Brera, Vorselung, s. 12 , tab. 1, fig. $3,7,13,14$, der unbewaffnete menschliche bandworm; Memorie du même, $8 \mathbf{1}-87$, toxia inerme umana.

Zeder, Anheitung, s. $347, \mathbf{n}^{0} .46$, halysis lata; s. $348, \mathbf{n}^{0} .47$, h. membranacea.

Rudolphi, Entoz., p. $7^{\circ}, \mathrm{n}^{\circ}$. 1, t. lata.

Bradley, A treatise, p. 84-86, tab. II, fig. 3-4, the broadt ope worm. Cuvier, Règne animal, p. 44 , le tania large.

De Lamarck, Anim. sans vert., t. III, p. 167, le bot. de l'homme.

Ce ver, nommé par Plater tonia prima, a reçu différentes dénominations : les Hollandais le nomment lindxorm, les Danois baandworm, baendelorm, les Suédois Binnike-mask, les Anglais the tape worm, jointed-worm, les Français le tania, le ver plat, et plus particulièrement toenia à épines, à anneaux courts ou à mamelons ombilicaux.

Le bothriocéphale se trouve dans les intestins grêles des habitans de la Pologne, de la Russie, de la Suisse et de quelques contrées de la France.

\section{DESCRIPTION.}

Ce ver, qui est plat en général, plus mince et trèssonvent beaucoup plus large et non pas plus étroit, comme on l'a prétendu, que le ver solitaire (tcenia solium), acquiert souvent une longueur de vingt pieds. Gœze assure cependant avoir reçu de Bloch un individu qui avait une longueur de soixante aunes un 
quart, et Boërhaave ${ }^{x}$ prétend qu'il en a fait revdre un, à un Russe, qui avait trois cents aunes. La partie la plus large du bothriocéphale est rarement de six lignes; il y a cependant des individus chez lesquels cette largeur s'étend jusqu'à un pouce, comme cela m'a été assuré par M. Rudolphi, qui en possède méme plusieurs de cette dimeusion.

Ce ver est ordinairement de couleur blanche, mais cependant il n'est jamais. d'un blanc parfait, et cette couleur se change en grisâtre quand on le met dans l'esprit-de-vin; d'où vient le nom de tania grisea employé par Pallas. L'échantillon que Sœmmerring a dounć ì notre cabinet d'histoire naturelle est également devenu gris, quoique ce ver fût mis dans de l'esprit de-vin qui avait été distillé avec le plus grand soin par Sommerring Jui-même: Cet échantillon se trouve dessiné d'après nature sur la planche 4 .

On peut clairement voir à sa tête allongée ( $V o y$. pl. 5, fig. $a, b, c$.$) des dépressions oblongues ou des fos-$ settes, que M. Rudolphi regarde comme des organes destinés à absorber la nourriture. Quant à moi, je crois que l'ouverture de la bouche proprement dite, qui conduit au canal alimentaire, est placée au milieu de ces deux dépressions. La fig. $c$ fait voir au moins une trace d'uue pareille ouverture; nais on peut voir celte dernière très-clairement sur lebotlliocéphale provenant du pleuronectes niaximus. Nous possédons cependant des bothriocéphales, appelés par M. Cuvier

Pre, ad institut., t. vı, ?. 18o. 
floriceps $^{x}$, provenant du requin et de la raie, qui présentent, selon l'apparence, quatre organes en forme de fleurs, ou bien quatre canaux destinés à puiser ia nourriture; mais il reste à savoir si ce ne sont pas des organes avec lesquels ces vers peuvent se cramponner pour sucer plus facilement leur nourriture au moyen de l'orifice de la bouche, qui se trouve, d'après ma supposition, au milieu de ces organes; au moins il y a assez de place en cet endroit pour une pareille ouverture. M. Rudolphi, qui a eu occasion die voir, lors de son dernier voyage en Italie, quelquesuns de ces vers vivans, nous donnera, sans doute bientôt, des éclaircissemens là-dessus; la limite entre la tête et le cou est, dans le plus grand nombre des cas, très-clairement marquée, comme on le voit sur les fig. $b$ et $c$. La fig. $a$ nous montre que la tête se confond quelquefois insensiblement avec le cou. Les helminthologues appellent cou, chez les cestoïdes, la partie qui suit immédiatement la tête et qui ne paraît pas être articulée; cependant le microscope fait souvent voir des traces d'articulations sur un cou, qui paraît à l'oeil n'être pas articulé; mais il y a des cas où les articulations peuvent être tellement rétractées, qu'elles ont l'air, vues même avec un bon microscope, de n'être qu'une surface continue. L'exis-

M. Jean Natterer, qui se trouve à présent au Brésil, a déjà envoyé, il y a quelques années, à notre cabinet, plusieurs espèces de ces vers, el il leur aväit déjà donné aupararant, c'est-à-dire avant M. Cuvier, le nom de tuliparia. 
tence ou la non-existence d'un cou ne peut pas servir, d'après moi, comme signes propres à établir la différence des espèces parmi les cestoïdes. Les fig. $a$ et $c$ font clairement voir un cou; il manque presque totalement dans la fig. $d$, et les articulations commencent immédiatement derrière la tête; mais cependant tous ces vers sont de la même espèce; la tête représentée dans la figure $b$ provient d'un individu qui n'avait que quatre pieds de long, et la dernière articulation arrondie faisait clairement voir que ce ver était entier, mais encore très-jeune. La tête de celui représenté dans la figure $c$ appartient à un échantillon de vingt-quatre pieds de long; c'était une femme de Pétersbourg qui l'avait rendu; l'extrémité de la queue arrondie n'existait pas; il s'était déjà probablement détaché plusieurs pieds de ce ver à une époque antérieure. Quelquefois on observe (à partir de la tête) un morceau filiforme de vingt pouces et plus, avant que le ver ne s'élargisse. Nous possédons un pareil échantillon, que nous devons à Jurine, de Genève. On ne doit pas cependant regarder, d'après moi, ce morceau filiforme conme un véritable cou, car quand le ver sort du canal intestinal sous forme de pelotte, cette partie filiforme ne s'aperçoit pas, mais bien quand il en sort en se déroulant peu à peu; il arrive alors que le cou et les articulations antérieures sont tellement distendus par la pesanteur du corps du ver, que l'on ne peut plus reconnaître les articulations. Comme le cou manque dans quelques cas, et qu'il existe dans d'autres, c'est la raison. 
pour laquelle on trouve dans la définition collo subnullo.

Les articulations sont en général plus larges que longues, quoiqu'elles forment quelquefois vers le milieu du corps un carré oblong; mais les côtés les plus longs de ce carré tombent toujours sur la largeur des articulations du ver, comme on le voit par, les figures. Les vers conservés en entier montrent que les articulations vers la partie postérieure du corps deviennent de nouveau oblongues ( $V$ oyez pl. 4). Les articulations des bothriocéphales, quand ils sont encore jeunes, se contractent quelquefois iellement, que l'on pourrait douter au premier abord qu'ils fussent articulés, d'où vient que Zeder a donné à ce genre le nom de rhitelminthus et plus tard celui de rhytis, en français ver ridé, en allemand runzelywurm ( $V o y \cdot \mathrm{pl} .4)$.

L'on peut voir facilement, au milieu des articulations complétement développées, une dépression, fossette, ou ouverture; quelquefois on en aperçoit une seconde plus petite, un peu plus en arrière, c'està-dire vers l'extrémité postérieure (Voyez fig. $h$ ); il sort dans quelques cas de la fossette un petit dard ( oy. fig. g représentée sous une éclielle plus grande que nature) que Bonnet a déjà remarqué. C'est probablement l'organe sexuel du mâle; les oviductes en forne de fleurs, entourent cette ouverture : on peut clairement voir ces oviductes en nettant un petit morceau de cet animal sous le microscope composé, et en passant en dessous, au licu d'une tablette noire, une petite plaque de verre sur laquelle le miroir réféchit Ja lumière. 
L'on trouve quelquefois une espèce d'incision sur l'extréınité large du ver, comme cela se voit dans la fig. $k$, pl. 5 , et que plusieurs médecins ont regardée comme la fin de la tête. Tulpius ${ }^{2}$ a représenté un pareil morceau large et détaché d'un ver sous le titre de genuinum lati lumbrici caput, parce qu'il prend les articulations postérieures fendues pour des têtes. Sa figure ressemble à une tête d'oiseau, et un trou superficiel (foramen superficiale) parait être l'œil de cette tête. Leclerc l'a copiée dans sa planche vir. Mais cette incision ou ces deux lèvres se forment quand on déchire les articulations, et ne sont qu'accidentelles; nous observons également souvent un ou plusieurs trous au milieu des articulations, mais cela ne nous met pas en diroit de regarder un ver ainsi conformé comme constituant une espèce particulière; car il me paraît probable, que ces trous provienuent de l'oviducte, qui s'est ouvert dans ces endroits là.

\section{REMARQUES.}

Plusieurs médecins anciens ont déjà observé que l'on rencontre dans l'homme deux différentes espèces de cestoïdes. Je ne veux pas cependant décider qui a remarqué le premier cctte différence; nous savons seulement que Sennert et Tyson ${ }^{2}$ en avaient déjà connaissauce; mais Bonnet, quile premier a fait dessiner

$=$ l.ib. 11, cap. 52 , p. 161 et 162 .

'Philosophic. transact., 1683 , p. $1 \times 3$. 


\section{7o SUR LES VERS INTESTINAUX}

la tête d'un cestoïde, qu'il regardaiı à tort comme appartenant à un tænia large, est cause que nos meilleurs helminthologues ont placé, même encore dans les temps modernes, ce ver dans le genre tæuia. Bonnet tomba par hasard sur une tête de tænia, qu'il croyait provenir (à cause des articulations courtes du cou) d'un tænia à anneaux courts ou bothriocéphale; comme on représente ordinairement la tête du véritable tæuia, pourvuc d'une couronne de crochets entre les quatre suçoirs ; et com me cette couronne manquait justement à la tête examinée par Bonuet (j'en possède moi-même plúsieurs semblables échantillons ), on regarda d'autant plus volontiers son dessin comme conforme à la structure d'un bothriocéphale, que l'on cherchait justement dans ce manque de couronne une différence essentielle entre la tête de ce dernier et celle d'un tania proprement dit (tonia solium). Bonnet rectifia cette erreur dans une nouvelle dissertation sur ce sujet, publiée en 1777 , c'est-à-dire trentequatre ans après la première; mais il paraît que l'on ne l'a pas regardée comme digne d'une attention particulière; car dans toutes les descriptions et dessins concernant le ţenia à anneaux courts, ou le bothriocéphale, qui ont paru depuis, on est toujours revenu à la première idée qu'il en avait donnée.

Brera ' a cependant copié une figure appartenant à la seconde dissertation de cet auteur; mais Brera a choisi la quatrième figure, c'est-à-dire la moins

Tab. I, fig. 7 , Vorlesungen. 


\section{DE L'HOMME.}

bonne, qui provenait d'un échantillon resté quinze ans dans l'esprit-de-vin, tandis que la troisième n'est pas mauvaise et est assez conforme à la nature. Lorsqu'en 1811 on avertit toutes les sociétés savantes de la formation de notre grande collection de vers intestinaux, on pria en même temps de vouloir bien nous envoyer un trenia à anneaux courts ou un bothriocéphale pourvu de sa tête, en cas que quelqu'un s'en trouvât en possession. Il'nous arriva, le 20 mars 1812 , de la part de M. Soemmerring, une boîte contenant plusieurs vases remplis de ces vers, parmi lesquels il y a un échantillon qui est représenté dans la pl. 5, et que M. Sœmmerring avait rendu lui-même plusieurs années auparavant. Mon étonnement fut à son comble, lorsque je pus remarquer, à l'aide d'une simple loupe, les deux fossettes ou dépressions oblongues qui existent à la tête de ce ver. Je compris alors surle-champ la différence qui existe réellemenı dass la conformation des deux cestö̈des, qui se trouvent dans l'homme, car ces animaux se ressemblent si peu, que non-seulement ils n'appartiennent pas à la même espèce, mais même qu'ils ne sont pas du même ğenre, et javoue que je fus fâché dc n'avoir pas placé plus tôt ce prétendu tænia à anneaux courts, par la simple conformation de ses articulations, dans le genre qui lui convenait; car l'ouverture des organes de la génération, chez ce dernier, se trouve sur la face des articulations, tandis qu'elle est placée au bord sur le véritable tænia. Je crois qu'aucun helminthologne qui aurail trouvé un morceau d'un ver parcil, même 
sans tête, dans un poisson ou bien dans un oiseau aquatique, n'auraic hésité un instant à le ranger dans le genre bothriocéphale; mais je me suis consolé en peusant que d'autres avaient commis la même erreur. Depuis, j’ai fait évacuer à une personne un ver de cette espéce pourvu de sa tête, et j’en ai aussi reçu quelques échantillons de la Suisse. J'ai choisi, pour le genre de ce ver, le nom de bothriocéphale, et celui de large pour son nom d'espéce.

Avant que nous nous occupions du véritable tænia, il faut que je fasse mention que notre collection possède un bothriocéphale difforme que M. Sœmmerring nous a procuré par un échange avec le cabinet d'histoire naturelle de M. Voith; c'est un fragment de bothriocéphale qui fait voir, à l'endroit où il a été déchiré (ce qui a pu avoir lieu dans toute la longueur de l'animal) deux fossettes sur chaque articulation; ces fossettes ne sont pas. placées l'une après l'autre, mais bieu l'une à côté de l'autre, comme cela est représenté par les fig. $k$, pl. 5 . Cette disposition des fossettes ne se trouve cependant que sur onze articulations, car au-delà de la onzième il n'y a, sur le reste du morceau, qu'une seule fossette sur ehaque articulation. Il ve faut pas cependaut regarder cela comme une monstruosité semblable à celle dans laquelle deux jumeaux sont accollés l'un à l'autre; mais il paraît plutôt que ces articulations, au moment de leur première conformation, se sont dérangées de lcur position naturelle, et sont devenues adtićrentes.

Ce fragment présente encore cela d'extraordinaire, 
que les articulations à sa partie postérieure se trouvent fendues pendant un assez long espace. Pallas ${ }^{i}$ a publié le dessin d'un semblable échantillon. Du reste, nous ne devrons pas regarder ce ver comme appartenant à une nouvelle espèce, car ce n'est qu'un bothriocéplale ordinaire. Nous avons encore une seconde difformité d'articulations provenant d'un autre échantillon (Voy. fig. $h$, pl. 5 ). M. Rudolphi observe que l'on n'a pas encore rencontré, autant qu'il s'en rappelle, debothriocéphale dans le cadavre d'un homme; j'ai entendu dire la même chose, il y a plusieurs années, à un médecin suisse. Les médecinsqui exercent dans le pays où ce ver séjourne, devraient bien nous donner des éclaircissemens à ce sujet.

J'étais au moment de faire imprimer cette feuille, lorsque je fis rendre, le 17 juin 1819 , un ver de cette espèce à un Suisse de vingt-six ans, du canton de Glaris, et cela m'a fourni l'occasion de faire encore quelques remarques sur le bothriocéphale; car la femme suisse incommodée par ce même ver, que j'ai traitée à Vienne, et à laquelle j'ai administré mon huile anthelmintique, ne l'a pas rendu d'une manière visible. La tête du bothriocéphale, que je fis évacuer entier à une femme de Pétersbourg, m'occupa alors trop pour avoir pu prêter une attention suffisante aux articulations: ce ver était, du reste, tellement gros et renflé, que l'on ne voyait les articulations que d'une manière très-peu distincte.

I. nord Beitr., I , tab. fig. ïI, fig. I6. 


\section{7f SUR LES VERS INTESTINAUX}

Le jeune Suisse dont nous venons de faire mention vivait déjà, depuis douze ans, hors de sá patrie, et ce ne fut que l'année passée qu'il fut averti, par l'évacuation de quelques morceaux, qu'il nourrissait un bothriocéphale dans son corps; il avait, du reste, toujours joui d'une bonne santé, et ce ne fut que depuis le traitement que l'on avait entrepris contre ce ver ( traitement qui consistait en médicamens vermifuges et drastiques, que l'on avait employés pendant plusieurs isemaines ), qu'il se trouva un peu affaibli; il cessa l'usage des médicamens, et se porta parfaitement bien. Cependant il vint me voir un jour, et me pria de m'assurer au juste si ce parasite existait encore dans son corps, ou non; car il n'avait pas examiné ses évacuations alvines depuis quelque temps.

Je n'hésitai pas à le soumettre à cet essai, en ce qu'il ne pouvait en résulter aucun danger pour sa santé. Je lui fis prendre un jour, le matin avant de se lever, trois gros de fougère mâle, choisie et fraîchement pulvérisée, en une seule dose, et je l'engageai à boire par dessus une tasse de café à l'eau, aussi chaud que possible (les médecins de Genève suivent cette méthode, par laquelle ils évitent souvent les vomissemens causés par cette racine); je lui ordonuai ensuite de prendre, deux heures après, une cuillerée à bouche d'huile de ricin frấchement exprimée et mêlée avec un peu de bouillon, de demi-heure en demi-heure, et jusqu'à la consommation de trois onces. Lorsque je fus le voir à deux 
heures après midi, il avait déjà dîné d'un très-bon appétit, et il n'avait eu, dans la matinée, que deux selles liquides très-copieuses, sans cependant être aqueuses. Il n'avait, du reste, rien senti qui pût lui faire présumer la sortie d'un ver; c'est pourquoi il ne s'était pas donné la peine de le chercher; cependant ce ver se fit voir après qu'on eut examiné les déjections alvines; elles eu contenaient trois morceaux, dont le plus long avait vingt-cinq pieds (mesure de Vienue); les deux autres étaient très-minces, ei avaient l'air de provenir du voisinage de la tête, et leur longueur était de six à huit pouces. Je n'ai pu déconvrir la tête, et l'extrémité postérieure n'était pas conservée en entier; l'on voyait clairement qu'un mơrceau en avait été déjà détaché quelque temps auparavant. Voici ce que j’ai remarqué sur ce ver, qui est le second que j'ai observé fraîchement rendu, et avant qu'il eût été mis dans l'esprit de vin. Le ver fut bien lavé avec de l'eau, et lorsque je l'ai examiné, il ne s'était passé qu'une heure depuis qu'il avait été rendu. Sa couleur n'étail pas parfaitement blanche, comme Jœrdens et Brera le prétendent ; mais il était d'un gris clair, tel qu'il est représenté sur la pl. 5 , fig. $d, f, i$. Les tænias sont en général beaucoup plus blancs que les bothriocéphales. Mais le docteur Gaede de Kiel, qui arriva justement dans le moment où mon dessinateur venait de terminer de dessiner ce ver, peut attester que cet animal a été réprésenté avec la plus grande exactitude; car M. Gaede l'a vu aussi avant qu'il ne füt mis dans 
l'esprit-de-vin. Quant aux articulations antérieures de ce ver, je n'ai rien remarqué qui ne puisse être vu sur la planche 5 , si ce n'est qu'elles diffé. raient dıns la couleur. Les articulations étaient transparentes dans le milieu, à l'endroit où le ver commence à s'élargir, et l'on pouvait clairement voir les ouvertures, en forme de petites fossettes rondes, qui conduisent aux organes de la génération.

Ces articulations n'étaient pas transparentes sur leurs bords; cependant on distinguait, dans leur intérieur, des points isolés de forme ronde; c'étaient peut-être des œufs non fécondés (Voyez fig. $h$, pl. 5). Un peu plus loin que les articulations dont nous venons de parler, il y en avait quelquesunes de fécondées : cela peut se voir sur låfig. $f$. Ces articulations ne sont pas dessinées d'une manière aussi élégante et symétrique que celles de Bonnet, copiées par Joerdens et Brera; mais je puis assurer que mon dessinateur les a représentées ficièlement et telles qu'elles se trouvaient dans la nature. On observait, sur différens endroits de ces articulations, à quelque distance de là, de petites protubérances ou papilles jaunâtres (Voyez fig. $i$ ). En ouvrant une de ces protubérances avec une épingle, il en sortit des oufs fécondés.

On peut les voir représentés, mais considérablement grussis, dans la fig. $l$. Ces œufs n'avaient pas tous la même forme, comme on le voit par la même figure.

A l'endroit où les œufs fécondés se trouvent pla- 
cés, le ver perd de sa largeur; les bords ou plutôt les parties latérales des articulations sout ridées, et l'on ne remarque pas dans ces dernières des points ou grains blancs, comme cela peut se voir sur la fig. $d$.

11 me paraît probable que toutes les articulations de bothriocéphale ne sont pas fécondées; car ou ne voit sur la fig. $i$ (qui représente un morceau de tænia de treize articulations) que deux protubérances ou papilles remplies d'œufs; une troisième protubérance se trouve sur le bord de la fente, et a l'air d'avoir été déjà vidée à moitié; je ne puis pas cependant affirmer que la chose soit ainsi.

Après qu'une articulation a élé fécondée, il me paraît probable que, dès ce moment, tout le travail de la nutrition n'influe que sur les œufs, qui se trouvent contenus dans cette articulation, et n'agit plus sur cette dernière elle-même; elle dépérit alors de plus en plus, se crispe, perd de sa largeur, se ride sur ses bords et meurt à l'époque à laquelle ses œufs sont parvenus à leur développement complet et oì ils en sortent. L'endroit perforé que l'on remarque sur la figg. $i$ paraît venir à l'appui de ma supposition; car il est probable que cette perforation ne s'est opérée qu'au moment où les articulations se sont rompues pour laisser sortir les œufs complétement développés dont elles étaient chargées. On voit sur la dernière figure mentionvée, que les articulations n'étaient pas loin de leur destruction totale, car clles avaient déjà perdu leur couleur naturelle et étaient devenues tout à faitbrunes; il est donc permis de sup- 
poser que ce morceau se serait déchiré sous peu en cet endroit, et que le ver aurait perdu deux pieds de sa longueur, car le morceau, qui faisait suite à ces articulations, était de cette longueur.

Les observations que je viens de rapporter ont été pour moi d'un grand intérêt, je désire qu'elles n'aient pas fatigué mes lecteurs.

\section{TENIA.}

Tania solium (en allem. der kettenwurm), pl. vi et viI.

TAN!A : capite subhemispherico, discreto; rostello obtuso; collo antrorsum increscente, articulisque anticis brevissimis, insequentibus subquadratis, reliquis oblongis, omnibus obtusiusculis ; foramimibus marginalibus vage alternis.

Pallas, Elench. Zoophyt, p. 405, $\mathrm{n}^{0}$. 1, t. cucurbitina.

Neuc, nord. beitr. du même, t. 1, p. 46 et 37 , tab. 11, fig. 4 et 9 , t. cucurb.

Bloch, Abhandl., s. 20 et 28 , der kürbiswurm.

Weruer, Verm. intest., p. 18 et 49 , t. solium; p. 49 et $5 \%$, fig. 47 et $57, t$. vulgaris.

Goze, Eingeweidew, s. 269 et 296 , tab. xx1, fig. 1, 7,9 et 12. Gmelin, Syst.nat., p. 3o62, $\mathrm{n}^{\mathrm{o}}$. I, t. solitum; p. 3o73, $\mathrm{n}^{\circ} .3$, t. dentata.

Batsch, Bandwürmer, s. 11 1-123, fig. 1, 6, 9, 11 , 21, 23, 54 , der kürbisbandwurm; s. 184-187, fig. 110 et I 13 , der geachnt: bandw.

C., In the Transact. of the Linn. soc., vol. II, p. 247 et 262 , tav. $\mathrm{XXv}$, fig. I et $8, t$.' solium.

Joerdens, Helminth., s. 40, tab. III, fig. I et 7 , derlangsliedrichte, bandwurm, s. 47 , tab. IV.

Brera, Vorlesung., p. 9, fig. I, 3, 8, io et I I; der bewaffnete 
menschliche bandxurm. Memorie du même, p. 64 et 80 , tab. I, fig. 1, 14, 17, 22, tenia armata umana.

Zeder, Anteitung, p. $359, \mathbf{n}^{0} .48$, halysis solium.

Rudolphi, Entoz. II, part. I, p. I6o, no. 56, t. solium.

Bradley, $A$ treatise, p. $7^{5}$ et 83 , pl. 11 , fig. 4 et 1o, tania osculis marginalibus. tape worm.

Cuvier, Règne animal, p. 43, tocnia à longs anneaux.

Olfers, De vegelativis, p. 35 et $37, t$. solium.

De Lamarck, Anim. sans vert., tome 111, page 164, tonia cucurbitain.

Le bothriocéphale et le véritable tænia, appelé par Plater tcenia secunda, portent, chez les auteurs, comme nous l'avons déjà remarqué, le même nom; cependant les Français désignent ordinairement le véritable tænia sous les noms suivans : le solitaire, le tænia sans épines, tænia à anneaux longs.

Le véritable tænia séjourne dans les intestins grêles de l'homme de toutes les nations européennes à l'exception de celles que nous avons mentionnées lorsqu'il était question du bothriocéphale. On rencontre aussi très-souvent le tænia.chez les Egyptiens.

\section{DESCRIPTION.}

Je crois que personne n'a encore vu un tænia entier, c'est-à-dire qui fût à la fois pourvu de la tête et de la queue; car souvent il arrive que les dernières articulations, qui sont ordinairement chargées d'œufs fécondés, se détachent et sont évacuées par les selles, avant que les articulations antérjeures, les 
plus près de la tête, soient encore complétement développées; c'est pourquoi on ne peut pas fixer au juste quelle longueur ce ver pourrait atteindre, si toutes Jes articulations restaieut ensemble. Les tæuias d'une longueur de vingt-quatre pieds ne sont pas très-rares : notre collection cependant n'en a pas de plus longs.

Reinlein, dans son ouvrage, parle souvent de ces vers, et porte leur longueur à quaraute jusqu'à cinquante aunes.

Dans les Dissertations " de Copenhague, on fait mention d'un tænia de huit cents aunes de long.

Robin raconte qu'il a trouvé dans le cadavre d'un homme (qui avait rendu, peu de temps avant de mourir, un morceau de tænia de plusieurs pieds de long), immédiatement au-dessous du pylore, un de ces vers reployć en forme de pelotte et qui s'étendait dans toute la longueur des intestins, jusqu'à six ou huit pouces de l'anus; Robin remarque que sa longueur a pu se monterà trente pieds, y compris le morceau qui avait été détaché auparavant. Si un tænia qui occupait presque tout le tube intestiual, comme - celui dont nons venons de parler, n'avait que trente pieds de longueur, il est alors bien permis de présumer que les antenrs cités plus haut se sont trompés dans l'indication de leurs mesures, et qu'ils ont compris, sans doute, plusieurs vers ensemble, comme cela a dû avoir lieu pour celui de huit cents aunes. Admettons en effet que la longueur du tube intes-

$=$ Acl. hamiens., rol. 1, p. 148 . 
tinal de l'homme ve soit que de trente pieds, et qu'une aune ne forme qu'une longueur de douzc pouces, c'est-à-dire qu'un pied, le ver de huit cents aunes de longueur aurait été, par conséquent, obligé de se reployer au moins vingt-six fois, pour trouver a s'y placer. Une teille quantité de replis aurait uaturellement rempli presque toute la capacité des intestins, et l'on ne conçoit pas comment les alimens et les matières fécales auraient pu encore s'écouler. Il est cependant facile de concevoir ce qui a engagé les médecins à croire qu'il y avait des tænias d'une longueur aussi extraordinaire; c'est qüils supposaient que tous les morceaux de tænias, rendus peu à peu par un homme, appartenaient à un scul individu.

Hufeland " fait mention d'un enfant de six mois, qui avait rendu pcu à peu trente aunes de tænia, sans éprouver la moindre altération dans sa santé. Supposons que cet enfant ev eût évacué une parcille quantité tous les six mois, jusqu'à l'âge de la puberté, la longueur du ver se seraic alors montée à mille quatre cent quarante aunes, à peu près; on aurait cependant bien tort de conclure de là qu'il y ait des tænias aussi longs.

La largeur de ce ver varie beaucoup : vers la fin de la tête, il n'a souvent qu'un quart ou qu'un tier's de ligne de largeur; mais elle augmente peu à peu jusqu'à trois, quatre, ou même six lìgnes; du reste,

'Journal Bd. 1 8 , st. x, p. 3. 
quand ou mesure ce ver, il faut toujours faire attention s'il se trouve dans un état de contraction ou d'extension; car sans cela la mesure ne peut pas être regardée comme conforme à la vérité(Voy. pl. 6, fig. 1). La grosseur du tænia varie aussi beaucoup; quelquefois il est très-mince et presque transparent, et dans d'autres cas il est gros, comme cela est démontré par les articulations de la fig. $a$, pl. 7 ; sa tête est en général très-petite (Voyez pl. 6, fig. 1); elle est cependant quelquefois assez grosse pour être vue à l'œil nu (Voyez fig. $a$ ).

Mais, à l'exception de l'individu, fig. $a$, j'avoue n'en avoir jamais va, depuis, un semblable, dans lequel une tête si volumineuse fùt placée sur une tige aussi mince, et qui se changeât si brusquement en un cou d'une largeur assez considérable. On trouve la forme de la tête, en général, variable, surtout lorsqu'on l'examine sur des individus morts, comme cela arrive presque toujours. Cette diversité de forme s'explique très-bien, quand on a observé un de ces vers vivaut hors du canal intestinal, comme j'en ai eu l'occasion. La tête et le cou de l'animal sont alors dans un mouvement continuel, et son corps se contracte et s'allonge alteruativement. Ces changemens de dimension influent également sur celles du cou et de la tête; et les têtes paraissent tantôt plus longues et plus étroites, et tantôt plus larges et plus courtes. En tuant subitement un individu obtemu vivant, au moyen de l'eau fraîche ou de l'esprit-devin, il conserve la forme qu'il avait au moment de 
mourir. On doit également remarquer que l'espritde-vin très-concentré a la propriété de rétrécir ou de contracter les individus morts. Le ver représenté sur la fig. $b$, pl. 6 , a été tué dans de l'eau tiède que l'on avait laissée peu à peu refroidir. L'on peut facilement se faire une idée de la diversité des dimensions dans la tête des cestoïdes, si l'on examine les tricuspidaires provenant du brochet, et les bothriocéphales provenant des barbeaux : l'on rencontre presque toujours vivantes ces deux dernières espèces de vers, daus ces deux genres de poissons. M. Rudolphi' a indiqué six différentes formes de tête sur les tricuspidaires : jen ai fait dessiner neuf des mêmes vers, et cinq du bothriocéphale provenant du barbeau. Je crois que les trois figures de tête de tænias, qui avaient séjourné dans l'homme (Voy. fig. $b, c, d, \mathrm{p}^{\mathrm{l} .6}$ ), et qui sont représentées sur une échelle plus grandi que nature, suffiront pour donner à mes lecteurs une idée claire de ces variations.

L'on remarque toujours à ces têtes, si variables dans leur forme, quatre suçoirs qui sont, daus l'état vivant, tantôt plus proéminens, tantôt plus rétractés. Le tæuia vivanı que j’ai examiné allongeait toujours les deux diagonalement opposés, tandis qu'il raccourcissaic les deux autres de la même manière.J'ai observé dans les tubes de deux tænias qui avaieut été rendus vingt-quatre heures auparavant, unematière noirître, représentée dans la fig. $d$, pl. 6 : cette matière se dis-

'Entoz, tals. 1x, fig. 6 el Ix. 
solvit et disparut après un court espace de temps; ce n'étaic rien autre chose qu'un peu de matière fécale qui s'était introduite dans les tubes des sucoirs de ces deux individus, et ce qui donnait à leur tête l'apparence d'avoir quatre yeux. C'est de là d'où provient, sans doute, que les auciens médecins représentaient toujours la tête des cestoïdes pourvue de quatre yeux; Audry, par exemple', défend encore l'opinion que les cestoïdes ont quatre yeux, contre Méry, qui regardait les suçoirs comme autant d'ouvertures nasales.

Quand la tête est tout ì fuit allongée, l'on voit, entre ces quatre suçoirs, une protubérance convexe (Voy. fig. b, pl. 6), sur laquelle se trouve toujours un cercle au milieu duquel est plácée une petite ouverture presque imperceptible. On observe quelquefois sur ce disque un double rang de petits crochets; l'on fait mention dans presque toutes les descriplions de ce disque pourvu de crochets, et on les trouve également représentés dans presque toutes les figures; mais cela ne s'observe pas toujours en réalité. J'avais déjà examiné cinq à six de ces vers, y compris celui qui était encore vivant, et dont jai parlé plus hant, sans quill m'eùt étć possible de découvrir une couronne de crochets sur la tête, malgré que je me fusse servi d'excellens microscopes. J'en fis part à M. Rudolphi, et peu de temps après il m'envoya un tænia pourvu d'une parcille couronne très-

Ourrage cité, p. Gog. 
visible (Vovez fig. c). Plus tard je reçus encore un semblable individu de M. Goergen.

11 me paraît probable que le ver donı nous nous occupons maintenant perd sa couronne de crochets en vieillissant. J'admettrais cette supposition d'autant plus volontiers que l'on rencontre assez souvent d'autres vers intestinaux chez lesquels les crochets se détachent : cela se remarque assez fréquemment sur le ver nommé par moi echinorlynchus polymorphus.

Notre collection possède un tænia très-long ( $t a-$ nia serrata) provenant d'un chien. La tête de ce ver est aussi dépourvue de crochets, chose qui n'arrive pas ordinairement chez les individus de cette espèce.

Le véritable tænia a le cou aplati ou déprimé, et variable en longueur. Je ne l'ai jamais vu manquer tout à fait dans aucun individu de cette espèce : après le cou dépourvu d'articulations, arrive le corps articulé. Les premières articulations sont d'abord trèsétroites, et toujours plus courtes qué larges; à mesure qu'elles s'élargissent, lęur longueur augmente proportionnellement beaucoup plus, et elles forment par la suite de vrais carrés qui deviennent plus loin des carrés oblongs, dont la longueur surpasse la largeur au moins du double; mais on rencontre souvent des individus qui sont conformés différemment, et sur lesquels l'on remarque des articulations plus larges que longues, suivies d'articulations qui sont plus longues que larges. Cet état de chose provient des contractions inégales de quelques parties du corps ; car les mouvemens de ces vers consistent, comme nous l'avons 
déjà remarqué, en contractions et en allongemens continuels de leurs articulations. Les contractions rendent ces dernières plus larges et plus courtes, et les allongemens, plus longues et plus étroites. J'ai fai dessiner un individu qui était d'une longueur de huit pieds, en omettant plusieurs longs morceaux d'articulations qui avaient la même conformation que les précédentes. Les lig. $b, c, e ;$ pl. 7 , font voir plusieurs anomalies dans la conformation des articulations.

Les difformités proviennent souvent, comme je l'ai déjà remarqué, de la manière subite dont on tue le ver, soit en le plongeant dans l'eau froide, soit dans l'esprit-de-vin.

On observe, près des bords des articulations, de chaque côté et le long de tout le corps de quelques in-

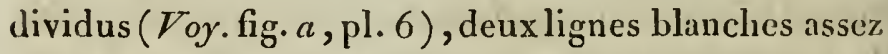
marquées; elles sont placées l'une sur l'autre, de manière que celle situéc supérieurement couvre et cache l'inferieure. M. Rudolphi regarde ces lignes comme des canaux alimentaires qui tirent leur origine des suçoirs de la tête. Je possède un tæuia très-mince et transparent, que l'on a fait rendre à un homme : ce ver n'était pourvu que d'un seul canal alimentaire, placé au milieu des articulations.

On observe encore, sur les bords des articulations bien développées, tantôt à droite, tan tôt à gauche, de petites protubérances papilliformes, qui sont pourvues, dans leur nilieu, d'une ouverture bien visible; on distingue clairement cette dernière sur les articulations du milieu (Voyez fig. 1, pl. 6), mais en- 
core mieux sur les fig. $d, e, f, i$, pl. 7 . On a cru pendant long-temps que le ver s'attachait, avec ses ouvertures latérales, aux parois des intestins, pour sucer sa nourriture; mais les naturalistes modernes sont tous d'avis que ces ouvertures, ainsi que les petits canaux qui en proviennent, conduisent aux oviductes : ceux-ci, dont la forme est assez variable, présentent cependant presque toujours une disposition phytoïde sur les individus dont le corps est mince, et sur ceux qui ont été déjà un peu macérés ( Voyez fig. $d$ ). Ils constituent presque tout l'appareil de la génération.

On observe sur quelques tænias, surtout. sur ceux provenant d'oiseaux aquatiques, des petits filamens qui sortent des ouvertures des ovaires, et que l'on peut, ce me semble, regarder comme les parties sexuelles des mâles. Je n'ai pas encore pu découvrir ces filamens sur des tæuias provenant de l'homme; la position des ouvertures latérales n'est pas soumise à un ordre ccrtain; quelquefois il y en a trois, quatre ou davantage du même côté, placées l'une après l'autre, sans interruption, tandis qu'il n'y en a qu'une ou deux de l'autre côté. Les personnes qui désirent conuaître la structure interne de ces organes, doivent étudier l'ouvrage de Werner.

REMIRQUES.

Les médecins les plus anciens font déjà mention de ce ver, mais sous deux noms différens; car ils 
distinguent le lumbricus latus, ou le tania solium, qui a été probablement confondu quelquefois avec le bothriocéphalus latus, des vers cucurbitains; quant au premier, ils croyaient qu'un homme ne pouvait être atteint que d'un seul individu à la fois, et, quant aux derniers (les vers cucurbitains), ces médecins les ont regardés comme appartenant à unc espèce particulière, ou, selon l'opinion d'Andry, conme des œufs du tænia; l'une et l'autre de ces suppositions sont erronées. D'abord, nous avons de nombreux exemples qui prouvent que plusieurs trenias peuvent se trouver en même temps dans les intestins du même homme. De Haë̃ x en a fait rendre dix-huit à une femme de trenteans, dans l'espace de quelques jours, et qui étaient tous très-minces ou filiformes à l'une de leurs extrémités. J'en ai vu moi-même deux ou trois dans le même individu, et j'en ai souvent rencontré soixante-dix ou quatre-vingts à la fois dans les intestins de jeunes chiens; et quant aux vers cucurbitains, les auteurs ont remarqué que ce ne sont que des articulations postérieures fécondées ou chargées d'œufs développés qui se sont détachés du tronc du tænia.

On a encore eu autrefois des idées tout à fait singulières sur la véritable nature de ces vers. Linné, par exemple, niait la présence de la tête du tænia. Blumenbach regarda pendant long-temps, comme

${ }^{2}$ Ratio medendi, vol. xu, p. 218 . 
les premières éditions de son ouvrage le prouvent, les articulations de ce ver comne autant d'animaux, et il pensait qu'ils n'étaient que collés l'un contre l'autre ${ }^{x}$; mais cet auteur a abandonné plus tard cette opinion. Carlisle prétend qu'il peut se développer un nouveau ver de chaque articulation.

Les personnes qui ont eu occasion d'observer beaucoup d'animaux dans lesquels le tænia séjourne souvent, et qui ont rencontré et observé des jeunes vers de cette espèce, n'admettent aucune des opinions que nous venons de rapporter. J'ai trouvé plusieurs fois, dans les intestins du cormoran (pelicanus carbo) de jeunes tænias appelés par M. Rudol phi toenia scolecina, qui n'avaient que trois on quatre lignes de long; leur queue etait arrondie et leur tête pourvue de quatre suçoirs et d'une couronne de crochets très-visibles, preuve que ces vers étaient entiers, quoique l'on ne pût y distinguer des articulations, pas même avec un microscope; on voyait néanmoins à leurs bords des filamens (lemnisci) dont nous avons fait mention plus haut. Cette observation prouve que les tæuias, et les cestö̈des en général, se forment d'un seul coup comme embryons, et qu'ils parviennent, comme tout autre animal, peu à peu à leur grandeur naturelle, sans qu'il soit nécessaire pour s'étendre, que de nouvelles articulations se forment après leur naissance.

Les naturalistes ont avancé beaucoup de closes et

- Gattingsche anzeigen von selehrlen sachen. 1774., st. 154, p. 1313 . 
souvent tout à fait chimériques sur cctte formation, ct nous verrons par la suite quel degré de confiance elles méritent.

La formation de nouvelles articulations peut être conçue de trois manières différentes, c'est-à-dirc qu'il est possible d'imaginer sur le corps du ver trois cudroits où cette formation puisse avoir lieu : $1^{\circ}$. à la fin de la tête, c'est-à-dire entre elle et l'extrémité anıérieure du corps; $2^{\circ}$. entre les articulations, dans un seul ou dans plusieurs endroits du corps à la fois : il faudrait alors admettre que les anciennes articulations se diviseraient en plusieurs, et que les articulations nouvellement produites acquerraient peu à peu la grandeur de celles entre lesquelles elles se seraient intercalées; et 3\%. à l'extrémité postérieure du corps, ou à la fin de la queue.

Si l'addition des nouvelles articulations pouvait avoir lieu à l'extrémité antérieure du corps, il serait plus naturel d'admettre que le cou recevrait une augmentation de la tête elle.même, et que pour conserver, malgré celte augnentation, sa longueur primitive, sa partie postérieure se diviserait en articulations, que de supposer que la totalité du cou se diviserait d'abord en articulations, et que les nouvelles pousseraient, pour ainsi dire, plus tard, de la tête elle-même. Cette hypothèse ne peut être conıbattue par l'observation, que nous avons faite plus liaut, que notre collection ne possède aucun tænia provenant de l'homme, qui ne soit pourvu d'un cou plus ou moins long, parce qu'il $y$ a, en eflet, une 
grande quantité de tæuias provenant d'animaux, auxquels le cou manque totalement; et, comme le tænia de l'homme leur ressemble pour le reste, il estalors permis de supposer qu'il n'en diffère pas non plus sous ce rapport.

Si le second cas avait lieu, c'est-à-dire s’il arrivait que des articulations (dans un endroit quelconque du corps) se divisassent en plusieurs autres, on aurait dû le remarquer déjà depuis long-temps, par la raison que la formation des nouvelles articulations produites par la division de l'ancienne, n'aurait pu s'effectuer que peu à peu, c'est-à-dirc dans un espace de temps plus ou moins long, et sans avoir été observé par les naturalistes.

L'on rencontre bien quelqucfois des articulations difformes et inégales, quant à la longueur des deux côtés; mais jamais on n'a observé, sur une articulation, des espèces d'incisions ou de rainures tranversales, qui auraient pu nous mettre en droit de présumer que cette articulation eût été sur le point de se diviser en deux, division que l'on peut opérer, par des moyens artificiels, sur les vertèbres des salamandres aquatiques.

Andry a fait, sur l'accroissement de nouvelles articulations à l'extrémité caudale, une observation qui lève, au premier abord, tous les doutes qui existent à ce sujet * . Ce médecin engagea un de ses malades,

2 J'ai extrait cette observation de l'ourrage de M. Rudolphi (Entoz., rol. 1, p. 337), n'ayant pu me procurer l'ouvrage 
qui avait déjà rendu, à plusieurs reprises, des morceaux de tænia de quatre à six pouces de longueur, mais sans la tête, $I^{\circ}$. de passer très-promptement, au moyen d'une aiguille, un fil à travèrs le corps de l'animal, aussitôt qu'il s'en présenterait, de nouveau, quelques parties hors du rectum; $2^{\circ}$. de déchirer tout ce qui serait au-dessous de l'endroit où le fil aurait été passé, et de laisser rentrer le reste dans le canal intestinal. Tout cela fut en effet exécuté, et , au moment de la rentrée, il n'y avait, du corps du tænia, qu'une longueur égale à quatre travers de doigt, et formée de cinq articulations. Après l'espace d'un mois, Andry ordonna une médecine qui fit évacuer le tænia pourvu de la tête. En examinant la portion du ver au-dessous du fil, on vit qu'elle était d'environ un pied de longueur, et composée de quarante articulations, au lieu de cinq qu'elle avait un mois auparavant. Malgré cela, cette expérience ne prouve rien, comme M. Rudolphi l'a également remarqué. D'abord, la proportion du nombre des articulations ne s'accorde pas avec celle de la longueur; car, si cinq articulations étaient de la longueur de quatre travers de doigt, longueur que nous n'évaluerons qu'à trois pouces, quarante articulations auraient dû être d'une longueur de deux pieds. En outre, il faut considérer qu'il était impossible de ne pas se tromper dans la mesure et l'évaluation du nombre

d'Andry, publié à Paris, ${ }^{7} 7 \mathbf{1} \delta$, sous le titre : Vers solitaires et autres espèces, etc. 
des articulations, lors de l'expérience. La piqûre a dû nécessairement irriter le ver, qui s'est contracté spasmodiquement, comme cela a lieu chez tous les animaux des classes inférieures; on le déchira, et il se contracta encore davantage. Les articulations allongées de l'extrémicé caudale, que le malade avait rendues en premier, ont probablement fait croire que le nombre de celles qui étaient inférieures au fil, n'allait pas au-dessus de cinq au moment de l'expérience. Un morceau de tænia de quarante articulations peut, en effet, se contracter de manière que sa longueur ne dépasse pas la largeur d'une main.

Les objections que nous allons faire maintenant à une asserlion avancée par Brera, peuvent également s'appliquer à l'observation d'Andry.

Brera s'est efforcé de montrer comment le tænia perd les articulations de la partie terminale de son corps, et comment d'autres se reproduisent à leur place, de manière que le ver devrait toujours conserver le même nombre d'articulations.

Cet auteur s'exprime ainsi ${ }^{x}$ : " 11 pousse, d'une des parties latérales de l'un des bords, par lesquelles les articulations sont jointes les unes aux autres, un petit bouton formé de la même substance que les articulations. Ce bouton grandit, s'élargit, repousse et sépare peu à peu l'articulation voisine, ét ne prend pas seulement sa place, mais encore sa forme, de

- Memorie, p. 46 . 
manièrc que l'articulation délachée se trouve tout à faic remplacéc."

Cependant cela n'ar rive pas ainsi en réalité, comme on peut le démontrer par la théorie et par l'expérience. Supposé que ces choses se passassent comme Brera l'a prétendu, on aurait remarqué que la queue ou plutôt la dernière articulation du ver se détacherait loujours en premier lieu, et, comme d'après l'opinion de ce nédecin, la nouvelle articulation prend la forme de celle qui vient d'être séparée, on devrait conclure de là qu'il ne pourrait sortir que des arliculations arrondies ou terminales. Cependant on voit journellement des individus rendre plusieurs articulations, sans qu'une seule portât le mêne caractérc ou la même formc que la dernière; outre cela, il n'est pas probable que tant d'articulations rendues par des hommes qui n'ont qu'un ou deux tzrnias, puissent se reproduire journellement, et si, par hasard, nn bouton, tel que le décrit Brera, se formait à la vingtième arliculation, à compter de lá quene, les dix-neuf autres, qui se trouveraient au-dessous, devraient également être séparées avec la vingtième, et, par conséquent, la nouvelle articulation, qui se scrait formée à l'endroit de cette dernière, ne pourrait pas servir d'équivalent, ou plutôt ne pourrait réparer la perte d'un morceau aussi considérable. Nous voyons encore que les articulations postérieures des vers parvenus ì leur développement, sont toujours chargées d'œufs fécondés, et qu'elles se détachent d'elles-mêtmes. Il faudrait alors suppo- 
ser, dans l'assertion de Brera, que les articulations nouvellement formées pourraient être chargées, dès leur origine, d'oufs fécondés, tandis que ceux qui se trouveraient dans des articulations précédentes ou anciennes resteraient, pour ainsi dire, immolsiles et sans pouvoir atteindre leur développement complet, chose absolument dépourvue de probabilité.

Le tænia, d’après mon opinion, est, dès sa naissance, entier, n'importe qu'il doive son origine à une formation spontanéc ou bien à un ouf. Cet animal commence alors à grandir; ses articulations deviennent de plus en plus marquées, surtont celles de la queue; ce sont aussi ces dernières qui se séparent d'elles-mêmes du tronc, sitôt qu'elles ont acquirs leur développement, et que les oufs dont elles sont chargées, ont atteint leur maturité. Ce dernier état de choses peut déjà avoir lieu avant que les articulations voisines de la tête soient encore visibles, et lorsqu'elles ne forment éncore qu'une espèce de cou allongé. Mais, par la suite, les articulations antérieures et la tête elle-même se développent à leur tour et se détachent successivement comme celles qui l'ont été en premier lieu. Je ne puis pas cependant indiquer au juste combien de temps il faut pour que cela s'opère; mais je doute très-fort qu'il faille dix ans et plus, comme on se croyait en droit de l'admettre, parce qu'on voit des hommes qui rendent, pendant cet espace de temps, presque continuellement des articulations dépourvues de tête *

"Vojez Carlisle. 
Il se forme, dans mon opinion, dans l'intervalle de temps que la natu:e emploie pour le développement d'un tænia, d'autres vers de son espèce, soit par des oufs, soit spontanément, supposé que la disposition pour cette dernière formation continuc à exister ; car, par exemple, on ne trouve point de tricuspidaires dans les brochets en automne, tandis qu'ils en sont renıplis au printemps.

On aurait souvent tort de croire que le tænia existe toujours dans le canal intestinal d'un homme, par la raison qu'il aurait rendu beaucoup d'articulations, sans que l'on ait pu remarquer la sortie de la tête.

Il arrive dans beaucoup de cas que cet animal se rompt dans le voisinage de la tête, et alors elle devient très-difficile à déconvrir dans les matières fécales. La meilleure manière pour atteindre ce but est la suivante: l'on fait verser de l'eau tiède en petite quantité sur les déjections, afin de les faire ramollir; quelques momens après on laisse découler avec. précaution tout ce qu'il y a de liquide; on répète ensuite celte opération jusqu'à ce que le ver et ses parties détachées restent seules au fond du vase. Je me suis procuré, de cettc manière, la tête d'un tænia qui se trouvail jointe à un morceau d'un pouce de long seulement.

Parmi plusieurs centaines de personnes tourmentées par ce ver, et traitées par moi, il n'y en a pas une seule qui ait vu sortir la tête de son tænia, et cependant je puis assurer que quatre-vingt dix-neuf sur cent se trouvent guérics. 
Outre les difformités dans la structure des articulations, que nous avons indiquées plus haut, et représentées par des figures, on remarque quelquefois des tænias avec des articulations percées. J'en ai fait rendre deux semblables; sur l'un on ne voyait que quelques articulations conformées de cette sorte, mais sur l'autre il y avait un morceau troué presque d'un bout à l'autre dans l'espace de plusieurs pieds. La fig. $f$ représente un petit morccau de ce ver. Masars de Cazeles a fait dessiner un tænia semblable, et il le regarde à tort comme appartenant à une nouvelle espèce. Il paraî̀ que les oviductes de ce vei étaient également crevés, ce qui a engagé cet auteur à le désigner sous le nom de tcenia fenestrata.

Notre collection possède un individu bien remarquable, c'est mu nıorceau de tænia de plusieurs pieds de long, et qui offre cela de particulier, qu'il y a de:Ix tienias fortement unis au bord d'une articulation. Les figures $g, k, i, p l .7$, représentenit différentes parties de ce ver. Il est à regretter que la tête ne s'y trouve pas. Il y a également dans cette collection un petit tæuia tout au plus d'un pouce de long, arné et pourvu de six suçoirs au lien de quatre. Cet individu a été rencontré dans les intestins d'un chat. La forme de ce ver était prismatique, et on observait des rainures le long de son corps. Il se peut bien que cette difformité se soit opérée par la réunion de trois tænias ou de trijumeaux accollés l'un contre l'autre.

Brera prétend avoir observé un tænia hybride, c'està-dire un ver qui tenait le milieu entre le bothriocé- 
phale et le véritable tania. Lindividu dont il est question ici se serait formé, d'après la supposition de cet auteur, par suite de l'accouplement de ces deux espèces de vers. La description que Brera donne de son prétendu tæuia hybride ne suffit pas pour en avoir uue idée claire, et il est à regretter, comme M. Olfers l'a également remarqué, que ce ver n'ait pas été dessiné. Olfers le regardc comme un tæuia ordinaire, et ajoute dummodo observationi credendum. Il est à la. vérité bien difficile de s'imaginer qu'un bothriocéphale et un tæuia aient pu se trouver dans lc canal intestinal du même homme.

\section{SECTION 11.}

Des vers qui séjournent hors du canal intestinal de l'homme.

VI. VER DE MÉDINE OU DE GUINÉE.

Filaria dracunculus (en allem. fadenwırm), pl. I!I, fig. I.

Filaria : Longissina, margine oris tumido, cauda acumine inflexo. Gmelin, Syst. nat. p. $5 \circ 39$, no. I , filaria Medinensis.

Joerdens, Helminth., p. $94, \mathrm{n}^{\circ} .2$, tab. I, fig. 1, der hautivurm, fil. med.

Rudolphi, Entoz., vol. 11, p. 56, fil. Med.

Brera, Memorie, p. 289 , spec. 2 , fil. Med.

Bradley, A treatise on worms, p. 1o3, the Guinea worm.

Cuvier, Regne animal, t. Iv, p. 3o, le ver de Médine ou de Guinée. Dc Lamarck, Anim. sans vert., t. I11, p. 217 , le fllaire de Médine. De Blainville, Dictionn. des sciences nat., tom. Xvil, p. 5 , le filaire de Médine. 
Noms et histoire de ce ver, avec les diverses opinions sur sa nature.

Gmelin est le premier qui ait rangé ce ver parmi les vers intestinaux; les Grecs l'appelaient $\Delta_{p} \times$ \%.\% \% $6 \%$, nom que les auteurs romains ont traduit par diacunculus.

Galien ${ }^{x}$ proposa d'appeler la maladie à laquelle il

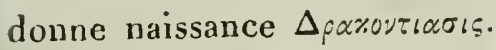

Aëtius ${ }^{2}$ le décrit sous le non de dracunculus leonidae.

Les Arabes l'indiquent sous celui d'ark, $a^{e} \mathrm{kr}$ ou irk almedini.

Ark signifie, d'après le dictionnaire arabe de Golius, radix , origo, stirps, genus, vena, arteria et simile quid.

Kunsemüller traduit ce mot par vermis, et dit que ce norn a aussi la siguification de nervus.

Ce ver porte encore le nom d'almedini, qui provient de celui de la ville de Médine, où on le rencontre souvent.

Almedini signifie de préférence civitas, parce que Mahomet s'y était réfugié de la Mecque.

La signification variée du substantif el de l'adjectif qui composent le nom de ce ver chez les Arabes, est canse que les traducteurs des auteurs de cette nation lui ont donné différentes dénominations, et qu'ils ont eu diverses opinions sur sa véritable nature.

- Ouvrage citć, inirod. , chap. xv11.

- Ourrage cité. 
Plusicurs de ces traducteurs ont entendu ark par vena : l'on trouve dans Halyabbas vena saniosa, ce que Guy de Chauliac a rendu, sańs doute par distraction, par vena famosa et vena meden.

Rhazès appelle ce ver vena medeme ou vena civilis, et Ebn-Sinah ou Avicenna, vena medinensis, comme Gérard et Velsch ont rapporté.

Bertapalia l'appelle vena civilis ou medena; Kæmpfer et Cartheuser, nervus medinensis.

Alsaharavius ou Albucasis le nomme vena cruris ou exiens ; Pedemontanus, vena egrediens; Avenzoar, vena medina et halalnachalaidini, ce que Velsch a traduit par serpens pulposus seu musculosus, medinensis, telce aranece in modum convolutus.

Montanus le nomme vena endimini.

A Haleb, d'après Niebuhr, il est connu sous le nom d'a rek el insil, ce qui se rapporte à vena exiens ou egrediens.

Niebulır, Cartheuser et Kæmpfer disent qu'on le nomme en Perse pejunk et naru.

D'après ces deux derniers auteurs et.Velsch, on lui donne le nom de nigritien ikon, sur la Côte d'Afrique, en Guinée.

A la Mecque, celui de farentit, selon Niebuhr.

Dans l'Inde, d'après Dubois, celni de narambo ou nurapoo chalandy.

En Bucharie, celui dè irschata, selon Sam. Gottl. Gmelin.

Kænpfer l'appelle dracunculus persarum, et Linné, gordius medinensis. 
Les auteurs allemands le désignent sous les noms suivans : der medina wurm, der guineische fadenwurm, hautwurn, beinwurm, pharaonswurm, der guineische drache.

Warenius propose de le nommer selnadernspulwurm.

Les Hollandais lui ont donné les noms suivans: huidworm, becnworm, traadworm, guineeische draakje. Les Anglais le nomment the hairworm, guinea-worm. Les Français, le dragonneau, le ver de Guinée, la veine de Médine, d'après Labat; ou bien le ver cutané. Les Portugais lui ont donné le nom de culcbrilla. Les Suédois, celui de onda-betet; Tagetmatk.

Le premier qui ait fait mention de ce ver est, autant que nous le savons, Agatharchides, né à Kuide; il était historien et philosophe, et il a vécu à peu près vers l'au cent quarante ou cent cinquante avant Jésus-Christ, du temps de Ptolomée Alexandre. D'après quelques auteurs, il était le gouverneur de ce dernier.

Plutarque parle de ce ver dans ses Propos de table, et s'exprimé ainsi : «Les peuples qui séjour- nent près de la mer Rouge ont été tourmentés, comme le raconte Agatharchides, par des accidens aussi extraordinaires qu'inouis : il sortail de leur corps des vers en forme de petits serpens $\Delta$ paxovtlo $\mu$ «xp $\alpha$, qui rongeaient leurs bras et leurs jambes; quand on les touchait, ils se reciraient, s'entortillaient dans les muscles, et causaient des souffrances horribles. " Tout cela se rapporte parfaitement à notre 
ver, et s'accorde avec les observations des uaturalistes modernes. Cromer dit, en effet, que, quand on les tourmente, ils causent des douleurs atroces qui surpassent celles de la goutte. Plutarque ajoute plus loin (je ne sais par quelle raison) « Ce mal avait été jusqu'alors tout à fait inconnu, et on ne l'a pas rencontré depuis chez aucun autre peuple, excepté chez celni dont je viens de parler, et qui était encore sujet à plusieurs autres incommodités. ")

Cette dernière phrase a engagé Licet, Nieremherg et Rcies, à regarder la maladie dont parle Agatharchides, comnie n'ayant rien de commun avec celle que produit le ver de Guinée; ces trois auteurs présument qu'Agatharchides avait probablement puisé cette description dans Moïse, et qu'il faut entendre par ce nom de petits dragons, les serpens ardens dont les enfaus d'Israël ont été tourmentés pour avoir murmuré lorsqu'ils étaient campés le long de la mer Rouge.

Bartholin est d'un avis contraire, et prétend que ces serpens ardens n'étaient rien autre chose que le ver en question.

Sennert s'efforce cependant de réfuter celte opinion, en disant que les Juifs avaient été attaqués de ces serpens extérieurement, et que par cette raison ils ne s'étaient pas formés dans Ieur corps.

Laissons aux docteurs en théologie à décider ce qu'étaient ces serpens ardens des Hébreux; il nous suffit de savoir que les habitans de la côte de la mer Rouge sont encore actuellement souvent incommo- 
dés par ce ver; et nous ne risquerons rien à adopter l'opinion d'A gatharchides. A près ce dernier, les médecins Soranus et Léonidas en parlent de nouveau; mais Soranus le regarde comme un plexus nerveux. Galien, qui ne l'avait pas vu, était assez modeste pour ne vouloir rien décider là-dessus. Les médecins grecs et arabes, qui ont eu occasion de l'observer, le regardent presque unanimement comme un animal vivant.

Les médecins qui ne l'ont pas vu, et qui ne le connaissent souvent que par de mauvaises traductions, ont eu des opinions assez singulières sur sa nature.

Ambroise Paré déclare que ce n'est qu'une tumeur ou qu'un abcès provenant du sang âcre. Velsch et d'autres ont réfuté cette opinion erronée.

Aldrovande et Montanus sont de l'opinion d'Ambroise Paré.

Lafaye le regarde comme un apostème occasioné par l'épaississement du sang, et Guy de Chauliac conme une veine allongée.

Polux croit que c'est une substance nerveuse corrompue.

Tagantius pense que c'est de la bile noire.

Wieurus prétend que ce ver est identique avec ceux nommés en Allemagne mitesser (comedones), masclous, Sauv.

Fiélitz a très-probablement observé un dragonneau; cependant il ne le regarde pas comme un animal vivant, mais il n'explique pas ce que cela pouvait être. Cet auteur doute de même que les masclous 
soient de véritables animaux ou bien de véritables vers vivant dans la chair; il prétend les avoir observés une fois, et croit que ce ne sont que des tumeurs enkystées ou bien des glandes de la peau.

Meyer a sûrement supposé à tort que le ver observé par Fielitz était un gordius aquatique.

Les médecins qui ne connaissaient le dragonneau que par ouï-dire ou par des descriptions, doivent être excusés quand ils portent des jugemens erronés sur sa nature; mais il est inconcevable que M. Larrey, qui aurait pu recueillir des renseiguemens exacts là-dessus pendaut qu'il était en Égypte, ait prétendu que ce ver n'est rien autre chose qu'un produit de l'opération que l'on pratique pour l'extraire, et que la chose que l'on regarde comme un ver n'est que du tissu cellulaire frappé de mort, d'où il conclut que la maladie n'est qu'un furoncle. Il tâcbe de soutenir son opinion en disant qu'il a opéré deux guérisons uniquement par des remèdes qui facilitaient la formation du pus, et sans faire l'extraction du ver. Mais nous verrons plus loin que cela ne prouve rien du tout, et qu'une pareille guérison s'itccorde trésbien avec l'existence du ver.

L'opinion de M. Larrey est réfutée par les observations de Kæmpfer, qui a extrait deux fois ce ver vivant du scrotum; elle est encore réfutée par les observations de Bajon, de Gallandat et de Dubois, qui ont remarqué que ce ver jouissait réelleneni de la vie ${ }^{\mathrm{x}}$.

2. Melorme a yu distinctement des mouremens d'ondulation 
Peré et Kæmpfer ont vu sortir une humeur blanchâtre quand on l'incisait ou quand on le déchirait.

L'expérience nous démontre encore qu'on rencontre des filaires, non-seulement chez les animaux à sang chaud, mais encore chez les reptiles et les poissons, et même dans les insectes et leurs larves. Quelle raison pourrait alors nous engager à douter de l'existence de celui qui se trouve dans l'homme?

La plupart des médecins et des naturalistes qui ont eu occasion de l'observer, conviennent qu'il faut le regarder comme un animal vivant, mais ils ne s'accordent pas quand il s'agit d'assigner à quelle classe ou à quel ordre d'animaux il doit appartenir; quelques-uns le regardent comme une larve d'insecte, d'autres le confondeut avec le gordius aquaticus.

Les défenseurs de la première opinion prétendent qu'un insecte dépose, dans quelqués cas, son cuf sous les tégumens sans que l'on s'en aperçoive, que sa larve y éclot, et qu'elle y parvient à une longueur, sans parler de la grosseur, qui diffère totalement de celle des larves d'insectes.

L'on pourrait objecter à cette opinion,

10. Que personne n’a encore vu l'insecte qui, comme on le suppose, pourrait donner lieu à la formation de pareilles larves, et si cette supposition était fondée, nous serions encore obligés de croire

sur ce ver, et un jeune nègre sentit de pareils mouremens provenant de la portion de ver çui était restée dans l'intérieur de son. pied. Voyez Journ. de phys., chimie, etc., par M. Ducrolay de Blainville. Août 1818, p. $155(B r$.$) .$ 


\section{SUR LES VERS INTESTINAUX}

qu'il y aurait autant d'espèces de ces insectes qu'il y a d'espèces de filaires.

Du reste, il n'y a aucune raison plausible pour admettre que les filaires qui séjournent dans les animaux n'aient pas la même origine que celui de l'homme.

Nous en rencontrons dans toutes les classes du règne animal. Dans les singes nous observons souvent des filaires qui ressemblent beaucoup à celui de l'homme. On en trouve également dans les cochons, les chevaux, les cerfs et les boufs; l'on remarque des filaires assez souvent dans la cavité thoracique et abdominale des oiseaux de proie, et dans plusicurs espèces de corbeaux. lls séjournent sous les tégumens dans le tissu cellulaire du rollier (coracias garrula, L.), et chez d'autres oiseaux dans d'autres endroits de leur corps. On en voit également dans les grenouilles, les serpens et les poissons, comme par exemple dans le cyprinus gobio el dans le cyprinus phoxinus; ils entourent le foie de ces poissons; il n'est pas même rare d'en rencontrer dans les insectes et leurs larves. Tous ces filaires forment des espèces distinctes, et s'ils n'étaient originairement que des larves d'insecte, il faudrait alors admettre, comme nous l'avons déjà démontré, qu'il y aurait autant d'espèces de ces insectes qu'il y a d'cspèces de filaires, el comme les animaux chez lesquels on les rencontre vivent les nns dans l'eau et les autres dans l'air, nous devrions par conséquent trouver également ces insectes dans ces deux élémens, car sans cela les poissons et les oiseaux ne pourraicut pas ĉtre incommo- 
dés des mêmes vers en même temps. Mais jusqu’à présent nous n’avons pas cucore rencontré cet insecte ni dans l'un ni dans l'autre de ces élénıens; par cette raison il est bien permis de douter de l'existence d'un insecte dont le ver de Médine serait la larve.

$2^{\circ}$. Chaque insecte dans l'état libre ne dépose ses œufs que dans un endroit où sa larve trouve non-senlement une nourriture convenable, mais aussi l'occasion d'arriver facilement de là à un endroit dans lequel elle puisse attendre son développement complet. Les oestres (oestri) nous fournissent une preuve remarquable de cela; leurs larves, conme quelques autres insectes ( par exemple les pous et les puces), ne peuvent conserver leur existence qu'aux dépens d'autres animaux. Les oestres, par cette raison, déposent leurs ouf's dans différens animaux, mais les individus de chaque espèce d'oestres déposent seulement leurs œufs dans les animanx de tel ou tel genre; ils placent leurs œufs dans des endroits différens, mais toujours dans ceux d'où leurs larves peuvent aisément, avant de se changer en chrysalides, parvenir dans la terre pour y passer à cet état, et par suite acquérir leur développement complet ${ }^{x}$. Mais cela se passe tout autrement chez les filaires. Un individu de cette espèce n'est jamais sorti spontanément du corps de l'homme, et ceux qui en ont été extraits vivans sont morts bientôt après. Du reste, nous ne connaissons aucune observation qui puisse nous

Voyes Bracy Clarck. 
mettre en droit de présumer que ces prétendues larves se changent en chrysalides dans l'intérieur du corps animal (comme par exemple les larves d'ichneu. mon), et qu'elles ne le quittent que sous la forme d'insecte parfait. Les larves elles-mêmes n'existent que momentanément sous cette forme. Il suit de là que le mode de génération des oestres ne peut pas être appliqué aux filaires, et que le corps humain ne peut pas être regardé comme l'eudroit que la nature aurait assigné à ces insectes pour le développement de leurs œufs; car sans cela leur espèce aurait dû être déiruite déjà depuis long-temps.

$3^{\circ}$. Si même l'on pouvait croire que ces insectes fissent une exception, en supposant qu'ils prépareraient, pour ainsi dire, un sort incertain à leurs descendans, en déposant les.œufs dont ils derraient provenir, ou dans des endroits qui nous sont inconnus jusqu'à présent, ou bien, par une sorte de caprice, dans un corps animal où ils devraient être nécessairement détruits comme larves; l'ou ne conçoit pas alors pourquoi chaque espèce de ces insectes choisirait toujours pour y placer ses oufs une espèce particulière d'animal qui servirait de tombeau à sa race. S’il était une fois décidé que l'insecte dût périr comme larve, il devait être alors indifférent que cela arrivât dans un mammifère ou dans un oiseau. L'opinion que les filaires proviennent de larves d'insectes n'est par conséquent appuyée sur aucune preuve valable.

J'ai été obligé de m'arrêter plus long-temps que je 
ne le voulais à réfuter cette hypothèse, parce qu'on ne l'a pas encore tout à fait abandonnée.

Brera est encore incertain sur ce qu'il doit en croire, et il termine sa définition, an haeruca?

Jœrdens combat l'opinion de ceux qui regardent le dragonneau comme une larve d'insecte, mais il croit que ce ver est identique avec le gordius aquaticus, $\mathrm{L}$. Jœrdens explique l'introduction de ce ver dans le corps animal d'une manière peu intelligible, comme on peut le voir par le passage suivant '. « Je regarde comme plus probable l'opinion de ceux qui confondent le gordius aquatique avec le filaire, qui le font vivre dans l'eau, mais qui ne le font cependant pas arriver par la bouche dans le corps animal. Des naturalistes prétendent aussi que ce ver s'introduit, étant très-petit, et non complétement formé, sous la peau pendant que l'on se baigne ou que l'on entre seulement dans l'eau les pieds nus, et qu'il y parvient à la longueur extraordinaire qui lui est propre; car il n'est pas probable qu'un ver aussi long, c'est-à-dire complétement formé, puisse s'introduire sans causer des douleurs, et sans que le malade s'en aperçoive, à travers les tégumens et les muscles, et avec une assez grande rapidité pour qu'on ne pût le saisir et l'extraire sur-le-champ. 11 doit être extrêmement fin à l'époque à laquelle il pénètre; l'extrémité antérieure ne doit pas être obtuse, mais a contraire capillaire, afin qu'elle puisse s'introduire dans les pores de la peau

${ }^{x}$ Ourrage cité, p. 99 . 
l'une manière insensible. Cette introduction doit avoir lieu dans un terrain sablonneux ou dans la poussière, mais non pas dans l'eau, car les mouvemens de cette dernière ne permettraient pas à un corps aussi léger de s'attacher à la peau. " Joerdens paraît avoir oublié à la fin du paragraphe ce qu'il a dit au commencement; du reste l'on ne concoit pas de quelle manière les jeunes ou bien les petits de ces animaux aquatiques pourraient arriver dans du sable sec.

Outre Jœerdens, il y a encore plusieurs antres personnes qui regardent le ver dont nous nous occupons comme identique avec le gordius aquaticus, et qui croyent qu'il arrive dans l'animal avec l'eau qu'il boit on lorsqu'il se baigne.

Loffler, qui a habité les contrées de l'A frique dans lesquelles les hommes sont incommodés par ce ver, a pris des informations exactes à ce sujet, et il n'a jamais appris qu'on l'ait observé dans l'eau.

Liud, qui a eu aussi l'occasion d'examiner l'eau de ces contrées, n'y a jamais vu de traces de ces vers ni de leurs oeufs.

Pallas * a rapporté qu'il n'a rencontré nulle part une aussi grande quantité de gordius aquaticus, que dans le lac de Waldei. "Cependant je n'ai jamais pu apprendre (ce sont les paroles de.cet auteur), que l'on ait trouvé de ces vers sous les tégumens de l'homme. "Si, par conséquent, l'on ne trouve pas le

- Reisen durch Russland, I. s. 3. 
dragonneau dans l'eau des régions où les hommes en sout incommodés, et si d'un autre côté les hommes ne sont pas non plus incommodés par ce ver dans les endroits où les gordius aquatiques se trouvent en grande quantité, il résulte de là que ces deux vers ne peuvent pas être identiques. Nous sommes alors obligés d'admettre que le dragonneau se forme de la nềme manière que les autres vers intestinaux, c'està-dire spontanément, dans le corps animal, et qu'il faut le regarder comme un ver sui generis, qui peut seulement se reproduire dans le corps de l'homme.

On remarque cependant une particularité touchant la génération de cet animal. D'abord on ne le rencontre pas dans toutes les contrées, ni dans toutes les parties du monde; ensuite ce ver n'incommode pas seulement les natifs des contrées dans lesquelles il séjourne, mais encore les étrangers qui y arrivent, de quelque nation qu'ils soient.

Il ne se produit jamais spontanément en liurope, mais on a souvent remarqué que les Européens qui revenaient de ces contrées l'en ont apporté. Le dragonneau ne se montra chez Cromer ${ }^{5}$ qu'après qu'il fut de retour en Suisse, de ses voyages.

La zone torride est, d'après Kunsenmüller, la véritable patrie de ce ver, cependant on ne le rencontre pas dans tous les pays situés sous le tropique. Il sc trouve principalement dans l'Arabie Pétrée, sur les bords du golfe Persique, de la mer Caspienne,

I Voyez Wrpfer, dans les Eph. nat. cur. 
du Gange, dans la Haute-Egypte, en Abyssinic, e乞 en Guinée.

D’après Lœffler on le trouve le plus souvent en Afrique dans les colonies anglaises et hollandaises. Cet auteur-ajoute que cette fréquence n'est pas la même partout. Parmi deux cent vingt esclaves que l'on avait achetés à Capmonte, à Messerade et à la Hou , il nè s'en trouva qu'un seul qui eût un dragonneaı dans le gros orteil; et parmi six cents esclaves africains achetés à Angola, il n'y en avait aucun qui en fût incommodé.

Sloane prétend également que les nègres qui arrivent à la Jamaïque d'Angola et de Gamba ne souffrent jamais de la présence du dragonneau.

D'après Bosmann, on l'observe sur toute la côte de Guinée, mais principalement à Cormantia et à Apan.

D'après Linchot on le reucontre dans l'île d'Ormus et surtout dans le château de Mourre.

D'après Lachmund et Arthus, on le trouve rarement à Acra. Dans le château appelé Joris de Minna et aux environs, il est tellement commun, que Hemmersan dit en plaisantant, que l'on éprouve déjà des démangeaisons sous la peau lorsqu'on ne fait que de passer à la voile devant ce château. Mais d'après Arthus on ne connait pas cet animal vingt-cinq lieues plus loin du côté du nord.

D'après Grégor, le quatre-vingt-sixième régiment anglais n'avait pas un seul homme incommodé de ce ver avant qu'il arrivàt à Bombay en septembre 1789 . Il en fut de même jusqu'à l'époque où règne la 
mousson; mais alors trois cents soldats en furent atteiuts. Le quatre-vingt-lsuitiène régiment, qui avait campé sur l'île de Coulabah depuis le mois de juin 1799 jusqu'au mois d'octobre 1800 , n'en fut nullement incommodé. Cette île n'est cependant éloignée que d'une lieue anglaise de Bombay, et ce n'est qu'après avoir remplacé le quatre-vingt-sixième, qui était en garnison dans cette dernière ville, qu'un homme en fut atteint. A près un séjour de deux mois dans ce dernier endroit, le régiment fut embarqué, et ce n'est qu'à cette époque que cette maladie se déclara d'une manière extraordinaire; parmi trois ceut soixante hommes, cent soixante.un en furent incommodés. Le séjour de Bombay paraît principalement donner lieu à cette maladie. Le vaisseau sur lequel Paton se trouvait, avait fait voile, le 15 août 1804 , de cette dernière ville pour se rendre en Chine; pendant la traversée, un homme fut incommodé d'un dragonneau; le 5 janvier le malade ful mis à terre. Le vaisseau partit le même jour de Canton, et dès ce moment personne de l'équipage ne mit pied à terre jusqu'à l'arrivée du vaisseau à Sainte-Hélène, qui eut lieu le 2 avril; le ver se moutra alors pour la première fois, le trente mai, chez un homme qui n'avait jamais débarqué. Cet animal n'est pas couvu dans cette île, et sur les bâtimens qui y mouillaient alors, il n'y a vait personne qui en fúl attaqué. Parmi deux cents hommes qui formaient l'équipage, vingl-six en furent incomınodés successivement. Il me parait probable qưils avaient gagné cette maladie à Bombay. 
Le dragonneau règne d'une manière endérnique au Sénégal, à Gabon, etc. , rarement à Gongo, d'après Peré; il se répand d'une manière endémique, d'après Dubois, dans les Indes.Orientales, et quelquefois, surtout dans les mois de novembre, décembre, janvier, la moitié de la population d'un village en est attaquée à Latimunculum et dans le district de Karnatik et de Madura .

Sloane remarque qu'il se montre plus souvent dans uve année que dans une autre, et Kæmpfer dit que plus il fait chaud, plus il y a de ces vers. D'après Niebulır', le dragonneau est très-commun à Jémen, dans la presqu'île de l'Inde, et à Gambron ou Bender Abbas, en Perse.

En A mérique il ne se montre que parmi les nc̀gres, qui ne font que d'y arriver de l'A frique. L'île de Curaçao fait cependant exception; car d'après Dampier, les blancs et les noirs en sont indistinctement atteints.

Le feu baron de Jaquin m'a également assuré que l'on peut admettre que le quart de la population de cette île, tant noirs qu'indigènes, en souffre. 11 m'a dit aussi que deux de ces vers s'étaient développés chez un de ses compagnons de voyage, qui était Européen, et qui n'avait jamais été auparavant ni en Asie ni en Afrique; il a ajouté que le dragonneau est inconnu dans les îles environnantes.

s Dans l'ouvrage cité il y a Madéra; ce que je dois regarder comme une faute d'impression, car Karnatik est bien dans le voisinage de Madura, mais non pas de Madéra. 
Les naturalistes ont des idées très-différentes sur la nature de cet animal, ou plutôt sur les causes de sà formation.

Plusieurs croient que la mauvaise qualité de l'eau favorise son développemenı; Bernier, Bruce, Chardin, Dampier, Dubois, Galandat, Linchot, Lister et Niebuhr sont de cetce opinion; ce dernier raconte que l'on fait filtrer l'eau à Jémen à travers de la toile afin de s'en garantir.

Arthus remarque que les habitans de l'île d'Ornus font par cette raison puiser l'eau de mer ì dix-huic toises de profondeur, et Galandat prétend que ceux qui ne boivent pas d'eau en Guinée ne sont pas attaqués par ce ver. D'autres attribuent les causes de sa formation à l'usage du vin de palmier, de certains poissons, du froment de l'Inde, du pain que les Iudiens appellent kaukiens, ou bien à un exercice immodéré de l'acte de la génération; d'antres regardent le vent ou les rosées du pays comme causes de sa formation. Mercurialis croit qu'on le gagne en mangeant des sauterelles. Le docteur Kier est de l'opinion que ces vers sont introduits dans le corps animal au moyen des vents et de la pluie, mais il n'explique pas de quelle manière leurs oufs ont pu arriver dans l'air ou dans lat pluie.

Ce médecin, ainsi que les docteurs Heath et Anderson observent que les officiers, qui ne se promenent ni ne se coucheut pas sur la terre, les pieds et les bras nus, n'en sont pas affectés.

Quelques personnes regardent celte maladie comme 
contagieuse, et Lind conseille aux Européens de ne pas fréquenter les nègres qui en sont attaqués, et de ne pas coucher dans la même chambre.

Grégor et Ninian Bruce sont également portés à croire que cette maladie est contagieuse. L'on peut très-bien expliquer pourquoi, dans l'exemple cité plus haut, elle ne se fit pas.voir aussi souvent sur le vaisseau, après que l'on eut séparé les malades de ceux qui se portaient bien, car cette maladie s'était déjà déclarée chez la plus grande partie de ceux qui l'avaient gagnée à Bombay.

Les faits suivans prouveront quel peu de cas l'on doit faire des causes que l'on a regardées comme prédisposantes de la production de ce ver.

Arthus raconte que quelques personnes qui avaient évité soigneusement ces causes, en furent néanmoins atteintes, tandis que d'autres qui s'y exposèrent hardiment ne le furent pas.

Anderson "a prouvé à Dubois que les hommes qui demeurent le long des rivières en sont aussi bien attaqués que ceux qui boivent de l'eau provenant des citernes.

Lorsque le baron de Jaquin arriva à Curaçao, on l'informa que l'on gagnait ce ver par l'usage de l'eau: très-bien, répondit le compagnon de voyage dont nous avons parlé plus haut, je promets de n'en pas boire une gontte; et, malgré qu'il ait tenu parole (ce qui ne lui a pas coûté beaucoup, comme on le prétend), il a été le seul qui en ait été atteint, tandis que Jaquin,

× Voyez l'ouvrage de Dubois. 
qui n'était pas accoutumé aux boissons spiritucuses, et qui par conséquent était obligé de s'en tenir à l'usage de l'eau, ne le fut pas.

Cromer, qui attribue la production de ce ver uniquement à un air malsain, fait mention d'un général hollandais qui demeurait à Angolax, et qui ne put s'en garantir, malgré qu'il'ne fît usage que d'alimens et de boissons provenant de l'Europe.

Chardin, qui a parcouru cinq ou six fois les régions de la Perse où l'on rencontre souvent ce ver, n'en a jamais été affecté.

Ninian Bruce dit par conséquent avec raison que son origine est encore couverte d'un voile épais.

\section{Description du dragonneau.}

L'on ne peut pas ajouter grand'chose à la définition que nous avons donnée plus haut de ce ver, à cause de l'extrême simplicité de sa conformation. Je crois que mes lecteurs pourront s'en former une idée exacte par la figure 1, pl. 8, qui est une copie fidèle d'un dragonneau très-petit que M. Rudolphi a fait dessiner d'après nature.

Notre collection en possède un individu que Fenger, professeur de Copenhague, a eu la bonté de nous envoyer, mais l'esprit-de-vin l'a tellement rembruni, qu'il n'était pas propre à être dessiné.

${ }^{2}$ Ce général a dú auparavant s'arrêter à une autre place de débarquement, car on n'observe pas de dragonneaux, d'après le témoignage de Loffler et de Sloane, à Angola. 
Ce ver est d'uue couleur blanche et presque d'une égale grosseur d'un bout à l'autre, si ce u'est à son extrémité postérieure, qui est plus amincie et un peu recourbée.

Kæmpfer observe que sa tête est pourvue d'une petite trompe, qui est appelée par les Perses la barbe, et qui, examinée au microscope, semble, à ce que l'on prétend, être formée par de petits poils.

Fermin, Hemmersan et Lachmund disent avoir observé à l'extrémité de la tête deux filamens que l'on pourrait appeler, selon eux, des poils ou des anteunes. Mais ces fllamens provenaient peut-être d'une lésion du ver, ou bien n'étaient que des restes d'un peu de tissu cellulaire. 11 arrive en effet souvent que le ver étant déchiré, se montre de nouveau dans un endroit assez éloigné de celui où il a d'abord páru. Ne pourrait-on pas alors croire que la fin de la queue ait pu sortir la première daus quelques cas, et que ces deux filamens n'étaient rien autre chose qu'un double pénis.

Le cas observé par Kæmpfer, et dans lequel le dragonneau se faisait voir au creux du jarret, quoiqu'il eut son point d'adhérence aũ gros orteil, paraît venir à l'appui du cette suppositiou.

Andry et Galandat se sont assurément trompés eu supposant que ce ver était pourvu d'une tête à chaque extrémité.

Les auteurs ne sont pas non plus d'accord quant à sa longueur.

Albucasis dit qu'il y a des individus de trois, dix, jusqu'à vingt pieds de long. 
Barère prétend que sa longueur est quelquefois de six aunes.

Dampier observe qu'elle est de cinq à six verges.

Dubois en a vu qui avaient plus d'une aune de longueur et qui étaient gros comme le la d'un violon.

Louis Franck indique sa longueur de quatre à six pieds, Gallandat de huit à douze, Gmelin de plusieurs aunes, Hemmersan d'une anne et demie, tantôt gros comme un fil, et quelquefois aussi gros qu'une ficelle. Celui que Gründler a reçu de Malabar avait trois pieds et demi ; il était de la grosseur d'une ficelle et d'une couleur jannâtre qui provenait peutêtre de l'esprit-de-vin dans lequel il était conservé. Celui qu'Isert possède a deux aunes de longueur, et il est de la grosseur d'un fétu de paille.

Kunsenmüller n'a jamais vu de dragonneaux dépasser la longueur de deux pieds.

Heath a observé soixante-quatorze malades qui en étaient incommodés, et il rapporte qu'il y en avait parmi eux plusieurs qui avaient deux, trois, quatre même cinq vers, dont la longueur la moins considérable était de neuf pouces, et la plus grande de quarante-deux.

Bajon a vu un ver de six aunes de longueur, et Bruce dit qu'il est rare d'en voir au-dessous d'un pied et demi et au-dessus de six. Cromer, dans l'ouvrage de Wepfer, indique sa lengueur de deux aunes, Labat de six, et Lister de six à sept aunes.

D'apres Schœeler, il ne dépasse jamars la longueur de deux à trois aunes. 
Hutcheson et Forbes en ont d'abord extrait à un malade trois aunes et demic (mesure anglaise) provenant d'une tumeur, et dans l'espace de huit semaines plus de trente aunes provenant de plusieurs autres tumeurs.

Arthus prétend qu'un second ver se présente souvent dans le même trou aussitôt que le premier en est sorti.

M. Rudolphi, en indiquant la longueur du ver de deux, huit et douze pieds, observe qu'il est possible que l'on ait mesuré plusieurs individus ensemble.

Cependant, si l'on juge d'après l'analogic, il est concevable que ces vers puissent atteindre la longueur la plus considérable dont il a été fait mention par les auteurs ; car nous possédons des filaires provenant de petites sauterelles qui sont de la longueur de quiuze pouces .

Ce ver séjourne ordinairement dans le tissu cellulaire, au-dessous des tégumens. M. Rudolphi présume cependant qu'il pourrait bien aussi se montrer dans l'intérieur du corps, comme cela arrive pour l'cspèce de filaire que l'on rencontre quelquefois dans la cavité abdominale des singes. Cette supposition, qui n'est pas dépourvue de probabilité, n'a cependant pas encore été prouvée par aucune observation.

On rencontre le dragonneau le plus souvent aux extréınités supérieures et inféricures, surtout aux inférieures, entortillé autour des malléoles; mais toutes

'Je possède maintenant un filaire provenant d'une sauterelle, qui est d'une lóngucur de ircrite pouces. $\left(B r_{\text {. }}\right)$ 
les autres parties du corps peuvent également en être affectées.

Kæmpfer l'a extrait vivant deux fois, d'une seule traction, du scrotum.

Baillie ${ }^{2}$ a vu un testicule sur lequel il y avait une petite tumeur qui contenait un de ces vers.

Peré l'a rencontré à la tête, sur le cou et au tronc.

Bajon l'a vu deux fois sous la membrane externe du globe de l'œil, et il est heureusement parvenu à l'extraire en totalité au moyen d'une incision.

Le ver que Mongin a retiré de l'œil d'une négresse, moyennant une incision, paraît, d'après la description de ce médecin, avoir été un filaire.

Grégor a publié un recueil de cent quatre-viugtune observations sur le dragonneau. Cet animal s'est montré dans ce nombre cent vingt-quatre fois aux pieds, trente-trois fois aux jambes, onze fois aux cuisses, deux fois au scrotum et deux fois aux mains.

Quelquefois il est placé assez superficiellement, et on peut alors aisément le sentir par le toucher, mais quand cela a lieu, il arrive bien souvent que l'on se trompe dans le diagnostic, et qu'on le prend pour un vaisseau variqueux; quelquefois il est caché plus profondément entre les muscles.

Cromer, en faisant des autopsies cadavériques, a observé qu'il entourait les nerfs et les tendons. Il cst très-souvent contenu dans un très-petit espace, côntourné en forme de cercle à la manière des serpens. Cela a surtout lieu lorsqu'il entoure les malléoles;

s Ourrage cité, p. 439. 
quelquefois on le trouve allongé le long des bras ę des cuisses.

Perél'a vu s'étendre en forme de serpent sous les tégumens de l'abdomen, èt en partie sous ceux du thorax.

Kǽmpfer a observé un cas ò̀ il se présenta au creux du jarret. Chaque traction que l'on exerçait sur lui mettait le grand orteil en mouvement, comme s'il eût été tiré par une ficelle, ce qui causait des douleurs très-fortes Il se forma par la suite de la suppuration en cet endroit, d'où le ver sortit en effet.

Chez un autre malade, il s'était creusé une route à travers le mollet, et entourait la malléole avec le milieu de son corps; l'autre extrémité s'était frayé une issue à travers la plante du pied.

Chardin assure qu'il u'a jamais entendu dire que l'on puisse être incommodé de plusieurs dragonneaux à la fois; cependant tous lesautresauteurs affirment le contraire; Bajon a diı même qu'il est rare de n'en rencontrer qu'un seul à la fois dans le même individu.

Bosmann raconte que leur nombre se monte quelquefois à neuf, à dix; et Arthus cite des cas dans lesquels il en a souvent vu dix à douze réunis, et qui se présentaient dans différens endroits du corps.

Andry ${ }^{x}$ a fait mention d'un cas oì il y en avait viugt-trois chez le mêne individu.

Un cuisinier qui se trouvait sur le vaisseau de

*. Ouvrage cité, p. 54 . 
Hemmersan en avait trente, dont trois ou quatre sortirent toujours à la fois.

Poupée Desportes ' a vu un exemple où il y en avait jusqu'à cinquante.

Du diagnostic de l'existence du dragonneau dans l'homme.

Gallandat, un des meilleurs auteurs qui aient écritsur cesujet, s'exprime en ces termes. "A Aussitôt que quelqu'un se plaint (dans le pays où ce ver séjourne), d'une démangcaison désagréable sur une partie quelconque du corps, surtout anx pieds, l'on peut déjà présumer la présence du dragonneau. Cette supposition gagne encore en probabilité s'il se forme une tumeur qui prend les caractères d'un furoncle."

Cependant il peut rester long-temps caché dans le corps sans causer la moindre incommodité; le malade éprouve tout au plus la sensation d'un corps qui rampe sous la peau.

La présence de cet animal ne se déclara chez Dampier qu'au bout de cinq à six mois, et chez Isert qu'au bout de huit mois après qu'ils eurent quitté le pays dans lequel le dragonneau se trouve. Ni l'une ni l'autre de ces deux personnes n'avait éprouvé la moindre incommodité avant ce temps-là.

Arthus, Bernier et Labat prétendent que cet ani-

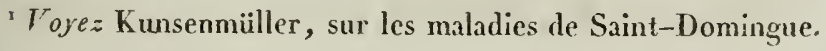


mal peut rester caché un an et même quinze mois sans donner le moindre signe de son existence, comme cela paraît avoir eu lieu chez le malade dont Paton fait l'histoire.

Cromer fixe cette époque à plusieurs années.

Kæmpfer cite un exemple où le ver ne se montra qu'après un espace de trois ans, sans que le malade eût éprouvé la moindre incommodité pendant ce temps-là.

Cependant il n'en est pas de même chez tous les malades.

D'après Peré ils meurent souvent cachectiques, sans cependanı éprouver ni fièvre ni perte d'appétit jusqu'au dernier moment de leur existence.

L. Frank dit également que quelques personnes meurent d'épuisement, si l'on ne vient pas à temps à leur secours.

Bajon prétend qu'il n'a observé aucun dépérissement chez les malades.

Peré cependant appuie son assertion sur une observation dont nous parlerons plus bas, et qui met la chose hors de doute. Cela est également démontré par la maladie dont Drumont fut atteint. Il s'exprime en ces termes : “Je sentis, à la fin de novembre 1791 , une roideur et un peu de douleur à la partie inférieure des jumeaux de la jambe droite, à l'endroit où les tendons de ces múscles se réunissent pour former le tendon d'Achille. Cette duuleur n'était jamais très-considérable, et elle ne me gênait pas en marcliant. Quelques jours après, jobservai à 
cette partic une tumeur qui cependant n'était accompagnée ni de changement de couleur à la peau, ni de douleur. Quelques jours après l'apparition de la tumeur, il se montra, à la partie interne de la jambe, à peu près un pouce au-dessus de la malléole du même côté, et à la partie musculaire de la jambe, derrière le tibia, une petite pustule rougeâtre, avcc un point noir au milieu. Je sentis en même temps, très-distinctement, sous la peau, une substance compacte et cyliudrique, et il m'était facile de suivre avec le doigt, sur un trajet assez cousidérable, la position de l'animal, qui s'étendait obliquement, et en serpentant, derrière la partie supérieure et postérieure de la jambe. Quoique je reconnusse alors la nature de cette maladie, je ne crus pas cependant nécessaire d'employer quelque chose contre elle, pour empêcher ses progrès, ou plutôt pour détruire le ver; mais, je l'avoue, je ne connaissais pas non plus un remède capable d'atteindre ce but. Je pensai que le ver chercherait un jour à sortir, et qu'il était plus prudent de ne pas le tourmenter. Dans la nui du 17 décembre, quelques jours après l'apparition de la pustule, et après m'être couché bien portant, je me réveillai à deux heures du matin, épronvant sur tout le corps une démangeaison insupportable, qui me forçait de me gratter d'une manière extraordinaire. Je sentis bientôt après une forte chaleur à la figure; je m’aperçus aussi, en me regardant dans une glace, que mon visage était d'un ronge foncé, et que les muscles étaient gonflés et dans un état convulsif. En 
touchant avéc les doigts les parties de mon corps, qui me démangeaient beaucoup, je déconvrais un épaississement, comme s'il était dans la peau même, et il me semblait qu'elle était remplie de nodosités. Pendant que je réfléchissais pour n’expliquer ces. accidens sur lesquels je n'avais aucune connaissance, $j e$ fus atteint de coliques violentes; $j e$ fis des efforts pour vomir, et je finis par le faire et par aller à la selle. Je rendis, par haut et par bas, de lit bile et uue matière acide, mais en si petite quantité, que je croyais que les accidens que j'éprouvais ne pouvaient pas avoir été provoqués par la présence de ces humeurs. Les vomissemens durèrent, autant que je me le rappelle, plus d'une demi-heure, saus presque discontinuer; et pendant tout ce temps, les douleurs continuèrent avec une égale force. Ces accidens furent suivis d'un froid très-violent qui dura pendant plusieurs heures, et qui ressemblait au frisson, d'une violence peu commnne, que l'on éprouve dans les fièvres intermittentes. Lorsque les vomissemens eurent cessé, je me couchai, et l'on me couvrit de plusieurs couvertures de laine. Je commençai à me réchauffer peu à peu, et je m'endormis. Je craignais que le froid ne fùt suivi d'une chaleur extraordinaire; mais cela n'eut pas lien, et en me reveillant le matin, je ne trouvai qu'un peu de moiteur aux pieds. La pustule avait crevé pendant la nuit, et il parut nue substance dure et blanche; mais elle était tellement profoude, qu'ou ne pouvait pas la saisir. L'animal avait changé de position pen- 
dant la nuit, et il s'était glissé sous les muscles à une profondeur assez considérable, de manière que l'on ne pouvait plus le sentir avec le doigt. Je pouvais encore le distinguer le 17 par le toucher; mais il avait disparu entièrement le 18 . L'accès éprouvé dans la nuit m'avait un peu affaibli; mais je ue fus nullement incommodéle jour suivant, et je n'eus pas même de rechute dans la nuit du 18 ; cependant il s'était formé une inflammation très-considérable autour de la malléole, ce qui m'empêcha de marcher le 19; et je fus même obligé de gardcr une position horizontale. Le 22, je passai, à tsavers la plaie résultant dc l'ouverture de la pustule, un fil, afin d'atteindre, par ce móyen, une des extrémités de :'animal, qui était très-dure au toucher, et fortement implantée dans les chairs. L'irritation provoquée par l'opération occasiona une évacuation considérable d'une humeur aqueuse; il resta une plaie très-douloureuse à l'endroit de la pustule, avec un écoulement de matières ichoreuses et teintes de sang. Cet état de choses aura jusqu'au commencement du mois de février 1792 , époque à laquelle la plaie se cicatrisa, sauf une très-petite ouverture. L'animal se montra alors de nouveau, et je fus assez heureux de poivoir le saisir à l'aide d'un fil. Quelques autres personnes et moi nous roulâmes le ver sur une baguette, et nous exerçâmes deux fois par jour des tractions à la manière accoutumée. Après l'espace de vingt jours, nous parvînmes à l'cxtraire complétement. Le ver avait plus de deux aunes de long, et sa grosseur 
égalait celle d'une plume de corbeau. Après que nous en eûmes retiré la moitié, sa grosseur diminua peu à peu. Je remarquai que l'application de feuilles d'aloës, aussi chaude que je pus l'endurer, sur la partie dure, douloureuse et gonflée du pied, facilitait sa sortie. Les frictions fortes produisaient le même effet. Pour pouvoir les supporter plus long-temps, et avec moins de douleur, je me fis frotter avec un corps gras; le ver s'était entortillé dans plusieurs endroits du pied en forme de petits pelotons, sur lesquels nous dirigeâmes surtout les frictions. Il m'a paru que l'emploi de feuilles d'aloës, et surtout les frictions, catsèrent une irritation au ver, et le forcèrent à quitter plus vite sa place, qu'il n'aurait fait sans cela. L'application de la chaleur sur la partie souffrante a dû occasioner une accumulation d'humeurs autour de l'animal, ce qui sans doute facilita sa sortie. Je suis porté à croire, continue Drumont, que les accideus extraordinaires que j'éprouvai dans la nuit du 17 décembre provenaient d'un changement de position de l'animal. Peut-être aurait-on pu prévenir ces accidens, si l'on avait fait une incision à la partie saine de la peau, sous laquelle le dragonneau se trouvait; et, en le saisissant à l'aide d'un fil, on aurait alors pu l'extraire très-probablement, sans le moindre danger, en entier, ou au moins une grande partie de son corps. »

Quaud le ver est prêt à sortir (ce sout les paroles de Dabois), il se forme une petite pustule, souvent sans 
le moindre accident antécédent, à l'endroit par où il veut se frayer une issue; mais quelquefois le malade sent, plusieurs jours avant la formation de la pustule, un nalaise, des maux de tête, des maux d'estomac, des nausées, et la douleur devient fixe, un à deux jours auparavant, à l'endroit par où le ver doit sortir. Il se forme en même temps de petiles vésicules qui causent de fortes démangeaisons : elles sont surtout très-vives à la place où l'animal perce la peau, et la douleur devient à la fin continuelle. La partie affectée se gonfle quelquefois très-fortement; elle s'enflamme, et passe à la suppuration. Si cela a lieu, le ver se présente aussitôt que la suppuration est établie, ou bien il ve se montre que quand la suppuration est sur le point de cesser. La partie au-dessous de laquelle le ver se trouve, se gonfle quelquefois et forme une espèce de poche, qui est remplie d'une humeur transparente; mais, dans d'autres cas, on ne sent, à cet endroit, qu'une dureté qui n'est pas nuême aecompagnée d'une inflammation considérable.

D'après Kaempfer, la formation de la pustule est précédée d'une fièvre ordinairement éphémère; mais plus souvent elle dure pendant trois jours. Si le ver est placé au-dessus d'une articulation, comme, par exemple, quand il s'étend de la cuisse à la jambe, en passant au-dessus du genou, les mouvemens de cette articulation deviennent quelquefois très-péuibles et même impossibles.

Ce sont à peu près là tous les accidens qui arrivent 
quand le ver veut se frayer un chemin au dehors. Du reste, comme les accidens qui ont lieu pendant la durée de cette maladie dépendent plus ou moins du. mode de traitement que l'on emploie contre elle, j’ai trouvé plus convenable de parler en même temps. et des accidens et du traitement de cette maladie.

Des accidens qui arrivent pendant la durée de la maZadie occasionée par la présence du dragonneau, et de. son traitement.

Quand, après l'espace de deux ou trois jours, le pus s'est accumulé daus la pustule, elle crève souvent d'elle-même; mais ordinairement on l'ouvre au moyen d'une lancette; il en découle alors du pus teint de sang, ou une matière ichoreuse très-liquide; et l'extrémité céphalique du ver, avec deux ou trois pouces de longueur de son corps, sortent en même temps aú dehors. On doit alors saisir la tête, et excrcer sur elle des tractious lentes avec beaucoup de précaution; souvent, par ce moyen, on en fait encore sortir quelques pouces de plus; mais, dans le cas où le ver ne cède pas facilement, l'on doit bien se garder d'employer la force, de peur de le déchirer, ce qui peut occasioner des suites très-fâcheuses. On roule la partie sortie antour d'un pelit cylindre de toile, ou bien autour d'une baguette de bois mince, et on la fixe avec une bandelette d'emplâtre agglutinatif, ou à l'aide d'une compresse; au-dessus de la plaie. Aveṇzoai', Rliasès et plusicurs autres roulaient la 
partie sortie autour d'un morceau de plomb du poids d'un gros; mais Paul d'Egine avait déjà désapprouvé ce moyen, parce que la pesanteur de ce métal était cause que le ver se déchirait facilement. On peut encore se servir, dans le même bui, d'un morceau de bois fendu à l'une de ses extrémités, avec lequel on serre le ver.

Velsch a proposé un appareil très-compliqué pour l'extraction de cet animal. Cet auteur a même donué la figure de cet appareil; mais un petit cylindre de toile cst suffisant pour obtenir ce résultat. On conseille en général de faire deux tractions lentes par jour sur le ver, jusqu’à ce qu'il soit entièrement enlevé. On ne peut pas déterminer au juste l'espace de temps qu'il faut employer pour atteindre ce but.

Kaempfer est parvenu, dans deux cas différens, à l'extraire en toialité du scrotum d'un seul coup.

Dubois remarque également que l'on trouve le dragomeau, dans quelques cas, en entier dans la plaic.

Isert en fut entièrement débarrassé dans l'espace de huit jours, quoique le ver fût de la Jonģneur de deux aunes et de la' grosseur d'un fétu de paille. Le mêne auteur rapporte que l'on est, en Afrique, souvent plusieurś mois avant d'obtenir la guérison de cette maladie. Dans le plus grand nombre de cas, on obtient sa terminaison dans l'espace de trois or' quatre semaines; mais, quand il y a plusieurs vers dans le mêne individu, le traitement peut alors durer plusieurs nois. On panse la plaie qui reste 
après l'extraction de l'animal, comme une plaie simple, et elle guérit ordinairement très-vite et sans difficulté.

Louffler a souvent suivi un autre mode de traitement : il consistait à faire une incision au milieu de l'endroit où le ver était perceptible au toucher, ce que Schocler n'a jamais pu remarquer, afin de le mettre a nu. Loeffler plaçait la partie du corps qui se présentait, dans un morceau de bois fendu à l'une de ses extrémités, et il exerçait ensuite des tractions tantôt sur l'une, tantôt sur l'autre moitié du corps de l'animal ; e $\iota$, par ce moyen, il est parvenu à le faire sortir une fois plus vite que s'il a vait suivi la méthode ordinaire.

Gregor rapporte que les médecins indiens enploient le même mode de traitement. N. Bruce et Peré le recommandent également. Ce dernier fut un jour chargé d'examiner à Saint-Domingue un bàtiment qui venait de Guinée; il trouva sur ce bâtiment un jeune nègre de dix à douze ans, qui était tellement maigre et affaibli, qu'il ne pouvait pas se tenir sur ses jambes. A près un examen attentif, il remarqua que cet enfant était incommodé par un dragonneau qui était perceptible au toucher, non-seulement sur presque toute la surface du bas-ventre, mais encore sur une grande partie de la poitriue. Le chirurgien du bâtiment avait pris les protubérances que l'animal formait à l'extérieur, pour des veines superficielles; cependant ces protubćrances provenaient de la position du dragonuean. Le chirurgien, 
après avoir employé inutilement toutes sortes de remèdes pour opérer la guérison, abandonna à la fin cet enfant comme un malade étique et incurable. Il est à remarquer que le petit nègre avait toujours conservé son appétit pendant la durée de la maladie. Peré l'acheta, pour une bagatelle, dans l'intention de le débarrasser de son ver, s’il était possible. Il commença, à l'aide d'une pince à disséquer, à soulever la peau à l'eudroit où il présuma que Je ver pouvait avoir le milieu de son corps. Il pratiqua une incision dę quatre lignes sur cette partie de la peau soulevée. Après avoir disséqué et écarté les lèvres de la plaie, il vit un corps blanc de la grosseur du la d'un violon, sur lequel, en exerçant une traction Jente, il donna lieu à la formation. d'une espèce d'anse. Quand le ver ne voulut plus céder à la traction qui était exercée sur lui d'un côté, le médecin le faisait tenir par un aide, et il essayait de tirer sur l'autre bout. 11 ordonua en même temps au malade de se tenir dans une position convenable, afin que les parties qui environnaient le ver se trouvassent dans un état complet de flexion ou de relâchement, de manière que la tension des muscles n'empêchât pas les mouvemens du ver, et par couséquent sa sortie. En moins de quatre heures, ce médecin fut assez heureux pour l'extraire entièrement. Le malade ne sentit aucune douleur pendant cette opération, et il voyait sortir le ver avec le plus grand sang-froid; il se rétablit ensuite à vue d'œil, sans prendre de médicamens, et il devint tellement gras et robuste, que 


\section{3- SUR LES VERS INTESTINAUX}

Peré put le vendie douze cents francs trois mois plus tard, éporque à laquelle il fut obligé de revenir en France.

Ces deux méthodes simpleś suffisent, dans les cas ordinaires, pour obtenir la sortie du dragonnean, et l'on n'a besoin d'employer de médicamens ni à l'extérieur, ni à l'intérieur; mais, quand le ver est placé dans des parlies très-musculeuses; quand il a déjà causé dans ces parties, avant son apparition, une forte inflanmation, une tuméfaction et de la douleur; ou bien quand il ne veut pas céder aux tractions que l'on exerce sur lui, ou enfin quand il s'est déchiré, c'est alors que le secours de la nédecine devicut nécessaire. J'indiquerai brièvemeut les médicamens que les médecins tant anciens que modernes ont cmployés dans le but d'accélérer la guérison.

Les médecins arabes et beaucoup d'autres recommandent en général les fomentations, les saignées ; et, parmi les purgatifs, les nyrobolans, et principalement l'aloës, taut à l'extérieur qu’à l'intérieur, et quelques onguens.

Anderson a dernièrement recommandé de nouveau l'aloës; il s'exprime ainsi à ce sujet : «Dans ma pratique, je n'ai rien trouvé de pliss efficace que les cataplasmes préparés avec l'aloë littoralis. Ce médicament m'a été communiqué par un Indien : sa qualité savonneuse paraît prévenir la gangrène, en ramollissant les tégumens enflammés, et, par conséquent, faciliter la sortic du ver." 
Aëtins " recommande, pour empêcher la rétrogradation du ver, la ligature du membre dans lequel il se trouve; outre cela, il conseille l'emploi des fomentations faites avec une infusion de graines de laurier dans l'huile.

Bajon propose des frictions mercurielles et des tisanes amères. Au cas que le ver ne cède pas facilement aux tractions, quand on a à craindre sa rupture et sa putréfaction, il conseille alors d'arroser la plaie à plusieurs reprises dans la journée avec des liqueurs spirituenses, comme, par exemple, avec de la teinture de myrrhe, d'aloës, ou avec de l'eau vulnéraire.

Bancroft a indiqué le mode de traitement suivant conme le meilleur : il conseule d'abord d'appliquer sur la tumeur un cataplasme fait avec des oignons, de la mie de pain et suffisante quantité de lait; toütes ces substances doivent avoir bouilli ensemble. Sitôt qu'on aperçoit la tête du ver, on l'enveloppe avec du coton, mais sans exercer de tractions. Le malade doit en ouire faire usage du mélange suivant : prenez poivre noir en poudre, ail pilé, fleurs de soufre, de chaque une once; rhum, une bouteille; mêlez et prenez une demi-tasse matin et soir. Un ou deux jours après l'emploi de ce médicament, on trouvera le ver contourné de diverses manières au-dessous du cataplasme.

Griffith Hughes, qui avait déja prop̣oséles mêmes remèdes que nous venons de mentionner, est tellement couraincu de leur bon effet, qu'il dit, que

Tetrabibl., Quart. serm. 11, cap. Lxxxy, p. 9oł. 
quand même un homme aurait un millier de dragouneaux, il en serait débarrassé d'une manière certaiue. Par ce procédé, chaque ver se contracte, se pelotonne et meurt; une tumeur se forme et perce par la suitc.

Hillary vaute les bons effets d'une composition semblable. La voici : Prenez soufre et ail, de chaque une once; poivre noir, une demi-once; camphre, deux gros; vinaigre, deux livres. Mêlez, faites digérer et passez dans un linge : à prendre deux cuillerées à bouche, deux ou trois fois par jour.

Barère recommande un liniment composé de feuilles brûlées de cotonnier mêlées d'un peu d'huile d'aoüara, que l'on extrait d'un palmier de ce nom.

Cet auteur rapporte encore que quelques personnes croient pouvoir faciliter l'extraction du ver en faisant tomber sur la plaie du jus de tabac provenaut des pipes à fumer.

Dampier, qui avait vers la malléole un dragonneau, dont il avait déjà extrait un morceau de deux pieds de longueur, alla consulıer, accompagné d'un de ses amis, un nègre qui traitait le cheval de ce dernier. Le nègre examina la malléole, la frotta dans différens sens, et appliqua ensuite une poudre que Dampier présume être celle de tabac. Le nègre lıi ordonna de n'enlever l'appareil qu'après trois jours; mais le jour suivant, comme il s'était détaché, on vit que le ver était déchiré et la plaịe cicatrisée. Dampier craignait des suites fâcheuses, qui cependant n'curent pas lieu. 
On s'est convaincu en Europe (ce sont les paroles de M. Louis Frank) par des expériences faites pour l'extraction du dragonneau, que la fumée de tabac souflée dans la plaie causait la mort de l'animal.

Un médecin indien a communiqué le remède suivant à Dubois : on prend sept panamdôr, pesant à peu près trois quarts d'une pagode, d'assa fotida d'une bonne qualité, puis le fruit très-connu dans toutes les Indes sous le nom de katricahe par les Tamuls, et de beringelle ( solanum melongena, L.) par' les Portugais, et enfin de l'huile de sésame, appelée par les 'Tamuls halla venie, en suffisante quantité pour frire le fruit du solanum.

On pile l'assa fotida, et après avoir divisé le beringelle en trois portions, de manière qu'elles soient encore adhérentes au pédoncule, on introduit dans chaque portion un tiers d'assa fotida; puis on entoure le fruit d'un fil, et on fait frire le tout dans de l'huile de sésame.

Le malade doit manger une portion avant de se coucher, une autre dans la matinée du jour suivant, et la troisième le surlendemain au soir. La partie dı corps où se trouve le ver doit être frottée trois fois par jour pendant trois jours consécutifs avec l'huile dans laquelle on a fait frire le fruit chargé d'assa foetida.

Ces moyens employés dans le commencement de la maladie empêchent le développement du ver, et celui qui est déjà complétement formé sort bientôt après. Dans tous les cas, ils calment la douleur dans l'espace de trois ou quatre jours. Mais si la maladie 
est très-opiniâtre, on est obligé de recommencer l'usage de ce moyen, etil est rare qu'il ne soit pas efficace.

Pour engager à son emploi, Dubois remarque que les Bramines qui assaisonnent très-fortement leurs mets avec de l'assa foetida ne sont jamais incommodés par le dragonneau.

Gallandat croiı qu'il y a plusieurs indications à remplir dans le traitement de cette maladie; il conseille aux malades, dans le but de faire cesser l'inflammation, chose essenticlle pour prévenir toute suite fâcheuse, $x^{\circ}$ de se faire saigner plus ou moins copieusement, selon la violence des symptômes inflammatoires, afin de diminuer l'afflux du sang vers la partie affectée; $2^{\circ}$ de boire des tisanes rafraîchissantes, avec addition d'un peu d'esprit de vitre, on bien d'esprit de vitriol dulcifié, et de faire diète; $3^{\circ}$ de se purger, selon les circonstances, plus ou moins copieusement; $4^{\circ}$ d'appliquer trois ou quatre fois par jour, sur la partie affectée, des cataplasmes émolliens e calmans, afin de diminuer la douleur et d'accélérer la suppuration; $5^{\circ}$ après l'ouverture de l'abcès, et après avoir roulé le ver à la manière accoutuméc, on doit exercer une légère pression, afin d'évacuer le pus, panser la plaic avec de la charpie trempée daus du miel rosat, et la couvrir avec un emplâtre; $6^{\circ}$ dans le cas où l'on aurait à craindre une vouvelle inflammation, ou bien dans le cas où lé ver offrirait trop de résistance, il faut alors continuer l'usage des remèdes, tant internes qu'externes, déjì mentionnés; en outre, on doit souvent arroser la 
partie affectée avec du miel rosat, faire deux tractions par jour sur le ver, et panser la plaie autant de fois.

Tont en faisant l'observation que des pilules mercurielles administrées jusqu'à exciter une salivation complète n'ont pas contribué à faciliter sa sortie, et qu'après cinq ou six semaines d'autres vers se sont présentés de nouveau, et ont donné des signes évidens de vie, Gallandat prétend que l'usage du sublimé corrosif, dissous dans de l'eau-de-vie à la manière de Van Swieten, contribue, $\mathrm{I}^{\circ}$. à faciliter la sortie du dragonneau; $2^{\circ}$. à diminuer la douleur et l'inflammation; $3^{\circ}$. à empêcher que le ver ne se déchire; $4^{\circ}$ a faire terminer le traitement avant le vingtième jour $; 5^{\circ}$. à causer la mort de l'a nimal.

Grégor croit que des frictions avec des onguens, et surtout avec l'onguent mercuriel, devraient être d'une graude utilité.

Des étincelles électriques dirigées à travers la partic affectée, n'ont produit aucun effet utile.

Hemniersan rapporte que les nègres se guérissent eux-mêmes; leur méthode est la suivante : Sitôt que le ver est sorti de la longueur d'un doigt, ils le conpent; ils frottent ensuite la plaie avec de l'huile de palmier, et mettent quelques feuilles vertes, au lieu d'un emplâtre, sur la partie malade; ils ouvrent la tumeur, en font découler le pus et lavent la plaie, dans l'inteution de causer une irritation, avec de l'eau dans laquelle ils ont fait infuser du poivre $c t$ d'autres plantes; ils calment ensuite cette irritation par l'emploi de l'huile de palmier et de quelques 
feuilles vertes. En général les nègres guérissent toutes leurs plaies avec ces derniers remèdes.

Isert croit pouvoir attribuer sa prompte guérison aux grands mouvemens qu'il se donnait, quoiqu'aveć peine, pendant la durée de cette maladie, et à un grand usage des bains.

Dans le cas où le ver ne cède pas aux tractions (ce sont les paroles de Kæmpfer), et que le malade éprouve de la douleur à l'extérieur, dans un endroit éloigné de celui ou le dragonneau se présente, on doit le laisser en repos et abandonner la guérison à la nature; il arrive ordinairement que le ver se fraye un chemin vers cet endroit. Kæmpfer ne désapprouve pas la méchode proposée par quelques personnes, et qui consiste à faire des affusions fréquentes d'eau froide sur les parties saines, dans l'intention de les garantir de la corruption, de diminuer l'accumulation des humeurs, el d'empêcher la formation d'un ulcère; méthode qui doit très-bien trouver son application dans les climats ehauds. Du reste eet auteur conseille de faire évacuer le malade au commencement de la maladie, et de lui faire observer un régime sévère pendant sa durée, afin que la plaie ne prenne pas un mauvais caractère, ce qui arriverait si on n'empêchait pas l'accumulation des humeurs vers la partie affectée. Kæmpfer avertit en mêmetemps, que l'on doit éviter l'emploi des corps gras, par la raison qu'ils amènent facilement la gangrène dans les pays chauds. Cet anteur regarde les eataplasmes conıme très-efficaces. Il rapporte encore, 
que le bas peuple n'a recours pendant la durée de cette maladie, qu'à l'usage d'oignous rôtis ; méthode qui n'est pas à mépriser.

Linscliot conseille l'application de beurre, et Leiter celle d'oignons et de feuilles de riz bouillies dans du lait.

Lœffler prétend que les frictions mercurielles sont plutôt nuisibles qu'utiles, par la raison qu'elles augmentent la douleur ct la tumeur. Le liniment volatil avec addition de laudanum liquide lui paraît plus convenable. Le sublimé corrosif, recommandé par Gallandat, ne s'est pas montré efficace dans ses expériences. Les esclaves, qui en avaient fait usage, perdaient l'appétit, et ils maigrissaient. 11 rapporte que l'aloës ne s'est pas non plus montré efficace, et que l'usage des purgatifs légers paraît produire de meilleurs effets.

Paul d'Egine recommande seulement des fomeutations chaudes; et, d'après toutes les recherches que j'ai faites sur le traitement de cette maladie, il résulte que les fomentations chaudes doivent être regardées comme le remède le plus convenable pour faciliter la sortie du dragouneau. Dans le cas où cet animal n'occupe qu'une petite place, comme par exemple une des malléoles, qu'il entoure souveut circulairement, l'emploi de l'aloës et des oiguons rôtis peùt être très-utile; car ces deux substances facilitent la suppuration. Dans les deux cas cités par M. Larrey les dragonneaux étaient probablement dans une semblable position; et c'est sans doute la raison 
pour laquelle ce médecin a pu détrnire ces animaux à l'aide de la suppuration et sans avoir besoin de les extraire. Il est aussi possible que M. Larrey n'ait eu à traiter que de simples furoncles, car on ne rencontre pas le diagouneau dans la Basse-Egypte. Du reste il n'est pas non plus constaté que cet animal, dans le pays où il séjourne, doive se trouver dans chaque furoncle. Krmpfer rapporte que les barbiers et les hommes ignorans causent souvent beaucoup de mal, par la raison qu'ils onvient la pustule en général trop tôt, et qu'ils saisissent, dans beaucoup de cas, quelque tendon au lieu du ver. Le même auteur racontc en effet, qu'il a connu deux personnes qui ont été estropiées par suite d'une bévue pareille. Dans les pays où l'on est accoutumé à rencontrer des dragonneaux dans l'intérieur des pusıules, il doiı aussi arriver quelquefois que l'on présume la présence de ces vers dans des tumeurs où il n'y en a réellement pas.

D'après ńa manière d'envisager la nature de cette maladie, il me semble que l'on ne peut pas heaucoup se pronettre de l'emploi des remèdes internes; leurs effets doivent se borner, à ce que je crois, à diminuer la trop grande tension des fibres. Je regarde l'assa foetida comme'un reınède préservatif plutôt que curatif. Il me semble aussi que l'on ne doit pas avoir besoin, au moins dans beaucoup de cas, de recourir à la saignée; elle pourrait cependant être indiquaée dans celui où plusicurs vers sembleraient sortir en méme temps, et quand l'inflammation menacerait de 
dégénérer en gaugrène. Du resıe le médecin doit diriger son traitement sélon la constitution du malade.

Il me reste encore quelque chose à dire sur les accidens qui arrivent après la rupture du ver; elle est ordinairement occasionée par une manœuvre trop brusque, ou bien par des tractions trop violentes. Cependant le ver se déchire dans quelques cas, malgré les plus grandes précautions possibles. Les suites en sont souvent bien malheureuses, et, quoique le malade, d'après le témoignage d'Avenzoar, n'en meure pas subitement, les cas ne sont pas cependant rares, comme cela a été attesté par Bancroft, Chardin, Gallandat, Labat et Lister, où cette rupture a causé la gangrène, et même la mort. Dubois n'a jamais vu arriver la gangrène dans ce cas, maịs il a observé que des raccourcissemens et des difformités des jambes en étaient quelquefois la suite.

Quoiqu'il soit prouvé que la mort n'arrive que rarement après la rupture du ver, néanmoins la plupart des auteurs conviennent que cet accident contribue à compliquer la maladie, à la traîner en longueur, et à donner lieu à des fistules très-difficiles à guérir. Il résulte de là, que l'on fait toujours trèsbien d'éviter, autant qu'il est possible, la rupture de cet animal. Le célébre voyageur James Bruce, à l'époque de son retour au Caire, fut atteint de cette maladic; malheureusement on ne connaissait pas alors dans cette ville le mode de traitement que l'on doit employer dans ce cas. Les médicamens qu'on lui administra restèrent sans effet; enfin on se dis- 
posa a faire l'extraction du ver, et en effet on parvint à faire sortir dans l'espace de huit jours trois pouces du corps de cet animal; cette opération ne causa â Bruce ni douleur ni fièvre. Ce voyageur s'embarqua alors pour la France, et confra son traitcment au chirurgien en chef du bâtiment. Ce chirurgien déchira un jour le dragonneau par une traction trop forte; il survint alors une inflammation très-violente avec tuméfaction, et la gangrène était à craindre; le chirurgien en second du bâtiment effectua plus tard la guérison par le débridement de la plaie. La maladie de Bruce a duré cinquante deux jours, et il éprouva pendant trente-cinq les douleurs les plus atroces; pendant une année entière, il se sentit trèsfaible, et il ne fut entièrement guéri qu'après avoir fait usage des bains de Poretta, situés dans les montagnes du Bolonnais.

Rhazès avait déjà recommandé le débridement de la plaie après la rupture du ver; Gallandat est d'un avis contraire, et il regarde cette opération, non-sculement comme inutile, mais encore comme dangereuse. L'expérience a prouvé, selon ce dernier, que cette opération augnente l'inflammation, la tuméfaction,, et donne lieu à la gangrène.

Une femme, habitante de l'A frique, avait un dragonneau au coude (ce sont les paroles de Gallandat); ce ver se déchira malgré toutes les précautions possibles, et il survint une inflammation accompagnée de fièvre et de délire. La malade était dans le plus grand danger; l'usage des cataplasınes énollieus, les 
saignées et de légers purgatifs firent cesser ces accidens, et le ver se fraya une autre route pendant l'emploi de ces médicamens. Dans un autre cas où un dragonneau avait également été déchiré, le res'e de l'animal, quinze jours après, put aussi prendre une nouvelle direction, sans causer cependant d'inflammation; plus tard on réussit à l'extraire en tota. lité, et Gallandat vit clairement des mouvemeus à l'une de ses extrémités. Cette observation sert à réfuter une assertion que cet auteur a avancée plus haut; il rapporte, "que les malades sont dans le plus grand danger quand le ver reste vivant après s'être déchiré," et il ajoute: "Quand le ver meurt après sa rupture, il ne se forme par la suite qu'une fistule, que lon parvient à guérir peu à peu." Hunter prétend le coutraire, et il s'exprime ainsi : "Si long-temps que le ver vit, il ne cause que peu d'embarras, mais lorsqu'il est mort, il irrite comme tout autre corps étranger, et la suppuration s'établit dans tout l'espace qu'il occupe. Gallandat rapporte encore un t:oisième cas, dans lequel la mort survint à la suite de la rupture d'un dragonneau, qui séjournait dans le scrotum.

Hemmersan raconte qu'il a été inconmodé par trois dragonneaux pendant son séjour dans le pays où cet animal se trouve: deux individus étaient placés à la jambe droite et le troisième à la jambe gauche. Celui qui était à la plante du pied droit l'empêchait de narcher. Cel auimal se déchira. Le second se fit sentir sous le calcanéum (c'était peut-être la contiunation du premicr ), et il se fit une ouverture à l'or- 
teil; des ulcères se formèrent en cet endroit, et Hemmersan ne fut guéri qu'après avoir éprouvé beaucoup de douleur; le troisième dragonneau parut sous le calcanéum du pied gauche. Après que cet animal en fut sorti de la longueur d'un quart d'aune, il se déchira et s'enfonça dans la jambe, ce qui occasiona une tuméfaction considérable dans cette partie. Hemmersan souffrit pendant quatre mois consécutifs.

Lister a été également incommodé par un dragonneau qui, pendant quarante ou cinquante jours, sortait par petites portions, sans causer beaucuup d'embarras. Quand cinq quarts d'aune de cet animal furent extraits, il se déchira par suite d'une trop forte traction; il s'enfonça alors plus profondément, et produisit au mollet une tuméfaction tellement considérable, que l'on craignait la rupture de la peau à cet endroit. Lister avait en même temps des insomnies accompagnées d'une forte fièvre, et il fut obligé de garder le lit pendant trente jours. Le dragonneau se montra dans différens endroits du pied; son chirurgien appliqua des remèdes qui causèrent probablement la mort du ver, et la guérison eut lieu.

Cromer éprouva, après la rupture d'un dragonneau, des douleurs tellement violentes, qu'il fut obligé de garder le lit pendant quatre semaines, sans pouvoir dormir et sans pouvoir éteindre une soif extraordinairc dont il était tourmenté.

Ces cas prouvent, d'uue manière suffisante, qque 
la rupture du dragonneau n'est pas une chose de peu d'importance.

Avant de terminer ce chapitre, je suis obligé d'ajouter encore une remarque. N'ayaut pas eu occasion de recueillir moi-même sur les lieux les observations que je rapporte sur ce ver, jai lu à son sujet tous les auteurs qu’il m'a été possible de me procurer; cependant je n'ai pu faire mention de tous ccux qui sont cités par Jocrdens dans sa description du dragonneau; la raison en est que plusieurs de ces auteurs n'en ont parlé que vaguement; plusieurs autres sont bien entrés dans quelques détails à son sujet; mais, comme ils ne connaissaient pas cet animal, par des observations qui leur fussent propres, ils se sont bornés à copier les auteurs qui ont écrit sur cette matière ex professo ; plusieurs autres enfin ont passé le dragonneau entièrement sous silence, ou biea. ils n'ont fait que de le nommer. Voici les noms des anteurs que l'on trouve dans l'ouvrage de Joerdens: Actuarius, Blumenbach, Borelli, Castelli, Doevern, Fallope, Freind, Corræus, Gruner : ce dernier a seulement tâché de prouver l'identité de la veine de médine (vena medinensis) et du dragonneau (dracunculus); Heurnius, lngrassias, Klein, Lange, Léoni, Lesser, Linné (Amẹit. academ.), Lorrey : la description que cedernier a donnée du dragonneau est assez coinplète, nais elle a été faite d’après celle de Kæmpfer ; Manardus, Med (Onomatologia, hist. nat.), Pollux, Sauvages, Schenk, Veiga, Vogel, Woyt. Les auteurs suivans ont entièrentent passé le 
dragonneau sous silence, ou bien, je le repète, n'ont fait que de le nommer. Voici leurs noms : $1^{\circ}$. Bauhin; cet auteur entend sous le nom serpigo des vers souscutanés (vermes subcutanei); $2^{\circ}$. Constantini : il parle d'un petit et d'un grand ver sous-cutané, dont le premier n'était qu'un herpes miliaris, et le second n'était qu'un erysipelas exulceratum; $3^{\circ}$. Donat : il rapporte qu'un homme a rendu, en urinant, un dragon ailé; $4^{\circ}$. Ettmüller : cet auteur remarque que Velsch a également écrit une dissertation sur les masclous, et que cette dissertation a été ajoutée, par ce dernier, à son rapport publié sous le tire Exercitatio de vená medinensi ; 50. Hannow a fait plusieurs recherches sur le gordius aquatique; $6^{\circ}$. Hasselquist parle d'un tenia solium, qui a été appelé, dans les annonces de Goettingue, nestelwurm; $7^{\circ}$. Joel dit expressément que les vers sous-cutanés (vermes subcutanei) n'ont rien de commun avec le ver nommé par Paul d'Egine (dracunculus); $8^{\circ}$. et $9^{\circ}$. Le Gentil et Olendorp parlenı du pulex penetrans (Linu.); $10^{\circ}$. Paracelse dit seulement qu'il y a des vers où il y a des apostèmes, et vice versa $\hat{}^{1} 1^{\circ}$. Plater " fait meution, à l'endroit cité, de pustules (plulictana), et cet auteur ne parle nulle part de flaires; $12^{\circ}$. Scholz rapporte seulement qu'il a vu des enfans incommodés par des masclous; $13^{\circ}$. Schwenkfeld confond le dragonneau avec le gordius aquatique; et $14^{\circ}$ Spiegel raconte qu'il a trouvé, sous la peau de la hanche 
d'un chardonneret, uu dragonneau d'un pied de long, et qui était roulé en forme de serpent.

Brera ${ }^{x}$ prétend à tort que Sœmmerring a trouvé un filaire de Médinè chez un mouton d'origine arabe. Sommerring ', à l'endroit cité, rapporte seulement qu'il a rencontré, daus l'estomac d'un mouton arabe, un ver qui ressemblait à un dragonneau; mais je réponds à cela qu'un ver qui ne fait que ressembler à un autre n'est pas pour cela de la même espèce. Brera, dans ses mémoires ${ }^{3}$, a encore cité et tronqué un passage de Pline, comme cela se voit quand ou confronte le texte latin avec les paroles de Brera. Pline dit : Nascuntur.... sicut intra hominem teniae tricennum pedum, aliquando et plurium longitudine. Brera l'a traduit : Nelle opere di Plinio trovasi pure fatto cenno di alcune sottili tinee, ô méglio tenie della lunghezza di tre pedi. Brera a retranché vingt-sept, mais il a ajouté, au contraire, quelques mots dont Pline n'a nullement parlé : Che in alcuni posi penetrano la pelle degli uomini.

Il me semble avoir assez parlé de semblables citations infidèlement rapportées. J'ai encore ajouté cette dernière, afin d'avertir mes lecteurs de ne pas ajouter foi trop légèrement aux citations, et de les engager à confronter toujours-le texte des auteurs dont on prétend les avoir extraites. Du reste, il me paraît probable que Brera a été induit en erreur par Kun-

- Memorie, p. 249 .

2 Ballic, p. 103 , note 218 .

${ }^{3}$ Memorie ; s. 2 4t. 
semïller, qui a également rapporté le passage men: tionné de Pline. Néanmoins, Brera a tâché de raconter, d'une manière élégante, l'introduction des tænias sous la peau, manière qui convient bien à un poëte, mais nullement à un naturaliste.

\section{Vil. Hamulaire.}

IIamularia : subcompressa (en allem. der fillhwurm), pl. 1II, fig. 2.

HAMULARIA : subcompressa, antice allenuala.

Treutler, Auctuar., p. 10-13, tab. II, fig. 3-7, Ham. lymphatica.

Joerdens, Helminthol., page 3i , tab. vi, fig. 9-ז2, ham. lymph. Zeder, Naturgesch, s. 45, Tentacularia subcompressa.

Brera, Mernorie, p. 225 , tab. 1v, fig. 1-3, amularia linfatica. Rudolphi, Entoz., t. II, part. I, pag. 8z, Ham. subcomp.

De Lamarck, Anim. sans vert., tom. III, pag. 216 , l'hamulaire de lhomme.

En disséquant, en 1790 , le cadavre d'un homme de vingt-lutit ans, qui était né avec une prédisposition héréditaire à la phthisie et à l'hydropisie, et qui s'était épuisé par la masturbation, par les plaisirs vénériens et par un usage démesuré du mercure, Treutler trouva, dans les glandes bronchiales d'un volume contre nature, des vers dont les uns avaient plus d'un pouce, et les autres beaucoup moins. Ces animaux étaient allongés, arrondis, un peu comprimés des deux côtés, de couleur noire bruńâtre, parsemée en partie de taches blauches, un peu amincis vers l'extrémité antérieure, à moitié transparens 
vers l'extrémité caudale, et recourbés (après leur mort) aux deux extrémités.

On remarqua sur la tête, qui était peu distincte, et qui se terminait en une pointe obtuse, deux crochets proéminens, qui pouvaient être soulevés par l'animal. L'extrémité caudale était également obtuse et peu distincte. Excepté les deux crochets, on ne voyait, sur toute la surface de ces animaux, aucune trace d'autres organes.

Ces vers, étant différens, selon l'opinion de Treuller, de ceux que l'on trouve dans les bronches des putois et des renards, cet auteur en a formé un nouveau genre, sous le nom d'hamularia, dont il a donné les caractères suivans :

Corpus lineare, teretiusculum, caput obtusum, infra duobus hamulis prominentibus instructum.

La. figure 2, qui représente ce ver huit fois * plus grand que nature, est une copie de Treutler. La figure $2 b$ représente l'extrémité de la tête encore plus grossie que dans la précédente.

J'ai déjà remarqué, quand j'ai parlé de l'indication des caractères des genres de vers, que ce genre me paraît douteux, et je le répète encore une fois ici. Zeder compte cependant des vers provenant de la cavité thorachique de la pie-grièche roursse (lanius collurio, L.), comme de ce genre. Mais ceux que

- Ce n'est pas ma faute si cette figure, grossic huit fois, nc s'accorde; is avec la mesure naturelle indiquée plus haut. 
i'ai trouvés moi-même dans cet oiseau appartenaient aux filaires. Quant aux vers provenant des gros intesins des poulets, et que Rudólphi range encore dans ce genre, ce sont assurément des capillaires. Excepté T'reutler, pessoune n'a encore trouvé ces vers dans les bronches de l'homme; cependant Brera prétend. que Vercelloni et Bianchi en ont fait mention; mais, comme Brera n'indique pas en quel endroit de leurs écrits, nous laisserons cette assertion dans le doute. Comme Treutler n'a trouvé ces vers qu'une seule fois, il est, par cette raison, possible qu'il se soit trompé, et qu'il ait pris l'extrémité caudale pour l'extrémité antérieure, et que ces deux crochets (hamuli) ne fussent qu'un double pénis, on bien peut-être des viscères qui pendaient au dehors.

Treutler dit lui-même que ces vers étaient tellement implantés avec leurs trompes dans les membranes, qu'il n'en a pu extraire presque aucun individu, saus déchirer ses trompes. Cette circonstance sert ì augmenter la probabilité que ces vers appartenaient à ceux que l'on rencontre assez souvent dans les bronches et les poumous des animaux du genre mustela, et dont Rudolphi, Olfers, Leuckart, Natterer et moi, n'avons non plus pu parvenir à extraire un seul individu entier des masses qu'ils forment, et c'est aussi la raison pour laquelle leur genre n'a pas pu être déterminé jusquä présent. Rudolphi n'est pas très-porté à croire que ce fut de véritables

Inemorie, 5. 226 . 
glandes dans lesquelles Treutler prétend avoir trouvé ces animaux.

Du reste, nous ponvons en toute confiance admettre que Treutler a effectivement rencontré des vers dans des capsules particulières, et qu'il ne nous a rapporté que ce qu'il a réellement vu ; mais comme il ne parle pas de leur structure interne, il serait à désirer que Brera eût la bonté de nous dire de qui il a appris que ces vers, trouvés dans l'homme seulement par Treutler, et décrits par lui seul, ont un système nerveux composé de ganglions :

\title{
VIII. Strongle géant.
}

Strongylus gigas, $R$, (en allem. der pallisadenwurm), pl. III, fig. 3.

Strongyuus : capite obtuso, ore papillis planiusculis sex cincto,

bursa maris truncata integra, cauda fenina truncata.

Rudolphi, Ento- , II, 1, p. 2 10, tab. II, f. 1-4.

Cuvier, Règne animal, tom. Iv, p. 34, le strongle géant.

De Lamarck, Anim. sans vert. , t. 11 , p. 202 , le strongle des rein.s.

Ce ver séjourne dans les reins et peut-être aussi dans les muscles qui les environuent.

\author{
DESCRIPTION.
}

Les strongles que M. Rudolphi a trọués étaient

'Memorie, p. 32. Ne' gurdi e nell' amularia linfatica si usserva pure questo sislema nerroso ganglionico, colla differenza che $i$ gangli 
de la longueur de cinq pouces à trois picds, et de la grosseur de deux à six lignes. M. Cuvier a eu la bonté d'envoyer à notre collection un strongle qui. est de la longueur de trente pouces et de la grosseur de quatre lignes, et qui provenait du r.ein d'une fouine. Les vers de celte espèce, que l'on rencontre dans les reins des animaux tués depuis peu, sont aussi rouges que du sang; mais ils perdent bientôt cette couleur, quand on les nret dans de l'espritde-vin.

Le mâle est plus petit que la femelle, et il est aminci vers ses deux extrémités; sa bouche est circulaire ( ooy. fig. $3 a$ ) et pourvue de six petites papilles; son corps, composé, pour ainsi dire, d'anneaux, offre plusieurs dépressions longitudinales; sá queue ( $V$ oy. fig. $3 b$ ) forme à son extrémité une vessie par laquelle sort un pénis extrêmement fin. Cette espèce de ver a cela de particulier, que sa vessie est entière (integra); mais elle est, au contraire, bifurquée et formée de différentes manières chez tous les autres strongles.

La femelle est plus grande; la fin de sa queue est droite et obtuse : on remarque à cet endroit un anus longitudinal. L'entrẻe du vagin est éloignée, selon la différente longueur du ver, d'un ou de plusieurs

sono piu piccioli; et p. 228 : Ho già fatto rimarcare, che ganglion ico ne épure il cordone nervoso, che nel suo interno scorre dall' altra estremitù, al pari di quello del lombricoide, colla sola differenza, che piu piccioli ne sono i gangli nell' amularia. 
ponces de l'extrémité de la quene. M. le professeur Otto croit avoir observé un système nerveux chez les strongles.

Ces vers, dont Brera et Jœrdens ne font mention qu'en passant, ont été souvent trouvés dans des fouines, des chiens, des loups, des bœufs, des cheraux et des phoques. 11 paraît qu'on les reucontre rarement dans l'homme.

Il faut bien se garder de vouloir compter, comme appartenant à ce genre de vers, tous les corps que les hommes ont rendus, ou que l'on suppose avoir été rendus par ces derniers en urinant; car ces corps ont été souvent qualifiés à tort, par les médecins, du nom de vers : ce n'étaient souvent que des larves d'insêctes, qui n'avaient pas été réellement évacués par l'urètre, mais s'étaient introduits sans doute, par lıasard, dans le vase de nuit des malades.

Dans le cas où il y a adhérence entre le rectum et la vessie, avec complication de sinus fistuleux, il est possible que des vers intestinaux aient pu être évacués par l'urètre. Il arrive encore que des femmes rendent des oxyures avec l'urine; mais cela n'a rien d'étonuant; car nous savons que ces vers, après avoir quitté le rectum, s'introduisent souvent dans le vagin, d'où ils sont rejetés en urinant. Il est de fait que'l'on a regardé, dans bien des cas, des concrétions polypeuses et mcmbraneuses, à cause de leur forme arrondie, comme des strongles. C'est aussi ce que Grimm a observé chez un homme qui avait des pierres dans les reius et dans la vessie; il croit 
que ces faux vers prennent leur forme arrondie ed passant dans l'urètre.

Le ver dont parle Tulpius ' n'était assurément rien autre chose que du sang coagulé, par la raison que ce prétendu ver se dissolvit entièrement. Je doute aussi des cas rapportés par Pauliu et Barry.

Decerf raconte le cas suivant : Un homme âgé de cinquante ans, avait eu souvent dans sa jemnesse des hémorragies violentes, qui cessèrent à l'âge de vingtcinq ans, époque à laquelle il commença à ressentir des douleurs dans l'hypochondre droit; on présuma que ce mal siégeait dans le foie : tous les remèdes employés restèrent infructueux; cependant quelque temps après, le malade sentit une diminution de douleur; il s'y accoutuma à la fin, et suspendic l'usage des médicamens. Le 15 juillet, il fut incommodé par une violente hémorragie de l'urètre, qui était accom. pagnée de douleurs horribles dans les cuisses et dans la vessie. L'application des sangsues, l'usage des bains et des boissons mucilagineuses, furent sans succès; le malade maigrissait à vue d'œil ; on consulta quelques médecius de Paris; ils ordonnèreut des médicamens, mais qui ne produisirent aucun effet. Dans les premiers jours du mois de septembre, le malade, après avoir éprouvé une hémorragie semblable à la précédente, accompagnée des même douleurs et d'un accès léger de fièvre, rendit en urinant un ver enduit de sang. Le ver était de la grosseur

- Ouvrage cité, chap. 49, p. $17^{2}$. 
d'un tuyau de plume, et de la longueur de quatorze pouces et huit lignes. Il est à regretter que cet animal ait été jeté. Immédiatement après sa sortie, le malade se sentit soulagé, les douleurs cessèrent subitement, ainsi que l'hémorragie. A dater du 15 septembre jusqu'au 2 décembre, il évacua par l'urètre au moins cinquante vers de différentes formes et grosseurs. La plupart de ces prétendus animanx étaient de la grosseur d'un tuyau de plume, de la longueur de six à huit pouces, et ressemblaient aux ascarides, surtout l'échantillon dont Tulpius fait mention ' ; d'autres n'avaient que dix-huit lignes de longueur, et ressemblaient plutôt aux filaires. Leur corps aplati se terminait par une queue allongée et très-fine. Il est à remarquer que le malade de Decerf, qui s'est trouvé parfaitement guéri, n'aurait rendu que des vers morts.

Lors de mon séjour à Paris, en 1815, je me suis entretenu, au sujet de ces vers, avec M. Duméril. J'ajoute ici, par parenthèse, que ce profosseur a eut la bonté de mettre sa bibliothèque à ma disposition à toutes les heures de la journée, et que j'y ai re. cueilli beaucoup d'observations qui se trouvent rapportées dans ce traité. M. Duméril eut la complaisance d'écrire à M. Decerf, et de le prier de vouloir bien nous communiquer quelques échantillons; nous en reçumes en effet six : deux avaient bien l'air d'avoir

- J'ai déjà remarqué plus haut que l'existence de ce ver ne me paraît pas avoir été constatée d'une manière suffisante. 
été séparés et de n'avoir formé auparavant qu'in senl et même morceau. Nous nous sommes convaincus, après un examen soigné, que ces prétendus vers n'étaient que des concrétions lymphatiques, parmi lesquelles il n'y en avait pas deux qui se ressemblassent, ce qui aurait dû avoir vécessairement lieu, si cela avait été de véritables vers. L'un de ces échantillons était mince et lonğ; un autre étail deux fois plus gros à l'une de ses extrémités que dans le reste de son étendue, et son extrémité opposće citait tont à fait amincie; un troisième échantillon était obıtus à ses deux extrémités, etc. Il est possible et même probable que le premier corps rendu par ce malade fut un strongle, et qu'après sa sortie tous les symptômes cessèrent.

Un cas semblable, observé par Barnett et rapporté par Lawrence, augmente la probabilité de mon opinion, que tous les autres morceaux, à l'exception du premier peut-être, que ce malade avait rendus, u'étaient que des concrétions lymphatiques.

Lawrence raconte qu'une femne, existante encore, fut incommodée, pendant plusieurs années, par des douleurs violentes dans les cuisses et la vessie; elle eut aussi une rétention d'urine. A près avoir enduré toutes ces souffrauces, elle commença par rendre des corps vermiformes; et, dans l'espace d'un an environ, elle en évacua à peu près huit cents ou mille. Barnett a envoyé à M. Rudolphi plusieurs de ces prétendus vers, et ce deruier a en la bonté de n’en céder denx échantillons. Ni M. Rudolphi, ni 
moi, ne sommes portés à les regarder comme de véritables vers, quoiqu'ils se ressemblent davantage entre eux, sous le rapport de la forme cxterieure, que ceux recueillis par Decerf. La forme allongée des corps dont parle Lawrence a dû provenir, daus ce casci, d'après mon opinion, plutôt des uretéres que de l'urètre, par la raison que ce dernier n'a que très-peu d'étendue chez les femmes. Il est vraisemblable que les concrétions Jymphatiques trouvécs par Decerf ont pu recevoir leurs différentes formes, pendant qu'ils traversaient l'urètre. On voit sur la planche 9, fig. $/ \mathrm{h}$ une figure pliée en deux; clle est de la longueur de cinq pouces, amincie dans sou milicu; à partir de là, elle devient plus grosse, et elle est pointue à ses deux extrémitćs. Cette figure a été faite d'après une copic de la figure originale de Barnett, et elle m'a étć envoyée par Nasse, conjointement avec son explication, qu'il a bien voulu extraire du journal anglais (Medico-chirurgical transactions), par la raison que ce journal ne se troure pas à Vienne. M. Rudolphi m'a envoyé également, à la même épc= que, un extrait du même journal auglais. Cette figure s'accorde assez bien avec mes échantillons, cependant je ne remarque rien de dentelé dans les dépressions longitudinales; ils ne sont pas non plus aussi régulièrement pointus que cela se voit sur la figure, et ils ont l'air d'avoir été déchirés; en un mot, il leur manque le caractère de l'intégrité.

Ce pseudohelminthe est représenté de grandeur naturelle, et Barnett assure qu'il n'a pu déconvrir 
sur lui aucune trace d'organisation, ni à l'aide du scalpel, ni au moyen du microscope. La même personne de laquelle provenait ce pseudohelmiuthe a rendu une autre fois plusieurs petits vers qui ne ressemblaient pas à ceux d'une dimession beaucoup plus considérable, qu'elle avait évacués antérieurement. Ces petits vers ont vécu dans de l'eau tiède pendant quarante-huit heures. M. Rudolphi eu a reçu six individus, et il a bieu voulu m'en céder deux. On les trouve représentés de grandeur naturelle ( Voyez pl. 5, fig. 4 et 5) et l'un d'eux très-grossi, même planche, fig. $5 a$; la fig. $5 b$ indique la tête, et la figure $5 c$, la queue encore plus grossie que dans la figure précédente. Ces vers, inmédiatement après avoir été rendus, étaient transparens; mais ils sont devenus opaques dans l'esprit-de-vin. Barnett assure qu'il les a examinés plusieurs fois à l'aide d'un microscope, mais qu'il n'a rien pu découvrir de leur structure particulière. Néanmoins, l'on ne peut pas nier. que ce soient de véritables vers; en effet, on n'a qu’à les regarder pour en être convaincu ; mais il n'est pas prouvé pour cela qu'ils appartinssent aux strongles dont nous nous occupons; il est cependant possible que ce fussent de jeunes strongles qui peút-être s'étaient formés spontanément peu de temps avant d'avoir été évacués, quoique leur structure extérieure ne vienne pas à l'appui de cette supposition; toutefois, M. Rudolphi remarque trèsjustement, dans une lettre qu'il m'a adressée, que les petits provenant des autres espèces de ce genre 
de vers, diffèrent beaucoup de ceux qui sont complétement formés. Nous n'avons pas encore observé jusqu’à présent les petits du strongle géant. Il se pourrait donc que les vers vus par Barnett fussent de ces petits; ce qui confirme encore cette opinion, est que l'on remarque sur la figure $5 b$ quelque chose qui ressemble asséz à une ouverture de bouche cylindrique pourvue de papilles. Cette bouche se présente en biais; mais cela peut provenir d'un effet de l'esprit-de-vin. L'on remarque encore sur la figure $5 c$, au milieu de l'extrémitć caudale, une petite vessie qui ressemble un peu à la fig. $3 c$, même planche; car la membrane détachée, que l'on voit sur les côtés de l'extrémité caudale, n'est pas la véritable vessie de la queue, ce n'est que l'épiderme décollé ou boursouffé, comme cela se remarque encore sur plusieurs autres endroits de la figure $5 c$. L'épiderme des inclividus morts de plusieurs espèces de nématoïdes, se décolle facilement quand on les laisse trop long-temps dans l'eau. Il résulte de ces réflexions, qu'il n'est pas impossible que ces petits vers dont nous venons de parler fussent de jcunes strongles. Mais on me fera la question : oì étaient donc leurs parens? Je réponds à cela que je n'en sais rien; mais je puis cependant m'expliquer là dessus : D'abord, $1^{\circ}$. il n'est pas du tout nécessaire qu'il existe en même-temps des vers tout à fait dévcloppés, où l'on remarque des petits, car ces derniers peuvent provenir d'une formation spontanée et récente; $2^{\circ}$. il est aussi possible que les parens de ces petits vers fussent déjà morts, 
et qu'ils eussent été rendus conjointement avec ces concrétions; $3^{\circ}$. l'on peut aussi supposer que leurs parens séjournaient encore, lors de leur évacuation, dans les reins de la personne citée par Decerf, par la raison que cette dernière, d'après le rapport de ce médecin, se trouvait bien un peu sonlagée, mais qu'elle n'étail pas encore tout à fait guéries.

Les cas que je vais maintenant ciler doivent être yegardés, ce me semble, conme ceux dans lesquels on a réellement observé des strongles plus ou moins grands, et dont les uns séjournèrent daus les reins, et les autres avaient été déjà rendus par l'urètre.

$\mathbf{1}^{\circ}$. On a trouvé une pierre et un ver dans les reins du grand-duc Ernest d'Autriche, qui mourut en 1595 dans les Pays-Bas, où il fut gouverneur. Hugo Grotius rapporte que ce ver était encore vivant lorsque l'on cut ouvert le corps de ce duc, et que cet auimal avait rongé les parties environuantes. $2^{\circ}$. Ruysch ${ }^{2}$, qui a souvent observé des strongles chez les chiens, trouva un jour un ver de cette espèce dans les reins d'un homme.

$2^{\circ}$. Blasius raconte qu'il n'a remarqué qu'une seule. fois, dans les reins d'uu vieillard, deux vers rouges de la longueur d'une aune, et il ajoute que l'on rencontre souvent ces vers chez les chiens.

$4^{\circ}$. Rhodius a connu un homme, atteint d'une

- Rudolphi (Syzops., p. 250) fait mentiou des petits vers dont il vient d'ètre question sous le nom de spiroptera hominis. (Br.)

2. Onvrage cité, $64^{e}$. observat. 
fiève maligue, qui avait rendu, en urinant, le cinquième jour de sa maladie, un ver vivant, de forme ronde et d'un pied de longueur. Cet honme n'a jamais éprouvé de difficulté à uriner, ni avant ni après la sortie du ver.

$5^{\circ}$. Albrecht fait mention d'un soldat qui avait éprouvé, depuis sept ans, uṇe difficulté d'uriner, et qui en fut entièrement débarrassé après qu'il eut rendu par l'urètre un ver de la longucur de trois doigts et de la grosseur d'un tuyau de plume.

$6^{\circ}$. Raisin rapporte qu'un homme de cinquante ans était incommodé, depuis deux ans, de coliques néplrétiques; son urine était sanguinolente et noirâtre. Tous les remèdes que l'on avait ẹmployés étaient restés infructueux. Un jour cet homme rendit en urinant un ver de la longueur de trois pouces; après quoi l'urine reprit sa couleur naturelle, et le malade guérit.

$7^{\circ}$. Duchâteau cite une observation semblable.

$8^{\circ}$. Le cas le plus remarquable a été communiqué par Monblet: Un cnfant de dix ans, du sexe masculin, à qui ce médecin avail déjà fait l'opération de la taille à l'âge de trois ans, éprouvait des douleurs très-forles à la région lombaire, et il s'y développa une tumeur. L'enfant était cn même temps incommodé d'une rétention partielle d'urine. Ḷa tumeur ful ouverte, il s'en écoula beancoup de pus, et la plaie guérit. Une nouvelle tumeur, accompagnée de violentes douleurs, nécessita une seconde onver-* ture. Pendant trois ans, celte tumeur s'ourrait et 
se fermait alternativement. A la fin, il parut, dans la plaie, un ver de la longueur de cinq pouces, et de la grosseur d'un tuyau de plume, qui fut bientôt suivi d'un second de même forme : sa longueur était de quatre pouces. Peu de temps après la sortie de ces deux animaux, la rétention devint complète, et la vessie fut très-distendue, accidens que cet enfant n'avait pas encore éprouvés. Tout à coup un troisième ver, semblable aux deux précédens, sortit, et il fut bientôt suivi d'un quatrième. A près l'évacuation des quatre vers, la santé du malade commença à s'améliorer. On employa un traitement couvenable, et cer enfant guérit complétement.

Chapotain, Monceau, Holler et Renner font également mention de vers que des hommes avaient rendus par l'urètrc. Schenk cite des exemples semblables.

Le ver qui a été trouvé dans la cavité thorachique d'un homme par Hæhne, était aussi trèz-probablement un strongle.

Mes lecteurs devineront facilement la raison pourquoi je ne suis pas entré dans plus de détails, dans ce chapitre, sur les causes particulières qui favorisent la formation de ces vers; ils sentiront également pourquoi je n'ai rien dit sur les symptômes à l'aide desquels on peut reconvâtre l'existence de ces animaux, et pourquoi je n'ai pas fait mention des remèdes propres à les expulser. Le fait est que nous n'avons pas encore de données certaines sur tous ces points. Du reste, les personnes versées 
dans l'art de guérir ont sans doute remarqué que lés symptômes qui ont précédé l'évacuation de ces vers avaient pu être attribués aussi bien à d'autres maladies des reins et de la vessie, qu'à la présence de ces animaux; et, quand on n'est pas au fait du diagnostic d'une maladie, il est alors bien difficile ou plutôt impossible de proposer un plan de traitement rationuel.

Les trois espèces de vers dont nous venons de nous entretenir, et qui séjournent hors du canal intestinal de l'homme, appartiennent tous au premier ordre des entozoaires, c'est-à-dire à celui des vématoïdes. Des vers provenant du second ordre, c'està-dire des acantocéphales, n'ont pas encore été trouvés dans l'homme, comme nous l'avons déjà remarqué.

M. d'Olfers, pendant son séjour au Brésil, a rencontré des échynorhynques chez un singe.

Jusqu'à présent on n'a encore découvert dans l'homme que deux espèces de vers de l'ordre des trématodes; et encore les rencontre-t-on très-rarement : ce sont la douve du foie et le polystome pinguicole.

\section{Douve DU FOIE.}

Distoma hepaticum (en allem. der leberegel), pl. IX, fig. I-Ic.

Distona : obocalum, planum, collo subconico, brevissino, poris orbicularibus, ventrali majore.

Gmelin, Syst. nat., p. 3o85, $\mathrm{n}^{\circ} .2$, fasciola humana.

Joerdens, Helminth., s. 64, tab. vis, fig. 13 et 14 , der leterblattrurm, fasciola hepaticu. 
Frera, Memorie, p. 92, tab. I, fig. 22 et 23 , fasciola epatica. Rudolphi, Entoz., vol. Ir, p. 352, Distom. hepaiti. Cuvier, Règne animal, tom. Iv, p. 4r, douse du foie. De Lamarck, Anim. sans vert., t.II, p. 182 , fasciole hépatique.

Ce ver porte les noms vulgaires suivans : les Allemands l'appelleut das leberdoppelloch, der leberwurm, schafegel, die egelschnecke, les Holiandais leverworm, botten, les Danois faareflynder, ikle, igler, iiler, souacgler, souigler, les Suédois lever-mask, les Angiais the liver fuke, les Italiens biscinola, les Espagnols caracolitlos, serillas, pujarillos.

La douve du foie séjourne dans la vésicule du fiel, ou peut-être aussi dans le foie de l'homme. On la rencontre égalemeut dans le foie des moutous, des bœufs, des cerfs, des gazelles, des chèvres, des chamois, des cochons, des chevaux, des lièvres, des kanguroos, etc.

\section{DESCRIPTION.}

Les vers de cette espèce sont de la longueur d'une à quatre lignes, et de la largeur d'une demie, jusqu'à une ligne; ils ont la forme d'une lancette, et ils sont obtus à leurs deux extrémités. L'ouverture antérieure est ordinairement dirigée obliquement en dcdans, le con est un peu arrondi, et d'un blanc jaunâtre; l'ouverture postérieure ou ventrale est un peu proémineute; sa direction n'est pas toujours la même. Un peu plus bas l'ou remarque quelques taches d'un blanc opaque et un paquel de vaisseaux ou de tubes d'une couleur jaune ou brune; ce sont probablement les oviductes. Les vaisseaux qui se trouvent le long 
de deux côtẻs paraisseut former les tubes alimentaires. On ne remarque pas les oviductes sur tous les individus, comme cela est démontré par les figures $1 a$ et $\mathrm{t} c$.

M. Otto croit avoir observé sur ces vers un système nerveux, ce que cenendant Gxdde ne veut pas admettre.

La description que nous venons de faire de cette espèce de vers se rapporte aus douves du foie, quel'on a trouvées jusqu'à présent daus la vésicule du fiel de l'homme. Les douves que l'on rencontre souvent dans le foie des animaux cités ci-dessus, sont ordinairement beaucoup plus grandes; leur longueur est d'un pouce à peu près, et leur largeur de quatre à six lignes. Ces animaux sont d'un jaune sale, ou brunâtre, et l'on ne peut que très-difficilement distinguer quelque chose de leur structure intérieure; leur peau est aussi plus compacte; cependant l'on rencontre souvent cliez les animaux des petites douves conjointement avec les grandes, et Zeder a prouvé d'une manière suffisante, que les douves d'une dimension moins considérable ne sont que les jeunes ou les petits des autres, et qu'elles ne forment nullement une espèce particulière; on est d'autant plus en droit de le croire, que l'on a trouvé chez les grandes des oufs tout à fait développés '.

Zeder $^{2}$ a rencontré dans le foie d'un lièvre, no-

"Voyez sur ce sujet, Nrau neue entdectiung, etc., s. 40.

- Erster Nachtras, s, $16_{7}$. 
tamment dans un des conduits biliaires, des vers de cette espèce, qui étaient de la longueur d'une ligne et un quart jusqu'à sept lignes et demie ; ces auimaux étaient placés, dans cet organc, dans un ordre en rapport avec leur grandeur, c'est-à-dire que ceux de la longueur d'un quart de ligne, et ceux d'une demi lignne se trouvaient réunis.

Mais comme les petites douves, que l'ou a observées dans l'homme jusqu'à présent ressemblaient tout à fait aux petits, ou aux jeunes douves que l'on voit souvent dans le foie des moutons et dans celui d'autres animaux, nous sommes alors également portésà croire que les douves que l'on trouve dans l'homme ne sout que des petits ou des jeunes individus de vers de cette espèce. Il paraît du reste, qu'on ne les rencontre que très-rarement; car les prétendus vers que des médecius assurent avoir remarqués dans le foie de l'homme n'appartemaient pas, pour la plupart, à l'espéce dout nous nous occupons à présent. Cependant il n'y a pas de doute que Malpighi savait déjà que l'on rencontre des douves du foie chez l'homme et chez les animaux; mais il n'est pas démontré d'une manière suffisante, que les vers dont parle Bauhin ${ }^{2}$ fussent de véritables douves du foie.

Bidloo $^{2}$, qui connaissait quel déraugement ces vers peuvent occasioner dans le foie des animaux, en a réellement observé dans celui de l'homme.

1 Voyez Boneti sepulchret.

2. Clericihist., la'i lumbr., p. I Ig. 
Wepfer" raconte qu'il a souvent vu le canal hépatique rempli de vers, qu'il a appelés sangsues ( $h i-$ rudines.)

Pallas rapporte " qu'il a trouvé des douves en disséquant à l'amphithéâtre anatomique de Berlin; ces animaux étaient comme enclavés dans le canal hépatique d'une femme.

Chabert $^{3}$ en a fait rendre à une fille de douze ans (à l'aide de son huile empyreumatique) une quantité innombrable; ces animaux étaient de la longueur d'une ligne et demie jusqu'à trois lignes.

Bucholz a trouvé une grande quantité de ces vers dans la vésicule du fiel d'un forçat mort d'une fièvre putride.

Brera nous assure qu'il en a également observé dans le foie d'un homme mort à la suite de scorbut compliqué d'hydropisie. Cet auteur remarque que ces vers étaient d'une dimension beaucoup plus considérable que ceux trouvés par Bucholz. Je ne doute nullement de la vérité de ce fait, mais je ne puis pas m'empêcher d'avouer que je ne concois pas pourquoi Brera ne les a pas fait dessiner d'après nature, et pourquoi il a préféré copier la figure tout à fait inexacte de Jœrdens; car les circonvolutions des oviductes représentées sur cette figure sont d'abord tout à fait mal dessinées, en outre il y manque les

\footnotetext{
'Dans les Ephem. nat. cur.

De infest. vivent., p. 252 et 270 .

${ }^{3}$ Rudolphi, Bemerk. , auf einer Reise II, s. 37.
} 
deux sucoirs caractéristiques du genre, que l'on découvre à l'aide d'une simple loupe jusque sur les plus petits individus. On les voit même sur mes fig. $x$ et $x a, p l .9$, qui représentent l'animal de grandeur naturelle, et je dois faire remarquer que les individus qui ont servi de modèle avaient la même origine, ou mieux, provenaient du même endroit que ceux de Jcerdens.

Lorsque son altesse royale le grand duc de Weimár visita notre collection en 1814 , je lai montrai le désir d'avoir quelques individus des douves du foie, que Bucliolz avait recueillies, et qui se trouvent à Jéna. A peine quinze jours s'étaient écoulés, que jen reçus une grande quantité de la part du professeur Lenz. Le cabinet impérial d'histoire naturelle de Viénne envoya en revanche à l'université de Jéna cent six vases remplis de vers intestinaux de tous les ordres, genres et espèces.

Je fais mention de cette circonstance, uniquement afin de prévenir les médecius qui voudront bien nous envoyer des objets rares concernant l'helminthologie, trouvés par eux dans les cadavres de l'homme, que nous serous toujours disposés à leur prouver notre reconnaissance.

Brera parle avec beaucoup d'éloge de la figure de Jœrdens et l'appelle eccellente figura; il désapprouve an contraire celle de Bidloo copiée par Leclerc. Les figures de Bidloo, représentant des vers grossis, ont en effet un air un peu singulier, mais celles faites de grandeur naturelle ne sont pas mal dessinées. 11 me 
paraît du reste, que Brera a regardé les vers recueillis par lui-même d'une manière bien superficielle, car sans cela il aurait dû remarquer que les suçoirs manquaient à la figure de $\mathbf{J} œ r$ dens.

Les exemples mentionnés ci-dessus de douves $\mathrm{d} u$ foie trouvées chez l'homme, sont les seuls qui soient venus à ma connaissance; cela prouve qu'on les rencontre bien rarement. Cependant il est possible que l'on ne reconnaisse pas toujours leur existence, ainsi que les désorganisations qu'elles produisent dans quelques cas dans le foie. Je n'ai jamais eu occasion d'observer cette espèce de vers chez les hommes; mais chez les ảimaux $\not$ cn ai rencontré bien souvent. Les douves siégent ordinairement, chez ces derniers, dans les conduits biliaires. Ces conduits s'élargissent quelquefois d'une manière extraordinaire, leur paroi interne est enduite d'une mucosité épaisse, d'un brun noirâtre, et leurs membranes s'épaississent fortement. Celte mucosité se durcit avec le temps et se change en une véritable substance osseuse. Quand on voit des inégalités sur la surface d'un foie, on peut presque assurer d'avance quelle espèce de désorganisations il a subies, et quand on entend une crépication en pressant l'organe, crépitation qui provient du brisement des lames osseuses, on est alors sûr que ce foie contient, ou bien qu'il a contenu des douves hépatiques. L’incrustation cst quelquefois tellement forte, que l'on peut obtenir, par la dissection, de véritables ubes osseux.

Lc docteur Freese de Meklembourg a rencontré, 
dans un foie de bœuf, une pareille désorganisation des conduits biliaires, qui avait presque la forme d'une main d'homme.

Quand ces conduits se trouvent désorganisés à ce point, alors les douves meurent peu à peu. Il peut bien se faire que l'on remarque dans un foie de semblables altérations, qui provenaient originairement de ces animaux, sans cependant, dans bien des cas, $\mathrm{y}$ trouver de ces derniers.

Je n'ai aucune notion certaine provenant de ma propre expérience, ou de celle des autres sur l'origine et sur le diagnostic de cette espéce de vers dans l'homme; Brera a indiqué une asthénie générale comme la cause prédisposante à leur formation : mais qu'entend-il par là? il me semble que cette explication est peu satisfaisante.

Si l'on était cependant convaincu de leur présence, je ne saurais proposer rien de plus efficace que l'huile empyreumatique de Chabert, et l'inventeur de ce remède a réellement eu occasion de sc convaincre de son efficacité dans des cas semblables. Je suis également persuadé que ce remède doit être d'une grande utilité pour les moutons, qui sont bien souvent incommodés par des douves qui les font quelquefois périr par milliers.

X. Poiystome plinguicole.

Polystoma pinguicola (en allem. das vielloch), pl. Ix, fig. 2.

Polystona : Depressum oblongum, antice truncatum, postice acuminatum : poris sex anticis lunation positis. 
Treutler, Auctuar., p. I9-20, tab. III, fig. 7-II, hexaihyridum pinguicola.

Joerdens, Helminth., p. 66, tab. 1, fig. 3-5, der fettbiattsurm. Zeder, Nalurgeschichte, p. $230, \mathbf{n}^{\mathrm{0}} .2$, polystom. pinguicola. Rudolphi, Lintoz. II, part. I, p. 458, polyst. pinguicola. Brera, Memorie, p. 100, tab. I, fig. 28 , tab. II, fig. I-2, exatiridlio pinguicola.

De Lamarck, Anim. sans yert., tome $\mathrm{In}$, p. 174 , la linguatule des ovaires.

En disséquant le cadavre d'une jeune paysanne de vingt ans, qui avait succombé à la suite d'un accouchement très-laborieux, Treutler a trouvé, dans la graisse qui entourait l'ovaire gatche (à l'endroit où le ligament large de la matrice commence), un cngorgement dur, à peu près de la grosseur d'une forte noisette et de couleur rouge. Cet engorgement était fixé légèrenuent dans le tissu cellulaire, de manière" que l'on pouvait le faire glisser dans tous les sens. Ce corps étranger n'était qu'un paquet de graisse endurcie, creux à l'intéricur, et contenant un ver libre semblable à celui qui est représenté tab. 9 , fig. 2 . La figure $2 a$ fait voir le ver isolé, et notamment par le côté où l'ou ne peut remarquer les suçoirs, parce que ses bords étaient repliés. On voit, au contraire très-clairement, ces organes, fig. $2 l$. Ces dessins, copiés fidèlement d'après ceux de Treutler, pourront suffire, ce me semble, pour donner à mes lecteurs une idée claire de ce ver. Je m'abstiens, pour cette raison, de toute autre description.

II est très-probable que les vers vésiculaires ou 
hydatides existent depuis aussi long-temps que les diverses maladies auxquelles le genre humain est assujéti. Les plus anciens médecins en font en effet déjà mention; mais ils ignoraient alors, presque tout it fait, que ces vers, ou, si l'on veut micux, ces vessies remplies d'eau, fussent d'une nature animale, ou bien qu'elles fussent donées d'une vie individuelle.

A retée' observe senlement quc l'on a sonvent trouvé de ces vessies dans la cavité abdominale de l'homme, et qu'elles obstruent, dans quelques cas, l'ouverture que l'on pratique quand on fait la paracentèse; mais cet anteur ne s'explique pas sur leur origine, ni sur la cause de leur formation.

A près cet auteur, les médecins émirent différentes opinions sur ce sujet : Pison croyait que ces vessies se forment, dans un endroit quelconque, par le mélange du serum avẹc de la mucosité, ou plutôt par le mélange d'une matière purulente.

Ruysch paraît avoir eu sur la nature des hydatides diverses opinions à différentes époques : tantôt en effet il les a regardées comme des glandes ${ }^{2}$, tantôt comme les extrémités de vaisseaux sauguins, qui auraient changé de nature ${ }^{3}$, et tantôt il croyait qu'elles se formaient par le tissu cellulaire qui se trouve entre les vaisseaux, et dans lequel il s'amasse de l'eau dans un

- Ouvrage cité, p. 5 r.

= Obs. anat. xxx!11.

3 Advers. anat., decad. I, p. 8. 
état contre nature. Cette eau, selon son idée, comprime les vaisseaux environnans, de manière à faire disparaịtre leur capacité, et à les oblitérer entière ment :

Grashuis était aussi de l'opinion que les hydatides se forment dans le tissu cellulaire, et principalement dans la turique adipeuse.

Aucun de ces auteurs n'est entièrement dans l'erreur dans sa manière d'envisager ce sujet, notamment quand il ne parle qu'en général de ces changenıens morbifiques qui s'opèrent dans le corps humain, et auxquels on donne ordinairement le nom d'hydatides; en effet, ce sont tantôt des vaisseaux sanguins et lymphatiques variqueux, tantôt des distensions du tissu cellulaire, et tantôt une toute autre désorganisation, qui produisent cette forme vésiculaire.

Le docteur Rust m'envoya, il y a quelque temps, un testicule avec le cordon spermatique, qui étaient gonflés d'une manière extraordinaire, et qui étaient remplis de semblables distensions vésiculaires.

Le docteur Schiffner a trouvé, dans lecadavre d'une femme, les deux reins gonflés d'une manière contre nature; il n'y avait plus de trace de la véritable substance des reins : l'un et l'autre ne formaient qu'un aggrégat de cellules ou de capsules, qui contenaient uno matière gélatineuse de différentes couleurs.

- Thes. anat. sext., N. xi, not. 1. Ibid., N. cIv, not. - Ads. anal., dec. IR, p. 24. 
Le chirurgien Rollet, a Baade, a observé une semblable difformité dans les reins d'une femme que l'on avait trouvée morte dans la rue. Excepté la désorganisation des reins, il n'y avait ancune autre anomalie sur le reste du corps; il n'y avait non plus aucune trace de lésion à l'extérienr.

Cependant ni les distensions vésiculaires dans le testicule et le cordon spermatique, ni celles des reins, dont nous venons de parler, n'étaient de véritables hydatides; car elles étaient fortement adhérentes à l'organe, ce qui n'a pas lieu pour les vers vésiculaires. On en a réellement trouvé dans le plexus choroïde dn cervean; mais, d'un autre côté, il fauı remarquer que l'on a aussi très-sonvent pris des vaisseaux lymphatiques variqueux pour des hydatides, ce que Baillie a déjà justement remarqué.

Notre collection possède deux semblables plexus variquenx.

J'ai trouvé, il y a quelque temps, un véritable cys. ticerque dans le plexus choroïde d'un singe ( simia cephus).

Le nom d'hydatide devrait seulement être donné, dans mon opinion, à une vessie (n'importe dans quelle partie du corps animal elle se trouve) qui est remplie d'un liquide aussi limpide que l'eau, ou bien d'une matière plus épaisse, complétement enfermée dans une capsule; mais où elle se trouve librement, c'est-à-dire sans avoir aucune adhérence avec les parois internes de la capsule. Cette dernière forme une partie intégrante de l'organe dans lequel 
elle est placée, de la mème manière que le cristallin l'est dans sa capsule.

La nature animale de ces hydatides enfermées dans des capsules particulières, comme nous venons deles décrire, a été découverte, vers la fiu du dix-septième siècle, par Hartmann, Malpighi et Tyson'. L'ou pent très-bien accorder à tous les trois l'honneur de cette déconverte; car il est très-probable qu'aucun de ces trois auteurs n'avait eu connaissance de la déconverte des deux autres. Les mouvemens de ces vessies, observés par ces trois naturalistes, les avaient conduitsà penser que c'étaient des versou de véritables aninaux doués d'une vie individnelle. Cependant ils n'avaient pas découvert leur extrémité céphalique.

Ce sont Pallas et Goeze qui ont les premiers démontré d'une nanière évidente l'existence de la tête de ces animaux. Nous devons égalemeut à ces deux auteurs une description claire, nou-seulement des cysticerques, ou des lydatides ordinaires, mais encore des polycéphales (ccenurus, Rud., polycephalus, Zed.) et des échinocoques.

Les hydatides sont ordinairec:-at remplies d'une liqueur limpide, mais elles contiennent aussi, daus quelques cas, une matière plus épaisse, et se transforment même quelquefois en une substance compacte; quand cela a lieu, on doit alors regarder l'hyda. tide transformée comme étant dans un état morbifique. J'ai eu souvent occasion d'observer dans les viscéı es,

'Lumbric. hydropic. 


\section{$27^{3}$}

principalement dans le foie des animaux bisulques, les désorganisations graduelles des hydatides, des cysticerques et des échinocoques. Ces désorganisatious s'opèrent de la manière suivante.

D'abord le liquide limpide commenee par perdre sa transparence, et la vessie, auparavant trèsdistendue, s'affaisse; le liquide s'épaissit de plus en plus, il devient jaunâtre et ressemble à du fromage mou. La vessie se ride, enfin la substance ancienuement liquide se durcit complétement. Quand on observe une semblable dégénération dans son origine, l'on trouve encore quelques traces oú parcelles de la vessie ridée. A la fin elle disparaît, et l'hydatide se transforme entièrement en une masse calcaire, que l'on pent quelquefois détacher, aussi facilement que l'hydatide saine, de l'organe dans lequel elle se trouve. Cette masse calcaire est aussi recouverte d'un épiderme particulier.

J'ai souvent rencontré dans le foie des bœufs à côté des hydatides compléternent développées et saines, tous ces degrés de désorganisation. L'hydatide saine, remplie d'un liquide limpide, forme à la surface de l'organe dans lequel elle séjourne, une protubérance convexe et élastique; mais si au contraire cet animal s'est déjà changé en une masse ossiforme, l'on trouve alors une dépression entourée de rides.

Ruysch avait déjà connu tontes les désorganisations dont cette espèce de vers est susceptible, et cet auteur ne doutait pas que ces animaux ne pus- 
sent se changer en athérome, stéatome, et mélicéris'.

J'ai toujours peusé, avant de connaître l'opinion de Ruysch, que les athéromes n'étaient que des hydatides désorganisées

Dehaen ${ }^{3}$ a trouvé dans une glande thyroïde d'un volume énorme, à côté d'hydatidès véritables, différentes espèces d'engorgemens. Je présume que ces derniers n'étaient que des hydatides dégénérées, comme j'ai eu souventoccasion dele voir, je le répète, chez les animaux. Les obstructions au foie observées par Mekel devaient aussi sans doute leur origine à des vers de cette espèce.

Les dissertations de M. Laënnec et de Ludersen sur ces animaux méritent d'être lues. M. Duméril a eu la bonté de me prêter la dissertation du premier, lors de mon séjour à Paris; j’en ai extrait plusieurs notes; les ayant perdues cn grande partie, je ne

${ }^{5}$ Dilucidat. valv. in vas. lymph., Obs. $\mathrm{xxr}$, p. 25 .

2 Dans le Journal de médecine de M. Horn (Archius fiir med. Erfahrung, iulius und august 1821 ), il est fait mention d'une observation très-remarquable d'une ossification de l'ovaire; c'était le docteur Behniz , à Lignitz, en Silésie, qui l'avait communiquée. La personne dans laquelle on avait tronvé cette désorganisation avait reçu de fortes contusions sur le bas-ventre à l'âge de douze ans, néanmoins elle a vécu jusqu'ì l'âge de soixante-quatre ans. Les prétendues dents que l'on a tronvées dans cet ovaire ossifié me paraissent être des hydatides dégénérées. Voyez également The american medical recorder, rol. III, p. 491-498; le même Journal, p. 37 I. Le ver dont il y est question n'était sans doute qu'une larve de mouclie. ( $\mathrm{Br}$.)

${ }^{3}$ Ratio medendi, vir, p. $\mathbf{1 3}$. 
pourrais en aucune manièe m'en servir avec ceriilude.

La dissertation de Ludersen m'a été communiquée par Osiander.

Jusqu'à présent l'on n'a trouvé dans l'homme que deux espèces de ver's vésiculaires, savoir les cysticerques (cysticercus), et les échinocoques (echinococcus).

\section{Cisticerque du Tissu celluialfe.}

Cysticercus.cellulosa, R. (en allen1. die finne oder der blasenschnanz), pl. vill.

CXSTICERCUS : capite tetragono; rostello terete uncinato, collo brevissimo; corpore cylindrico longiore, vesica caudali elliptica, transversa. Gmelin, Syst. nat. p. $3 \circ 59, \mathrm{n}^{\circ} .6$, tania cellulusa; p. $3063, \mathrm{n}^{0}$. 27, toenia finna.

Jœrdens, Helminith., s. 57 , t. v, fig. 12-16, tonia muscularis, seu finna humana; der muskelblasenwurm, s. 39, t. v, fig. I 7-2 I. tania pyriformis; der birnfarmige blasenwurm, s. 6r , tal. v, fig. I-2, tonia albopunctate; der sxeisspunctirte blasenwurm.

Brera, Vorlesung., s. I4, tab. II, fig. 89 , tab. III, blasenwurm. Memorie du même, p. 130 , tab. In, fig. 5 , fischiosonı globoso ; p. I38, fischiosoma pyriforme; p. 153 , tab. II, fig. I I-13, tab. III, fig. 6-1o, fina muscolare.

Zeder, Anleitung, p. $407, \mathrm{n}^{0} .2$, cystic. finna; p. $414, \mathrm{n}^{0} .6$, cystic. pyriformis; p. $42 \mathrm{I}, \mathrm{n}^{0} .2 \mathrm{I}$, cystic. albopunctatus.

Rudolphi, Entozcolog. p. 226 , cystic. cellulosce

De Lamarck, Anim. sans vert., t. III, p. 154 , hydatide lancéolée.

Ces vers séjournent dans le tissu cellulaire des muscles et du cerveau; on les observe souvent dans.

'Ce dessin n'est qu'une copie de la vingt-septième figure de Hartmann. 
les cochons, mais non pas dans les sangliers. On les trouve rarement chez l'homme; on les a également rencontrés dans les singes.

J'ai déjà remarqué que j'ai trouvé moi-même un cysticerque chez un animal de ce genre.

Comme j'ai fait dessiner un de ces vers avec la plus grande exactitude, non-seulement dans sa position et sa grosseur naturelles, mais encore sur une ćchelle beaucoup plus considérable que nature; il m'a scmblé qu'une explication détaillée des figures serait plus instructive que toute autre description qui en serait privée. La figure 1 a représente un morceau de muscle dans lequel la capsule contenant le cysticerque est fixée. La figure $1 b$ représente un petit morceau de graisse sur lequel le ver est placé, et la figure $1 c$ fait voir le ver avec sa capsule. Cette dernière n'a pas appartenu, d'après mon opinion, au ver, mais bien à l'organe dans lequel il avait séjourné; car on observe des vaisseaux qui passent à la surface de cette capsule. En général, on ne peut en effet séparer cette dernière qu'après avoir coupé ou déchiré les fibres par lesquelles elle est fixée à l'organe ou aux muscles qui l'entourent.

La formation de la capsule s'opère probablement par l'irritation que la présence du ver cause à l'endroit de l'organe dans lequel il se développe. Cette formation de la capsule par l'irritation du ver (supposé qu'elle se développe ainsi) ressemble à celle de la noix de galle.

Eṇ ouvrant la capsule, le ver, qui y est placé li- 
brement, se présente, comme cela se voit dans les figures $1 d$ et $1 e$. L'intérieur de la capsule est lisse et contient un peu de liquide. Aussitôt que le ver se présente hors d'elle, il rétracte ordinairement la tête, le cou et même le corps dans la vessie. L'on reconnaît le siége de ces parties par une tache qui est blanche comme du lait, opaque et un peu dure au toucher. En pressant la vessie avec les doigts ou au moyen de l'instrument de Goeze, dont je ne me suis jamais servi, l'on peut forcer le corps, le cou et la tête à sortir de la vessie; mais, en employant ces moyens, on déchire toujours la vessie du cysticerque; car celui-ci, en se rétractant, prend une forme plus ou moins ovalaire, et les différentes partics de son corps occupent alors le diamètre transversal de la vessie ( Voyez figure $1 f$ ). Pour éviter cét inconvénient, on fera mieux de mettre les vers extraits de leur capsule daus de l'eau tiède, et en tâchant d'entretenir ce liquide, pendant quelque temps, à la même température, il arrive alors que l'un ou l'autre de ces cysticerques fait sortir spontanément la tête et le cou, comme cela se voit sur la figure $1 \mathrm{~g}$. Le ver représenté sur la figure $1 f$ avait déjà le cou allongé; mais aussitôt que l'eau commença à se refroidir, il se rétracta. Les personnes qui ont la vue bonne remarcjuent, même à l'oeil nu (sur un individu complétement développé, et tel qu'il a été représenté fig. $1 \mathrm{~g}$ ), la tête, le cou très-court, le corps ridé et la partie improprement appelée vessie transparente. Je dis improprement appelée, par la raison que ce n'est en effet qu'une 
continuation distendue du corps ridé, ayant la forme d'une vessie. Cela peut surtout clairement s'apercevoir dans le cysticercus fasciolaris ${ }^{x}$ provenant du foic des souris, et qui a probablement donné lieu à la dénomination de tout ce genre de vers. Ce ver vésiculaire, souvent de la longueur de quatre à cinq pouces, et de la largeur d'une à deux lignes, est presque ridé d'un bout à l'autre, et pourvu d'une très-petite vessic à l'extrémité postérieure. Quelquefois cet animal est tellement aplati, et les rides sont souvent rapprochées les unes des autres si régulièrement, que l'on serait porté de regarder ce ver comme étant récllement articulé, si l'on ne voyait pas quelquefois, même au milieu de ces articulations apparentes, des endroits qui sont tout à fait boursoufflés, et qui servent en mêmc temps à prouver que la vessie et le corps sont formés de la nuême membrane. Cette circonstance est cause que Brera a entièrement rejeté la dénomination de cysticerque. Je l'ai conservée $1^{\circ}$. parce que les meilleurs helminthologues l'ont adoptée; $2^{\circ}$. parce que l'on doit avoir différentes dénominations pour indiquer divers genres; $3^{\circ}$. parce que la dénomination de fischiosoma proposée par Brera s'adapte aussi bien que celle d'hydatis à toutes les hydatides; et $4^{\circ}$. parce qu'elle désigne l'ordre et non pas le genre. Le tablean suivant prouve que Brera s'est servi de la dénomiuation fischiosoma, aussi bien comme nom d'ordre que comme nom de genre ${ }^{2}$.

" Himly l'a appelé faussement douve du foie.

- Memorie, p. 8. 


\section{Ord. II, Fischiosomi (vermi vesicolari).}

Gen. I, Eremili.

Spec. I, fischiosoma globoso.

Spec. II, fischiosoma pirifurme.

Spec. III, ditrachierosoma.

Gen. II, sociali.

Spec., fischiosoma policefalo.

Gen. III, capsolari.

Spec. 1, fina misscolare.

Spec. II, fina epatica.

Spec. III, fina viscerale.

Spec. IV, fina idaloide.

Il résulte de ce tableau qu'il y à, selon Brera, hui différentes espèces d'hydatides dans le corps humaiu. Cependant, dans la grande quantité d'animaux de différentes classes, ordres et espèces examinés par moi, je n'ai peut-être pas trouvé eu tout autant d'espèces d'hydatides, différentes les unes des autres par des signes caractéristiques particuliers; aussi je prends la liberté de douter de l'énumération de Brera.

L'on peut clairement voir , sur la figure $1 h$, les différentes parties de l'extrémité céphalique très-grossies. Au-dessus de quatre suçoirs, sort une protubérance tantôt plus courte, tantôt plus longue ou plus ou moins conique, selon qu'elle est plus ou moins étendue. Cette protubérance est pourvue, dans son milieu, d'une double couronne de crochets. L'on 
voit un de ces derniers considérablement grossi dans la figure $1 i$; le cou et le corps, de même considérablement grossis, sont représentés sur la figure $1 h$. La figure $1 g$, qui fait voir la vessie caudale dans sa grosseur naturelle, est dessinée de manière à donner une idée très-claire de cette partie du ver, et il m'a semblé inutile de la faire représenter sur une plus grande échelle que nature.

A l'exception de Treutler et Brera, aucun des anteurs qui ont donné une description de ce ver, n'a fait dessiner en entier, autant du moins que je me le rappelle, la vessie de la queue; et ce que Zeder dit, $\$ 366$, corfernant les hydatides, est tellement obscur et inintelligible, que celui qui n'a pas étndié luimême l'économie de ces animaux pourrait être porté à croire que le ver est attaclié à la capsule par la vessie de sa queue; cependant cela n'a pas lieu, et le ver, y compris sa vessie, se trouve au contraire libre dans la capsule. Les passages suivans, que l'on trouve dins l'ouvrage de Zeder, comme, par exemple, "lesvers s'enfoncent dans la vessie", ou bien "les vers sortent de la vessie de la queue ", n'ont nullement un sens clair, parce qu'ils peuvent donner lieu à faire croire, à un homme peu instruit sur celte matière, que le ver et la vessie sont deux choses différentes. Quand on veut parler des mouvemens de cet animal, il faut s'exprimer ainsi : La tête et la partie antérieure du ver se rétractent dans la vessie caudale; car la vessie elle-même appartient au corps de l'animal, et en effet on voit seulement qu'une partie du 
ver s'enfonce ou se rétracte dans l'autre. Cette contraction s'opère de la manière suivante: le pointle plus saillant de la protubérance, qui se trouve au milieu de la couronne de crochets, s'affaisse en premier lieu; la couronne de crochets suit après; les suçoirs rentrent plus tard, età la fin toute la partie ridée du corps, de manière que lorsque celui-ci est entièrement contracté, la tête est la partie qui se trouve la plus profondément située dans la vessie caudale. Quand la tête veut sortir, les parties mentionnées se présentent dans un ordre inverse. Lues plis du corps paraissent d'abord; viennent après les suçoirs, la couronne de crochets, et à la fin la protubérance conique, qui , quand elle n'est pas entièrement sortie, a sonvent l'air de former une fossette. La figure if donne une idée assez claire de la contraction et de l'extension du ver. Du reste, le mécanisme de la contraction du ver ne peut être mieux comparé quà la rétroversion d'un doigt de gant, oì la partie pointue se trouve rentrée la première; et, en la repoussant, elle sort la dernière.

Les cysticerques, observés chez les cochons depuis long-temps, ont été découverts dans l'homme, pour la première fois, par Werner ${ }^{5}$. Il les a rencontrés dans le cadavre d'un soldat de quarante ans, qui avait péri dans l'eau. Cet auteur rapporte que presque tous les muscles de cet homme contenaient de ces vers.

"Verm. intest., cont. II, p. 7 . 
Fischer " , après Werner, en a trouvé vingt-trois dans les deux plexus choroïdes du cerveau d'un jeune homme. Ces vers étaient fortement attachés à ces plexus, et Fischer prétend qu'il n'a pas observé sur eux de vessie extérieure. M. Rudolphi a répondu à cela que le tissu cellulaire est extrêmement fin à l'endroit où ces animaux se trouvaient, et c'est probablement la raison pour laquelle Fischer n'a pas remarqué la vessie extérieure.

Quant à moi, je suis de l'opinion que les vers, ayant été mis dans l'eau tiède, se seront gonflés au point de faire crever la vessic, probablement trèsfine, et qu'clle se sera rétractée ensuite de manière à ne pouvoir plus être apercue.

Cependant il est de fait qu'il y a quelquefois des cysticerques qui se trouvent placés librement dans de grandes cavités du corps animal, comme j’ai eu occasion de l'observer daus la cavité thorachique des campagnols.

Dans le cadavre d'une femme morte par suite d'une hydropisie, Treutler ${ }^{2}$ a remarqué dix-sept cysticerques dans les plexus choroïdes, quinze d'un côté et deux de l'autre. Cet auteur les appela tania albopunctata, et il croit qu'ils différaient, par leur structure, de cenx rencontrés par Werner et Fischer. Cependant M. Rudolphi peuse, et même avec raison, que c'étaient des vers de la même espéce;

${ }^{2}$ Tarn. hydatis., p. 28.

'Ouvrage cité, p. I. 
du reste, il n'est pas probable que ceux trouvés par Treutler n'eussent qu'un seul suçoir et qu'une couronne simple composée de six crochets. Il paraît plutôt que leur tête n'était pas entièrement développée : cette seule observation, par conséqueut, ne nous mettrait pas eu droit d'admettre qu'il y a dans le cerveau une secoude espèce de cysticerg̨ues d'une structure différente.

Brera a également trouvé des individus de cette espèce daus le plexus choroïde, et Steinbuch en a rencontré vingt-cinq daus les muscles du cou et du dos.

Loschge a encore observé quelques individus dans le plexus choroïde du même cadavre que Steinbuch avait déjà examiué.

Isenflamm a remarqué un individu de cette espèce sous l'aisselle d'un autre cadavre.

Himly a reucoutré des cysticerques nou-seulement daus les muscles, mais evcore dans plusieurs viscères d'un cadavre humain; il y en avait en effet à la surface du cerveau, quelques-uns étaient suspendus à la pie-mère, et enfin d'autres étaient enfermés dans le cerveau lui-même; un seul iudividu fut remarqué dans les poumons: le foie, la rate et les autres viscères n'en contenaient point. Le niême Himly a encore trouvé des cysticerques sur quelques muscles d'un autre cadavre.

M. Rudolphi m'a fait savoir, par une lettre qu'il m'a adressée, que jusqu'ici, chaque hiver, il a observé quelques vers de cette espèce à l'amphithéâtre de Berlin. 
$J$ 'ai fait mes effor!s depuis dix ans, mais en vain, pour m'en procurer dans le grand hôpital de Vienne et dans l'amphithéâtre anatomique de la même ville. Notre collection doit plusieurs individus à la bonté de M. Rudolphi.

Les ohservations qui ont été faites jusqu'à présent sur les cysticerques ne mous ont pas encore fourni de certitude sur les symptômes d'après lesquels on peut présumer leur présence, et elles ne nous ont pas fait connaître non plus les accidens qu'ils peuvent occasiouer pendant la vie. J'indiquerai ici brièvement quelques renseignemens que l'on a recueillis sur cette espèce de vers.

Werner raconte que le sujet dans lequel il en a tronvé était un homme fort et robuste.

Fischer ' rapporte qu'un de ses amis, mort par suite d'une fièvre maligne, était très-porté à la mélancolic; quelques aunées avant de mourir, il se plaignait souvent d'une fatigue et d'une pesanten. dans les membres; et quoique Fischer ne veuille pas décider que ces accidens aient été réellemen sionés par la présence des cysticerques, il remarque cependant qu'il les a justement trouvés dans les parties qui avaicnt fail lc plus souffrir cet homme pendant sa vie.

Treutler a examiné le cadavre d'une femme qui avait succombé par suite d'une hydropisie, et qui avait éprouvé, pendant long-temps, beaucoup d'accidens très-graves. On était d'avis qu'ils provenaient

$\therefore$ Const. 15, p. 47 . 
d'une affection de la tête. Outre les cysticerques que l'on a tronvés dans les plexus choroïdes, on a remarqué dans le cerveau une désorganisation d'une étendue considérable; il y avait en même temps des excroissances osseuses d'un volume énorme à la base du crâne.

Brera a observé des cysticerques dans le cadavre d'un homme de cinquante-cing ans, mort par suite d'aploplexie; les ventricules dn cerveau étaient remplis d'une sérosité sanguinolente.

Treutler en a rencontré dans le cadavre d'un homme de soixante-cinq ans, qui était mort de consomption.

Les cysticerques décrits par Himly provenaient des muscles, du cerveau, et des poumons d'un hommequi avait succombé à la suite d'un cancer au visage; cependant cet auteur a examiné d'autres cadavres de personnes mortes de la même maladie, mais elles n'avaient pas de traces d'hydatides. Celles que Himly a vues dans une seconde observation provenaient d'une personne qui n'avait pas non plus succombé par suite d'un cancer, mais bien par celle d'une toute autre maladie; et les accidens qu'elle avait éprouvés avaut de mourir ne pouvaient pas être attribués, avec certilude, à la présence des hydatides.

Le singe dont j’ai parlé plus haut, et dans lequel j’ai rencontré quelques vers de cette espèce, avait souvent des accès convulsifs, et mourut un jour snbitement.

Comme on sait si peu de choses sous le rapport de l'étiologie, de la pathologie et du diagnostic sur les 
cysticerques, on peut encore moins dire quelque chose de positif sous le rapport thérapeutique.

Les cysticerques, dans le premier cas observé par Hinıly, se sont fait remarquer, à l'extérieur, sur la poitrine et le bas-ventre, par des espèces de boutons . de la grosseur d'une lentille.

Si un homme se présentait chez moi avec de semblables boutons, je tâcherais d'abord de m'assurer, par une incision, si j'ai réellement à combattre de véritables cysticerques. Convaincu de leur existence, je n'ordonnerais cependant rien autre chose qu'un changement de régime ${ }^{\mathrm{x}}$; car j'ai raison de croire que ces vers se détruisent souvent d'eux-mêmes. La dégénération d'hydatides dont j'ai parlé plus haut, me le fait également présumer; car on peut très-bien conclure de l'étendue des rides du foie qui entourent les cysticerques transformés en une masse calcaire, que le volume de ces animaux a dû égaler au moins celui d'une noix, à l'époque de leur distension la plus considérable. Cette dégénération du ver, qui est sou-

'Je crois que ce serait ici le cas d'employer les vermifuges à l'extérieur sous forme de frictions. Ėn effet, l'expérience ayant prouvé que ces médicameus administrés de cette manière agissent sur les vers qui séjournent dans le canal intestinal, à plus forte raison doivent-ils se montrer encore plus efficaces sur ceux qui se trouvent sous les tégumens. Il me paraît que les huiles empyreumatiques, par exemple, employées en frictions, devraient être d'une grande utilité; il serait seulement à craindre que leur mauvaise odeur n'empêchât beaucoup de personnes d'en faire usage. (Note du trailucleur. 
vent telle qu'il se trouve réduit, probablement an moyen de la résorption, à n'avoir plus que la grosscur d'un pois, nous met en droit de croire que tout le reste de celte masse calcaire peut être entièrement résorbé.

I. fe fait suivant vient encore à l'appui de ma supposition, que les cysticerques disparaissent quelquefois d'eux-mêmes.

Pour obtenir ces animanx en grande quantité, dans le but d'étudier leur écop̣nomie aussi exactement que possible, on avait donné ordre aux experts pour la vente des animaux, il y a à peu près quinzc aus, d'acheter, pour lc compte du cabinet impérial d'histoire naturelle, un cochon qui se trouverait rempli de cysticerques; en effet on nous envoya un individn qui avait été censé en contenir une grande quantité. A vaut de faire iuer cet animal, nous le nourrimes pendant quelque temps (probablement avec des substances autres que celles qu'il avait maugées auparavant) dans l'intention de faire augmenter le nombre de ces vers; mais nous fùmes trompés dans notre attentc, car au lieu d'en rencontrer un nombrc considérable, nous ne trouvâmesque douze ou quinze individus.

Il reste à savoir si les experts ne s'étaient pas trompés, et si cet animal en avait réellement un grand nombre; ou bien si les cysticerques n'avaient pas disparu spontanément, peut-être par le changement de nouriture.

Outre le cysticerque dont nous venons de parler, Gmelin (Syst. nat., p. $5059, \mathbf{n}^{\circ} .5$, tonia visceralis), 
Treutler (Observat. path.-anat., pag. 14, fig. 1 et 4), Jardens (Helminth., pao. 56, tab. v, fiğ. 8 et 11), et Zéder (Anlcit., s. 418, n. I I), font encore mention de cysticerques viscéraux. M. Rudolphi remarque très-justement que les observations de ces auteurs se rapportcnt ou aux hyclatides en général, que l'on a trouvées dans les animaux, ou bicn à d'autres vers, que l'on doit compter parmi les échinocoques.

La fina epatica de Brera est peut-être, d'après inon idće, un cysticerque viscéral, si tontefois c’est atutre chose qu'un cysticerque celhleux. M. Rudolphi ne veut pas cependant nier l'existence d'un eysticerque viscéral dans l'homme, et je n'ai rien non plus à objecter contre clle. J'engage les naturalistes, en cas qu'ils aient occasion d'observer un de ces vers, a indiquer exactement les caractères par lesquels il se distingue, principalement à l'extrémité céphalique, des autres espèces de ce genre; car tous les cysticerques se ressemblent surtout par la forme de la tète, qui est constante; la forme de la vessie caudale n'est qu'accidentclle, par la raison que sa structure dépend, dans mon idée, de l'organe dans lequel le ver se dé-. veloppe.

XII. ECHNoconie DE T'UOML.

Echinococcus hominis (en allem. der hülsenisurm), pt. vir!, fig. 2-2è.

Jordcns, Helminith., s. 62, tab. vil, fig. 2 e et 23 , dermenscherbvielloopf, polyccphalus hominis. 
Zeder, Anheitung, s. 43r, no. 2, tab. Iv , fig. 7-8, polyceph. humanus; s. $432, n^{\circ} 6$, polycephal. echinococcus.

Drera, Memorie, p. 149, fischiosoma policefalo; p. 164, tab. Iu, fig. I-3, fina idatoide.

Rudolphi, Entoz., 11, 2, p. 247, tab. xı, fig. 4, echinococcus humanus.

De Lamarck, Anim. sans vert., tom. I11, p. 157, l'échinocuque de lhomme.

M. Rudolphi distingue les hydatides en vivantes et en non vivantes; il regarde l'échinocoque provenant des intestins des bisulques (echinococcus vetcrinortan ) comme une hydatide vivante, par laraison que l'un trouve dans le liquide qu'elle contient les échinocoques proprement dits, c'est-à-dire de petits corps microscopiques, pourvus de quatre suçoirs et d'une couronne de crochets. Ainsi il paraît que Rudolphi ne regarde pas la vessie dans laquelle ces petits corps sont contenus comme un véritable animal; à plus forte raison il ne doit pas non plus regarder toutes les autres vessies, qui ne contiennent point de petits corps pourvus de suçoirs et de couronnes de crochets, comme de véritables animaux; cependant comme ces vessies 'ressemblent, sous tous les rapports, aux échinocoques, je me crois pour celte raison en droit de regarder aussi comme de véritables animaux toutes les hydatides qui, selon la définition ci - dessus établie, sont contenues librement dans des capsules particulières, sans être adhérentes ni à celles-ci, ni à l'organe dans lequel elles se trouvent. 
Home croit que l'hydatide sphéroidale est l'animal le plus simple, et qu'elle est pour ainsi dire tout estomac.

Himly est l'auteur dont la manière de voir à ce sujet me paraît atteindre le plus directement le but. Voici ses propres expressions: "On trouva des vésicules isolées (hydatis simplex) flottantes et libres : plusieurs médecins les regardèrent comme de simples dilatations de vaisseaux lymphatiques. Cette hydatide est en effet un être extrêmement simple et peut-être le plus simple de tous les animaux; je dois cependant la considérer comme un animal parce qu'elle vit, puisqu'elle ne pourrit pas et qu'elle n'a aucune adhéreuce avec le corps de l'homme ou de l'aninal dans lequel elle s'est produñte : ainsi les humeurs n'étant pas douées de vie, elle doil être regardée comme un: ĉtre vivani individuellement, et par conséquent comme un animal particulier. On ne doit pas s'attendre à ce qu'elle soit pourvue de tête, de bouche ou de quelque organe analogue, et encore moins qu'elle soil douée d'intelligence. La lymphe épanchée entre deux parties d'un animal, par exemple entre les surfaces enflammées, et par conséquent plus aclivement productrices du poumon et de la plèvre, reçoit aussi bien des vaisseaux sanguins que les parties avec lesquelles s'établit l'adhérence, tandis que Ja même substancc animalisée sans communicatiou ou adhérence avec les parties du corps dans lesquelles clle s'est produite, et par conséquent individuelle, n'a pas la nature des animaux parfaits à sang chaud, 
mais constitue un animal aquatique ${ }^{x}$ Ces lyydatides sont, lorsque leur enveloppe est un peu épaisse, d'une substance semblable à celle du cristallin, et lorsqu'elle est plus épaisse, elles présentent alors l'aspect d'une membrane cartilagineuse, transparente et incoinplétement incolore, tirant un peu sur la couleur de l'opale, et qui s'affaisse quand on vient à l'inciser. Par l'analyse chimique elles se comportent complétement comme les substances animales; ainsi ce sont des individus particuliers vivans qui n'ont rien du tout de commun avec les plantes, ce qui nous oblige de les regarder comme des animaux, d'où résulte que la définition ordinaire de l'animal, tirée de la présence d'une bouche et de la faculté d'y introduire la nourriture, de son propre mouvement ${ }^{2}$, est

3 " Le développement de la vie individuelle dans ce cas-ci n'esi pas pour nous un plus gुrand problème que le déreloppement d: la vie en général, qui restera toujours pour nous insoluble. Je prie de croire que je n'ai pas la prétention de voulcir le résoudre; j'observe senlement que l'on ne doit pas s'imaginer ètre beaucoup avancé dans son explication en admetłant que la vie se developpe par le moyen d'un cuf. Je suis étonné de ce que M. le professcur Oken, qui n'aime pas cependant à être gêné par dles entraves quelconques dans sa manière de penser, ait pu, dans son ouvrage sur la génération (die zelızung), revenir sur les monades, qui ne seraient pour ainsi dire que des ceufs primitifs, ce que l'on n'a pas besoin d'admettre, à moins qu'on ne veuille supposer que l'esprit pri : mitif soit mort, et qu'il ne vive que par ses créations. ”

2 Blumembach dit seulement dans son onvrage ayant pour titre: Handbuch der Naturgechichte 8te auflase, Gottingen, 1807 , 
beaucoup trop restreinte. Quelques-unes des hydatides splséroïdales contienneut cncore une autre membrane plus mince qui offre épars cà et lia, et le plus souvent par groupes, des corpuscules l'uve apparence glanduleuse; enfin il est des lydatides sphéroïdales qui en renferment d'autres plus peties, et qui ainsi réalisent, ponr ainsi dire, le système d'emboitement. Toutes, contenantes et contenues, se sont-elles formées à la fois? on bien la plus grosse a-t-elle produit la plus petite? on peut-être enfin les corpuscules d'apparence glanduleuse se comportent-ils comme les gemmules sphéroïdanx de la conferve des fontaiues, qui se détachent et vont former de nourelles conferves. "n

Cette derniẹre opinion me paraît la plús probable, comme il résultera encore davantağe, ì ce que je crois, de l'explication des fisures; les liydatides représentées dans la planche 8 provenaicht ioutes de la mêne poche d'un volume énorme qui s'était formée dans le foie d'une femme que l'on avait amenée mourante à l'hôpital de Vicune. Toutes les hydatides se trouvaient placées librement dans cette poche, très-lisse dans son intéricur. La figure $2 a$ représente une des petites hydatiades, mais uon pas lit plus petite, et les figures 2 et $2 e$ représentent deux individus un peu plus gros. En regardant la figure $2 e$ on est

p. 36, "Ces animaux paraissent awn: lous une bouche" Mais roume it adinc à la mềne nage une exception pour les infusoires, les lydatides ne pournient-clies pas être regartécs seneme tids? 
porté à croire qu'un petit ver est contenu dans un autre plus grand. Cependant il n'eu est pas ainsi; car la niembrane interne du ver (cet animal est composé de deux membranes) s'était seulement détachée et rétractée de l'externe; cela est prouvé par les deux figures 2 et $2 e$, qui ont été faites d'après le même échantillon. Isa figure 2 fut dessinće aussitôt que le ver cut paru hors de la poche, il fut placé dans l'eau, et le lendemain matin la membrane iuterne s'était détachée, comme cela est indiqué dans la figure $2 e$. Ces vessies avaient l'air d'être couvertes de granulations très-fines qui, représentées sur une échelle plus grande que nature, ressemblaient à de petites boules transparentes (Voy. fig. 2c). Quelquefois lcs petites boules contenues dans celles d'un plus grand volume sont assez volumineuses pour être aperçues à l'oeil nu, comme cela se voit dans la figure $2 b$. La figure $2 c$ en représente une très-grossic. En supposant que l'hydatide représentée dans la fig. 2 était déjà le produit d'une autre hydatide plus grosse et plus àgée, comme je vais le démontrer tout à l'heure, il faudrait par conséquent considérer les petites boules contenues dans l'hydatide ( fig. $2 c$ ) comme provenant de la troisième génération, et celles représentées dans la figure $2 b$ comme provenant de la quatrième génération, à partir de l'hydatide primitive '

Voyez Meckels, Archiv. fiir physiologie, vol. vi, heft. 2. Etwas iiber den echinococcus, Rud. par le docteur Bremser; voyez Mrcckels, Archiv., vi, heft. 4. Jaegers Beobachlung ïbier déll hillsenwurm. (Br.) 
J'ai remarqué plus lıaut que les échinocoques de l'homme ressemblent, sous tous les rapports, à ceux que l'on rencontre dans les intestins des bisulques. Je vais maintenant tâcher de prouver cette assertion.

L'on observe souvent, surtout dans le foie des bisulques, des échinocoques très-volumineux, qui nut quelquefois une forme irrégulière. Ces animanx sont remplis, dans l'état sain, d'une liqueur limpide, qui dépose cependant une matière trouble. Si l'on place une goutte de cette matière sous un microscope, on y voit nager une grande quantité de petits corps de différentes forme et structure; i] y ell a qui sont ronds, ovalaires, presque cylindriques, cordiformes, claviformes, et d'autres sembleut être divisés en deux parties d'une struclure différente. L'on remarque clairement sur quelques-uns de ces corps quatre sucoirs et une couronne de crochets. On voit encore nager dans cette matière une grande quantité de petits crochets détachés, absolument de la même forme que celui représenté dans la figure $1 i$ et qui provient d'un cysticerque. Ces faits nous prouvent jusqu'à l'évidence, que les crochets se détachent. Les suçoirs disparaissent également, et les petits corps de tant de formes différentes prennent avec le temps celle de petits globules lisses. J'en possède, qui sont de la grosscur des graines de coquelicots. Aussi long-temps que les choses restent dans cet état, la mère hydatide se laisse encore facilement séparer du sac daus lequel elle est contenue; ou plutôt elle sort d'cllcmême si on fend ce dernicr avec précaution au moyen 
d'une incision d'une étendue convenable ; mais quand ces animalcules, transfor'més eux-mêmes en hydatides, commencent à se développer davantage, l'hydatide primitive devient adhérente au sac dans lequel elle est contenue, et elle ne s'en laisse plus séparer ; en ouvrant un de ces sacs on voit sortir librement au deliors ces petites hydatides, et on trouve déjà dans leur intérieur les animalcules de différentes formes, dont nous venous de parler; mais comme leur nombre dans une vessie est très-considérable, ils n'y trouveraient pas assez de place, si tous arrivaient à leur développement complet; il me paraît alors probable, que les animalcules développés en premier lieu doivent empêcher complètement, ou au moins en partie, l'accroissement des autres; aussi voyons-nous également un grand nombre de ceux-ci dans un état de difformité. La figure $2 d$ nous en fournt un exemple; on y reconnaît facilement la forme sphérique primitive, et il est très-probable qu'une hydatide voisine volumineuse a empêché son développement complet. Dans quelques cas on n'observe pas dans les vessies une accumulation considérable de ces animalcules; dans d'autres, au contraire, ceux-ci se muliplient probablement trop tumultueusement, de manière que la mère hydatide tombe dans un état maladif, ou môme finit par en mourir; si cela a lieu, elle est alors soumise à tous les changemens mentionnés plus haut, et elle se dessèche à la fiu en une masse dure et calcaire.

La mème chose s'opère chez les hydatides que l'on 
rencontre dans l'homme; ì l'exception que l'on n'a trouvé jusqu'à présent, dans leur intérieur, que de petites boules lisses, et non pas encore des petits corps pourvus de suçoirs et d'une couronne de crochets. J'avoue que je ne me fie pas entièrement à l'observation faite par Goëze ', qui prétend avoir observé chez les hydatides que Meckel lui avait communiquées, de petits corps de forme olivaire, pourvus d'une simple couronne de crochets. Je crois que l'instrument de compression dont il se servait volontiers dans les observations de ce genre, a été cause de quelque illusion d'optique. J'ai également trouvédes corps d'une forme olivaire, dans des hydatides qui provenaient d'un homme ${ }^{2}$; mais ces corps n'étaient point pourvus d'une couronne de crochets : je dois ces hydatides à la lonté de M. Sommerring.

Néanmoins ces petites vessies se déreloppent, quoiqu'elles n'aient de communication dirccte avec ancun autre corps, absolument comme les hydatides des bisulques, et elles sont aussi sujettes aux mêmes désorganisations; nous devons, par cette raison, ranger les hydatides de l'homme et celles des bisulques dans le même genre. Peut-être trouvera-t-on les conronnes des crocliets et les suçoirs, quand l'occasion se présentera d'examiner une hydatide primitive. Ce qui m’a surtout engagé à ajouter foi à cette supposi-

Z Zeder nachtrag, s. 3 ro.

${ }^{2}$ De hydatidibus in corpore humano, presertim in cerebro repertis; Diss. anct. Car. Rendtorf. Berolini, 1822 , in-\$०. ( $P r$. 
lion, c'esı la circonstance que les petits vers provenant des échinocoques des bisulques de la seconde et troisieme génération, perdent plus vite leur forme particulière, et s'approchent de la forme globuleuse. Il me paraît également qu'aussitôt que l'acte de la génération se trouve une fois bien en vigueur chez ces animaux, le développement des petits de la troisième et quatrième géuération s'opère plus vite. 'Toutes les hydatides de l'homme, examinées par moi, provenaient, pour le moins, de la seconde génération. Je n'ai pas encore rencontré, dans les cadavres d'hommes, des hydatides primitives, c'est-àdire où une seule hydatide volumineuse remplissait un seul sac, ce que j’ai observé très-souvent chez les bisulques.

Il est probable que Félix Plater ${ }^{x}$ a vu des hydatides primitives daus le foie et dans le mésentère d'un homme; car il fait mention de vessies remplies d'une cau claire, qui étaient de la grosseur d'une pomme.

L'hydatide dont parle de Haen ${ }^{2}$ était aussi certainement une hydatide primitive. Cet auteur rapporte que le foie très-volumineux d'un homme de vingtquatre ans, était rempli de tumeurs qui ressemblaient à des squirres. On fit une incision sur une de ces tumeurs, et il en sortit environ une pinte de liquide; après sa sortie, on put facilement extraire la vessie affaissée; car elle n'avait pas la moindre adhé-

Ohs., lib. 111, p.6r7.

- Ratio meiendi, ru, p. 125. 
rence avec la concavité dans laquelle elle se trouvait; ainsi l'hydatide primitive, dans ce cas, était la senle qui s'y trouvât dans un état sain. Plusicurs des autres tumeurs en contenaient beaucoup, de grosseur différente; d'autres étaient remplies d'une matière grasse (crassa et pinguis amurca), et d'antres enfin contenaient une matière qui semblait être sablonneuse au toucher; il y avait par conséquent dans le même organe une mère hydatide, beaucoup d'individus de la seconde génération, et quelques-unes qui étaient désorganisées.

J'ai observé, il y a quelque temps, absolument la même chose dans le foie d'un chameau (camelus bactrianus, L.)

Comme on n'a pas encore constaté d'une manière positive la présence d'une couronne de crochets chez les échinocoques de l'homme, il me semble par conséquent, que le nom d'échinocoque n'est pas bien choisi pour cette espèce, en ce que ce ver est plutôt un liocoque (liococus); mais si l'on choisit cette dernière dénomination, on ferait alors des échinocoques de l'homme un nouveau genre, ce qui cependant ne conviendrait pas; je propose par conséquent d'appeler le genre splanchnococcus, une des espèces echinatus, et une autre lavis, jusqu'à ce que l'on ait découvert les couronues de crochets chez les individus de ce genre. M. Laënnec appelle le ver dont nous nous occupons maintenant, acephalocystis.

Excepté dans le canal intestinal, on a rencontré des hydatides dans tous les organes de l'homme; ce- 


\section{3o' SUR LES VERS INTESTINAUX}

pendant il est de fait, que beaucoup d'auterurs ont crit avoir observé ces animaux, et que ce n'était pas de véritables hydatides, je n'excepte pas même le cas observé par Kelch, et rapporté par Lüdersen. Parmi toutes ces liydatides, il paraît qu'il n'y en avail qu'une seule adhérente au foie, qui méritât d'être qualifiée comme telle. Je prie de croire que je n’ai pas relevé cettc erreur de Lïdersen dans l'intention de nuire à sa mémoire. Cet autcur a travaillé avec un zèle extraordinaire, et il a recueilli tout ce que les médecins ont publié sous le nom d'hydatis : du reste, dans sa classification des hydatides, il s'est amplement justifié de sa manière d'envisager ce sujet.

Les cas observés par Persius et Coiter ${ }^{\text {x }}$ ò̀ tous les organes de la poitrine et du bas-ventre étaient couverts d'hy datides, doivent être regardés comme conformes à la vérité.

Morgagni, dans sou ouvrage (De caus. et sedib. morb. ), rapporte qu'il a rencontré des hydatides dans le cerveau, dans la moëlle épinière, et leurs membranes; cet auteur a également trouvé celte espèce de vers dans d'autres cavités et d'autres parties du corps humain; plusieurs de ces animaux appartenaient sans doute au genre cysticerque; je possède aussi quelques échinocoques provenant de la glande pituitaire (glandula pituitaria) que je dois à la bonté de M. Sommerring; ces vers sont encore plus petits que les graines de senevé.

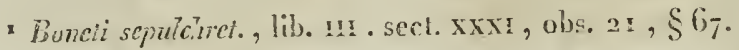


Morrach rapporte qu'une fille de seize ans avait toutes les trois semaines deux accès de syncopes trèsviolens; à la fin, elle perdit l'ouie, la vue et l'odorat; elle devint ensuite paralytique de tout le côté gauche; huit mois après, à compter de la première syncope, elle mourut d'une apoplexie; on trouva dans J'hémisphère droit du ccrveau une hydatide de la longueur de trois pouces et de la largcur de deux.

Adam Schmidt a observé une hydatide dans la glande lacrymale.

Morgagni' a trouvé un de ces vers d'un volume très-considérable, qui était adhérent au cœur. Le même auteur ${ }^{2}$ cite encore de semblables olsservations faites par Cordæus, Fontanus, Persius, et Ballonius.

Geoffroi rapporte qu'il a trouvé daus la cavité thorachique deux hydatides d'une grosseur extraordinaire.

Collet raconte qu'une femme de quarante-sept ans avait évacué, en toussant, depuis le 6 sepiembre ${ }_{177} 1$ jusqu'au $\alpha^{\text {er }}$ janvier $177^{2}$, cene trente-cinq liydatides de la grosseur d'un pois, jusqu'à celle d'un œuf de poule; toutes ces hydatides étaient déchirées. Cette femme avait également une tumeur dans la région du foie; il est possible que ces vers provinssent de cet endroit.

Le Journal dé médecine publié à Edimbourg ${ }^{3}$ fuit

$=$ De causis et sedibus, $\mathrm{xxr}, \mathrm{I} 5$.

'Ibid., xxxvin, 35.

3 The Edinburgh medical and surgical Journal, vol. v! , 18 ir, p. 490. 
mention d'une fermme de vingt-quatre ans, qui avait évacué, en toussant, après avoir éprouvé une inflammation des poumons, une grande quantité de membranes, quel'on a dû regarder, d'après leur structure, comme des dépouilles d'hydatides.

Monro rapporte un cas ou la fumće du tabac facilita l'évacuation d'hydatides qui siégeaient dans les poumons.

Nous avons déjà fait mention plus haut de l'hydatide que Dehaen a rencontrée dans la glande thyroïde.

M. le professeur Kern a extirpé, il y a quelques années, une tumeur qui était placée au.dessus du sternun et qui contenait plusieurs hydatides; M. Kern a eu la bonté de me les communiquer.

Ruysch ${ }^{5}$ a examiné un foie qui ne consistait, pour ainsi dire, qu'en hydatides, et qui contenait une matière sablonneuse transparente; l'on ne voyait plus dans ce foie la moindre ramification provenant de la veine porte ni de la veine cave; les conduits biliaires avaient disparu ainsi que les artères du foie.

Veit a observé un abcès entre la dixième et la douzième côte du côté droit, par lequel plusieurs centaines d'hydatides de la grosseur d'un pois, jusqu'à celle d'uu ouf de pigeon, étaient sorties dans l'espace de quatre à cinq jours.

Pemberton a trouvé sur l'épiploon ten de ces vers de cinq pouces de diamètre; le même auteur a vu â foie un abcès qui s'était étendu jusqu'aux pou-

Thescur. I, n. xil. 
mons, et qui contenait au moins cinq cent soixante hydatides d'inn diamètre de deux pouces et demi jusqu'à celui d'une tête d'épingle. Le même auteur a observé dans le parenchyme de la rate deux hydatides globuleuses de trois pouces de diamètre chacune, qui contenaient une liqueur très-limpide, dans laquelle nageaient de petites hydatides ${ }^{\text {. }}$.

Jüdersen a cxaminé le cadavre d'un homme de quarante ans, mort d'hydropisie; la rate de cet individu s'était transformée en un sac d'un volume énorme, et qui contenait une quantité extraordinaire d'hydatides; la plus volumineuse était de la grosseur d'un citron, et pesait à peu près trois onces; les plus petites étaient de la grosseur des graines de moutarde.

Boudet $^{2}$ a rencontré entre les muscles abdominaux et le péritoine, un sac qui contenait à peu près quatre mille vessies remplies d'eau.

Maclay raconte qu'il se forma dans le mésentère d'uu homme, dans l'espace de dix-huit mois, une tuneur énorme, qui occupait toute la capacité du bas-ventre, et qui contenait à peu près trente-cinq pintes d'hydatides, parmi lesquelles il y en avait plusieurs de la grosseur d'une orange.

Lecat a publié une observation semblable.

M. Cullerier a rencontré dans le tibia une conca-

- David Price a trouvé des hydatides dans la substance du cour chez une personne morte subitement. Voyez Med. et chir. Trans. , vol. XI, p. I. (Br.)

-Giornale di medicina pratica compilato da V. L. Brera, v. Il, Padua, 1812. 
vité de la longueur de trois ponces, et de la largeur d'un pouce et demi, qui contenait une hydatide de plus d'un pouce de diamètre, et dans laquelle il y avais d'antres petits vers semblables.

Il me serait facile d'augmenter d'une cinquantaine le nombre des observations que l'on a faites sur ce sujet, si je ne craignais pas de fatiguer mes lecteurs. J'avoue qu'il serait beaucoup plus satisfaisant pour moi, et beaucoup plus intéressant pour mes lecteurs, si je ponvais indiquer quelque chose de certain concernant le diagnostic de l'existence de cette espèce de ver dans lhomme, ou bien concernant les causes prédisposantes de sa production, et un mode de traitement propre à la combattre.

Lassus nous a indiqué les symptômes suivans, par lesquels on peut, selon lui, présumer l'existence des hydatides dans le foie; il dit que les personnes qui en sont incommodées ont le tciut pâle, mais non pas jaune ou bilieux; elles éprouvent de temps à autre des douleur's violentes au foie et de la constipation; le bas-ventre est cependant souple. Elles out des nausées et vomissent quelquefois; elles croyent qu'elles seraient débarrassées de la sensation de pesanteur dont elles sont inconimodées, si elles pouvaieut vomir fortement; la région épigastrique se gonfle un peu, et elle est presque toujours douluureuse; les malades éprouvent à cet endroit une sensation de pesanteur, qui les menace d'étouffement; cette sensation rend leur respiration difficile et provaque unc toux sans expectoration; ces malades sont 
à peu près incommodés des mêmes symptômes quu'éprouvent les asthmatiques; à la fin il se forme peu à peu à la région épigastrique une tumeur uu peu élastique avec une surface inégalc; son volume augmente insensiblement; et l'on y sent plus ou moins distinctement une fluctuation; les malades ne peuvent pas facilement rester couchés sur le dos, ils se reposent de préférence sur le côté droit, surtout quand l'hy datide a son siége daus le grand lobe du foic, et ils ne peuvent pas être assis sans se courber en avant. En général l'appétit manque, la digestion est mauvaise, la languc est pâle sans être chargée. Cependant cet âteur a qbservé quelques malades, également incommodés par des hydatides, qui avaient conservé leur appétit jusqu’à la mort, leurs pieds ne se gonflaient que dans le cas où il y avait complication d'ascite et vers la fin de la maladie. L'on ne remarque pas non plus, d'après cet auteur, des signes d'une inflammation précédente ni de suppuration; le pouls, qui n'indique point l'existence de la fièvre, est lent, petit et tendu.

J'avoue franchement. que je ne regarde pas le tableau de ces symptômes comme très-exacl: je crois, du reste, que Lassus les a puisés dans une observation faite par Roux '; mais, dans le cas rapporté par ce dernier, il y avait non-seules:en $\mathrm{des}$ hydatides an foie, mais il y avait aussi des désorganisations dans le cour et les parties environnantes, désorga-

- Journal de Hédecine, octobre 177 , p. 3.'. 
nisations qui ont pu facilement causer des accès d'asthme, et à la fin une mort subite.

Malgré le grand nombre d'observations recueillies par moi sur les hydatides, il me serait réellement impossible d'indiquer les symptômes par le moyen desquels on peut reconnaître, avec certitude, l'existence de cette espèce de vers. 11 me serait également aussi difficile de dire quelque chose de satisfaisant sur les causes qui favorisent leur formation. Cependant, je ne puis passer sous silence que l'on a souvent trouvé des hydatides justement dans des organes qui avaient éprouvé auparavant des contusions. Les septième et huitième observations de Lassus font mention de deux filles qui avaient reçu en tombant de fortes secousses sur la région épigastrique.

Corvisart et Leroux ont rapporté l'histoire d'un cordonnier qui avait également reçu un violent coup dans l'hypocondre droit; cet homme éprouva, par suite de cet accident, une douleur très-forte, qui cependant se dissipa peu à peu entièrement; trois ou quatre mois après, le malade remarqua un léger gonflement du bas-ventre et surtout à l'endroit où il avait reçu le coup; cette tuneur augmenta pendant six aus de plus en plus, de manière à le forcer d'abandonner entièrement ses travaux.

Le malade dont parle M. Cullerier avait également reçu un coup sur le tibia, en montant à une échelle.

En supposant que des violences dirigées à l'extérieur de notre corps favoriseraient le développement de l'especce d'hydatide dont nous nous occupons, 
il serait alors également permis d'admettre que toute autre commotion violente, comme par exemple un saut d'une hauteur considérable, une toux très-grave, des éternuemens violens, etc., pourraient avoir les mêmes résultats. Cette supposition ne me paraît pas dépourvue de bon sens; car s'il est de fait qu'une commotion violente sur la colonne vertébrale peut occasioner une hydropisie dans les ventricules du cerveau, ne serait-il pas aussi bien admissible qu'une goutle de substance plastique et vivante, sortie de la circulation, pût être épanchée dans un tout autre organe, et que cette goutte se formât subitement en un tout individuel? Ce tout prendrait alors la forme primitive de tous les corps organisés (c'cst-à-dire la forme sphéroïdale), se développerait par la suite aux dépens de l'organe, et finirait par jouir d'une vie indépeudante.

Aussi long-tempsque le diagnostic et l'étiologie de ces parasites seront couverts d'un voile aussi épais, on ne pourra pas établir une thérapeutique rationnelle.

Lassus est de l'opinion que l'ouverture de ces sacs ou bien des hydatides a toujours été suivie de mort; mais il cite cependant lui-même un cas 'tiré de l'ouvrage de Guattani, où un semblable sac s'ouvrit spontanément, et duquel sortirent plus de trois cents hydatides ${ }^{2}$; il resta une fistule pendant six ans, qui se

${ }^{2}$ Ourrage cité, p. 137 .

'Rheinische Jahrbiicher fïr med. und chir., r. Harles. 13.d. 111, st. I, blasenmolen. $\left(B r_{r}\right)$ 
cicatrisa à la fin, et le malade guérit. Lat personne dont il est question dans la première observation, faite par Lassus, vécut encore pendant une année, après que l'on eut pratiqué une ouverture dans la tumeur.

'Tyson 'parle d'une femme, chez laquelle il fit pratiquer, dix ans auparavant, une incision au défaut des côtes; il en sortit une grande quantité d'une eau claire, et plus de cinq cents hydatides, ciont la plus grandé partie étaient entières et remplies d'un liquide limpide; les plus grosses, qui ne pouvaient pas passer à travers l'ouverture, furent déchirées. A près l'évacuation de ces animaux, cette femme se porta mieux qu'avaut.

On a aussi des exemples que des hydatides ont été quelquefois évacuées par le caual.intestinal.

Bidloo ${ }^{2}$ fait mention d'une observation semblable; il est probable que le sac qui entourait les hydatides était dans ce cas-ci adhérent auxiutestins, et qu'après la rupture du sac ces auimaux s'y sont épauchés; car l'ou conçoit aisément que ces vers n'ont pas pu se produire et se multiplier dans le canal intestinal.

11 faut que je fasse encore ici mention d'une espèce d'hydatide, qui diffère, sous plus d'un rapport, de l'échinocoque, je veux parler des hydatides qui se forment souvent dans la matrice, soit seules on conjointement avee un foetus, ou bien seulement

- Lumbricus hydropicus.

- Ourrage cité, p. 28. 
dans le placenta, et qui ont été appelées par Weissmantel hydrometra hydatica.

Le docteur Helm a eu la complaisance de m'en envoyer un échantillon avec l'histoire de la maladie que je vais rapporter ici.

Barbe Sı. était, dès son enfance, d'une constitution faible et maladive; elle était sujette aux engorgemens glanduleux et aux nualadies de la peau; ses règles se montrèrent dès sa neuvième année, et tonjours tellewient abondantes, qu'elle les gardait chaque fois une quinzaine de jours. Llle éprouva plusieurs maladies, entre autres une blennorrhagie, dont elle fut guérie, et le flux menstruel diminua; elle se maria à l'âge de vingt-deux ans, dans le mois de juin 1815 ; ses règles cessèrent au mois d'octobre, et plusieurs symptômes firent présumer une grossesse.

Cette femme fit appeler le docteur Helm le 7 janvier I816; elle se plaignit de fortes douleurs dans les reins, qui duraient déjà depuis un mois; elle perdait en même temps par le vagin une plus ou moins grande quantité de sang noil; du reste, cette personne assura à Helm que tous ces accidens n'influaient pas trop sur sa santé; qu'elle n'avait pas beaucoup d'appétit, mais une grande soif.

L'on pouvait facilement sentir au-dessus du puhis la matrice qui était distendue. La moindre pression que l'on y exerçait causait une douleur violente.

Les médicamens qu'on lui avait ordonnés firent cesser l'hémorrhagie; mais, après l'espace de quel-. ques jours, elle reparut conjointement avec les dou- 
leurs de reins; à dater de cette époque, elle se porta tantôt assez bien, et tantôt plus`mal.

Le 3o janvier, elle fut de nouveau incommodée par de grandes douleurs, et elle éprouva en même temps une hémorrhagie par le vagin.

L'orifice de la matrice, que l'on ne pouvait pas atteindre auparavant, était dilaté, mais il était impossible de sentir la présence d'un enfant. A neuf heures, les douleurs devinrent violentes, et une mole, de la grosseur d'une tête, sortit; elle c'ait entourće d'une nembrane, que la sage-femme déchira, et quelques milliers d'hydatides se présentèrent.

Deux jours après, cette femme fut atteinte d'une fièvre, ses seins se gonflèrent, et il en sortit du lait; un traitement converable a promptement produit la guérison complette : dans la mème année, cette femne devint de nouveau enceinte, et accoucha d'une fille saine et bien conformée.

Je reçus, le lendemain de leur sortie, leshydatides observées par M. Helm; elles étaient attachées, au moyen de pédoncules, à une espèce de placenta; les plus volumineuses étaient de la grosseur d'une noisette, et les plus petites de celle de grains de chenevis : elles étaient transparentes et remplies d'un liquide limpide. Je versai sur-le-chaınṕ, sur une partie de ces vers, de l'esprit-de-vin, pour mieux conserver cette espèce de placenta, qui semblait devoir se dissoudre facilement dans l'eau. Ces vessies prirent bientôt une couleur rouge. La même chose arriva pour celles qui se trouvaient dans l'eau, conjointe- 
ment avec les annexes sanguinolentes, et elles ne se décolorèrent que peu à peu.

Il paraît résulter de là que les hydatides sont réellement douées d'une vie individuelle, et qu'elles forment des animaux particuliers. Cette supposition u'est nullement réfutée par la circonstance qu'elles se trouvent implantées, au moyen de pédoncules, sur un corps commun, ou bien qu'elles sont adhérentes et placées les unes sur les autres; car il y a dans le grand règne aquatique un nombre infini d'animaux qui ont le corps ainsi fixé. En outre la circonstance que pendant leur vie elles n'admettent dans l'intérieur de leur corps que la quantité nécessaire d'un liquide convenable, tandis que ce n'est qu'après la mort que peut y pénétrer celui qui les entoure, est encore en faveur de leur vie individuelle. La même chose a lieu pour les échinocoques, quand on les met dans un liquide coloré. Une môle en forme de grappe ne peut pas être regardée comme une simple distension ou boursouflement des vaisseaux lymphatiques, ou d'autres vaisseaux, par la raison que les pédonculcs ne sont pas creux. On peut se convaincre de ce fait, si on remplit de mercure une de ces vessies un peu volumineuse, dans laquelle d'autres plus petites se trouvent placées plus profondément; ce liquide ne pénètre pas dans ces dernières.

Je ne prétends cependant pas que l'on doive ranger dorénavant ces vessies, d'après ma seule autorité, parmi les vers intestinaux; c'est par cette raison que je fais placer la figure qui représente une petite par- 
tie de la môle, examinée par moi, parni les pserrdohelminthes (Voy. pl. 9 , fig. $f$ ).

Une semblable môle, assez bien dessinée, se trouve dans Bidloo .

Clarke cite un cas qui paraît tout-à-fait semblahle à celui rapporté par Helm; il s'était également formé $\mathrm{du}$ lait, dans une circonstance pareille, dans le sein d'une femme, de manière qu'elle aurait pu alaiter un enfant.

Moreau rapporte un cas analogue.

Watson raconte le suivant : "Une femme de quarante-huit ans, qui avait eu plusieurs enfans, se crut de nouveau enceinte dans le mois de novembre; depuis le mois de février jusqu'à la fin du mois de mars, elle eut chaque nuit des pertes de sang par le vagin. Cependant, comme elle ne remarqua pas un gonflement du ventre, ni des seins, elle s’imagina qu'elle était arrivée à l'époque où elle perdrait tout-à-fait ses règles; elle rendit, le premier avril, après avoir éprouvé auparavant de fortes douleurs de reins, beaucoup d'hydatides, qui étaient de la grosseur d'une noix muscade jusqu'à celle d'une tête d'épingle; quelques-unes étaient remplies d'une lymplie transparente, et d'autres d'une lymphe sanguinolente; peu de temps après, elle fut complétement rétablie. »

Mougeot a aussi écrit sur ce sujet; mais les observations qu'il rapporte ont été faites par M. Percy. Ce dernier regarde également les hydatides comme

S Ouvrage cité, tab. 2. 
des animaux vivans, et il ajoute qu'on les rencontre sonvent dans le placenta et qu'on les observe presque toujours dans les animaux ruminans. Il établit les symptômes süvans, comme ceux d'après lesquels on pent présumer une grossesse d'hydatides. Les femmes éprouvent souvent de petites pertes de sang ou de glaires, à dater du second mois jusqu'à l'accouchement. L'orifice de la matrice est toujours dilaté, et il ne change presque pas de forme ni de place; le basventre est en effet gonflé, mais il est plutôt souple que dur.

L'époque de l'accouchement des hydatides n'est pas fixe; leur sortie a lieu quelquefois dans le troisième mois, et quelquefois dans le dixième, mais rarement plus tard.

Pour accélérer la sortie de ces animaux, Percy conscille de faire des injections d'eau de mer, ou d'une dissolution saturée de sel marin, avec du vinaigre.

Une seconde observation que nous allons rapporter a prouvé à ce médecin qu'une semblable formation de môles peut avoir lieu dans l'état de virginité.

Une chanoinesse de vingt-six ans éprouva un retard de règles dans le mois de juillet 1788 ; son ventre se ballonna, etc. Le 5 avril 1789 , elle rendit par le vagin deux pots de chambre d'eau, conjointement avec des hydatides, qui étaient en partie cntières et en partic déchirées. 
Le docteur Guillaume Schmidt a rapporté trois cas semblables.

Dans le premier, les hydatides ressemblaient toutà-fait à celles que le docteur Helm avait examinées; dans les deux autres cas, qu'il a eu occasion d'observer plus tard, les boules ou vessies étaient beaucoup plus petites, et en général d'une grosseur très-inégale; elles se trouvaient entassées par-ci par-là dans une substance gélatineuse, qui ressemblait beaucoup au frai des grenouilles. Toutes ces vessies étaient couvertes d'une membrane qui avait la consistance du chorion.

Ne pourrait-on pas supposer que les hydatides, dans ces deux derniers cas, se trouvaient dans un état maladif?

Plusieurs naturalistes parlent de l'évacuation de vers vésiculaires, sans cependant nous faire savoir s’ils avaient été réunis par le moyen de pédoncules.

Ne pourrait-on pas admettre que les hydatides se séparent, avec le temps, de leur pédoncule, de la mème manière que les œufs se séparent de l'ovaire de la poule?

Je crois la chose possible, et elle me paraît même très-probable; justement au moment où cette feuille allait être livrée à l'impression, je reçus de M. Brera

- Tabula anatomico-pathologica ad illustrandam historiam vernium in visceribus abdominis degentium, hydropem ascitem, vel graviditatem simulantium, cum epicrisi, auctore Valeriano Aloysio Brera, D. M. Viennce Austrice, 1818 , in- $4^{\circ}$. 
une petite dissertation, accompagnée d'une grande planche, où se trouvent représentées de semblables hydatides.

Autant que je puis en juger par le texte, elles étaient placées entre les muscles abdominaux ct le péritoinc. La plus grande partie consistent en vessies isolées plus grosses et tout à fait libres. Dans trois différens endroits, se trouvent cependant plusieurs hydatides beaucoup plus petites, qui ont l'air d'être unies les unes aux autres par le moyen de pédoncules.

\section{SECTION 111.}

Des pseudohelminthes, ou des corps, animaux ou non, regardés à tort comme des vers intestinaux.

Je donue cette dénomination à tous les corps étrangers, soit qu'ils aient appartenu au règne animal ou à tout autre, que des hommes ont rendus, ou bien que l'on a trouvés dans les cadavres, et que les médecins ont regardés à tort comme des vers intestinaux. Cependant, je passerai seulement en revue quelquesuns des pseudohelminthes, que les auteurs modernes nous ont fait connaître; car si je voulais m'occuper de ceux qui sont cités par les anciens médecins, et qui en général ne sont rien autre chose que des insectes ou leurs larves, je pourrais remplir un volume en entier.

On trouvera un catalogue très-détaillé de semblables observations dans l'ouvrage de Brera, sous 
lc titre de vermi metastatici, notamment dans la seconde section, oì cet auteur traite des insectes.

Je ne crois pas que beaucoup de personnes ajoutent foi à ce que cet anteur a avancé sur les vers accessoires, vermi accessori. Il y est fait mention, entre autre choses, d'une femme qui aimait beaucoup la viande de moutou, et qui a renda un tænia que l'on ne trouve que dans cet animal; il yest encore question d'un lomme qui faisait un grand usage de viande de porc, et qui a rendu un échynorhinque géant que l'on ne rencontre que dans cet animal; mais je reviens à mon sujet.

\section{Ditrachycíne rude.}

Ditrachyceros rudis( en allem. das rauhe doppelhorn), pl. Ix, fig. 3 .

Karl Sulzer's Beschreibung eines neu-entdeckten Fingeweide-svurms im menschlichen Koerper. mit drei kupfertafeln. Strasburg und Paris, 1802 .

Zeder, Anleitung, s. 421 , cysticercus bicornis.

Rudolphi, Entoz., 11, 2 , p. 238 , tab. xi1, fig. 5, diceras rude. Brcra, Memorie, p. 140 , tab. 111 , fig. $11-13$, ditrachiserosoma. De Lamarck, An. sans vert., t. In, p. ז5o, le bicorne hérissé.

De Blainville, Dic. des Sc. Nat., tom. xur, p. $36 \mathrm{~g}$.

Une demoiselle de vingt-six ans, qui avait déjà éprouvé plusieurs maladies, fut incommodée un jour d'une esquiuancie ; le huitième jour de cette maladie, elle prit un purgatif, composé de manne et de 
sel de Glauber, qui lui fit rendre pendant deux jours, conjointement avec les matières fécales, une quantité extraordinaire de petits corps, que Sulzer regarda comme des animaux.

A près avoir fait usage des amers pendant quelque temps, cette demoiselle évacua de nouveau plusieurs corps semblables, mais qui n'étaient pas entiers.

On envoya tous ces échantillons, conservés dans l'esprit-de-vin, à Sulzer, qui , après les avoir examinés très-soigneusement, publia une description détaillée, accompagnée de plusieurs dessins, sur une échelle très-grande, qui représentaient non-seulement le ver en entier, mais également plusieurs de ses parties. Un de ces échantillons est figuré de grardeur naturelle, fig. $d$, pl. 9. Les antennes recourbées, qui avaient l'air d'être velues sous le microscope, n'avaicnt pas la même direction dans tous les échantillons. Parmi la grande quantité de ces corps, il n'y en avait que quatre qui fussent pourvus de ces organes. Cependaut on voyait nager plusieurs de ces derniers dans l'esprit-de-vin.

Sulzer a raugé ces petits corps parmi les hydatides.

Zeder ne veut pas les reconmaître pour telles.

M. Rudolphi est de l'opinion qu'on devrait plutôt les ranger (supposé qu'ils fussent de véritables vers, ce qui lui paraît encore douteux) parmi les acantocéphales, et qu'ils devraient former un genre particulier, sous le nom de dirhynchus. Quant à moi, je ne me suis pas encore inquiété quelle place ils doivent occuper dans un système d'helminthologie; car je 
n’ai pas encore pu ue convaincre que ce fussent de véritables vers; il me paraît plus probable (je puis cependant me tromper) que ces corps n'étaient rien autre chose que des graines d'une plante que cette demoiselle avait avalées; mais je ne puis pas dire de quelle plante ils provenaient. Les antennes de ces corps, telles qu'on les voit représentées dans les figures de Sulzer, ont l'air de n'être que les germes de ces graines. Ceux qui ont lu avec attention tout ce que cet auteur a dit sur leur structure extérieure et intérieure, et ceux qui ont bien examiné les figures qu'il en donne, seront peut-être de mon opinion.

\section{Ascaris stephanostona.}

(En allem. das kronenmaul), pl. Ix, fig. c.

Jordens, Heiminth., s. 29, tab. vı, fig. 5-8.

Brera, Memorie, p. 189 , tab. II, fig. 14-17, ascaride stephanostoma.

\section{Ascaris concsoua}

(En allem. der kegelwum), pl.xı, fig. e.

Jordens, helminth. , s. 3o, tab. v11, fig. 9-12.

Erera, Memorie, p. 193; tab. II, fig. I8-21, ascaride conosoma.

Je réunis ces deux vers ensemble, parce qu'ils proviennent de la même source, et qu'ils apparticunent à la même fanille.

Quclques médicamens ordonnés par le professeur 
Bretsthneider, de Jena; à un jeune homme, firent rendre à ce dernier les animaux dont nous nous occupons maintenant. Bretsthneider, voyant que ce n'était pas des oxyures, les envoya au professeur Lenz; ce médecin donna aux plus considérables le nom de stephanostoma, et il appela les petits conosoma. Il communiqua quelques échantillons à Jcerdens, qui les rangea dans le genre ascaride.

M. Rudolphi s'exprime à ce sujet en ces termes ${ }^{2}$ : "Jœrdens a décrit et dessiné, sous le nom d'ascaris stephanostoma et conosoma, des corps qui ne sont rien autre chose que des larves de mouches. A près avoir examiné la description et les figures de cet anteur, je conclus sur-le-chanp qu'il ne s'agissait ici que de larves d'insectes. Cependant je priai le professeur Lenz de me communiquer quelquesuns des échantillons de Jœrdens, et, en effet, il eut la bonté de m'en envoyer. Je me trouvais à cette éporque à Berlin, qui possède un grand nombre d'entomologistes très-habiles. Je leur fis voir ces prétendus vers, et ils les regardèrent également comme des larves.

"On devrait supposer qu'un homme qui s'est décidé à publier une helminthologic du corps humain, serait en étal de distinguer une larve de mouche d'ua ver; je ne conçois pas comment Jœrdens put adapter à ces deux larves les signes caractéristiques propres au genre ascaride. En général, cet auteur n'a

W'iedemanns archic., $\mathrm{ni}, 2$, s. . 
jamais fait attention aux caractères dans ses observations; tout son ouvrage porte le caractère de l'ignorance et de la légéreté; il a même ignoré que son prétendu ascaris conosoma se trouve dessiné dans l'ouvrage de Phelsum, qu'il a cité cependant quelquefois.

"Il est arrivé assez souvent que des larves de mouches ont séjourné dans le canal intestinal de l'homme. Acrel, Osiander et beaucoup d'autres en citent des exemples; mais il est assez étrange, et cela mérite d'être relevé sévèrement, qu'un helminthologue du dix-neuvième siècle ait regardé des larves comme des oxyures."

Brer: , qui a également reçu, par la bonté de M. Gautieri, quelques - nns des échantillons dont nous venons de parler, les regarde aussi comme des larves de mouches, et il désigne même l'espèce à laquelle èlles appartiennent. Il a attribué les premières (stephanostoma) à la musca carnaria, et les autres à la musca domestica; mais néanmoins il leur a assigné une place parmi les ascarides, sans doute par la raison qu'il ne voulait pas contredire un autre naturaliste, qui les avait rangées avant hi dans ce geure. C'est pousser, réellement, la condescendance un peu trop loin, et elle est cértainement très-mal placée dans un cas pareil.

La figure $c$, pl. 9, représente le stephanostoma, eı la figure $e$, même planche, le conosoma. 
IV. Cercusoma, pl. IX, fig. b.

Cercosona : species nova; capile distincto; labio amplissimo, quadricuspidato, quatuor papillis insignito; corpore oblongo, subdepresso, nodoso, spirce adinstar fibroso ; retrorsum caudato ; margine superiori ac inferiori dentritico; dorso punctato; poro caudali; ; cauda longissima, tereti, subcirrosa. Frabitat in vesica urinaria.

Brera, Memorie, p. Io6, tab. I, fig. 26-27.

Nous devons au professeur Canali de Perouse la découverte de cette nouvelle espèce d'un prétendu ver intestinal qui, étant encore vivant, a été rendu par une femme pendanı qu'elle urinait; je crois que l'on aurait dû plutôt dire, que l'on a trouvé dans le pot de chambre d'une femme.

Après que ce ver eut été examiné et disséqué avec soin, on en fit une description détaillée que l'on envoya à Fabbroni à Florence, qui la fit insérer, par les soins de M. le professeur Gatteschi, dans le Giornale litterario de Pise. Brera a eu le bonheur d'enrichir sa collection de cet échantillon rare et unique; quant à moi je n'en suis pas envieux, et je crois que mes lecteurs ne le seront pas nón plus, quand ils en auront vu le dessin (Voyez fig. $b$, pl. 9 ). Tous les entomologistes sans exception, ce me semble, regarderont sur-le-champ cet animal comme une larve d'insecte; cependant, pour lever tous les dontes sur la nature de cet animal, je consultai mon collègue M. Zieggler, et voici la réponse qu'il m'a faite : Cet animal n'est qu'ume larve d'éristale, ct très-probablement de l'eristalis pendulus, Fabric. (Syst. Enlliat., 
$11^{\prime \prime} .7$, p. 233); car les autres espèces d'éristales connues eu Europe sont plus petites, et par conséquent leurs larves doivent être également moins grosses; la remarque ajoutée par Fabricius à la description qu'il a donuée de cet animal paraît confirmer encore davaniage celte supposition; il s'exprime ainsi : "Habitat in Europae aquis stagnantibus larva tubo filiformi respiratorio suspensa." "

Brera a regardé également cet animal dans le commencement conme une larve de syrphe, dénomination synonyme de celle d'éristale; mais en l'examinant au microscope, il trouva que les signes caractéristiques propres à ces larves manquaient au cercosome; il ajoute que les caractères qu'il avait observés sur cet animal appartenaieut an contraire, selon lui, au genre linguatula ou polystoma; mais je crois que personne, excepté cet auteur, ne trouvera de semblables caractères sur une larve d'éristale. L'on ne peut pas s'expliquer comment Brera a vu au microscope autre chose que ce qu'il avait aperçu si exactement auparavant à l'œil nu; je présume que cet auteur a encore, dans ce cas-ci, adopté par condescendance lopinion des trois autres médecins, qui avaient mal observé, ou qui avaient pour le moins mal envisagé le sujet en question. Cependant une chose essentielle manque à ce fait, c'est-à-dire la preuve que la femme ait réellement rendu cet animal en urinant; quant à moi, je ne puis pas m'imaginer qu'il provenait de la vessie, il est plutôt probable qu'il était tombé par hasard dans le pot de chambre de cette femme. 


\section{Hexathiordum vevarum.}

(en allem. der vencnblattsurm), pl. $\mathbf{x}$, fig. $u$.

HEXATHY RYDIUM : conporc depresso lanceo!ato, poris anticis sex intra labium.

Treutler, Auctuar., p. 23 , tab. 1v, fig. $\mathrm{x}-3$.

Joerdens, Helmintli., s. 67 , tab. vı, fig. 6-8, der venenbluttwurm. Zeder, Anleit., s. 23I , $\mathbf{1}^{\circ} .4$, palysioma venainm.

Rudolphi, Entoz. II, I, p. $456, \mathrm{n}^{\mathrm{n}} .6$, pol. venar.

Brera, Menumie, p. $10 \mathrm{I}$, tab. 11, fig. 3-4, exatiridio sangaicola. De Lamarck, Anim. sans vert., tom. 111 , pag. 174 , limsuatule des veines.

De Blainville, Dictionn. des sciences nitur., tom. xxı, pag. 144 .

Cet animal doit être placé sans contredit parmi les vers; mais il est encore bien douteux si on doit le ranger parmi les vers intestinaux.

Treutler avait à traiter un jeunc homme de seize ans qui, d'après ce quill croyait, était tournenté par des oxyures. Comme ce jeune homme était extrêmement malpropre, Trentler lui conscilla de se baigner souvent däns la rivière (firequenti lavatione in flumint? uti admonitus est); ce sont les paroles de ce médecis.

Un jour ce jenne homme entra lentement dans l'eau (cum aliquando pedetentim aquam intrasset); peine y était-il depuis une minute, que la veine saphève du pied droit se rompit sulsitement et spontanément (sponte rupta est vena). Il s'ensuivit une hémorthagie qui s'arrêta ct se renonvela de momens à autres. Dus remedes styptiques et une forte ligature 
ne purent pas la faire cesser entièrement. Treutler, qui fut appelé, vit sortir de la plaie une substance un peu compacte, qu'il prit au commencement pour du sang coagulé, mais un examen plus exact le convainquit que c'étaient deux animaux vivans, qu'il enleva saus peine, et l'hémorrhagie cessa. Cependant la plaie ne se cicatrisa que trois semaines après. Le malade se sentit un peu soulagé, mais il retomba bientôt après dans son ancien état maladif. Les meilleurs vermifuges furent vainement employés, aucun ver ne fut rejeté, et Treutler conclut de là que les accidens que ce jeune homme contiuuait à éprouver, provenaient sans doute des vers qui séjournaient dans les vaisseaux sanguins.

On aurait tort de douter un instant de la vérité du fait rapporté par ce médecin; cepeudant il n'est pas prouvé que ces vers provinssent réellement de l'intérieur d'un vaisseau sanguin; Rudolphi et Zeder ne le croyent pas. Ces deux auteurs sont plutôt portés à regarder ces vers comme des planaires (plariaria) qui vivent dans l'eau, et qui peuvent facilement s'attacher à notre corps el causer une hémorrhagyie.

Si ces vers étaient venus réellement de dedans au dehors, il me paraît qu'ils auraient dû en premier lieu présenter la tête et une petite partie du corps; unais sortis de cette manière, ils n'auraient pas pu long-temps se soutenir au dihors; car leur tête unc fois passée à travers les tégumens (comme elle est chez ces animaux le seul organe avec lequel ils peuvent s'accrocher), leur corps aurait dû être cntraîné par l'eau ou bien par l'ćcoulcment du sang. 
Brera rapporte aussi cette observation, mais il s'est permis de le faire avec quelques changemens afin de pouvoir mieux l'adapter à sa théorie des vers dans lc sang. 11 raconte que le malade de Treutler a pris un bain tiède, et qu'il s'est ouvert une veine avec une esquille en entrant dans la baignoire.

J'ai rapporté plus haut les propres paroles de Treutler, afin de pouvoir les mettre en comparaison avec la traduction de Brera, dans laquelle il cite exactement les pages de l'ouvrage de cet auteur " $U_{n}$ giovane.......... entrato essendo in un bagno caldo urtò col piede destro in una scheggia del recipiente, che era de legno, rimasse ferito nella saffena anteriore, étc."

En lisant cette traduction, comment peut-on ajouter foi aux paroles d'un homme qui se permet de dénaturer ainsi les observations faites par d'autres auteurs; que doit-on penser des observations qu'il cite comme siennes et sur lesquelles on ne peut pas le contrôler? L'on ne s'étonnera pas alors, si je ne crois pas, entr'autres, à celle qui a été rapportée dans le commencement de ce traité, où il nous raconte que des milliers d'oxyures se sont développés dans la cavité abdominale d'un chien, dans laquelle il avait introduit, au moyen d'une incision, dix prétendus œuls de vers. Ces œufs, grossis dix fois, selon cet auteur, et copiés d'après sa figure, se trouvent représentés dans notre pl. 9 , fig. $i$. 
VI. Diacakthos polycephalus, pl. Ix, fig. 9 .

Meckels, Deutsches archiv. firr dic physiologie. bd. Iu, heft. 2. s. 17 -

Le docteur Stiebel avait à traiter un enfant de onze ans, dı sexe masculin, qui avait déjà éprouvé, dès l'âge de deux ans, des accès spasmodiques. Ces accès étaient d'une nature tout-ì-fatit singulière, et méritent d'être connus. Un jour, en se frappaut vers la région épigastrique, cet enfant s'écria : "comment une aussi petite chose me peut-elle tant tourmenter?"Une autre fois il dit: "quand viendra donc le vrai remède qui pourra m'en débarrasser?" I Le lendemain il ne se rappelait rien de tout ce gu'il avait dit pendant la nuit. Les symptômes cessèrent tout-à-coup le premier novembre, et le leudemain il rendit un petit animal particulier enduit de mu-cosités; dès ce moment, cet enfant fut complétement guéri.

Cet animal se.trouve représenté de grandeur naturelle sur notre planche 9 , fig. 9 .

Stiebel a fait dessiner différentes parties de ce prétendu animal sur une échelle très-grande, parmi lesquelles on peut voir des tentacules, arnıés de griffes cornées, des lèvres pourvues de petits crocliets, des trompes susceptibles d'être alongées et rétractées.

L'original a été communiqué à Blumenbach.

Stiebel a regardé ce corps comme le tronc (stamm) d'un ver intestinal. Je n'ai jamais été de cette opinion, 
et $j^{\prime a i}$ cru, au premier abord, que cet échantillon n tait rieu autre chose qu'un tronc d'artère, provenant d'un petit animal, d'un oiseau peut-être, avec des ramifications rongées ou déchirées; de semblables échantillons, auxquels on avait donné le nom de vers, mont été déjà communiqués bien souvent; cependant les parties dures que l'on a observées sur l'échantillon en question, m'ont convaincu que cela ne pouvait pas être un trone d'artère. M. Rudolphi me fit part, dans une lettre datée du 18 juillet 1818 , que l'animal nommé par Stiebel diacanthos polycephalus ", n'était rien autre chose qu'une petite tige de plante, peut-être celle d'une grappe de ritisin. Rudolphi ajouta qu'il était allé voir lui-même Blumenbach, qui possède ce prétendu animal, dans l'intention de l'examiner. "Je ne l'avais jamais regardé ni comme un animal, ni comme un ver intestinal de l'lomme (ce sont les paroles de Rudolphi); je l'avais pris plutôt pour la dépouille d'une larve d'insecte; mais un examen particulier me fit voir qu'il tirait son origine d'un végétal, et, au moyen d'uue petite incision, j'ai trouvé même les vaisseatux spiraux. "

Nous voyons par là que Stiebel s'était trompé, comme il peut arriver à tout le monde, et il est ì présumer que ce médecin a été induit en erreur prar la cessation subite des souffrances au moment où cet cufant avait rendu ce corps; il est également possible

× Journal complémentaire du Dietionaire des scicuces médicales, tom. I , cah. 2,18 18. (Br.) 


\section{2}

que ce dernier se fùı fixé à un endroit très-sensible; et qu'il ait occasioné les accidens mentionnés.

Je saisis ici l'occasion d'observer que l'on m'a déjà envoyé souvent des restes non digérés de tendons, de membranes, de ligamens, de vaisseaux, des filores de plantes quelquefois provenans des asperges et des champiguons, etc., substances auxquelles on avail donné le nom de ver.

Il est souvent très-facile de reconnaître la véritable nature d'un pseudohelminthe; dans d'autres cas, il est très-difficile, même impossible de la deviuer; car l'homme se nourrit de tant de substances différentes, que l'on ne peut pas savoir de laquelle il a fait usage en dernier lieu. Je ne puis pas non plus m'enpêcher d'avertir ici mes collègues que le microscope composé, dont on se sert en géuéral pour faire des recherches, peut très-facilement induire en erreur, si l'on n'a pas l'habitude de s'en servir. Le microscope simple offre moins d'inconvéniens, et il montre les choses sous un point de vue plus vrai. Cependant il y a beaucoup de cas dans lesquels on ne peut pas se passer du premier.

\section{DES VERS DES DENTS.}

Je puis bien présumer qu'il n'y a pas un de mes lecteurs qui n'ait déjà entendu dire que l'on rend quelquefois avec la salive des ver's, après avoir fait usage de certaines fumigations, vers que l'on prétend provenir des dents creuses; mais, d'un autre côlé, il 
est aussi de fait que tout le monde ne sait pas ce que l'on doit penser de cette assertion.

Il y a quelques années qu'un médecin présenta à notre société de médecine le couvercle d'une tabatière noire, sur lequel on avait desseché des prétendus vers, qu'un homme avait rendus après avoir fait des fumigations préparées avec les semences de jusquiame. Ce médecin était présent lorsque'le malade les avait rendus, et il a également observé qu'ils se remuaient dans l'eau.

Nous n'avons pas douté de la vérité du rapport de ce médecin; mais nous avons seulement présumé qu'il avait mal observé, et qu'il avait regardé à tort les mouvemens de ces corps comme des monvemens spontanés d'animaux vivans. Nous avons également présumé, et avec raison, que ce médecin était encorc dans l'erreur, en supposant que ces corps étaient de véritables vers sortis des dents de son malade.

Les prétendus vers des dents ne sont rien antre chose que les germes des graines avec lesquelles on prépare les fumigations.

Au moment où on jette la graine sur des charbons rouges, sa capsule crève, et le germe saute loin d'elle. S'il tombe dans l'eau, il s'opère, à cause des contractions inégales des fibres, un mouvement circulaire, que l'on peut regarder aisément, mais à tort, comme un mouvement volontaire.

Il y a à peu près un demi-siècle que Schaeffer a déjà donné des éclaircissemens sur ce sujet, et il a même publié une dissertation sur les vers inaginaires des dents. 
Chacun de mes lecteurs peut préparer à volonté ces prétendus vers, au moyen des graines de jusquiame. Pour les obtenir, on n'a qu'à mettre une petite barre de métal, rougie ou très - chaude, audessus d'un vase rempli d'eau, et poser sur cette barre une petite quantité de ces semences. Cela fait, on couvre le vase sur-le-champ avec un entonnoir. En l'ôtant un moment après, on verra nager dans l'eau les germes de ces graines. Ces germes se heurtent en sautant contre la paroi de l'entonnoir, et retombent dans le liquide.

Schaeffer a prétendu à tort que cette expérience ne réussit qu'avec le fruit nommé alkekengi ou coqueret, et après avoir enduit ces semences de cire. La fig. $k$, pl. 9, est une copie de la figure de Schaeffer, elle représente les germes de ce fruit. La fig. $l$ fait voir les germes de graiues de jusquiame que jai obtenus moi-même par le même procédé.

\section{Appennice.}

Le chapitre précédent peut servir à prouver à mes lecteur's que l'on ne doit pas regarder trop précipitamment les corps que les hommes rendent, ou que l'on suppose avoir été formés dans leur intérieur, et évacués par eux conjointement avec les déjections, pour des nouvelles espèces de vers intestinatix. Avaut de porter un jugement dans un parcil cas, il faut examiner soi-même les objets avec beaucoup de soin, ainsi que les circonstances qui les ont accompagnés; car il arrive souvent que des personnes, guidées par des intérêts particuliers, veuleut 
faire croure des choses qui n'ont pas réellement existé. $S i$ je vonlais citer tous les récits mensongers de cette nature qui sont venus à ma connaissance, je pourrais facilement en remplir des feuilles entières; cependant, qu'il me soit permis de rapporter le suivant.

Une femme d'une quarantaine d'années éprouvant des indispositions de toute espèce, on présuma qu'elle avait le ver solitaire, et l'on employa des vermifuges. A près l'avoir fatiguée par beaucoup de médicamens peudant six semaines, cette femme éprouva une nuit, une oppression violente, accompagnée de nausées, etc. En effel, elle vomit quelques matières, dans lesquelles se trouvait un petit crapaud et des membranes. A près cette évacuation, tous les symptômes qui avaient précédé le vomissement cessèrent sur-le-champ; mais, du reste, il ne s'était opéré aucune amélioration dans sa santé.

Ce crapaud, y compris les membranes, furent communiqués à M. le baron de Tï̈rkheim, ct c'est cliez lui que j'ai vu, quelques jours après, ces objets couservés dans de l'esprit-de-vin.

C'était un crapaud ( rana bombyna var., Linn., Gmel. ; bufo igneus, Daud.) qui avait les pattes dederrière cassées. Il y avait également quelques menbranes, qui cependant n'ont pas pu appartenir à cet animal; car elles étaient d'une texture très-compacte, ce qui n'est pas du tout propre aux membranes des crapauds.

Pour découvrir la véricé de ce fail, j’ai pris toutes les informations possibles; je me suis adressé à cette 
femme elle-même; mais comme elle était trop obstinée et trop réservée dans sa manière de se prononcer, je n'ai pu tirer d'elle rien de satisfaisant. Son mari m'inspirait trop de confiance pour ne pas ajouter foi à ce qu'il me disait; ilassura que sa femmeavait réellement vomi, en sa présence, un crapaud dans une cuvette vide. Je ne savais que dire à cela; cependant, il ne paraissait pas du tout probable que cette femme eût pu avaler cet animal en buvant de l'eau sans s'en apercevoir, ou bien que ce crapaud fût arrivé dans l'estomac, sous forme de tétard, ou bien sous celle de frai, et qu'il s'y fût développé peu ì peu. Je fus fâché de n'avoir pu déconvrir le fond de la chose; mais j'avais cependant l'espoir que le temps dévoilerait cette énigme. En effet, cela est arrivé comme je l'avais présumé. On s'aperçut un jour que cette femme avait, de temps à autre, des accès de folie, et on a même été obligé de la mettre plus tard dans une maison d'aliénés. Avant de devenir tout-à-fait folle, elle avait encore quelquefois des intervalles lucides. Dans un de ces derniers, elle avoua à quelques personnes de sa connaissance qu'elle avait avalé ce crapaud, venimeux, selon son idée, entouré d'une membrane, qu'elle avait ramassẻe dans une boucherie, dans l'intention de mettre un terme à sa vie, dont elle prétendait être lasse.

Son estomac supporta très-bien ce prétendu poison pendant toute la journée jusqu'à minuit, heure à laquelle elle vomit ce crapaud, conjointêment avec la membrane, qui était déjà à moitié digérée. 


\section{DEUXIEME PARTIE.}

\section{CHAPITRE QUATRIEME.}

Des causes de la Jormation des vers dans le canal intestinal de l'homme.

Si nous admettons comme prouvé que les vers intestinaux n'arrivent pas du dehor's dans le corps de l'homme, qu'ils n'y sont pas innés, et qu'ils doivent par conséquent leur existence à une formation primitive $^{2}$, nous ne pouvons chercher ailleurs la cause première de leur production, que dans une altération de la nature et du mélange des substances propres à l'entretien ou à la nutrition du corps en général, ou bien dans une surabondance de ces substauces, auxquelles les vers intestinaux doivent probablement aussi souvent leur production qu'à toute autre cause. Cependant la cause première d'unc pareille altération de la nature, ou bien de la dispro-

x Dans toute cette seconde parlie il n'est question que des espèces de vers qui séjournent dans le canal intestinal de l'homme, et non de ceux qui se trourent dans quelques autres parties, les causes de leur formation, leur diagnostic et leur traitement élant trop obscurs pour pouroir donner lieu à des généralités, ont éié rapportées à chacun d'eux en particulier.

2 Voyez Mémoirc de Robert hnox inséré dans l'ourroge de Froriep, ayant pour titre: Notizen etc., n. 8, 1821, p. 121 . 
portion des substances propres à la nutrition du corps ne peut provenir que d'une faiblesse relative des organes en particulier, et non pas d'une faiblesse générale de tout le corps; car celle failslesse, généritlement parlant, peut être regardée anssi peu comme une maladie que comme une cause de la production des vers, quand du reste l'harmonie existe entre toutes les fonctions. Une maladie ne résulte que d'un défaut d'harmonie dans les fonctions des organes. Un semblable état a lieu sans doute quand des vers doivent se former; car, par exemple, $1^{\circ}$. s'il ne se prépare, par le moyen des alimens arrivés dans l'estomac, ni plus et ni moins de substance nutritive qu'il n'est nécessaire pour le remplacement des humeurs excrétées, pour la conservation et pour l'accroissement du corps; $2^{\circ}$. s'il ne s'animalise pas dans le même organe plus de subsiances que les vaisseaux lymphatiques ne peuvent en absorher, ou qu'ils en absorbent réellement; et $3^{\circ}$. s'il ne se sécrète pas de la part du corps animal plus d'humeurs provenạut de sa propre masse, qu'il n'est nécessairc pour animaliser ou pour organiser les substances qui se trouvent dans l'estomac, alors la production des vers ne pourra pas se faire dans le canal intestinal. S̈il cxiste an contraire une disproportion, c'est-à-dirc s'il s'animalise plus de substance qu'il ne peut en être absorbé, alors rien n'est plus facile que cette prodnction. C'est pour cette raison que nous observons souvent des personnes qui paraissent parfaitement saines et robustes, et qui ont néanmoins des vers dans le canal 
intestinal. Ici il paraît que l'estomac et les intestins, ou, si l'on aine mieux, les premières voies, se trouvent dans un état d'activité vitale plus graud qu'il n'est réellement nécessaire à la conservation du corps, et que l'activité des vaisseaux lymphatiques qui absorbent seulemeat autant qu'il faut pour réparer la perte des humeurs, se trouve en disproportion avec cette activité vitale, ct que par conséquent l'appareil alimentaire animalise plus de substances que les vaisseaux lymphatiques ne peuvent en absorber; ensorte que la substance animalisće ici stagnante est déterminée à se transformer en un tout existant par luimême, ou bien en un ver; c'est pourquoi la prédisposition (opportunitas) à la formation des vers, ainsi qu'à beaucoup d'uutres maladies, pent être héréditairc innée ou bien acquise. On explique même par là pourquoiles enfans sont plus disposés que les adultes, les femmes plus que les hommes, à la formation des vers; car le système lymphatique des enfans et des femmes se trouve en général dans un état particulier de faiblesse. On observe souvent que des enfans, surtout quand ils ne sont pas nourris par le sein de leur mère, se développent très-lentement, malgré la quantité d'alimens qu'on leur donne. Cela ne dépend pas ordinairement du peu de substance nutritive contenue dans ces alimens, mais bien de la mauvaise élaborition de ceux-ci et du chyle qui s'en sépare, et gui n’est pas absorbé en sufisante quantité. Chez les enfans scrofuleux et atrophiés, la circulation libre dans les vaisseaux destinés à absorber les substances. 
nutritives, est interceptée ou an moins très-gênce; une grande quantité de suc uutritif reste par conséquent en stagnation dans le canal intestinal. Je comprends sous le nom de suc nutritif non-senlement celui qui s'est formé dans l'estomac anx dépens des alimens, mais encore son mélange avec les hımeurs propres du corps, en un mot un suc déjà animalisé, qui se prête facilement à la formation des vers, si toutefois il u'est pas rejeté par les selles conjointement avec les substances qui ne sont pas propres à la nutrition; aussi ces enfaus serofuleux et atrophiés sont-ils très-souvent, comme l'expérience nous le clémontre, sujets aux vers.

Cette manière d'envisager le mode de formation des vers intestinaux me semble s'approcher un pen plus de la vérité que l'opivion des Aëtius ${ }^{x}$, des Paul d'Egine, des Riolan et des Cabucinus; ces auteurs croyaient que le ver solitaire n'était autre close que la membrane interne détachée des intestins grêles et transformée en un corps vivant. On rencontre encore aujourd'hui des liypothèses de la même force.

Si cependant les vers intestiuaux se produisent fréquemnent dans les conditions qque nous venons de mentionner, il ne suit nullement de là qu'ils doivent se former absolument partont où ces conditions se trouvent réunies. Une pareille assertion aurait même l'expérience contre elle, car toutes ces circonstances ont quelquefois lieu, il y a même encore sonvent tous les sýmptômes par lesquels on se croit ordinai-

- Tetrabiul. III, serm. I, cap. xL, p. $5_{97}$. 
rement en dtoit de pouvoir présumer la présence des ver's, et cependant il n'y en a point.

Pour que la génération primitive des vers ait lieu, il faut admeitre l'action de deux agens (factor), l'un que nons pouvons concevoir, on peut l'appeler agent matéricl, et l'autre dont nous ne pouvous pas nous laire d'idée, que nous ne connaissons pas, et qu'en attendant je demanderai la permission de nommer agent spirituel. La coopération simultanće de ces deux agens est absolument indispensable lorsqu'il doit se développer, de la matière animalisée sans forme, un nouvel animal. Vouloir approfondir la nature du second agent, qui n'est autre chose que l'esprit vivifiant de l'univers qui domine déjà dans la substance animalisée amorphe, mais pas cncore au degré de tension nécessaire au développement de la vie individuelle, serait une vaine entreprise pour l'espèce humaine tant ciu'elle restera ici bas. Nous ne connaissons sir présence que par ses effets, mais nous savons qu'elle donne lieu à des conditious sans lesquelles, quoique l'autre agcont puisse exister, la matière animalisée ne peut s'individualiser, et c'est probablement le cas dont nous venons de parler.

Nous pouvons compter parmi les causes éloignces qui favorisent la formation des vers, une vie sćdentaire ec inactive, une habitation humide et non aćrée, une nourriture de laquelle se prépare un clayle trop nutritif, l'usage des corps gras, des farineux, du laitinge, etc.

Feuilićc croit pouvoir attribuer la fréquence des 


\section{$3\}^{2}$}

vers chez les sauvages de l'A mérique méridionale à un trop grand usage du sucre.

La vie sédentaire des femmes est probablement une des causes éloignées pour lesquelles elles ont plus souvent des vers que les hommes.

Un séjour prolongé dans une habitation lumide (où la suppression de la transpiration agit indirectement d'une manière nuisible sur les fonctions du système lymphatique propre au canal intestinal) angmente la disposition à cette maladie. Si de plus les alimens sont d'une nature à favoriser la production des vers, il y a alors tont ce qu'il faut du côté de l'agentmatériel pour faciliter la formation de ces animaux.

11 est connu que le pâturage dans des endroits marécagenx détermine souvent la formation des douves du foie chez les moutons; c'est pour cette raison que dans les établissemens bien administrés, on rend les bergers responsables des ravages que cette maladie fait dans un troupeau, car on peut alors présumer, presqu'avec certitude, que l'on a fait paître ces animaux dans des lieux marécageux, et qu'on leur a donné, surtout en hiver, des fourrages lummides et malpropres; il arrive cependant dans des années pluvieuses que la maladie vermineuse fasse de grands ravages parmi les moutons, sans que cela soit par la fante des hergers; l'emploi prompt de substances amères et fortifiantes, comme par exemple la gentiane, le calamus aromatique, etc., peut encore arrêter dans quelques cas les progrès de cette maladie.

De même que des maladies vermineuses peuvent 
régner d'une manière épizootique chez les animax (ear, outre la douve du foie, nous voyons souvent que les strongles (strongylus filaria, Ptud.), qui séjournent dans la trachée-artére et ses ramifications chez les moutons, agissent également daus beaucoup de cas comme une cause de maladie, et font périr une grande quantité de ces animaux, ainsi que j'ai eu occasion de l'apprendre très-souvent d'agriculteurs); de même, dis-je, ces maladies peuvent se montrer d'une manière cndémique et épidćmique chez les hommes; car si par exemple une disposition particulière de l'atmosplère peut contribuer et même oceasioner, dans quelques contrées, à certaines époques, des fièvres bilicuses ou d'antres maladies non contagieuses, nous pouvons égalenent admettre avec juste raison, que la formation des vers et des accidens qu'ils font naître peuvent être occasionés dans quelques cas par des causes générales, ou bien par des causes qui agrissent d'une manière endémique ou épidémique : les épidémies vermineuses par cosséquent ne doivent pas être regardées comme des ehimères.

Marie a observé à Ravennes et aux environs une épidémie de ce genre qui fut très-remarquable, et durant laquelle tous les malades rendaient des vers par haut et par bas.

Cependant il ue faut pas regarder toutes les maladies que l'on a qualifiées de vermineuses comme de véritables épidémies. Je ne puis pas non plus m'imaginer que la présence des vers ait pu occasioner 


\section{3.' SUR LES VERS INTESTINAUX}

des fièvres putrides régnantes d'une manière épidémique, comme Beruard est porté à le croire. 11 me paraît plutôt probable quệla maladie vermineuse a régné quelquefois d'une manière endémique dans les contrées qu'il habitait, ę qu'elle s'est montrée conjointement avec nue fièvre putride à l'époque dont il parle.

Bonnevault a donné sans doute à tort ì une fièvre putride ordinaire le nom de fièvre putride vermiǹeuse épidémique, par la seule raison que quelques personnes qui en étaient atteintes rendaient des vers. On observe avec juste raison, dans le Journal de médecine de Paris, où il est encore question d'une semblable maladie décrite par Dufour, que l'on aurait dû plutôt la désigner sous le nom de fièvre avec complication vermineuse. Les épidémies vermineuses ne peuvent par conséquent se montrer que dans les contrées où les vers doivent être regardés comme appartenant à une constitution épidémique; l'expérience nous prouve même qu'il y a réellement dés pays dans lesquels on observe des vers intestinaux beaucoup plus fréquemment que dans d'autres. Daquin prétend que l'on pourrait difficilement s'imaginer un pays où il $\mathrm{y}$ ait une plus grande quantité de vers intestinaux qu'en Savoie, auprès de Chambéry; et, d'après ce qu'il assure, on n'y olserve pas seulement ces animaux chez les pauvres, mais encore chez les riches, et on ne peut par conséqueut regarder dans ce cas-ci la mauvaise nourriture comme ayant fayorisé la formation des vers; anssi Daguin ne sait à quoi on pourrait l'attri- 
buer. Outre les habitans de beaucoup d'allires contrées, on regarde surtout ceux de la Hollande et de la Suisse comme très-sujets aux ver's intestinatux. L'explication de ce fait n'est pas facile chez les habitans de ce dernicr pays; on ne peut guère en effet l'attribuer à la disposition de l'air atmosphérique, qui du reste, dans ce pays montagneux, diffère tont à fail de celui de la Hollande. On ne peut pas non plus l'attribuer à la qualité des alimens; car la manière de vivre, au moins celle des grandes villes, ne diffère pas essentiellement de celle des pays limitrophes; peut-être doit-on attribuer la fréquence des vers intestinaux à l'usage du fromage, et principalementà celui du lait, non-seulement par la raison qu'on en hoil beauconp, mais parce qu'il est d'une si bonne qualité dans ces deux pays, et qu'on le vend sans être mêlé avec de l'eau? J'ai dit peut-être, parce que je régarde cette idée comme conjecturale, et je l'abandonnerai volontiers si quelqu'un pent m'expliquer d'une manière plus satisfaisante la fréquence des vers intestinaux chez les Suisses, et surtout celle des bothriocéphales, que l'on ne remarque presque jamais chez les habitans des pays voisins.

Parmi les Allemands et la plus grande partie des habitans de la France, chez les Italiens et même chez les Tyrolieus, on n’observe en géuéral que le tænia, tandis qu'un véritable Suisse, c'est-à-dire celui qui est né d'une mère suisse, n'a peut-être jamais été incommodé par cette espèce de ver. En Russie et en Pologne on ne rencontre que le bothriocéphale, et 
M. Rudolphi assure qu'il u'a reç que des tæuias de la Suède. Cette deruière circonstance pourrait bien être attribuée à une certaine particularité de nation, ou bien à la différence d'origiue qui existe entre le peuple russe et celui de la Suéde. Mais d'où vient que les Suisses et les Russes sont sujets aux mêmes vers? c'est un problème qui probablement ue sera pas résolu de long-temps.

Chez les Hollandais on pourrait peut-être avec raison attribuer la fréquence des vers à la disposition particulière de l'air atmosphérique et du climat, qui ont déjà une si grande infuence sur le tempérament de ce peuple, mais on aurait tort de vouloir l'attribuer a l'usage fréquent du poisson. D'après M. Rudolphi, d'autres habitans des côtes mangent également beaucoup de poisson, et ils ne sont pas extrêmement sujets aux vers.

Feu le professeur Reinlein" a été pendant dix ans médecin de chartreux, qui ne mangrent ni viande vi lait, et qui se bornent en général à l'usage du poisson; cependant il n'a vu aucú de ces moines être incommodé par le bothriocéphalę. Les plus a nciens de ces pères ne se rappelaient pas non plus avoir counu un de leurs confrères attaqué par un ver de cette espèce; du reste la qualité de la nourriture peut assurćment contribuer beaucoup à la formation des vers; Reinlein a même rapporté quelques faits très-remarquables sur ce sujet: voici ses paroles ${ }^{2}$ : "J'avais à

${ }^{x}$ Uebersetzung , p. 25.

${ }^{2}$ Ibid., p. 2 r. 
traiter, il $y$ a quelques années, un cólibataire de soixante-quatre aus, quii menait une vie très-r'égulière: aussi avait-il fort bonne nine, et il jouissait d'une santé parfaite. Lorsque je fus appclé chez lui, sept mois s'étaient à peu près écoulés depuis l'époque à laquelle un de ses anis lui avait donné le conseil, bien malà propos à la vérité, de changer sa manière de vivre, vu son âge avancé, et de faire usage de läitage de préférence à toute autre chose. Ce vieillard suivit ce conșeil, et supporta même pendant plusieurs semaines ce changement de nourriture sans en éprouver le noindre malaise; mais bientôt après il commença à sentir, surtout après le dîner, une plénitude dans le bas-ventre, une gêne vers la région précordiale, des palpitarions de cœur, et une diminution d'appétit; sa mine ordinairement bonne șaltéra, et il éprouva en outre souvent des nausées. En arrivant chez ce vicillard, on m'informa de tout ce qu'il avait éprouvé depuis son changement de nourriture. Je trouvai les hypochondres et la région ombilicale très-tendus. Je conclus alors de là que j'avais à combattre un embarras gastrique, et je prescrivis par conséquent une médecine composée de cing onces de la potion purgative ordinaire de Vienne, avec addition de six gros de tartrate de soude. Le lendemain j'allai le voir de nouveau. Le domestique qui servait déjà depuis trentedeux ans ce vieillard, m'attendait avec impatience pour me faire voir la grande quantité d'excrémens, mêlés, selon lui, de graines de citrouille ou de melon, que son maître avait rendus. Je demandai à ce domes- 
cique šil en avait déjà observé une antre fois clans les déjections. Il we répondit qu'il n'y avait trouvé rien de semblable depuis un grand nombre d'années, excepté il y avait deux ou trois semaines, éporque it liaquelle il en avait vu des traces par-ci par-là.

"Je dirigeai en conséquence par la suite mon traiteunent contre le tania, et je fus assezheureux pour le fiire évacuer entièrement au bout de sept jours. Ce vieillard a repris depuis son ancienne manière de vivre, et il jouit maintenant d'une santé parfaite. Peut-on s'imaginer, continue Reinlein, que le germe de ces vers ait pu rester plus de soixanie ans dans le corps de cet homne avant de se développer?"

Le même auteur m'a communiqué une observation non moins intéressante sur ce même sujet. La voici : Je connais une dame (ce sont les paroles de Reinlein) depuis plus de trente ans, qui a encore fort bonne mine, et qui a mis au monde douze enfins, lont six du sexe masculin, et six du scxe féminin. Chaque fois qu'elle fut enceinte d'une fille, elle éprouva toujours une envie irrésistible de manger de la crême et des alimens farineux, mais chaque fois clle fut aussi incommodée par les symptômes qui indiquent ordinairement la présence des vers, et clle évacua réellenent des ascarides de temps à autré jusqu’’̀ la fin de la gestation. Les mêmes symptômes n'avaient pas licı lor'squelle était enceinte d'un enfant mille; elle avait alors an contraire le plus grand dégout pour les alimens que nous venons de mentionner. Six accouchemens terminćs avec de pareilles cir- 
consiances lui avaient appris à prédire exactcment le sexe du foetus qu'elle portait dans son sein dans les grossesses suivantes. $»$

Quoique ces deux observations pussent nous engager à regarder un trop grand usage du lait et des farineux, comme une cause prédisposante à la formation des vers, nons ne pouvons cependant considérer ces substances que conme un des agens, ou plutôt que comme la moitié de l'un des agens, c'est à dire du matériel; mais il reste encore à considérer la constitution du corps; car ce n'est que lorsqu'clie existe à la fois avec une cause prédisposante que l'agent matériel peut ètre complet pour la production.

Un plat de pois ou de lentilles avec lequel un ouvrier assouvit sa faim, ne donne pas moins lien aux flatnosités, que la purée de pois ou de lentilles de laquelle un hypocondriaque ne fait que goûter; cependant le premier supporte ces substances sans éprouver le moindre dérangement, tandis que le dernier en est tellement incommodé, par le développement de quelques pouces cubiques d'air, 'qui par hasard ne tronvent pas aisément une issue, qu'on croirait qu'il va rendre l'ame. En général les alimens agissent de même sots le rapport de la production des vers, c'està dire que leurs effets sont différens sur différens individus. Les paysans du 'Tyrol ne souffrent pas souvent de la présence des vers, autant que je me le rappelle, et cependant ils ne mangeut de viande que quatre ou cinq fois dans toute l'année. La nourriture des forcats daus la maison de correction de 
Vienue consiste en farineux et légumessecs, mais ces hommes sont en même temps obligés de travailler constamment, souvent en plein air, et du reste on a toujours soin de faire renouveler l'air, autant que cela est possible, dans les lieux qu'ils habitent. M. de Guldener, qui fut médecin en chef de cette maison pendant quatorze ans, m'a assuré que ces hommes étaient très-rarement incommodés par des vers intestinaux.

Le lait et ses préparations, telles que le beurre, le fromagc, etc., seraient alors, comme nous l'avons remarqué, les seuls alimens que l'on pourrait considérer, de préférence, comme cause occasionelle, ou lien comme ofirant plus de matière propre à la formation des vers que beaucoup d'autres; mais le lait doit être anssi regardé, parmi les alimens provenant du règne animal ou végétal, comme celui qui contient le plus de substance nutritive. La formation si subite de vers daus le fromage paraît venir à l'appui de cette opivion, que je ne regarde, du reste, que comme une supposition, et non pas comme une preuve de ce que je viens de dire.

Je suis cependant convaincu que l'usage d'alimens qui ne contiennent pas beaucoup de substance nutritive, ne favorise pas la production des vers viscéraux, encore moins celle des vers intestinaux, et je suis persuadé que ceux-ci se trouvent fort mal dans un corps animal qui éprouve un manque d'alimens.

Nous avons cxaminé près de deux cents carpes (cyprinus carpío, L.), et à peu près cinq cents tan- 
ches (cyprinus tinca, L.); ce ne fut que dans six de ces dernières que nous avons rencontré des vers.

Nous en avons bien également observé dans des carpes, mais ce n'élaiı que dans celles qui nous étaient parvenues directement du lac Neuside, dans lequel on les avait pêchées depuis peu, tandis que les carpes provenant du Danube n'en avaient point. Les tanches et les carpes qui servent à l'approvisionnement de la ville de Vienne son télevées daus les étangs; mais, avant de les porter au marché, on les conserve pendant long-temps daus des réservoirs placés dans le Danube, afin que ces poissons perdent le goût bourbeux occasioné parl'eau dans laquelle ils avaient vécu auparavant. Ces animaux mançuent de nourriture pendant qu'ils sont enfermés dans ces réservoirs, c'est pourquoi l'on ne trouve souvent dans lcur canal intestinal pas même de trace de cette mucosité si abondante dans d'autres individus de lenr espèce. Leur canal intestinal est comme lavé, tandis qu'il est, dans les poissons fraîchement pêchés daus le Danube, comme, par exemple, le barbeau (cyprinus barbus, L.), fortement enduit de mucosités; mais aussi ce canal cst-il souvent rempli de vers. On a conservé, il y a plusieurs années, quelques dorades de la Chine (cyprinus auratus, L.), provenant d'un réservoir de Schœenbrun, dans des bocaux de verre remplis d'eau pure. La plus grande partie de ces animanx mourut au bont de quelques jours. Chez un individu de ces deruiers, un échynorhinque (echinorh. claviceps, Rud.) s'était frayé une route, non-seulement it 
travers les parois du canal intestinal, mais encore a travers les muscles et les tégumens. Ce ver, après la mort du poisson dans lequel il séjournait, aura voulı saus donte se procurer de la nourriture au-dehors; mais, ne trouvant pas dans l'eau ce qu'il lui fallait, et voyant qu'il était arrivé à la limite de son propremoude, il se décida, probablement pendant que la partie postérieure de son corps était encore fixée dans celui du poisson, à y rentrer de nuuveau ; car on voyait qu'il avait essayé à se frayer un chemin du dehors dans une autre partie du corps que celle d'où il était sorti. Cet échynorhinque est conservéainsi attaché à la surface du poisson dans notre collection. Chez d'autres de ces poissons morts, des vers de la même espèce avaient seulement perforé le canal intestinal, et ils se trouvaient implantés aux parois internes de l'abdomen, ou bien à la surface extérieure des intestins. Dans lo calbinet impérial d'histoire vaturelle de Vienne, on nourrit, pendant tonte l'annce, beaucoup d'oiseaux de différens ordres ct espèces, dans le but de chercher particulic̀rement des. vers. 11 est rare de trouver dans ces oiseaux, surtout dans ceux qui ont été enfermés pendant long-temps, un ver intestinal, et cependant, malgré la peine que l'on se donne, on ne penı pas procurer à ces animaux une nourriture semblable à celle qu'ils trouveut en état de liberté.

On dit ordinairement que la production des vers est causée par l'usage d'alimens d'une mauvaise quallité. Je ne veux pas niercette assertion, mais je me permets seulement d'observer ici que l'on regarde 
souvent à tort des alimens comme mauvais, tandis qu'ils sont réellement bons; et, en effet, une sulsstance peut être excellente pour l'un, et être trèsmauvaise pour l'autre. Par exemple, les légumes secs et les farineux, qui conviennent en général si bien à la classe laborieuse, causent souvent toutes sortes de malaises, et favorisent même, dans quelques cas, la production des vers chez les personnes qui mènent une vie sédentaire, ou bien qui ont les voies digestives très-faibles; je crois aussi que les écolès modernes ont avancé à tort que les alimens tirés du règne végétal contiennent moins de substance nutritive que ceux tirés du règne animal. Les Tyroliens, race d'hommes extrêmement robustes, qui ne se nourrissent, comme nous l'avons dẻjà remarqué, que de végétaux, et qui ne mangenı dans toute l'année que quatre ou cinq fois de la viande, lèvent tous les doutes à ce sujet.

On a encore faussement prétendu autrefois que les œufs des vers étaient introduits dans le corps de l'homme et dans celui des animaux, par l'usage des fruits véreux, parce qu'on supposait que les excrémens déposés dans ces fruits par les larves qui y séjournent, étaient les œufs de ces animaux. Un pareil préjugé n'a pas besoin, ce me semble, d'être réfuté de nos jours.

Une fois que les vers se sont formés spontanément dans un corps animal, ils peuvent alors se régénérer et se multiplier par l'acte de la génération, quand même les causes qui avaient favorisé lcur développe- 
ment auraient cessé, par la raison que les véritables ver's intestinaux sont tous pourvus d'organes sexuels: néanmoins, il faut que les circonstances soient favorables à cette génération ; car nous voyons, par exentple, que les vers qui avaient auparavant résisté ì l'emploi des meilleurs remèdes, disparaisseut spontanément cliez les enfans, quand ils arrivent à un âge mûr ; ces aninıanx disparaissent aussi dans beaucoup de cas chez les hommes adultes, quand ils clanggent de climat ou de régrime. Il arrive également quelquefois que certaines maladies de l'homme déterminent la mort des vers, comme nous aurons occasion de le montrer plus loin par des exemples.

\section{CHAPITRE CINQUIEME.}

Du diagnostic de la présence des ver's dans le canal intinal, et des dérangemens qu'ils peuvent occasioner.

On reconnaît en général la présence des vers intestinaux aux signes suivans :

Le visage des personnes qui en sont affectées est changé; elles sont ordinairement très-pâles, leur teint est même plombé, cependant leur figurure s'anime souvent tout à coup, et on croit encore avoir observé que la rougeur se borne à un seul côté; les yeux perdent leur brillant, ils deviennent ternes, la pupille est élargie et les paupières inférieures sont ceruées par un cercle blcuâtre. 
Les malades ont le nez souvent enflé; ils y éprouvent une démangeaison presque continuelle, et telle qu'ils ne peuvent s'empêcher de le gratter ou dè le frotter; ils saiguen souvent au nez, et ils éprouvent de temps à autre un mal de tête accompagné d'un bourdonnement d'oreilles. I. langue est chargée; il s'accumule souvent beaucoup plus de salive dans la bouche que dans l'état naturel. L'halcine est fétide, surtout à jeun.

L'appétit est très-variable; tantôt il a l'air d'avoir disparu entièrement, et dans un autre moment les malades sont comme affamés. On observe encore chez eux des nausées ou des envies de vomir, et mênse des vomissemens d'un liquide aussi limpide que l'eau; des coliques souvent très-violentes ", et principalement dans la région ombilicale; des excrétions alvines glaireuses, et souvent teintes de sang; une urine trouble, sédimenteuse ou ressemblant à du lait ćtendu d'eau ; un ballonnement et une dureté du basventre; un amaigrissement général du corps. Le sommeil est troublé, et souvent accompagné de grincement de dents. Les malades sont en général paresseux, tantôt de bonne, et tantôt de mauvaise humeur ${ }^{2}$.

J'ai traité des personnes incommodées par des vers intestinaux qui éprouvèrent de temps à autres des coliques tellement forles qu'elles se ronlaient par terre; l'emploi des vernifuges efficaces a fait cesser cet état de choses et aidé à ramener la santé. (Note du traducteur.)

2 J'ai remarqué dans ma pratique particulière que des attaques 23. 
Courbon Perusel croit avoir observé que l'aphonie est souvent causée par la présence des vers, et Girandy nous assure que la cecité, la surdité, et le délire dérivent dans quelques cas de cette même cause. On remarque enfin plus rarement dans les matières vomies, mais plus souvent dans les matières stercorales, des morceaux de vers ou même des vers entiers. Je ne crois pas avoir besoin de faire observer à mes lecteurs, que tous ces symptômes ne se trouvent que très-rarement réunis dans un individu; il me semble aussi inutile de remarquer qu'aucun de ces symptômes, à l'exception du dernier, n'indique réellement et infailliblement l'existence des vers, et que chacun de ces symptômes peut aussi bien faire présumer d'autres affections, comme par exemple l'hydrocéphale, maladie qui se caractérise également par une pupille élargie, par des envies de vomir, par un abattement d'esprit, etc. ' Quand cependant plusieurs

de nerfs, des syncopes et la diarrhée avaient été provoquées par la présence de vers intestinaux ; les enfans quị en étaient incommodés se réveillaient en sursaut en poussant en même temps de grands cris. Des oxyures qui se trouvaient en grand nombre dans le rectum de trois hommes âgés de dix-huit, vingt et quarante ans, causèrent non-seulement des démangeaisons très-fortes à l'anus, mais encore firent naître les désirs vénériens jusqu'à porter ces hommes à se masturber. L'auteur, en parlant des oxyures, cite des cas où ces vers, qui s'étaient introduits dans le vagin, excitèrent également des désirs vénériens à un très-haut degré. Avis important pour le médecin. ( Note du traducteur.)

- On conçoit aisément que des médecins peu versés daus l'art de guérir aient pu se tromper dans le diagnostic de l'hydrocéphale 
de ces signes se trouvent réunis, et quand on n'a dı reste aucune raison de les attribuer à une affection idiopathique de la tête, mais plutôt à un dérangement dans les fonctions des organes du bas-ventre, on se trompera rarement si l'on en conclut l'existence d'une maladie vermineuse'; et même dans le cas où une

chronique, et le regarder comme une maladie vermineuse, ’̀ cause de la ressemblance de quelqués symptômes qui sont propres aux deux affections; mais il est inconcevable que des praticiens aient pu confondre, comme on en connaît des exemples, l'hydrocéphalc aiguë (encephalitis exudatoria, hydrocephalus acutus) avec une fière nerveuse ou bien avec une affection vermineuse. Mon ancien ami ct professeur le docteur Wendt s'exprime sur ce sujet (Voyez son ouvrage ayant pour titre : Die kinderkrankheiten systematisch darGestcllt, Breslau, 1822 , p. 197.) de la manière suivante : " Ia faculté de porter un diagnostic médical juste est un don d e la nature celui qui n'en a pas été doué sera toujours embarrassé dans ses jugemens, et les meilleures monographies des maladies ne peuvent pas non plus suppléer à ce défaut. Je ne peux m'imaginer comment un médecin habile peut confondre l'lydrocéphale aiguë avec une maladie de nerfs ou bien avec les accidens occasionés par la présence des vers intestinaux; et, comme cela a été rapporté par Henke, il est possible que quelques médecins aient pu commettre de semblables erreurs de diagnostic, mais on peut présumer avec raison que ces médecins n'étaient pas très-familiers avec les symptômes qui caractérisent si bien l'affection inflammatoire du cerveau. Je ne puis nullement concevoir comment la marche toute différcute d'une maladie de nerfs, ou bien les accidens causés par des vers, accidens qui ne sont jamais ni aussi violens ni aussi continus que ceux occasionés par l'existence d'une hydrocéphale aiguë, pourraicnt ĉtre confondus avec celle-ci. " (Note dutraducteur.)

- Si dans un cas douteux pareil le praticien ne prescrit à soıı ma- 
affection de la tête ne pourrait pas être méconnue, on fera toujours très-bien d'avoir égard au bas-ventre, c'est-à-dire aux dérangemens probables dans les fonctions de ses organes; car j’espère qu'aucun médecin ne me contredira, si je prétends que les affections de la têle sont occasionées dans beaucoup de cas par les affections ou dérangemens des vicères du basventre, et vice versa. Mais est-on toujours en élat de désigner exactement quelle est l'affection primitive? Quand même cela serait délerminé, nous sommes wéanmoins toujours obligés d'avoir égard aux denx affections, à cause des rapports mutuels qui existent réellement entre elles.

Je n'ai parlé jusqu'alors que de la maladic vermineuse et non pas des vers, par la raison que, malgré l'existence de presque tous les symptômes rapportés ci-dessus, malgré l'emploi continu de vermifuges les plus efficaces, on a vu des cas où l'on n'a pu faire rendre aucune trace de vers ni même en trouver dans le canal intestinal ouvert après la mort. J'entends, sous le nom de maladie vermineuse, un dérangement ou bien une disproportion dans les fonctions des organes destiués à la digestion et à la nutrition; pendant la durée de ce dérangement il se prouluit ou bien il s'accumule dans le canal intestinal

lade que des purgations douces, assurément on ne pourrait pas hlàmer son plan de traitement. Il n'en serait pas de même s'il ordonnait au hasard des drastiques ou bien l'huile empyreunatique de Chabert, dans le cas surtout ou le canal intestinal se trouverail dans un état inflammatoire. (Note du traducteur.) 
des substauces à l'aide desquelles il peut se former, dans des circonstances favorables, des vers; mais cependant il n'y a pas nécessité absolue que cette formation doive en résulter. L'accumulation de ces substances, en pareil cas, nc constitue que l'agent matériel propre à la production des vers; l'existence de ces animanx dans le canal intestinal ne forme pas par conséquent une maladie primitive; il ne faut pas même les regarder comne constituant une maladie (excepté dans quelques cas dont nous parlerons par la suite ); ils sont plutôt un prodnit de l'état maladif des organes propres à la digestion et à la nutrition, ou bicn ils sont le product d'un dérangement de l'équilibre et de l'activité réciproque de ces organés, ce qui peut alors occasioner tous les sympiômes rapportés plus haut, sans que pour cela la présence des vers doive avoir nécéssairenent lien. L'expérience a démontré en eflet fréquemment qu'une maladie, n'importe laquelle, ne se développe pas toujours dans un corps animal, quoiqu'il y existe des vers; cc fait n'est pas seulement démontré par l'évacuation de ceux-ci chez des hommes se trouvant bien ', mais il est encore constaté, principalement par l'existence d'une grande quantité de vers dans le canal intcsti-

"Il faut soigneusement distinguer se trouser bien ou plutôt se sentir bien, d'être bien, car très-sonvent les anomalies ou l'état malarlif des organes ne sont nullement senties. Par cela je ne s cus rien dirc de unureau aux módecins qui ont fait beaucoup d'autopsics cadaverigurus. 
nal des animaux qui ne sont pas morts par suite de maladie, mais bien qui ont été tués d'une manière violente; et cependant on ne remarque pas pour cela chez eux un changenent contre uature dans les organes, et ils n'ont pas non plus l'air de s'être trouvés dans un état de dépérissement pendant leur vie, ce que du reste nous avons déjà remarqué dans le premier chapitre de ce traité. Cependant il pourrait paraître à quelques personnes que je suis ici en contradiction avec moi-même, car d'un côté j'attribue la cause de la production des vers à un dérangement dans les fonctions des organes précités, et de l'autre je suis obligé de convenir que l'on remarque souveut des vers où l'on n'avait pas observé auparavant de dérangemens dans les fonctions. En réponse à cela, je commence par dire qu'il existe souvent dans le corps animal des lésions ou des dérangemens beaucoup plus considérables que ne sont ceux qu'y occasione la production des vers, et cependant ces dérangemens ne se font pas apercevoir clairement par une sensation désagréable ou maladive. Cet état de choses dépend, dans beaucoup de cas, de la constitution particulière, ou plutôt du degré d'irritabilité de l'individu; le cas suivant peu servir à confirmer cette assertion: J'ai soigné pendant plusieurs années un homme qui mourut par suite d'une paralysie des poumons. Je fis l'autopsie cadavérique, et je trouvai dans le rein gauche une pierre d'une grosseur considérable, et cependant sa 
présence ne s'était fait remarquer par aucun symptôme pendant la vie. Tous les anciens praticiens peuvent citer de semblables observations.

Mais la circonstance, que des vers sont fréquemment rendus sans avoir causé auparavant des dérangemens, et que, d'un autre côté, la production des vers fait néanmoins toujours présumer un état contre nature, quoiqu'il ne soit pas toujours senti, a engagé quelques naturalistes d'admettre que les vers sont quelque chose de salutaire, et qu'ils sont destinés à se nourrir des substances qui surchargent ou incommodent le caual intestinal.

Goeze était de cette opinion. Cet auteur s'est cru encore obligé de prouver que chaque être était d'une utilité plus ou moins directe pour l'homme, ou, plutôt, que tous les êtres avaient été créés seulement à cause de lui. Cependant cette assertion serait bien difficile à prouver; car nous trouvons toujours dans le canal intestinal, conjointement avec les vers, une surabondance de glaires, et il est même très-probable que la sécrétion des mucosités est augmentée par l'irritation que ces animaux y causent.

Abilgard présume bien que la production des vers s'opère originairement par suite d'une inertic ou inactivité du canal intestinal, mais d'un autre côté il croit que ces animaux réagissent d'une manière salutaire sur ce canal, en ce qu'ils augmentent ses mouvemens par l'irritation produite par leur succion.

Gauthiéri * affirme même que la (prétenduc)

3. Ourrage cité , p. 66. 
consommation des glaires est lit moindre des utilités yue le corps humain tire de la présence des vers. Daprès cet auteur, les mouvemens de ces animaux aiden tà mieux développer les poumons et à déprimer les intestins du bas-ventre; il ajoute que les enfans qui ont des vers se grattent souvent le nez, et provoquent par cela assez fréquemment des éternûmens, moyen très-efficace, selon lui, au développement des poumons, à la dépression des intestins, à la descente des testicules, à l'évacuation de l'urine, des matières stercorales eı mêne des vers. Lorsque Ganthiéri écrivit cela, il paraît qu'il n'avait pas réfléchi que si, dans sa supposicion, la présence des vers dans le tule intestinal était réellement aussi avantageuse, l'évacuation de ces animaux ne devrait pas par conséquent être regardée comme quelque chose d'utile.

Quand même les vers, considérés comme un produit vivant et individuel provenant d'une activité anomale, ne devraient pas être regardés comme aussi directement favorables au corps animal, on a cependant tort de les considérer comme des êtres des plus malfaisans, et comme les plus grands ennemis de la santé de ce corps, ce que Fortassin a cherché a soutenir dans sa dissertation. D'après ce médecin, il n'y a pas une maladie qui ne puisse être provoquée par ces animaux; il les cousidère en effet comme la cause des affections du cerveau, des ophtalmics, des maladies de poitrine, des vomissemens, des nausées, des éructations, de la gangrène, de la paralysic, etc. Ce médecin prétend également que les vers peuvent 
devenir une cause prédisposaute des maladies périodiques et spasmodiques, en un mot, si l'on en croit M. Fortassin, il n'y a rien au monde de plus nuisible et de plus pernicieux que les vers intestiuaux.

Marteau de Grandvilliers les regarde aussi comme la cause des apoplexies, des sueurs colliquatives, etc.

Beaucoup de médecins accusent encore ces animaux d'être la source d'un grand nombre d'autres maux; on ne rencontre guère, par exemple, d'ćpileptiques ou d'hommes affectés de la danse de Saint-Guy ou d'autres maladies nerveuscs, maladies pour lesquelles la médecine théorique et pratique éprouve si souvent des échecs, auxquels les médecins n’ìient pas donué quelquefois des vermifuges '. Si un de ces malades rend un ver, on peut dire dans quelques cas pour son malheur, un morceau de tænia, ou s'il en a rendu seulement dix ans auparavant, on regarde exclusivement les vers comme étant la cause de la maladie, et très-souvent on ne se doune plus alors la peine d'examiner si elle a été réellement déterninée par la présence des vers ou non.

- Ce n'est pas déjà si mal agir que d'avoir recours dans des cas pareils aux vermifuges, après que d'autres moyens thérapentiques ont été inutilement employés, quand même l'indication àt l'emploi de ces remèdeś ne paraitrait pas ćtablic d'une manière suffisante, car la présence des vers, comme il a été déjà démontré plus haut, a souvent lieu sans que l'on s'en aperçoive, et occasione maintes fois toutes sortes d'accidens plus ou moins graves. Un pareil plan de traitement dirigé avéc prudence, loin de pouvoir ĉtre nuisible aux malades, pent au contraire ĉtre couronnć dans bicu des cas d'un plein succès. (Note du traductcur:) 
Je citerai ici, dans le but d'éclairer cette matière; quelques cas, les uns observés par d'autres médecins et les autres par moi-même, cas où il est au moins très-problématique si ces animaux avaient été en effet la cause de la maladie ou même de la mort.

Courbon Perussel a rapporté dans le Journal de médecine de Paris plusieurs cas analogues : je me bornerai à citer les suivans. Le premier a été publié sous le titre : "Rapport de l'ouverture du cadavre d'un homme que l'on croyait avoir été assassiné, mais dont la mort a été probablement occasionée par la présence des vers intestinaux." Un homme de vingt-cinq aus et d'une bonne constitution, fut battu le 13 mars; les coups ne parurent pas avoir dérangé sa santé, car il continua ses travaux jusqu'au 19, jour où il commença à se sentir malade. Le 21 Courbon fut appelé : le malade, qui était au lit, avait perdu la parole, mais il conservait sa connaissance; il s'était plaint (comme on le rapporta à ce médecin) depuis le 19 et les jours suivans, d'un grand mal de tête et d'envics de vomir; l'on ne voyait à l'extérieur aucune. lésion sur la tête. Courbon lui ordonna une tisane rafraîchissante. Le troisième jour le malade mourut sans avoir repris la parole. Le cadavre fut examiné le 24. Excepté quelques légères contusions sur le dos, l'on ne trouva aucun dérangement ni à l'intérieur ni à l'extérieur de la tête ; les poumons et le cour étaient sains; les intestins du bas-ventre paraissaicnt également être dans l'état naturel. En ouvrant le canal intestinal, on vil beaucoup de vers longs et gros; ils 
étaient cntortillés ensemble dans un endroit où ils semblaient avoir obstrué le canal intestinal; Courbon en retira quarante-deux; la mauvaise odeur l'empêcha de pousser ses recherches plus loin; l'estomac n'en contenait cependant aucun, et il n'y en avait que très-peu dans les gros intestins, qui, du reste, n'étaient nulle part enflammés.

Courbon avoue, dans une note ajoutée à ses observations, qu'il n'a jamais examiné, dans les cas qu'il rapporte, la moelle épinière, et qu'il u'avait pas même ouvert les ventricules du cerveau du cadavre dont nous venons de parler.

Une autre observation du même médecin est intitulée : "Rapport sur l'ouverture d'une femme que l'on croyait avoir été assassinée par son mari, mais dont la mort paraît avoir été occasionée par la présence des vers. "Le cadavre de cette femme, ágée de vingt-un ans, avait quelques écorchures légères à la partie antérieure du cou, et une petite excoriation à la joue droite. La tête fut rasée, et l'on trouva le crâne, le cerveau et ses membranes sans lésions; les poumons et l'estomac étaient sains; ce dernier contenait des alimens à moitié digérés; le duodénum était également sain, mais le jéjunum était rempli de vers; dans plusieurs endroits ils étaient réunis en forme de peloton, et paraissaient obstruer le canal intestinal; Courbon en retira cent quatre; les autres intestins n'en contenaient point; aucune membrane n'avait l'air d'être dans un état inflammatoire. Courbon finit par conclure que la mort avait été causée par 
la présence des vers, et il présume que les excoriations que l'on remarquait sur la figure et sur le con avaient été probablement faites sur la femme par ellemême dans des mouvemens convulsifs. L'on ohserve encore cependant daus ce rapport que cette femme avait prić le juge de paix, quelque temps avant sa mort, de faire enfermer son mari parce qu'il la maltraitait. Le juge de paix lui ayant refusé sa demande, elle s'en alla fort tristement en disant qu'elle serait assassinée sous peu. Ne pourrait-on pas croire, ajoute Courbon, que l'état maladif de cette femme ait pu influer sur son imagination? Je le présume, dit-il, et ma supposition se base, du reste, sur l'axiome d'Hippocrate : Ubialiqua parte dolent, neque dolorem sentiunt iis mens agrotat. Quant à moi, je doute très.for t qu'un tcl rappor de médecine légale ait pu engager un juge criminel à regarder les vers conme cause de la mort de ces deux personnes.

Je ne crois pas non plus que beancoup de médecins soient tout à fait de l'avis de Courbon dans le cas suivant, qu'il a publié sous le titre de: "Mort subite probablement causée par la présence des vers." Une jeune fille de dix-neufans, bien portante et non menstruée, jouissait encore d'une bonne santê le 5 . avril, comme dans les jours précédens; le 6 avril, à onze heures, elle fuc saisie de frissons et commença à vomir; à midi elle perdit la parole et la faculté d'avaler. Courbon alla la voir à sept heures du soir : le pouls v'était pas trop fréquent, pas trop faible, et était assez régulier; la pupille était élargie et ne se con- 
tractait presque pas à l'approche d'une lumière. La malade ne parlait pas et ne répondait à rien. On voulut Ini faire prendre une cuillerée d'une potion éthérée, mais sitôt qu'elle̊ sentit le liquide dans la bouche, elle: s'agita avec violence, fit entendre quelques plaintes, pendant lesquelles elle semblait faire beaucoup d'efforts. Courbon ordonna un bain et des vermifuges, sitôt que la malade serait en état d'avaler. La malade ne prit pas de bain et elle mourut à neuf lienres dı soir , c'est-à-dire dix heures après le commencement de la maladie. Courbon pensait que cette fille avait succombé à une maladie vermineuse.

Un cas à peu près semblable a été rapporté par Krause'. "Une fille de treize aus, après avoir étć guérie d'une gale six mois auparavant, se plaignit qu'elle rendait de temps à autre des vers, et qu'elle se trouvait un peu incommodée. On lui administra des vermifuges pendant quelque temps, et un jour elle mourut subitement à midi pendant qu'elle était occupéc à coudre. A l'exception d'une faiblesse de la vue, elle n'avait éprouvé la veille de sa mort aucune incommodité allarmante."

M. Serres a publié une observation sous le titre : Affection vermineuse simulant la rage. Uin enfant de treize ans, du sexe masculin, fut mordu par un clien enragé; six mois après, le 2 septembre, tous les symptômes de l'hydrophobie se déclarèrent, et il mourut deux jours après. L'autopsie cadavérique prouva que le cerveau, la moclie épinière, les pou- Voyez préfacc, à l'ouvrage de Vandocvern. 
mons et le larynx étaient dans un état sain, l'estomac ne contenait rien d'extraordinaire, mais les intestins grêles étaient remplis d'ascarides qui obstruaient tout à fait leur cavité. Le nombre de ces animaux était très-considérable. MM. Serres et Bosquillon présument que l'on doit attribuer la maladie et la mort de cet enfant à la présence des vers et non pas à la morsure du chien. Ces médecins remarquent encore que l'hydrophobie se montre souvent peu de temps avant la mort.

Geischloger rapporte qu'un enfant scrofuleux rendit deux ascarides peu de temps avant de mourir. Cependant cela ne prouve pas, ce me semble, que ces vers aient été la cause de la naladie et de la mort.

Quoique je sois très-peu porté à regarder les vers dans les différens cas que je viens de citer comme ayant causé la mort, je ne puis cependant pas nier que ces animaux ou bien la maladie vermineuse n'aient souvent déterminé ou n'aient au moins beaucoup contribué à causer des accidens graves et même tout à fait extraordinaires.

Krause rapporte le cas suivant : "Je fis connaissance, il y a quelques années, d'un homme de trenteun ans, d'une constitution robuste, qui était trèssouvent obligé, depuis plusieurs années, et même encore à l'époque où je le vis pour la première fois, de rire aux éclats (cachinnum) malgré lui; il éprouvait en même temps à chaque accès un malaise dont il ne ponvait se soulager qu'en se couchant à plat ventre dans son jardin. 
Les médecins qu'il avait consultés jusqu'alors n'avaient pas supposé qu'il eut des vers, c'est pourquoi les médicamens qu'on Ini avait ordonués restèrent sans èffet. Cet homme me demandat mon avis. Après lui avoir adressé plusieurs questions, je lui fis entendre que je présumais chez lui la présence des vers; son teint était en effet pâle, et ses yeux étaient terues. Sa réponse fut qu'il n'avait pas été seulement incommodé par des vers dans sa jeunesse, mais qu’il en avait encore reudu il y a quelques anvées. L'usage de vermifuges très-efficaces l'a au moins débarrassé alors de tous ces accidens désagréables; mais je ne peux pas dire avec certitude s'il n'en a pas été incommodé de nouveau, car je n'ai pas reçu de ses nouvelles; il habitait en effet une ville assez éloignée de cclle où je résidais.

Parmi plusieurs observations très-curienses cilécs par Girandy, la suivante mérite surtout d'être raplportée : Un jeune homme de seize ans avait, entre autres accidens très-singuliers, cela de particulier, qu'il lui était impossible de marcher sur un corps quelconque, fût-ce même une feuille de papier. Chaque fois qu'il voulait vaincre cette difficulté, il tombait en syucope; des vermifuges qu'on lui administra opérèrent l'évacuation de plusieurs vers, et la guérison en esı résultée.

M. Hufeland "rapporte le cas suivant: Un homme tourmenté par les vers voyait, étant à jeun, pendant des quarts-d'heure entiers, tous les objets en

\section{${ }^{\prime}$ Juarnal Baad.1v , p. $25 \%$.}

$$
34
$$


junne; cepeudanı il n'avait aucun symptôme de jaunisse, et ses yeux étaient dans un état sain. Le malade fut débarrassé de cel accident par l'évacuation des vers.

Ackard a publié une dissertation (que je n'ai pas pu me procurer) où il est question d'au homme chez. lequel la présence des vers provoquait une véritable rumination.

Delisle fait mention d'une jeume persoune qui rendit spoutanément pendant une année entière des ascarides et des morceanx de tania; pendant tout ce temps elle ne pouvaiı supporter de musique ni vocale ni instrumentale.

Désarneaux rapporte l'observation suivante : "Un jeune lomme éprouvait des convulsions horribles, qui contimuèrent jusqu’à sa mort; il avait été également incommode par des vers. Un jour un accès convulsif fut par hasard calmé par de la musique vocale, et plus tard on s'est convaincu que ses convulsions diminuaient chaque fois qu'on jouait du violon."

D'après Hannæus, une petite fille de quatre ans, gui avait perdu la faculté de voir et de parler, fut rétablic par l'usage des vermifuges; et Hannes rapporte qu'une jeune fille de onze ans, qui ne pouvait plus parler ni marcher, fut guéric par l'usage du tartre stibié, qui produisit l'évacuation de vers intestinaux.

Marchal de Rongères a publié six observations de maladies vermineuses très-compliquées. Je prie mes lecteurs de lîcher de se procurer l'ouvrage dans icquel il en parle, et d'en croire ce qui leur plaira. 
Un malade qui a fourni matière à une de ces observations, éprouva les symptômes suivans : doulcur insupportable et engourdissement de tous les menbres, difficulté de parler et d'avaler, mal de tête violent et beaucoup de fièvre. Une saignce calına un peu le malade, mais bientôt après tous les accídens se renouvelèrent avec plus de violence, et ils fureut même accompagnés de convulsions. On pratiqua une seconde saignée sans en tirer aucun avantage; le malade ne guérit complétement qu'après l'usage du tartre stibié ordonné par Marteau de Rocquemont. Ce médicament fit évacuer beaucoup de bile et plus de trente vers, tant par liaut que par bas.

Remer a guéri deux personnes attaquées d'une amaurose, par l'évacuation des ascarides, et Rosière de Lachassagne a débarrassé un homme d'un étourdissement subit, qui avait augmenté pendant trois jours, par l'emploi d'une infusion purgative, composée de follicules de séné et de tamarin; elle fit évacuer au malade par la bouche deux ascarides, et tous les symptômes cessèrent sur-le-champ.

Kichard fait mention d'une diarrhée occasionée par la présence d'un tænia. L'usage des purgatifs, de la fougère mâle et de l'éther sulfurique a produit la guérison.

Thomassen à Thuessink, a guéri une petite fille scrofuleuse, de six ans, de la danse de Saint-Guy, par des remèdes qui firent évacuer beaucoup de glaires et de vers.

L.e même a observé, chez un canonnier de vingt

$$
2 \text {. }
$$


ans, la métamorphose remarquable d'une fièvre rinoridienne en une épilepsic. Il présuma chez cet homne la présence de vers intestinaiux, et ordonna l'électuaire vermifuge de Stoerk, qui en effet fit rendre par haut des paquets de vers, des glaires, et par bas beancoup de matières glaireuses, aprés quoi la fièrre reprit son ancien type; mais comme elle était toujours accompaguée de légers accès d'épilepsie, Thomassen ordonna de nouvean un purgatif, quelque temps apres une infusion de geoffrea, el ì la fu du quinquina en poudre. L'emploi de ces médicamens prodnisit une gुuérison complète.

Thomassen cite encore le cas suivant: "Un homme robustc el sanguin, âgé de vingt ans, avait des accès de manic, que ce médecin qualifie du nom de manic vermineuse. La mère de ce malade était également folle ei elle a continué de l'être jusqu'à sa mort. Ce jeune homme fut guéri par l'usage de la belladonne en pondre, dont il prit d'abord deux grains matin et soir, et plus tard jusqu'a huit grains. "Thomassen ne dit pas que son malade ait rendu des vers, et il ne s'explique pas nou plus sur ce quilui a faii présumer que leur présence fiut réellement la cause des accès de manie.

Le docteur Suck, à Wolmar en Livonic, a observé une rétroversion du globe de l'œil causée par l'irritation des vers; une jeune paysanne âgée de douze ans, fuclout à coup atteinte de maux de tête très-violens; cinq henres après élle tomba daus un délire furieux accompagne de convulsions qui semblaient 
devoir meure fin à scs jours. Elle se réveilla, vingtquatre hemres après, délJarrassée des douleurs; les cavités orbitaires étaient comne remplies de chairs crues (rohem fleische) et la pupille avait disparu; le globe de l'ueil s'était contour ué de bas en haut, de manic̀re que les parties qui reposent sur l'orbite étaient tournées, conjointement avec les muscles, par devant entre les paupières; la cornée et lit pupille étaient entièrement cachées sous la voúte orbiłaire. On présuma que la présence des vers étail cause de ces accidens, et on administra des vermifuges conjointement avcc des purryatifs. A près en avoir fait usage pentant trois jours consécutifs, la malade comuevęa a rendre quelques oxyures; elle en évacua encore davantage le quatrième jour, époque à laquelle les yeux éprouvèrent des contractions convulsives, et l'on aperçut de tempsen temps une petite partie de la conjonctive; Je sixième jour clle ne rendit pour ainsi dire que des vers; après cette évacuation extraordinaire, les yeux. reprirent leur position naturelle et la vue fut rélablie.

A près une évacuation semblable, d'à peu près trois cents vers, Sylvestre a vu cesser entièrement des convulsions violentes, qui ressemblaicnt à la danse de Saint-Guy.

Dufan a également guéri cette même affection par l'usage des purgatifs, qui firent rendre beaucoup de vers par haut et parbas; cependant il faut remarquer que cette maladie avait déjà cossé une fois d'ellemêne à une époque antéricure.

Monnich a traité un confant de deux à trois ans, 
qui, ayant au paravantl'air robuste et bien portant, fut atteint tout à coup d'une paralysie des extrémités inféricures, il était en même temps strabite de l'œil gauche, et toute sa figure était décomposée; après l'emploi de vermifuges, il rendit dix-huit ascarides avec une grande quantité de glaires, et sa santé se rétablit.

Delacroix a vu disparaître un vomissement presque continuel accompagné de hoquets et de convulsions, après que le malade eut rendu par la bouche sept ascarides très-longs.

J'ai observé moi-même les deux cas suivans: On me présenta, en 1816, un evfant de ueuf ans, du sexe masculiu, qui avait depuis deux ans des accès trèsviolens et très-fréquens d'épilepsie, il rendait en même temps des morceaux de tænia. Je fus assez heureux pour le débarrasser du reste de l'animal, et dès ce moment il n'eut plus d'accès d'épilepsie ${ }^{\text {. }}$.

Lne jeune fille de onze ans était tourmentée par une toux sèche et presque continuelle. Ayant observé qu'clle rendait des articulations de tæenia, on lui fit faire usage d’anthelmintiques; elle évacua un grand morceau de l'animal, et la toux se calma pendant deux mois, époque à laquelle elle reparut de nouveau. Une nouvelle évacuation d'un morceau de tæuia eut lieu, et la toux cessa encore une fois nomentanément. Cette fille éprouva encore par la suite les mêmes accidens trois ou quatre fois, jusqu'à ce qu'enfin je parvins, il

' Le même eufant est venu me voir le 4 février $\mathbf{1} 821$, il a toujours joui, drnuis mon traitcment, d'une santé parfaitc. $\left(B r_{0}\right)$ 
y à peu près huil ans, à délruire complétement son tienia, et depuis ce temps la toux n’a plus reparu.

Lepelletier attribue les accidens suivans à la présence des vers : "Une fenme de trente-six ans, d'une constitution cachecligue, fut subitement attcinte d'une doulcur violente au côté gatuche, accompañuce de toux sèche, d'une forte oplression, d'une soil' excessive, de naux de tête et de fièvre; sa figure était en nême temps trèsanimée; on lui fit une saignée, pnis on administra un vonitifet un purgatif qui Iui firent rendre par haut quelquess ascarides. C'est pourquoi on enploya des purgatifs et des vermifuges d'une uature luileuse, qui firent encore évacuer quelques vers avec beaucoup de matière glaireuse, ct la santé se rétablit." Je pense qu'on aurait dû appeler cette naladie pneumonie et non pas maladie vermineuse.

Sumcire attribua aussi la cause d'un point de côté à la présence des vers, par la raison que la mousse de Corse en avait fait évacuer quelques-uns; ce médecin remarque cependant qu'on avait fait précéder de quelques saignées l'usage de ce médicament; en outre il observe dans son rapport que la mousse de Corse est sonvent très-efficace dans le cas de coliques subites, quoiqu'il n'y ait pas de vers.

Il me serait facile d'auguenter le nombre des observations de ce genre tirées des ouvrages tant anciens que modernes, mais toutes ces obscrvations réunies ne prouveraient pas davantage que celles que je vicus de rapporter, linfluence nuisible des vers 


\section{$3-6$ SUR LES VERS INTESTINAUX}

sur le corps animal; d'autre part, tout médecin ima partial conviendra que dans ces exemples une accumulation de glaires et de matières fécales dans le canal intestinal, en un not un dérangement daus ses. fonctions, ou bien une disproportion de son activité, relativement à celle des autres organes, a pu donnerlieu, aussi bien que l'irritation appelée vermineuse, aux symptômes les plus singuliers.

Les faits que nous venons de rapporter parlent en faveur de cette supposition. Nous avons d'abord vu qu'une grande quantité de vei's séjourne quelquefois dans le corps animal sans se faire sentir en aucune manière. D'un autre côté, nous avons remarqué que tous lès symptômesà l'aide desquels on suppose ordinairement l'existence des vers, se trouvent souvent réunis sans cependant qu'on puisse en découvrir. Ces animaux sont eux-mêmes le produit ou bien le résultat d'un état morbides ou contre nature. Ainsi donc tout ce qui peat contribuer à la production des vers peut également devenir la cause prédisposante des accidens les plus extraordinaires. Combien de fois n'arrivet-il pas que la manie, l'hypocondrie et d'autres affections mentales sont le résultat d'un dérangement dans

1 Nathanicl Ramsey cite des cas par lesquels il paraît démontrer que quelques personnes sont en mêne temps incommodées de vers intestinaux et de crachemens de sang. Le mème auteur remarque qu'il est probable que ces denx affections sont dans u rapport mutuel. Voy. Medic. chir. transact. of London, vol. 1X, part. It, 18 8 , 1 $^{0} .5 .\left(B r_{\text {. }}\right)$ 
Ifs fonctions des organes du bas-ventre, naladies que nous parvenons cependant, dans quelifues cas, à guérir par la méthode évacuante, sans que nous voyons de traces de vers; quoiqu'il soit arrivé dans plusieurs des cas cités que l'évacuation de quelques vers fût suivie d'une diminution des symptômes graves, il ne résulte nullement de là que la présence de ces animaux fût la seule cause de ces symptômes. Lc cas ohservé par moi-même ne fournit pas non plus une prenve suffisante quele tenia fut la cause première de l'épilepsie; car ce ver fut détruit par un usage continu de mon huile anthelmintique, composée en yrande partie d'huile de térébenthine, substance qui en effet a été employée par les médecins anglais Pcrcival, Latham eı Philipps dans plusicurs cas avec le plus grand succès contre l'épilepsie, non compliquée de maladie verminense.

Qnand il s'agit de déterminer la cause d'une maladie, jengage les praticiens à ne pas attacher trop d'importance, sauf quelques exceptions, à la présence des vers el encore moins à une évacuation de ces animaux à une époque très-éloignée.

Lorsque j'étudiais à Jéna, feu mon ami le docteur Schleussner me fit faire la connaissance d'une femme hystérique qui avait rendu un jour, je peux dire pour son mallseur, un tænia; elle avait déjà cousulté beaucoup de médecins qui ayant tous dirigé leur plan de traitement contre ce ver, avaient employé inutilement toutes les méthodes comnues pour le faire sivacuer; elle me fit mème voir un verre de mercure 
coulanı qu'on lui avait adusinistré dans le nóme but; et quelle avisit rendu par bas. Je plaignis beaucoup celle pauvre femme, et je fus étonné de ce que l'on n'eut pas pu parvenir, après l'emploi dés médicnmens les plus efficaces, à la débarrasser de son ver. Maintenaul que jai appris à envisager un peu mieux cetle matière, jai raison de la plaindre encore davantage, et je ne m’étonne plus pourquoi les remèdes qu'elle avait pris n'avaient pas opéré l'évacuaion d'un tæuia; car je suis presque convaincu qu'elle n'en avait pas.

Je counais quelqu'un à Vienne qui ayant rendu un tenia vingt-cing ans auparavant, saus avoir éprouvé le noindre dérangement de santé, en fit part un jour a son médecin; celui-ci regarda cela comme une cliose d'une hatue importance, et il lui annonça yu'il voulait le soumettre à un traitement complet, alin de le débarrasser entièrement de son ver; ce dont cet homme n'avait ni le temps ni l'envic. Le traitement liut par conséquent remis à un autre moment. Le malade reçut tout-ì-coup ordre de se rendre à l'armée dans les Pays-Bas; pendant son absence de Vienne, qui a duré plusicurs années, il n'a plus peusé à son tæuia, et il n’en a pas même vu la moindre trace, il faut avoucr que ce füı un vrai bonheur pour lui d'avoir pu se soustraire a la guérison que son médecin lui avait promise avec tant d'empressement.

Le cas suivant mérite encorc d'être cité. Je fus consulıé par écrit, il y a trois ou quatre aus, sur l'ćtat de santé d'un prêtre qui habitait la Moravic. II 
élait dit dans le rapport, que cel homme avait toljours joui d'une sauté parfaite, jusqu'au momeut où il avait rendu un tænia il y avait trois ans. Depuis ce temps il avait essayé tous les remèdes connus proposés par des médecins et par des charlatans pour se débarrasser de son ver, mais aucun de ces remèdes n'en avait pas même fait rendre un seul norceau. Il était également dit dans ce rapport, que cet homme, jadis robuste, arait tellement maigri, qu'il ressenblait à un squelette couvert de la peau, et que sa faiblesse élait telle qu'il pouvait à peine se tenir sur ses jambes.

Je lui conseillai, comme on peut bien se l'inaginer, de s'abstenir sur-le-champ de toute espèce de vernifuges, étant convaincu que son mauvais état de santé ne provenait pas de la présence d'un tænia, mais bien de l'abus de vermifuges drastiques. Je ne puis pas dire si ce prêtre a recouvré la santé, n'ayant pas reçu depuis de ses nouvelles.

Quoique la présence des vers ne cause dans beaucoup de cas que très-peu ou même point de dérangemens (ce sont les paroles de quelques médecins), nous ne devons cependant pas nier d'un autre côté qu'une grande quantité de ces animaux ne puisse ubstruer d'une manière mécaniquele canal intestinal, et causer par cela des coliques nortelles; voilà ce donı on se plaint, et dont nous avons nêne déjà rapporté quelques exemples, c'est-à-dire où l'on se croyait en droil de présumer que les vers avaient réellement causé de pareils accideus: en voici quelques autres. 
Daquin nous a conmuniqué l'observation suivance : Un eufant du sexe masculin, de dix i douze ans, fut amené à l'hôpital le 14 novenbre. Il s'était déji plaint de coliques depuis plusieurs jours; on lui arlministra une polion huileuse vermilinge, qui lui fit rendre des matières glaireuses et jaunâtres pa. hatut et par has. Le 15 les coliques étaient augmentécs de heaucoup, il vomissait tout ce qu'il prenait; il n'y avait pas de traces de hernie; le bas-ventre élait souple et aplati. Le malade sentait une douleur violente audessous de la région du foie, quand ou y exercail ume pression. L'huile d'amandes douces, gu'on lui avait ordonnée, fut vomie. sur-le-champ, et les souffrances du malade continuèrent. Dans l'aprèsnuili il ava it l'air d'avoir perdu complétement la raisou; il sauta de son lit, ôta sa chemise el se roula ì terre, etc.; il ne ponvait rien garder dans son corps. L.e 16 il étail sans pouls, sans conuaissauce, dans un état comateux et complétement amaurotique. II succomba à une heure aprés midi. Son corps était desséché; l'épiploon avait presque entièrement disparu. On trouva dans l'estomac un ascaride trèsyros, de la longueur de l'avant-bras; une des cxtrémités de cel animal se trouvait bien haut dans l'oesoplage, tandis que-l'autre étail descendue très-bas dans le duodénum. Cet intestin, ainsi que les autres intestius grêles et le coecum, étaicnt tellement remplis de vers, qu'ils paraissaien y avoir été mis par force; l'on en trouva également quelquies-uns dạns les gros 
intestins. Malgré la présence d'me si grande quantité de vers, il n'y avait pas de traces de phlogose dans le catnal intestinal. La tête de cet en fant ne fut pas ouverte."

Au premier abord on serait tenté de croire que l'obstruction causée par celte grande quantité de vers fut l'unique et principale canse de la mort de cet enfant, mais l'on doit prendre en considération que ces vers n'ont pas pu se produire pendant nne nuil, et si nous voulions même adınettre que cela ait cu lien, ces animaux n'auraient pas pu, dans tous les cas, atteindre leur développement complet assez suhitcment pour obstruer totalement les intéstins. Du reste, si l'obstruction avait été la principale canse de la mort, chose que nous ne pouvons admettre, par l:t raison que cet enfant avait encore eu une évacuation le 14 , une inflammation et la gangrene auraient dit alors avoir lien aupararant, comme on le voit en parcil cas, mais il n'y avait pás même la moindre trace d'inflammation dans les intestins du cadavre. Pendant la vie, cet cnfant pouvait en outre supporter une pression sur le bas-ventre, qui était même aplati, par conséquent les intestins étaient susceptibles d'une distension beaucoup plus considérable.

Il résulte de l'ensemble de cette histoire, que ni la grande quanité de vers existant sans doute depuis long-temps dans le corps du malade, comme cela paraît être prouvé par sou extrêne maigreur, ni cnfim l'obstruction apparente des intestins, attribuéc à la préseuce du grand nombrede ces animaux, ne peuvent être regardés avec certitude comme la causc des 
accidens et de la mort decet enfant. 11 me paraît trèsprobable que le ver que l'on a trouvé dans l'estomac a provoqué les vomissemens; cependant il reste encore à savoir si ce ver n'avait pas pris la position dans laquelle on l'a reucontré seulement après la mort de l'enfant ou peu de temps auparavant; je nue plais à admettre cette supposition par la raison que cet animal aurait dû être déplacé et arrachéde sa position par les efforts que l'estomac fait ordinairement en pareil cas dans le vomissement. Du reste, je ne conçois pas comment la présence d'un seul ver dans l'estomac aurait pu irriter cet organe au point de causer une mort subite; il est toujnurs à regretter que l'ou n'ait pas ouvert la tête du cadavre.

Le cas suivant, rapporté par Campedou, paraît pronver davantage qu'une mort subite pent être occasionée par une très-grande quancité de vers. "Un homne succomba après avoir éprouvé pendant vingtquatre heures des coliques violentes. L'autopsie calavérique fut faite, et l'on trouva l'intestiu coecum et une partie du colon remplis et entièrement distendus par un peloton d'ascarides (il y en avait trois cent soixante-sept de la longuenr de cinq à six pouces), de manière que ces intestins ont dît s'enflammer et tomber en gaugrène."

Les observations suivantes prouveront cependant qu'une grande quantité de vers peut séjourner dans le canal intestinal sans causer des accidens graves.

J'ordonnai un jour à un honnetier d'A polda (à trois lienes de Jéna) l'électuaire vermifuge de Stocrk; 
quelques jours après je fus le voir, et il me conduisit dans son jardin afin de me montrer les matières stercorales qu'il avait rendues et anxquelles était mêlée une quantité extraordinaire de vers. Si je ne les avais vus de nes propres yeux, je n'aurais jamais cru gu'un aussi grand nombre ait pu se trouver à la fois dans le corps d'un homme, et cependan le malade, autant que je me le rappelle, n'en était pas très-incommodí. $\mathrm{Si}$, a cette époque, il avait succombé par hasard i la suite d'une colique violente occasionée par le refioidissernent, par l'usage d'une mauvaise bière, etc., on aurait, sans le moindre donte, regarlé les vers comme la cause de sa mort.

Dall' Olio raconte qu'il a rendu par la bouche, dans l'espace de quinze jours, quatre cent einquante ascarides qui étaient de la longueur de la main *

Marteau de Grandvillers a connu un soldat die vingt-trois ans qui a évacué trois cent soixante-sept ascarides dans l'espace de six jours.

Il résulic de ces faits, que sil'on trouvait une grande quantité de vers réuvis dans une partie quelconque du canal intestinal, l'on porterait un jugement au moins très-ilasardé, en les regardant comme ayamt été réellement la cause de la maladie ou de la mort. Je crois aussi que les accidens observés par Murạlı sur une femme en couche attaquée d'un iléus violent provenaient de sa hernie ombilicale et erurale, et nou pas de vers intestinaux. Cette fenme se trouva soulagée après qu'elle eut fait usage de bains tièdes, et

1 Memorie, p. 215. 
immédiatement après elle rendit plusieurs vers par hant et par bas.

Lion ne peut pas nier que les vers ne deviennent beancoup plus incommodes quand ils sortent du lieu que la nature leur a assigné, et quand ils se rendent dans d'autres organes. Dans le cás où les ascarides, par exemple, qui, cliez l'homme, vivent dans les intestins grêles, se portent à l'estomac, ils y causent alor's beaucoup d'accidens désagréables, lesquels continuent jusqu'ì ce que l'cstomac, par un effort considérable, les ait rejetés. Dans plusicurs des cas ci-dessus rapportés, il y avait des vers dans l'estomac, et il est très-vraisemblable que Palmer se trompe en croyant qu'ils peuvent séjourner daus l'estomac longtemps sans donner lieu à des accidens.

Désarneaux a observé un accès très-grave d'épilepsie qui, d'après la relation de çet auteur, avait été produit par un seul ascaride qui s'était glissé dans l'estomac .

Cependant il n'est ici question que des vers intestinaux de l'homme, car il y a chez quelques animanx des vers que l'on rencontre toujours uniquement dans l'estomac; il esi très-probable qu'ils s'y dé-

- Dr. Joseph Klapp Leber die W'airmer des magens. Voyez The American recorder I'hilatelphie, vol. $\mathrm{un}, \mathrm{n}^{\mathrm{0}}$. In, april $\mathbf{1} 320$. Ibid., Haguer, Wiirmer, in der lcber einer salhnsinnuigen (rers trowés dans le foic d'une femme aliénée). Celte femme arait également des lydurites daus le plexus choroide ct d'antres désorganisations dans le cerveau. Foyez Zeilsinift fur phy sische Aeräte pon Aasse. 4 tes heft is 8 , Leipziy, page $51-4$. ( $B r$.) 
veloppent aussi, et qu'ils ne causent en cet endroit pas plus de gêne que les autres vers dans les intestins, si long-temps qu'ils ne quittent pas l'endroit que la nature leur a assigné. Il vit en effeı dans l'estomac de la souris un ascaride assez gros, et j'en ai trouvé une fois vingt-trois réunis, une autre fois vingt-quatre qui, mis dans l'eau, et conservés après dans l'espprit-de-vin, causent l'étonnement de tous ceux qui les voient, parce qu'on ne pent pas comprendre comment tous ont pu trouver place dans une cavité aussi petite.

Quand les vers se portent d'un endroit du canal intestiual dans un autre, ou, pour mieux dire, quand ils changent leur siégre naturel, il est à présumer qu'ils ont été forcés à se déplacer par des circonstances extéricures indépendantes de leur volonté.

Quand les sucs contenus dans le canal intestinal, dont ces animaux se nourrissent, changent de nature, quand les intestins se contractent spasmodiquement, il est probable qu'ils ne se trouvent pas à leur aise, et qu'ils tâchent alors de découvrir un endroit qui leur soit plus convenable. S'ils se portent par hasard de bas en haut, ils arrivent, a leur grand désavantage, dans l'estomac; car cet organe n'étant pas accontumé à la présence d'un corps de cette nature, ferat tous ses efforts pour le rejeter au dehors.

Mais on a certainement tort d'admettre gu'ils perforent les intestins, el que par là ils causent la mori. Félix Plater ainé, dont les idées sont conformes aux nôtres sur la formation des vers intestinaux, a déja 
douté de ce fait, par la raison qu'il fandrait des organes particuliers, tels quine trompe pointue ou bien des dents, organes qu'il n'a pas pu remarquer sur ces animaux, pour opérer une semblable perforation, et il ajoute qu'une simple succion ne peut pas l'effectuer.

Bianchi et Wichmann" sont de la même opinion, et le dernier range les lumbrici effractores parmi les fables de la pathologie. M. Rudolphi, qui a fait des recherches très-étendues à ce sujet, a prouvé que les vers intestinaux de l'homme ne peuveut pas perforer les intestins ni les tégumens en général, et il remarque que tout lomme impartial peut se convaincre qu'ils manquent des organes nécessaires pour le faire. Il a, qui plus est, démontré que des espèces de vers étrangères à l'espèce humaine, comme les acanthocéphales, qui peuvent parvenir à perforer l'intestin, ne produisent pas même d'inflammation dans cet organe, sans doute à cause de la lenteur avec laquelle la perforation a lieu ?

Cependant, comme on parle souvent de semblables

- Ourrage cité, p. 84.

2 On ne doit pas regarder l'exemple cité par moi plus haut concernant les dorades (cyprinus auratus) comme une preuve contre cette assertion. Quoiqu'il y soit dit que l'on a trouvé dans le bocal plusieurs de ces poissons morts et perforés par des échynorhinques; car on ne peut pas admettre que ces poissons soient morts par suite de cette lésion, je crois plutôt qu'ils avaient succombé, ainsi que les échynorhinques mêmes, par le manque total d'alimens, ce qui met une fin naturelle à la vie dans tous les animaux. 
perforations, je vais citer seulement quelques exemples ( car si je faisais mention de tous je remplirais un volume) afin de mettre mes lecteurs à même de juger quel degré de confiance ils méritent.

Gramaun raconte le cas suivant: Une femme sentait une tumeur de la grosseur d'une noix dans la région inguinale; un chirurgien y appliqua pendant quinze jours des émolliens; à la fin la tumeur s'ouvrit, des matières fécales sortirent d'abord, et un instant après cinq vers de forme ronde, qui furent suivis successivement de beaucoup d'autres. Gramann, que l'on avait appelé en consultation, déclara sur-le-champ cet accident comme étant le résultat de la perforation des intestins causée par les vers intestinaux. L'emploi des amers produisit, dans l'espace de quatre jours, la sortie de plus de cent vers de la longueur d'un pied, et pour chacun d'eux la malade éprouva dans la plaie une sensation semblable à celle d'une morsure ; néanmoins elle fut complétement guérie dans l'espace de trois semaines. Gramann remarque qu'il avait déjà observé un cas analogue, mais où il ne sortit que trois vers.

Vollgnad rapporte l'observation suivante : Une femme qui avait déjà éprouvé des symptômes qui avaient fait présumer la présence des vers, et dont elle avait en effet vomi quelques-uns, fut tout à coup effrayée par uné sensation douloureuse, qu'elle éprouva en tendant fortement le bras en haut, pendant qu'elle travaillait; il lui sembla que quelque chose s'était détaché de l'ombilic, et s'était porté vers 25. 
la région inguinale. Cette femme fut obligée de garder le lit, et elle s'aperçut bientôt qu'il se formait à ce dernier endroit une tumeur qui augmenta peu a peu jusq rn'ì la grosseur du poing. Les douleurs étaient contimuelles et semblables à la sensation qu’on éprouve quand on est pincé. Elle croyait aussi remarquer d'une manière distincte que quelque chose de vivant se renuait. La tumeur creva le troisième ou quatrième jour, et il sortit une grande quantité d'une matière très-fétide dans laquelle se trouva un ver qui resscmblait à un lombric (lumbricus), d'un pied de long, et pourvu d'une trompe. Pendant trois semaines leancoup de matière purulente s'échappa de la plaie, ct la fenme succomba." Cependant je ne pense pas que sa murt puisse être attribuée à la présence du ver.

Schelhammer tâche de réfuter l'opinion de Plater, dont il a été fait mention plus haut, par l'observation suivante: Une paysanne de quarante-six ans avait éprouvé depuis long-temps des coliques violentes. II se forma dans un de ses hypocondres une tumeur qui s'enflamma et tomba en gangrène. A près la rupture de la tumeur il en sortit d'abord du pus très-fétide, ct, ensuite, c'est-à-dire dans l'espace de huit jours, vingt-quatre ascarides plus ou moins gros. Quelquesuns de ces vers se présentèrent par la tête, d'autres, étant ployés, se montrèrent par lc milieu du corps, ce qui permit de les extraire avec quelques précautions dans la forme qu'ils avaient. De l'huile que l'on avait domnée à boire à cette fenme sortit peu de temps après par l'ouverture. La plaie se cicatrisa bien une 
première fois, mais elle s'ouvrit de nouveau, et lorsque Schelhammer fit la conuaissance de cette fonne, cet état de choses avait déjà duré dix-huit ans.

Si Schelhammer conclut de tout cela [u'il y at cu réellement dans ce cas-ci perforation des intestins, personne ne pourra nier ce fait, mais il n'a pas prouvé que cette perforation avait été produite par les ver's que la malade avait évacués, et la circonstance rapportée par lui-mênıe, que ces animaux s'étaienı présentés par le milieu du corps, prouve plùtêt contre que pour son assertion.

Tous mes lecteurs approuveront sans doute Marcus d'avoir voulu garantir une femme malade, alleinte de la danse de Saint-Guy, du soupçon qu'on avait qu'elle était possédée du diable; mais c'est une chose qui restera tonjours problématique, de savoir si les quasante un ascarides qu'elle avait reudus en partie avaut de mourir, et dont le reste fut trouvé dans les iutcstins sphacélés, avaient été la cause de sa maladie ct de sa mort.

Lüdücke donne, à tort, ce me semble; à un cas particulier qu'il cite, la dénomination de perforation des intestius causée par des vers; car depuis plusieurs mois la tumeur avait déjà crevé dans la région inguinale, et ce ne fut que douze jours avant la mort de l'individu qu'un ver apparut daus la plaie.

M. Godot me parait avoir commis une crreur de mème sorle. Un abcès considérabie au foit fint onvert; au huiticue pausement on aperent daus la plaie un asearile qui lut suivi de quelques autres. Godot 
croit que ces vers avaient perforé l'estomac; mais il est plus vraisemblable que c'était le pus qui avait causé cette perforation.

Hünerwolf a publié l'observation suivante sous le titre : De ileo lethali a vermibus. Une femme de trente ans vomit, après avoir éprouvé de fortes coliques, scize ascarides fort gros, et mourut bientôt après. Les intestins grêles étaienı sphacélés et percés a plusieurs endroits. On ne dit cependant pas dans l'histoire que l'on y ait trouvé des vers.

Dans le cas rapporté par Fischer ${ }^{\mathrm{x}}$, oü un ascaride fut trouvé dans la cavité pelvienne, mais où un autre pendait à moitié hors du cœcum, il y avait également inflammation des intestins, et les trous dans l'intestin avaient été probablement causés par la gangrène dont cette partie se trouvait déjà frappée.

Wichmann affirme en effet qu'il a souvent trouvé des trous d'une forme ronde dans les intestins de vieillards chez lesquels il ne s'était pas manifesté de traces de vers ni pendant leur vie, ni après leur mort.

J'ai prévenu mes lecteurs dans le commencement, que je ine bornerais à leur faire connaître un petit nombre des cas dans lesquels on a cru pouvoir attribuer la mort à la perforation des intestins par les vers; les personnes qui voudront prendre connaissance de plusieurs autres cas semblables peuvent les trouver dans les onvrages de Lebeau, Borellus, Girard, Goekel, Heister, Marteau, Moulenq, Offred, Schmidt, Tulpius, etc. , et j'abandonne à leur juge-

- Fon. hydatig., p. 4 o. 
ment à décider si c'est a vec raison que l'on a regardé les vers dans les cas cités comme ayant causé la mort. Cependant je ne puis m'empêcher de rapporter ici les propres expressious d'un célèbre médecin allemand," Baldinger; elles servent de complément à l'histoire d'une maladie rapportée dans son Journal', etqui ne diffère de celles que nous venons de citer que par une plus grande complication; les voici : "On me communiqua l'histoire de cette maladie afin d'avoir mon avis. Malgré toute l'exactitude avec laquelle elle a été rédigée, j'ai pu seulement reconnaître que la fistule en question descendait obliquement entre les muscles abdominaux. Il me parait probable que l'intestin se trouvait dans le commencement étranglé dans l'anneau inguinal, et que le chirurgien appelé en premier lieu aura pris une hernie pour un abcès, ce qui arrive assez squvent, et ce dont Heister cite plusieurs exemples. L'intestin par conséquent fut frappé de gangrène, et des ascarides sortirent alors par l'abcès, ce qui a été déjà observé un grand nombre de fois.

Hirsch, dans ses remarques faites sur un cas rapporté par lui-même, et qui est semblable à ccux que nous venons de citer, ne croit pas que les vers provinssent du canal intestinal. Voici ses propres expressions : "En considérant l'amas énorme de vers qui s'était accumulé dans cette femme, surtout à l'époque de la grossesse, où leur contact a vec les parois du canal intestinal a dû être favorisé par la pression de la mat:ice, et où, par la double irritation

$\therefore$ Ncucs mağazin für acrste, bd. 6 , st. I, s. 75 . 
des vaisseaux lymphatiques, leur activité fut augmentéc, il paraitra concevable que l'absorption de la matière plastique de ces zoogénites ait pu avoir lieu par les vaisseaux lymphatiques des intestins. Lorsque la substance absurbée par les plus fins de ces vaisseaux arrive promptement à sa formation, elle agit aussi d'une manière destructive sur eux, et parvicnt après leur rupture clans des cavités qui diffèrent du séjour ordinaire des vers, c'est-à-dire hors des intestins. Les larves de ces animaux se pourrissent, dans l'endroit oil elles se trouvent déposées, de la vapeur animale et de l'humeur exsudée par les vaisseaux lymphat:ques; elles parviennent bientôt, à l'aide de cette nourriture, à se multiplier, et elles forment alors un amas qui doit réagir d'une manière anomale sur les parties envirounantes. ")

Cependant M. Hirsclr n'aurait pas eu besoin d'avoir recours à cette explication hasardée pour démontrer comment les vers auraient pu arriver dans la plaie, s'il n'avait pas mis trop d'importance à la circonstance qu'il ne s'était pas forméà l'endroit affecté un anus arificiel, qu'il regarde comme une suite certaine dans le cas d'une perforation de l'intestin. Des guérisons, à la suite de liernies étranglées compliquées de gangrène, ne sont pas du tout impossibles. J'ai observé moi-même un cas semblable étant encore étudiant en médecine. Voici le fait : Une femme à A poldà avait une hernie qui s'étrangla un jour et tomba en gangrène; des maières stercorales (qui cependant ue contenaient pas ide vers), sortirent par ja plaie. Comme cependant 
ces matières ne pouvaient s'écouler par l'anus qu'à l'aide de lavemens, je me proposai, secondé par deux de mes amis, de fixer l'intestin au péritoinc, ou bien aux autres tégumens, afin de prévenir l'épanchement de ces matières et de couserver la vie de cette femme, dût-elle même garder un anus contre naturc. Cependant l'essai échona, et nous ne pûmes parvenir, malgré la dilatation de l'ouverture de la plaie, à saisir l'intestin déchiré.

Qu'arriva-t-il alors? la nature, si puissante dans ses effets, effectua spontanément une cicatrice, et la femme fut rétablic sans avoir d'anus artificie!.

Ces cas sont, ì la vérité, très-rares : en voici encore quelques autres.

Needham, médecin à North-Wolsham, a rapporté l'observation suivante très-remarquable : Un enfant de treize ans, du sexe masculin, fut renversé par une voiture qui lui passa sur le corps; une grande partie des intestins et du mésentère était sortie par le rectum, et bicutôt après la gangrène s'y déclara. Needham enleva une longueur de cinquante-sept pouces d'intestins sphacélés, et le malade guérit.

Borell et Marteau parlent de hernies ombilicales, et Goeckel et Moulenq, de hernies inguinales, où les plaies se cicatrisèreut entièrement sans laisser ni fistule, ni anus artificiel.

Roudier rapporte aussi un cas de hernie étranglée avec conplication de gangrène; il se forma à l'endroit affecté une ouverture de laquelle sortirent dix-neuf ascarides et toutes les matières stercorales; l'excré- 
tion par l'anus avait entièrement cessê : nonobstant le malade guérit.

Baillie fai également mention de plusicurs exemples d'anus artificiels par suite de gangrène, qui ont été guéris.

Nous ne nous tromperons par conséquent pas, si nous admettons que les vers, dans l'observation citée par Hirsch , provenaient également du canal in. testinal, dont ils sortent en pareil cas, et qu'ils n'avaient eu aucune part à sa rupture. Cependant ceute dernière assertion n'est pas généralement admise, et si l'on ne peut pas accuser ces animaux de la perforation des intestins, puisqu'ils ne sont pas pourvus d'organes propres à l'effectuer, on les regarde au moins comme une cause d'étranglement de hernie.

Richter comprend les vers intestinaux parmi les causes qui peuvent produire cette espèce de maladie. L'explication de la manière dont il conçoit que cela puisse s'effectuer, s'accorde avec celle de Wedekind; ce dernier a même publić une dissertation sous le titre des étranglemens de hernies occasionés par les vers.

Wedekind admet bien plus que l'on doit attribuer les cas très-multipliés, et presque endémiques de hernies avec complication de vers dans le conté de Diephols, où il était autrefois médecin en chef, à cette cause, et à la manière de vivre des habitans de ce pays. D'après cela, il cherche à démontrer avec beaucoup de sagacité, comment les vers peuvent produirc de deux manières ces étranglemens. D'a- 
bord, notamment par irritation consensuelle (étranglement spasmodique), et puis par obstruction ou compression des intestins contenus dans le sac herniaire. Le premier genre d'étranglement, d'après lui, est une suite d'irritation vermineuse. Mais qu'est-ce que l'irritation vermineuse? un terme de médecine, comme beaucoup d'autres, par lesquels on s'imagine avoir désigué quelque close, sans cependant pouvoir en rendre compte ni à soimême ni à d'autres. Comment et de quelle manière irritent les vers, surtout les ascarides, car ce sont sans doute eux doutil est ici exclusivement question? J'avoue que je ne saurais l'expliquer.

Tous les ascarides que j'ai rencontrés dans des animaux fraîchement tués se trouvaient libres, isolés, ordinairement enveloppés de mucosités dans l'intestin, et ils étaient loin de toucher sa tunique muqueuse (tunica villosa). Placés de cette manière, je ue conçois pas comment ils penvent causer une irritation semblable à celle dont parle Wedekind; la sensation, comme s'il rampait quelque chose dans le corps, dont les malades se plaignent assez souvent, est aussi illusoire que celle des femmes hystériques, qui croyent bien des fois que la matrice se porte réellement vers la gorge.

J'ai traité depuis plus de dis ans plusieurs centaines de malades tourmentés par des vers, ou qui croyaient l'être, et j’ai toujours remarqué que ceux qui en avaient réellement dans leur canal intestinal, 
se plaignaient le moins de ces sensations singulieres, surtout lorsqu'on ne les avait pas encore fatigués de. beaucoup de vermifuges; mais les personnes au contraire qui avaient rendu un jour des vers, et dont il ne restait probablement plus de traces depuis un long espace de temps, ou enfin celles auxqueỉles les médecins avaient mis dans la tête qu'elles en étaient incommodées, se plaignaient en effet que les vers les pinçaient, les mordaient, les suçaient ou les fatiguaient par leurs mouvemens, etc.

Quand on examine de pareils malades, ils nous disent souvent qu'ils sentent les vers tantôt dans le duodénum, et tantôt dans l'estomac, dans le jéjunum, dans le rectum, dans la gorge, dans les épaules, etc. Qui voudrait admettre qu'un ver intestinal, naturellement très-lent dans ses mouvemens, puisse parcourir aussi promptement le canal intestival, si long et si tortueux? qui pourrait encore croire, que les mouvemens d'un tel animal, si souple, seraient sentis plus distinctement que, par exemple, le roulement l'un noyau de cerises, dont plusieurs centaines parcourent souvent le canal intestinal, sans cependant se faire remarquer par une sensation quelconque?

Aucune de semblables sensations morbides ne nous autorise par conséquent à conclure la présence des vers; il nous est seulement permis de soup. conner une maladie vermineusé, généralement parlint, si toutefois les circonstances mentionnées plus Latut ont réellement lien; car, je le répète, il y a des 
hommes qui out souvent une très-grande quantité de vers dans le corps et qui cependant ne se plaignent jamais d'aucune de ces sensations.

On m'objectera sans doute que cette assertion peut être vraie seulement dans le cas où les vers séjourneraient dans un canal intestinal parfaitement sain, mais non pas dans celui qui se trouverait dans un état d"irritation contre nature. Mais alors la cause de la maladie ne doit pas être directement attribuée à la présence des vers, qui existaient peut-être déjà ici depuis des années, mais bien à quelque autre chose (peutêtre aux remèdes employés contre les vers) qui a produit cette irritation considérable du canal intestinal.

Le second genre d'étranglement de hernies, d'après Wedekind, est celui que les vers occasionent, ou par une obsiruction, ou bien par une pression des intestins contenus dans le sac herniaire. Ce médeciu raisonne là-dessus de la manière snivante; "les ver's, en obstruant le canal intestinal, peuvent donner licu an miserere (on bien au vomissement de matieres stercorales), par conséquent ils peuvent bien plus facilement produire cette maladie, quand ils se trouvent placés dans le sac herniaire." Mais je réponds à cela: l'antécédent de cette conclusion n'est que présumé et nullement constaté (je renvoie mes lecteurs aux réflexions que jai faites plus hatit sur les obstructions du canal intestinal causées par la présence des vers); quant à la conclusion elle-même, clle doit être naturellenient inexacte.

Cependant Wedekind défend son opinion comme 
de coutume, avec beaucoup d'esprit, et il prétend que les vers contenus dans le sac herniaire provoquent d'abord, par leur irritation, une contraction de l'intestin, et il ne regarde l'étranglement que comme une suite de l'inflammation. Il tâche de prouver son assertion en disant, que la douleur dans la hernie précède toujours l'inflammation.

En admettant, même pendant un momeut, la possibilité de ce fait, il me semble cependant que les antécédens ne sout pas établis d'une manière tout à fait exacte; car les ascarides, conformément à la structure de leurs organes destinés à recevoir la nourriture, peuvent tout au plus s'implanter dansla tunique muqueuse des intestins. (Jen'admets celá que comme une chose possible, car je vieus de remarquer que je n'ai jamais rencontré un ascaride qui fùt implanté dans cette tunique, et encore moins dans la tunique appelée nerveuse); mais il ne me paraît nullement probable que cela pourrait produire dans l'intestin, supposé même qu'un, deux ou trois vers s'y trouvassent implantés, une constriction capable de faire naître un iléus.

J'ai trop d'exemples à ma disposition pour être convaincu du contraire. Les échynorhinques s'accrochent quelquefois par centaines sur les intestins des poissons, des oiseaux aquatiques et même des pores, et tellement profondément, que l'ou peut reconnaître le siége de chaque ver par une petite protubérance sur la paroi externe de l'intestin. Dans la cigogne sc trouve un distome qui se forme des excavations très- 
profondes dans les parois du canal intestinal : nous voyons souvent dans les intestins des brochets une quantité énorme de tricuspidaires, qui s'y implantent également profondément : chaque endroit où un individu avait été accroché est marqué par une protubérance papilliforme qui est creusée par une concavité dans son milieu.

Le docteur Gœlis a bien voulu me céder un jour le cadavie d'un enfant mort raclitique et par suite d'une carie presque générale des os, dans les intestins duquel j'ai trouvé un tænia vivant fortement implanté par son orifice buccal à la paroi interue de l'intestin ; mais il n'y avait ni constriction, ni inflammation, ui aucun autre changement norbide. Il en est toujours de même pour les animaux cités plus haut. Comment, par conséquent, les ascarides, qui sont justement les moins propres a s'accrocher, pourraient-ils causer tant de dérangemens. N'expliquera-t-on pas le fait rapporté par Wedekind beaucoup plus naturellement en regardant la circoustance, qui, d'après l'opinion de ce médecin, donne lieu à la fois à la production des vers et à la formation des hernics, comme la cause primitive des coliques et de l'étranglement? Du reste, il me paraît qu'une hernie contevant un ou plusieurs ascarides vivans devrait toujours être plus facile à réduire qu'une autre qui ne renfermerait que des matières stercorales endurcies. Le ver comprimé par les doigts que le chirurgien emploie pour opérer le taxis, se détachera non-seulement ( chose que je suppose, mais que je n'admets pas) et 
levera par là la cause de l'étranglenent; mais encore il s'en ira par le chemin par où il est venu. Cela fait, la circulation libre du chyle et des matières fécales sera rétablie, si toutefois aucun autre obstacle ne s'y oppose.

Des vers morts offriront à peu près autant d'obstacles que des matières stercorales endurcies; cependant ceux-là, à cause de leur superficie lisse et dé leur structure cylingdrique, qui se termine en cône aux deux extrémités, seront toujours plus faciles ì éloigner que celles-ci.

Mais à quoi bon poursuivre plus long-temps la défense de ces animaux : tout homme qui a la certitude d'en avoir désire d'en être débarrassé ; c'est pourquoi nous avous indiqué dans le chapitre suivant les moyens thérapeutiques propres à les combattre.

\section{CHAPITRE SIXIEME.}

Du traitement hy'giénique et thérapeutique contre les v'er's. intestinaux.

SECTION I. Des remèdes en général.

L'espèce hnmaine est sujette à peu de maladies pour lesquelles on ait proposé autant de remédes que pour les affections vermineuses. Cette observation en donnera sans doutc une idée très-défavorable en réflé- 
chissant que ce sont en général les maladics les pỉts incurables, telles que la phthisie pulmonaire, les scrofules, etc., contre lesquelles on cherche encore tous les jours des remèdes plus efficaces que ceux que l'on connaissait et que l'on avait d'abord regardés comme infaillibles, tandis que le traitemen de la péripneumonie, par exemple, est aussi simple dans le dix-neuvième siècle qu'il l'étaiı du temps d'Hippocrate. Ce n'est cependant pas l'opiniâtreté avec laquelle les vers résistent aux remédes dont l'expérienc e a sanctionné l'efficacité, qui est la cause principale que ces remèdes ont été rejetés ponr être remplacés par d'autres, qui souvent même ne jouissaient pas de la propriété vermifuge; cela tient à plusieurs autres raisons.

D'abord avec l'emploi de ces remèdes on n'a pas eu égard a la cause déterminante de la formation des vers, et par conséquent à son éloignement, en sorte que toutes les fois où, après l'emploi efficace d'un médicament, les vers ont reparu, on en a substitué un autre; ajoutons 'que l'usage du premier a pu aussi n'être pas contiuué assez long-temps. C'est ainsi que Dianière, dans un casqu'il rapporte, où tout semblait indiquer l'existence de vérs intestinaux, et oì des vermifuges administrés deux ou trois fois conjointement avec des purgatifs, n'avaient déterminć la sortie d'aucun de ces animaux, ayant trouvé à l'ouvertnre du corps un grand nombre d'ascarides réunis par pelotons dans différens endroits du caual intestinal, en conclut un système particulier pour le traitement de 
l'affection vermineuse. L'administration des vermifuges doit ctre, suivant Jui, continuée long-temps et avoir lieu deux ou trois fois par jour, immédiatenent avant ou après le repas, afin, dit.i], que les vers affamés se jettent sur ces substances avec plits d'avidicé. 11 faut leur associer des matières sucrées et propres à rendre le poison plus agréable; enfiu le iraitement doit être terminé, lorsque tontefois rien ne dénote plus la présence des vers, par l'emploi des purgatifs; car dans le cas contraire on doit recommencer le même systeme de traitement quii, abstraction faite de l'idée d'affamer lés vers et d'édulcorerles vermifuges, n'est pas à dédaigner.

Une autre raison pour laquelle on a quelquefois eu recours à de nonveaux vermifuges, c'est que leur emploi est souvent déterminé par l'existence de quelques symptômes généraux qui ont pu induire en erreur, comme nous l'avons démontré plus haut, et sans avoil des prenves. certaines de l'existence des vers, ou bien qu'après l'évacuation de quelques-uns de ces animaux on a continué de leur atuibuer des accidens dont la cause réelle aurait dû être cherchéc ailleurs.

Les deux observations que j'ai rapportées plus laut, savoir l'histoire de la maladie de la femme de Jéna el celle du prêtre de la Moravic viennent à l'appui de ce que je viens de dire.

Il est anssi arrivé que l'on a attribué la propriété vermifuge à des substances qui ne l'out réellement pas, au moins d'une manière directe. Ainsi, par 
exemple, si pendant le traitement d'une maladie quelconque, le malade a rendu des vers, il est souvent arrivé que l'on a attribué cette évacuation à la substance médicamenteuse qui venait d'ĉtre employée et qui dès-lors a été vantée comme un vermifuge infaillible; mais dans ce cas et dans d'autres semblables, les vers ont puêtre évacués accidentellement, comme cela arrive souvent; ils ont pu être expulsés par un effet même de la maladie, ce qui donne l'explication du grand nombre de fièvres épidémiques dites verraineuses, dans des pays où les affections de ce genre sont véritablement endémiques, et où les vers disparaissent naturellement par la corruption des humeurs contenues dans le canal intestinal; enfin ce médicament a pu faire cesser entièrement la disposi tion unorbide (opportinitas) qui avait favorisé la production des vers; ceux qui se trouvaient alors dans le canal intestinal ont été rendus, et il ne s'en produisit plus d'antres. Ainsi, par exenple, Vandovern a placé, page 329, l'écorce de simarouba parmi les vermifugucs, parce que Hempel, qui l'avaiı employée contre une diarrhée chez un malade attaqué depuis quinze jours de la pelite vérole, avait observé une évacuation considérable d'ascarides.

On a donc pu croire que les derniers remèdes employés dans un traitement quelconque fussent de véritables vermifuges; cependant il n'en est pas tout it fait ainsi, car il faut faire une distinction importante entre les remèdes qui produisentl'évacuation des vers 26. 


\section{SUR LES VERS INTESTINAUX}

existant dans le canal intestinal, et cenx qui empếs. chent la formation de nouveanx vers, parce quils font cesser la cause essentielle et primitive de leur reproduction.

Un médecin, doni le nom m’est échappé, demeurant à Brünn, m'a raconté qu'il avait aḍministré plusieurs fois, pendant un assez long espace de temps, des vermifuges et des purgatifs, sans cependant pouvoir effectuer une évacuation de vers qui n'eut lien que lorsqu'il ent ordonné le fer et d'autres médicamens fortifians.

Cependant je me croirais aussi pen engagé par cette observation à commencer le traitement d'une affection vermineuse avec le fer ou autres remèdes semblables, dans un cas oì le canal intestinal se tronverait chargé de glaires et de veŕ, qu'à employer dans le commencement d'une inflammation des poumons le polygala sénega ou le camphre.

En sommettant les vers intestinaux hors du canal intestinal à l'action de différens remédes, on s'imagina que ce serait le meilleur moyen de connaître cenx qui causeraiert la mort la plus subite à ces aninaux. Redi fut le premier qui fit des expériences de cette nature; elles ont été répétées par Baglivi, Andry, Torti, Coulet, Arnemann et Chabert; cependant toutes ces expériences n'élaient pas propres à conduire à un résultat certain; car il faut considérer que la plus grande partie des vers intestinaux meurent très-vite quand ils se trouvent éloignés du lieu 
de leur séjour naturel, quoiqu'il y ait quelques exceptions, le spiroptère" (spiroptera cystidicola, Rud.) qui provient de la vessie abdominale de la truite se conservant vivant dans l'eau froide pendant huit jours au moins. Exposés à l'influence de l'air atmosphérique, les vers intestinaux ineurent tous sans exceptiou très-promptement, et ils se contractent.

Les expériences que l'ou fuit sur les vers intestinaux de l'homme sont encore moins exacies, par la raison que l'on ne peut employer que des vers rendus avec les niatièrcs stercorales, et par conséquenı déjà affaiblis, ou bien parce qu'on se sert de ceux que l'on a recucillis dans li a cadavres. Dans le dernier cas on peut, généralement parlant, présumer que leshommes a vaient succombé par suite d'une maladie qui a également pu agir sur les vers d'une manière nuisible; comme d'ailleurs nous pouvons rarement ouvrir des cadavres avanıque vingt-quatre heures ne soient écoulées, à compter du moment de la mort, il est alors permis d'admettre que les vers commençaient aussi à perdre la vie.

Enfin il faut considérer que les remèdes administrés à l'intérieur passeni d'abord, avant d'être en contact avec les vers, par l'estomac, où ils subissent de grands changemens, de manière que ceux qui sont contenus dans l'intestin ne sont jamais directement soumis a leur itifluence, ce qui au contraire a lien quand on emploie les remedes sur ces animaux hors du canal iutestipal.

- Nouvean genre créc par M. Rudolphi. 
Ces remarciues, an premier abord de peu d'importance, méritent cependant d'être prises en considération, quand on veut faire des expériences de ce genre; du reste celles de Redị et d'Arnemann nous ont appris que les huiles grasses n'agissent pas sur les vers intestinaux d'une manière aussi nuisible que l'on s'était cru en droit d'admettre, d'après l'influence délétère que ces huiles ont sur les insectes, et surtout sur leurs larves. Ce fait est facile à expliquer : l'huile bouche, chez les insectes, les organes respiratoires, ce qui n'a pas lieu pour les vers intestinanx, qui n'ont pas ces orgaues. Il résulte encore des expériences d'Arnemann que l'huile de ricin, si vantée comme agissant d'une manière délétère contre le tæuia, n'a. git réellement que comme un purgatif, en mème temps qu'elle rend le canal iutestinal glissant; car des ascarides, provenaut des cochons, mis dans cette huile, ont conservéla vie pendant trente-six heures, ct des ascarides provenant de l'homme, depuis quarantequatre jusçu’à quarante-lıuiı heures. Dans aucune autre huile, à l'exception de l'huile d'amandes douces ", les vers n'ont vécn aussi long-temps.

Les expériences ont appris que la manière la plus prompte et la plus sûre de canscr la mort aux vers intestinaux consiste à les soumcttre à l'influence du froid, de l'esprit-de-vin ou d'autres liqueurs spiritueuses et des huiles empyreumatiques.

r Je n’ai pas lis l'ourrage d'Arnemann: Commentatio de olcis unguinusis, Geelling., 1735 , sect. IY, et j’ai tirć ce que je viens de rapporter de Rutulohi, Lntoz., vol. I, p. 487 et 488 . 
L'emploi du froid et des liqueurs spiritueuses sur les honmes offre souvent, comme on peut bien se J'imaginer, beaucoup de difficultés, mais il n'en est pas de mème de celui des huiles empyreumatiques; ou peut en faire usa ge très-facilement, et leur utilité a élé déjà souvent constatée par l'expérience, comme nous le verrons par la suite.

Prenons maintenant en considération les remèdes que l'on a essilyés on enıployés contre les vers sur l'homme vivant. On peut, ce me semble, les diviser d'après leur mode d'action $1^{\circ}$. mécanique, $2^{\circ}$. spécifirgue, $3^{\circ}$. purgatif, $4^{\circ}$. fortifiant.

\section{Des remèdes qui agyissent sur les vers d'une mantière mécanique.}

Le zinc, tant sous forme de limaille qu'en grains, occupe le premier rang parmi ces médicamens. Il a été d'abord reconmandé par Alston, et, plus tard, par Pallas ${ }^{2}$ et Bloch ${ }^{2}$.

Le zinc doit être pur, c'est-à-dire débarrassé de tout alliage de plomb et d'arsenic; il agit d'une manière purcment mécanique; ecpendant il se moutre beaucoup plus actif contre les vers, sous forme de limaille, que sous celle de grains; mais la limaille irrite trop le canal intestinal, c'est pourquoi Pallas a préféré l'usage du zinc en grains.

Nous ferons encore une fois unention de ce médi-

Nord.beilr., st. $5 \overline{5}$.

2 I'rcisschirift, s. 56 . 
cament quand nous iodiquerons les méthodes de traitcment spécifiques recommandées par divers auteurs contre les cestoïdes.

Le strzolobium, appelé communément dolichos pruriens, a été beaucoup vanté par Chamberlaine, et M. Rudolphi a remarqué que les huil espèces de ce genre de plante sont douées des mêmes propriétés.

Lion se sert seulement des petits poils qui se trouvent à l'extérieur des gousses dans lesquelles les graines sont contenues. Ces poils appliqués sur la peau de l'homme occasionent une démangeaison insupportable, cependant dans les Deux-Indes l'on s'en sert déjà depuis très-long-temps comme d'un vormifuge, sans que l'on ait vu en résulter le moindre accident. Toutefois, on a soin de donner ce remède dans un vélicule mucilagineux, ou bien dans un sirop épais, qui, conjointement avec les mucosités de l'estomac et des intestins, mitige singulièrement l'action mécanique de ces poils. Ce serait it tort cependant, si l'on croyait que les vers vivant dans ces mucosités sont garantis également de l'action mécanique de ce remède, puisque, d'après les nombreuses expériences de Chamberlaine et l'assertion de beaucoup d'autres médecins, il fait évacuer des ver's toutes les fois quil en existe dans le canal intestinal. Cet effet avantageux s'étend surtout aux ascarides; car, d'après ce qu'cu dit Chamberlaine, il ne s'observe pas d'une manière aussi satifaisante, lorsqu'il s'agit de combattre le tæuia.

C'est de ces poils, dont il n'indique point la pra. 
portion, qu'il fait faire, avec suflisante quantité de sirop commun, un électuaire dont il ordonne matin et soir une cuillerée à café aux enfans de six à luit ans, une petite cuillerée aux enfans de huit à quatorze ans, et une cuillerée à bouche à tous les individus au-dessus de quatorze ans. Ce médecin administre en outre tous les trois ou quatre jours un purgatif.

Palmer a égalemeni vanté ce remède, notamment comme agissant d'une manière purement mécanique; quant à moi je n'ai aucune expérience personnelle à cet égard, attendu que les autres vermifuges m'ont toujours paru suffisans.

Chardon pulvérisé. Suivant Pallas ' cette substance est employée en Islande comme vermifuge, et il dit avoir opéré par ce moyen l'expulsion d'un long morceau de tænia.

Les carottes jaunes, que l'on râpe, et que l'on mange le matin à jeun, constituent un autre vermifuge dont on fait usage dans plusięurs contrées d'Allemagne.

11 est hors de doute que les remèdes qui n'agissent que mécaniquement irritent les vers, ct les disposent à quitter le corps dans lequel ils sont renfermés; c'est ainsi que l'on trouve souvent des vers dans le canal intestiual des oiseaux, qui en été se nourrissent d'insectes, au lieu qu'en automne, où ces oiseaux se nourrissent de graines mêlées de sable, leur canal intestinal ne présente aucune trace de vers. Ceite circoustance senuble faire croire que l'on pourrait encore employer comme vermifuges agissant d'une manière

- N. n., beitr., I, s. 64. 
410 SUR LES VERS INTESTINAUX

mécanique, une foule d'autres substances, dont cependant il faut s'abstenir, à cause de l'effet nuisible qu'elles pourraient produire sur la santé du malade. Toutefois, je dois faire remarquer quil n'est nullement nécessaire de recourir à l'usage de substances dont l'effet se borne à éloigner le produit de la maladie en laissant subsister la maladie ellemême. Quant à moi, je n'ai jamais eu recours à de tels moyens malgré le grand nombre d'individus affectés de vers que j'ai ens à traiter dans l'espace de plus de dix ans.

11. Des remèdes qui agissent d'une manière spécifique contre les ver's intestinaux.

Ea f frorde. Rosenstein et Pallas ont recommandé l'emploi de ce liquide. Il est certain que l'impression du froid agit d'une manière très-nuisible, et même délétère, sur les vers intestinaux. C'est pourquoi, s'il y avait possibilité de faire arriver l'eau à une température très-basse jusqu'au séjour des vers, ce moyen devrait être regardé comme étant à la fois très-simple et très-cfficace pour opérer leur expulsion; mais il est à remarquer que ces animanx séjourneot chez l'homme dans les intestins, et non pas dans l'estomac, ct quand même on boirait l'eau à une température très-basse, clle se réchaufferait déjà dans cet organe, et n'arriverait par consécquent pas assez froide jusqu'au séjour du ver. Cependant comme il esı constaté par l'expérience que l'eau prise à froid, et sur- 
tout en grande quantité, a opéré lien souvent, nonsenlement l'évacuation des ascaricies, mais encore celle des trenias, M. Rudolplii s'est efforcé d'expliquer, bien ingéniensement à la vérité, de quelle manic̀e elle agit. Il croit que l'inpression faice par ce liquide sur l'estomac s'étend jusqu'aux intestins; il s'imagine en outre que l'eftet de cette impression doit consister en une espéce de commotion violente. Puis il remarque que les vers qui se trouvent pour ainsi dire inondés par l'enploi d'une grande quantité d'eau, doivent facilenient être entraînés avec elle, par la raison que ces animanx, mis dans l'ean, en absorbent une plus ou moins grandequantité, ce qui les gonfle, et les rend presqune roides; el que réduits à cet état, ils ne peuvent plus résister à l'affluence de ce liquide.

L'eou salcée agit encore d'une manière plus efficace. Pallas raconte, qu'il y a à St.-Chat, petit village près de Londres, et pas loin de Sadlerswell, un hôlel et un jardin publics, dans lesquels il se trouve une source nommée Battelbridge-Wells, coiteviant une eau chargée d'un peu de sulfate de soude. Cette eau est regardée comme un remède très-efficace contre le ver solitaire. L'on fait voir dans cet hôtel une collection de plus de cinçuante hocanx remplis de tæuias de différentes espèces, surtout provenant de l'espèce large et memlraneuse, qui ont ćté rendus en entier par l'usage de celle eau.

Cependant l'histoire de la maladie d'une dane, * N. n. beitr., I, s. 64 . 
rapportée par Rosenstein, prouve que les caux minćrales froides ne doivent pas être regardées comı nie un remède souverain contre les cestoüdes; car quoique cette dame eût déjà fait usage de l'eau de la source, dont il vient d'être parlé, pendant plusieurs anuées consécutives, elle rendait toujours des morceaux de tænia.

Van Swieten " croit que des lavemens d'eau froide doivent être très-utiles contre les oxyures, et Loeffler conseille d'avaler de petits morceaux de glace, dans le cas où l'on supposerait des vers dans l'estonac.

ValÉRIANE (valeriana sylvestris). La racine pulvériséc de cette plante doit être regardée comme la subsıance la plus active contenue dans l'électuaire vermifuge de Stoerk, dont voici la formule ${ }^{2}$ :

R. salis polychrest., pulv. rad. jalapp., valerian. sylv., de chaque 3 j; oxymel scillit., $\mathrm{j}_{\mathrm{iv}}, m$. s. Les adultes en prendront une demi-once quatre fois par jour, et les enfans depuis un jusqu'à deux gros.

Daus le mode de traitement proposé par Laģène comme iufaillible contre les cestoïdes, c'est aussi la valériaue qui est le médicament principal, car le reste n'est composé que de purgatifs.

Tous les médecins connaissent les grandes vertus de cette plaste; clle mérite d'être employée surtout daus les maladies vermineuses; en effet elle agit d'ahord, à cause de son odeur spécifique, comme un Lon vermifuge, et ensuite comme combattant avec

- Comment., t. xvir, $\S$ г 37 I.

'Ann. med., , , p. 103, 162, 228 et 386. 
beauconp d'efficacité les symptômes nerveux qui accompagnent ordinairement la présence des vers.

Oicnon (allium cepa) et AIL (allium sativum). Ces deux substances sont employées depuis long-temps contre les vers.

Rósenstein a obtenu, même dans deux cas, l'évácuation d'un tænia par l'usage d'une gousse d'ail prise à jeun et légèrement mâchée. Ce médecin observe ccpendant que cette substance n'agit contre les vers que dans le cas où l'on n'en aurait pas mangé habituellement auparavant.

Cranz a au contraire attribué la grande quantité de vers qúu'il a trouvés dans les intestins d'un homme, à l'usage immodéré que cet individu avait fait de l'ail pendant sa vie. Cependant Limhard a justement réfuté cette assertion. On administre aussi souvent du lait dans lequel on a fait bouillir de l'ail, comme un remède contre les vers.

Emhard rapporte que Binninger l'a également employé dans ce but sur un homme qui était, pour ainsi dire, à l'agonie, et qui, après avoir rendu des vers, se trouva soulagé.

Baglivi a connu un jeune homme de vingt ans, qui était chargé un matin de couper de l'ail; tout à coup l'odeur de cette substànce l'incommoda tellement, qu'il manqua d'étouffer; quelques minutes après il commença à vomir d'une manière violente, et rendit un ver rond, de trente aunes de longueur, et qui élail contourné en forme de peloton.

' Ourrage cilé, p. 696 . 
Dans ma jeuuesse j’ai été égalcment obligé de boire pendant longr-temps du lait chargé d'ail, mais la raison pour laquelle ce reméde ne me débarassa pas de mes vers, doit être attribuéeà la manière irrégnlière dout je l'ai pris; en eflet, an lieu de le boire, je me suis permis bien souvent de le jeter, ayant toujours eu une répugnance extraordinaire pour l'ail. Je fais mention de ce fait seulement afin que l'on ne regarde pas tout de suite nu bon vermifuge comme ineffcace, quand il n'opère pas l'évacuation de quelques vers, et afin que l'on s'informe d'abord si le malade la régulièrement pris; car les ordonnances que nous prescrivons contre les vers sont en général suivies d'une manière très-inexacte : cela dérive ordinairement de ce que les vermifuges ont presque toujours un très-mauvais goùt, ct de ce que les malades, qui ne croyent pus leur vie en danger, se perneltent d'autant plus volontiers quelques négligences.

Lorsque je me fus décidé à employer l'huile empyreumatique de Chabert contre le trenia, je préparai ce médicament moi-même, et je le distribuai gratis. Plusieurs de mes malades n'en ressentirent arcun effet, par la raison tonte simple qu'ils ne l'avaient pas pris; cependant tout cela s'explique assez facilement. D'abord les remèdes qui ne coûtent rien n̈inspirent pas beaucoup de confiance à certaincs personnes, et puis elles se disent: si nous arouons quc le remède a manqué son effet, le médecin nous en donnera bien un autre ųui sera peut-être d'un meilleur gout. Depuis que ce sont les apothicaires qui 
fournissent l'huile de Chabert à mes malades, et que heaucoup d'cotre eux ve veulent pas avoir inutilement dépensé leur argent, ils la prennent régulièrement et ils guérissent. Mon ami le docteur Albert doit se rappeler encore qu'ila trouré un jour, lors de son séjour à Jéna, trois pots remplis d'électuaire de Stoerk sous le lit d'un jeune homme qui était soigné aux frais de la clinique. Je demande pardon à mes lecteurs pour cette digression, mais l'occasion en était bien naturelle.

Le semen contra (artemisia judaïca, L., semer santonici, semen cince), et les graines ou plutôt les fleurs bien développées de la Tanaıse (tanacetun a'ulgare).

Le semen contra est connu depuis long-temps comme un remède contre les vers, surtont contre les ascarides; cependant il peut très-bien être remplacé par la tanaisie.

Avant d'administrer l'une ou l'autre de ces plautes, il faut être sù: de lcur bonne qualité; en effel, quand on les prescrit telles qu'elles se tronvent ordinairement dans les pharmacies, c'est-à-dire très-finement pulvérisées, peut-être couservées depuis plusienrs nois, et par conséquent dépourvues de leur odeur spécifique, on en obticudra bicn pel: d'effei.

J'en ai pris, étant enfant, une grande quanticé, mais aussi je ne fus pas débarrassé de mes vers. J'cn fus encore incommodé à l'âge de treize ou quatorze ans, et on attribua la pâleur de mon teint à la présence de ces animaux; cela m'engagea à les combattre 
de nouveau, et en effet j'avalai, tous les matins à jeun, autanı que le creux de ma main en pouvaiı contenir, de semen contra, après l'avoir légèrement mâché. L'effet a répondu à mon attente, je fus entièrement et assez promptement guéri, sans éprouver de rechute. Il est cependant également possible que le changement dans ma manière de vivre ou bien la période de la vie à laquelle j'arrivais alors aient coutribué à ma guérison. En conséquence de cela, je ne prescris le semen contra jamais autrement que grossièrement pulvérisé, et je présume qu'il agit sous cette forme à la fois d'une manière spécifique et mécanique.

$\mathrm{Je}$ ne crois pas que les graines confites de cette plante (confectio semen-cince) soient très-efficaces : prises à fortes doses, elles agissent alors, dans mon opinion, plutôt comme un remède mécanique que spécifique; car la chaleur à laquelle elles sont soumises dans la chaudière pour les cnduire de sucre, doit leur ôter leur odeur particulière, et en ontre après que le sucre s'est dissous, la graine restée entière se comportera comme telle, et sortira de même entière par l'anus.

La mousse de CoRse (helminthocorthon) appelée conferva helminthocorthos ou corallina corsicana; c'est une espèce de conferve provenant de la Corse.

D'après Sumeirc un Grec nommé Stephanopoli, fut le premier qui apporta ce remède de la Corse, et il le publia en 1777 dans un mémoire. Depuis, la mousse de Corse, conuue dans le pays de ce nom 
depuis très-long-temps comme un bon remède contre les vers et surtout contre les ascarides, est devenu le remède favori des médecins français. On peut l'administrer en poudre à la dose d'un scrupule jusqu'à un demi-ǵros; mais on la donne plus volontiers en décociion à peu près à la dose d'une demi-once, bouillie dans suffisante quantité d'eau, jusqu'à réduction de quatre onces, et que l'on fait prendre dans la journée. On peut aussi l'administrer sous forme de gelée. L'efficacité de cette plante dépeud peut-être du sel marin dont elle est imprégnée.

Chenopodium anthelminticum. Les semences de cette plante sont, d'après ce que l'on dit, souvent employées en Amérique contre les ascarides'.

Cortex angeline. L'on fail bouillir une once de cette écorce dans trois livres d'eau, jusqu'à réduction d'une livre, et les malades en prenuent une à deux onces tous les matins. On prétend que ce remède canse des coliques, mais provoque l'évacuation des. ver s

Grana tiglie (croton tiglium ou ricinoides). Ces graines devraient plutôt être placées parmi les purgatifs que parmi les vermifugès proprensent dits ${ }^{3}$.

Spigelia antielmia el spigelia marilandica. L'on se sert depuis long-temps en Amérique de la première de ces plantes; Bergius regarde la seconde comme beaucoup plus efficace : l'une et l'autre ont

× Brera, Torlesung, s. 97.

2 Même ourrage, s. $\mathbf{g}^{3}$.

${ }^{3}$ V andeevern, s. 299 . 
une proprićté narcotique, et, prises à grandes doses, clles causent des vertiges, troublent la vue et excitent des nouvemens convulsifs dans le globe de l'œil, c'est pourquoi nous devons être circonspects dans leur enploi.

Vau Swieten les a regardées comme très-venimcuses, et rapporte que les Français appellent le spigelia Brainvilliers, nom d'une empoisonneuse trèsconnne. Ce médecin a remarqué que ce remède cause des évacuations très-fortes par haut et par bas, et quil fait rendre les vers d'une manière certaine.

D'après mon idée, on poutrait très-bien se passer de cetic plante.

Non-seulement les feuilles, mais encore les racines de la spigelia sout employées tant sous forme de poudre, à la dose de dix grains pour les enfans, que sous celle d'infusion à la dose d'un demi-gros.

Browne conseille de faire bouillir deux poignées de cette plante dans deux livres d'eau, jusqu'à réduction de la moitié; il y fait ajouter suffisante quantité de jus de citron et de sucre pour rendre la décoction plus agréable an goût. Les malades doivent en prendre deux, trois, jusqu'à quatre onces, toutes les six à douze heures, pendant trois jours consécutifs; Browne ordonne ensuite un purgatif.

$J_{e}$ ne sais pas pourquoi ce médecin administre ce dernier, puisque, d'après Van Swieten, la spigelia jouit elle-mème de vertus purgyatives très-fortes.

Rosenstein " recommande également ce remède;

- Ouvrage cilé, p. 56 . 
quant à moi je n'ai pas eu occasion de l'employer.

Geoffrea surinamensis. Ouemploie l'écorce de cet arbre.

Bondı, Eggert et Schwartze ont publié des dissertations sur sa propriété vermifuge.

Je ne possède que la dissertation du dernier, que je dois à la bonté de M. Osiander : les observations qu'elle renferme concermant l'évacuation du tæuia opérée par cette écorce, ne me paraissent pas suffi-. santes; Schwartze l'ordonne de la manière suivante:

R. Pulv. gross. cort. geoff. sur., unc. ij, infund. aq. font. comm., libr. ij, spir. virl. rect., unc. iv; stet. v'ase clauso in digest. per sex dies; dein coque leni igne donec post colaturam remaneat libr. j. A prendre pendant deux jours à jeun deux cuillerées toutes les heures, pendant trois heures consécutives; le troisième jour on doit boire le reste par verres, et le quatrième on se purge avec du calomélas et du jalap.

CÉvadille (semen sabadilli.) Les semences, y compris les capsules de cette plante pulvérisées, sont employées depuis très-long-temps coutre les poux.

Seeliger a administré ces semences avec succès contre le trenia, à la dose d'ua demi-gros par jour, avec addition d'nue conserve convenable el de suffisante quantité de miel pour en former un bol, et tous les cinq jours il faisait interrompre l'emploi de celte sulstance par un drastique.

Comme les graines de cévadille ont elles-mêmes la propriété drastique, il faut les employer avec une grande circonspection, et il me semble qu'il ne fau- 
drait pas en donner aux enfans plus de trois ou quatre grains à la fois.

Ce remède a été également recommandé en lavement contre les oxyures; mais même employé de cette manière, il cause des nausées et des vomișsemens.

Ceux qui désirent avoir des renseignenens plus étendus sur l'emploi de ce médicament, doivent lire l'ouvrage de Schmucker ${ }^{\text {. }}$.

Juglans regia. On ordonne le brou de noix non mûres en infusion, on bien on en prépare un extrait, que l'on fait prendre dissous dans une eau aromalique.

Hippocrate et Dioscoride avaient déjà remarqué que l'usage de ce remède agissait sur le tænia; Andry l'a surtout beaucoup recommandé.

Rosenstein ${ }^{2}$ conseille de faire dissondre deux gros de l'extrait de cette substance dans une demi-once d'eau de canclle et d'en donner cinquante gouttes pendant six jours aux enfans de deux ou trois ans. Il Jes purge ensuite, du sixième au huitième jour, avec suffisante quantité de pilules mercurielles.

Assa fetida (ferula asa foetida). Ce remède est très en usage contre les vers, probablement parce qu'il sent mauvais. Je l'ai vu souvent employé sans succès contre le tæenia. Mellin ${ }^{3}$, cependant, rapporte qu'il s'est montré efficace contre ce ver.

On administre ordinairement l'assa foetida sous

"Vermischte chir. schiff. Bd. II.

$=$ Ourrage cité, p. 536.

${ }^{3}$ MIateria medica, s. $9^{\circ}$. 
forme de pilules. Rosenstein en fit préparer du poids d'un grain, el en donna cinc à un enfant contes les trois ou quatre heures pendant deux jours consécutifs, et le troisième jour il le purgea avec suffisante quantité de rhubarbe.

D'autres médecins prescrivent l'assa fœlida conjointement avec des purgatifs. Leclerc l'employait dissous dans du vinaigre ou daus l'eau.

Camphre (camphora). Baldinger, Leclerc, Hirschel, Mcbius, de Pauliz, Prange, Zacharias Vogel, Wedel vantent beaucoup les vertus vermifuges de ce remède.

D’après Brera ', le célèbre Moscati préfẻre en général le camphre à tous les autres vermifnges, surtout quand il s'agit de faire évacuer des ascarides.

Rosenstein ${ }^{2}$ s'exprine sur ce remède de la mavière suivante : CComme les vers ne peuvent pas supporter le camplire, et que ce remède, avec addition, de vinaigre, est très-efficace contre les fièvres malignes, la potion suivante peut être cmployée avec avantage dans ces affections.

"Pr.camplire, un gros; esprit-de-vin; quinze gouttes; mêlez, hroyez, et ajoutez vinaigre d'une bonne qualité, cinq onces; sucre en poudre, une demi-once; mêlez avec soin. A prendre une cuillerće à bouche toutes les heures ou toutes les deux heures ${ }^{3}$. »

Vorlesungcn, s. 99.

Ouvrage cité, p. 57 r.

${ }^{3} \mathrm{Je}$ ne crois pas que leraucoup de praticiens se décident à nettre en usage à la lettre l'ordonnance de Rosenstein. Une si 
Cependant Arnemann présume'que l'évacuation des vers dans le cas des fièvres malignes doit être attribuée plutôl aux accès de ces fièvres qu'à l'usagge du camphre.

Fougère male (polypodium filix mas.). Galien ' et Pline ${ }^{2}$ connaissaient déjà les vertus vermifuges de cette plante, qui est encore actuellement employée, et qui entre dans presque toutes les compositions contre les cestoïdes. C'est en effet un excellent remède contre le bochriocéphale, mais non pas contre le véritable tænia; car tout en provoquant l'ćvacuation de quelques morceaux de ce dernier, elle ne cause pas sa destruction, et on aperçoit ordinairement de nouvelles traces de ce ver trois mois après. Cependant on peut administrer la fougère comme remède indicateur contre le tænia, dans le cas où l'on n'a pas des preuves évidentes de sa présence, et où l'on voudrait acquérir une certitude sur ce point. Dans ce but je fais prendre an malade, dans la matinée, à jeun, deux ou trois gros de la racine pulvérisée, et quelques heures aprés un léger purgatif, n'importe lequel, mais on doit avoir égard aux deux circonstances suivantes :

$l^{\circ}$. Il fant que la racine de fougère soit saine, que l'on ait coupé la partie inférieure trop vieille et la grande quantité de vinaigre, tel qu'on le trouve à Paris, par excmple, causerait sans doute de violentes coliques. (Note du traducteur:)

- De simplic. medicam. facult., lib. viu, p. $5 \mathbf{2} 2$.

- Lib. xxvir, cap. Ix, p. 43 o. 
partie supérienre encore verte ${ }^{x}$.Il faut, en outre, que l'on enlève l'écorce avant de la piler, et que l'on n'emploie pour l'usage interne que de la fougère fraichement pulvérisée.

$2^{\circ}$. L'expérience peut facilement tromper, si peu de temps auparavant le malade avait rendu spontanément, ou bien après l'usage de vermifuges, plusieurs aunes de tænia pourvues de la tête ou seulement d'articulations voisines de cette dernière. Dans le cás où les intestins n'auraient contenu qu'un senl ver de cettc espèce, le malade n'en rendra pas même de traces, ct cependant il est possible qu'il évacue de nouveau spontanément quelques articulations de tenia denx ou trois semaines après. La raison en est facile ì concevoir; mais si l'on ne trouve pas, pendant deux mois ou plus, des traces de ce ver dans les matières stercorales que le malade rend journellement, et s'il éprouve, apiès cet espace de temps quelque nouvelle incommodité qu'il pourrait attribuer à la présence d'un tænia, on peut alors, à l'aide de la fougère, mettre le malade au fait presque d'une manière certaine, si ses inquiétudes sont réellement fondées ou non; car si, après l'emploi de ce médicameuı, il ne rend pas des morceaux de tæuia, ou peut alors parier dix contre un qu'il en est entièrement débaṛassé.

Les anciens n'osaient pas administrer cette plante aux femmes, parce qu'ils croyaieut qu'elle provoque l'avortement en cas de grossesse, ou bien qu'elle leur ôte la faculté prolifique. Quant à cette dernière

- Saus doute avant de la dessécher. (Note du traducteur.) 
'q2' SUR LES VERS INTESTINAUX

assertion, Spiegel avait déjà démontré d'une manière évidente quielle n'est nullement fondée.

J'ai ordonné moi-même la fougère à une jeune fermme qui, sans le savoir, était enceinte, pour la première fois, de deux mois, et qui était en même temps incommolée d'un bohtriocéphale. L'emploi de ce médicament n'a pas agi sur elle d'une manière nuisible, car elle accoucha à terme d'un eufant bien développé.

L'ACIDE Prussigue a été également employé contre les vers ${ }^{*}$.

Petrole (petroleum). Leclerc, Rosenstein, Wedel et beaucoup d'autres médecins l'ont recommandé surtout contre le tænia.

Lors de son séjour en Égypte, et notamment au Caire (où le tæenia est tellement fréquent, que l'on peut admettre que les trois quarts de ses habitans, surtout les juifs et le bas peuple, en sont incommodés), Hasselquis $\iota^{2}$ a vu chez un chirurgien français nommé Foumace, trois morceaux de tænia qu'il avait fait rendre à différentes époques à une femme à l'aide du pétrole. Un de ces morceaux était de la longueur de quarante pieds français, un autre de quinze, et le troisième de dix; ils étaient de la largeur du petit doigt.

On fait prendre ce médicament à la dose de vingt à trente gouttes pendant trois jours consécutifs, et Je quatrième on purge le malade.

I Brera, Nuosi commentalori di med. ct chir., 1813 , semestro secundo, p. ig3. ( $\boldsymbol{B r}_{r}$.

$=$ Ouvrage cité, p. 587 . 


\section{DE L'HOMME.}

Le cas de la femme traitée par Foumace prouve que le pétrole ne détruit pas le tænia entièrement.

Quelques médecins donnent le péirole conjointement avec l'huile de térébenthine.

HuILE DE TÉRÉBENTHINe (oleumterebinthina). Lues Anglais recommandent beaucoup cette huile contre les cestoïdes, et surtout contre le tænia.

Fenwik l'emploie à jeun, à la dose de deux onces, et en cas qu'elle ne produise pas de selles, il en fait prendre encore une à deux onces; il n'a jamais vu arriver d'accidens fâcheux après l'usage de cette liuile, et dans six cas clle a fait évacuer très-promptement le tænia.

1l est de fait que l'évacuation de ce ver peut être aussi bien effectuée par de fortes doses d'huile de térébenthine et de pétrole que par celles de fougère; mais il n'est pas encore constaté par l'expérience qu'un traitement terminé aussi promptement puisse pour tonjours débarrasser les malades de leur tæuia; car dans toutes ces observations on rapporte bien combicu d'annes de ce ver ont été rendues dans l'intervalle de tant et tant d'heures, mais on a toujours négligé de nous faire savoir si les malades n'en ont pas été incommodés cie nouveau deux ou trois mois après; cependaut on nous a rapporté qu'un boucher de Duraham avait évacué, quatre mois après avoir fait usage d'huile de térébenthine, des morceanx de tænia. Un cordonnier a été obligé de reprendre cette huile à quatre époques différeutes. Le troisième malade du docteur Osann s'est vu éga- 
lement dans la nécessité de prendre ce remède trois fois. Les deux autres observations rapportées par le même ne prouvent pas non plus en faveur de ce nrédicament; et quoique des doses aussi grandes de cette huile n'aient pas produit de suites fâcheuses pour l'état de santé des personnes qui étaient incommodées par des vers, elles ont néanmoins donné lieu, comme il résulte de plusieurs rapports, à un maJaise général, à des douleurs dans le bas-ventre, à dés étourdissemens, à des nausées, à des vomissemens, à une chaleur dans l'uretère et daus le rectum.

L'IIUILE DE CAJEPUT (oleum cajeput) a été recommandée par M. Rudolphi.

Huile animale de Dippel (oleum animale Dippeli) ${ }^{\mathrm{S}}$. Dans un des cas rapportés par Moutin, et dont

× Je dois à l'huile empyreumatique de Dippel que j'ai prise pendant six semaines, matin et soir, à la dose de cinq jusqu'à quinze gouttes, d'ètre entièrement débarrassé de mon tæenia, après en avoir été affecté pendant vingt-deux ans consécutifs. Le médecin qui ćtait chargé de me soigner daus mon enfance, m'a fait prendre le remède de madame Nouffer, dans l'espace de dix ans, pour le moins une vingtaine de fois, sans pouvoir détruire ce ver. Fatigué par tant de remèdes, surtout par l'usage des drastiques, auxquels j'atribue en grande parlie l'ctat de faiblesse dans lequel je me trouve actuellenrent, j'ai renoncé pendant une douzaine d'années d'autant plus volonliers à l'usage des médicamens, que mon tænia ne m'a jamais incommodé le moins du monde. Arrivé à l'àge de trente ans, et voyant toujours des traces de ce ver, je me décidai à le détruire à l'aide du remède déjà mentionné, dont les grandes vertus anthelmintiques m'étaient connues, et je fus assez heureux pour atteindre mon but; car depuis luit à neuf ans il n'a plus reparu. ( Note du traducteur.) 


\section{DE L'HOMME.}

nous avons fait mention plus haut, l'usage de cette huilc continué pendant long-temps a produit de bons effets, et M. Rudolphi qui, à défaut d'huile de Chabert, employa celle de Dippcl trois fois par jour, à la dose de cinq à dix gouttes mêlées dans une tasse de bouillon, obtint non-seulement l'expulsion d'ascarides, mais encore celle de quelques longs morceaux de tænia. M. Rudolphi regarde cependant lc médicament suivant, c'est-à-dire l'huile de Chabert, comme plus efficace.

Hulle empyreumatique de Chabert (oleumempyreumaticum Chaberti). L'inventeur de ce remède a indiqué la manière suivante pour le préparer :

Pr. huile empyreumatique de corne de cerf, une partie; huile de térébenthine, trois parties; mêlez. Au bout de quatre jours on fait distiller ce mélange au bain de sable dans une connue de verre, et on en retire les trois quarts. Le liquide qui se trouve transvasé doit être employé à l'usage interne.

Le contact de l'air rend cette huile noirâtre, épaisse et plus dégoûtante à prendre, c'est pourquoi on fait bien de la mettre dans de petits flacons (contenant une once ou bien une once et demie) bien bouchés et coiffés avec des morceaux de vessies de cochon.

Nous parlerons plus loin des vertus et de l'emploi de cette huile.

Mencure covlant (mercurius vivus). L'eau dans laquelle on a fait bouillir ce métal a été regardée depuis long-temps comme ayanı des vertus verni- 
fuges; c'est dans ce but que Baglivis conseille de faire infuser une once de mercure coulant dans trois onces d'eau de chiendent, et dans autaut d'eau de pourpier, de remuer ce mélange et de le décanter ensuite. Ce liquide forme, d'après le témoignage de Corge Bateus, un vermifuge très-eflicace.

Cependant il est prouvé que ce métal, étant bien purifié, n'est pas susceptible de dissolution dans l'eau; il faudrait alors seulement attribuer la propriété vermifuge au mercure, tel qu'on le trouve dans le commerce, et qui n'ayant pas été préalablement purifié, contient du plomb; mais comme la quantité de ce dernier métal contenu dans le mercure ne pcut pas être calculée au juste, ce remède doit être regardé conme tout à fait incertain.

Du reste, l'expérience a prouvé que le mercure n'agit pas sur les vers intestinaux d'une manière spécifique; on a même des exemples que des personnes lont pris jusqu'à salivation, et que cependant elles n’ont pas été débarrassées de leurs vers.

Scopoli croit que l'on ne peut guère rencontrer une plus grande fréquence d'ascarides que chez les ouvriers qui travaillent à Idria dans les mines de vif argent.

Ce médecin employait bien aussi le mercure pour faire évacuer les vers, mais il ne se servait de ce métal que comme purgatif; envisagé sous ce point de vue, il en sera fait mention encore une fois plus.

× Ouvrage cité, 1. 49. 
loin; mais de vouloir administrer du sublimé-corrosif contre les vers, c'est réellement plus qu'imprudent.

Le múconate be BARYte agit, comme il résulte des expériences de M. Sertuerner à Einbeck, contre les vers intestinaux de l'homme et des animaux ; mais comme l'acide méconique est un poison des plus violen; il ne sera probablement jamais placé parmi les vermifuges; il en est de même de la solution arsenicale proposée par Hill.

Plusicurs des médicanens dont nous venons de parler ont été employés extérieurement et mênse avec succès dans le but de faire évacuer des vers; mais il ne faut pas s'imaginer qu'étant, ce que l'on croit à tort, implautés dans les parois des intestins, ils aient été forcés par ce moyen de s'en détacher. Quant aux ascarides, il est prouvé $1^{0}$. qu'ils ne s'accrochent pas aux intestins, au noins parmi plısieurs milliers de vers de cette espèce que j'ai observés dans les cadavres de l'homme et surtout dans cenx des animaux récemment tués, je n'en ai rencontré jamais un seul ainsi implanté. Ce fait a été également constaté par M. Rudolphi. $2^{\circ}$. On tronve souvent d'autres nématoïdes, plus particulièrement encore des échynorhinques, ainsi que des trématodes et des cestö̈des attachés fortementaux intestins desanimaux, quelquefois même avec perforation, chez lesquels on n'a pas pu apercevoir, pendant leurvie, le moindre signe de douleur. Le tæuia même, propre à l'homme, s'implante aussi dans les parois desintestins, comme jai eu occasion de le remarquer dans des autopsies cadavériques; 
mais parmi des centaines de personnes attaquées de ce ver et traitées par moi, je ne me rappelle pas en avoir rencontré une seule qui se soit plaint de douleurs que l'on aurait pu attribuer à la succion du tænia. ll est encore de fait que l'on ne rencontre dans le canal intestinal de l'homme qu'un, deux, ou tout au plus trois tænias à la fois, et conmme ce ver peut seulement s'accrocher par son extrémité céphalique trèspetite, la douleur résultant de la succion devrait toujours partir d'un seul point, mais, je le répète, je a'ai jamais entendu porter une plainte semblable. Ceux de mes malades qui étaient dans un état de souffrance se plaignaient des accidens généraux dont nous avons parlé plus laat; plusieurs autres n'éprouvaicnt aucun malaise, et comme on peut bien supposer que leur tænia se sera également attaché ou accroché aux parois internes de leurs intestins, on a raison, ce me semble, de présumer que cette succion ne doit pas causer de douleurs aussi atroces que colles que l'on remarque dans le cas de coliques on de spasmes dans le bas-ventre. Enfiu il est encore de fait, et comme cela a été également remarqué par M. Rudolphi, que les vers qui se tronvent accrochés aux parois des intestins ne s'en détachent pas même, du moins pour la plus grande partic, après avoir été tués dans l'esprit-de-vin. Notre collection possède plusieurs morceaux d'intestins et d'estomacs, qui prouvent ce fait d'unc manière incontestable.

Le bien qui résulte de l'emploi externe des remé- 
des, dans des cas de prétendues coliques vermineuses, doit être ordinairement attribué à l'influence que ces remèdes exercent sur le système nerveux en général, et surtont sur celui du bas-ventre en particulier; c'est pourquoi l'évacuation de quelques vers, qui aurait lieu, après avoir administrédes vermifuges en frictions, ne peut pas servir de prenve que ces animaux avaient été réellement la cause des souffrances. 11 est cependant possible que les médicamens $\mathrm{cm}-$ ployés ainsi, aient agi contre les vers, et qu'ils aient en effet provoqué leur évacuation; car nous connaissons beaucoup de médicamens, qui, administrés à l'extérieur, montrent leur efficacité aussi bien que si on les avait fait prendre par la bouche.

L'application des vernifuges à l'extérieur est surcout à recommander dans les cas où les malades ne sont pas disposés à les prendre à l'intéricur; c'est pourquoi je vais indiquer quelques formules de verinifuges vantés par des auteurs, comne se prêtan£ facilement à l'emploi externe.

M. Rudolphi recommande contre les coliques, dites vermineuses, des frictions avec l'huile de cajeput, et l'usage de bains tièdes; du reste ce médecin ne s'inquic̀te pas s'il y a, en pareil cas, réellement présence de vers ou non, et je crois qu'il a parfaitement raison.

Rosenstein conseille de frictionner le bas-ventre avec du pétrole mêlé d'ail, à l'endroit où les vers, d'après son idée, veulent perforer le canalintestiual. 
Mellin fait encore entrer dans ce même mélange du fiel de bouf récent.

D'après Crato de Kraftheim, Jean Næsius s'est servi avcc succès contre les vers d'un onguent composée d'une once de pétrole nuir mêlé avec un gros et demi de cire nouvelle.

Lower et Schicnk vantent, contre les prétendus vers du cour, l'ail à l'extérieur, sous forne de cataplasme, dans lequel ils font entrer la linaire, la tanaisie et l'absinthe. Le tout doit être bouilli dans du vinaigre.

Vandoevern conseille l'usage de.l'onguent nommé unguentum Agrippee, et celui appelé unguentum Arthanithce, sive de cyclamine, mêlés ensembles à parties égales, et employés en frictions ".

Brera propose, dans le même but, les deux formules suivantes":

I. Pr. Fiel de bouf, un gros; savon de Venise, autant; mêlez et faites, avec suffisante quantité d'huile de tanaisie, un liniment.

II. Pr. Fiel de bouf, deux onces; aloës en poudre, pulpe de coloquinte préparée, de chaque une demionce; faites digérer dans un endroit cliaud ${ }^{3}$ pendant vingt-quatre lieures dans suffisante quantité de suc

$\times$ Ourrage cité, p. 345.

- Voriesung, p. I29.

${ }^{3}$ On trouve dars la traduction allemande : faites digérer daus de l'huile chaude. Je regarde cela comme une faule d'impression; je n'en suis cependant pas sûr, n'ayant pas eu l'original italien à ma disposition. 
gastrique ou de salive, et ajouter à la fin suffisante quantité de graisse purifiée, pour former uu onguent.

Le même auteur a encore fait connaître une formule pour préparer un esprit d'ail, avec lequel on peut rendre les deux linimens précédens plus actifs, ainsi qu'une autre formule pour la préparation d'un emplâtre; les voici :

I. Pr. Ether sulfurique, six onces; ail pilé, une once; camphre pulvérisé, un gros; mĉlez avec soin.

II. Pr. Assa foetida, emplâtre de céruse, cire jaune, de chaque, pariies égales; galbanum purifié, la moitié autaut; faites selon les règles un emplâtre.

On emploie aussi sous formo de lavemens plusieurs des remèdes que nous venons d'examiner. Nous aurons encore occasion d'en parler, quand nous indiquerons les modes de traitement qu il falit employer pour combattre chaque espèce de vers en particulier.

III. Des remèdes purgatifs.

Quand on est parvenu à faire périr les vers avec les remèdes que nous venons de mentionner, il est alors nécessaire, si toutefois ces remèdes n'ont pas déjà produit d'augmeutation daus la sécrétion du canal intestinal, que l'on tâche de faire évacuer non-seulement les vers morts, car ceux-ci s'en vont naturellement, mais encore les glaires qui se trouvent presque toujours en pareil cas en grande abondance. Pour atteindre ce but, l'on doit avoir recours aux 
remèdes qui jouissent de propriétés purgatives, tels que, par exemple, les sels neutres dont on emploie de préférence, en pareil cas, le sulfate de potasse et le sulfate de soude, de même que les caux minérales contenant beaucoup de ce dernier sel. Weigel vante même le sulfate de soude comme un remède presrjue infaillible contre le tænia. Nous ferons conuaître plus loin la méthode que cet auteur emploie contre cette espèce de ver.

Le SEL MARıN, pris à fortes doses, et dissous dans l'eau, agit de la même manière, comme cela résulte d'un cas tiré des dissertations de médecins de Londres, et rapporté par Mellin s. Le voici : U Un homme qui se plaignait depuis quatre ans d'une gêne. dans le bas-ventre, et qui maigrissait à vue d'œil, prit, par le conseil d'un de ses amis, deux livres de sel marin dissous dans deux pintes d'eau de foutaine; il s'en suivit une forte oppression daus la poitrine, et à la fin il rendit par haut et par bas beaucoup de glaires et de vers. La grande soif et la dysurie, qu'il éprouva en même temps, furent calmées par l'usage d'eau pure, et par celui du lait de beurre, et peu de temps après la santé fut rétablie." Ce muême lıomme prit encore une fois trois ou quatre jours ivant la nouvelle et la pleine lune, ce sel comme remède prophylactique, à la dose d'une demi-livre.

LE TARTRE STIBIÉ a été surtont recommandé par Mellin' contre les maladies vermineuses, et il rap-

- Ouvrage cité , p. $9^{3 .}$

- Ouvrage cité, p. 20. 
porte que Ludovici a fait évacuer par hasard un tæniàà l'aide de ce remède.

Après avoir employé inutilement beaucoup de médicamens, Marci a guéri avec le même renède une jeune fille de onze ans, atteinte d'une maladie de nerfs très-violente, qui avait été occasionée par la présence d'un ver solitairè. Brouset et Hirschel font aussi mention de cas semblables.

Scheid raconte qu'Aulbert admivistra un jour, avec le plus grand succès, un remède (composé d'un grain et demi de tartre stibié, d'un peu de résine de jalap et de cinabre) à un enfant de onze ans, du sexe masculin, affecté d'épilepsie causée par la présence d'ascarides.

Dans le cas de complication de maladie vermineuse avec l'épilepsie, Armstrong et Tode préfèrent le tartre stibié à tout autre remède.

Muteau de Rocquemont, Lepelletier et bėaucoup d'autres médecins français se servent exclusivement de ce médicament contre les vers.

D'après notre manière d'envisager les épidémies dites vermineuses, l'emploi du tartre stibié nous paraît, du moins dans beaucoup de cas, très-conrenable; car dans ces épidémies, ou plutôt dans ces fièvres, il y a toujours en même temps un embarras gastrique, que nous devons combattre par la méthode évacuante. Le tartre stibié remplit très-bien ce but, car il provoque à la fois par haut et par bas des excrétions qui entrainent souvent les vers avec elles.

Quant au mercure doux donné souvent contre les 28. 
vers, il est très-vraisemblable qu'il n'agit que comme tout autre purgatif, toutes les fois qu'il détermine l'évacuation des 'rers. Clossius dit expressément qu'il s'est convaincu, par des essais réitérés, de l'inefficacité des remèdes mercuriels dans le cas de maladies vernineuses. Ce médecin pense qu'un usage continu de ces médicamens, à petites doses, par lesquelles la sécrétion des intestins ne serait pas augmentée, produirait plutôt la salivation que la destruction des vers intestinaux. Cependant il arrive quelquefois que des enfans scrofuleux rendent des vers après l'usage du calomélas; mais on aurait tort si on voulait attribuer directement l'évacuation de ces animaux à la propriété vermifuge de cette substance, car cela peut aussi bien être attribué à un effet du hasard, ou bien à ce que la constitution de ces enfans s'était améliorée, et que les vers ont disparu naturellement, comme cela a lieu en pareil cas.

En outre, comme nous devons également admettre que la vic des vers intestinaux doit avoir un terme, quand il sera atteint, le corps de ces animaux sera alors rejeté conjointement avec les matières fécales. $\mathrm{Si}$ dans ce moment, par hasard, le canal intestinal n'a plus la disposition à la formation de ces parasites, alors l'homme n'en aura plus. Presque tous les médecins connaissent des hommes qui, ayant été incommodés, dans leur enfance, d'ascarides, n'en ont plus offert de traces dans un âge mûr, sans cependant pouvoir dire avec certitude à quelle époque les derniers de ces animaux ont été rendus, ou à quel re- 
mède on doit attribuer leur destruction. Quand un médecin parvient à guérir une maladie scrofulcuse, il ne manque pas non plus, du moins, dans beancoup de cas, de débarrasser en même temps le malade de ses vers, n'importe quels remèdes il aura employés. Ce que je viens de dire s'applique, ce me semble, au muriate de baryte, proposé comme un bon vermifuge par Hufeland ', Willis, Bucholz, Stark, Müller et Sulzer.

Huiles grasses. Passerat de la Chapelle est le premier quiait recommandé l'HUILE DE NOIX comme un remède certain contre le tænia; il conseille de prendre à jeuv, pendant quinze jours consécutifs, cinq onces de ce liquide, et de boire, deux heures et demie après, quatre onces de vin d'Alicante. Binet a confirmé par sa propre expérience les bous effets de ce traitement. Je ne crois pas qu'il puisse obtenir une grande faveur parmi les Allemands : d'abord le prix élevé du vin d'A licante offrirait déjà Jeaucoup d'obstacles; mais, ce qui est plus. essentiel, je crois que beaucoup d'estomacs ne supporteraient pas une aussi grande quantité d'huile. Postel de Francière, qui a du reste des idées très-erronées sur la nature et le séjour du tænia, n'envisage cependant pas trop mal la manière dont ces deux liquides agissent; roici ses expressions : "l'huile obstrue les suçoirs des vers (ce qui reste encore à démontrer), elle rend le canal intestinal glissant, et, prise à aussi grandes doses, elle agit comme purgam -Ueber die salzscure schwererde, s. $8 \mathrm{~g}, \mathrm{f}$. f. 
tif. Le vin d'Alicante sert à corriger l'effet nuisible que l'huile produit sur l'estomac, et, en outre le vin est, en pareil cas, un remède préservatif contre la reproduction du ver solitaire. $\Delta$

L'HUILE DE RICIN (oleum ricini) est plus souvent employée dans ce but que l'huile dont il vient d'être question. Dunant et Odier sont les premiers qui ont recommandé et administré l'huile de ricin contre le tænia, en place du bol drastique de madame Nouffer.

Il est cependant de fait que c'est Odier qui l'a employée le premier contre le tænia, et Dunant n'a fait que devancer ce médecin dans la publication de ce remede, ce qu'Odier n'a pas non plus passé sous silence.

On ne devrait pas se servir de l'huile de ricin, telle qu'on la trouve dans le commerce, par la raison quelle est presque toujours rance ou qu'elle le devient bientôt, surtout lorsqu'elle n'a pas été préparée de la manière la plus convenable; celle-ci consiste à enlever aux graines, avant de les exprimer à froid, la pellicule externe, qui est d'un goût très-âcre et mordant ${ }^{2}$. La grande quantité de substances mucilagineuses et aqueuses contenues dans cette huile, est

× MNI. Boutron-Charlard et Henri fils, dans un mémoire qu'ils ont lu à l'Académie royale de médecine, le 17 avril 1824 , combatient par des expériences positives les opinions de certains auteurs qui attribuent à l'embryon et à la partie corticale l'àcreté qu'on remarque dans l'huile de ricin. Nous croyons devoir transcrire ici les principales conclusions de leur intéressant mémoire :

$\mathrm{I}^{\mathrm{n}}$. Que l'enveloppe corticale ne contient aucun principe capable 
cause qu'elle se gâte très-facilement; c'est pourquoi on devrait la faire préparer nouvellement autant de fois que l'on en aurait besoiv. Une huile faite de cette manière agit comme un purgatif très-doux, et sans causer les moindres coliques.

Cependant ies expériences du docteur Arnemann ont prouvé que cettc huile'n'a pas de vertus spécifiques contre les vers; c'est pourquoi l'on pourrait la remplacer par l'huile d'amandes douces ou par toute autre huile grasse dans laquelle on aurait dissous un peu de résine de jalap. Au reste, quand il ne s'agit que de faire évacuer des vers, je me sers de préférence, pour atteindre ce but, de follicules de séné et du jalap. J'ordonne plus volontiers les follicules de séné en substance, c'est-ḋ-dire saus forme de poudre,

de pouvoir communiquer à l'huile de ricin une saveur âcre et désagréable.

$2^{\circ}$. Que l'embryon ou germe, qui jusqu'à ce jour avait été regardé comme le siége d'un principe âcre et vénéneux, ne renferme au contraire qu'zne huile douce ayant un goût agréable analogue à celui du café vert.

$3^{\circ}$. Que le périsperme est la parlie du ricin qui contient le principe purgatif.

$4^{\circ}$. Que les procédés par la claaleur développent dans l'luile de ricin une âcreté qui n'existe pas dans celle préparée à froid et par expression.

$5^{\circ}$. Enfin que l'huile de ricin préparée à froid et par expression étant la plus pure, est la seule qui doive être employée en médecine.

L'huile de ricin extraite à froid peut se conserver fort longtcmps sans être susceptible d'altération ni de rancidité. (Nole du (raducteur.) 
qu'en infusion, parce que je crois qu'une partie de la poudre peut arriver dans le canal intestinal sans avoir été entièrement digérée, et qu'elle agit alors, par son goût âcre, plus directement sur les vers que si on l'avait administrée en infusion.

La racine de Jalap est sans contredit, dans le cas de maladies vermineuses, un des meilleurs purgatifs, et qui peut-être possède en même-temps plus de vertus anthelmintiques que tous les autres.

Wepfer ${ }^{x}$ vante cette racine comme un vermifuge excellent. Van Swiéten 2 l'a également employée avec succès contre le tænia.

Je ne me sers jamais de la résine de jalap, quoique Arnemann ${ }^{3}$ la regarde comme agissant plus efficacement que la raciue de cette plante; ce médecin prétend qu'en donnant une dose de la racine, l'on ne sait pas au juste, n'étant pas sûr de la quantité de résine qu'elle contieut, combien on a administré de celle-ci. Cependant le raisonnement de ce médecin n'est pas propre à me convaincre que la résine doit être préférée à la racine; car, toute réllexion faite, on ne sait jamais au juste, à l'avance, combien de résine tel ou tel canal intestinal peut supporter; mais en administrant avec circonspection la poudre de jalap, il peut tout au plus arriver qu'elle n'agisse pas au degré que l'on s'était proposé, et l'on peut facilement remédier à cet inconvénient, si c'en est un,

' Cicut. aquat. hist., p. 224.

- Loca cilato, $\$ 13372$, p. 540 .

${ }^{3}$ Ouvrage cité, p. 476. 
en domnant la racine à lus forte dose. 11 n'en est pas de même avec la résine; car si, par hasard, cette substance s'est précipitée dans un seul endroit de l'estomac ou des intestins, ce qui peut arriver par une faute dans la diète, ou par l'emploi d'une boisson trèsfroide, le malade éprouvera de fortes coliqnes qui, quoiqu'elles ne soient pas tonjours accompagnées de suites graves, peuvent néanmoins beaucoup inquiéter et le médecin et le malade.

Pour faire rejeter les vers hors du canal intestinal, je ne me sers jamais d'aloès, non plus que de gratiole ni d'ellébore, de gomme-gutte, de scammonée, ni d'autres drastiques semblables.

J'ordonne cependant bien souvent l'aloës, mais seulement à la fin d'un traitement, à des doses trèspetites, et plutôt com me tonique que comme purgatif.

Werlhof et d'autres médecins regardent la gommegutte comme un remède spécifique contre le tænia.

Bisset surtoút vantebeaucoup ce médicament, mais je ne sais pas trop pour quelle raison, car il l'administra un jour à un marin à des doses très-fortes, qui firent en effet rendre quelques morceaux bien longs de tænia, néanmoins ce ver se montra de nouveau au bout de quelques mois, c'est pourquoila gomme-gutte fut employée de nouveau, et même à plusieurs reprises; cependant les résultats furent toujours les mêmes; enfin Bisset l'ordonna encore une fois à son malade au mois d'octolse; et, à dater de celte époque jusqu'au 18 décembre, conıme il résulte de l'histoire de la maladic, le ver n'a plus reparu; mais il reste it 
savoir si le malade n'en aura pas rendu d'autresmorceaux le mois suivant.

\section{Des remèdes fortifians.}

'A près avoir détruit et fait évacuer les vers à l'aide des vermifuges et des purgatifs convenables, il est souvent d'une grande utilité d'employer des médicamens forlifians afin de prévenir une nouvelle production de ces animaux; j'avoue cependant, que la méthode que j’ai suivie jusqu'à présent contre les affections vermineuses, m'a presque toujours dispensé d'avoir recours à ces remèdes.

Ce sont ordinairement les amers et le fer que l'on emploie en pareil cas ; ce dernier peut être administré, tant sous forme métallique que sous celle d'oxide et de sel ncutre. On peut également se servir dans le même but des eaux minérales ferrugineuses.

Werlhof rapporte une observation où il administra à une femme, deux fois par jour, de la limaille de fer, qui lui fit rendre beaucoup d'ascarides (c'étaient probablement des oxyures); plus tard elle fit usage d'eau de Pyrmont, qui produisil d'abord l'évacuation de quelques morceaux de tænia, et ensin un tænia eutier; après quoi elle fut débarrassée, comme par enchantement, de toutes ses souffrances.

$J_{c}$ crois inutile de parler ici en détail de l'emploi des remèdes fortifians, car je dois présumer que tous les médecins, à l'usage desquels cet ouvrage a été écrit, connaissent la manière de les administrer. 
Les remèdes dont nous veuons de parler jusqu'ì présent doivent être regardés, généralement parlant, comme les meilleurs que l'on doive employer contre les vers. Les personnes qui désirent en connaître davantage, n'ont qu'à lire les ouvrages de Vandoevern et de Leclerc; mais comme probablement peu de mes lecteurs possèdent l'ouvrage du dernier, c'est ce qui m'a décidé à leur donner un petit échantillon de l'arsenal vermifuge de ce médecin.

Medicamenta simplicia adversus lumbricos petila ex animalibus.

'Alcis nungula.

Anseris adeps.

Apri urina.

Avium quarumcunque pennarum combustarum cinis.

Bezoar.

Bovis talus ustus, ejus et stercus ustum cum castoreo suffitum.

Butyrum.

Caprinum stercus, aridum tritun, ex melle potui datum tineas ontnes radicitus eximit. (Plin. Valer.)

Caseus veteratus.

Castoreum.

Cantharides.

Cervi cornu et medulla.

Ebur.

Fel variorum animalium.

Gallines adeps, item ejus ovorum putamen contritun.

Hominis urina, et ossa, prasertim combusta.

Ichneumonis pilorum suffitus.

Lumbrici terreni.

Lumbrici intestinorum human. exsiccati, contriti, ore assumpti. Mel.

Monocerotis et rhinocerotis corntua. 


\section{4' SUR LES VERS INTESTINAUX}

Muris stercus, triduo bibitum.

$P$ isces, muria conditi.

Secundina mulieris primipara pulvis.

Scorpiones.

Vermiculi spongia bedegar.

I ipera.

Mon recueil vermifuge tout entier ne consiste pas dans la moitié des remèdes que l'on voit rapportés dans ce tableau, qui ne contient cependant que ceux tirés du règue animal.

SECTION II. Du traitement particulier qu'il faut employer contre chaque espèce de vers ${ }^{x}$.

Nous passons maintenant à l'indication du traitement ou des moyens que l'on doit employer contre chaque espèce de vers intestinaux en particulier, où nous devons ajouter ce que l'on peut dire des signes par lesquels on peut reconnaître la présence de telle ou telle espèce de vers.

LE TRICHOCÉPHALE (trichocephalus dispar) séjourne principalement daus le cœcum, mais on le remarque aussi dans les autres gros intestius. Je ne conuais réellement aucun signe par lequel on puisse soupçonner sa présence, et uéanmoins on le rencontre dans presque tous les cadavres de l'homme, comme Wrisberg' l'a aussi remarqué; le plus souvent ọn ne trouve qu'un petit nombre d'individus de cette espèce dans le même cadavre ; cependant M. Rudolphi en a trouvé une fois

× Félix Pascal. Voyez Nouveau Journal de médecine, rédigé par Béclard, Chomel, etc. Mars I8ı8.

'Dans la préface ajoutée à l'ourrage de Roderer et Wagler. 
plus de mille dans celui d'une femne. On en observe au contraire quelquefois une quantité extraordinaire dans les intestius de quelques bisulques, comme par exemple dans ceux du chamois et du mouton.

Aucun médecin, autant que je me le rappelle, n'a encore observé qu'une personne ait rendu le trichocéphale pendant la durée de sa vie, ce que cependant j'ai eu occasion de remarquer une fois dans le cas suivant :

J'ai traité, il y a à peu près dix ans, une petitc fille de six ans qui élait affectée du tænia. Pendant l'emploi des médicamens que je lui avais ordonnés, elle rendit à plusieurs reprises des ascarides et des oxyures, et une senlc fois un trichocéphale.

Cependant comme on trouve ce ver, je le répète, dans presque tous les individus de l'espèce humaine, et que cependant la plupart ne s'étaient jamais plaint d'avoir étéincommodés par les vers, il résulte de là que les trichocéphales doivent rester dans le cœcum sans déterminer aucun accident, de manière que l'on n'a pas besoin de s'occuper de leur expulsion.

Si cependant une personne én rendail, et s"il y avait des symptômes que l'on pût attribuer à leur présence, je conseillerais d'employer les mêmes renièdes que j'indiquerai tout à l'heure contre les oxyures.

Oxyure vermiculatre (oxyuirisvermicularis). Les oxyures séjournent ordinairement dans le rectum, cependant j’en ai rencontré même dans le cocunn.

11 est de fait que les vers de cette espèce incommodent les malades plus que tous les autres, et 
saus excepter même le tænia, car il reste encore à savoir s'il donne réellement lieu aux accidens que l'on se plaît à lui attribuer, mais au contraire on ne peut nier que les oxyures n'incommodent maintes fois les hommes à un très-haut degré. Il y a cependant des cas où il existe dans le même individu des milliers de ces vers, sans qu'ils annoncent leur présence par aucun symptôme désagréable. J'avais à traiter, il y a quelques années, un jeunc homme de douze ans, atteint d'une fièvre nerveuse: un lavement que je lui avais ordonné fit rendre une quantité considérable d'oxyures, et cependant le malade n’en avait jamais été incommodé : il en fut de même après son rétablissement. Mais dans beaucoup de cas les oxyures ne se comportent pas d'une manière aussi indifférente; ils se tiennent, il est vrai, ordinairement assez tranquilles pendant la journée, mais à l'approche de la nuit ils commencent presque toujours à causer des démangeaisons insupportables dans le rectum; il paraît que la chaleur du lit et l'élévation de la température du corps animal les excitent considérablement. On les rencontre le plus souvent chez les enfans, cependant les adultes n'en sont pas toujours exempts. J'ai connu un vieillard de quatrevingts ans qui en rendit jusqu'à sa mort. Les démangeaisons auxquelles ces animaux donnent lieu provoquent souvent, chez les enfans, des convulsions qui ressemblent à l'éclampsie. Les oxyures s'introduisent aussi dans le vagin, et ils excitent alors les femmes à la nasturbation; je connais même plusicurs exemples 
où ils avaient presque causé une véritable nympliomanie. Scharf rapporte une observation où les oxyures qui s'étaient introduits dans le vagin d'une femme de cinquante ans, produisirent dans cette partie pendant long-temps une démangeaison et une chaleur très-vives. Elle rendit en offet, à plusieurs reprises, une quantité extraordinaire de ces vers.

Becker a observé le même accideut chez une femme de soixante-dix ans : les démangeaisons qu'elle éprou vait dans le vagin firent naître en elle certains désirs à un tel point, qu'elle renouvelait presque les turpitudes de Messaline ${ }^{x}$. Des injections composées de plantes amères dirigées dans le vagin firent évacuer beaucoup d'oxyures, et tous les symptômes cessèrent sur-le-champ.

Dans le traitement des affections des parties sexuelles chez les femmes, les praticiens feront bien de se rappeler les cas que nous venons de rapporter.

Cette espèce de vers n'est pas seulement, je le répète, la plus incommode pour l'espèce humaine, mais elle est aussi la plus difficile à combattre; car on a beau faire évacuer des milliers de ces animaux, il en reste toujours dans les replis des intestins, et comme ils se régénèrent avec une rapidité étonnante, les mênes inconvéniens ne tardent pas à recommencer.

Les vermifuges ordinaires pris par la bouclie per-

Et resupina jacens mullorum absorbuit ictus.

Et lassata viris, sed non satiala recessit. JUY'ĖAL. 
dent dans l'estomac et les intestins grêles, du moins en grande partie, leur propriété essentielle; c'est pourquoi, en arrivant dans les gros intestins, séjour naturel des oxyures, ils agissent d'autant plus faiblemeut, que ces animaux se trouvent toujours entourés de beaucoup de matières fécales. Les vermifuges administrés sous forme de lavement opèrent bien sur les oxyures qui séjournent dans le rectum, mais non pas sur cenx qui siégent dans le cœecum; c'est pourquoi le but que l'on s'était proposé n'est pas complétement atteint.

Quoique leur destruction totale ne puisse être obtenue que très-difficilement par des médicamens, car dans l'âge adulte les oxyures disparaissent souvent naturellement, il faut cependant employei quelques moyeus contre eux, ne fùt-ce que pour soulager momentanément les personnesqui en sont incommodées.

La méthode que j'ai nise en pratique contre ces vers jusqu’à présent avec plus ou moins de succès est la suivante : Je commence par faire prendre, matin et soir, une cuillerée à café de l'électuaire no. 1 (indiqué dans la dernière section) dans l'intention de forcer les oxyures qui se trouvent le plus rapprochés des intestins grêles à descendre plus bas; j’ajoute volontiers à cet électuaire le jalap à une assez forte dose pour causer une légère évacuation. Outre cela, j’engage les malades à prendre par jour deux petits lavemens compiosés de plantes amères (on pent suivre la formule $\mathrm{n}^{\circ}$. 2, qui se trouve aussi dans la dernière section). Il est à remarquer que le malade ne doit 
prendre le lavement qu'après avoir été à la selle, et dans ce cas on peut 'espérér qu'il restera pendant quelque temps dans le canal intestinal, condition essentielle pour qu'il puisse produire de l'effet. Chez les personnes peu irritables, je fais ajouter quelryuefois aux lavemens une cuillerée de fiel de bouf frais. Les malades doivent faire usage des médicamens que je viens d'indiquer pendant plusieurs semaines consécutives, après quoi ils resteront souvent pendant long-temps en repos, et se trouveront même quelquefois radicalement guéris. Un lavement d'huile grasse ${ }^{x}$ fait souvent cesser sur-le-champ les démangeaisons insuportables causées par les oxyures ${ }^{2}$.

Pallas ${ }^{3}$ ą vu employer dans le méme but, avec suc. cès, la fumće de tabac.

Van Swieten conseille les lavemens d'eau froide.

Le meilleur moyen pour débarrasser les femmes des oxyures qui se seraient introduits dans le vagin, consiste dans une injection d'eau froide, avec addition d'un peu de vinaigre.

Le docteur de Vest, de Gratz, m’a assuré qu'il n'emploie contre les oxyures que les fleurs de soufre,

* MI. Sømmerring a également employé avec succès l'huile d'clive en lavement à la dose de deux ou trois onces. ( $B r$.)

- Une dame de vingt-cinq ans, qui était tourmentée de ces vers depuis son enfance, s'en débarrassa à la fin en prenant deux fois par semaine (mercredi et vendredi) une botte d'ail coupce menue dans une demi-tasse d'huile d'olire. $(B r$.

${ }^{3}$ De infest. viventib., p. 258. 
it la lose de dix à quinze grains. Ce médicament doit être pris à jeur, et pendant uu certain temps.

Depuis que M. de Vesı m’a fait connaître ce remede, je n'ai eu occasion de l'administrer qu'à une seule personne, mais l'ayant perdue de vue, je ne puis dire quel effec il a produit. J'ai engagé un jour le docteur Fechner à le donner à un de ses malades, chez qui il avait déji essayé inutilẹment beaucoup de remèdes pour le débarrasser des oxyures; mais ce médecin mobserva que son malade avait déjà fait usage pendant long-tem ps de fleurs de soufre mêlées avec de la crême de tartre, pour combattre une autre affection; cependant les vers n'avaieut pas été détruits pour cela. 11 ajouta qu'il employait ordinairement avec succès contre les oxyures des lavemens composés d'une infusion de plantes amères avec addition d'huile empyreumatique. Je crois en effet que ce mélange doit être très-actif. L'huile de Chabert' ne pourrait guère être employée en lavemens ${ }^{2}$, a cause de l'huile de térébenthine dont elle est en grande partie composée, néanmoins on pourrait fort bien l'administrer par la bouche en mênie temps qu'on emplojerait les lavemeus dont

I. Rollet, chirurgien à Baade, a enployé arec succès l'hnile de Chabert en lavemens, à la dose de deux cuillerées à café mêlée arec une décoction de graines de lin ou une toute autre décoction mucilagineuse. $(B r$.

2 Je ne congois pas pourquoi l'auteur a pu hésiter un moment d'administrer cette hnile en lavement, tandis qu'il la donne à si larges doses par la bouche. Je m'étendrai dans une note plus loin sur ce sujet. (Notc du traducteur.) 
nous venons de parler, afin d'augmenter leur effet. Je me suis aussi proposé de donner l'huile de Char bert, si efficace contre le tænia, dans le but de combattre des oxyures; mais excepté la personne chez laquelle j'ai essayé les fleurs de soufre, il ne s'cn est pas présenté d'autre qui en fùt incommodée. En général on s'en plaint dans ce pays-ci beaucoup plus rarement que des ascarides et des tænias.

Les Ascarides (ascaris lumbricoüdes) séjournent dans les intestins grêles, cependant ils se glissent aussi quelquefois dans l'estomac, où ils donnent lieu à des accidens plus ou moins graves. L'estonıac, irrité par la présence de ces animaux, fait ordinairement beaucoup d'efforts pour les rejeter promptement au dehors. Dans les commentaires de Leipzigr on rapporte un cas où trois de ces vers s'étaient introduits dans la vésicule du fiel par le canal cholédoque.

Cependant je dois remarquer ici que ceux des nématoïdes d'une forte taille que l'on a souvent trouvés dans les reins, ou que l'on a vu sortir par l'uretère n'appartiennent pas au genre d'ascarides dont il est question ici, mais bien à celui des strongles.

Les ascarides font soupçouner leur présence par les symptômes décrits plus haut; on peut les combattre par tous les remèdes que nous avons aussi déji indiqués comme les meilleurs vermifuges, et parmi lesquels chaque médecin pourra choisir suivant sa pré-

" Commentarii de rebus in scientia naturali et medicina gestis. Tom. xıv, Lipsice, 3767, p. 66\%. (Voyez la note.) 


\section{3}

dilection. Nous avons rapporté à quelle dose et sous quelle forme on doit les adninistrer; du reste, en cas de besoin, on n'a qu'ì consulter les matières médicales. Aucun de ces remédes n'est absolument à rejeter. La chose principale consiste toujours, dans le traitement contre les ascarides, à avoir non-seuleneut égard aux vers, mais encore aux causes qui avaient fuvorisé leur production.

Ma métıode d'agir contre les asearides est tréssimple. Si lon me présente un enfant chez lequel on remarque plusicurs des signes pathognomoniques qui caractérisent ordinarement la maladie vermineuse, je lui ordonne l'électuaire $n^{\circ} \cdot \mathbf{1}_{j}{ }^{\mathrm{z}}$, ì la dose d'une cuillerée à café, matis et soir, peu m’importe que cet enfant eût réellement rendu auparavant des vers ou non; après un usage de ce médicament contiuué pendant trois ou quatre jours, les excrétions alvines commencent à devenir plus copieuses et plus liquides, elles sont presque toujours chargées de glaires, et quelquefois de vers. Dans le cas où cet électuaire ne produit pas ect effet, je l'administre à plus forte dose. Pendant l'usage de ce médicament, si toutefois on ne s'était pas trompé dans le diagnostic, et si la maladie était réellement le résultat d'un dérangement, ou plutôt d'une inaction dans les fonctions des intestins, le rétablissement du malade s'opère à vue d'œil. Cela se caractérise surtout par le re.

- La formule de cet électuaire et des antres médicamens numérotés cst indiquée daus la demière section. 
tour de la gaîté, que l'on remarque ordinairement clicz les enfans bien portans.

Si la première portion de cet électuaire n'était jas suffisante pour rétablị la santé, j'en ordonve nue seconde, en réglant toujours la dose de maière qu'elle augineute l'évacuation des matières fécales et des glaires, mais qu'elle ne provoque pas d'excrétions aqueuses ou séreuses.

Dans le cas où les circonstances paraîuaient exiger que le malade soit purgé, je préfère de suspeudre l'usage de l'électuaire et jordonne ì sa place la pondre purgative $n^{0}$. 3 ; car, en ne purgeant le malade qu'une seule fois, cela ne peut pas autant affaiblir le canal intestinal que s'il se faisait pendant plusieurs semaines une perte contiunelle des humen's nécessaires à une lonne digestion. Je ne ne rappelle pas avoir eu besoin d'employer plus de deux portions de l'électuaire pour faire disparaître tous les accidens. 11 m'est du reste indifférent si, perdant ce traitement, le malade rend des vers ou non; jai remarqué quelquefois que les malades n'avaient évacué quelques ascarides qu'après un rétablissencn complet.

Si j’ai à traiter une personne d'une constitution Iymphatique, j'ordonne pendaut quelque temips l'usage des gouttes $n^{\circ} .4$, pour prévenir les rechntes.

A l'égard du régine que l'on doit observer pendabı le traitement, je défends l'usage des farineux, des légmmes secs et des substances grasses; jengage également les malides i ue pas manger trop de pain. 
Je ne me suis jamais trouvé dans le cas d'avoir bescin de recourir it d'autres noyens pour combattre lez ascarides.

Le вот̈riocéphare (bothriocephalus latıs), et le TENAA (tcenia solium.)

Je suis olıligé de parler de ces deux genres de vers à la fois, parce que les praticiens, en indiquanı les remèdes propres à les combattre, n’ont pas eu égard a la grande différence qui règne réellement entre ces deux animaux. L'un et l'autre séjournent dans les intćstins grêles, quoique Postel de Francière prétende qu'ils ne séjournent que daus le ccecum. Cette assertion a valu ì ce médecin une dispute savante avec IMM: Rolin et Binet.

La présence de ces denx animaux dans le canal intestinal n'est pas caractérisée par des signes particulier's et qui ne puissent aussi bien indiquer l'exis-. tence des ascarides. Mais les tænias s'annoncent, dans beaucoup de cas, plus aisément que ceux-ci par l'évacuation de quelques articulations; au moins cela a lieu pour le véritable tænia : quant au bothriocéphale, je ne puis rien dire avec certitude, faute a'une expérience suffisante. Celui-ci ne se découvre pas ordinairemént par la sortie de petites parties d'articulations détacbées, mais bien par celle de morcearix d'une certaine longueur.

Comme ces deux espèces de vers ne cèdent pas en général à l'emploi des vermifuges ordinaires, et quoiqu'ils soient quelquefois rendus spontanément, beaucoup de médecins et de charlatans ont proposé 
plusieurs remèdes, en général très-compliqués, qu'ils ont tenus ordinairement secrets pendant longramps, et qu'ils ont vautés comme de vrais spécifiques.

Afin de ne choquer personne, nous allons firre connaître, par ordre alphabétique, les différentes méthodes proposées jusqu'a présent contre le tæuia.

SECTION HI. Des difiérentes méthudes de traitenteut contre le trenia

Méthode d'Alston. "Après avoir purgè le malade, un jeudi avant le chingement de lune (ce sont les paroles d'Alston), avec suffisante quantité de follicules de séné et de nanne (substances que j'il fait infuser dans une décoction de raciue de chiendent), je lui donne, le vendredi suivant, une once de ziuc pur (passé au tamis), dans quatre onces de sirop ordinaire ${ }^{2}$; le samedi, une demi-once de zinc daus decix onces de sirop, et autant le dimanche; le malade doit être purgé le lundi avec la même médecine. Quoiqu'il soit probable que le jour el l'éporjue à laquelle on doit administrer ces médicamens n'influent flas sur leur eflicacité, je me suis cependant temu strictement à la prescription proposée par un charla-tan, et comme l'omploi de ces remèdes avail répondu à mon attente, je n'ai rien voulu changer à son mode de traitement. "

- Common treacle ne peut signifier ricn au tre chose dans ce cas-ci ๆ̨ue du sirop hollandais et non pas de la thríraque, comme on le toure indicué dans la traduction allcuand, + "lourage d'Alston el thans la Malière médicale de M!llin; car ine pareille dose de thcriaque causerait la mort d'un homme. 
Pallas l'approuve beaucoup, cependant il a vu un cas où il n'a pas répondu à son attente.

A vant de mettre ma méthode actuelle en pratique, j'ai essayé celle d'Alston sur plusieurs de mes malades incommodés du tænia; mais aucun n'a été radicalement guéri : tous sont revenus, après un espace de trois mois, se plạiguant de l'inefficacité des remèdes qu'on 'leur avait administrés, car le tænia s'était montré de nouveau.

Métrode de Béck. Nous en devons la publication à M. Langge '. In voici l'exposé :

Ric. Mercurii dulcis, scrupulum unum; cornu cervi usti, cinnabaris an'imonii, ana grana decem; m., f. pulv.d. s. (A.)

Rc. ol. amygdal. dulc., uncias duas, d.s. (B.)

Iic. radic. flicis maar., drachnam unam; jalapp., summi-gutte, T.eru. cardui benedict., eburis usti, ana drachn. semis., m., f. pulv. subtiliss. diside in iij part. ceq. d. s. (C.) ou spécifique.

Le malade tourmenté par le tænia commence par prendre, ì quatre ou cinc heures de l'apiès-midi, la poudre marquée $\mathbf{A}$ dans une cuillerée d'eau commune, ou bien dans une cuillerée d'eau de gruau; le soir, après avoir nłangé un potage, il doit boire deux onces d'huile d'amandes douces; le matin suivant, il prendra un des trois paquets de la poudre du spécifique marqué $C$, dans un peu de thé édulcoré avec une cuillerée de sirop de fleurs de pêcher. Cette poudre détcrmine ordinairement, dans l'espace de denx heures, deux ou trois vomisseniens; le malade freutalors faire usage d'un peu de thé. Tout ce qu'il a

FIufeiands Journal, t. Xin, st. 2 , p. 153. 
rendu par le bas, pendant la nuit et dans la matinée, duit être examiné, et si le tænia ne s'y trouve pas en entier, on doit administrer au malade un second paquet, c'est-ì-dire deux heures après avoir pris le premier, et enfin le troisième : dans le cas où cés remèdes ne produisent pas leffet désiré, on firit passer un lavement, composé d'une décoction de plantes amères et d'une suffisante quantité de sulfate de magnésie, et, si le ver n'est pas évacué par ce moyen, on donnera alors an malade, dans l'espace de trois hesres, la poudre suivante, divisée en trois paquets.

Ric. pulv. radic. jalapp., diachm. unam; herb. gratioles, scinpul. unum; m., f. dos.tres. (D.)

Cette niéthode peut être utile contre le bothriocéphale, mais non pas contre le véritable tænia. Le mélange de la racine de fougère mâle avec les purgatifs u'est point du tout convenable.

Méthode de Bucilanan. Buchanan a reçu du doctcur Roussel la formule de ce remède indien. L'ou fait bouillir une demi-livre d'écorce fraiche de la racine de grenadier, dans trois pintes d'ean commune, jusqu'à réduction de deux ‘. Outre cela, l'on faiı un mélange de graines pulvérisées de seca dana (convulvulus nil), avec celles de putas papara (erythina monosperma), de chaque substance, un deni-gros. A près avoir fait fondre un peude sucre dans la bouche,

" Breton (Voy. med. chirur. transact., vol. xi, part. I1) fiut bouillir deux onces de celter racine frầche daus nue pinte of demie d'au jusqu'a réduction de moilié; it en domie un serre boules les ilemi-heures. ( Jir.) 
on avale cette poudre, et on boit par dessus une tasse de la décoction précédente pendant qu'elle est encore chaude; on doit en faire usage à des intervalles trèscourts, jusqu'à ce qu'elle soit entièrement prise. Ces médicamens produisent des évacuations par haut et par bas.

Les graines de seca dana et celles de putas papara sont ordinairement employées comme purgatifs par les médecins de l'Inde. Buchanan regarde la décoction comme le reniede le plus actif de ce traitement.

Méthode de Clossius. Cette méthode a été décrite en premier lieu dans les Annales de Fritze. Le fils de Clossius l'a publiée de nouveau dans le Magrasin de Baldinger, telle qu'il l'avait apprise de feu son père, qui l'avait découverte par hasard. Voici comment il s'exprine: "Mon père avait à soigner une dame en Hollande, qui était malade depuis longtemps, et qui avait en nême temps le veutre extrênıement ballonné; il lui ordonua, je ne me rappelle plus po' 'quelle raison, de la térébenthine de la manière suivante :

Ric. terebinth. Venet., drachm. j; solv. in vitell. ovior. q. s.; add. $u_{i}$, menth. piperit., unc. iv, $S$. , à prendre peu à peu.

Il fut appelé tout à coup le soir, et on lui dit que la malade était expirante. En effet, il la trouva sans connaissance, et le front couvert d'une sneur froide. lufin, elle se remit peu à peu, et lui dit qu'elle iprouvaic une sensation semblable à quelque chose yui descendait dans le ventre, eı qui lui donnait en mème temps envic d'aller à la selle; unc évacuation 
de nuatières stercorales eut récllement lien, ec qui produisit l'aflaissement presinne complet du basveutre. Lion vit dans les déjections un paquet blanc, qui n'était a utre chose qu'un bothriocéphlıle, mais qui n'élait pas pourvu de trompe. Quelque temps après, le ventre se ballonua de nouveau, et presque aussi fortement que la première fois; il ordonna alors sou drastique (que nous ferons connaître plus bas), qui fit évacuer le ver en entier, et il n'a plus reparu.

"Depuis ce temps, mon père administra ì tous les malades qu'il croyait affectés du tænia, son remède explorateur, c'est-ì-dire de la térébenthine, en réglant tontefois les doses selon l'âge, l'irritabilité, etc., desindividus. A près s'être convaincu par ce moyen de l'existence du ver, il commençait par faire observer aux malades un régime partieulier; ils étaient obligés de ne manger, pendant un mois, que des choses piquantes et salécs, comme, par exemple, du fromage, du poisson salé, da saucisson, de la viande silée, du jambon, etc. En outre, ils devaient aussi boire plus de vin que d'habinde; quelques jours avant de faire usage de son drastique, il administrait anx malades, tons les soirs, un grain d'opium, ou bien un peu de laudanum liquide de Sydenbam. En suivant cette méthode, il m’assura qu'il avait été obligé, dans bien des cas, de ve domner qu'une seule dose de son drastique pour faire évacuer le txnia en entier. Voici la formule du drastique et des antres nédicaneus dont il se servait dans le cours du traitement. 


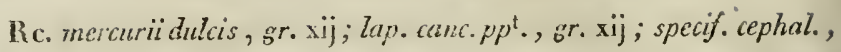
M. gr. vi; m. f. pulv. S. (No.1.)

Rc. ol amygdal. dulc. unc. $S_{s: S .}$ ( No. 2.)

Ric. s.-guttce, gr. xxxvj, rad. angel., gr. viij, pulv. card. bened., pulv. cpileps., ana scrup. $\mathrm{j}$, m. , f. pulv. subliliss. div. in iij $p . \alpha q . S$. (No.3.)

"Le malade prend, à quatre ou cinq hemres de l'après-midi, la poudre préparatoire marquée $n^{\circ} .1$, mêlée dans une cuillerée d'eau; il doit souper très-légyerement, et boire, avant de se coucher, à la dose prescrite le remède $n^{\circ} .2$. Le matin suivant, le malade étant encore au lit, ou levé (l'auteur prélère cependant qu'il soit couché, comme on peut le conclure d'après sẹ remarques), doit prendre une dose de la poudre marguće no. 3 , dans une petice tasse de thé ou d'eau tiède, ou bien, s’il le préfére, il peut l'avaler enveloppée daus du pain à chanter. Cette dose occasione ordinairement, dans l'espace de deux heures, deux ou trois vomissemens et quelques selles. Lion peut faciliter ces évacuatious par l'usage de bouillons légers, ou de quelques tasses de thé fiible; deux heures après on examine les excrémens, et si le ver ne s'y trouve pas encore en entier, le malade avalera une seconde dose de la même poudre. Si deux henres et demie après le ver n’est pas encore évacué, le smalade doilfaire usage de la troisiéme dose. Cette dernière ne manque jamais de faire sortir le ver en entier, qui, s’il esı rendu de bomue heure je mêne jour, donuera des signes devic; dans le cas contraire on letrouvera mort le lendemain daus les déjections du malade. » 
L'anteur de cette méthode ajoute encore dans ses remarques, qu'il y a des malades auxquels ces renèdes ne causent ui romissemens ni selles, et qui cependant rendent le ver dans l'espace de vingt-quatre heures par une selle naturelle. Cette remarque no m'étonne pas, car je crois avoir observé que de trèspetites doses d'aloës et de remêdes drastiques en gé-néral produisent unc plus grande abondance d'excrétions alvines aqueuses on séreuses, que des doses plus fortes de ces médicamens ${ }^{x}$.

J'ai copié textuellement, non-seulement les ordonnances, mais aussi toute la méthode de traitemeni de Clossius, parce que, dans l'emploi de médicamens anssi héroïques, il importc beaucoup d'être informé de toutes les mesures de précautions que l'on doit prendre, et dont la négligence pourrait mettre la vic du malade en danger.

Eu général, quand il s'agit de donner son avis sir l'efficacité ou la non efficacité d'une méthode curative quelconque, je crois qu'il faut la suivre telle que son inventeur l'a proposée; mais" ordinairement on se permet de la modifier, de la moderniser, pour ainsi dire, de manière qu'il ne reste à la fin qu'une sorte de squelette.

Nous devons sans donte anx progrès de la chimie qu’il n'y a plus de médecins aujourd'hui assez igno-

- J'aroue avoir observé constamment le contraire de ceque l'auteur avance concernant les effets de l'aloề et des autres drasliques. ( Note dutraducteur.) 
rans pour prescrire ensemble le tartre vitriolé, l'arcanum duplicatum et le sel nommé sal polychrestum Glaseri, parce que ce serait indiquer la mêne chose sous trois noms différens. Mais il y a des composés d'autres substances médicamenteuses qui ne forment pas exactement un tel contresens chimique (chemisches neutrale), et qui dans leur composition agissent d'une manière toute différente que chacnn des composans pris à part; aussi différente est l'action des sels neutres de l'action des acides et des alcalis, dont ils sont composés; aussi différente est, par exemple, l'action de l'opium et celle des acides minéraux quand on les donne mêlés ensemble ou bien chacun séparément. La même chose doit avoir plus ou inoins lieu pour d'antres mélanges moins hétérogènes. C'est pourquoi les médicamens indigènes que nous voulons substituer aux exotiques se montrent si peu efficaces. On ne cherche ordinairement l'efficacité d'un médicament que dans sa propriété la plus saillante. Si l'on trouve par liasard cette même propriété dans une autre substance indigène, on s'imagine qu'elle pent remplacer l'exotique, mais on n'a pas égard au mélange d'autres parties constituantes, par la raison qu'on ne les considère pas comme essentielles, tandis que ce sont peut-être elles qui modifient justement le remède de manière qu il produise tel cflet et non pas tel autre.

La vanille, le poivre, la canelle, sont connus sous le nom d'épices; mais qu'elle est grande la différence de leur action seuleneat pour le gontl, faculté inhé- 
rente à notre individualité; peut-on douter que ers trois substances n'agissent pas anssi d'une manic̀re toute différente sur la totalité de notre économie animale. Quelle substance pourrait remplacer, par excmple, le gingembre? je n'en connais aucune, et cependant on se permet souvent de le retrancher dans la préparation de l'elixir acide anglais.

Métiode de Desault. M. Brera ${ }^{2}$ s'exprime it ce sujet de la manière suivante : "Le fameux docteu" Desault avait observé à Bordeaux que les tænias (sans doute ceux qui sont armés) s'attachent souvent aux parois des intestins, de manière que leur expulsion est très-difficile à obtenir; cela lui fit naîtrè l'idée aussi spirituelle que hardie d'administrer alternaticement aux malades incommodés du tænia, des frictions mercurielles sur le bas-ventre, et de leur donner un remède purgatif chargè d'une forte dose de calomélas. "

D'après ınon idée, cette métlode n'est rien antre chose qu'uue méthode évacuante et qui n'offre aucun avantage réel en pareil cas; car j’ai déjà dil avoir vı des personnes qui avaient fait un usage même imnodéré de préparations mercurielles sans avoir pu se débarrasser de leurs tænias.

Méthode de Richard de Hautesierck. Il propose les remèdes suivans pour détruire le tænia.

Bolus gunzmi-guttce. - Ric. gummi-sutice, gr. x; semin. colocjnth., $\mathrm{n}^{0}$. iij, cum amygdal. amar. $\mathrm{n}^{\mathrm{o}}$. x.; triturentur et cum syrupo ab-
synth. f. bol. ij.

'Vorlesungen, p. I18, oủ il cite Venel (Précis de matière mé- 
Le malade doit prendre ces deux bols en une fois; et réitérer leur usage tous les hnit jours.

Pilula foctidee. - Rc. aloüs soccotrina, asce foetida, ana unc. j; salis absynihii, semi-unc, ; olei roris marini, drachm. ij; cum elix. ppt.f. pil., ${ }_{s} r . \mathrm{x}$, pund. S. ; à prendre matin et soir deux pilules, et boire par dessus six onces d'une décoction de fougère mâle.

Opiata jovialis. - Re. stanni purissini, inercurii vivi, ana unc. j; stcnno liquefacto, adde argentum vivam, posiquan mixtura refrixerit, in pulverem cum concharum ppt. unc. $\mathrm{j}$, redigatur.

R. hujus pulieris, conserve absynthii, ana zunc. ij, cum syrupo absynth.f. opiata; à prendre deux gros dans la journée.

11 est à remarquer que le gros en France contient soixante-douze grains, c'est pourquoi il faut toujour's retrancher un sixième du poids, si l'on veut que la dose des médicamens corresponde à la mesure allemande.

Méthode de Herrenscirwand. Après avoir fait un secret de sa méthode pendant long-temps et dans des intentions qui ne sont pas très-lonables, Herrenschwand la communiqua enfin à plusicurs médecins, mais les différentes formules qu'iḷ en a donuées ne s'accordeut pas très-exactement entre elles. Pallas assure que l'examen chimique que l'on avait faic à Pétersbourg des remédes anthelmintiques de Herrenschivand a fait voir qu'ils contenaient non-seulement du mercure, mais aussi de l'arsenic et une terre absorhante. Herrenschwand a indiqué le traitement suivant dicale, augmenté de notes, etc., par Carrère. Paris, 17 18, १. II, p. 337. ) 
daus sa dissertatiou, et en ces termes: "Le moyen le plus efficace qui soit venu à ma connaissance pour expulser les deux espèces de tænia et qui agit sans nuire à la santé, consiste à prendre, supposé que l'estomac soit en bon état, pendant deux jours consécutifs, et à deux reprises, c'est-à-dire le matin à jeun, et le soir, après avoir soupé légèrement, un gros de fougère mâle pulvérisée, délayée daris de l'eau ou enveloppée dans du pain à chanter. Faute de fougère mâle, on peut se servir de l'autre espèce de fougère, mais il faut qu'clle ait été cueillie en automne, et qu'on l'ait fait sécher à l'ombre. Ce remède provisoire ne cause que peu ou point de gêne. Le troisiène jour le malade doit preadre à jeun la poudre suivante :

Ric. summi gutice gr. xij; sal. alsinth. neutr. gr. xxx; sapon. Starkeigr. ij; misce intime, d. ad. chart.

Cette poudre excitera, pendant l'espace de deux ou trois heures, un ou deux vomissemens, et produira autant de selles. On peut faciliter ces évacuations en Juvant, après chacunc, un vẹ̦re d'eau liède ou quelques tasses de thé. Trois heures après le malade doit faire usage d'une tasse de bouillon, dans lequel on aura versé une once d'huile de ricin provenant de l'Amérique, qui vaut beaucoup mieux que la nôtre; cependant l'on peut également se servir de la dernière, fante de l'autre. Une heure après le malade prendra de nonveau une même dose de cette huile, et si, après un intervalie de deux heures, le ver n'était pas cucore rejeté, le malade sera obligé d'avaler une troisième dose. Ce remède purge très- 
doucement, et le ver ne tardera pas à être évacué; mais en cas que cela n'ait pas lieu, on doit administrer vers le soir un lavement composé de parties égales d'eau et de lait, avec addition d'huile de ricin, qui fera rejeter le ver en entier. "

Dans cette prescription il n'est question ni de la gratiole, ni du mercure doux, ni de la scammonée, ctc. , qui se trouvent cependant dans les prescriptions publićes par l'autcur antérieurement. Il parait résulter de. l'emploi de médicamens si variés, que toutes les méthodes lui ont paru insuffisantes coutre le véritable tænia; c'est sans doute la raison pour laquelle il a eu aussi recours à l'huile de ricin proposée par Odier, mais cette huile n'a pas non plus, comme cela a déjà été observé plus haut, de vertu spécifique contre cette espèce de vers.

Méthode de Hufeland. M. le professeur Hufeland a publié sa métlıode dans son Journal de médecine (vol. $x$, calı. 3, p. 178 ). Il fait boire aux malades, tous les matins à jeun, une décoction d'ail dans du lait, et il leur administre dans la matinée, dans l'après-midi ct le soir, une cuillerée à bouche d'huile de ricin. Outre cela les malades doivent prendre journellement une demi-once de limaille de zinc mêlée avec de la conserve de rose, et faire plusieurs frictions avec du pétrole sur le ventre. Le soir on doit administrer du lait en lavement. Les malades sont en outre obligés de ne manger que des substances salées et âcres. Cette méthode doit être continuée pendant plusieurs semaines consécutives, et mêrnc plus long- 
tensps, jusqu'à ce qu'enfin la tête du tænia ait été rendue. Dans le cas où cela n'aurait pas lieu, les malades sont alors forcés de prendre de nouveau les remèdes déjà cités, mais à plus fortes doses. Ce médecin conseille également l'usage de l'eau de Pyrmont et de Driburg.

Nous iguorons si cette méthode a été souvent employée avec succès, néanmoins nous pouvons conclure de la durée du traitement, que M. Hufeland n'a pas une grande confiance aux remedes que l'on dit expulser le tænia au bout de trois heures; et nous croyons qu'il a parfaitement raison.

MÉthode de LAGene. L'auteur assure, dans une lettre adressée à $\mathbf{M}$. Minaur, qu'il n'a jamais fait un secret de son remède contre les cestoïdes. Il regarde, comme contraire aux devoirs d'un médecin, de tenir un remède caché quand il peut être utile à l'humanité souffrante : son mode de traitement contre ces espèces de vers est le suivant. Le malade commencera par prendre, avant de se coucher, un lavement fait avec une décoction de figues; le matin suivant, à jeun, il fera usage de la poudre suivante délayée dans un ver de vin blanc:

Rc. Radic. valerian. s. recent. puls. drachm. $\mathrm{j}$; putamin. ovor. calcinat. et ppt. gr. $\mathrm{xx}$; misce.

Le malade restera couché et se couvrira bien ; il s'o. père alors ordinairement une douce transpiration. On ne donne au malade, pendant trois heures, ni à boire ni à manger; ensuite il lui est permis de prendre un potage; en général, il est obligé d’observer un régime 
sévére pendant tout le temps du traitement. Le malade doit prendre la même poudre pendant trois jours consécutifs, et le quatrième jour le purgatif suivant:

Rc. mercurii dulcis gr. $\mathbf{x}$; panac. mercurialis gr. iv; diagrydii sulfurat. ${ }^{r} r$. xij; syrupi flor. persicor. q. s.ut fat bollus. d. s.; à prendre à jeun.

Deux heures après le malade boira un verre de la tisane suivante :

Re. fol. senn. mund. unc. semis; infund. in aq. fervid. libr. ii; adde salis tartari fixi ${ }_{6}{ }^{r}$. viij; diger. per noct. et col. ad usum.

Unc heure après on Jui donnera un bonillon. L'usage de cette tisane doit être continué on suspendu, selon qu'elle purge plus ou moins fortement; du reste, on gouvernera le malade comme quelqu'un qui a pris médecine. Le soir on lui administrera de nouveau un lavement composé de la manière déjà citée plus haut. Si l'on a à traiter des personnes robustes, ou d'autres, qui ont la langue chargée, et chez lesquelles se remarque un embarras gastrique, on commencera par les faire vomir avec le tartre stibié donné en lavage, c'est-à-dire étendu dans une grande quantité d'eau. Je fais, dit Lagène, répéter ordinairement l'usage de la poudre vermifuge pendant trois jours, et j'ordonne ensuite la poudre purgative; il m'est arrivé, ajoute-t-il, plusieur's fois d'ètre obligé de revenir sur l'emploi de ce reméde une troisième fois; mais ces cas sont très-rares. La dose des remèdes est calculée pour une personne adulte, et on la varie selon l'âge et les circonstances.

Je ne doute pas que, par ce tritement, on ne 
fasse souvent évacuer les deux espéces de cestoïdes; mais il ne me paraît pas certain que les malades en soient radicalement guéris.

MÉthode de Lieutaud. M. Reinlein' l'a fait connaître telle qu'elle se trouve indiquée dans le Précis de matière médicale de Lieutaud. (Voy.t. 1, p.432.) Elle consiste dans l'emploi des remédes suivans:

Rc. diagryd., crentor. tartar., ana scrup. semis.; antimonii diaph. gr. xïj; pulv. rad. filic. mar., mori fructu nigro, ana drachm. semis ; m. f. pulvod. s.; à prendre à la fois.

Rc. pnlv. sabin., semin. ruth., ana ${ }^{g} r$. viij; mercurii dulcis ${ }_{g} r$. iv ; olei essent. tanacet. guttl. vj; m.f. cum syrupo persicor. bolus. s. ,à prendre à la fois le matin, et boire après un verre d'une infusion vineuse de noyaux de pêches.

Méthode de Mathine. Ellea été long-temps tenue secrète jusqu’’a ce que S. M. le roi de Prusse l’acheta; ellē fut publiée d'abord dans les éphénérides de Formey, et, plus tard daus le journal de Hufeland. Voici de quoi elle se compose.

A. Re. limat. slam. anolic. pur. unc. j; rad.filicis mar. drachm. vj; pulv. semin. cince unc. dimidian; pulv. rad. jalapp. resinos., salis polyuirest., ana drachm. $\mathbf{j} ; \mathrm{m} . f$. cum mellis communis sufficiente quantitate electuarium.

B. Re. pulv. rad. jalnpp. resinos., salis polychrest., ana scrup. ij;

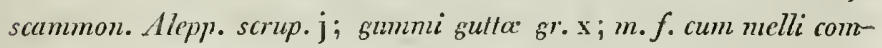
mune electuarium.

Avant de faire usage de ces remédes, le malade est obligé d'observer un régime sévére pendant plusieurs jours. Il se tiendra à l'usagge de choses salées, comme des harengs, eıc., d'un potagre de pain léger et de légumes d'une facile digestion. On commence le tarite-

${ }^{1}$ Vebersetzung, p. 179 . 
ment par l'électuaire $\mathbf{A}$, à la dose d'une cuillerće à café toutes les deux heures, pendant deux ou trois jours consécutifs, et même plus long-temps, jusqu'à ce que le malade sente les mouvemens du ver dans les intestins. Lorsque cela a lieu, il doit alors faire usage de l'électuaire purgatif marqué $B$, à la inême dose que le précédent, et dans les mêmes intervales de temps jusqu'à l'évacuation du ver. Dans le cas où cette évacuation tarde trop à s'effectuer, on donne au malade quelques cuillerées d'huile de ricin fraîchement préparée, ou bien on lui administre cette huile en Iavement. On doit varier la dose de ces médicamens selon l'âge, le sexe et la constitution.

Il me semble que cette méthode ne valait pas beaucoup la pcine d'être tenue secrète. ${ }^{2}$

MÉthode de Nouffer. Madame Nouffer, demeurant à Morat, dans le canton de Berne, a fait beaucoup de bruit pendant vingt ans consécutifs avec un remède contre le ver solitaire, dont elle avait hérité de son mari, et qu'elle a administré à beaucoup de personnes qui s'étaientrendues auprès d'elle pour se faire guérir. Pour le bonheur de cette dame il se trouva dans ce nombre un prince russe nommé Baratinski, qui rendit, après avoir fait usage de son remède, quatre aunes de bothriocéphale; cependant ce ver reparut six mois après : le prince, qui se trouvait alors à Paris, invita cette dame à venir le trouver, ce qu'elle fit en effet, et elle fut assez heureuse pour lui

x Mongany et Ritter. Voyez Rust magazin fiir dic gesammte heilkunde. 8 ter band, 2tes heft 1820, p. 352. ( $B r$.) 
faire évacuer un ver d'une longueur de huit auncs. Plusieurs autres personnes furent encore traitées par elle avec un égal succès. Ces cures si heureuses firent grand bruit et parviurent même jusqu'aux oreilles du roi, qui convoqua une commission dans le buc d'examiner le remède de madame Nouffer. Sur le rapport que fit la commission, le roi l'acheta une somme de dix-huit mille livres. Madame 'Nouffer indiqua à MM. Lassonne, Macquer; Couriez de la Motte, A.-L. de Jussieu, J.-B. Carburi et Cadet, nembres de la commission, la manière de préparer son remède et le mode d'emploi suivant, que nous allons rapporter dans ses propres termes".

«Les malades n'ont besoin d'aucune préparation particulière jusqu'à la veille de l'administration du reméde. Ce jour ils doivent se priver de tout aliment après le dìner, et prendre seulement, sur les sept ou huit heures du soir, la soupe $\left(n^{\circ} .1\right)$; un quartd'heure après je leur donne un biscuit et un gobelet ordinaire de vin blanc, pur ou détrempé avec de l'eau, ou même de l'eau toute pure à ceux qui ne sont pas habitués au vin. Si le malade n'a pas été à la garderobe ce jour-là, ou qu'il soit échauffé ou sujet aux constipations, ce qui est rare quand on a le ver plat, je lui fais prendre le lavement simple $\left(n^{\circ} \cdot 2\right)$, qu'il doit garder le plus long-temps qu'il pourra, ensuite il se couchera et reposera de son mieux.

- Voyè Traitement contre le ténia ou ver solitaire, pratiqué ì Morat, en Suisse, examinć et approuvé à Paris, publić par ordrc du roi. Paris, ${ }^{7} 776$, de l'inmprimerie royalc. 
"Le lendemain de grand matin, environ huit ou neuf heures après la soupe, il prend dans soa lit le spécifique $\left(\mathrm{n}^{\circ} .3\right)$, et pour faire passer les nausées qui viement quelquefois à la suite, il mâche du cítron on autre chose semblable, ou se gargarise la bouche avec quelque liqueur', sans rien avaler, ou il se contente de respirer du bon vinaigre ${ }^{3}$. Si, malgré ces précautions, les nausées sont trop fortes, si les efforts dı malade pour garder le spécifique sont impuissans, il en reprendra une nouvelle dose dès que les nausées seront passées, et tâchera de s'endormir aussitôt après.

"A u bout de deux lieures, il se levera pour prendre le bol purgatif $\left(n^{\circ} .4\right)$ en une ou plusieurs prises, et boira pardessus une ou denx tasses de thé vert peu chargé; il se promenera ensuite dans sa chambre. Lorsque la purgation commencera à faire effet, il prendra de tomps à antre une nouvelle tasse de thé léger jusqu'ì ce que le ver soit rendu; alors, et pas avant, je lui donne un bouillon qui est bievtôt suivi d'un autre ou d'une soupe, si le malade la préfère; il dine comme on fait un jour de purgation; après le dîuer il se repose sur son lit, ou va faire un tour de promenade, se conduisant tout ce jour avec ménagenient, soupant peu et évitant les alimens indigestes.

"La guérison est alor's parfaite, mais elle ne s'opère pas avec la même promptitude dans tous les sujets. Celui qui n'a pas gardé tout le bol, ou que ce bol ne

' Les médecins de Genève el de la Suisse française ordonuent, pour aiteindre le même but, une tasse de caié à l'eau bien chaud. 
purge pas assez, prend, au bout de huit heures, depuis deux jusqu'à huit gros de sel de Sedlitz, ou, à son défaut, de sel d'Epsom dissous dans un petit gobelet d'eau bouillante. On varie la dose selon le tenpérament et les circonstances.

"Si le ver ne tombe pas en peloton, mais qu'il file (ce qui arrive particulièrement quand le ver est engagé dans des glaires ienaces qui ont peine à se délacher), le malade doit rester à la garde-robe sans le tire: ; et boire du thé léger un peu chaud. Quelquefois cela ne suffit pas, et l'on a recours à une dose de sel de Sedlitz, sans changer de position jusqu'à ce que le ver soit rendu '.

" ll est rare que les malades qui ont gardé le spécifique et la purgation ne rendent pas le ver avant l'heure du dîner : ce cas particulier a lieu lorsque lc ver tué reste en gros pelotons dans les intestins, de facon que les matières, ordinairement plus elaires sur la fin de la purgation, passent au travers et ne l'entraînent pas. Le malade peut alors diner, et l'on a observé que le manger, joint à un lavement, concourait à la sortie du ver.

"Quelquéois le ver sort par l'action seule du spćcifique, avant qu'on ait pris le bol, alors on ne lui donne que deux tiers de celui-ci, ou on lui substitue le sel.

' Sitôt que le trenia paraît hors du recium, il faut plonger surle-champ Ja partie sortic dans du lait ou de l'eau tiède; ce seul moyen suffit souvent, dans le cas oì le ver jouit encore de la vie, pour opérer promptement la sortic du reste. (Note dut traducteur.) 
"Les malades ne doivent pas s'inquiéter des chaleurs et des malaises qu'ils éprouvent quelquefois pendant l'action du remède avant ou après une forte évacuation, ou lorsqu'ils sont prêts à rèndre le ver; ces impressions sont passagères et se dissipent d'ellesmêmes, ou à l'aide du vinaigre respiré par le nez.

"Ceux qui ont vomi le spécifique et le bol, ou qui n'en ont gardé qu'une partic, ne rendent quelquefois pas de ver ce jour-là; je leur fais reprendre le soir la soupe $\left(n^{\circ} .1\right)$, le biscuit, la boisson, et, suivabt les circonstances, le lavement $\left(n^{\circ} \cdot 2\right)$. Si le ver ne sort pas dans la nuit, je donne, le lendemain de bon matin, une nouvelle dose de spécifique, deux heures après six à huit gros de sel, el je dirige du reste mon malade comme le jour précédent, à l'exception da bol, que je supprime.

«J'ai remarqué que les grandes chaleurs diminuent un peu l'action de mon remède, aussi j'ai toujours préféré de l'administrer daus le mois de septembre. Quand je u'avais pas le choix de la saison, et que je me suis vu obligée de traiter des malades dans les jours les plus chauds de l'été, je donnais le spécifique de très-grand matin. Avec cette précaution je n'ai remarqué aucune différence dans les effets ni dans les suites, "

Le ver solitaire ${ }^{x}$ est le seul sur lequel le remède de madame Nouffer a une action certaine, quoiqu'elle le

${ }^{x}$ Les membres de la commission out appelć de préférence le bothriocéphale ver solitaire, pour le distinguer du ver cucurlitain, nom qu'ils ont appliqué au tenia solism. 
regarde aussi comme très-utile contre le véritable tænia; elle avertit pourtant que ce dernier est beaucoup plus difficile à déraciner, et que, pour en guérir, il faut répéter le traitement plus ou moins souvent.

A près cette déclaration, il fut décidé que le 23 juin on procéderait aux premières expériençes qui devaient constater la bonté du remède de madame Nouffer. Cette dame, de concert avec M. Móttet (ancien conseiller secret et chirurgien de la ville de Morat, et depuis environ huit ans confident de sa méthode de guérir cette maladie), administra, en présence des membres de la commission, suivant le procédé indiqué, la soupe, le lavement, le spécifique et le bol purgatif à cinq sujets différens, en prévenant qu'un seul avait décidément le ver solitaire, et qu'elle ne proposait que celui-là pour l'essai de son remède. Le second et le troisième malades ćtaient incommodés du véritable tænia, et les quatrième et cinquième étaient seulement soupçonnés d'être affectés de vers.

Ce premier essai ayant prouvé que le remède proposé était efficace dans le premier cas, et en général nullement dangereux, les membres de la commission jugèrent qu'il convenait de répéter eux-mêmes les expériences sur de nouveaux sujets, et pour les. mettre à portée de les faire, madame Nouffer leur donna les recettes suivantes :

N . r. La soupe. Prenez une livre et demie d'cau ordinaire, deux à trois onces de bon beurre frais et deux onces de pain coupé en petits morceaux, ajoutez-y la quantité suffisante de sel pour l'as- 


\section{SUR LES VERS INTESTINAUX}

saisonner, cuisez le tout à bon feu, en le remiant souvent jusq̨u้à ce qu’il soit bien lié et réduit à une bonne panade.

$N^{\circ}$. 2. Lavement. Prenez feuilles de mauve et de guimauve, de chacune une petite poignée, faites-les bouillir dans suffisante quantité d'eau, mêlez-y une pincée de sel ordinaire, et après avoir coulé, ajoutez deux onces d'huile d'olives.

No. 3. Spérifique. Prenez deux ou trois gros de la racine de fougère mâle, cueillie en automne et réduite en poudre très-fine, donnez cette poudre dans quatre à six onces d'eau de fougère ou de fleurs de tilleul. Il faut que le malade passe deux on trois fois de cette même eau dans son gohelet, et qu'il la boive après s'en être rincé la bouche, pour n'y rien laisser ${ }^{3}$.

N`. 4. Bol purgatif. Prenez panacée mercurielle sublimée quatorze fois, résine de scammonée d'Alep bien choisie, de chacune dix grains ${ }^{2}$; gomme gutte bonne et fraîche, six à sept grains; réduisez chacune de ces substances en poudre fine, ensuite vous les mêlerez ensemble pour en faire un bol avec de la bonne confection d'hyacinthe.

J'a: rapporté la méchode de madame Nouffer dans toute son étendue, parce qu'on la trouve souvent dénaturée dans les ouvrages qui en ont fait mention. Les membres de la commission font la remarque, que la fougère mâle, comme nous le savons tous trèsbien, est conuue depuis très-long-temps comme un bon remède contre les cestoïdes, et que beaucoup de

${ }^{x}$ L'eau 'de tilleul ou celle de fougère ne sont pas absolument nécessaires et peuvent être remplacées par l'eau simple. Madame Nouffer ne regardait pas cette différence comme fort imporlante.

2 Dans quelques preseriptions l'on trouve indiqué douze grains au lieu de dix ; cela provient, comme nous avons déjà remarquó, de la diffërence qui existe entre la mesure allemande et la mesure française. 
mnédecins célébres l'ont recommandée de temps à autre; ils ajoutent que ce remède est tombé dans l'oubli, comme beaucoup d'autres, parce qu'il n'a pas toujours répondu à l'attente que l'on s'eu était promise, ce qui a pu bien provenir de ce qu'on n'avait pas bien décrit la manière et le mode de son emploi, ou bien que l'on s'était écarté de la route prescrite, dans le but de faire de prétendues améliorations.

Je crois plutôt que cela dépendait de ce que l'on ne savait pas toujours distinguer les deux genres de vers, comme cela arrive encore très-souvent aujourd'hui.

Celle remarque ne peut cependant pas atteindre messieurs les membres de la commission, qui ont observé très-expressément, à la fin de leur rapport, que l'on a fait différens essais de ce remède, ct même à plusieurs reprises, sur des personnes incommodées par le véritable tænia, et qu'il a toujours manqué son effet.

N'éanmoins, beaucoup de médecins allemands administrent encore actuellement le remède de madame Nouffer, quoiqu’ils n'aient en général que le véritable tacnia ì combattre. Il u'y a pas même longtemps, que l'Observateur antrichien nous a fail connaître en entier comme un secret nouvellement découvert, après qu'un charlatan français venant de Pétersbourg l'eut débité pendant quelque temps ì Vienne, le mode de traitcment de cette dame, à la différence près, que ce charlatan employait l'huile de ricin au lieu du bol purgatif. 11 est bien vrai qu'au 
grand étonnement de tout le monde, ce prétendu médecin fit évacuer plusieurs fois, dans l'espace de quelques heures, des morceaux de tænia plus ou moins longs, mais avant que trois mois fussent écoulés, les malades en reudirent spontanément de nouveaux. Plusieurs personnes qui avaient pris le remède jusqu'à deux ou trois fois sans. être guéries, se sont par la suite adressées à moi.

Qnoique quelques malades incommodés du bothriocéphale n'aient pas été entièrement guéris après avoir fait usage du remède de madame Nouffer, comme par exemple le prince Baratinsky, et une autre personne observéc par Odier, néanmoins il faut avouer que ce remède est efficace dans le plus grand nombre de cas, si toutefois on l'administre exactement selon les prescriptions ci-dessus indiquées; car c'est a tort, (comme cela a été justement remarqué par messieurs les membres de la commission) que l'on a coutume de donner des purgatifs conjointement avec la fougère. Celle-là doit d'abord détruire le ver, ou le forcer à se détacher des parois des intestins avant de l'expulser. En joignant ces deux remèdes ensemble, le purgatif entraîne le vermifuge avant que celui-ci puisse agir efficacement contre le ver. Sennert ${ }^{\text {* }}$

${ }^{x}$ Ideoque fortioribus medicamentis opus est, ut interficiantur ( $s c$. lumbrici lati) quapropter etsi in teretibus purgantia cum interficientibus commode admisceantur : prostat tamen, nulla iis purgantia primun admiscere, cum purgantia non sinant medicamenta vermes interficentia diu in intestinis hoerere, sed ea cilu per aloum secum educant. Si vero prius cxhibcantur medicamenta, quce ipsum debilitant, totus rotundus 
avait déjà fait cette obscrvation. Si l'on donne au contraire le purgatif plus tard il entraînera facilemenı le ver tué, ou qui, sil est encore vivant, se sera pour le moins déja détaché des parois des intestins, si toutefois on a tâché auparavant de rendre le canal intestinal glissant. Cela s'opère ici à l'aide de la panade grasse, qui offre encore, dans mon opinion, l'avantage que le bol drastique n'irrite pas trop les intestins; car les membres de la commission ont remarqué qu'ils n'ont jamais observé de suites fâcheuses après l'emploi de ces remèdes, et que les malades étaien decins n'avaient pas donné, dans les premiers essais, le bol en entier, ce qui avait retardé beaucoup son effet, de manière que le ver ne fut évacué que dans la nuit ou même le lendemin. Le bol, administré entier, l'expulsa très-vitc, et sans danger pour le malade.

Méthode d'Odier. Cette méthode ne diffère de celle de madame Nouffer, qu'en ce que M. Odier prescrit, en place du bol purgatif, trois onces d'huile de ricin, dont il fait prendre une cuillerée à soupe, de demi-heure en demi-heure dans un peu de bouillon. Avant la publication du remède de madame Nouffer, Odier avait déjà employé l'huile de ricin avec quelque succès contre le bothriocéphale; mais il n'en fut pas de même contre le tania solium.

factus ad pilce figuram exil et homo sanus evadit. Voyez ouvrage cité, p. 420. On oublie avec le temps de pareilles règles. 
Métilode de Rathler.

Ric. puls, herb. sabin. gr. $\mathrm{xx}$; semin. ruth. gr. $\mathrm{xv}$; mercurii dulcis ${ }_{g} r . \mathrm{x}$; olei dest. tanaceti gr. xij ; syrup. for. persicor. q. s. utf. mass. ex qud form. bol. n. ij.

Le malade prend un de ces bols le matin et un le soir, mêlé avec un peu de sirop de fleurs de pêcher. Une demi-heure après il boil un verre de viu, dans lequel on a fait iufuser vingt noyaux de pêches pendant douze heures.

Cette méthode est la même que celle de Licutaud, à la différence près que les doses sont plus fortes.

Méthode de Schucker. Ce médecin s'exprime, sur l'emploi des graines de cévadille contre les vers, de la manière suivante:

" $\mathrm{L}^{\circ}$. Je fais réduire en poudre très-fine les cosses jaunes et oblongues de cévadille, avec les graines d'une forme pointue et d'une couleur noirâtre.

"J'cn prends cing grains dont je fais faire, avec suffisante quantité de miel, une grosse pilule. De cette manière je sais au juste la quantité que j'ordonne an malade. Je désigne ces pilules sous le nom de pilules vermifuges.

"Je commence par purger le malade avec une suffisante quantité de poudre de rhubarbe et de sel de Glauber, en réglant toutefois la dose selon l'îge et la constitution. Le jour suivant $\mathrm{j}$ 'administre aux individus d'un âge adulte, surtout au moment où ils se plaignent de beaucoup de nausées, un demigros de poudre.de cévadille mêlée avec une égale quantité d'olceosacharum de fenouil, après quoi il doit 
boire d'abord une à deux tasses d'une infusion de flcurs de camomille ou de fleurs de sureau, et une heure après une tasse d'eau d'orge. "

"Sil'estomac contient des vers, ceux-ci, irrités par la poudre de cévadille, se meuvent considérablement; ils augnentent par là les nausées et le vomissement, et ils finissent par être rejetés. Les ascarides et les lombrics ordinaires, vivans, soumis à l'action immédiate de cette poudre, se tordent aussi d'une manière convulsive, et meurent très-promptement. "

"J'ordonne le jour suivant une même dose de cévadille qui provoque également des vomissemens; quand le malade ne rend plus de vers, il ne doit prendre les troisième et quatrième jours, matin et soir, que six grains de ce remède, et il se purgera le cinquicine jour dans la matinée avec uu demi-gros de rhubarbe el huit grains de résiue préparée, ce qui produira l'évacuation de vers morts ou vivans. Si les matières fécales ne conticnuent pas de ces animaux, elles seront néanmoins chargérs de beaucoup de glaires, ce à quoi il faut faire attention. Le sixième jour, le malade doit prendre, matin et soir, trois de nos pilules vermifuges, et boire après une tasse d'unc infusion de fleurs de camomille on de fleurs de surean; le huitième jour il se purgera de nou: eau avec le remède dejà indiqué ; dans le cas où les matières fécales seraient encore chargées de beancoup de glaires, le malade sera obligé de prendre, les jours suivans, trois pilules vermifuges matin et soir, et il continuera ainsi jusqu’à ce que les glaires aient disparu, et jus- 
qu'à ce que les matièe' es fécales aient repris leur aspect naturel; en un mot il continuera l'usage de ces pilules jusqu'à ce qu'il ne soit plus incommodé par les mêmes accidens qu'il éprouvait auparavant. J'ai été obligé dans quelques cas de continuer les mêmes moyens, vingt jours de suite, avant de faire disparaître entièrement les glaires. Pendant la durée du traitement, le malade ne doit presque pas manger de viande; il doic au contraire se nourrir de légumes et de laitage."

«La dose des médicamens dont nous venons de parler convient seulement aux personnes de l'âge de vingt ans et au-dessus; pour les enfans de l'àge de deux à quatre ans, je réduis la poudre de cévadille, à la dose de deux grains."

Smucker prétend avoir obtenu l'expulsion des tænias à l'aide de son remède, je ne l'ai jamais employé.

Méthode de Weigel. L'on fait dissoudre une demi-once ou tout au plus une once de sel de Glauber dans deux livres d'eau de fontaine, et l'on en boit tous les soirs une tasse; le malade prend, outre cela, dans la journée, deux fois trente gouttes de l'élixir vitriolique de Mynsicht ou dix gouttes del'élixir acide de Haller, dans une demi-tasse d'eau commune, ou bien d'eau sucrée; l'on continue, selon les circonstances, pendast plusieurs mois, l'usage de ces médicamens, et l'expérience a prouvé qu'ils avaient été efficaces dans plusieurs cas.

Kortum a rapporté une observation où l'usage du lait de jument a effectué l'évacuation d'un tænia; une paysanne conseilla à une demoiselle de trente à qua- 
rante ans, qui avait un dégoût contre toute espècc de médicament, de boire de ce lait. En effet elle en fit usage pendant quelque temps quoiqu'il lui causât de fortes coliques, et enfin elle rendit un tænia à moitié pourri. Celte seule observation, ce me semble, ne doit pas nous engager à substituer ce lait à d'autres vermifuges plus actifs.

Le journal intitulé Allgemeiner Anzeiger der deutschen, année $1817, \mathrm{n}^{\circ} .295$, p. 3332, fait mention d'un nouveau remède anthelmintique que l'on avait tiré d'un journal de Londres intitulé the News, du 5 octobre.

Un jeune homme, qui était souffrant depuis longtemps, et qui avait déjà fait usage inutilement de beaucoup de remèdes, but tous les matins, par le conseil d'un maréchal-ferrant, une certaine quantité d'eau dans laquelle on avait fait infuser du lin vert pendant une dixaine de jours, et peu de temps après il rendit un tænia d'une longueur de huit pieds et demi.

Cependant toutes les méthodes dont nous avons fait mention jusqu'à présent sont insuffisantes pour détruire eutièrement le véritable tænia, et en effet l'on est encore à la recherche d'un moyen plus efficacex 2.

En Suisse personne ne se donne plus la peine d'es-

- Le docteur Kipke, à Breslau, vient d'inventer un nouveau remède très-efficace contre le tænia : il y a lieu de croire que ce remède sera publié sons peu pár ordre du gouvernement prussien. (Note du traducteur.)

2 Voyez Notice sur une nouvelle plante de la famille des rosacées, employée avec le plus grand succès en Abyssinie contire le 
sayer contre le bothriocéphale un autre renéde que celui de madame Nouffer, et après l'avoir pris tout au plus deux fois, on se trouve ordinairement guéri.

\section{Quoique je regarde ces différentes méthodes comme}

tænia et apportée de Constantinople par Brayer, D. M. P.; c'est M. le professeur Kunth, botaniste célèbre, qui s'est chargé d'examiner cette plante, presque réduite en poussière. A force de patience il a reconnu qu'elle appartient à la famille des rosacées, et qu'elle en forme un nouveau genre. Je joins ici la description qu'il en a donnee, et dont il a fait lecture à la société d'histoire naturelle, il y a environ quinze mois.

“M. Braycr, médecin distingué, a apporté de Constantinople les fleurs d'une plante originaire d'Abyssinie, vantées dans ce pays-là comme un spécifique certain contre le ver solitaire. Il a été lui-mème témoin de scs prompts et heureux effets dans un cas extrêmement opiniàtre. Il a eu la complaisance de me remettre des fragmens de ces fleurs; $j$ 'y ai reconnu la structure suivante :

„Quatre flcurs pédicellées, entourées d'autant de bractées membraneuses. Calice tubuleux, persistant, rétréci à son orifice ; limbe à dix lobes, dont les cinq extérieurs plus grands. Cinq pétales très-petits, linéaires, insérés au limbe du calice. Étamines, 12 à 21 , insérées au même endroit, filets libres. Anthères biloculaires. Deux ovaires attachés au fond du calice, parfaitement libres, uniloculaires, monospermes. Ovule pendant. Deux styles terminaux. Stigmates élargis, légèrement lobés. Fruit point observé.

"D'apres ces caractères, cette plante doit être rapprochée du genre Agrimonia, dont elle ne diffère que par son limbe double, par ses pétales extrêmement petits, et par ses stigmates élargis, différences qui suffisent pour constituer un genre distinct. Je fruit doit être semblable à celui des agrimonia.

"Je propose de donner à ce nouveau genrele nom de Brayera, en l'honneur de M. Brayer, à qui nous devons la première con- 
plus ou moins insuffisantes contre le tæuia, nciamoins je les ai rapportées toutes, d'abord pour com-

naissance de cette plante. Le nom spécifique de anthelmintica doit rappeler ses propriétés anthelmintiques.

Brayera (Kunth). Genus novum ex rosacearum familid, agri-. moniæ proximum, distinctum : calycis limbo duplici, utroque 5-partito; petalis parvis, squamaformibus; stigmatibus peltato-dilutatis et inflorescentia ramosa.

Species unica (Brayerà anthelmintica). - Descriptio. - Calyx persistens; tubus turbinatus, externè sericeus, internè glaber et decemnervius; nervi laciniis oppositi; limbus decempartitus, membranaceus, patens ; lacinia quinque exteriores magna, oblonga, venosa, quinque interiores cum exterioribus alternantes iisque duplo triplode breviores, spatulato, trinervia ; faux conico-prominens, membranacea, apice pervia, slabra.

Petala 5, ima basi limbi imposita, cum laciniis inierioribus allernantia iisque triplo breviora, squamaformia, iinearia, slabra, aqualia, decidua.

Stamina 12, 13, 18, 21, ibidem inserta, subarualia, petalis breviora. Filamenta linearia? libera, glabra. Antherce subrotunda, didyma, bilorilares, glabra, longitudinaliter dehiscentes.

Ovaria duo, in fundo calycis sessitia, libera, lineari-oblonga, apice pilosa et in stylum desinentia, zinilocularia. Oculum solitarium (in unico ovario vidi ocula duo apposita), angulo centrall affixum, pendulum, ovato-oblongum, glabrum. Styli tot quot ovaria, exserti, slabri. Stigmata magna, subpeltata, crenato-lobata et undulata, carnosa. etpapillosa.

Fructus.....

Arbor. Pedinculi ramosi, teretes, molliter pilosi, flexuosi, bracteati; ramis alternis. Bractea solitaria, integra. Pubes simplex. Flores quaterni, pedicellati, bracteis quatuor involucrati. Bradea subrotundo-elliptica, obtusa, concaice, tenuiter membranacea.

Flores in Abyssinid contra loniam adhibentur. (Note du traduet.) 
pléter mon ouvrage, ensuite pour empêcher que le public ne se laisse tromper et n'achète un anthelmintique secret, prétendu nonveau, connu peut-être depuis Jong-temps, et enfin pour donneraux médecins la facilité de pouvoir choisir l'un ou l'autre remède, en cas qu'ils rencontrent des malades qui se refuseraient à employer ceux que je vais indiquer ci-après, et comme cela m'est arrivé dans la personne d'un médecin, qui préféra expulser quelques morceaux de son tænia tous les trois mois, à l'aide de la fougère et de l'huile de ricin, que de prendre de ihuile empyreumatique.

SECTION IV. Méthode de traitement de l'auteur.

Je u'ai eu à traiter que trois personnes incommodées par le bothriocéphale; la première était une femme suisse que j'ai guérie radicalement à l'aide de l'huile vermifuge de Chabert, quoique l'on n'ait trouvé aucune trace de ce ver dans les matières stercorales. La seconde était une femme de Pétersbourg, qui vint me consulter en 1812 . Je lui administrai lá fougère et l'huile de ricin, afin d'expulser son bothriocéphale entier, ce qui eut lieu en effet. J'ai déjà fait nention plus haut de la troisième personne à laquelle $\mathrm{j}$ ai également fait rendre un ver de cette espèce.

Je puis assurer avoir traité plus de cinq cents personnes de différens âges et sexes, mêne deux enfans de l'âge d'un an et demi, incommodées par le véritable tænia. Parmi cette grande quantité de malades, il n'y en a eu que quatre qui furent obligés de prendre l'huile de Chabert une seconde fois, savoir 
trois dans l'année 1814 , et le quatrième dans l'année 1817 . Il se présenta en outre, il y a plusieurs années, un cinquième malade qui, après en être resté délivré pendant deux ans, fut de nouveau incommodé par le tænia ; mais cette personne n'en a plus remarqué de traces depuis qu'elle a fait dènouveau usage du même remède; tous mes autres malades, autant que je le sache, n'ont pas eu de rechutes depuis le traitement que je leur ai fait subir.

L'on me demandera peut-être si j'ai eu occasion de n'informer auprès de toutes ces personnes, au bout de trois ou quatre mois, si elles n'ont pas rendu depuis le traitement quelques morceaux de tænia. Je suis obligé d'avouer franchement de n'eu avoir vu à cette époque, et encore toujours par pur hasard, que le plus petit nombre; mais on me demandera encore: d'où savez-vous qu'elles soient restées débarrassées? Je le sais, $1^{\circ}$. par la raison qu'elles ne se sont pas fait voir chez moi ; car un homme soulagé de souffrances doit à son médecin de la reconnaissance, mais en général on aime assez à pouvoir s'en dispenser. Si un malade au contraire est inutilement tourmenté par les nédicamens que son médecin lui a prescrits, il ne manque jamais de lui dire, avec une espèce de satisfaction: vous m'aviez promis de me guérir et vous n'avez pas tenu parole, ce qui m'est arrivé en effet bien souvent aussi long-temps que j'ai suivi la méthode d'Alston. $2^{\circ}$. Je conclus du nombre assez considérable de malades que j'ai eu souvent occasion de revoir depuis le traitement, aux autres que j'ai perdis 
de vue, et de la santé desquels je reçois maintes fois des nouvelles par de nouveaux malades qui m'ont été adressés par eux.

Je commence le traitement par l'électuaire $\mathbf{n}^{\circ} .1^{*}$; je le donne de la manière ${ }^{2}$ que j’ai indiquée lorsque j'ai parlé du traitement qu'il faut employer contre les ascarides. Sitôt que la première portion de cet électuaire est finie, je commence l'emploi de l'huile empyreumatique de Chabert, à la dose de deux cuillerées à café matin et soir, mêlée avec un peu d'eau. Ce médicament a une odeur que beaucoup de personnes n'aiment piss; cependant son goût ne me paraît pas désagréable: je conseille aux personnes qui, après en avoir fait usage, veulent se débarrasser de son odeur, d'avaler quelques gorgées d'eau avee un certain effort, et je leur conseille en outre de ne pas se rincer la bouche; car il pourrait arriver alors que quelques parcelles de ce médicament fussent poussées dans les fosses nasales, et l'on ne serait pass alors quitte de longtemps de sa mauvaise odeur.

Pour corriger le goût désagréable de ce médicament, l'on fera bien de mâcher, après son usage, un peu de canelle ou un clou de gérofle; mais que l'on se garde bien de manger quelque chose qui causerait des renvois, comme par exemple des pastilles de menthe et d'autres choses semblables, car on aurait

-Voyez la cinquième section, qui contient la formule des médicamens employés par l'auteur. (Note du traducteur.)

2 C'est-à-dire à la dose d'une cuillerée à café matịn et soir: (Note du traducteur.) 
le désagrément de renouveler le goût de ce médicament avec chaque éructation.

La dose de deux cuillerées à café deux fois par jour ést en général supportée très-bien par des personnes de tout âge ${ }^{x}$; il est cependant des personnes qui ne

- Il me semble que l'auteur emploie ce médicament, notamment dans le commencement, à des doses trop fortes. Toute réflexion faite, il est beaucoup plus prudent, et on ne s'exposera pas à faire naître d'accidens fàcheux, surtout chez les enfans, si on commence l'usage d'un médicament aussi violent à petites doses, que l'on est toujours à même d'augmenter quand on voit qu'elles ne causent aucune incommodité au malade.

Dansle seul but de saroir quel ffet cette huile produirait sur mon corps, je m'élais décidé, il y a à peu près unan, à la prendre de la manière prescrite par l'auteur, quoique je prévisse, et avec raison, comme on verra par la suite, qu'elle ne me conviendrait pas. Je dirai, par parenthèse, que l'huile de Chabert dont je fis usage avait été préparée avec le plus grand soin par M. Faguer, très-habile pharmacien à Paris. A peine en arais-je pris deux cuillerées à café, que je sentis un grand malaise qui fut suivi de coliques, de nausées et d'éructations fréquentes, symptômes qui ne cessèrent que pen à peu vers le soir. Je m'étais proposé de répéter cette expérience le lendemain, mais je me trouvai trop fatigué, et je la remis au surlendemain. Quoique je n'eusse pris ce jour-là qu'une cuillerée à café de ce remède, cependant les mênıes symptòmes, quoique moins violens, se renouvelèrent, ce qui m'engagea à ne faire usage, le quatrième jour, que de la moitié d'une cuillerée à café, et cette fois je fus quitte pour quelques éructations qui me rappelèrent le goùt et l'odeur détestables de ce médicament. J'ai rapporté ce fait, non pas dans l'intention de faire croire que l'huile de Chabert, à la dose que je l'ai prise, causcrait à tout le monde les mêmes accidens, une telle assertion anrait les expériences multipliées de M. Bremser contre elle; mais ce fait prouve 
peuvent pas prendre une aussi forte dose de ce médicament sans éprouver une grande irritation et même de légers étourdisseniens; en pareil cas on diminue un peu la dose. Ces accidens n'arrivent souvent qu'au commencement, et le malade supporte par la suite le remède plus aisément. Quelques personves peuvent Je prendre très-facilement à jeun, mais celles auxquelles il causerait des nausées feront biẹ de n'en faire usage qu'une heure et demie après avoir déjeuné. 11 est des cas où ce remède cause aussi une chaleur dans les voies urinaires et dans les intestins '; quelques verres d'orgeat ou quelques cuillerées d'une émulsion huileuse, font assez facilement cesser ces accidens.

cependant que j'aurais agi beaucoup plus prudemment et que j'aurais probablement évité touș les accidens, sij'arais commencé par ne prendre que la moitié d'une cuillerée à café.

Qu'il me soit encore permis une remarque. Je ne puis pas croire que l'huile de térében thine ajontée à l'huile animale de Dippel (nélange qui forme l'huile de Chabert) puisse augmenter de beaucoup les vertus vermifuges de cette dernière, qui, prise seule, agit dejà d'une manière si efficace contre toute espèce de vers. Ce qui paraît confirmer mon opinion, ce sont les cas rapportés par plusieurs médecins, où l'huile de térébenthine donnée, même à la dose de plusieurs onces, n'avait pas opéré la destruction des vers. Du reste Chabert, comme je viens de l'apprendre de M. le docteur Schwediaur, n'a ajouté l'huile de térébenthine à celle de Dippel que dans l'intention de rendre le goût de cette deruière un peu moins mauvais, et de former par ce mélange un médicament à la fois purgatif et vermifuge. (Note du traducteur.)

${ }^{3}$ Ces accidens prouvent assez que nous devous être circonspects dans l'emploi de cetto huile. (Note du traducteur.) 
A près que le malade a pris deux onces et demie ou trois onces de cette huile, cequ'il peut faireà peu près dans l'espace de dix à douze jours, je lui ordonne un léger purgatif, par exemple les pondres $\mathbf{n}^{\circ} .3$; ensuite il doit recommencer l'usage de l'huile. J'en fais prendre ordinairement quatre à cinq onces dans le cours d'un traitement; mais dans le cas où le ver aurait déjà résisté à beaucoup d'autres médicamens, j'emploie six à sept onces de l'huile empyreumatique. J'avoue que le traitement est un peu long, mais son résultat est certain; il ne cause aucune incommodité, et n'offre pas le moindre danger. Du reste, il paraît constaté par l'expérience qu'il faut toujours continuer le traitement pendant long-temps, si l'on veut entièrement débarrasser quelqu'un du tænia.

Lengsfeld et Geischlœger, médecins de Vienne, qui avaient gardé leurs vermifuges secrets, les donnaient à leurs malades pendant un mois consécutif; Dyanière a également observé que l'usage des vermifuges doit être continué pendant long-temps, si on veut obtenir la destruction entière des vers intestinaux.

Le mode de traitement des deux médecins de Vieune m’a déterminé à administrer pendant longtempset à petites doses l'huile anthelmintique de Cliabert. Il est possible que, par ce moyen, la disposition pour la formation des vers soit entièrement détruite. Mais il est aussi possible quie ce remède n'agisse pas sur les œufs des vers qui se trouvent dispersés çà et là dans la mucosité du canal intestinal; de même que le 


\section{$49^{2}$}

suc gastrique n"agit pas non plus sur une graine entière, c'est-à dire sur celle qui n'a pas été átlaquée par l'ébullition, et qui est rendue sans être digérée. En ne donuant qu'une scule fois, mais à forte dose, Thuile anthelmintique, il se pourrait donc bien que cela ne produisît que la destruction des vers et non pas celle de leurs eufs; en continuant au contraire l'usage d'un remède vermifuge pendant longtemps, les vers sortiront, sur ces entrefaites, des œufs, et le remède agira alors efficacemeut sur eux, du moins cela ne me paraît pas impossible. Ma méthode d'agir contre le tænia a'a pas ordinairement besoin d'être suivie d'un traitement secondaire; c'est seulement dans le cas où il y a prédisposition à la formation des glaires, et par conséquent à celle des vers, que j'administre pendaut quelques semaines la teinture fortifiante marquée $n^{n} .5$.

Je ne soumets pas mes malades pendant le traitement à un régime particulier, et je ne les force pas non plus à faire usage de harengs ou de viandes sas lées, etc.; cependint je les engage à ue pas faire abus de farineux, de légumes secs et de viandes grasses; je leur conseille eu général d'éviter tout ce qui favorise la formation des glaires et des vers.

ll faut que je fasse encore une remarque. Après l'usage des anthelminthiques ordinaires, vantés souvent à tort comme très-efficaces, on est accoutumé de voir sortir le tænia par longs morceaux. Je préviens que cela n'arrive que rarement après l'usage de l'huile 
de Chaberı. Son efficacité consiste à détruire le ver et à empêcher qüil ne s'en forme de nouveau; c'est pourquoi il est rendu pendant les premiers jours, a moitié ou même entièrement digéré, de manière que l'on a souvent beaucoup de peine à reconnaître dans les déjections des traces de sa forme primilive. Du reste, quant à la tête de l'animal, peu m'importe de savoir si on l'a trouvée ou non dans les matières évacuées; car deux ou même trois têles peuvent avoir élé rendues, et les malades n'être pas dćbarrassés pour cela de leur tania, par la raison que plusieurs peuvent se trouver à la fois dans le canal intestinal.

Le seul indice certain que le malade en est entièrement débarrassé, consiste à savoir qu'il n'a pas reodu dans l'espace de trois mois des traces de ce ver. Si on voyait de nouveau, deux ou trois ans après, des traces de tienia, elles proviendraient alors bien certainement d'individus nouvellement formés, qui cependant ne doivent pas être regardés comme les petits de ceus contre lesquels on avait employé le remède.

SECTION V. Des formules de médicamens de l'auteur.

No. I. Re. Semin. cince. S. tanacet. rudit. contus., ३ß; pulv. valerian. $S ., 3 \mathrm{ij}$; jalapp. $3 \mathrm{j} ß-3 \mathrm{ij}$; tartar. vitriolat., $3 \mathrm{j} ß-3 \mathrm{ij} ; O x y-$ mel. scillit., q. s., ut f. elertuar. $d . S$; à prendre deux ou trois cuillerées à café par joul ${ }^{3}$.

${ }^{2}$ Cet électuaire, ainsi que l'huile empyreumatique de Chabert, sont sans doute les meilleurs vermifuges que nous connaissions, mais ils offrent l'inconvénient d'être d'un trèss-mauvais goût, ce 
Je me sers de cet électuaire depuis plusieurs années avec le plıs grand succès contre toutes les espèces de vers intestinaux. Cette composition me paraît une des plus convenables, sauf meilleur avis, pour atteindre le but que l'on se propose pendant le traitement de la maladie vermineuse.

Le semen contra, ou en sa place les graines ou plutôt les fleurs bien développées de tanaisie, ont tou. jours été regardées comme de bons vermifuges. Le semen contra, tel qu'ou le trouve ordinairement dans les pharmacies, c'est-à-dire très-finement pulvérisé,

qui fait que beaucoup de personnes, et surtout les enfans, ne veulent pas les prendre. Pour le premier de ces médicamens, on peut remédier à cet inconvénient en donuant la dose prescrite par l'auteur, qui est de deux cuillerées à café (environ deux gros pesaut ) par jour sous forme de pilule. Quant à l'huile de Chabert, elle ne peut guère être employée sous cette forme; d'abord elle s'évaporerait à la longue entièrement; en outre il faudrait une trop̀ grande quantité de masse pilulaire pour absorber les quatre cuillerées à café formant la dose à consommer dans une journée. Ne connaissant aucun moyen propre à administrer cette huile d'une manière plus convenable, je me suis adressé à MI. Page, pharmacien distingué (successeur de M. Faguer). Après plusieurs essais qu'il a eu la bonté de faire, il a trouvé que deux parties de sirop de limon ajoutées à une d'huile enyyreumatique, corrigeaient assez bien son goût désagréable. Un autre moyen que M. Page n'a indiqué, atteint plus directement le but que je m'étais proposé; mais il est un peu plus embarrassant dans l'exécution ; il consiste à ajouter à l'huile enpyreunatique, au moment où on veut en faire usage, suffisante quantité d'une poudre vermifuge, de manière à former une espèce d'électuaire qui, divisé par petits bols, pourrait être pris dans du pain à chanter. (Note du traducteur.) 
conservé depuis long-temps et privé par conséquent en grande partie de son odeur et de son goût particulier, n'est pas un remède très-actif. Je n'emploie ces graines que légèrement concassées, et, données sous cette forme, je m'imagine qu'elles ne sont pas tout à fait décomposées dans l'estomac, et qu'elles agissent par conséquent sur les vers d'une manière mécanique. Supposé même que cela ne se passerait pas ainsi, il est toujours permis de présumer que des graines ainsi préparées auront encore conservé, en arrivant dans les intestins grêles (séjour des ascarides et des cestoïdes) une bonne partie de leur vertu spécifique.

La valériane n'est pas seulement connue comme un excellent vermifuge, mais aussi comme un des meilleurs antispasmodiques; ajoutez à cela que son emploi offre si peu de contre-indications. Comme du reste le systène nerveux est ordinairement plus ou moins affecté dans les maladies vermineuses, je crois que personne ne voudra retrancher la valériane de cette formule.

Le jalap donné avec les précautions convenables est, ce me semble, parmi tons les purgatifs, celui qui affiiblit le moins le canal intestinal, et il méritcrait mêne qu'on l'appelât un purgatif tonique. Le jalap est, en un mot, un des remèdes les plus propres à dissoudre et à expulser les glaires et les matières fécales stagnantes.

Le sulfate de potasse occupe depuis long-temps une place parmi les meilleurs purgatifs. Jc le préfere 
à tous les autres, parce qu'il se dissout très-difficilement, ce qui eșt la raison pour laquelle il agit d'une manière plus lente, mais d'autant plus certaine. Le sulfate de potasse doit être regardé comme un remède qui dissout les glaires tenaces et qui provoque, en irritant le canal intestinal, des excrétions séreuses à l’aide desquelles les glaires détachées et atténuéẹs sont entraîuées. Du reste, en l'administraat à une dose aussi petite que celle indiquée dans la formule, on n'a pas à craindre quilil produise des selles aqueuses qui affaibliraient le malade.

L'oxymel scillitique n'a pas été clıoisi non plus au hasard comme véhicule. Cette substance est connue depuis long-temps comme un excellent dissolvant, mais elle est encore douée de propriétés à l'aide desquelles elle se montre ici utile d'une manière indirecte. L'oxymel scillitique augmente, comme nous le savons, la sécrétion des reins, de la peau et des poumons. A mesure que ces organes opèreront une augmentation dans la sécrétion des matières qui molestent le corps animal, les intestius en seront d'autant moins chargés, par conséquent il s'accumulera non-seulement dans ceux-ci une moins grande quantité de nıatières qui peuvent contribuer en partie à la production des vers, mais encore lechyle provenant des alimens será alors aussi plus pur ct plus propre à rétablir les forces physiques et l'harmonie dans les fonctions.

11 est possible que ma manière d'envisager l'action dechacun de ces médicamens en particulier ne soit pas 
tout-à-fait exacte, mais je puis toujours assurer que le remède, tel qu'il a été indiqué, ne m'a jamais trompé dans mon attente.

No. 2. Rc. herb. absinthii, rad. valerian. S., ana unciam unam; semen. tanacet., cortic. aurant., ana unciam dimidiam, c.c. m. d. S. On verse sur deux cuillerées à bouche pleines de ces substances, une livre d'eau bouiliante; cela fait, on couvre le vase, et on laisse infuser ces substances pendant une nuit; ensuite passez, exprimez et employez l'infusion pour deux lavemens. On ajoute à chacun une cuillerée d'huile empyreumatique de corne de cerf.

No. 3. Rc. pulv. rad. jalap., scr. j; fol. senn., drach. dimidiam; tartar. vitriol, drach. unam; m.f. pulv. divid. in iij vel iv part. oq. $d$. S. Prendre toutes les heures un paquet, ou toutes les demiheures un dcmi-paqnet, jusqu'à ce que cette poudre opère.

$\mathrm{N}^{\circ}$. 4. Huile anthelmintique. (La manière de la préparer a été indiquée page 427.)

Ce remède a été inventé par Chabert, qui l'a souvent administré avec succès aux animaux, dans le but de les débarrasser des vers de toute espèce; il a également fait rendre, à l'aide de cette huile, des douves du foic à une petite fille de douze ans, comme cela a été rapporté par M. Rudolphi. (Voy. Voyage, vol. Ir , p. 37.)

Goëze avait déjà recommandé cette huile aux médecins, afin de la soumettre à un examen particulier. Il en est de même de Brera ${ }^{3}$, et Rudolphi a la reregarde comme le vermifuge par excellence.

Chabert a bien indiqué à quelle dose il' faut la
I I'orlesungen, p. 1 זा.
2 Entoz. I, p. $49^{3}$. 
donner aux animaux, mais personne n'a encore indiqué jusqu'à présent celle qu'on peut administrer aux hommes. Ayant grande envic de la faire prendre à mes malades, sans cependant vouloir les exposer à des accidens qui auraient pu me mettre dans l'embarras, je me suis décidé à l'essayer d'abord sur moimême, quoique je ne fusse pas incommodé de vers. Je commençai par en prendre de très-petites doses, que j'augmentais peu à peu; le goût et l'odeur de ce remède n'ont rien de désagréable pour moi. Voyant qu'il ne me causait pas la moindre incommodicé, je l'ai ordonné à mes malades, et je n’ai jamais remarqué qu'il ait causé d'accidens fâcheux, si toutefois il avait été administré avec les précautions convenables. Une cuisinière s'avisa d'en boire un flacon entier, c'est-à-dire plus d'une once dans une nuit, ce qui lui causa en effet des coliques assez violentes, qui se dissipèrent cependant entièrement vers le soir, après l'usage d'une émulsion huileuse.

№. 5. Teinture fortifiante. - Rc. tinctur. alves compos. pharm ausir., drach. unam; tinctur. martis pomat., uncian unam; elix. vitriol. anglic. pharm. I.ond., unciam dinuidiam, M. d. S.; à prendre dix, vingt, trente et plusieurs gouttes, trois à quatre fois par jour dans un peru d'ean our de vin.

Le remède connu sous le nom de tinctura aloes composita, est le même que l'on appelait autrefois elixirium proprictatis dulce; il est composé d'aloès, de myrrhe et de safran; et, donué à petites doses, il agit conme un excellent tonique, et tient le ventre libre.

Le fer, surtout le sulfate de fer, a été placé par 
plusieurs médecins parmi les vermifuges; mais nous avons déjà fait remarquer qu'il mérite seulement ce litre, en tant qu'il empêche, par le rétablissement des forces physiques et du mélange convenable des humeurs, la production des vers; mais en pareil cas il se montre comme un remède des plus exquis, si toutefois le canal intestinal avait été préalablement débarrassé de glaires tenaces et épaisses.

L'élixir connu sous le nom d'elixirium vitrioli anglicanum porte également celui d'elixirium vitrioli Mynsichti. Je le fais toujours préparer d'après la pharmacopée de Londres, eı non pas d'après celle d'Autriche.

Je me sers de ces gouttes fortifiantes que l'on peut saus doute appeler un remède très-compliqué nonseulement à la fin d'un traitement contre les vers; mais je les administre aussi avec beaucoup de succès dans les cas de chlorose, de blennorrhagie et de maladies semblables, bien entendu lorsqu'il ${ }^{\circ} \mathrm{y}$ a indication.

Afin qu'un critique n'ait pas occasion de m'objecter que la teinture appelée tinctura martis pomata se trouve décomposée dans le mélange ci-dessus indiqué, je dois le prévenir que je le sais très-bien, et que je le savais déjà lorsque je l'ai employée pour la première fois. J'avoue cependant n'avoir pas encore cherché à savoir combien de fer se trouve transformé, par l'acide sulfurique contenu dans l'élixir anglais, en sulfate de fer, et combien de malate de fer se pré- 
cipite dans ce mélange. Tout ce que jai appris par l'expérience, c'est que l'emploi convenable de ces gouttes change la couleur pâle des lèvres en rouge, et donne au teint une couleur plus animée, rend les muscles plus vigonreux, en un mot fortifie tout le corps, et cela me suffit. 


\section{APPENDICE}

\section{PAR M. H.-D. DE BLAINVILLE,}

L'ouvalie de M. le docieur Bremser, dont nous venons de donner la traduction anssi littérale qu'il a été possible au docteur Grundler, a été apprécié comme il devait l'être par les journaux de médeciue de presque tontes les nations européennes, et tous en ont fait un éloge mérité; un seul peut-être en a donné une critique évidenment passionnée : et c'est en Italie, dans ce pays où toutes les connaissances humaines ont repris naissance, où l'helminthologie a évidemment commencé, comme le prouve le Traité de Redi, que cette eritique a été publiée; elle a été cnsuite répanduc avec profusion en France, en Angleterre et même en Allenagne; mais ce n'est que dans ces premières contrées que, par un abus blàmable, elle a passé dans les journaux de médecine en extrait et sans en indiquer la source. C'est M. Raickem qui l'a traduile en français en Italie, sans doute sous les yeux de son auteur, en passant sous silence plusicurs passages de l'analyse italieme, qui lui ont avec raison paru peu convenables; et il l'a introduite dans le Noureau Journal de médecine de Paris, octobre 820 . Un exirait de cette même eritiúue a été insérédans le Medical Journal de Londres, novembre 1820 et janvier $182 \mathrm{r}$; en effet il est sans nom d'autcur, il contient les mêmes objections, fausses ou vraies, et, bien plus, les mêmes inexactitudes et oublis, comme l'a démontré clairement M. Bremser, dans un article en réponse à cet extrait du nême journal anglais. M. James est le seul qui ait pris la peine, bien inutile à ce qu'il nous semble, d'en faire une réfutation qui aurait pu aiscment être plus vigoureuse. Nous ne nous arrêlcrons pas à relever nous-mêmes les nombreuses imputations erronées qu'elle contient, et entr'autres, que M. Bremser regarde le cinquième chapitre de son ourrage comme un clief- 
d'œurre; car dans tout le livre cette expression n'est employée qu'à l'occasion de l'homme, qu'il appelle le chef-d'oeuvre de la création; nous nous bornerons à dire que, quoique fort longue, puisqu'elle contient soixante-dix-neuf pages d'une justification in- $8^{\circ}$ assez serrée, nous l'avons lue avec toute l'attention que méritaient son objet et son titre: Analisi ragionate delle opere recentamente publicate sui vermi del corpo umano e degli animali dai chiarissimi sisnori dottor Bremser di Vienna ei cons. Rudolphi di Berlino, mais nous n'y avons rien trouvé de cette critique éclairée et de la bonne foi qui devraient guider tout homme qui s'est arrogé à tort ou à raison le droit de juger et de porter publiquement un jugement sur les ouvrages d'autrui. Nous croyons au contraire y avoir remarqué presqu'à toutes les pages les indices d'un amour-propre blessé qui se regimbe contre la vérité des observations critiques que MM. Bremser et Rudolphi ont faites sur beaucoup de points de l'ouvrage de M. Brera sur les vers intestinaux “. L'analyste tâche en effet de défendre plusieurs des opinions de ce dernier, et il l'exalte presque constamment aux dépens des he'minthologues allemands. Nous n'y avons au reste trouvé qu'un très-petit nombre d'observations justes dont nous ayons pu nous servir dans cet appendice, où nous allons successivement passer en revue chacun des chapitres de l'ouvrage de M. le docteur Bremser, en y ajoutani ce qui nous a paru plus convenable, ce qu'il nous a indiqué luimême pour le rendre plus complet, ce que nous avons puisé dans une excellente analyse de l'ouvrage de M. le docteur Bremser parfeu le docteur Albert, insẻré dans la Gazette de Salzbourg ( $n^{\text {os }}$ I I et 12 , février 1820 ), et en proposant quelque doute sur ce qui nous semblera plus ou moins erroné.

\section{Sur la formation des vers intestinaux.}

La question de physiologic générale que traite M. Bremser, dans le premier chapitre de la première partie de son ouvrage, ne doit

- Au sujet de ces critiqnes, nous sommes cependant obligés de souscrire au jugement de l'auteur de l'Algmeines repertorium, qui dit que celle de M. Bremser est mordante, et que le style dans lequel elle a été conçue manque souvent de noblesse. 
fas être placéc an nombre de ces questions oiseuses qui il imporie fort peu d'envisager. En effet, quand mème on ne parviendrait pas à résoudre complétement le problème qu'elle renfcrme, il est aisé de voir que la formation spontanée des vers intestinaux dans l'être vivant qui les nourrit doit avoir une influence considérable sur les moyens thérapeutiques propres à les combatire, puisqu'il ne suffit plus, comme on l'a fait long-temps, de chercher à détrnire le ver lui-même; mais qu'il faut essentiellement combattre la diathèse vermineuse, ou l'ensemble des causes de leur reproduction.

Malgré l'importance de cette question, et malgré les argumens négatifs et positifs apportés dans sa discussion par M. Bremser, il faut convenir que la génćration spontanée d'animaux aussi com-pliqués que l'ascaride lombricoïde, ou les sexes sont distincts sur des individus séparés, est quelquc chose qui parait répugner à la raison, surtout lorsqu'on croit que la génération yar l'accouplement d'individus semblables soit plus aisée à comprendre.

En étudiant l'histoire de l'esprit humain sur ce point, nous trouvons que, comme dans tant d'autres, il oscille perpétuellement d'une opinion à l'opinion opposée. Les anciens physiologistes, et Aristote à leur tête, admirent la génération spontanée pour un très-grand nombre d'êtres organisés. La combinaison de l'humidité, de la chaleur et du limon leur paraissait suffisante pour former de toutes pièces non-seulement des vers, mais des insectes ; mais comme ils avaient compris dansle nombre des animaux qui se: reproduisent spontanément des êtres dont la génération n'est nullement équivoque, comme les mouches, il fut aisé à Redi, par des expériences positives, de renverser l'opinion d'Aristote; mais, comme il fut aussi entraîné trop loin, il conclut absolument l'opposite de l'opinion d'Aristote, et tout animal fut déclaré provenir d'un animal semblable à lui et d'un œuf. Cependant, les obscrvations s'accumulant, s'appliquant à un plus grand nombre d'êtres, la plupart des physiologistes prirent dernièrement une opinion intermédiaire, et semblèrent admettre qu'à l'origine de la série animale il y a des générations spontanées; enfin aujourd'hui plusieurs personnes pensent et commencent à professer l'opinion que toute génération est spontanée. Comment concevoir autrement la production des animaux microscopiques, quel'on roit pour aiusi 
dire nâttre sous le champ du microscope dans les infusions végétales ou animales?' mais dans celte opinion on a été eucore beaucoup plus loin, en prétendant que des mélanges de gaz̉ pouvaient donner naissance, en les exposant, dans un ballon bien fermé, à la chaleur d'un fumier, à des animaux, ct même à des animaux assez élevés, comme M. Frey l'a soutenu dans un ouvrage publié une première fois en Allemague, et depuis peu d'années à Paris. Quoique les pliysiologistes ne pussent certainement pas adopter les faits qui y sont rapportés, cependant comme ils semblent appuyés par des expériences et sur l'autorité imposante, sous tous les rapports, le M. Berthollet, nous devons dire que ce savant même les regarilait celles-là comme complétement inexactes.

Avant d'établir sa manière de concevoir le mode de génération des vers intestinaux, opinion qui s'accorde parfaitement bieu arec ce que dit à ce sujet MI. Treviranus dans le second volume de sa Biologie, $\mathbf{I I}$. Brenser passe en revue et réfute d'une manière assez sevère, souvent même trop acerbe, mais irrécusable, les opinions des auteurs qui l'ont précédé. Etablissant ce dilemme, admettant ce principe rigoureusement vrai, que ces vers ne peuvent provenir que de deux sources ou extérieure ou intérieure, il combat successivement l'opinion de ceux qui veulent que les vers intestinaux proviennent :

$x^{0}$. De vers de terre ou d'eau qui se seraient introduits dans le corps animal, en supposant que les rers intestiraux se trouvent également dans l'eau ou dans la terre.

$2^{0}$. De vers de terre et l'eau, mais ne prenant la forme spécifique des vers intestinaux qu'en arrivant dans le corps animal, opinion qui est celle de MI. Brera, et qui se réfute presqu'avec autant de facilité que la première; $\mathbf{r}^{\circ}$. car s'il est quelques vers intestinaux qui pourraient laisser quelques doutes, comme les nématoïdes et les trématodes, il est évident qu'il n'y aura plus de doute pour les échinorhynques, les cestoïdes ct les cystoïles, dont la forme et la structure n'offrent rien de comparable arec ce qui existe dans les autres animaux; $2^{\circ}$. plusieurs animaux ont des vers qui leur sont propres, quoique bien certainement il y ait des espèces communes à plusieurs animaux; $3^{\circ}$. on trouve des entozoaires dans toules les parties de l'animal les plus éloignées du canal intestinal; 
je ne vois cependant pas qu'on en ait encore trouvé dans le canal médnllaire des os, ni même dans le canal vertébral; $4^{\circ}$. certains genres et certaines espèces d'entozoaires ne se trouvent jamais que dans les mêmes parties et dans les mêmes organes du corps animal; $5^{\circ}$. tous les entozoaires meurent plus ou moins vite quand on les a retirés du lieu où ils vivaient et se multipliaient, tandis que les insectes qui y subissent une partie de leur développement sont forcés d'en sortir pour le reste, et, à plus forte raison, ne penvent y multiplier; $6^{\circ}$. il existe souvent une grande quantité d'entozoaires dans un animal, sans que sa santé en souffre; $7^{\circ}$. on a découvert des entozoaires dans des foetus nouvellement nés.

Les entozoaires ne sont donc pas des animaux extérieurs qui auraient pénétré accidentellement dans le corps animal, ou qui y auraient subi des changemens dépendans des circonstances nouvelles dans lesquelles ils se seraient trouvés.

Mais quelle est leur origine?

Ont-ils ćté communiqués à l'état parfait ou à l'état d'oeufs d'un animal à l'autre, par les alimens solides, liquides ou aériformes? ce qui au reste ne ferait que reculer la question.

Cette opinion, soutenne surtout par Pallas, nous paraît solidement réfutée par M. Bremser; cependant l'auteur de l'analyse de l'ouvrage de ce dernier inséré dans la Gazette de Salzbourg pense qu'elle n'a pas été suffssamment déreloppée. Ne serait-il pas possible, dit-il, que les œufs des entozoaires, ainsi que les graines de quelques végétaux pussent rester dans des circonstances particulières pendant un long espace de temps sans se développer, comme les expériences de Leuwenøeck, Spalanzani , Fontana, etc., l'ont prouvé pour le rotifère. L'analyste aurait pu ajouter le gordius aquatique, comme je l'ai observé moi-même, et surtout les ocufs de la limace agreste, d'après les expériences de MI. Leechs, sans que cela rendit l'opinion de Pallas plus admissible, puisque dans toutes ces expériences il y avait dessiccation préalable, ce qui ne peut avoir lien quand l'œuf ou le germe du ver est dans le corps animal. Quant à la confirmation qu'on pourrait tirer de l'observation rapportée par M. Joln Miller Barry, dans les Transactions des médecins du collége du roi et de la reine en Irlande, vol. II, I818, qu'une famille dans le voisinage de Macrony en Irlande, 
avait été tellement tourmentée par les ascarides provenant de l'cau d'une source dont elle faisait usage, il parait qu'clle peut encore aller avec les autres observations analogues rapportées par M. Bremser, puisqu'il résulte du récit que ce n'étaient que des larves.

Ainsi, après avoir successivement montré que cctte communication ne peut avoir lieu par les alimens ni par la boisson, M. Bremser a recherché si cela ne pouvait pas avoir lieu par l'air ; et pour réfuter cette opinion, il ajoute que cela ne pourrait se faire que dans le cas où les oufs seraient extrêmement desséchés, et alors pourraient-ils conserver leur faculté de se développer? Oui, d'après les expériences de Leechs ; mais cela ne fait rien à la force des raisonnemens de Bremser.

Si donc ce n'est par aucun des alimens que les germes des vers s'introduisent dans le corps animal, ce ne peut plus être que par la transmission des parens, soit dans l'acte de la génération, soii par la nutrition dans le sein de la mère, soit par l'allaitement. Or il n'est pas difficile à M. Bremser de jeter à bas tous les raisonnemens qu'on a employés pour soutenir cette hypothèse; car il prouve que des enfans ont eu des vers, ce que leurs parens n'avaient pas, et que l'explication de Brera est bien gratuite.

Il prouve que les germes n'ont pu être introduits par le sperme du mâle, qu'ils n'ont pu l'être par la circulation dans la mère, et qu'ils ne peuvent l'être davantage par l'allaitement.

Ainsi ayant rapporté successivement les preuves que les entozoaires ne peuvent provenir du dehors dans le corps animal, reste par voie négative qu'ils proviennent de l'intérieur, qu'ils y naissent; en un mot leur génération spontanée.

- Il admet cependant que quelquefois une espèce introduite avec un animal maugé peut vivre quelque temps, mais non se reproduire, et l'expérience de Pallas, du développement d'œufs d'un ver propre à une espèce, quand on les a introduits dans un autre individu de la même espèce, lúi semble plutôt à l'appui de son opinion que contraire. C'est à ce sujet qu'il réfute arec vigueur, d'une manière irréfragable, un fait cité par Brera, dans lequel il est question de prétendus vers, qui n'étaient que des graines de fraises, et qui cependant introduites dans la cavité abdominale d'un chien, ont donné naissance à des milliers de petits vers que celui-ci regarde comme des ascarides vermiculaires, quoiqu'ils n'en eussent aucun des caracidres. 
Ici l'auleur, pour faire mieux concevoir son idée ou la manière dont il conçoit la chose, remonte à la formation du globe, et nous aurons peut-être besoin de nous faire excuser d'avoir traduit tout ce clıpitre, qui n'est qu'une pure hypothèse, et qui contient même quelques erreurs ; comme celle-ci, que, dans les terrains de transition, il existe peu de traces de corps organisés; car on y connaît maintenant beaucoup de végétaux, des coquilles, des trilobites, et même un grand nombre de poissons, etc.; mais nous avons saisi cette occasion de faire connaître en France la manière de philosopher de l'école allemande, et nous convenons même que ce n'a pas été sans quelque peine que M. le docteur Grundler et moi sommes parvenus à bien saisir le sens de l'autcur, et à lier ses idées dans notre langue. Il se pourrait même que nous n'eussions réussi qu'assez incomplétement, ce qui nous forcerait dans ce cas de prier M. Bremser de ne pas trop nous en vouloir.

L'auteur expose d'abord comment il conçoit la formation de la terre.

C'était au commencement une masse liquide sans forme, mais vivifiée, c'est-à-dire, douée de l'esprit vivant ou de l'esprit universel; peu à peu il se développa un noyau et les terrains primitifs : la cause principale de cette formation du noyau n'est pas dans une force morte inhérente à la matière ou gravitation, mais bien dans l'esprit même, dans sa tendance à dominer la matière et à former par sa liaison intime avec elle des touts clos existant par eux-mêmes. Alors l'esprit sépara d'abord la matière brute et la rejeta au centre, d'où les terrains primitifs; après que la plus grande partie de la matière la moins propre à la matière fut cristallisée, l'esprit fut plus libre. Il y eut alors une révolution ou fermentation dans toute la masse, et les terrains de iransition se déposèrent en un grand nombre de fois; il n'y avait cependant pas encore de vic particulière.

Ce ne fut qu'à cette époque qu'elle commença. Une nouvelle fermentation eut lieu; la première création fut détruite; la terre fut peuplée de nouveaux animaux. Sans pouvoir cstimer le nombre de ces révolutions, chaque précipitation fut suivie d'une nouvelle création, et l'homme fut le produit de la dernière, c'est-à-dire, l'être où l'esprit domine le plus la matière. 
D’après cela, M. Bremser ne peut adınettre que les espèces animales soient provenues les unes des autres par des perfectionnemens successifs; mais bien qu'elles ont été créées d'un seul jel, et sans doute à l'ćtat complet, du moins sous le rapport des organes nutritifs. Ne serait-il pas plus rationnel de penser que ce ne sont pas les animaux eux-mèmes qui se fornent spontanément, mais seulement leurs œufs et leurs germes?' Il y aurait plus d'analogie avec ce qui existe dans la reproduction des animanx les plus élevés.

Cela posé, notre auteur divise les substances dont se compose en ce moment notre terre, en

${ }^{\circ}$. Corps morts inorganiques (minéraux);

$2^{\circ}$. Corps vivans organisés (végétaux et animaux);

3o. Corps sans forme, sans limites (air et eau).

Les minéraux ne peuvent être considérés comme ahsolument privés de vie; clle est seulement chez eux à un degré de tension très-faible.

C'est au sujet des corps vivans qu'il recherche ce que c'est que la vie, et il la trouve dans ce qu'il a nommé l'esprit.

D'après cette idée, que la totalité de la terre existail à l'état amorphe avant l'existence des corps organisés, et que ceux-ci se sont développés successivement de la même substance également à l'état amorphe, il ne doit pas paraître étonnant que la même chose se reproduise encore aujourd'hui, et que partout où il se trouve de la matière animalisée, ou à l'état sans forme, il se déreloppe de nouvelles vies individuelles ou bien de nouvelles orgauisations; et si une de nos infusions ne produit pas des baleincs ou des chênes, cela tient, dit notre auteur, à ce que la masse en fermentation est infiniment petite comparativement avec ce qu'elle a dû être à une époque ancienne de l'état de la terre.

Il n'y a pas de véritable mort dans le règne organique, la mort n'étant que le passage à une nouvelle vie.

Convaincu par les observatious et les expériences de Treviranus sur la génération spontanće des infusoires, il conclut que, s’il est évident que la moisissure et des infusoires peuvent se former par le moyen de corps organisés privés de vie, cela peut encore plus aisément avoir lieu dans les organisations vivantes.

Dans les organisations virantes, le noureau produit est tou- 
jours déterminé par la nature de l'organisation de laquelle il a été formé; ainsi, des lichens et des mousses croissent sur les plantes, des vers intestinaux, des poux et des cirons dans le corps animal.

Mais comme on pourrait douter que les poux pussent aussi se reproduire spontanćment, étant des animaux déjà élevés dans l'échelle, M. Bremser rapporte quelques faits à l'appui de son opinion, soit pour les poux, soit pour l'acarus de la galc.

Au reste, aux personnes qui, disent-elles, ne peuvent pas concevoir la génération spontanée des vers intestinaux et des cirons, il demande si elles concoivent mieux celle par la succession des parens, qu'il montre avec raison être tout aussi spontanée, avec la seule différence que c'est dans un lieu déterminé de la mère. C'est ce qu'il fait voir surtout pour l'oisean chez lequel l'ouf se produit dans la capsule de l'ovaire sans communication réelle avec lui. Quant à ce qu'il ajoute, que le jaune et le blanc sont également produits par cet organe, il se trompe. Le dernier n'est qu'une excrétion de l'oviducte à mesure que l'œuf le traverse et constitue ce que j'ai nommé les membranes adventives.

Après cette démonstration indirecte, M. Bremser se voit pour ainsi dire forcé de conclure que les vers intestinaux se forment spontanément; alors, appliquant ici sa théorie générale de la formation des individualités du globe terrestre, et admettant que dans chaque organisation animale il existe une fermentation cont.nuelle, pendant laquelle de nourelles substances sont admises, précipitées, appropriées, dissoutes, décomposées et excrétées; et réfléchissant à la grande quantité de substauces animalisées sans forme qui se trouvent dans chaque animal, il ne lui paraît pas étonnant qu'il puisse se former de l'excédant de ces substances à sa nutrition, ou même de celles qui n'y sont pas propres, un tout existant par lui-même ou un ver, comme dans le monde se forma autrefois le ver de terre : parmi ces vers offrant toutes les espèces de génération. comme les animaux extérieurs, il s'en trouvera qui, étant pourvus des deux sexes, pourront se reproduire ensuite par l'acte de la génération; mais cela ne devra jamais aroir lieu que dans le même individu; dans chaque animal noureau il y aura une nouvelle génération spontanée, et celte production 
pourra différer non-seulement suivant les espèces, mais encore suivant l'àge du même individu. En effet, les ascarides et les oxyures sont plus communs dans le jeune âge, et les cestoïdes dans l'âg e adulte.

A tontes ces preuves négatives ou analogiques en faveur de son opinion, M. Bremser en ajonte encore de directes en rapportant les observations curieuses qu'il a eu l'occasion de faire sur le développement des géroflés dans les poissons, en sorte qu'il reste bien convaincu que les vers intestinaux ne pouvant provenir de l'extérieur, se forment de tontes pièces dans les différentes parties de l'animal, en sont pour ainsi dire le produit, comme dans les mammifères et les oiseanx le foetus est le produit de l'ovaire. La formation spontanée des vers intestinaux s'opère donc probablement de la même manière que celle des infusoires dont l'origine, pendant la fermentation des substances organiques, a été mise hors de deute par les belles expériences de Treviranus, l'organisation d'une plante ou d'un animal retombant pour ainsi dire ici en plusicurs organismes.

Cette théorie de la formation spontanée des vers intestinaux paraît si peu extraordinaire aux physiologistes allemands, que M. Oken, dans la Chronique de la litiérature autrichienne, $n^{\circ} \cdot 9$, nov. 1819 , dit que M. Bremser aurait pu tirer des preures plus concluantes en sa faveur de la formation de l'organique de l'inorganique, puisque, dit-il, il aurait pu démontrer par des faits qu'il se forme avec de la chaux, du charbon, du sel et de l'eau de la mucosité qui est déjà, eo ipso, un animal qui se divise en globules et en infusoires.

Sur la distribution systématique des vers intestinaux.

M. Bremser, ayant démontré que les entozoaires ne peuvent être rapportés à aucun des animaux extérieurs, en a conclu trop rigoureusement suivant nous qu'ils doivent former un type particulier; car c'est un principe de toute classification zoologique, que le séjour ne doit avoir ancune influence sur la place d'un animal dans la série, mais seulement l'eusemble de son organisasion. Or, comme il est évilent que celle d'un ascaride est extrê- 
mement différente et isupérieure à celle d'un échinoccoque sous tous les rapports, il en résulte que, dans un système général de zoologie, ces animaux ne doivent pas même appartenir à la même classe, et doivent au contraire être répartis dans des types différens, par conséquent être entremêlés avec des animaux extérieurs, comme l'ont fait MMI. Oken, de Lamarck, Cuvier, et encore plus M. de Blainville.

Le système de distribution des entozoaires de M. Bremser est calqué exactement sur celui de M. Rudolphi, qui n'est lui-même qu'une imitation de celui de Zeder, comme nous l'avons déjà dit, en grécisant les dénominations allemandes que celui-ci avait données. Avant Zeder cette distribution éta:t presque nulle.

M. le professeur Oken, dont le Manuel d'histoire naturelle a paru à Leipzick en I 8 I 5 , a ajouté pen de choses à la distribution systématique des vers intestinaux du premier ouvrage de $\mathbf{M}$. Rudolphi; mais, par une singularité assez remarquable, son système differe sensiblement dans le corps de l'ouvrage, de ce qu'il est dansle tableau analytique qui est au commencement: comme nous présumonsque celui-ci est postérieur à celui-là, nous allons commencer par son analyse.

Dans l'ouvrage les vers intestinaux forment le premier ordre de la quatrième classe, ou mieux le dernier, à cause de l'ordre de composition croissante que suit M. Oken, entre les beroès qui commencent la cinquième, et les mollusques acéphales qui terminent la troisième, et ils sont entremêlés de plusieurs genres d'animaux extérieurs. Il suit ici le système de divisions ternaires, c'est-à-dire, que cet ordre est partagé en trois tribus divisées chacune en trois familles contenant chacune trois genres.

TRIBU PREMIERE.

Fam. I. Echinoccoque, comure, cysticerque.

- II. Toenia, botllriocéphale, rhytis.

- III. Lisule, tricuspidaire, prionoderme.

TRIBU SECONDE.

Fam. I. Polystome, scolex, distome et planaire.

- II. Porocéphale, tetrarhynque, échinorhyngue.

- III. Phylline, schisture, lernée. 


\section{TRIBU TROISİ̀ME.}

Fam. I. IIamulaire, liorhynque, cucullan.

- II. Gordius, trichocéphale, oxyure.

- III. Strongle, ophiostome, ascaride.

Dans le tableau analytique, les vers intestinaux forment les trois premières tribus de la première division de l'ordre des vers, qui est le premier de la quatrième classe, comprenant tous les animaux articulés, en sorte qu'ils sont avant les sèches, qui commencent la classe des mollusques, et après les chétopodes ou vers à sang rouge de M. Cuvier, qui forment les premières tribus du même ordre. Une autre différence, c'est qu'ici MI. Oken adopte le système quartenaire. En voici le tableau.

\section{TRIBU PREMIÈrE.}

Fam. I. Echinoccoque, coenure.

- II. Cysticerque, cysture ${ }^{\mathrm{I}}$, vésicaire ${ }^{2}$.

- III. Halysis, tonia ou bothriocéphate, fuimbriaire ${ }^{3}$.

- IV. Tricuspidaire, rhytis 4 , ligule.

\section{TRIBU SECONDE.}

Fam. I. Scolex, caryophyllée.

- II. Monostome, amphistome ou strigéen distome ou fasciole, festucaire ${ }^{5}$.

- III. Polystome, linguatule.

- IV. Porocéphale ${ }^{6}$, hèruque, tétrarhynque, échynorhynque.

Nouveau genre établi avec le cysticercus fasciolaris de Rudolphi, et qui correspond au genre hydatigère de M. de Lamarck.

2 Nouveau genre établi avec le cysticercus lucii de M. Rudolphi.

3 Nonveau genre étabii pour le tania malleus de M. Rudolphi.

4 Nouveau genre adopté pour quelques espèces de bothriocéphale, et entr'autres pour le bothriocephalus solidus.

5 Genre adopté de Shrank pour le distoma trigonocephalum, qui parait n'être qu'une espèce de monostome.

6 Genre adopté de M. de Humboldt, pour un anjmal qui paraît ètre une espèce de polystome de M. Rudolphi, ou de linguatule de M. de Lamarck. 


\section{TRIBU TROISIÈME.}

Fam. I. Prionoderme ${ }^{x}$, schisture ${ }^{2}$.

- II. Filaire ${ }^{3}$, capsulaire 4 , hamulaire ${ }^{5}$, liorhynque.

- III. Capillaire, trichocéphale, oxyure.

- IV. Cucullan, strongle, ophiostome et fusaire ou ascaride.

Ainsi, dans cette nouvelle classification des vers intestinaux, les lernées, les planaires, les gordius en ont été retirés.

La distribution de M. de Lamarck est un peu différente.

Les entozoaires, qui terminent la division des animaux apathiques, sont tous compris dans la cinquième classe du système des animaux sans vertèbres, entre les ascidies et les diptères; mais ils n'en constituent que les deux premiers ordres, sous le nom de vers mollasses et de vers rigidules, le troisième et dernier étant formé par les vers hispides, qui sont des animaux extérieurs. Voici le tableau de la distribution des genres.

\section{ORDRE PREMIER。}

VERS MOLI.ASSES.

Vers nus, d'une consistance nolle, sans raideur apparente, diversiformes, et la plupart irréguliers.

\section{première section. - Les I'́siculaires.}

Leur corps est vésiculaire, ou se termine postérieurement par une vessie, ou adhère à la vessie qui le contient.

- Proposé par M. Rudolphi, mais qu'il a reconnu depuis être établi sur son polystoma tuenioides.

${ }^{2}$ Genre furt douteux établi par M. Rudolphi sur un animal incomplétement décrit par Redi.

3 Genre de Zeder, peu ou point distinct des véritables filaires.

4 C'est le genre trichosoma de M. Rudolphi.

5 Genre établi par Treutler sur un animal décrit à l'envers et supprimé par M. Rudolphi. (On peut voir ce qu'en dit M. Bremser, p. 250) 
Bicorne", hydatide", hydatigère ${ }^{3}$, coenure, échinocioque.

\section{DEUXıÈne SECTıON. - Les Planulaires.}

Leur corps cst toujours aplati.

Tania, bothriocéphale, tricuspidaire, ligule, linguatule 4 , polystome ${ }^{5}$, fasciole ${ }^{6}$.

\section{TROISiÈME SECTION. - Les Hetéromorphes.}

I.eur corps est tantôt aplati, tantôt cylindracé, et souvent difforme.

Monostome, amphistome, géroflé 7 , tétragule ${ }^{8}$, massette 9 , tentaculaire $^{\mathrm{x}}$, sagittule ${ }^{\mathrm{x}}$.

\section{ORDRE DEUXIEME.}

\section{VERS RIDIGULES.}

Ils ont un peu de raideur, ce qui les rend presque élastiques, et sont nus, cylindracés, filiformes, la plupart réguliers.

$$
a \text {. intéricurs. }
$$

Porocíphale, échinorhynque, strongle, cuculian, fissule ${ }^{22}$, oxyure, trichiure, ascaride, hamulaire, liorlynnifue, filaire.

$$
\text { b. extéricurs. }
$$

\section{Dragonneau.}

- Genre fort douteux établi par Sulzer sous le nom de ditrachyceros pour des corps de la nature animale desquels M. Breinser parait peu persuadé (Voy. p. 320).

${ }^{2}$ Ce sont les cysticerques de M. Rudolphi.

3 Genre distinet q:ıe MI. de Lamarck forme avec les espèces de cysticerque à corps allongé.

4 C'est le genre polyst one de Rudolphi.

$5 \mathrm{M}$. de Lamarek a adopté ec genre de Delaroche; il est établi sur un animal de la famille des sangsues, suivant uous.

6 C'est le genre distome de Rudolphi.

? Caryopliylixus.

8 Ce genre, admis de MI. Bosc, est établi suivant nous sur le polystoma serratum de Rudolphi, linguatule de M. de Lamarck.

9 Scolex.

10 Tetrarhynchus.

"Nouveau genre dent nous parlerons plus tard.

1s C'est le genre ophiostome de MI. Rudolphi. . 


\section{APPENDICE.}

M. G. Cuvier fait des vers intestinaux la deuxième classe de ses zoophytes, et les place après les échinodermes, entre ses échinodermes sans pieds ou siponcles, et les actinies, qui commencent sa classe des acalèphes; il y réunit plusieurs genres d'animaux extérieurs. Sa distribution est la suivante :

\section{ORDRE PREMIER.}

\section{INTESTINAUX CAVITAIRES.}

Qui ont un canal intestinal flottant dans une cavité abdominale distincte, une bouche et un anus : ce sont les $E$. nematoidea de M. Rudolphi.

Filaire, hamulaire, trichocéphale, oxyure, cucullan, ophiostome, ascaride, strongle, prionoderme ${ }^{2}$, lernée, némertes ${ }^{2}$.

\section{ORDRE DEUXIÈME.}

\section{INTESTINAUX PAREXCHYMATEUX.}

Dont le corps renferme dans son parenchyme des viscères mal terminés et ressemblant le plus souvent à des ramifications vasculaires, ne s'apercerant même quelquefois pas du tout.

Cet ordre comprend les quatre derniers ordres de M. Rudolphi. première faMille. - Les Acanthocéphales.

Héruque ${ }^{3}$.

\section{Echinorhynque.}

DEUXIÈMe FamilLE. - Les Trématodes.

Douce.

Festucaire (monostoma, R.), strigée (amplistoma, R.), gérofí, polystome 4 , tristome ${ }^{5}$.

\section{Planaire.} marck.

' C'est le genre polystome de Mr. Rudolphi, linguatule de M. de La-

2 Genre ronveau établi pour un ver marin, que M. Oken avait déjà distingné sous le nom de Borlasia.

${ }^{3}$ Genre adopté de Gmelin, pour un ver que M. Rudolphi confond parmi les échisorhynques.

${ }^{3}$ Sous ce nom II. Cuvier réunit les polystomes de Rudolphi et le poIystome de Delaroche.

5 Nourcau genre que M. Bosc arait nommé capsule pour un animal 
Troisı̀̀ie FAMiLLE. - Les Taniö̈les.

Tania.

Tricuspidaire, bothriocéphale, floriceps ${ }^{x}$, tétrarlyynque, cysticerque, cocnure, échinoccoque.

\section{Scolex.}

Quatrième Famule. - Les Cestö̈les.

Ligule.

M. de Blainville regarde les vers intestinaux comme appartenant à différens degrés d'organisation, et par conséquent à différens types de la série animale; aussi dans son système général de zoologie en place-t-il une partie parmi les entozoaires, une autre dans le sous-type des sub-annelidaires, intermédiaire an type des animaux articulés et à celui des rayounés; enfin, en admettant que les acéphalocystes soient de véritables animaux, il en fait un genre d'amophozoaires. Voici le tablean de ses divisions, et comme il y distribue les genres.

\section{CLASSE DES ENTOMOZOAIRES APODES.}

\section{ordre I. - Les Poljporidés.}

I.e corps court, déprimé, très-contractile, pointu en avant, elargi en arrière el garni au-dessons de son bord postérienr de plusięurs paires de pores contenant chacun un on denx crochets; houche à l'extrémité pointue du corps; une ouverhure abdominale pour l'appareil générateur.

\section{Hexathiridium $^{2}$.}

parasite sur les branchies des poissons, que je regarde comme de la fainille des sangsues.

r Nouveau genre nommé anthocéphale par Rudolphi, établi par M. Cuvier pour un ver intestinal que M. Bremser pense n'être qu'un degré de développentuent d'une espèce de bothriocéphale.

z Je doute beaucoup que ce genre puisse être regardé comme véritablement intestinal; il est extrêmement rapprochédu polystome de Delarochs: anssi pensais-je qu'il a été décrit a l'envers, ce que l'on voit dans ma défunition des caractères de l'ordre. 
URDRE II. - Les Onchocéphales.

Corps peu allongé, sub-cylindrique, sub-articulé ; canal intestinal complet; bouche orbiculaire inférieure, pourvue de chaque côté de deux crochets cornés, recourbés, dirigés en arrière et rétractiles chacun dans un tubercule; anus terminal et postérieur; sexes distincts.

\section{Linguatule.}

Lingualule, pentaslome ${ }^{\mathrm{X}}$, tétragule, porocéphale, prionoderme, crucullan $^{2}$, nettorhynque ${ }^{3}$, caryophyllée 4.

OPDRE III. - Les Oxycéphates on Ascaridiens.

Corps médiocrement allongé, élastique, cylindrique, atténué aux deux extrémités, sans presqu'aucune trace d'articulations; caual intestinal complet; bouche terminale orbiculaire, nue on pourvue de quelques tubercules radiairement disposés; anus plus ou moins terminal; sexes séparés.

a. Bouche en suçoir, à lèvres simples on tuberculeuses.

Filaire, trichosome, oxyure, trichocéphale, ophiostome, pleurothynque ${ }^{5}$, physaloptère, spiroptère, liorhynque ${ }^{6}$.

' Je préfère, pour réunir les c̀nq premiers genres, qui n'en sont évidemment qu'un, la dénomination de linguatule à celle de pentastome, qui donnerait une idée fausse.

${ }^{2}$ Ce genre est trop mal connu pour assurer sa place.

3 J'établis ce genre avec un ver intestinal observé par Paisley, et dout il sera parlé plus loin.

4 C'est encore un genre trop mal connu pour juger ses rapports naturels.

${ }_{5}$ Ce genre est élabli par M. Nau pour unc espèce d'opluiostome, O. spharocephalum.

${ }^{6} \mathrm{C}$ 'est encore un genre bien ilouteux. 
๖. Bouche close et armée de nodules ou d'espèces de dents radiaircs.

Capillaire , fusaire ${ }^{2}$, ascaris, théla $\approx i^{3}{ }^{3}$, strongle, sclérostome ${ }^{4}$, sclérotrique ${ }^{5}$.

\section{CLASSE DES SUG-ANNELIDAIRES ov GASTRORHYZAIRES.}

\section{ordRE I. - Les Porocéphales.}

Corps très-ınou, sans traces d'articulations, très-déprimé ou cylindrique, avec un ou deux grands pores médians, l'un antérieur, le plus souvent terminal, et l'autre plus ou moins reculé en arrière, servant de ventouses; canal intestinal incomplet, vasculaire, commençant par un très-petit orifice percé dans le fond de la ventouse antérieure; sexes réunis sur le mêne individu; l'organe femelle se terminant dans la ventouse postérieure; l'organe mâle saillant en avant snus forme d'un tentacule.

IIypostome ${ }^{6}$; festucaïre 7 , fasciole, alaire ${ }^{8}$, hirudinelle 9 , lobostome $^{\mathrm{I0}}$, echinostome ${ }^{\mathrm{15}}$, strigée ou amphiostome, holostome ${ }^{\mathrm{I2}}$.

I J'appelle capillaire les trichocéphales qui ont trois nœuds ou tuberculcs à la bouche, comme le tricocephalus nodosus, Rud.

${ }^{2}$ Ce sont les filaires, dont la bouche est pourvue de tubercules, comme le $F$. coronita, R.

3 Genre élabli par MI. Bosc (Journ. de phys., 1819), ponr une trís petite espice d'ascaridiens trourée sur la cornée d'un bocuf, et qui scrait fort remarquable, si en effet elle offrait l'organisation qu'on lui assigne.

$4 \mathrm{C}$. sont les strongles beaucoup moins allongés et pltis raid's que les autres, et dont l'exırémilé antérieure un peu renflée on tronquée carrément, est armée d'une sorte de courone cornée diviséc en six parties, comme le strongle du cheval.

5 J'admets ce genre arec MI. Rudolphi pour la singulière espèce de trichocéphale, qu'il a nommée $T$. echinatum.

6 C'est un genre bien douteux, peot-être un degré de déreloppement.

7 Ce sont les monostomes cyliadriques et à boache terminale de MI. Rudolphi.

\& Je propose de rétablir ce genre pour quelques espèces de fascioles cylindriques, avec une assez longne queue, el qui ont des espèces d'ails de chaque eòté du corps : $\mathrm{j}$ 'en as décrit une trouvée dans le pancréas du Simia muimon de Linné, à l'art. fasciole dn Dicciono. des sciences nat.

$9 \mathrm{~J}$ 'ad mets ce genre pour les fascioles cylindrique's qui ont queique ressemblance avec les sangsues, et entre auires le fiusc. clavata, Lin.

10 Ce sont les fascioles cylindrigues dont la lèrre de la ventouse antérieure c'st lobée, comme le dist. luureatum de Rudolphi.

" Ce sont les espèces de fascioles cylindriques qui out des crochets vers le pore antérieur, comme le distoma cchinalum, Rud.

12 Nouveau genre établi par Nitzsch ( Encycl. Halensis, pour les an- 
ondre II.-Les Acanthocéphales.

Corps plus ou moins sacciforme, peu ou point articulé, obtus aux deux extrémités; l'antérieure avec une sorte de renflement céphalique ou de trompe céphaloïde garnie d'aiguillons recourbés et percée d'un pore médian extrêmement petit; la postérieure percée par un orificc mćdian également terminal, souvent trèspetit; canal intestinal? sexes sćparés?

Héruque, échinorhynque.

ORDrE III. - - Les Bothriocéphales.

Corps de forme très-rariable, avec un reuflement céphaloïde, sans bouche proprement dite, mais pourvue de quelques organes saillans et surtout de fosseltes disposées à sa circonférence et subradiairement; canal intestinal nul ou subvasculaire; sexes non distincts; tous les individus semblables.

Famile I. - Les Polyrhynques.

a. Le corps court et sacciforme.

Dibothriorhynquex, bynnurhynque, tétrarhynque, hépatoxylon, anthocéphale.

b. Le corps très-long, tænioïde, articulé.

Rhynchobotris ${ }^{2}$, onchobotris ${ }^{3}$, tricuspidaire 4.

phistomes à têtc non distincte de Rudolphi, et les d. alatum et excavatum du nême.

' J'élablis ce nouveau genre prour un ver intestinal trouvé dans les intestins du poisson appelé sabre ou ceinture d'argent sur nos côtes (lepidopus Guanii), attaché en assez grand nombre à d's paquets de cellulosilés renfermant des filaires. Il est caractérisé aisćment, parce que son corps courl, sacciforme, portc en avant une sorte de renflement céphaloïde allongée, de chaque còlé duquel cst une longue fosselle, un pcu bipartite en arrière; à la partie antérieure est en outre de chaque eôté un tubereule rẹ́tractile, pédieulé, sphérique, ga ni de petits aiguillons recourbés. Cettc combinaison de deux suçoirs ou fosselteset de dcux trompes, b'existant dans aucun groupe de cette faunille, méritait d’être distinguée.

${ }^{2}$ Secion générique établie pour le b. corollatus de Rudolphi.

${ }^{3}$ Division des Lothriocéplıales, les $b$. uncinati, et, entre autres, le $b$. coronatus.

4 Je place ici ee genre, parce que je suppose que les dents tricuspides sont l's analogucs des trompes aiguillonné es des genres précédens. 
Famlde II. - Les Stéphanorhynques.

a. Le corps très-long, tænioïde, articulé.

Aly sclminthe ${ }^{2}$, halyse ${ }^{2}$, tania, hydatigère.

b. Le corps court, hydatoïde.

Cysticerque, coenure, échinoccoque ${ }^{3}$.

FsMille III. - Les Céphalorhynques.

a. Quatre fossettes ou appendices nus.

Scolex, tentaculaire, tétrabotris 4 .

b. Deux fossettes sans appendices ni trompes.

Bothridium ${ }^{5}$, rhythis ${ }^{6}$, ligule.

\section{CLASSE DES MONADAJRES.}

Acéphalocyste, hydromètie.

Quant au genre schisture, dont il n'est pas question dans ce tableau, et qui est établi sur une description et une figure trèsincomplettes d'un animal trouvé par Rédi dans le canal intestinal d'un tétraodon mola, ne serait-ce pas le singulier siponcle dont M. Rolando a fait son genre Bonellie?

- Les tænias sans trompe ni couronne de crochets, comme le $t$. plicata, R.

- Les tænias à trompe non armée, comme le $t$. farciminula, Lin.

3 J'aroue que, malgré les nouveaux détails que nous a donnés MI. Rentdorf sur ce singulier animal, je ne conçois pas trop comment la vessic qui contient les individus en fait réellement partie.

$4 \mathrm{Je}$ réunis dans ce genre les espèces de bothriocépale qui ont quatre fossettes bien distinctes, que le corps soit articulé ou non, comme les b. auriculatus et macroceplaalus de Bremser.

5 Je donne ce nom générique à un ver tænioïde bien régulièrement articulé, à articulations droites, serrées, nombreuses, comme dans le tania plicata, et dont l'extrémité antérieure ovale est formće par deux. fossettes, ou mieux par deux espèces de petites capsules ouvertes en araut sculement par un orifice arrondi; il provenait d'uae grande espèce e. serpent du genre pithon, qu'on montrait virant à Paris en 1823 .

5 Je réserve ce nom aux bothriocépliales qui n'ont que deux fossettes ieu marquées, comme le b. de l'homme (tconia lata). 
Sur la description des vers intestinaux propres à l'espèce liumaine.

Les descriptions que M. Bremser donne des différentes espèces d'entozoaires qui ont été obserrées jusqu'aujourd'hui dans le corps de l'homme ne sont pas torjour's très-étendues, mais elles sont cependant presçue constamment suffisantes, quoi qu'en ait dit son critique italien, surtout accompagnées qu'elles sont d'excellentes figures originales détaillées. On pourrait plus justement lui reprocher de n'aroir pas parlé de l'organisation. Cela me semble une véritable lacune qui nuit à la conception de tout ce qu'il dit sur lâ formation de ces animaux.

Parmi les espèces qui séjournent dans le canal intestinal, M. Bremser décrit et figure successivement :

$\mathrm{I}^{\circ}$. Le trichocéphale de l'homme.

$2^{\circ}$. L'oxyure vermiculaire.

$3^{\circ}$. L'ascaride lombricoïde.

$4^{\circ}$. Le bothriocépbale large.

$5^{\circ}$. Le trenia de l'homme.

Et parmi celles qui se trouvent hors du canal intestinal:

$6^{\circ}$. Le ver de Médine.

$7^{\circ}$. L'hamulaire subcomprimè.

8. Le strongle géant.

$9^{\circ}$. La douve du foie.

${ }^{\prime} 0^{\circ}$. Le polystcme pinguicole.

${ }^{1} \mathbf{I}^{0}$. Le cysticerque du tissu cellulaire.

$12^{\circ}$. L'echinoccoque de l'homme.

Nous n'avons rien à ajouter à ce qu'a dit Mr. Bremser sur les espèces de vers qui vivent dans le canal intestinal de l'homme; nous nous bornerons seulement à faire remarquer que c'est aux observations assidues et délicates de l'auteur que la zoologie doit être éclairée sur la véritable place de l'oxyure vermiculaire et du bothriocćphale large, dont on faisait jusqu'ici du premier un ascaride sous le nom d'ascaride vermiculaire, et du second un teria sous la dénomination de taenia large ou à anneaux courts, et qua î a aussi observé le premicr le mầic de l'oxyure vermiculaire x .

- Nous derons cependant dire que M. Hippolyı Cloquet a figuré dans sa Faune des médecins un ver intestinal qu'il regarde comme ap- 
Nous ajouterons cependant à ce chapitre l'indication d'un ver intestinal auquel nous avons donné le nom de nettrorhynque, et qui, quoique observé depuis long-temps, a été passé sous silence par les meilleurs helmin thologues modernes. C'ést dans le tome II des Mémoires de la Société de medecine d'Edimbourg qu'il en est question dans un mémoire intiulé Description d'un ver extraordinaire, par J. Paisley, chirurzien à Glassow. Ce ver était fort considérable, puisqu'il avait deux pieds six pouces de longueur sur un pouce et demi de diamètre; il élail formé d̉e plusieurs grands anneaux semblables à ceux du ver de terre; les intervalles entre chaque articulation étaient de couleur brune, les arliculations mêmes de couleur de chair livide. La tête, beancoup plus petite que le corps, quoique formée également d'anneaux, ressemblait beaucoup au bec d'un canard, étant aplatie en dessus. La bouche était triangulaire comme celle d'une sangsue. Ce ver fut rendu par l'anus avec une très-grande quantité de sang. Le malade eu rendit un second encore plus gros, mais par morccaux. Le premier fut dessiné en présence de plusieurs docteurs de l'université. II était d'abord beaucoup plus gros; mais, aussitôt que le malade l'eut rendu à l'aide d'une personne qui le lui tira en partie du corps, un assistant plongea un ou deux fois un canif dans le ver, et il en sortit une immense quantité de sang.

Nous en donnons la figure, planche I, de l'appendice, fig. I et 2, copiée de l'ouvrage cité.

Quant aux entozoaires qui vivent hors du canal intestinal, nous allons joindre quelques observations à celles de l'helminthologue viennois.

partenant au genre ophiostome, et qu'il nomme ophiostome de Pontier, $O$. Pontieri, du num du médecin qui le lui a envoyé. Ce serait doné la premi're fois qu'un ver de ce genre aurait été découvert dans l'espèce lımıaine. Il avait été rendu par le romissement provoqué par l'ellébore dans une épilepsie désignée sous le noun de rermineuse. Ce ver avait neuf pouces de longueur el la grosseur d'une corde à violon; à l'une de ses extrémités était une petile tête garnie de denx mâchoires, dit l'observateur original. Ne se pourrait-il pas que ce fût un gordins aquatique, auquel tous ces caractères conviennent assez bien? Nous derous aussi đire que le même médecin, M. Cloquet, annonce aroir observé une 
$V e r$ de Médine ou dragonneau. Je possède dans ma collection un individu de celte espèce qui m'a été envoyé par M. Delorme, ariquel nous devons les observations dont M. Bremser a parlé, page 204, dans la note. Quoique bien évidenment différent du gordius aqquatique par sa longueur ei sa grosseur ${ }^{x}$, on ne peut nier qu'il soit d'un genre bien voisin. Mais est-il absolument certain que cet animal ne provienne pas de l'extérieur. M. Brenser combat fortement cette opinion. Certainement ce ne peut être une larve d'insecte; car, comme nous le ferons observer plus loin, une larve d'hexapodes, seuls insectes qui éprouvent de véritables métamorphoses, n'a aueune ressemblance avec ee ver. C'est cependant l'opinion d'un médecin praticien anglais, le doeteur Chisolm, qui l'a énise d'abord dans la préface de son ourrage intitulé : Essay on the malignant pesilentiel ferers introduced in to west indian Islands from Bulllan on the coast of Guinea as it appeared in $179^{3-1} 79^{6} ; 2^{\mathrm{e}}$ édit. , Londres, $\mathrm{r} 80 \mathrm{r}$, mais plus particulièrement dans un mémoire inséré dans l'Édinburggh-medical and surgical journal, 1815, vol. 15, pag. 145-16\%. Ce médecin, qui se vante d'avoir traité dans l'espace de trois ans plus de mille mâlades attaqués du dragonneau, pense que l'on pourrait admettre, mais seulement comme une hypothèse, que ce ver est introduit dans le corps par le moyen des ocufs que l'insecte a déposés sous la peau, ou qu'on a gagués en buvant de l'eau, et que dans ce dernier eas les oenfs avalés sont conduits par les organes de la sécrétion dans les interstices de la peau et des muscles.

Un aulre auteur de la même nation, Thomas Healh, dans un mémoire inséré dans le même journal (Observations on the generation of the Guinea Worm ), vol. 12, pag. 120, réfute l'opinion de la contagion par l'cau, et croit que le ver s'introduit dans la peau pendant quinil est encore très-jeune ou à l'état d'embryon.

Hamulaire subcomprimé. Cet animal, dont le seul Trentler a dé-

cspèce notuvelle d'hamulaire qui avait déterminé des accidens nerveux très-graves. Or nous arons vu que ce genre rst bien douteux.

${ }^{2}$ M. Bremser possède cependant aujourd'hui un filaire provenant d'une sauterelle, qui a trente pouces de longueur. Tous les gordius que j'ai vus ont l'extrémité antérieute divisée en forme de pince, ce qui n’a pas licu dans les filaires. 
couvert deux seuls individus dans l'intérienr d'un raisscan lympliatique de la glande bronchiale d'un homme, est décidément reporté par MI. Rudolphi dans son synopsis parniles trichosomes, admettant que les deux crochets (hamuli), décrits par Treutler, comme servant à l'animal pour se cramponner et sucer sa nourriture, ne sont que les organes mâles.

Comme MIM. Bremser et Rudolphi n'admettent pas l'existence de ce ver comme distinct des trichosomes, et que le premier a paru douter qu'il eût été vu anciennement par deux médecins italiens, Vercelloni et Bianchi, ainsi que le dit M. Brera, et encore plus que celui-ci ait pu en faire l'unatomie, le critique italien, en répondant à ces doutes de M. Bremser, cite de Vercelloni la dissertation intitulée : De glandulis as sophagis conglomeratis, succo vero nutritivo et vermibus. Cesta, 17 I 1 , in- $8^{\circ}$. , et la page 349 de l'ouvrage de Bianchi, De naturali in humano corpore vitiosa, morbosaque seneratione, et ajoute que la descriptiou extérieure et anatomique de Brera est faite sur l'hamulaire cylindrique commun dans les pies-grièches, et qu il regarde comme au moins du même genre que l'hamulaire lymphatique on subcomprimé: mais, en admettant ce rapprochement, il est assez difficile de penser qu'on ait pu voir le système nerreux ganglionique ou non sur un animal aussi grềle.

Strongle géant. J'ajouterai à ce que dit M. Bremser sur cet entozoaire, ce que j'ai vu sur un individu trouvé par moi dans le rein d'uue marte et à peine mort, lorsque j'en fis la description et la figure. Le corps presque cylindrique et s'atténuant insensiblement, mais très-peu jusqu'aux deux extrémités fort obtuses, avait une largeur totale de vingt-neuf pouces trois lignes, sur deux lignes et demic de largeur dans son plus grand diamètre. Sa couleur était d'un brun-clair tirant sur le jaunâtre, parsemé d'une très-grande quantité de taches jaunes oblongues ou transverses. On remarquait dans toute la longueur du corps huit stries étendues de la bouche à l'anus, dont quatre alternatircment plus grosses et quatre plus petites. Celles du dos et du reutre paraissaient faire des espèces de circonrolutions, mais pen profondes. Les deux latérales offraient chacune unc série régulière de pelits tubercules percés à leur sommct, au nombre de quinze it scize par pouce. 
I'extrémité antérieure on luccale était un peu plus étroite, un peu plus atténuée que la postérieure. La bonche était entourée de huit tubercules très-courts portés sur un anneau glanduleux. L'anus, an contraire, formait une ouverture coupée obliquement et assez large. Le canal intestinal, vu à travers la peau de l'animal, m'a paru libre et s'étendant d'une extrémité à l'autre sans circonvolutions. On ne voyait aucune trace des organes de la génération, comme cela a lieu dans les ascarides.

Le rein dans lequel était contenu ve ver beaucoup plus gros que l'autre dont la longueur était de vingt lignes, sur neuf de largeur, était réduit à nne minceur d'une demi-ligne environ, et n'offrait plus aucun indice de son organisation normalc.

Polystome pinguicule. C'est encore à Trentler que nous derons la connaissance de ce singulier entozoaire dont il n'a vu qu'un seul iudividu, trouvé dans la graisse qui entourait l'ovairc d'une jeune paysanne, et qui n'a été observé par aucun autre naturaliste. J'ai dit plus haut que je soupçnnnais fortement que cet animal a été décrit à l'envers, et que ce pourrait bien n'être autre chose que le polystonia integerrimum trouve dans le poumon et la vessie urinaire d'une grenonille, que je suis tenté de regarder comme une espèce de sangsuc; mais conment s'est-il trouvé si profondément dans le corps de la jeune fille de Treutler.'

Echinoccoque de l'honme. An moment où M. Bremser écrivait son ourrage, il paraît qu'il n'arait pas encore eu l'occasion d'observer complétement l'échinoccoque de l'homme, puisqu'il dit, page 3o3, qu'on n'a pas encore constaté, d'une manière positive, une couronne de crochets chez ces animaux; depuis ce temps il a été plus heurenx, et MI. Charles Rendtorff, dans sa thèse intitulée: De hydatidibus in corpore humuani presertim in cerebro repertis, nous a donné une description et des figures qui laissent peu de chose à désirer. Nous allons rapporter ici la traduction de ce qui regarde l'animal qae les figures $\mathrm{a} a, 1 b, \mathrm{I} c$ de notre planchex: représentent.

Le sac qui contenait toutes lés ligdatides était d'un blane de lait, semblable à de la lymphì plastique, pelíncide et de l'épaisseur d'une demi-ligne environ. Il uc renfermail rien antre shose yue de l'eau avec les sćsicules, et remplissitit tout le ventricule 
droit du cervean qu'il avait dilaté, an point que la matière cérébrale qui le recouvre n'avait plus que l'épaisseur d'une demiligne. Le nombre des hydatides extraites du ventricule latéral était de soixante-onze; clles n'araient elles-mêmes aucune odeur, et différaient beaucoup de grosseur. Quelques-unes avaient un diamètre d'un pouce et demi; les autres étaient plıs petites, la plupart égalant la grosseur d'un grain de raisin de Malaga. Elles étaient globuleuses, ovales ou pyriformes. Tonte la masse des hydatides avec le sac qui les contenait pesait deux livres trois gros (poids médicinal); et la plus grosse des hydatides denx onces deux gros. Les plus petites vésicules avaient leur enveloppe un peu plus épaisse que les grosses. Elles étaient toutes indépendantes les unes des autres, sans ancune trace de vaisseaux, de fibres vu de ligamens qui les réuniraient. Le fluide qu'elles contenaient était limpide et pellucide. Ia face interne ne paraissait pas partout de la mème couleur, quelques parties étant blanchâtres et celles qui étaient couvertes par des échinoccoques tont-à-fait blanches. En agitant un peu les hydatides, les petits animaux se détachaient de la membrane interne et se répandaient dans le fluide.

Csuantà la structure de ces animaux, M. Rendtorff ne nous fournit presque que ses figures. On voit que' les pelits vers sont épars d'une manière fort irrégulière dans l'intérienr de la vessie, et que chacun d'eux en forme de petit corps ovalaire est un peu attenué aux denx extrémités et renflé au milieu. L'extrénité céphalique est armée d'une couronne de crochets. Mais il ne paraît pas qu'il y ait de suçoirs, comme dans les cœnures, du moins d'après la figure. Quant aux rapports des animalcules avec la vessie, M. Rendtorff dit positivement : Mihi autem animalcula, qui propriam desun: vitam, neque vesicula partes habenda videntur, et paraît n'avoir pas entendu M. Bremser, lorsqu'il ajoute, page 22, chap. Io: Hos quidem vesica quam habitant particulas existimat esse; car celui-ci, dans une lettre qu'il nous a écrite, assure n'avoir pas dit un seul mot de cela, ni dans les Archives de Mekel, ni dans sơn livre, où il s'est exprimé clairement. Il entend, sons le nom d'échinoccoque, les pelits corps pourvus de quatre suçoirs et d'une couronne de crochets, qui nagent librement dans l'hylatide. A mesure que ces petits corps microscopiques grossissent, 
iis prennent peu à peu la forme sphérique, les crochets se détachent, et il se produit de nouveaux échinoccoques dans ces petits corps changés eux-mêmes en hydatides. Les nouveaux échinoccoques sont des petits fils de l'hydatide primitive, qui ne fut ellemême probablement d'ahord qu'un corps microscopique semblable.

Coenure cérébral. Puisque je viens de parler de ce singulier anjmal, j’ajouterai ici une explication des figures qu'en a données M. le docteur C. Fischer de Vienne dans sa dissertation De entozois sive vermibus intestinalibus, dont nous avons parlé déjà plus haut, et qu'à cause de leur beauté nous arons copiées dans notre planche $10,2 a, 2 b, 2 c$, et des observations faites par moi sur un chamois, né et élevé au Jardin du roi, et mort du tournis. Son abdomen contenait plusieurs cysticerques, mais son cerveau surtout renfermait une très-grande quantité de cœnures. Les vessies de grosseur très-variable, ainsi que de forme, étaient remplies d'un fluide séreux, plus ou moins enfoncées dans la substance du cerveau, et quelquefois même encore recouvertes de cette substance, ce qui prouve qu'elles se développent de dedans en dehors. Il y en avait surtout une fort remarquahle occupant l'extrémilé an lérieure de l'hémisphère gauche, et qui semblait avoir rongé la table interne de la partie de l'os frontal qui répond aux sinus frontaux, en sorle qu'il n'y avait qu'une simple membrane entre les deux cavités.

Les animaux composans étaient irrégulièrement répandus sur ces vésicules et réunis par groupes plus ou moins considérables; mais il n'y avait nullement de rapports entre leur nombre et la grosseur de la vessie commune. A l'œil nu, ils ressemblaient à de petits points cartilagineux qui commenceraient à se développer à la surface de la membrane externe d'un organe. Mais au microscope, ils formaient de petiles tètes blanches, en partie rentrées ou sorties, portées sur un cou plus ou moins long, et pourvues d'une couronne de crochets terminale et de quatre suçoirs arrondis. On voit dans la figure $2 a$ un cœnure avec un grand nombre de têtes disposées par petits groupes subréguliers; $2 b$ en est un antre. beaucunp plus petit trouvé dans la substance du cervelet; $2 c$ offre deux têtes grossies, dont l'une est rentrée et l'autre sortie.

Sur les hydatides en général. Luedersen, en envisageant son 
sujet d'une manière complète, et plutôt, il est vrai, pathologique que zoologique, partage les hydatides en sept groupes distincts:

$I^{0}$. Les résicules ayant des corpuscules saillans pourvus d'organes de différentes sortes, contenus quelquefois dans une capsule externe.

$2^{0}$. Les vésicules uniformes, globuleuses, complétement libres, et entourées d'un sac extérieur.

$3^{\circ}$. Les vésicules adhérentes à une partie du corps, le plus souvent petites, et contenant de petits vers sans aucuue connexion avec elles.

$4^{\circ}$. Les vésicules simples, de structure variable, adhérentes à une partie grande ou petite du corps sans capsule externe.

$5^{\circ}$. Les vésicules réunies entre elles en séries continues, et se terminant pour ainsi dire par un filet au moyen duquel elles sont attachées au corps.

60. Les vésicules de forme variable adhérentes au corps par un pédoncule, et réunies en grappes.

$7^{\circ}$. Les vésicules irrégulières, simples ou celluleuses, adhérentes au corps d'une manière si forte, qu’on ne peut les en détacher sans dilacération.

M. Rudolphi a traité ce sujet un peu plus zoologiquement; il divisait en effet d'abord toutes les hydatides en denx ordres, les unes inanimées et les autres contenant des animaux. Dans le premier étaient trois genres, suivant que l'hydatide est solitaire, ou qu'elle est groupée, ou enfin qu'clle est contenue dans un sac; dans le second il y en avait cinq, suivant que l'animal contenu dans le sac était un strongle, un tricuspidaire, une fasciole, des échinoccoques, ou enfin un ou plusieurs tænias; mais dans son dernier ouvrage, admettant en principe que les vessies pleines d'eau que l'on rencontre souvent dans l'homme et les animaux ne sont pas du domaine de la zoologie, il réunit dans le même ordre (cystici) toutes les hydatides el les partage en quatre genres, anthocéphale, cysticerque, cœnure et échinoccoque, division que nous avons vue adoptée par M. Bremser, mais qui pourrait bien ètre fort peu naturelle, puisque les anthocéphales paraissent n'ètre que de jeunes échinorhynques ou bothriocéphales, et que les échinoccoques diffèrent beaucoup des coenures. 
On a pu voir, en lisant ce que dit MI. Bremser des hydatides en général, et avant de traiter particulièrement des cysticerques et des échinoccoques de l'homme, depuis la page $2{ }_{2} 3$ jusqu'à la page 280 , que, contre la manière de voir de MI. Rudo!phi, il regarde comme une hydatide animale toute vessie pleine d'eau qui est renferméc, mais complétement libre, dans une capsule faisant partie intégrante de l'organe dans lequel elle est, ce qui comprend évidemment les acéphalocystes de M. Laënnec; mais alors il me semble que ces animaux ne pourront être rangés, ni avec les tænias lydatigères, ni avec les coenures, ni même avec les échinoccoques, mais qu'ils derront être rapprochés des monadaires dans le type des amorphozoaires, quoiqu'ils puissent parvenir à une bien plụs grande taille.

Comme M. Bremser n'a pu parler du travail de M. Laënnec, parce qu'il nel'avait pas en sa possession, nous allons y suppléer en donnant l'extrait du mémoire de celui-ci sur les vers vésiculaires, et principalement sur ceux qui se trouvent dans le corps humain, qui fut lu à la Société de l'Ecole de médecine en 18 r. 4 . Il y distingue, comme nous l'avọns dëjà dit, les corps organisés dont il est question, sous le nom générique d'acéphalocystes, en les regardant comme de véritables animaux, et il en caractérise trois espèces: $\mathbf{I}^{0}$. l'A. A curs, A. ovoidea. A. pyriformis, simplex, vesicularis, corporibus ovatis prodita intus; $2^{\circ}$. 1'A. A Bourgeovs, $A$. surculigera. A. pyriformis, simplex, vesicularis, surculis prodita intus; et 3'. l'A. A Grans, A. granulosa. A. pyriformis, simplex, vesicularis, granulis intus prcedita. Ainsi ces trois espèces ne diffèrent que parce que la première offre dans ses parois de petits corps sphériques, blancs, opaques, peu adhérens, et souvent creux dans le centre; que la seconde présente à ses deux surfaces de petits hourgeons d'une forme très-irrégulière et très-variée, à peine visibles, ou de la grosseur d'un grain de chenevis, et enfin que la troisième est parsemée intérieurement de granulations transparentes de la grosseur d'un grain de millet. Quoiqu'en apparence peu différentes, ces trois acéphalocystes ne se trourent jamais dans le même kyste. Logées dans le tissu des parties, elleś sont enfermées dans des poches dont l-j parois les isolent absolument du parenchyme de l'organe; celles-ci sont de naiture fibreuse, sou-

$$
3 \text { 't }
$$


vent avec quelques points cartilagineux et même osseux, et leur surface interne est tapissée par une sorte de fausse membrane. Le liquide dans lequel nagent les acéphalocystes est souvent semblable à de l'eau; mais quelquefois il est plus ou moins épais; Quant à celui de l'acéphalocyste elle-mếme, il est presque toujours parfaitement transparent. Ces kystes augmentent quelquefois de manière à contenir plusieurs pintes de liquide; il paraît qu'on en trouve dans presque toules les parlies du corps de l'homme, mais surtout dans le foie, le rein, les poumons, etc.

M. H. Cloquet, dans l'article de sa Faune des médecins, qu'il a consacré à l'histoire de ces animaux douteux sous bien des rapports, a proposé d'ajouter aux trois espèces de M. Laëmnec une quatrième sous le nom de A. Granuleux, $A$. granulosa, pour des granulations lenticulaires, hydatiformes, trouvées dans la capsule du tendon du grand fessier.

Sagittule. Adoptant la manière de voir de M. Bremser, qu’il serait inutile de parler de tous les corps que les médecins ont regardés à tort comme des vers intestinaux, je me bornerai à donner l'histoire du prétendu ver intestinal, dont M. de Jamarck a fait son genre sagittule. C'est un médecin italien, M. le docteur Bastiani, qui a induit en erreur le célèbre zoologiste français, dans un mémoire assez étendu, intitulé : Historia medica illustrata con reflessioni sopra un animale bipede evacuato per secesso in cardialgia verminosa, et inséré dans le tome VI, page 2/4 des Actes de l'Académie de Sienne, avec figures, pl. xiı, fig. 3-4. Le malade était un ecclésiastique de cinquante ans, qui était réellement tourmenté par des ascarides lombricoïdes, et qui rejeta, avec quelques-uns de ces animaux et les matières stercorales, un corps que tous les médecins et naturalistes de l'Académie, après un examer attentif extérieur et intérieur, regardèrent à l'unanimité comme un nouveau ver intestinal. Cependant il est aisé de s'assurer, par la description comme par la figure, que ce n'était que l'appareil hyolaryngien tronqué de quelque oiseau. La trompe n'est sans doute qu'un reste de la langue; la bouche, l'ouverture de la trachée, dont le docteur Bastiani décrit même les cartilages arythénoïles, les ailes ou nageoiras cartilagincuses, les dentelures de la base de la langue, le fémur, le genou, le tibia, les cornes de 
l'hyoïde; et enfin la queue, probablement un reste de la trachéeartère. Ce prétendu animal était percé d'outre en outre par un canal qui ne contenait ancun viscère, et en effet c'était la trachéeartère. Voy. sa figure, pl. I de l'Appendice, fig. I 4 .

\section{Sur les pseudo-helminthes.}

Dans cette section de son ouvrage M. Bremser s'est borné, comme il a soin d'en prévenir ses lecteurs, à parler des principaux faits apocryphes rapportés par les auteurs, et qui deviennent de moins en moins nombreux à mesure que l'helminthologie fait plus de progrès; mais il n'a pu entrer dans son plan d'analyser et de critiquer tout ce qu'on trouve à ce sujet dans les recueils d'observations de médecine. ILes exemples qu'il a choisis suffiront sans doute pour faire que les médecins se tiennent dorénavant sur leurs gardes. On y voit que le plus souvent ce sont des larves d'hexapodes, comme pour les prétendus ascarides stephanostome, conosome, cercosome, ou des animaux que l'on peut tout au plus rapprocher des entozoaires, comme le ditrachycéros de Sulzer que M. Bremser parât regarder comme une graine, mais réellement sans en donncr de preuves, comme me paraît justement le lui reprocher le critique italien, l'lexathyridium des veines qui pourrait bien n'être qu'une espèce de planaire ou de sangsue, ou enfin quelques parties de plantes, comme le diacanthos polycephalus de Stiebel, qu'on est étonné de trouver dans le savant recneil de plysiologie de M. Meckel, ainsi que dans le Journal complémentaire des sciences médicales, $t$. I, cah. 2, I 81 8 , et qui n'était qu'une rafle de raisin, comme l'a moutré M. Rudolphi, et les prétendus vers des dents dont M. Bremser donne une étiologie hors de doute, en faisant voir que ce ne sont que des germes de graines d'alkekengi ou de jusquiame.

M. Bremser joint à ce chapitre comme appendice quelques observations sur les corps que peuvent rejeter les individus de l'espèce lumaine, et sur la grande attention que l'on doit apporter à leur cxamen, avant de décider que ce soit une nouvelle espèce de ver intestinal. Nous allons nous-mêmes y joindre quelquıes considérations propres à rendre cet examen plus facile. 
Il faut d'abord fairc la plus grande attention à la source ou à l'endroit d'où est sorti le corps, regardé à tort ou à raison comme un entozoaire, savoir s'il n'y a pas quelque plaie simple ou fistuleuse; ainsi lc critique allemand de l'ouvrage de M. Bremser rapporte le cas d'un paysan qui a rendu pendant plusicurs années consécutives et de temps à autres des larves d'insectes avec beaucoup de difficulté, par le canal de l'urètre, et l'on trouve dans les recueils médicaux une foule d'exemples semblables.

On devra ensuite, avant de soumettre le corps rendù à un examen attentif, le suspendre dans une assez grande quantité d'eau, pour le laver et le débarrasser des matières qui pourraient l'envelopper et pour permettre son extension complète : alors ou pourra avoir égard aux observalions suivantes.

Les corps rendus par l'espèce humaine avec les évacuations nasales, salivaires, pulmonaires, stomacales, dnodénales, alvines ou urétrales, peuvent être de deux natures très-différentes, végétale ou animale.

Dans le premier cas, la structure seule du corps rejeté doit suffire pour reconnaître sa nature, surtout si l'on joint à cela la considération de la forme extérieure, qui ne peut être régulière ou symétrique si ce n'est pour les fleurs, tandis que dans un entozoaire comme dans tout autre animal, elle l'est constamment.

Dans le second cas, ce sont des produits animaux, des parties d'animaux, ou enfin des animaux tout différens des entnzoaires.

Quand ce sont des produits animaux, comme ce qu'on nońme des concrétions lymphatiques, la structure non celluleuse et seulement gélatineuse, ainsi que le défaut de symétric dans la forne, ne penvent laisser long-temps dans le doute un observateur de bonne foi; mais, pour en faire l'examen, il faut mettre le corps dans l'eau.

Des parties d'animaux sont encore peut-être plus aisées à reconnaitre, pour peu qu'on ait quelques connaissances grossières d'anatomie, et l'on est réellement étonné de roir que le docteur Annibal Bastiani et le corps tout entier des médecins et des naturalistes de l'académie de Sienne, devant lequel en fut fait, en 777 , l'examen extérieur et intérieur, n'ait pas reconnu que son prétendu animal bipède n'était autre chose que l'appareil hyola- 
ryngien d'un oiseau, quoique le secrétaire de cette académie, dans une note ajoutée au mémoire de Bastiani, ait dit: $E$ provato abbattanza essere il verme vero, verissimo animale vivente.

Si ce sont des animaux plus ou moins tronqués, un peu de sagacité et quelques bonnes figures d'animaux devront suffire pour reconnaître aisément l'erreur.

Enfin, quand ce seront des animaux complets, quelques connaissances de zoologie, et surtout pour les médecins qui en manquent, ce qui est malheureusement trop commun, de bonnes figures d'animaux faciliteront la reconnaissance.

Ils auront aussi bientôt reconnu si c'est un animal vertébré, et par conséquent une supercherie ; car, quoi qu'on en ait dit, il n'est guère admissible que même un reptile, lézard ou couleuvre, ou un amphibie, grenouille, crapaud, ou salamandre ent pu s'introduire darss le canal intestinal d'un homme, par accident, et sans que celui-ci s'en fìt aperçu.

Si c'est un animal articulé extérieurement, ce qu'il est fort aisé de voir, on pourra se guider d'après la distinction du nombre des articulations, el l'existence ou nón des appendices sur leurs côtés.

Tous les entozoaires connus jusqu'ici ont les articulations de leur corps peu ou point marquées, mais toujours fort nombreuses et sans traces d'appendices locomoteurs; par conséquent on ne peut les confondre ni arec les myriapodes, ni avec les clıétopodes ou néréides qui, ayant des articulations très-nombreuses, les ont très-distinctes, et pourvues d'appendices complets ou incomplets. Par la même raison ils ne peurent l'être avec les larves ou vers d'hexapodes qui, si quclquefois leurs articulations sont peu marquées et sans pattes, n'ont jamais des premières au-dessus de quatorze, ni des secondes au-dessus des trois paires.

On ne peut cependant nier qu'il soit à peu près impossible de ne pas confondre plusieurs entozoaires avec plusieurs animaux extérieurs, par exemple les filaires arec quelques gordius, certaines fascioles avec des planaires, et peut-êtrc même quelques siponcles avec plusieurs ascarides. Il n'y a peut-être alors que la coulcur presque constamment blanche, du moins à l'extérieur, qui puisse servir à assurer qu'uu de ces animaux similaires présenté à l'examen d'un médecin soit un yer intestinal ou non. 
La question des réritables hydatides n'est peut-être pas moins difficile à résoudre, à moins que d'admettre la définition tranchée de M. Bremser, ce que je suis assez porté à faire. Ainsi, toutes les fois que, dans une partie quelconque de l'homme, on trouvera une ou plusienrs vessies pleines d'eau, de forme ou de grosseur variable, libres et flottantes dans une sorte de vessie adhérente au reste de la partie, et en contiunité de substance avec elle, on pourra les regarder comme des hydatides simples ou des acẻplialocystes; mais, s'il y avait une adhérence quelconque vasculaire on celluleuse entre la vessie et le kyste, ce ne serait plus une véritable hydatide animée. On conçoit cependant que, par suite de la mort d'une lydatide simple et de sa désorganisation, l'adhérence puisse avoir lieu. C'est peut-être ainsi qu'il faut considérer les hydropisies enkystées de l'ovaire, à moins que de croire que les espèces de kystes qu'on remarque souvent dans ses parties, et surtout dans les vieilles filles, ne soient dues au développement contre nature des calices des germes qui n'auraient pas pu suivre leur accroissement normal par défaut d'action du fluide spermatique.

Quant aux hydatides en grappe de l'utérus, s'il n'y avait point de continuité de substance avec les parois de cet organe, et s'il y avait seulement adhérence, on pourrait croire que ce serait le germe ou le foetus lui-même, ou son placenta, qui se serait pour ainsi dire développé en hydatides simples.

Au sujet de cette singulière espèce d'hydatides qui se forment souvent dans la matrice, tantôt isolées, tantôt avec adhérence avec le foetus ou avec le placenta, et que M. Weismansel a nommé hydrometra hydatica, il est très à regretter, dit le critique allemand, que M. Bremser n'ait pas connu l'excellent mémoire de Gregorini : De hydrope uteri et de hydatidibus in utero viris aut ab eo exclusis, Halo, r 794 ; car il en aurait au moins fait mention dans sa bibliographie. La figure qu'il cite de Bidloo n'est pas à comparer avec celle de Gregorini, qui représente une très-grande môle vésiculaire dans laquelle est contenu un fotus, et qui existe dans la collection de M. Meckel. Quoique Gregorini ait pu recueillir toutes les observations qui ont trait à son sujet, il en a omis luimême un très-grand nombre, comme M. le professeur Sprengel 
l'a fait voir dans un mémoire ex professo sur cette matière. Le dernier cas de ce genre se trouve consigné dans un artiele de M. Lemon, dans le vol. xI, p. 96-10o de l'Edimburgh medical and surgical Journal, et qui a pour titre: Case in wich a mass ressembling a placenta without a foetus, was discharged from the womb.

M. I. Cloquet a aussi rapporté dans sa Faune des médecins, p. I33, un cas analogue; il y figure, pl. Iv, la masse d'lydatides sous la dénomination d'acéphalocyste en grappes, acephalocystis racemosa. Dans ce même ouvrage M. Cloquet a recueilli avec soin toutes les observations qui ont un rapport plus ou moins inmédiat avec son artiele un des acéphalocystes, qu'il envisage d'une manière assez complète.

Des causes de la formation des vers intestinaux.

Dans ce chapitre, qui commence la seconde partie de son onvrage, M. Bremser ne parle que des vers qui séjournent dans le canal intestinal; les causes de la formation, de nềme que le diagnostic et le traitement de ceux qui vivent ailleurs paraissant jusqu'iei beaucoup trop obscurs.

Sans admettre la théorie de notre auteur, qui du reste n'cst que le développement de la manière dont il conçoit la génération des entozoaires en général, on ne peut nier qu'elle ne se trouve assez bien concorder avee les faits et les lier d'une manière rationnelle et fort satisfaisante; par conséquent elle mérite d'être prise en considération. Quel ques personnes ont cependant regardé comme peu vraisemblable que des vers puissent se former des humeurs versées à la surface du canal intestinal; et l'au tenr de la Clironique autrichienne, qui admet cependant bien complétement la génération spontanée des éntozoaires, pense que ce sont les parties même de l'intestin, comme son tissu cellulaire, ses villosités qui s'allongent, et qui jouissent peu à peu d'une vie indépendante; cu sorte qu'il voit dans cette transformation une ramifieation de l'intestin, comme chez les polypes. Les liydatides ne sont írès-probablement, suivant lui, que du tissu cellulaire pourvu de suçoirs.

On a pu voir, p. 349 , que M. Dremser paraît assez portó à croire que l'usage du lait peut contribuer à la formation des vers 
intestinaux. Le critique allemand de la gazette de Salabourg, trouvant que cetie assertion n'est pas dépourvue de vraisemblance, citeà l'appui l'observation, que la plupart des enfans des paysans de la contréc qu'il habite sont très-tourmentés par les vers, et qu'ils se nourrissent principalement de pain de seigle, de pommes de terre, et surtout de lait, dont ils boivent régulièrement trois fois par jour.

Une autre opinion de MI. Bremser, que la nourriture maigre, comıosée de substances peu nutritives, n'est pas propre à favoriser la formation des vers intestinaux proprement dits, et que leur plus grand eunemi est la faim de l'animal dans lequel ils séjournent, s'accordé parfaitement avec l'expérience du même critique, qui pense même que l'on peut en tirer l'explication, pourquoi surtout les ascarides sortent par les deux orifices du canal intestinal de la personne malade, qui n'avait encore pris aucun médicament. Il se pourrait aussi que certaiues maladies déterminassent la sortie naturelle de certains vers; par exemple, il paraît que le tæenia ne peut rester dans le canal intestinal des personnes affectées de fièvre intermittente, et que les ascarides lombricoïdes sortent de celui des enfans atteints de fièvre intermittente ou plutôt encore du typhus, ce qui a porté quelquefois à penser que c'étaient ces vers qui avaient occasioné ces maladies.

\section{Du diagnostic de la présence des vers intestinaux.}

Quoique l'auteur de l'analyse italienne citée plus haut ait fortement critiqué M. Bremser au sujet de cette partie de son ouvrage, qu'il regarde comme fort incomplète, en la comparant avec ce qu'a dit M. Brera sur le même sujet, il est évident qu'elle est bien suffisante, et qu'aucun des symptômes importans n'a été oublié.

Après avoir donné tous les caractères qui indiquent la diathèse vermineuse en gẻnéral, il rapporte successivement ceux qui peuvent faire présumer la présence de telle ou telle espèce de ver intestinal. Nous n'avons douc que très-peu de chose à ajouter à ce que dit M. Bremser, surtout parce que M. le docteur Grundler l'a fait au fur et à mesure que s'en est présentée l'occasion.

Nous allons uous borner à rapporter quelques faits qui sem- 
blent prouver que les ascarides lombricoides peuvent percer le canal intestinal, et pénétrer dans la cavité péritonéale.

A ce sujet M. Bremser, adoptant la manière de voir de M. Rudolphi, pense que c'est certainement à tort que l'on admet que les ascarides lombricoïdes perforent les intestins, et par là causent la mort (p. 385). La preuve tirée de ce que ces animaux n'ont pas d'organes propres à effectuer cette perforation n'est pas tout-àfait irrécusable; car, sans admettre avec Jacopi, comme le fait le critique italien, que la bouche de ces animaux est armée de trois pointes cornées, formant par leur réunion un instrument trèsaigu, il est cependant rrai que les espèces de valvules qui l'entourent sont assez dures et même un peu tranchantes, du moins à̀ leur bord. D'ailleurs les vers de terre qui ont leur bouche encore moins armée que les ascarides, ne percent-ils pas la terre, il est vrai humide, par la forme pointue et l'espèce d'érection dont l'extrémité antérieure de leur corps est susceptible; les lombricoïdes ne pourraient-ils pas en faire autant, surtout si les parois du canal intestinal étaient préalablement ramollies par la suite de quelque inflammation, ou mieux peut-être si le ver a introduit l'extrémité de son corps dans quelque orifice des glandes de Peyer, qui sont le plus souvent les parties qui s'enflamment les premières dans les affections du tube intestinal, et lorsque la maladie dont le sujet est atteint force ces animaus de désirer un séjour qui leur convienne davantage?

Le critique italien cite, à l'appui de son opinion contraire à celle de MIM. Rudolphi et Bremser, des observations de MI. Gautier de Claubry, insérées dans le Noureau Journal de médecine de Paris, juillet 18 I 8 , où celui-ci, en rapportant plusieurs exemples de perforation de l'estomac occasionée pàr des ascarides, conclut que chaque rer effectue une ourerture particulière, et que jamais deux individus ne passent par la même; que les bords de ces orifices tombent en suppuration et en gangrène, et que par là ils arrivent dans la cavité abdominale, où l'on en rencontre d'une grosseur extraordinaire, puisqu'il en a vu qui avaient de six à onze lignes de circonférence.

Il aurait pu citer également le fait observé par M. Fischer ễ inséré dans sa Dissertatiou sur l'hydatide du plexus clıoroïle, d'unc 
vicille femme de soixante ans, qui s'était laissée mourir de faim, et sur le cadavre de laquelle il trouva le cœcum percé de deux trous ronds assez grands, dans l'un desquels un ascaride avait son corps à moitié engagé, tandis qu'un autre individu, qui sans doute avait passé par l'autre trou, était déjà dans la cavité abdominale.

Il aurait troavé contraire à cette manière de voir l'observation faite sur l'évacuation des lombricoïdes à travers les tégumens de l'abdomen par Mécanly, et qui a été publièe daus le tom. II, p. $49 \mathbf{I}-49^{8}$ de l'American medical recorder.

Nathaniel Ramsey cite plusieurs cas qui prouvent que des vers et des hématodes ou pierres de sang (concrétions sanguines endurcies sans doute) peuvent se trouver en même temps dans les intestins, et il ajoute qu'il est probable que ces deux affections sont en rapport entre elles. Voy. Medico-chirurgical transactions de Londres, vol. IX, part. $\mathbf{I}, \mathbf{1 8} 8$. )

On trouvera une dissertation particulière sur les vers de l'estomac par le docteur Jos. Klapp, dans le journal de Philadelphie intitulé The American medical recorder, vol. III, $\mathrm{n}^{n}$. II, 1820 , où Ie diagnostic de cette maladie est analysé.

Quant aux vers trouvés dans l'estomac d'une maniaque dont parle Hagner, dans le journal intitulé Zeistchrift fiir psychischc aerzte von Nasse. $4^{\mathrm{c}}$ heft, 1818 , Leipzio, p. 514 , il faut remarquer qu'il y avait aussi des hydatides dans le plexus chorö̈de et et d'autres anomalies dans le cerveau.

Quoique M. Bremser, dans ce chapitre, n'ait parlé que des vers qui séjournent dans le canal intestinal, nous allons donner l'hisloire des phénomènes qu'a présentés la malade sur laquelle M. Rentdorff a observé les échinoccoques, et dont nous avons parlé plus haut, parce qu'elle nous a paru aussi complète que curieuse.

\section{Historil morbi.}

Guilelma Ohle, Berolinensis, anno x81 г, parentibus, bona valetudine gaudentibus, nata est. Partum difficilem, et forcipc absolutum esse commemorare non alienum existimavi. Puella, matre affirmante, tenerrima atate bona, si nounullas levioris mo- 
menti ægritudines excipias, valetudine gravisa est. Quinto et sexto xetatis anno bis terve laborabat capitis impetigine, qux, nullo curante medico, paulo post sponte evanuit. Mensc julio anni proximi ægrota, septem annos nata, febre corripiebatur variolosa, quæ cum graviora non præe se ferret signa; matri adeo videbatur benigna, ut medico opus 'non esse existimaret. Decimo quarto morbi die puella convalescens vehementioribus ita vexabatur subito convulsionibus, ut mentis impos fieret. Neque de hoc morbo mater consuluit medicum, quia convulsiones, quxe primo quidem die, quo videbantur, ter, altero totidem redierant, proximis omnino remiserant. Eodem anno ægrota, e mensa precipitata, tam vehementer in occipite offendebatur, ut totum caput doleret; qui casus, doloribus postero die cessantibus, symptomata nulla reliquit. Quod ad animi puellse indolem pertinet, usque ad annum retatis octavum satis exculta videbatur. Ab ${ }^{\circ}$ hoc autem tempore filiam initio minores, postea nullos in iis, quibus puellæ institui solent, progressus fecisse mater animadrertebat Puella usque ad octavum annum ita robusta et sana erat, ut optima eam gaudere valetudine dixeris; post sæpe laborabat levioris momenti ægritudinibus; alvus ei plerumque vel oppressa, vel, si ducebatur, pituitosa erat, ejusque ventriculus interdum adeo expletus sordibus, ut hæ vomitu sponte redderentur. Mense aprili anni abhinc proximi puella, octo annos nata, valde refrigerata, hora præterita tam vehementes pede sinistro sentiebat dolores, ut claudicare cogeretur. Qui, cum per quatuor hebdomades durassent, etiam brachium sinistrum capiebant, ut ad nullum officium perficiendum idoneum remansisset.

Quæ cum esset morbi conditio, ægrota die VII mensis julii anni preteriti medici auxilium in instituto polyclinico, quod viro perill. C. W. Hufeland rectore hac urbe floret, quæsivit; et contigit, ut curatio morbi ejus mihi committeretur.

Quamquam morbi conditio, qualis sit, jam ex relatis cognosci potest, oportet tamen, ut etiam horum faciam mentionem; pulsus paululum frequentior, in utraque arteria radiali idem sentiebatur, tussis nulla, respiratio bona, caput non affectum, pro totius quidem corporis ratione paululum justo majus, oculorum pupillic naturali conditioni convenientes, visus et auditus sani, functiones 
intestinorum non læsæ, dolores abdominis nulli, vermes denique nunquam erant excreti. Itaque morbus hemiplegia rheumatica nominandus, et usum eorum postulare videbatur remediorum, quxe oppressam culis functionem restituerent, nervosque incitarent. Quæ ut amoverem indicia, ægrotæ rationem vivendi morbo aptam commendavi, præter hoc eam infuso flor. arnic., rad. valeriau. min. cum spirit. sulph. æeth. et syrup. simp. uti jussi ; simul pulveres, ex calomel., sulph. antim. aur. et extr. aconit. paratos, sumendos ei dedi, neque alienum existimavi partes doloribus affectas unguent. nerv., cui tinct. canth. admixta esset, bis quotidie perfricare, et emplastrum vesicatorium in scapula sinistra ponere.

Tribus præteritis diebus, medicamentis bene adhibitis, morbus in melius mutatus videbatur, dolores remiserant, corporis partes afflictæ facilius poterant moveri; neque erat, quod remedia, qux adhibenda præscripseram, cum aliis mutarentur.

AF grotam die XII mensis julii aggressus, eam pedem quidem facilius movere, brachium autem minime posse observavi. Alvus ei erat oppressa, quamobrem, ut duceretur, enema injici jussi.

Sequentibus quatuor diebus ægrota bene, si hemiplegiam excipias, se habebat; quinto autem die cibum assumptum unacum magna pituitæ copia bis sponte vomens excernebat; frequenter laborabat ructibus, cupiditate edendi carebat, linguam ostendebat pituita obtectam, ejusque pulsus frequens et durior febriculam adesse indicabat; de capitis autem doloribus filia non querebatur. Hæc cum animadvertissem symptomata, ægrotæ vomitorium, quo ventriculus sordibus liberaretur, sumendum præbui, quod sumptum quater excitabat romitus, quibus nagna pituitæe copia ejiciebatur.

Postero die ægrota melius se hahebat; pulsum cjus conditioni normali convenientem, linguam minus, quam pridic, sordidam observavi, et alvum satis frequenter ductam esse mili denuntiabatur; ab omnibus igitur eam remediis internis abstinere, externis autem continuo uti jussi.

Die XIX mensis julii ægrota, lectulo affixa, quinque convulsionibus vehementer vexabatur, qux maxime brachium et pedem sinistrum corripiebant, et quarum quisque impetus per horæ quadrantem durabat; pollices in volam flexi, animi defectus, mañ 11 . 
denique corporis lassitudo et sopor, quemque impetum secuti, epilepticas eas fuisse perspicue probabant. Peilsus inæqualis erat et parvus, urina tenuis et alba. Vespere, convulsionibus remittentibus, puella ter sponte vomebat, dolorem in regione cardiaca sentiebat, linguam habebat flavescentem, pituita obtectam. Vonitorium igitur prescribendum videbatur.

Postero die ægrota, quæ vomitorio sumpto ter vomuerat, denuo convulsionum impetu concutiebatur, qui binis interpositis loris, quater redibat. Pulsus vespere erat frequentior, inæequalis, debilis. Paralysis pedis sinistri, cum valde remisisset, puella ambulare, simul brachium sinistrum melius movere potuit. Edendi cupiditas erat depravata, lingua albescens, urina ex albo flavescens, alvus adstricta, quare solutionem ex elect. lenit., tartaro tartaris. cum vino stibiat. paratam sumendam dedi; præter loc partes resolutas ung. nervin. cum tinct. canth. perfricari, et ulceri arte effecto ung. irrit. quotidie imponi jussi.

Die XXII mensis julii puella, quæ nocte præterita bene dormierat, et mane alvum bis duxerat, melius se habere et hilaris videbatur; pomeridiano tempore denuo corripiebatur courulsionibus, quæ per decem circiter sexagesimas horæ durantes, minus antecedentibus erant vehementes. Vespere doloribus partium resolutarum auctis, ægrota, neque manum sinistrum ad caput movcre, neque meare potuit; quamobrem cruri etiam emplast. vesic. imponebatur. Coeterum morbi conditione non mutata, non erat quod remedia prescripta mutarentur. Cum convulsionum causa nondum remota videretur, intestinorum functio læsa, et oculorum pupillæ dilatatæe essent, eam vermibus, qui intestina occuparent, tribuendam suspicans, puellæe, pulvere ex rad. jalapp. et hydrarg. muriat. mit. composito, purgate, decoctum, ex semin. santon. cum tart. tartaris. paratum, sumendum prebui.

Die XXIII mensis julii ægrota, convulsionibus non vexata, meliori gaudebat valetudine, caput ei non dolebat, et cibum assumere cupiebat; lingua quidem pituita erat obtecta, et alvus, bis quotidie ducta, nullos excreverat vermes.

Tribus diebus sequentibus morbus melior videbatur; partes hemiplegia affectie facilius poterant moveri, stercoris parun, idque mucosum, vermes nulli dejiciebantur. 
Itaque die XXVII mensis julii, ne convulsiones redirent, solutionem e sale mirab. Glaub. et elect. lenit. paratam sumi, simul partes resolutas bis quotidie ungi, et ung. irrit. ad ulceris suppurationem sustentandam adhiberi jussi.

Proximis ad secundum usque mens. august. diebus, valetudo puella, si hemiplegiam excipias, in melius convertebatur; caput non dolebat, et cerebri functiones, lingua color, appetitus edendi, pulsus, alvus denique naturali conditioni convenientes videbantur.

Die XVI mensis augusti puella magnam pituitæ copiam ter sponte vomebat, linguam ostendebat muco obtectam, et ructibus frequenter vexabatur; quamobrem emeticum aptum existimavi, quo sumpto, melior valetudinis conditio efficiebatur. Morbus sequentibus diebus non mutatus, nullam mutandorum medicaminum causam afferebat.

Die XXV mensis augusti paralysis partium correptarum tanta erat, ut ægrota neque brachio, neque pede sinistro uti posset, simul de visu hebetiori querebatur, et pupillæ oculorum luce allata non diminute erant. Per quinque igitur hebdomades remediis accurate adhibitis, morbus tamen gravior, quam ineunte curatione exardebat. Quare de alia, quam initio, causa, quæ morbum sustineret, suspicatus, et alrum frequenter adstrictam, capitis magnitudinem pro corporis ratione paululum majorem respiciens; in opinionem discessi, ab hydrope cerebri interno morbi symptomata esse deducenda. Iterum, iterumque, quibus causa morbi detegi possit, ex rgrota quasivi, nihil autem investigare potui. Morbum, quo se conversurus esset nullis remediis sumptis, per nonnullos dies observarem, necesse existimans, agrotam onnibus antea adhibitis abstinere jussi; ut autcm amblyopiæ, quæ frequenti asu florum arnic. fortasse affecta esset, mederer, camphor. cum gumm. mim. ct acet. vin. illi prebui.

Primo dic mensis septembri puella, cum nounullis diebus ante refrigerata esset, et cibo se obruisset, febrc corripicbatur gastrica, qux ex nausea, lingua flavescente, pulsu frequenti et plenn, urina crocea cognosci poterat. Itaque vomitorium praescribebatur, ct postero dic, cxcretis sordibus gastricis, febris symptomala mitigata observabantur. Tunc, ut alrus duceretur, solutionem, 
ex elect. lenit. cum tart. tartaris. paratam prabui ægrotæ, quæ proximo die, febre omnino liberata, melius se habebat.

Cum remedia bene quidem, sed frustra essent adhibita, et ambyopia aucta, morbi symptomata ab hydrope cerebri interno esse deducenda certior eram factus; aliam igitur curandi methodum, qua serum, præter naturam in cerebro accumulatum resorberetur, inire mihi proposui; et agrotam pulveres ex hydrarg. muriat. mit., herb. digit. purp. flor. arnic. et sacch. alb. compositos sumi jussi, simul in scapula sinistra et in crure sinistro emplastrum vesicat. perpetuum ponere, et resolutas partes ung. nervino cum tinct. canth. quotidie perfricare non alienum putavi.

Quar quam ægrota die III mensis septembri, quod ad morbi naturam, bene se habuerat, postero tamen vehementer quater corripiebatur couvulsionibus epilepticis, et, cum remisissent, sopore tenebatur, vehementer stertebat, pulsus ei debilis erat, inæequalis et parvus. Proeterea urina pallida erat, et alvus per biduum oppressa, quare clyster injiciebatur, et inf. fol. sennæe cum sal. mir. Glaub. et vino stibiat. sumebatur. Postero die, purgataægrota denuo ter vesabatur convulsionibus, quibus cessantibus, morbus multo gravior factus videbatur; paralysis enim gravior et amblyopia utriusque oculi aucta erat, urina insciente ægrota excernebatur, et tanta ipsi erat virium debilitas, ne lectulo se surgere posset.

Die IV mensis septembri convulsiones plures faciebant impetus, singulum quemque priori minus vehementem. Paralysis partium aucta videbatur, urina ægrota inscia reddebatur, lingua erat sordida, alvus oppressa ; quamobrem medicamentum purgans adhibendum erat.

Septimo mensis septemliri et sequentibus diebus ægrota indies paululum melius se habebat, incontinentia urinæ omnino remiserat, alvus quotidie ducebatur, et partes resoluta facilins moveri poterant; visus autem non melins videbatur. Usa tunc erat pulveribus ex hydrarg. muriat. mit., floribus arnic. et herb. digital. purp. compositis; simul partes resolutie unguent. nervin. cum tiuct. canth. ungebantur, et ulcera continuo suppurabant.

Die IV et sequentibus mens. octob. diebus ægrota, quamquam meliuscule se habebat, nullam tamen convalescendi spen prebuit. 
Utebatur tunc infus. flor. arnic. cum spirit. sulph. æth. et syrup. simpl., simul pulveribus ex lydrarg. muriat. mit., herb. digit. purp. camph. et sacch. alb. paratis.

Die XV mens. octob. æogrota epilepticis subito concutiebatur convulsionibus, quibus cum hora praterita ter sponte vomuisset, liberabatur ; alvum, per duos dies oppressam, ut duceret, infus. fol. sennæ cum tartar. tartaris. sumebat. Postero die alvus bis ducebatur, neque convulsiones redibant. - Qıæ cum ita essent, omnibus, excepto unguenti, quo coitus materiæ ex ulceribus fieret, usu, medicamentis puella per nounullos dies abstinebat.

Die XVIII mens. octob. ei proximis morbus, quaraquam convulsiones nou redierant, multo gravior videbatur; pulveres igitur supra laudati denuo, et majori quidem portione sumebantur, et alvus clysteribus adhibitis sæe erat ducenda.

Die XXVI mens. octob. die ægrota adeo erat debilis, ut per se neque stare, neque in lectulo recta sedere posset, caput enim dextrorsum in pulvinar recidebat. Urina plerumque, excrementa alvi interdum inscia puella reddebantur; amblyopia adeo creverat, ut amaurosin eam dixeris, cum clarior etiam lux percipi vix posset; caput frequenter scabebat æegrota; sopor gravior et animi lentitudo aucta videbantur. Ne quid negligeretur, occiput tonsum unguento, e tartar. stibiat. drachmis duabus et axungiæ porcin. uncia dimidia parato, quotidie perungebatur. Quo remedio per quinque dies adhibito, pustulo, pure repletæe, neque dolentes, nascebantur; morbus tamen nullo modo levior, neque amaurosis sublata videbantur.

Die XXXI mens. octob. die preter morbi signa, quæ antea fucrant, oscitationes frequentes, respiratio inxqualis et stertens, pulsus intermittens et frequens observabantur. Oculi et os ægrotæ dormienti late patebant; lingua ei muco erat obtecta, appetitum ciborum ipsa non habebat, et ructibus frequenter vexabatur. Vomitorio sumpto melius se habebat.

Primo die mens. novemb. nulla de morbo removendo spes aderat; excrementa enim dejiciebantur insciente agrota, oculorum bulbi erant collapsi, cutis coloris pallidi, et rerum memoria traditarum postera dic puella erat immemor. 
Die XIV mensis septembri amblyopia valde erat progressa, pupillæ non nisi clariori luce incitante, paululum tantun, contrahebantur; oculi speciem propriam, quam amauroticorum solent, præ se ferebant; paralysis lateris sinistri eadem remanserat, memoria ita imbecillis videbatur, ut puellarum sodalium æegrota vix recordari posset. Symptomata morbi respiciens, magnan fluidi alicujus copiam in cerebro esse accumulatam et pessimum illius exitum exspectandum existimabam. Ut vitam, etsi bona non crat recuperanda valetudo, quam diu fieri posset, sustentarem, ad methodum roborantem et incitautem adhibendam confugi. liaque puellam alimenta facilia concoctu et nutrientia sumere, preterea decoctum rad. arnic. cum rad. valerian. minor. et extr. aloes aquos. haurire, porro pulveres ex hydrarg. muriat. mit., herb. digit. purp., camph. et sacch. albo paratos adhibere, denique in cervicibus emplast. vesicat. ponere, et cæeteris remediis iternis continuo uti jussi.

Die XVI mens. septemb. et sequentibus diebus conditio morbi, remediis bene adlibitis, indieis pejor fiebat; æegrota res minuas oculis oblatas non cognoscebat, lecto semper affixa tenelatur, caput dextru?n sæpe scabebat, de doloribus ejus autem non querebatur, solito magis dormiebat, olfactus et auditus hebetiores erant, matris amorem et curam leute accipiebat, et de appropinquante morte haud eritanda sæpius loquebatur. Pulsus plerumque erat, satis frequens, debilis, maxime in membris sinistris, vicesinus quisque intermiltebat, partes resolutie panlisper erant sanis frigidiores, alvus, quotidie ducta, stercora magna muci copia obtecla excernebat. Cum in curandi methodo nihil mutandum videreiur, vivendi ratio et remedia, qư præscripseram, continuo adhibebantur.

Die III mens. octob. gravior, quam præterito, videlatur morbus, cujus rei causa detegi non poterat. Lethargus et animi lentitudo valde erant aucta, pulsus frequens, parvus et intermittens, cutis arida, lingua ex albo flavescens, urina pallida, ejusque copia satis magna, alvus denique per biduum oppressa. Quare injiciebatur clyster, et cum remediorun adhibitorum optima viderentur vesicantia, ulcera, ut suppurarent, unguent. cantharid. irritabantur. 
Die III mens. noyemb. xgrota, sponte vomens, jus sumptum reddidit.

Die VI mens. novemb. convulsionum impetu corripiebatur, et per horam dimidiam mentis non erat compos. Facultas loquendi sublata videbatur, interrogata enim non nisi signis datis respondere studebat; adeo difficile audiebat, ut clariorem tantum vocem excipere posset; me jubente linguam, non nisi maxima opera, porrigebat. Amaurosis ad summum usque erat aucla, membrana conjunctiva bulbi inflammata. Partes sinistræ corporis non nisi vehementiori digitorum vellatione afficiebantur dolore, et mox calidæe erant, mox frigidæ, plerumque tamen cæeteris frigidiores; multum sudorem tenacem et odoris acidi emitiebat, scabebat sapius caput dextrum, pulsus ei debilis erat, inæqualis et frequeus, duodevicesimus quisque internittebat, spiritum illa stertens et difficilius reddebat; excrementa insciente ipsa excernebantur. Qux cun esset morbi conditio pulveres, ex calomel., florib. arnic., herb., digital. purp., camph. et sacch. alb. parati, sumebantur ; simul ulcera in occipite, cervicibus, scapula et crure arte effecia irritabantur.

Die VII mens. novemb, agrota mane per horam dimidian convulsionum impetu concutiebatur, quem gravis sopor sequebatnr; vespere et postero die convulsiones multo leviores antecedentibus redibant. De vehemente siti ducta, multa bibebat, brevi post autem evomebat; interrogata non voce, sed signis respondebat. Alvus per biduum oppressa clystere injecto ducebatur.

Die IX mens. novemb. ægrota mane vexabatur convulsionibus, qux postero die denuo faciebant impetum. Morbi conditio quam maxime pessima erat, pulsus parvus, inæqualis, intermittens et tardus; urinam et stercus inscia excernehat ægrota ; noribunda in inferiorem lectuli partem prolabebatur, dentibus sæepe frendebat, spiritum stertens trahebat, potum vix devorare poterat, omnia mortis instantis signa præseferebat. Vespere puella levioribus iterum corripiebatur convulsionibus, quibus remittentilus facultatem loquendi recuperabat; morbum sum brevi transactun fore æquo animo profitebatur, et matri interruptis verbis valedicebat. Hand multo post sopore tenebatur, et secunda hora post mediam noctem convulsionum impetu correpla, animum eddidit. 
Ve iis qua in funeris capite dissedo observata sunt.

Die altero post mortem cerebri conditionem, præsente viro $\mathrm{cl}$. Busse, medico aulico, et assistentibus viris doct. Otto, M. D R et Grape M. D D. ut morbis causa cognosceretur, dissecando investigavi. - Priusquam autem de iis agam, quæ in cerebro nobis apparuerint, de corporis symptomatibus nonnulla dicenda videntur. Membra totius corporis rigidissima, vix flecti poterant. Volæ manuum et ungues coloris erant cærulei. Digiti manuum pedumque, precipue pollices valde erant flexi. Integumentum abdominis maculis non erat obductum ; partes, quibus nisum jacebat corpus, imprimis eæ scapularum, coccygis et natum, ut solent, fuso sanguine observabantur repletæ. - De capitis magnitudine jam supra mentionem feci. Integumenta ossium cranii integra reperiehantur. Fonticuli omnino erant clausi ; suturæ calvariæ nilil præseferebant, quod naturali conditioni non convenisset. Cranium serra circumfissum adeo adhærebat duræ matri, ut ab hac vix sejungi posset, ipsum miræ erat tenuitatis et levitatis, crassioribus partibus lineæ fere et dimidiæ, tenuissimis vix dimidice. - Cerebrum paulisper erat sueto firmius, coloris flavescentis, pallidi, et vasis sanguiferis repletum. Hemisphærium cerebri dextrum propemodum teria parte sinistro majus animadvertebatur. Cum dura mater latere dextro incisa et remota esset, cerebri partem, quæ ventriculum lateralem tegebat, crassitudinis invenimus lineæ tantum dimidiæ ; hac parte remota, tanta hydatidum copia in conspectum nobis veniebat, ut ventriculi cavum mire esset dilatatum, neque cornu ejus anterius discerni posset. Omnis hydatidum copia tunica propria erat circumdata, quæ ob tenuitatem suam una cum hydatidibus e ventriculo tolli non poterat. Omnibus remotis hydatidilus, cerebri, pars, quæ ventriculum dextrum constitueret, propemodum omnino deesse videbatur, ipsa enim ad latus utrumque crassitudinis erat linearum trium usque ad quatuor, et in fundo membranam tendineam æquabat. - Uterque plexus choroideus coloris pallidi, et sanguine carens repericbatur; dexter autem multo erat sinistro major. Nuspiam vasa cerebri sanguine valde impleta, ne35. 
que sanguis ex vasibus progressus videbatur. - Ventriculus lateralis sinister aquæ continebat circiter unciam ; etiam ex meduliæe spinalis canali aqua profluebat. - In basi encephali nervi optici, acustici, trigemini, olfactorii a conditione naturali non cedebant. - Glandula pituitaria, quam in epilepticis mortuis majorem se reperisse contendit Wenzel, integra videbatur. - Glandula pincalis nullum continebat acervulum, qui in hominum setate provectiorum cadaveribus plerumque reperitur. - In cerebello niliil notatu dignum observalsatur. Neque thoracis, neque abdominis viscera dissecari permitiebat mater.

\section{T'raitement hygiénique et thérapeutique contre les vers infestinaux.}

I.es considérations de notre auteur sur les moyens hygiéniques et thérapeutiques propres à combattre la diathèse vermincuse ex qénéral, et les vers en particulier, sont en rapport avec l'étiologie de la maladie; M. Bremser insiste avec grande raison, ce nous semble, sur le principe qu'il est moins important de combattre les vers actuellement existans, que les causes qui peuvent en déterminer la formation ou la reproduction, et sur cette observation que, très-souvent, les remèdes inconsidérément administrés sont plus nuisibles au malheurenx malade que la présence des vers intestinaux regardés comme les plus dangereux. Dans un cours spécial sur les vers intestinaux, que nous fìmes en 1813 à la faculté des sciences, nous avions été assez heureux pour être conduits aux mêmes résultats, beaucoup plus, il est vrai, par la théorie que par la pratique. Au reste, on ne peut guère nier que M. Brera n'ait admis à peu près les mêmes conséquences, quoiqu'il n'ait pas suivi la même division des médicamens vermifuges.

Dans ce chapitre, M. Bremser parle successivement des différentes espèces dẹ remèdes qu'on a proposés successivement contre les vers, en les partageant suivant leur mode d'action; après quoi il rapporte avec toute la bonne foi convenable les systèmes de traitement que les médecins ont employés contre telle ou telle espèce de vers.

On peut sans doute trouver quelque chose à reprendre dans sa 
classification des remèdes vermifuges, parce que dans la nature il n'y a rien d'absoln; et il a pu en oublier quelques-uns, parce qu'un ouvrage humain ne peut être parfait.

Il est évident en effet, comme le fait justement remarquer le critique allemand, que M. Bremser range à tort les carottes, mangées crues ou râpées, comme un remède dont l'action est mécanique, puisque le suc de cette racine agit également comme vermifuge, et que d'ailleurs des pommes et autres substances mangées crues devraient agir d'une manière mécanique aussi bien que les carottes.

Il ne paraît pas probable, ajoute ce même observateur, que la propriété vermifuge de l'helminthọcorton, ou mousse de Corse, dépende du sel marin qui s'y trouve adhérent; car il y en a en si petite quantité, que c'est à peine si on le sent au goût.

Le camphre lui semble un excellent remède contre les ascarides, car on observe qu'ils sont évacués chez les enfans auxquels on administre cette substance.

Le même critique a employé souvent avec le plus grand succès l'huile de téréljenthine contre le tænia. Il est vrai cependant que cette espèce de vers reparaît souvent après l'emploi de ce remède; mais ne peut-on pas faire la même objection pour beaucoup d'autres vermifuges.

Il ne partage nullement l'opinion de M. Hiremser, suivant lequel le mercure doux ne serait qu'un simple purgatif privé de véritables propriétés vermifuges; car il a observé que de très-petites doses de cette substance qui n'avaient pas d'action purgative ont cependant produit l'évacuation d'ascarides. 11 cite, à l'appui de cette manière de voir, la grande confiance que les médecins anglais, et spécialement lc célèbre docteur Latham, ont dans le calomélas employé comme vermifuge, comme le prouve le mémoire de celui-ci intitule : Some observations respecting the medicines usually given in worm cases, with remarks upon the collateral advantages some times derivedfrom thern in cases of epilepsy (Médical. trans., Lond., $5^{\mathrm{e}} \mathrm{vol}$. )

Nous venons de dire que, sans aucun doute, parmi un si grand nombre de substances réputées vermifuges, M. Bremser a dû en oublier quelques-unes, comme le lui reproche amèrement le critique italien. En effet, on tronve qu'il a passé sous silence l'huile essentielle d'ail, les graines du papayer (carica papaya, L.), le 
deléa officinale, le lonicère, le tabac, la noix vomique, le phellandrium aquatique, le psoralier, le diagrède sulfuré, le muriate d'ammoniaque, l'antimoine, le sulfure d'étain d'après le procédé d'Alemanni, le zinc, le soufre, les eaux sulfureuses; substances, il est vrai, assez généralement peu employées, ou dont le mode d'action rentre dans celui de plusicurs des médicamens dont Nî. Bremser a parlé.

Quant à l'étain, que le critique italien dit aussi avoir été passé sous silence, il est certain que M. Bremser en a parlé à l'article de la méthode d'Alston, p. 455.

L'acide prussique a été également oublié. Voyez à ce sujet Brera, Nuovi commentari re medic. et chir., ann. 1818, sem. seg., p. $19^{3}$.

Il paraît qu'il n'a pas non plus rapporté absolument toutes les méthodes de traitement qui ont été proposées contre les vers. Du moins, le critique italien lui reproche d'avoir oublié celles de Rosenstein et Meier, dont Brera a parlé, qui consistent, l'une à faire avaler au malade une grande quantité d'ean froide après un purgatif (ne vaudrait-il pas mieux que ce fùt avant ?), et l'autre à donner toutes les heures une cuillerée à café de carbonate de magnésie, et anssitôt après une autre cuillerée de tartrite acidule de potasse. Quant à l'oubli de l'emploi de l'huile de ricin si vantée par Odier, M. Bremser en a réellement parlé à l'article de cette huile, en rapportant la méthode de ce médecin, p. 479 .

On pouvait sans doute aisément allonger beaucoup la liste des méthodes de traitement et des médicamens proposées contre les ver's en général, et contre chaque espèce de ver en particulier qu'a donnée MI. Bremser; mais, comme cela serait sans beaucoup d'avantages, nous nous bornerons à ajouter quelques-unes de celles qui nous sont presque tombées sous la main.

Colin Makensie, dans un ouvrage anglais intitulé Operative cyclopedia, qui contient cinq mille procédés ou recettes, annonce qu'un médecin, d'une grande antorité en Italie, a donné avec le plus grand succès l'essence de bergamotte à la dose d'un ou deux gros mêlée avec du miel; il ajoute que ce remède agit plus effcacement que l'essence de térébenthine et que le naphte dans le traitement contre le trenia ou telle autre espèce de ver que ce soit. 
On trouve rapporté dans le même ouvrage, que le doctetir Mongeny, médecin de l'hôpital de la colonie Ferdinéenne à l'île de Cuba, a administré avec un succès étonnant, contre le trenia, les feuilles d'une planle aromatique très-commune dans le pays et connue sous le nom de baume. On les réduit en pâte, fraîchement cueillies; l'on en prend à jeun trois onces, en y ajoutant une certaine quantité de micl : une heure après on avale deux onces de miel pur, puis d'heure en lieure une once de la même substance; au bout de six à sèpt heures le tænia est expulsé.

D'après ce que M. le comte de Champigny a dit à M. le docteur Grundler, il paraît que ce baume de Cuba n'est rien autre chose que la menthe poivrée (mentha piperata, L.) ; mais il est plus probable que c'est la balsamitc odorante, dont les feuilles et les graisres sont vermifuges; car la menthe poivrée est d'Eurnpe.

Bourdier, médecin de l'Hôtel-Dieu de Paris, faisait usage avec succès, comme nous en avons été plusieurs fois témoin, de l'éther sulfurique dans un verre de décoction de racine de fougère mâle à jeun; cinq minutes après il faisait prendre un lavement de. la nème décoction, contenant deux gros d'éther, et une heure après il administrait deux onces d'huile de ricin et une once de syrop de fleurs de pêche. Il continuait ce traitement pendant trois jours.

Enfin, pour ne rien oublicr, nous citerons encore le moyen anmoncé dans les journaux par M. de La Bernardière, naturalistevoyageur, et qui consiste à avaler dans l'espace d'une heure une livre et demie d'huile d'olives or: d'amandes douces.

\section{Traitement particulier de l'auteur.}

Nous n'avous pas grand'chose à dire sur la dernière section de l'ouvrage de $\mathbf{M}$. Bremser, dans laquelle l'auteur détaille la méthode dẹ traitement qui lui est particulière, d'autant plus que M. le docteur Grundler a parlé dans ses notes des précautions à prendre dans l'administration de l'huile empyreumatique de Chabert, dont la dose indiquée par M. Bremser serait trop forte chez nous, de la manière de la préparer, et d'en former un médicament moins repugnant aux malades, etc. Voyez en effet p. $4_{42} 6$ et 489 . Nous allons nous borner aे rapporter textuellement les observations 
faitcs par le critique allemand, parce que, comme toutes les autres, elles sont marquécs au coin du savoir et de la bonne foi.

M. Bremser u'avait hésité d'employer l'huile empyreumatique de Chabert en lavement, qu'à cause de l'buile de térébentline qu'elle contient; mais cette hésitation a dû être détruite par les expériences des médecins anglais qui ont administré cette dernière huile en lavemens dans plusieurs maladies, et entre autres dans un cas de trismus, rapporté par le docteur Phillips dans le vol. v, p. 65-72 des Transactions medico-chirurgicales de Londres. J.e crilique ne sait cependant pas qu'elle ait été employée comme vermifuge.

M. Bremser donne dans certaines circonstances un purgatif; le critique allemand lui conseille très-expressément d'employer alors le calomélas comme le font les mélecins anglais, non pas comme simple vermifuge, mais comme faisant cesser le plus sûrement l'état morbide des intestins qui a licu dans la maladie verminense.

Quant à la précaution que prenait M. Bremser de donner l'huile vermifuge à petites doses pendant long-temps, parce qu'il lui semblail que de grandes doses tuent bien le ver, mais ne détruisent pas ses œufs; il pense à prèsen ‘ qu'il scrait au contraire possible que les vers sortissent à la longue pendant un usage prolongé du médicament, et que ce soit pour cela qu'il se montre efficace dans ce mode d'administration. Je critique $M$. Albers pense que l'emploi de l'huile de térébenthine, continué de même pendant long-temps à petites doses, produirait absolument lemême résultat que l'huile empyreumatique ; et, en effet, il est bien convaincu d'avoir entièrement guéri plusieurs personnestourmentées de tænias par l'usage de celte substance, en imitant les expériences nultipliées des Anglais sur ce remède, qui ont été rapportées dans leurs différens journaux de médecine, comme dans l'Edimburgh medical and surgical journal; London medical repository; London medical and physical jounnal, e!c.

\section{Figures.}

La plupart des figures que donne M. Bremser sont originales et faics avec le plus grand soin sous ses yeux. Tout le monde est d'accord pour les regarder comme excellentes et comme tres-supé- 


\section{APFENDICE.}

rieures à ce que nous avions déjà à ce sujet; aussi le critique italien ne pouvant sans doute y trouver à redire, puisqu'il les déclare nitide, venuste e chiare, s'est borné à ajouter que le mérite en était au dessinateur et au graveur; que la manière de rendre les vers en blanc sur un fond noir n'est pas de l'invention de M. Bremser; ce qui est sans doute vrai, puisque Gaspard Asello avait déjà fait ainsi représenter, quoique grossièrement, les vaisseaux lactés, et surtout que Hopper (Robert) avait employé le même moyea pour les planches figurant les vers intestinaux dans son mémoire intitulé Observations on human intestinal worms, etc.; Mem. of medic. soc. of Lond., vol. $\mathrm{v}$, Londres, $\mathbf{r} 799$, in- $8^{\circ}$, comme le fait justement observer le critique italien ; maís cela n'empêche pas que les figures de Bremser ne soient bien plus exactes même que celies de Jœrdens, ce qui est ici la chose importante. Nous ne puivons en dire positivement autant des planches de Hopper, quoique nous soyons fortement portés à le croire, parce que nous n'avons pu trouver à Paris les Mémoires de la Société médicale de Londres, pas plus que MI. Bremser n'a pu le faire à Vienne, quoique le critique italien ait écrit que l'on sait (si sappia) qu'ils existent dans la bibliothèque impériale et royale de cette ville, en lui reprochant amèrement de n'aroir pas cité le mémoire de Hopper.

J'ai fait copier avec soin les figures de M. Bremser, et j'ai employé pour cela la lithograplie; ; jespère qu'on n'en sera pas trop mécontent : il n'en existe certainement pas de meilleures dans aucun ouvrage français sur les vers intestinaux.

Dans le but de rendre cet ouvrage plus complet et plus utile aux médecins qui ne peuvent pas toujours aisément recourir aux ouvrages de zoologie, j'ai cru devoir ajouter aux neuf planches copiées du Traité de M. Birenser, non-seulement la planche $\mathrm{x}$, qui renferme la copie des bonnes figures de cœuure et d'échinoccoque donnée par MM. Fischer et Reutdorff, mais encore deux planches đe supplément ou de l'appendice : j'y ai représenté une espèce de cliaque genre d'entozoaires, en sorte que les praticiens qui rencontreraient une espèce qu'ils croiraient nouvelle, pourrout s'aider de nos figures pour les rapprochemens et la description qu'ils cu ferout; c'était d'ailleurs une conséquence de l'cxposition du systcme de classification des entozoaires de MI. Breuser el du mien. 


\section{TABLE}

\section{ALPHABETIQUE}

\section{DES NOMS DES AUTEURS}

\section{CITES DANS CET OUVRAGE.}

Je n'ai pas lu ceux désignés par un astérisque.

ABILDGAARD (P.-c.), Allgemeine Betrachtungen über Eingeweidewürmer. In den Schriften der naturforschenden Geselischaf $i$ i Copenhagen, B. I, Abtheil. I. a. d. Daen Copenh. 1773, in-So. S. 24 , etc. Abynzonr, Abhuneron, 'Theizir; in-fol. Venetiis, r497, lib. 1I, c. 20. Ackard, praside Goldhagen; Dissert. de ruminatione' humana, singulari quodain casu illustrata; Halce, $1783 .{ }^{*}$

Actuarir (sо.) Methodi medendi libri sex; in- $4^{\circ} . V_{\text {enetiis, }} 1554$, lib. IV, p. 173 .

A eтrr Tetrabilos; in-fol. Lugduni, 1549. Tetrabili quarta, sermo II, cap. 85 , p. 9\%4. De brachiorum ac crurum Dracunculis Lconide.

Altrecht, Die Wurmkrankheiten; in- $8^{\circ}$. Hamburg und Altona, Uhne Jarzathl.

-- Jон. Рет., Vermis per meatum urinarium exclusus. Ephem. nat. cur. , dec. II, ann. I, p. 183 , obs. 77.

Alвuсdsis Methodus medendi; in-fol. Basilea, 1541, c. 91, p. 162. De extractione vence crutis.

Aldrovandr (ulyss.), Serpentum et draconum historia; in-fol. Bononice, 16 fo, hib. II , p. 328 et 329 .

Alsaharavi Liber theorica necnon practica; in.fol.' Aug. Vindel., 1519, tr. 1, 28, c. 12, fol. 118. De passione vence exeuntis.

Alston (Karl.), Powder of tin an anthelminthic medicine. Med. cssais and observ. by a Society in Edinburgh, the fourth edition; in-8\%. Edinl., 2752 , vol. V, parl. I, p. $\% 7$. 
Alston (karl), Zimnpulver cille artsney wider die Würmer. Voycz mediz. Versuch, und Bemerk., welchev.e. Ges. in Edinburgh herausgegeben werden, 5 ten bandes iter theil; in-\$o. Altenlurg, 1-52, S. 97. ff.

Amati Lusitani Curationum medicarum centuria septen $;$ in- $4^{\circ}$. Burdigalce, 1620 , cent. VII, cur. 64, p. $7^{5} 7$.

ANDRY (xicol.) von Erzeugung der Würmer im menschlichen Leibe, a. d. Franz; in-So. Leipzig, 1716.

Aretar $\mathrm{CAPP}_{\mathrm{A}}$ docrs de causis et signis acutorum et diuturnorum morborum libri quatuor; in-fol. Lugd. Bat., I 755 , lib. II, c. I, p. 51.

Arnemann (ग.), Pratisclie arzneinittellehre, 2te auflage; in-So. Go'ttingen, 1795 .

Arthus, Gotardi Dantrscani, Indice orientalis, pars $V I$, veram $e^{\iota}$ historicam descriptionem auriferi regni Guinea, ad Africam pertinentis, quod alias littus de Mina vocarl, continens, etc.; in-fol. 1604 , Francofurti ad Moen., c. 48, p. 101. De vermibus, etc.

AVICENNe arabum medicorum principis ex Gerardi Crenionensis versione et Andrea Alpagi Bell.nensis castigatione; in-fol. Venetïs, 1595 , tom. II, lib. IV, fen. III, tr. 3, c. 21 et 22 , p. 132 et 133.

Azara (don Félix de), Voyages dans l'Amérique méridionale, depuis I-81 jusqu'en i 8 or, pabliés par C.-A. Walkenaer; Paris, iSog, I. I, p. 217 .

Baguivil (Georg.) Opera omuia medico-practica; in $4^{\circ}$. Antwerpice 179.

Bajon, Mémoires pour servir à l'histoire de Cayenne et de la Guiane française; in- $8^{\circ}$. avec des planches, Paris, 1777, tome 1. Richtens chir. bibl. b. 5 , s. 169 .

Barlute (mathew), Analomie des krankhafien baues von sinigen der wichtigsten theile im menschlichen hoerper. Aus dem Engl. nı. Zuzaetzen von S. Th. Soenumering; in-S․ Berlin, 179 .

Baldinger ( Irnest-gottfr.), Neues magazin fuer Aerste; in-So. Leip$z i g, 1784$, b. 6 , st. 1 , s. 57 , contient une hisioire d'un abcis au basventre, avec complication de lombrics perforans.

Bancroft (eduard), Naturgeschichte von Guijana in Süd-America in vier Briefen; in-So. Franlif. u. Leipz., 1769, 4ter brief., s. 239 , 24,

Barère ( pierre), Nouvelle relation de la France équinoxiale; in- $\mathrm{S}^{\circ}$. Paris, 17$\}^{3}$.

- Beschreibung von Guiana, in der Berlinisch. Samml. zur Befoerder. de. Arzeneiwissensch., Naturgeschichte, etc.; in-8 . Berlin, $177^{5}$, b. 7 , st. 3 , s. $2 \nmid 2$.

BARry (Edward), An account of bloody urine for a worm in the bladder. Medic. ess. and Obscrv. by a Society in Edinburgh; in-8 . Edinb., 1752 , rol. V, part. II, p. $25.9 .--$ Mediz. versuche and Bemerk. einer gescllsch. in Edinburgh; Altenburg, 1752, 5ter band, 2 th., 3. 288. 
Bartionin (rhom.), De lue hominum et brutorum libr. III; in.8०. Hafrice, 1669, p. 260.

-- De morbis biblicis miscellanea medica; in-8 ${ }^{\circ}$. Francofurt., 1674 , P. $2 \tilde{5}$, edit. 2. Voy. aussi Thesaur. antiquitat, sacrar., auctore Blasio Ugolino; in-fol. Venetiis, 1765 , vol. 3o, p. 1533-1537.

Bлтsсн (A.-s.-G.-c), Naturgeschichte der bandwurmgattung ueberhaupt und ilerer arten insbesondere, etc., mit 5 Kupfert; in-8 ${ }^{\circ}$. Halle, 1786.

Bammin ( . ), Historia fontis admirabilis Bollens., lib., , , c. 5, p. 2 \%.

BEAU, fils (Le), Sur des vers sortis de l'aine d'une paysanne. Journal de médecine de Roux, tome VI, page 96 .

Beckers (nicol.-guil.), De ascaridibus uteri; Ephem. nat. cur., dec. I, ann. VIII, obs. 75 , p. 121.

Beireis, Dissert. de febribus et variolis verminosis, proside Godofi.-

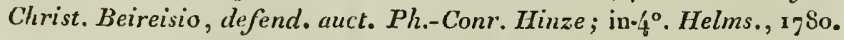

Bernard, Beschreibung eines epidemischen wurmfiebers, das im jahre 1796 in Kurland herrschte. Hu felands Journal, b. 4, s. 692.

Bernier (Franç.), Voyages; in-12, Amsterdam, iz23, t. II , p. 212.

Bertapalie (Leonardi), Recollecto super quarto canonis Avicenne; infol. Venetiis, 1498, tract.I, cap. 27 , fol. 242 ; de vena civili vel medena.

Bısссн ( эо.-варt.), De naturali in humano corpore vitiosa morbosaque generatione listoria; in-8. August.-Taurin., 174 r, p. 353.

Bidloo (Godefrid.), Opera omnia anatomico-chirurgica edita et inedita; in $-4^{\circ}$. Lugd. Bat., $17 \times 5$.

Brnet, Sur les effets de l'huile de noix et du vin d'Alicante contre le ver solitaire; Journal de Roux, tome XV, page 214 .

-- Remarques sur le tænia, adressées à M. Postel de Francière. Journal de Roux, tone XXXIV, p. 217.

Bisset (charles), Medical essays and nbservations; in- ${ }^{\circ}$ Newcastle upon Tyne, 1766 , p. I86. Of the jointed tape-worm, with an effectual method of expelling it.

Blası (cérard), Olservata anatomico-practica in homine brutisque variis ; in-So. Lugd. Batav. et Amstel., ${ }^{6} 674$, fig. 9, p. 125; lumbrici in renibus.

B Loch's ( Marc-eliezer), Alhandlung von der erzeugung der eingeweidevvermer; in- $4^{\circ}$. Berlin, 1782.

Bonetr (Théophile), Sepulchretum sive anatomia practica ex cadaveribus morbo denatis; in-fol. Genev., 1779.

Bonnet (charles), Dissertation sur le ver nommé en latin tcenia et en frauçais solitaire, etc., dans les Mémoires de mathématique et de pihysique présentés à l'Académie royale des sciences par divers savans et lus daus ses assemblées; in. $4^{\circ}$. Paris, 1750 , tom. I, p. 478.

- Nouvelles recherches sur la structure du tæuia, dans les Observations 
sur la physique, l'histoire naturelle et les arts, par Rozier; in $-4^{\circ}$. Paris, 1777 , t. IX, p. 243-267.

Bonsevault, Observation d'une fic̀re putride, verminense, épidémique, qui affligeai: la ville d'Arbois en Franche-Comté pendant l'année ${ }_{1}$ 7 66. Voyez Recueil de Rich. de Hautesierk, etc., tom. II, ก. 228.

Borelur ( petri), Hist. et obscrvat. medico-physicarun, Cent. IV; in-8。. Francofurti, 1770, obs. XL, P. 48. Vernies ex umbilico.

- Hist. et obs. medico-phys. Cent.; Francofurti et Lips., 16-6, cent. I, obs. XXVIII.

Bosmann (guill.), Voyage de Guinéc; in-12. Utrecht, 1 705 , lett. VIII, p. 116.

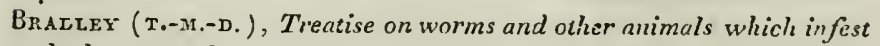
the human body; with the most speedy, safe, and pleasant means of cure; in-8०. London, $18 \mathrm{r} 3$, m. abbild.

BrERA (V.-L.), Medicinische-practische Vorlesungen ueber die vorneltmsten eingeweidewuermer des meischlichen lebenden koerper's und die sngenannten wurmkrankheiten. Aus dem Italienischen ucbersetzt und mit zusaetzen versehen von $F_{0}-A$. Weber. Mit 5 Kupfern; in- $4^{\circ}$. Leipzig, 1803.

- Memorie fisico-mediche sopra i principali vermi del corpo umano vivente $e$ le cosi dette mallatie verminose per servire di supplemento $e$ di continuazione alle lezioni; in- $4^{\circ}$. Crema, 1811 , arec figures.

Bruce (James), Voyage aux sources du Nil, en Nubie et en Abyssinio pendant les années 1768 et $177^{2}$; in $-4^{\circ}$. Londres, 1791 , 1. VII, p. 6i.

- ( vinian ), Remarks on the drucunculus or Guinea worm, as it appear's in the peninsula of India; Edinb. medic. and surgic. Journ., rol. II, 1806 , p. 145 .

Buchanan (Francis), Account of an indian remedy.for the tape-worm, medical and surg. Journ., vol. III, fasc. IX, $n^{\circ} .8$.

CALDANI (Flor.), Osservazioni sopra la trasformazione di un inselin $e$ sopra le idatide delle ranocchie; in Memorie della Societa italinna; in $-4^{n}$. Verona, 1794, tom. VIII, tab. VII, fig. 7 el 8, p. 3 г 2-3ıs. *

Campenos, Colique du bas-ventre occasionée par une quantité prodigieuse de vers contenus dans la partie inférieure du colon eı dans tout le cœcum, qui bouchaient esactement ces deux iutestins. In Ricl. de IIautesierk obs., vol. II, p. 472.

CARLisle (Anthony), Observations upon the structure and xeconomy of those intestinal worms called tania. Transact. of the Linuean Society; in- $4^{\circ}$. London, I794, vol. II, p. '24́-2C2.

Cartheuser ( jo.-Fried.), De morbis endemicis libellus; in. $8^{\circ}$. Francofurti al Viadium, 1771 , p. 207-220.

Castelr. (вarth.), Lexicon medicum srreco-latinum; in-4º. Lipsice, $17 \mathrm{r} 3, \mathrm{p} .273$.

LE CAT, An observation on hy-datides, with conjectures of their forma- 
tion. Plillosopls. Transact. for the rears 1739 et 1740, vol. LXI, n०. 460, P. $7 \mathbf{2}$.

Caulatco (cuido de), Chirurgia; in-8. Lugduni, 1559 , tract. II, cap. VIII, p. 129 sqq.

Chabert, Abhandlling von den wurmkranklieiten der europaeischen hausthicre aus der saugthier-classe. A. d. Franz. m. Anmerck. und Zuzuetzen von F.-A.-A. Meyer; in- $8^{\circ}$. Gottingen, 1789 , s. 153 .

Chamberlaine (william), A practical treatise on the superior efficacy and safety of dolichos pruriens or cowhage internally administered in diseases occasioned by worms; in- $8^{\circ}$. London, I 812 , the tenth edition, cnrrected and enlarged.

Cuapotain (ch.), Topographie médicale de l'Ile-de-France; Journail de médecine, vol. XXIV, p. 410.

Chardin (chevalier), Voyages en Perse et autres lieux de l'Orient; in- $4^{\circ}$. Amsterdam, 17.35, tom. II, p. 2 I3, nouv. édit.

Clarke (вracy), Observat. on the gemus oestrus, Transact. of the Linnean Society; in $-4^{\circ}$. London, I797, vol. III, p. 289 , и. 303.

Cla Acke (sames), Medical report for Nottingham from march 1 So to march 1808 , in Edinb. med. and surg. journ., 1809 , vol. V, p. $257 \mathrm{~s}$. Clerici (Dan.), Historia naturalis et medica latorum lumbricorum intra hominem et alia animalia nascentium, etc., cum variis figuris; in-4 $4^{\circ}$. Genev., 1715.

Clossuvs, Mittel gegen den bandivurm, in Baldingers neuem magazin fuer aerzte, 13 ten bds. 2tes stueck, s. 1 亿S.

Collet-Meygret, Mémoire sur un ver trouvé dans le rein d'un chien, dans le Journal de physique, de climie, d'histoire naturelle et des arts, par Delaméthrie; in- $4^{\circ}$. Paris, $1 \mathrm{Soz}$, tom. LV, p. 458.

Colier ( sohn), A cases of hydatids disrharged by coughing, etc., Med. transact.; London, I772, vol. II, p. 486.

Constantini ( 7 r.-Gerh., In dem Hanoevrischen magazin; in- $4^{\circ} .177^{3}$, Sten st.

Corvisart et J.-J. Leroux, Observation sur une hydropisie enkystée du foie avec hydatide, dans le Journal de méd., chir. et pharm., par Corvisart, Leronx et Boyer; Paris, an IX, tom. I, p. 21 strq.

Courbon-Predssel, Observations sur les vers, dans le Journal de médecine; Paris, 1807 , tom. XIII, p. 315 . sqq.

Cromer (Ant.), Man sehe Wepfer in Ephem. nat. cur.

Croix (De la), Vomissement accompagné du hoquet et de convulsions, occasioné par les vers, In Rich, de Hautesierlk Obs., t. II, p. 463.

Cullerier, Observation sur une tumeur du tibia qui contenait une grande quantité d'hydatides, dans le Journal de médecine, 1806 , tom. XII.

Curier's, Elementarischer entwn'f der naturgeschichte der thiere, aus 
Lem franz. von Wiedemann; in-8o. Berlin, 1800 , Ld. II, s. 521. Der nerven fadenwurm.

Cuvier, Recherches sur les nssemens fossiles; in- $4^{\circ}$. Paris, $181 x, 4 \mathrm{rol}$. -Le Règne animal distribué d'apris son organisation; in-So. Paris, isiz, 4 vol. avec fig.

Dampter ( guillaume), Supplément du Voyage autour du monde; in-12. Amsterdam, 1714, tom. III, part. II, p. 135.

DAQurn, Sur des affections vermineuses; Journal de Foux, t. XXXIV, P. $15 \mathrm{r}$.

Decerf , Observation snr des vers rendus par l'urètre; Journal de médecine par Corvisart, etc., 1810 , vol. XVII, p. $9^{2 .}$

Deltsce (victor-Amédée), Dissertation zoologique ct médicale sur le tænia humain ou rer solitaire; Journal de méd. de $1 S_{12}$, tom. XXIII, p. 218, t. XXIV, p. 364 .

Desaraeadx, Convulsions occasionées par des ters, dont on calmait sensiblement la violence par le son du violon et par le chant; in Rich. de Hautesierk Obs., t. II, p. 469.

- Attaque d'épilepsie produite par la présence d'un ver; ibid., , $\Gamma .465$.

Dianyere, Sur les maladies rermineuses; Journal de médecine de Ronx, t. V, r. 252 .

Deverex (walthervan), Abhandlung von den wuermern in den gedaermen des menschlichen hoerpers, aus dem latein. Mit zusaetzen von Theod.-Thomas Weichart; in-8 ${ }^{\circ}$. Leipzig, 1776 .

Donatus (marcellus), De medica historia mirabili libri sex; in-4 $4^{\circ}, \mathrm{Te}_{\mathrm{e}}$ netiis, 1597 , lib. IV, cap. XXVI, n. 167 .

Duвоrs, Brief an dr. Anderson nebst dem Antwortschreiben des let:teren; mitgetheilt von herrn dr. de Carro in Hufelands Journ., nov. und dec. 1813, s. I1 2, ff.; Voy. aussi Edinb. medic. and surgic. Journ., v. II, fasc. $7, \mathrm{n}^{\circ} .5$.

DuchateAu, Beobachtungen von wuermern, welche in den harnwegen enthalten, und durch die harnroehre w'sgegangen waren, dans le Journal de méd., chir., pharm., etc, contenant les iraraux de la société médicale d'émulation, par M. Leroux, r $\$ 16$, t. XXXV; Voyez Salzb. med. chir. zeit., 1816 , bd. 4, s. 106.

Dufat, Sur une hystérie vermineuse; Journal de méd. de Roux, tome XXIX; p. I 20 .

Dufour, Observations diverses sns la fièvre putride vermineuse; Journ. de méd., chir. et pharm., Paris, 1 786 , Didot, tom. LXVI.

Dunast, Lettre aux auteurs de ce Journal; Journal de Roux, t. XLIX, p. 44 .

Emhard (samuel), Dissertatio medica inauguralis de allio; in- $4^{\circ}$. Tence, 1718 , p. 17.

Eттыoller (mich.), De crinonibus sive comedonibus infantum; $A$ cl., eruclit., Lips., 1682 , obs. 1. 
Fatropn (cabr.), Libelli duo; in-\{́c. Venetiis, 1563 , c. XXIV, p. 6\%。

Fenwick (John-Ralph.), Ueber den gebrauch des terpentinoehls beim bandwurm, in einem briefe an M. Baillie; a. d. medico-chirurgical transact., the II edit., 18 ، 3 , vol.II, p. 24 ; Voy. Salzb. med. chir. zeit., r8 5 , bd. 4 , s. 130 .

Fermin (phil.), Description générale, historique, géographique et phy sique de la colonie de Surinam; in $-8^{\circ}$. Amsterdam, ${ }_{7} 69$, ch. XXIV, des vers.

FeúLtÉ (Lonis), Journal des observations physiques, mathématiques et botaniques, faites par ordre du roi sur les côtes orientales de l'Amérique méridionale et dans les Indes occidentales, depuis l'année I 707 jusqu'en 1712; Paris, :714, t. I, p. 421 .

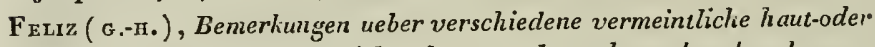
fleischwuermer in menschlichen koerper, besonders ueber den dracunculus oder dic venamedinensis; Voy. Neues magazin de Baldinger fuer aerzte, in $-8^{\circ} .1788$, bd. 10, st. 6 , s. $49^{2-50} \%$.

Fischer ( Jo.-Leonh.), Tanice lydatigenre in plexu choroideo inventa historia; in-8 ${ }^{\circ}$. Lipsiae, ${ }_{7} 79$.

Fonrassin, Considérations sur l'histoire naturelle et médicale des vsrs du corps de l'hourme, présentées et soutenues à l'Ecole de médecine de Paris le 22 ventose an XII; in- $8^{\circ}$. Paris, $180 \%$.

Frank (Louis), Collection d'opuscules de médecine pratique, avec un

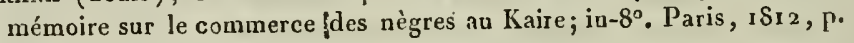
235.

Freind (s.), Histoire de la médecine, traduite de l'anglais par Etienne Coulet; in-12. Leyde, 1727 , part. I, p. $75-81$.

Fromansi (so.), Observ. de verminoso in ovibus et juvencis reperto hepate; Eph. nat. cur., ann. VI et VII, Francof. et Lips., 1677, p. 249 , obs. 188.

Gabucivo (Hieron.), De lumbricis alvum occupantibus ac de ratione curandi eos qui ab illis infestantur commentarius; in- $8^{\circ}$. Venet., 1547 , c. III, p. 6 .

GAnd's (Pet.-Andr.), prof. der chemie zu Abo, physikalisch-oekonomische beschreibung des noerdlichen theils der kreise von Satakunda in Finnland; Voy. Dan.-Gottfr. Schreber's neue Caineralschriften, th. V, Halle, ${ }^{7} 766$, s. $30 x, \$ 6$.

Game (henr.-Maur.), Dissertatio inuuguralis sistens observationes quasdam de insectorum vermiunzque structura; in- $4^{\circ}$. Kilixe, 1817 .

GALENI (claud.), De simplicium medicamentorum facultatibus, libri $\mathrm{XI}$; in- $8^{\circ}$. Lugduni, 15 亿 7 , lib. VIII, p. 5 r2.

- Opera; in-fol. Basilece, $156 \mathrm{r}$, vol. II, de locis affectis, 1. VI, c. III, P. 76, spaxovtiagis.

Gariandat (nav.-rent.), Diss. de dracunculo sive vena medinensi; 


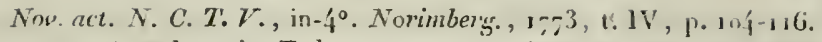

Garmann ( L.-ch.-Fr.), Eph. nat. cut., ann. I, scil. 16 ;o; in-40. Lijs., obs. I $\{5$, vernes intestina perforantes restituto cegro, $\mathrm{p} .321$.

Gautieri.(Giuseppe), Slancio sulla genealogia della terru e sulla costruzione dinamica della organizzazione. Seguito da una ricerca sull origine dei vermi abitanti le interiora degli animali; in-So. Iena in Sas. sonii, 1805 .

Geischloegrer (ant.), Unerwarteter ausgang einer complicirten skrofelkranlikeit, nebst beinerkungen ueber wuermer und wurmmittel; Journal de Hufeland, bd. 10, st. r, s. s/3, ff.

Gruinex ( эо.-Nep.•^nt.), Diss, inaug. medica de entozois lonini familiaribus; in. $4^{\circ}$. Prage, 1812 ,

Gentix (le), Noureau voyage autour du monde; in-12. Amsterdam, 1728 , t. I, p. I1.

Groffroy ( Dr.-M.) , Estrait d'une observation snr deux hydatides d'un volume considérable trourées dans la cavilé thorachique, etc.; Bulletins de l'Ecole de médecine de Paris, an XIII, première asuée, n. 11, 1. 164 .

GiradDy, Observations sur les maladies vermiaeuses; Journal général de médecine, Paris, 180ł, neuvième année, t. XXI.

Gmelin (sam.-Gottl.), Reisen durch rufsland zur untersuchung der dre Naturrei:le, dritter theil; in $-4^{\circ}$. Petersburg, 177', s. :302, tab. 3o.

- (so.-Fried.), Caroli a Linné Systema natura; in-8. Lips., 1790, t. I, part. VI.

Goechel (chr.-Ludw.), De abscessu inguinis periculoso curato; Ephem. nat. cur., dec. II, ann. IV, obs. 112, p. 219.

GnDor, Sur un dépôt enkysté dans le rentricule, arec perforation de ce viscère, dont l'adhérence s'est propagée au petit lobe du foie, aux muscles du bas-rentre, et y a formé un dépôt externe; Journal de Roux, t. XL, P. I 45 .

Gosze (Joh.-Aug. ephraïm.), Versuch einer naturgeschichte der eingeweideswermer thierischer koerper; in $-4^{\circ}$. Leipzig, $17 \delta 7$, avec 44 planches gravées.

Gonraei (so.), Opera definit. medicar. , libr. XXIV; in-fol., Parisiis, $1622,1) .16$.

Grashurs (so.), De natura et ortu hydatidum; Act. nat. cur., 1744, vol. VII, p. 408-424.

GreGor (James-ñ.), Medical sketches of the expedition to Egrpt from India; in-8 ${ }^{\circ}$ London, 1804, p. 202.

Griffith (uughes), The natural history of barbaros in ten books; in-fol. London, $1750, p .4 \mathbf{r}$.

Grimm (Jos.casp.), De viro qui excrevit per urinam substantian glandulosam membranaceam instar vermis renalis, s. lumbrici terrest, i; Act. nat. cur., t. I, p. 22\%; obs. 11 f. 
Groти (hugonis), Annales et 7istoria de rebus belgicis; in-fol., Amstelodami, 1657, p. 209.

Gruxnerr (Godefr.-Aug.), In Commerc. litter. ad rei medic. et scient. nat. increment. institut.; in $-4^{\circ}$. Norimberga, $1740, \mathrm{p} 329$.

Grunerr (chr.-Godefr.), Morborum antiquitates; in-8․ Vratislavice, 1774, P. 216-226.

HaEHNe (Tob.-Henr.), Vermis in pectore inventus cordique adfixus; Act. nat. cur., vol. V1I, p. 53.

$\mathrm{H}_{\text {AнN }}, I_{n}$ Pallas neuen nord. beitr., bd. 1 , s. 160.

HALIFiluUs ( $\mathrm{bb}$ bas), Liber totius medicina necessaria continens, etc.; in- $4^{\circ}$. I 553 , lib. IV, pract., cap. XVI, p. 201 ; De passionibus qua in manibus pedibusque fiunt et primum de saniosa vena.

HANNsus (Georg.), Obs, e vermilus cœea et muta restituta; Eph. nat. cur., dec. II, ann. V, olss. $2 S$, p. $3 j 6$.

Hannes, De aphonia aliisque incommodis vermium ejectione sanatis; Nov. act. nat. cur., t. VI, p. $26 \mathbf{1}$.

Hanow's (mich,-christoph), Seltenheiten der natur und œkonomie, pu . blié par J.-D. Titius; erster-bund., in-8 ${ }^{\circ}$. Leipzig, 1 783 , s. 586.

HartaAn ( ph.-Jac.), Obs. vermes vesiculares sive hydatweles in caprearum omentis et in pulmonilus alterius furfuracea; Eph. nat. cur., dec. II, anv. IV, obs. 73 , p. 152-159, fig. $25-28$.

- Anatome glandiorum; Eph. nut. cur., dec. II, ann. VII, obs. 2f́, p. 5 s.

Hasseleurst's ( Filed.), Reise nach Palaestina in deil Jahren $1749^{+1}, 52$, herausgegeben von Carl. Litnaus, traduit du suédois; in. $8^{\circ}$. Rostoch, I 762 , s. 58\%.

Hautesierc (rich. de), Recueil d'observations de médecine et des hôpitaux militaires; in $4^{\circ}$. Paris, 1766 , 1. I, 1772 , t. II.

НеАтн (George-тhom.), Observ. on the Guinea worm, Edinl. med. and surg. Journ., janv. $1 \$ x 6$, p. 120.

Heister ( Laur.), De lumbricis in cavo abdominis repertis, intestinisque $a b$ eis perforatis; Act. nat. cur., t. I, obs. 172, p. 391.

Hemmersam (mich), Guineische und West-Indianische reisebeschrbibung de an. $1639-16 \nmid 5$; in- $8^{\circ}$. Nürnberg, 1669 , kap. 13 , von wuermern so aus dem menschen kommen.

Herrenschwand (J.-Fr.-v.), Abhandl, von den vornehmsten und gemeinstein innerlichen und reufserlichen krankheiten, zum gebrauch junger aerate und Wundierzte u. s. w. A. d. Franzoesischen; in- To $^{\circ}$. Bein., 1788 , s. 4. 44千.

Heurnius (э.), Opera omnia, Lusd., I65S, Ł. II. - De morbis noris et mirandis epist. ad Henr. a Bra, p. I 19.-Derselbe brief in Pet. Foresti obs. et curat. medic. op. onm.; in-fol , Francofurti, 1619, I. XX, obs. 39, p. $30 y$. 
HiLL (G.-N.), On the effects of ursenic; Edinb. med. and surg. journ, $\mathbf{r}-\mathrm{S}_{9}$, rol. V, p. 312 .

Hillary's (wilh.), Beobaclitungen ueber die veraenderungen der Luft und die damit verbundenen epidemischen krankheiten auf der Insel Barbados, tradnit de l'anglais par J-Ch.-G. Ackermann; in-So. Leipzig, xэ76, s. 3jz-383.

Hrmuy, Beobachtung und beschreibung des Finennenwurms; Journal de Hufeland, dccemb. ISog, s. I 15 , folg.

Hirsci, Einige gedanhen ueber erzeugung der wuermes in individuellen organismus, veraniafst disch die beobachtung einer sehr seltenen wurmkrankheit; dans les Ephémérides de médecine, par Markus, $4 \mathrm{r}$ bd., 2tes heft, s. 136 .

Hollerr (sac.), Omnia opera practica; in-fol., Parisïs, r664. - De morb. intern., lib. I, cap. 54; de vermilus, p. 419, in Schol.

Hons (eterard), Lectures on comparative anatomy; in which are explained the preparations in the Hunterian Collection; illustrated by En gravings ; London, $\mathrm{ISI}_{4}$, in two volumes. Dans le Hall. allg. lit. zeit, april, 1816. s. 753 .

Hopkinson (F.), Account of a worm in a Horse's Eye; in Transact, of the american philosoph. Society held at Plitadel,hia, in-4․ vol. II, 1786 , p. 183 , n. 18.

Hurezand (chr.-wilh.), Tollstaendige darstellung der nedicinischen krcefie und des gebrauchs der salzsauren schwererde; in-So. Berlin, 1794.

- Ueber die ihm am besten gelungene methode den bandwurm ubatreiben; dans son Journal, bd. 1o, st. 3 , s. rz8.

Нuзводтт (Alex.-v.), Ansichten der natur mit wissenschafilichen Erlaeuterungen. Erster band.; in-S०. Tubingen, 1808 , s. 142 f.

Hunters (John.), Versuche ueber das blut, die entzuendung und die schusswunden; a. d. Engl. von Hebenstreit, Leipzig, 1797, 2ten bds. Ite Abth., s. 34 , dans la note.

Huenertrolf (Ir.-Jac.-Aug.), De ileo lethali a vermibus; Epl. nat. cur., dec. II, ann. V, obs. I9, P. 32 .

Hutcheson (rob.) et Forbes (Georg.), Ulcers from dracunculi; Med. essays and observ. publ. by a Sociely in Edinburgh, in-8 ${ }^{\circ}$. Edinb., 1752 , vol. V, part. II, p. 3og. - Dans le med. versuche und bemerl., einer Gesellsch, in Edinburgh; Altonn, $\mathrm{x} 752$; 5 te bd. 2te th. s. 1022.

Ingennousz (ло.), Vermischte schriften physisch-medic. Inhalts; uebersetzt von Molitor, in $8^{\circ}$. Wien, 1754 , 2te Auflage, 2ter bd., s. $173 \mathrm{ff}$.

Ingrassis (so-phil.), De tumoribus prater naturam tomus printus; in-fol.

Neapoli, 1553 , c. r, p. $\mathbf{9}$.

Joel (s.), Opera medica; Amstelodami, r zor, lib. X, secı. 3.

Joerdens (गо.-нeinr.), Entomologie und helminthologie des menschlichen hocrpers oder beschreibung und abbildung der bewoluner und feinli'e 
desselben unter den insekten und wucrmern, zwciter band, mit sieben colorirten kupfertafeln; in-4․ Hof., 1802.

Iserr's (paul-erdmann), Reise nach Guinea und den Caraibischen inseln in Columbien, in Briefen an seine Freunde geschrillsen; in- $8^{\circ}$. Copenhagen, 1785, s. 369-371. - Ainsi que Isert (P.-E.), Voyages en Guinéc, etc., traduits de l'allemand; in-8. Paris, $179^{3}$, p. 335 .

Ka enprer (Engelbert), Amœnitatum exoticarum politico-physico-medicarum fasc. $V$; in $-4^{\circ}$. Lemgovice, 1712 , fasc. III, obs. IV, dracunculus Persarum in littore sinus persici, p. 534 sqq.

KArG (so.), Ueber den steinbruch $\approx u$ Oeningen bei stein am Rheine und dessen petrefacte; dansles Denkschriften dèv vaterlıendischen Gesellschaft der aerzte und naturforscher schwabens, erst. bd.; in-8०., Tub., 1805 , s. $1, \mathrm{ff}$.

KELCH, Zergliederung eines sehr ausgedehnlen und mit schleim gefuliten osariums; Journal de Hufeland, bd. 25 , st. 3 , s. I9' ff.

Kerhringir (rhéod), Spicilegium anatomicun; Amstelodani, 1670, obs. 79, p. 154 .

Kier (Dr.), Journal of a residence in India by Maria Graliam; in $-4^{\circ}$. Eclinburgh, $1 \$_{13}$, p. 23.

KLeıx (sac.-rheod.), Tentanen Herpetologia, Lcida et Gattinga; in. $4^{\circ} \cdot 1755, \mathrm{p} .60$.

- Untersuchung unterschiedlicher meinungen von dem herkommen und der fortpflanzung der in menschlichen loerper befindlichen wuermer; Voy. Iamburgisches megazin, bd. 18, st. I, s. 19-58.

Kortur, Die stutenmilch, ein mittel gegen den bandwurm; Hufelands journ., April $1 \$ 12, \ldots 119$.

Kunsemuller (Fr.-guil.), De morbo yaws dicto et de vena medinensi, pries. Curt. Sprengelio; in-4 Hala, 1797. In Brera Sylloge opuscul. select., Ticini regii, 1799 , rol. VIII, p. $27 \mathbf{1}$ sq $\urcorner$.

LAват, Voyage du chevalier des Marchais en Guinée, etc., in-12. Amsterdam, I 73 r , t. II, p. I ro, vers cutanés.

Lachmund (Fried.), Von wunderbaren würmern, welche in Guinea in den Fuessen, lenden und horlensack der menschen wachsen; Voy. Ablaandl. der roern. liais, Akademic der naturforscher; in-4.4. Nurenberg, 1757 , 4ter th., s. 279 .

LAENnec ('Théoph.), Mémoire sur les vers vésiculaircs, et principalement sur ceux qui se trouvent dans le corps humain, lu à la séance du 26 pluviose an $x 11,1804$.

Lafare, Principes de chirurgie; in- $S^{\circ}$. Haris, 1756 , part. V, sect. I, chap. I, P. 220.

LAGENe, Remède contre le tænia; Journal de médecine de Roux, r. XLV, p. 220 .

LANGE, Veler die tvirlisanticit des mittels des $\mathbf{h r m}, G_{.}-H_{0}-R$. Beck; dans le Journal de Hufeland, bd. I7, st. 2, s. 153. 
LAxGn (Lembergii-so.), Epist. medic. velum. sripartit. ; in-80. Francof., I $5 S_{9}$, lib. I1, ep. 42, 1. 756.

Larrex (c.), Note sur le prétendu ver de Guinée, dans le Bulletin des sciences, par la sociélé philomatique, septiène année, n 83 , p. r-8 sq.

Lassus, Recherches el observations sur l'hydropisie enkystée du foie, Journal de médecine, chirurgie et pharmacie, par Corvisart, etc.; t. I, p. I $5 \mathrm{sq}$.

Lawrence (w.), Case of a woman, who voided a large number of worms by the uretina with a description of the animals; Medico-chirurgical Transact. published by the med. and cluir. Society, in-So. London, $18 x 3$, vol. II, p. 385-95, tab. 8, deux. édıt.

LeEuwenhoen, Part of a letter from authory van Leeuwenhock concerning worms observed in sheeps livers and pasture Ground; Philosoph. transact., vol. XXIV, for the years 1 ;of et 1705, p. 1522.

Lengsferd (Jos.), Beschreibung ler bandwürme und deren Heilmittel; in- $8^{\circ}$. Wien, х791.

Leoxi (Dom.), Ars medendi; in-fol. Bononice, г583, sect. III, lib. IV, c. $9, \mathrm{p} .575$, de vernilus.

Lesser, Théologie des insectes; in.S . La Haye, $174^{\circ}, \mathrm{t}$. II, liv. II, part. III, chap. 2, p. 223.

Licetus (fortunat.), De spontaneo viventium ortu; in-fol. Vicet., 1613, lib. III, cap. 5i, P. 2 \{́2.

Liñ (sam.), An Essays on diseases incidental to Europeans in hot climates; in-8 ${ }^{\circ}$. London, $1755, \mathrm{p} .53$.

Linné (car.), Amoenitates academice, vol. $V$, curante Schrebero; in-So. Erlanga, $1788, \mathrm{p} .103$, exanthemata viva, etc.

Linshor (Jean-rughes de), Histoire de la navigation, avec annotations de B. Paludauus; in-fol. Amsterdam, 1638 , c. VI, p. r7.

Listen (nartin), Part of a letter fiom fort St. George in the East-Incites, giving an account of the long worm which is troublcsome to the inhabitans of those parts; Philos. Transact.; in-4\%. London, 1698 , vol. XIX, p. $4 \mathrm{I} 7,4 \mathrm{i}$.

LoEfFleus (Adolph.-Ftied.), Beitrage zur arzenei und wundarzencihunst, erster theil, in-8०. Leipzig und Altona, 1791, s. 59-65.-Dracunculus oder vena medinensis, ainsi que dans Richter's chir. bibl., bd. 7 , s. 785 , u. b. 12 , s. 33 千.

-Eispillen; Journal de Hufeland jul. I 810 , s. 110.

Lorry (ann.-car.), Tractatus de morbis cutaneis; in-40. Parisiis, 1777 , p. 556-592, de dracunculis Gracorum, vena medinensi Arabum. Herrn Ann.-Carol. Loory's Able. von den hrankheiten der haut, traduit Uu latin par le docteur Chr.-Fr. Held, Leipziğ, 1779,2 r bd., s. 383.

Ludersen (nenr.-car.-Lud.), De hydatidibus diss. inauguralis; in· $4^{\circ}$. Gottingae, 1808 .

Lunuckx, Von einer toedlichen durchlohrting der gedaerme, welche 
von wuermern verurs acht worden; Voy. Joh.-Lebrecht Sclimuckers vermischt. chit. schriften, 2 te bd. 2te, auflage, in-S ${ }^{\circ}$ Berlin und Stettin, 1 -36 , s. 235 .

M ACLFAY, Fallvon balgivassersucht mil hydatiden; Voy, the Edint. med. und surg. joum., bd. II, heft VI, n. 5, allg. lit. zeit. febr. I815, n. 45 .

MaLfighil (marcelii), Opera posthuma; in-4 $4^{\circ}$. Amstelodami, 1698 , tab. X, p. 112, fig. $5, n$. I et 2.

Manardi (Jo.), Epist. medic, , tibr. XX; in-fol. Basilea, i 549, 1. VII, ep. 2, P. 124 .

Marcus (dr.-rh.-Marc.), De lumbricis ; Epl. nat. cur., dec. II, anr. I, obs. 120, p. 306 .

Maréchal de Rougères, Sur quelques maladies compliquées, Journal de Roux, t. XXX, p. 44 .

Milnie, Obscrvation sur une fière putride vermineuse qui a rég é à Rar ennes, St.-Alberto, etc.; Journal général de médecine, 1804, t. XXI, [1. 250, nsuvième année.

Marteav, Sur une ouverture à l'ombilic, qui donnait passage au cliyle et à dès vers contenus dans les intestins grêles; Journal de Roux, t. V, 1). 100 .

Marteau de grandvilliers, Sur quelques fidvres vermineuses singulières, accompagnées de symptômes siaguliers; Junrn. de Roux, tom. XVII, p. $24 \mathrm{~s}$.

Martin (Anton.-rolandson), Gordier, Knoten-oder Fadenwuermer bei fischen und menschen gefunden u. s. w; Voy. Schwedische abhandl., année 1771, bd. 33, s. 258.

Masars de cazeles, Sur le tænia ou ver solitaire, et pius particulièrement sur un tænia percé à jour; Journal de Roux, t. XXIX, p. 26.

Matuieu, Mittel gegen den bandwurm; in Hufelands journ., bd. 10, st. 2 , s. 199, tiré des Ephémérides de Formey.

Mead (nich.), Monita et pracepta medica; in-8०. Parisiis, 1757 , sect. III, cap. 7, j. 74 , de lumbricis.

Meckel (J.-F.), Ueber einige ungewoehnliche erscheinungen an leberḱnolen; Voyez deutsches Árchiv fuer die pliysiologie, bd. I, heft 3 , s. $432 \mathrm{ff}$.

Melin (chr.sac.), Plaktische inateria nedica, 4te auflage; in- $\delta^{\circ}$. Franhfuit a $M 1 ., 1789$.

Mercunals (nier.), Variarmm lectionum, lïri quatuor; in- $4^{\circ}$. Venct., I57 $\mathrm{x}, \mathrm{lib} . \mathrm{II}, \mathrm{c.} 20, \mathrm{p} .6 \mathrm{o}$.

Meyer (F.-A.-A.), Einige zweifel gegen herrn chirurgus Fieliz uebel. ver'schiedene haut und fleischwuermer im menschlichen hoerper; dans Laidinger's neuen maguzin fuer aerste, bd. I1, st. 2, s. 156.

Monceau, Observation sur des iers urinaires; Joura. de néd., ch., etc., Paris, an' $\mathbf{x} 1 \mathrm{v}, \mathrm{t} . \mathrm{XI}, \mathrm{P}$. II. 
Monern, Observation sur un ver trouvé sous la conjonctive, d Mar:barou, île Saint-Dorningue; Jou nal de Roux, t. XXXII, p. 335.

Monncr (conr.), Systentatisehe lehre von denen gebraeuchlichsten einfachen und zusamınengesetzten arzney-mitteln, 3te auflage; in-So. Marbourg, 1795.

MoENNica, I'underbare und verkannte zufaelle durch wuermer, ein beweis ihrer grossen pathologischen wichtigkeit; Hufelands journal, september $181 \mathrm{z}$, s. 114 .

Monro (Alex.), The morbid anatomy of the human gullet, stomarh and intestins; in-S ${ }^{\circ}$. Edinburgh and London, 1811 . Voyez Leijziger lit. zeit., .. 75 , u. 76 , des jahrs $18 \imath 5$.

Montani (so.-варt.), Libri de excrementis, etc.; in-8o. Venetïs, 1556 , de morb. gallic., p. 121, Endimini.

Montin (Lorenz), Auszug eines falles von einer fasciola intestinali mit mancherlei wuermer bei einer hranken; Voyez abh. der schived. akad. der wiss. auf das jahr 1763 , Leipzig, 1766 , bd. 25 , s. 122.

Moreau, Journal de médecine, t. XXV, p. 435 .

Morgagnt (Jo.-Bapt.), De sedibus etcausis morborum per anatomen indagatis libri quinque; in- $4^{\circ}$. Lugd.-Batav., 1767.

Morgan (sohn), Of a living snake in a living Horse's Eye, and of other unusual productions of animals; in Transact. of the American philosoph. Society held at Philadelphia; in- $4^{\circ} \cdot 1786$, n. 43 , vol. II, p. 383.

MorRAH (mich.), Ueber eine hydutide im gehirn; medico-chi, urg. trans. publ. by a med. and chir. Society of London, $\mathrm{I}_{13} 3$, vol. II, edit. II. Voyez Salzb. med. chir., zeit 2515 , bd. 4, s. 136.

Moublet, Sur des vers sorlis des reins et de l'uretère d'un enfant, avec des réflexions sur la néphrotomie; Jonrnal de Roux, $\imath$. IX, p. 244 s., et $\mathrm{p} .33 \mathrm{~s}$.

Movgeot (з.в.), Essai zoologiquc et médical sur les hydatides; in- $8^{\circ}$. Paris an $\mathrm{xr}, \mathrm{r} 803$.

MovlenQ, Sur un tænia sorti par l'aine d'une femme; Jourual de Roux, เ. LVI, p. 330 .

Muli er (ollo-Fricd.), Vermium tervestr. et fluviat., etc., succincta historia; in-4" Havnice et Lipsice.

Muracto (Joh. de), Ileos a vermibus in puerpera natura; Ephem. nat. cur., dec. II, ann. I, obs. is , p. 300.

Mutead DE Roceuemont, Sur une maladie vermineuse accompagnée d'accidens extraordinaires; Journal de Powx, t. XXI, p. 243.

Nau (Bernh.-scb.), Neue Entdeliungen und bcobachtungen ats der physik, naturgeschichte und oekonomie, ersterband; in $8^{\circ}$. Frankfurt a $M$. $179^{3}$.

Nerdiam aus Nortr-Walshar, Ein jall von einem vorfalle der gedaerme darch die offiuns des mastulurms, a. d. Medical transact. of 
phys, in London, r8г3, vol. IV, n. 24; Voyez allg. lit. zcit., janr. $18 \mathrm{5}$, n. 8 , s. 63.

Nie в un (carstcn), Beschreibung von Arabien; in- $4^{\circ}$. Copenlag. , $177^{2}$ s. 133 .

Nierembergir (jo.euseb.), Historia nature maxime peregrina lib. XVI distincta; in-fol. Antverpice, 1635 , lib. X1I, cap. 24, de norbo vermium, 1). 280 .

Odrer, Sur l'usage de l'huile douce de ricin, particulièrement contre le ver solitaire; Journal de Roux, t. XLIX, p. 333 et 450.

OfFren (car.), De fecibus alvinis et lumbricis ex abscessu abdominis prodeuntibus; Eph. nat. cur., dec. II, ann. I, ohs. 126, p. 3 г8.

Окек, Die Zeugung; in-S०. Bamberg und Wurzbourg, 1805.

Oldennonss (c.-G.-A), Geschichte der mission der evangelischen brueder auf den Carribischen inseln St.-Thomas, St.-Croix und St.-Jean, publié par J.-J. Bossart; in-So. I Th. Burby, 1777, buch. II, abschn. 7, s. $125,126$.

Olfers (J.-Fr.-M. de), De vegetativis et animalis corporibus in corporibus animalis reperiundis commentarius; in-80. Berolini, 1816, pars I, c. tab. aën.

-Onomatologia hist. nat., oder vollstardiges lexikon, das alle Zenennungen der kuntswoerter der naturgeschichte nach ihrem ganzen umfange erklaert; in-S ${ }^{\circ}$. Frankf. und Leipzig, $177^{3}, 4$ ter bd., s. 32.

Osann (c.), Beobachtungen ueber den innerlichen gebrauch des terpentinoehls gegen den bandwurm; Hufelands journ., sepı. I $\delta \mathbf{1 6}, \mathbf{s .} 3$ I ff.

Oтто (A.), Ueber das nervensystem der eingeweidewuermer; Magazin der Berlin. naturf. gesellsch, 7ter jahrang 3 tes quarı., s. $223 \mathrm{ff}$.

Pallas (pet.-simon), De infestis viventibus intra viventia; in Sandifort Thesaurus dissertationum; in- $4^{\circ}$. Roterdami, 1768, v. 1, p. 247 s.

- Reisen durch verschiedene provinzen des Russischen reichs, 3 theile; in- $4^{\circ}$. St.-Petersburg, $1771-1776$, th. I, s. 9 , th. II, s. $54_{1}^{\prime} 3$.

- Neue nordische beitraege, erster band. ; in-8 ${ }^{\circ}$. Petersburg und Leipzig, 1781 .

Palmer (ro.-Fysche), Tentamen medicum inaugurale de vermibus intestinorum 1766; in thesaur. med. Edinburg. nov.; in-S . Edinburgi et Londini, 1785 , tom. III , p. 42 sqq.

PAracelsi (A ur.-phil.-rheophr.), Opera ommia; in-fol. Geneva, 1658. Theoretica fisura universalium morborum, tab. VIII, 1. 738. Generatio morborum et aposteniatum, lib. $11 \mathrm{I}$, cap. 6, p. 6o, de apostemamatibus.

Parei (ambros.), Opera; in-fol. Parisiis, 1582. De tumoribus contra natıram particularibus, lib. VII, cap. 31, p. 253-256, de clracunculis.

Passerat de la chapelie, Effets de l'huile de noix et du rin d'Alicante contre le ver solitaire; Journal de Roux, 1. VI, p. 305. 
PAToN, Cases of Guinea worms, with olservations; Edirb. med. and surgical joumal, 1806 , vol. II, p. $15 \mathrm{I}$.

Prullinr (chr.-Franc.), Disquisitio curiosa an mors naturalis plerumque sit substantia verminosa? in-80. Francofurti et Lipsiae, 1503, p. 105.

Pauli aleinete, libri septem; in- $4^{\circ}$. Lasilea, 1533 , cap. 59 , p. $32 \imath$.

Pelcetier (le), Sur une maladie singulière produite par des vers; Journal de lioux, tome XXXIII, page 3 f́.

Pemberton (chr.-rob.), A practical treatise on various diseases of the abdominal viscera; in-8\%. London, 1\$14. Voyez Goetting. sel. anz. 6otes, st. I 816 , s. 59 \%

Percy, Mémoire sur les hydatides utérines et sur le part liydatique; Journal de médecine, t. XXII, p. I 71.

Pent, Mémoire sur le dragonneau, Journal de Roux, t. XLII, p. 121.

Prsonis (car.), Selectiorum observationum et consiliorum liber singularis; in-4․ Lugd.-Bat. , 1733 , p. 242.

Plateri (felic.), Praxeos tomus tertius, de vitiis; in- $4^{\circ}$. Basil., 1625 , cap. 3 , p. 182. De extulerantia phlyctena, cap. 13, p. $8_{7}$ เ-896. De animalium excretione.

- Observationum libri $I I I$, in-So. Basilea, $\mathbf{6} 68 \mathrm{o}$, lib. III, p. 6r $7 . H_{y-}$ drops ascites ob hep. et lienis fissuras et vesiculas.

Plene (sos.-sac.), Hrgrologia corporis humani; in-8o. Vienna, 1794, p. 179. Usus putrefactionis cadaverum.

Plinir (c.), Historia nuturalis libri $X X X V I I$, cum notis Harduini; in-fol. Parisiis, 1723 , lib. XI, cap. 33, p. 611.

Plutarchi cheronensis, Opcra qua extant omnia, cum interpretatione Hermanui Cruserii; ia-fol. Francofurti, 1599 , t. II, Symposiacon, lib. VIII , quast. 9, p. 733 .

PLUtakch's Moralisch-philosophische werke, traduit par $J_{0}-E_{0}-S$. Kaltwasser 5ter theil; in-8 ${ }^{\circ}$. Wien und Prag, 1797 , s. 495.

Pollucis (Julii), Onomasticum graece et latine; in-fol., Amstelodami, 1, o6, lib. IV, cap. 25, sigm. 205, p. 472.

Postel de francière, Sur le tænia, vulgairement appelé ver solitaire; Journal de Roux, ı. XVIII, p. 4 r6.

- Réponse à la lettre de M. Robin; ibid., t. XXVI, p. 4 I5.

Raisin, Observation sur un ver rendu par les urines; Journal de Roux, t. XXIX, 1.458 .

Rathier, Reunde contre le ver solitaire; Journal de Roux, t. XXVIII, p. 44 .

RAUH (Dan.-corn.), Diss. inaus. med. de ascaride lumbricoide Linn., vermium intestiualium apud homines vulgatissimo; in $4^{\circ}$. Gottingce, г 779.

REDI (FRANC.), Osservazioni intorno agli animali viventi che si trovano negli unimali viventi; in.8`. I'chesia, 1741. 
Rieies franco (GAspar A), Elysius jucundartum qurestionum campus; in-40. Francofurti aul $M ., 1670$, quast. $36,1 \% .426$ sq.

fiernlein (sAc), Ainimadversiones circa ortum, incrementum, causas, symptomata et curan tcenice late in intestinis humanis nidulantis casibus practicis illustrata, cum figuris; in-So. Viennce, ISI I.

Reinlein's Bemerkunhen ueber den ursprung, die entwickelung, die ursachen, syniptomen und heilart des breiten bandwurmes in den gedae!men des menschen, durch practische faelle erlaeutert, mit 1 hupfer, nach cien lateinischen uebersetzt; in-S ${ }^{\circ}$ Wien, 1812.

ReMer, Beobachtungen am Krankenbette; Hufelands journ., b. 17, st. 2 , s- $106 \mathrm{ff}$.

Rinaze (abubetri manethi), Opera exquisitoria; in-fol. Basit., 1544 , ad Munsor de re snedica, lib. VII, cap. 24, p. 179, de vena medeme sive civili.

Rrchand, Journal de médecine, t. XIX, p. 3r3.

Richten's (Aug.-Gottl.), Abhandlung von den bruechen; .in-8º. Gout., 1785, s. $271-274$.

Piosin, Lettre à M. Postel de Francière; Journal de Roux, tome XXV, p. 222.

Resel vel Rosenhof (Aug-Jo.), Historia ranarum nostratium; in-fol. Norimberga, 1758.

Rosenstein, nils rosen von, Antveisung zur kenntnifs und kur der hinderkrankheiten; in- $8^{\circ}$. Gottingen, $179^{8}$.

Rovdier, Tumeur considérable sur l'hypogastre; Journal de médecine de Roux, r779, t. LII, p. 124-126.

Roux, Sur une hydropisie enkystée du foie trouvée dans le cadavre d'un homme mort suffoqué ; Journal de médeciac, t. XLII, p. 314.

Roziène DE LACHASSAGNe, Sur un vertige vermineux; Journ. de Roux, tom. XXVI, p. 430 .

Ruvolphi (кarl.-Asmund), Bemerkungen aus dem gebiet der naturgeschichte, medizin und thierarzneykunde auf einer reise durch einen theil von deutschland, holland und frankreich; in-8 ${ }^{\circ}$. Berlin, $180-1$. ISo5, 2 theile.

- Entozoorum sive vermium intestinalium historia naturalis; in-8 . Anstclodami, 1808-181o, vol. III, c. tabb. aën.

Rurschir (Fred.), Thesaurus aratomicus; in-4 $4^{\circ}$. Amstelodami, 1;01, llies. I, n. 12 .

- Opera omnia anatomico-medico-chirurgica; in- $4^{\circ}$. Amstelod., 1737 , vol. III.

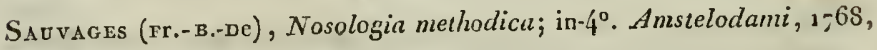
t. II, p. 553 .

Sch 玉FFer (sac.-christ.), Die egelschnecken in den lebern der schafe un $a^{\prime}$ dic von diescn wuermern entstehende schafkrankheit, nebst einer hupferplatte; in- $4^{\circ}$. Regensburg, 1753 , s. 29. 
- Die eingebildeten wuermer in zaehnen, nebst denz vermeintlichen huelfsnittel wider dieselben, nebst einer kupfertafel in Farben; in- $4^{\circ}$. Regensb., 1757 .

Scharfr (вenj.), De vermibus uteri, Eph. nat. cur., dec. I, ann. IX et $\mathrm{X}$, obs. 7, p. 44.

SchelHaMmel: (Günth.-chr.), Lumbrici ex abscessu in inguinali regione erumpentes; Eph. nat. cul', dec. II, ann. V, obs. 10, p. 19,

Schexcкir (Jo. a grafenberg), Observat. nedicar. ravior, libri VII; in-fo!. Francofurti, 1665, lib. V, de pltthiriasi, obs. 6, pag. 701, de dracunculis Athiopice et Indice propriis.

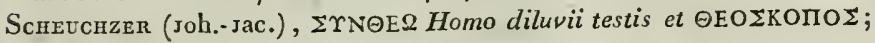
in $-4^{\circ}$. Tiguri, 1726 , p. 2 .

Sснмирт (Joh.-Adam.), Ueber die krankheiten des thraenenorgans; Wien, 1803, tab. II, s. 73 .

Schinest (Joh.), De hernia exulcerata, unde excrementa sine alia sanitutis noxa egerebantur; Eph. nat. cur., dec. 1, ann. III, obs. 122, p. 19 '.

Schmucker (Jo.-Lebrecht), Praktische amerkiungen von dem nuetzlichen gebrauch des sabadillsamens in allen arten von wurmkrankheiten des meuschlichen koerpers; in seinen vermischten chir. schrift, in- $8^{\circ}$. Berlin und Stettin, 1782, bd. 3.

SchøLER (ud.), Diss. inaug, med. sist. observationes super morbis Surinamensium; in $-4^{\circ}$. Goeltinga, $178 \mathrm{t}$.

Scholzir (caur.), Epistolaruin philos. medic. ac chymicar. volumen; in-fol. Hanovia, 1610 , epist. XXVII, p. 32 sqq.

Sciraxi (Fr.-v.-paula), Verzeichniss der bisher hinlaenglich bekannten eingeweidewuermer, uebst einer abhandlung ueber ihre anverwandtschafien; in- $8^{\circ}$. Muenchen, $\mathrm{x} 788$.

Schwartze (avg.-jac.), Observationes de virtute corticis Geoffrece Surinamensis contra tceniam; in $-4^{\circ}$. Gotting., $179^{2}$, p. 16.

Schwenckfeld (casp.), Theriotropheum Silesice; in- $4^{\circ}$. Lugduni, 1603 , p. 556 , seta aquatica.

Scopolr (Jo.-Ant.), De hydrargiro idriensi tentamina; in-8 $8^{\circ}$. Venetiis, ${ }_{17} 6 \mathrm{~s}$, p. 155 .

SEELIGER, Nutzen des sabadillsamens wider den bandwurm. Schmuckers vermischte chirurg. schrifi; Berlin und Stettin, 1786,2 ter bd., ste auflage, s. 27 r.

Senvert (dan.), Operum; in-fol. Parisiis, 1641, t. I, hypomn. V, cap. $8, \mathrm{p} .233$, serpentes in deserto Israelitis immissi non fuerunt dracunculi. Tom. III, lib. III, pars II, cap. 5, De lunbricis.

SERres, Affection vermineuse simulant la rage; Jourual de médecine, tom. XXV, p. 25, p. 258.

Sertueraner ( F. $_{\text {-w. }}$ ), Ueber eines der fuerchterlichsten gifte der pflanFonwelt, als ein nachtug zu sciner abhandlung ueber die rehonsaeure 
und des morphium; mit bemerkungen, der anderen extractivstoff des opiuns und seine verbindungen beiriffend; Voy. Gilberts annalen der. physik, neue Folge, jahrgang 1817 , si. 1o, s. IS3.

Slonde (nans), Voyage to the Islands, Madera, Barbados, etc., with the natural history; in-fol. 1725 , vol. II, p. 190.

Spalaxzani, Tersuche ueber die erzcugung der thiere und planzen, a. d. Franz; in-So. Leip zig, 1791, s. I So ff.

Splegerir (Adrian.), Opera ommia; in-fol. Amstelodami, 16 5 , de luntbrico lato liber.

Sposits (Jac.), In Boneti sepulchr. anat., t. II, p. 530.

Siteinich (jo.-Georg.), Commentatio de tcenia hydatigena anomala, adnexis cogitatis quibusdam de vernium visceralium physiologia, cum tab. an.; in-8 . Erianga, 1802.

Streber, Liucanthos polycephalus, ein intestinalwurm des menschen; in Meckels deutschem archiv fuer die physiologie, dritten bandes zwaitem hefte, Halle und Berlin, ${ }_{1} 8_{17}$, s. $174 \mathrm{ff}$.

$\mathrm{S}_{\text {tenr }}$ (Ant.), Annus medicus, quo sistuntur observationes circa morbos acutos et clironicos, etc.; in- $8^{\circ}$. Vindobonce, 1759 , ann. II, $\mathbf{1}-6 \mathbf{1}$.

Suck, Merkwuerdiger fall einer durch wurmreiz bewirkter unstuelpung cles augapfels, a. d. russ. samml. fuer naturw. und Heilk; Voyez Alls. med. annal. $18 \mathrm{r} G$ bd. I, hft. I, s. 8 ; , maerz, s. $35 \%$.

Sumerre, Sur des donleurs pleurétiques dépendantes des vers, et sur la vertu de la coralline (appelée leeinithocorton) dans ces sortes de cas et dans d'autres; Journal de Roux, t. LII, p. 331.

Swieten (Gerardi-B. van), Commentaria in omnes aphorismos Bocrhaal'e; in $-4^{\circ}$. Venetiis, $1764, \mathrm{t}$. VI.

Sxlvestre, Sur des mouvemens convulsifs occasionés par des rers; Journal de Roux, 2. XXXIV, p. 424. Tagautr (गо.), De chirurgiä institutione, libri $V$; in- $S^{\circ}$. Lugduni, 156 ;
lib. I, P. S.

Гнопаs, Sur le ver solitaire ou tænia; Journal de Roux, tom. XIIII, p. 6s.'

Thomasse. à Thuessink, Journal de méd., t. XIX, p. 77, 18 ro.

'Tissot (s-A.), Epistolce medico-practicce, auctee et emendutce; in-S ${ }^{\circ}$. Luusanne, 17\%o, p. 132 .

'Traldraxi (Alex.), De lumbricis epistola, nunc primum grace et latine edica; Venetis, 15 ;0."

'Trevirands (G.-R.), Biologie oder philosophie der lebenden natur fuer naturfoscher und aerzte bd. $1 I$; in-So. Coltingen, 1 So3, s. 264 ff.

'Turpir (Nicol.), Observationes medice, editio nova, libro quarto auctior; in-8 $8^{\circ}$ Amstelodami, 1685 ; lib. II, cap. 42, p. 161, genuinum lati lumbrici caput; cap. 49 , p. $17^{2}$, mictus vermis cruenti; lib. III, cap. 12, p. 199!, lumbricus, ex inguine.

Txsox (Edward), L:mbricus latus, or a discourse of the jointed worm, etc.; 
Phylos. transact. for the year' 1683 , p. I 3 ff. - Lumbricus teres, or the round worm bred in lzuman bodies; ibid., p. $153 \mathrm{ff}$.

- Lumbricus hydropicus or an cssays to prove that hydatides often met with in morbid arimal bodies, are a species of worms or imperfect animals; Philos. transact, rol. XVII, for the year $169^{3}, \mathrm{n}$. $\mathbf{1 g}^{5}$; March. I6gI, p. 506 ff. ; et dans Act. erudit. Lips., ann. $169^{2}$, p. 435 et 4 40.

Unzer, Beobachtung von den breiten wuermern (vermes cucurbitini); Voy. Hamburger magazin, bd. 8, s. 312-3ı5.

Vaupuelin, Annales de chimie, vol. XXIX, p. 3 s; et dans Scherers allg. Journ. der chesnie, band. III, s. Ig9 ff.

VEIGA (Th.-Rodericia), Opera omnia; in-fol., Lugluni, I586, locor. affect., lib. VI, p. $38_{9}$ et $39 o$.

VeIt, Einige bemerkingen ueber die entsteliung der hydatiden; Voyez Reils Archiv. fuer die physiologie, hd. II, I797, s. 486 ff.

Venschil (Gcorg.-Hier.), Exercitatio de vena medinensi, ad mentem ebnince, sive de dracunculis veterum. Specimen exhibens nova versionis ex Arabico cum commentario uberiori, etc.; in $-4^{\circ}$. August. Vind, I679.

Vogel (rud--nug.), De cognosc. et curand. corp. human. affect.; in-Sº. I $78 \mathrm{r}$, pars II, p. 327 , dans la nole

VoIGt (F.-s.), Grundzuege einer naturgeschichte als geschiohte der entstelung und weiteren ausbildung der naturkoerper, mit 3 kupfert; in-8 $8^{\circ}$ Frankfurt a $M ., 1817$.

Vollgadi (Henr.), Obs. Vermes intestina perforantes pereunte cegra; Epl. nat, cur., dec. I, ann. I, p. 322.

Warfanus (henr.), Nosolngia hermetica et gallenica; in-So. Lipsia, 1605, disp. XXI ; de lumbricis', d:acunculis et crinonibus, thes. $13, \mathrm{p}$. 218.

WArson (will.), A/l observation of hydatides voided per vaginam; in Philos. transact., for the year's I739 et I 7fo, v. XLI, n. 460, p. 7 I I .

WEDEKIND, Von der einkleinmung der brueche, die durch wuermer verursacht wird; in Richters chir. biblioth., bd. 8, s. 79-9i.

WEIGEL, Ncue methode den bandwurm abzutreiben; Voy. IH felands journ., bd. 1 , s. 439 .

Werssmanters, Vermischte plyysikalische beitraege; in- $8^{\circ}$. Leipzig, I777, st. I, hydrometra hydatica. Trauben-molen-schwangerchaft.

Wexpenstant, Bemerhungen ueber spulwuermer und bandwhermer; Voy. Hufelands journ., b. I1 , st. 3, s. I $18 \mathrm{ff}$.

Weprer (jo.-jac.), Cicutre aquaticre historia et noxa; in-4․ Basilea, 1679 , cap. 15, de Gialappa, P. 221 , in schol.

-Intestini ilei ruptura integro abdonine; Eph.nat. cur., dec. II, ann. X, obs. 21, scliol. 5, p. 315-317, de vena medin. 
Werlhof (paul-gottl.), Observationes de febribus; in- ${ }^{\circ}$. Hanoverce, 1732, p. 142 .

WERNer (panl-christ.-Fried.), Vermium intestinalium prasertim tanice lzumance brevis expositio; in- ${ }^{\circ}$. Lips., 1782 , tab. VII.

- Continualio secunda edita a Jo-Leonh. Fischer ; in-8. Lips., i 786.

Wichrann (Joh.-ernst.), Ideen zur diagnostik, 3ter bond.; in-8. Hannover, 1802 , s. 85 .

Wiedentann, Archiv fuer zoologie und zootomie; in-8 ${ }^{\circ}$. Braunschweis, I801 u 1802 , 2ter uod 3 ter band.

Wierr (so.), Opera ommia; in-4º. Amstelodani, 166o, obs. lih. II, p. 947 sqq.

Wirmand (Ј.в.), Ueber die classification der thiere, eine von der alademie zu Harlem mit der goldenen medaille gekroente preisschrift; in-8 ${ }^{\circ}$. Giessen, 1814 .

Worт's (sо.-эac.), Gazophylaceum medico-physicum; in-4 . Leipzig, $\mathbf{1 7 6 7}$, p. 2386.

Zener (Joh.-Georg.-Heinr.), Erster nachtrag zur naturgeschichte der eingeweidewuermer, von $J .-A . \cdot E$. Coeze, mit zusaetzen und anmerkungen, mit 6 kupfertafeln; in $-4^{\circ}$. Leipzig, 1800 .

- Anleitung zur naturgeschichte der eingeweidewuermer, fuer aerzte und naturforscher, mit 4 kupfertafelı; in-8 $8^{\circ}$. Bamberg, 1803. 
Tisez, pagc 26 , ligne 2ł, fig. $i$, au lieu de fig. I.

47, 20, capsules au lieu de apsulcs.

5r, 3o, lucio perca au lieu de perca lucio, L.

157, 8, fig. 3-4, au lieu de 3-3.

il.

8 , lumbricoides an lieu de lombricodes.

161, 10, fig. 4, au lieu de fig. 3.

164,8 , anleitung, au lieu de anheituug.

ib.,

167 , $x$, the broad tape au lieu de the broadt ope:

ib., s, fig. $b$, au lieu de $c$.

I7x,

172 , 5 , fig. $c$, au lieu de $d$.

12, pl. 4, au lieu de pl. 5.

16 , ce qui a paru avoir lieu au milieu de toute la longueur de l'animal, au lieu de ce qui a pu avoir lieu dans toute la longueur.

176,

ib., 3 , pl. 4, uu lieu de 5 .

186 ,

187 ,

197 ,

13 , fig. $d$, au lieu de $h$.

16 , pl.5. 7 , au lieu de 6 .

$\mathbf{x}$, fig. $g$, au lieu de $f$.

18, dans toute la longueur d'un côté, an licu de au bord d'une articulation.

ib.,

217 ,

261 ,

282 ,

297 ,

298 ,

ib.,

ib.,

320 ,

327 ,

330 ,

384 ,

534 ,

538 ,

$x_{9}$, fig. $h$, au lieu de $k$.

19 , pl. 3, au lieu de pl. 8 .

17, fg. $5 a$, au lieu de $5 c$.

16 , fig. $1 e$, an lieu de $\mathrm{s} f$.

26 , fig. $2 a$, au lieu de $2 e$.

17 , fig. $2 d$, au lieu de $2 b$.

18 , fig. $2 b$, au lieu de $2 c$.

19 , fig. $2 d$, au lieu de 2 .

14 , fig. $d$, au lieu de fig. 3 .

2. fig. $a$, au lieu de lig. $u$.

I et 18 , fig. $g$, au lieu de fig. 9 .

note 3 , wahnsinnigen, au lieu de wahnsiumuigen.

3o, visis au lieu de viris.

12 , Il est question de crachemens de sang ct non pas d'hématodes, comme au restc cela a dejà été dit, p. 376, dans la note. 




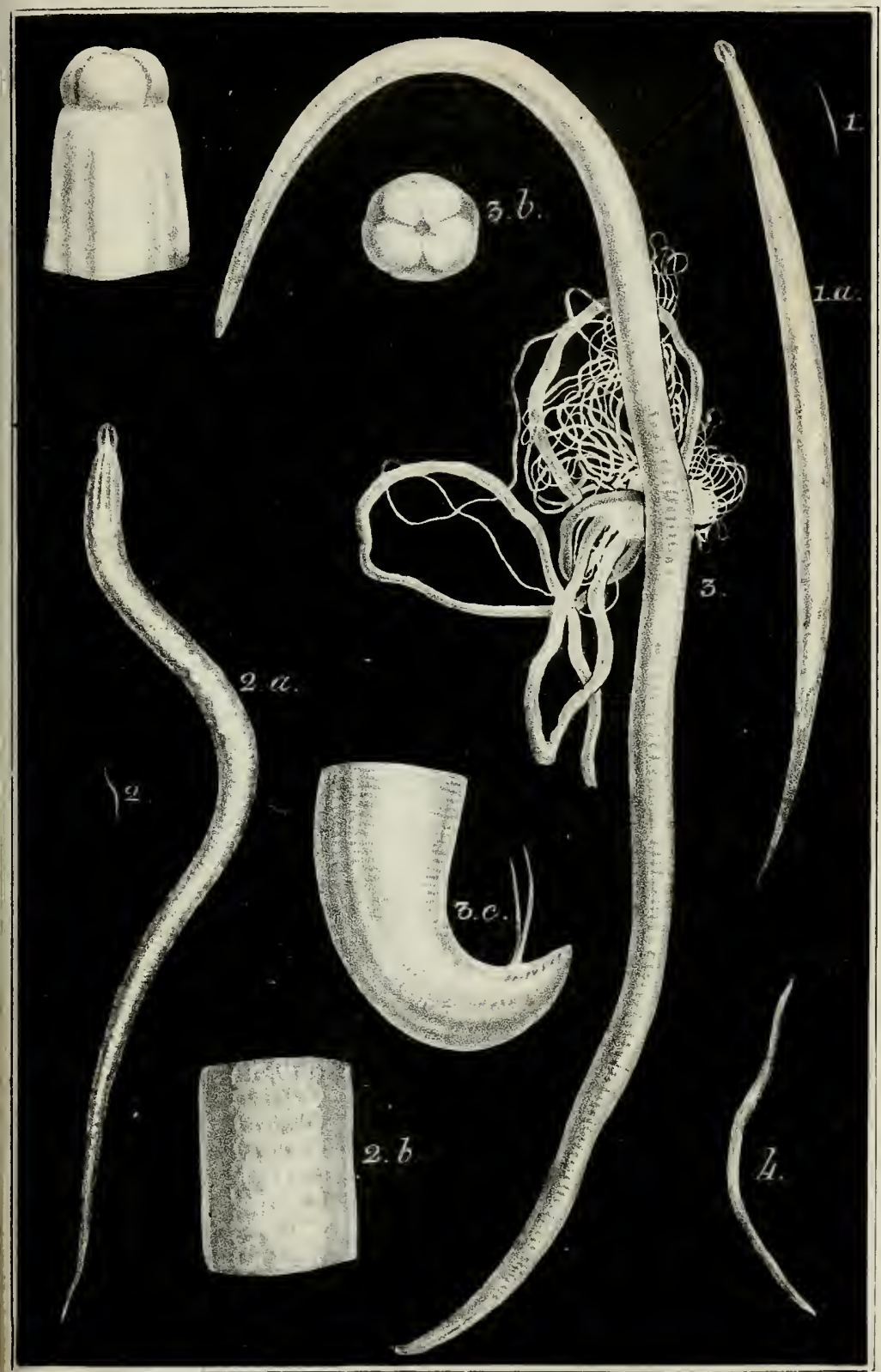





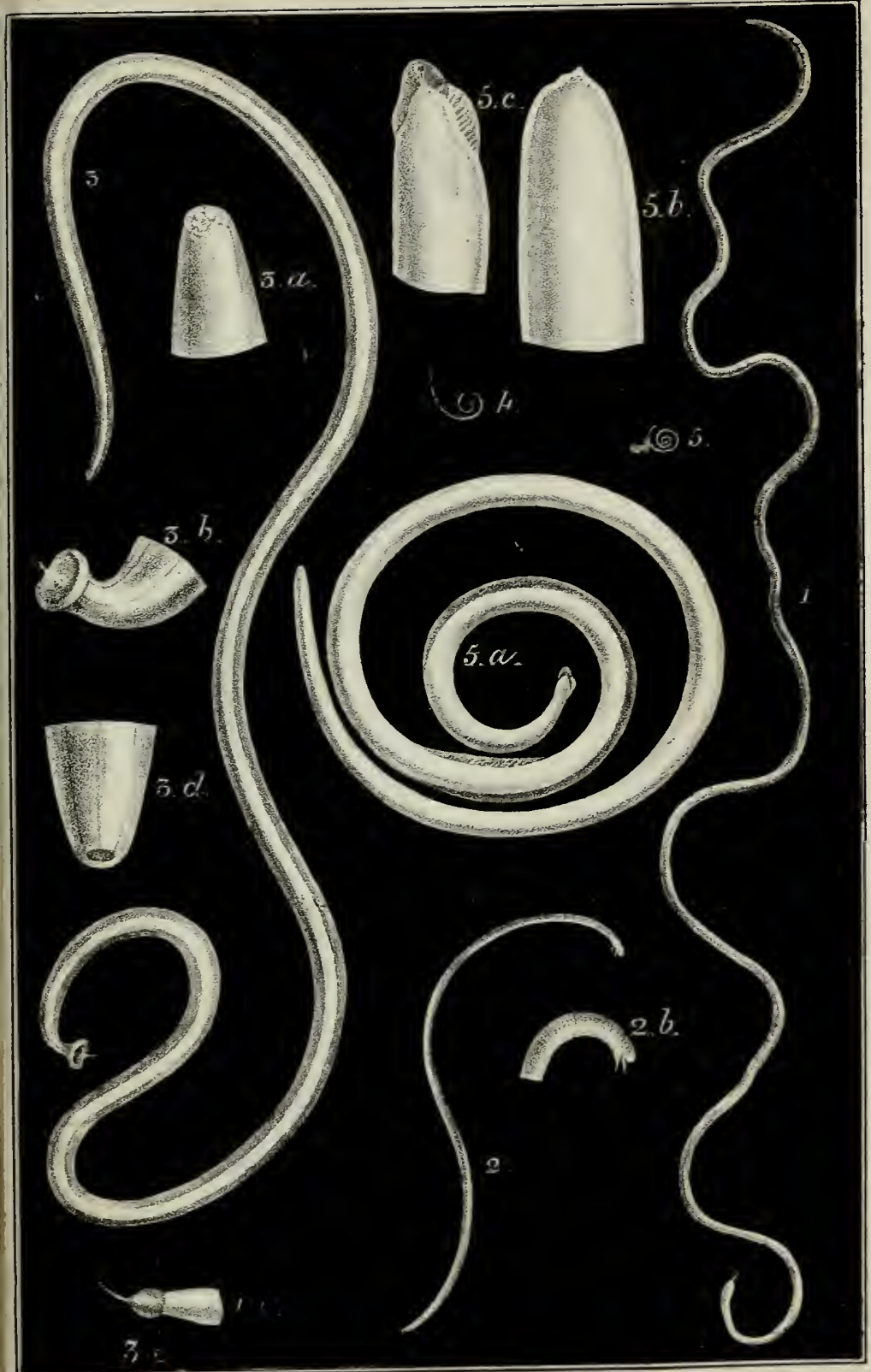





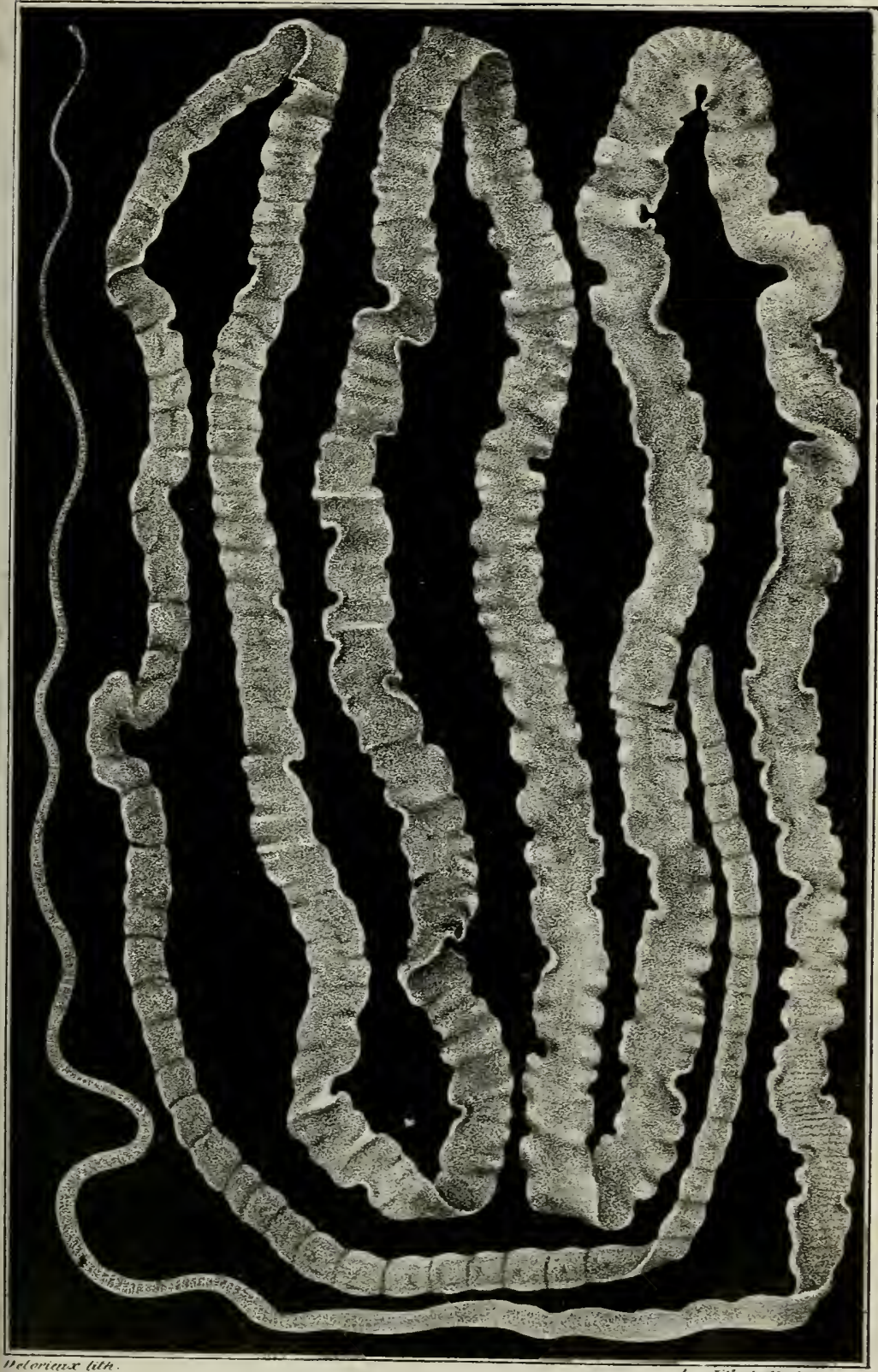





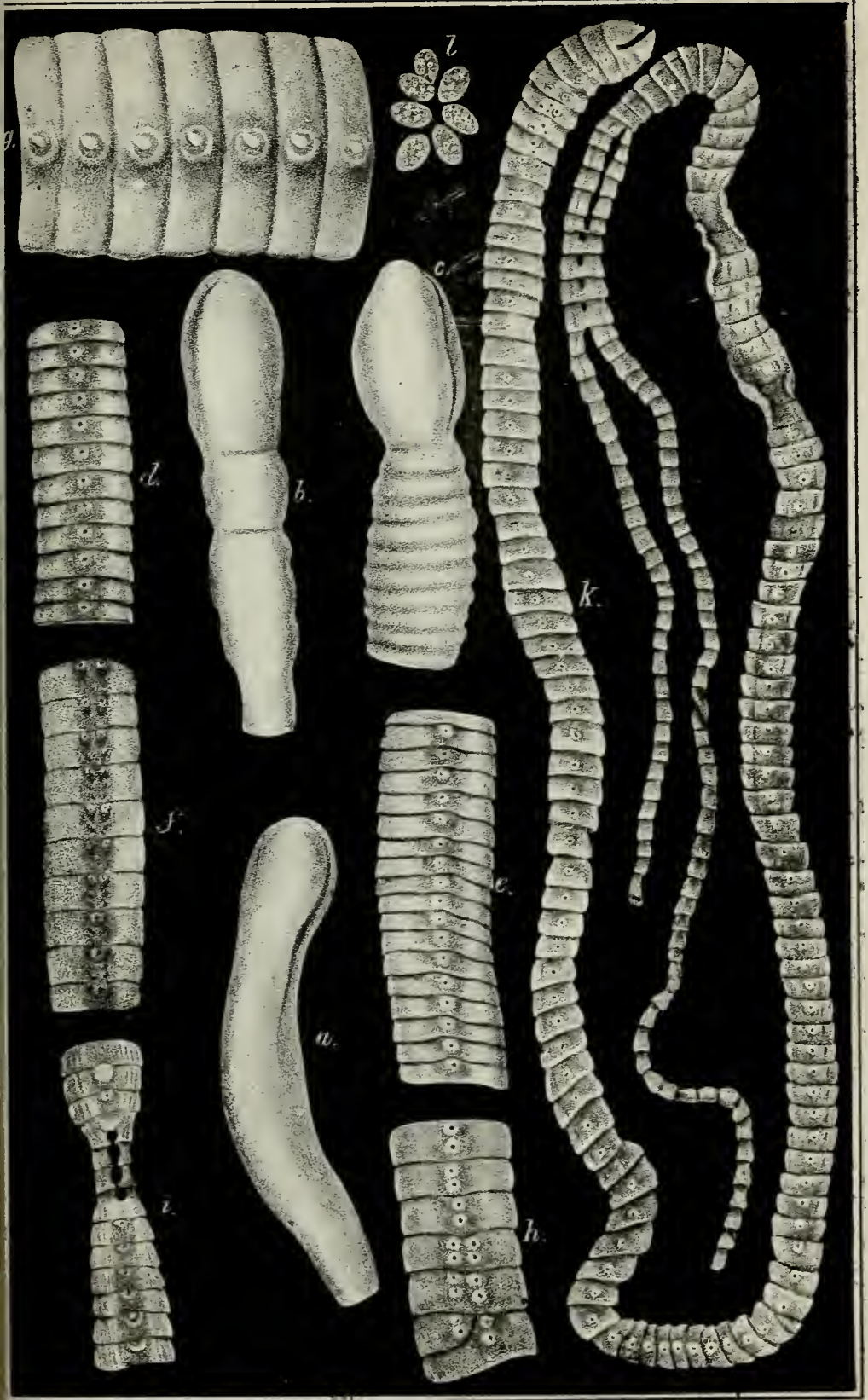





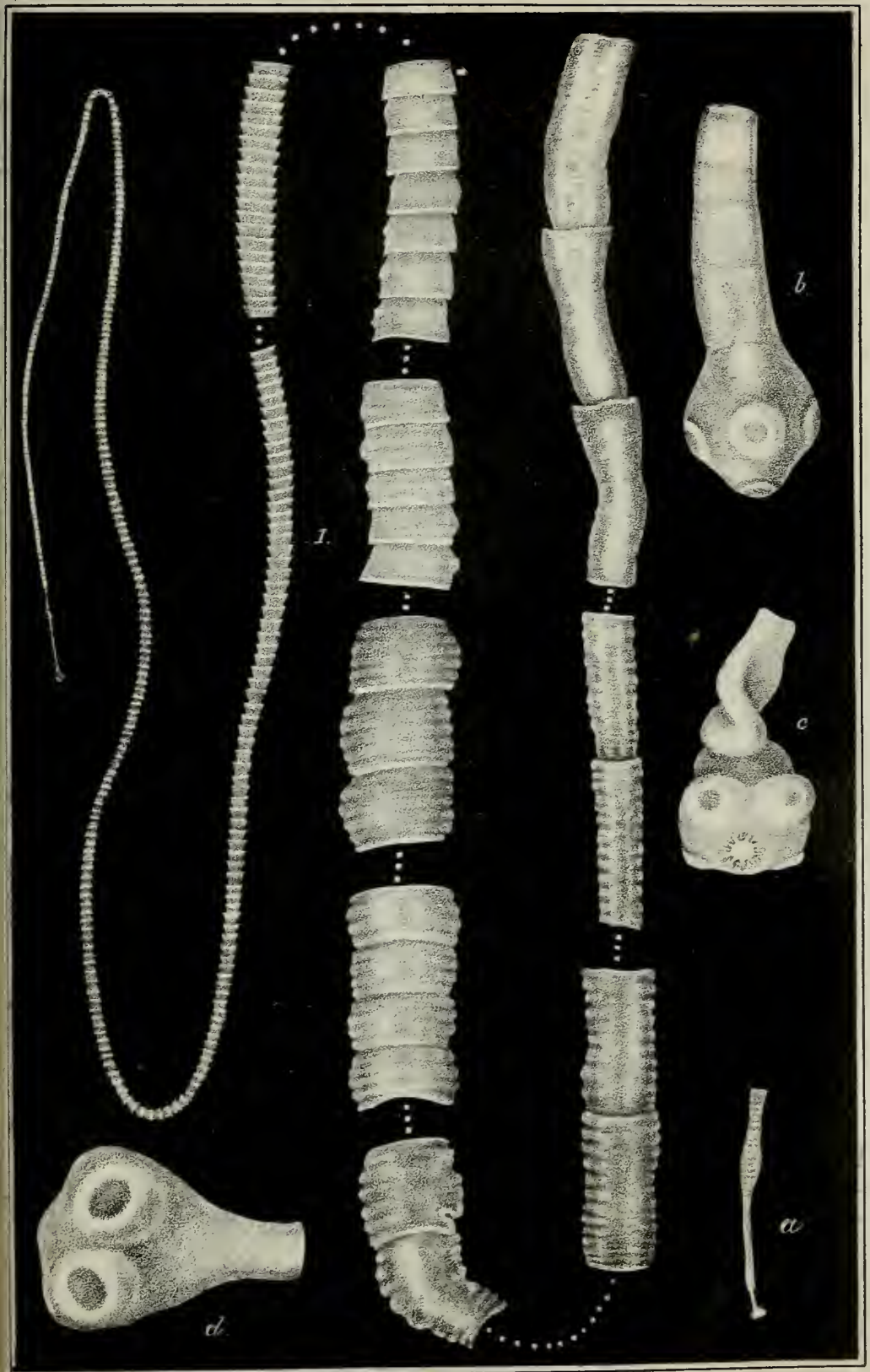






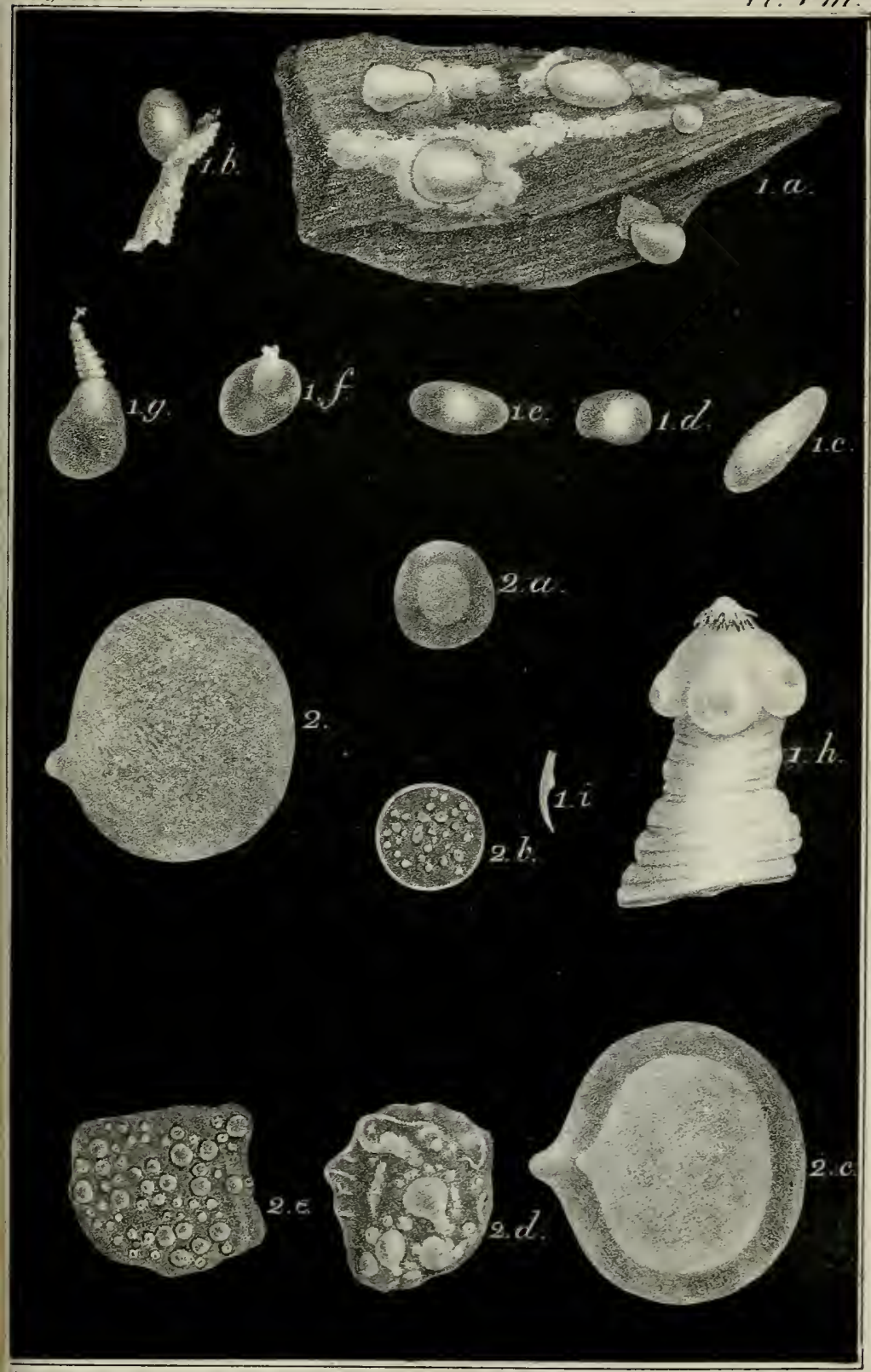




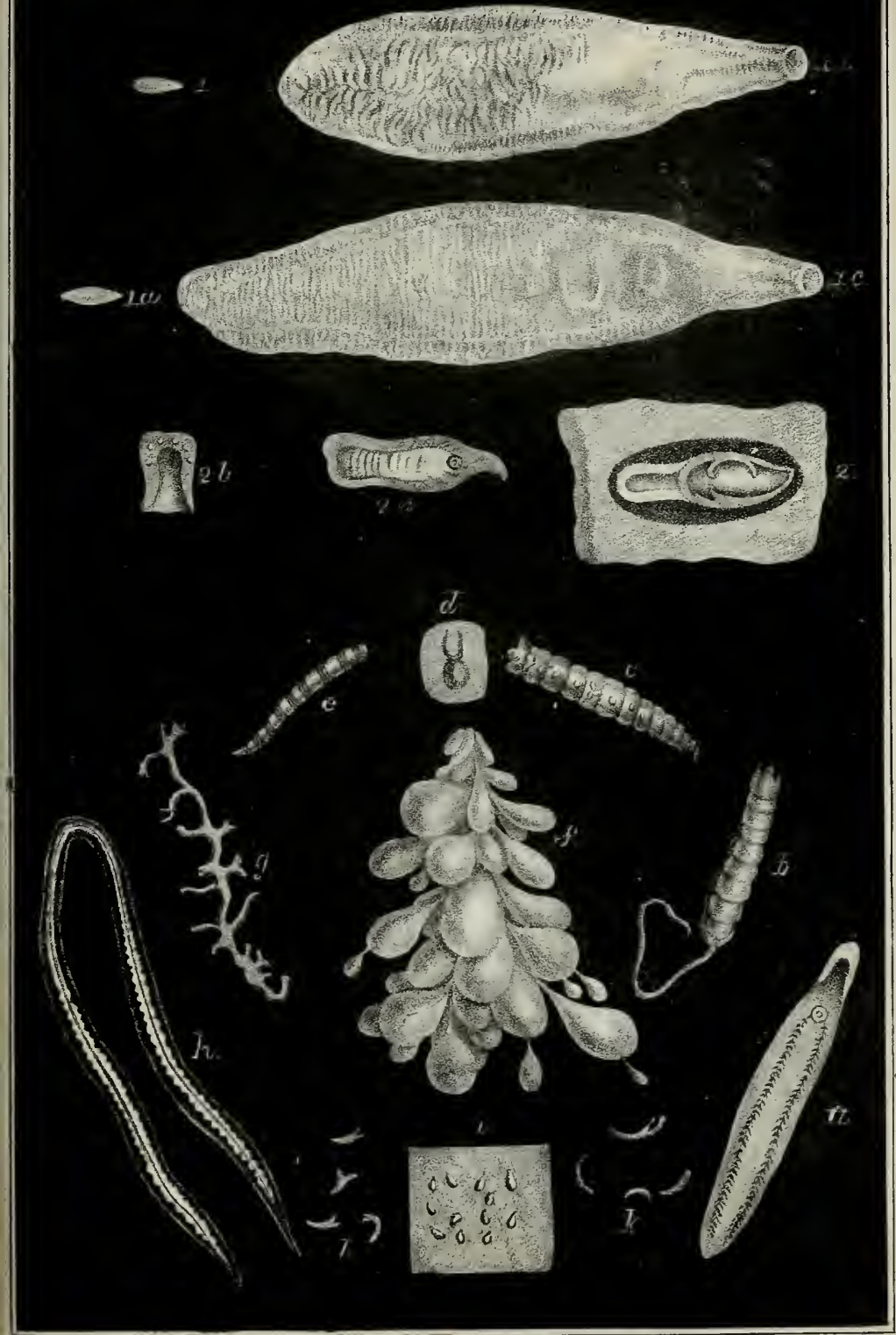

$2 \times 12$
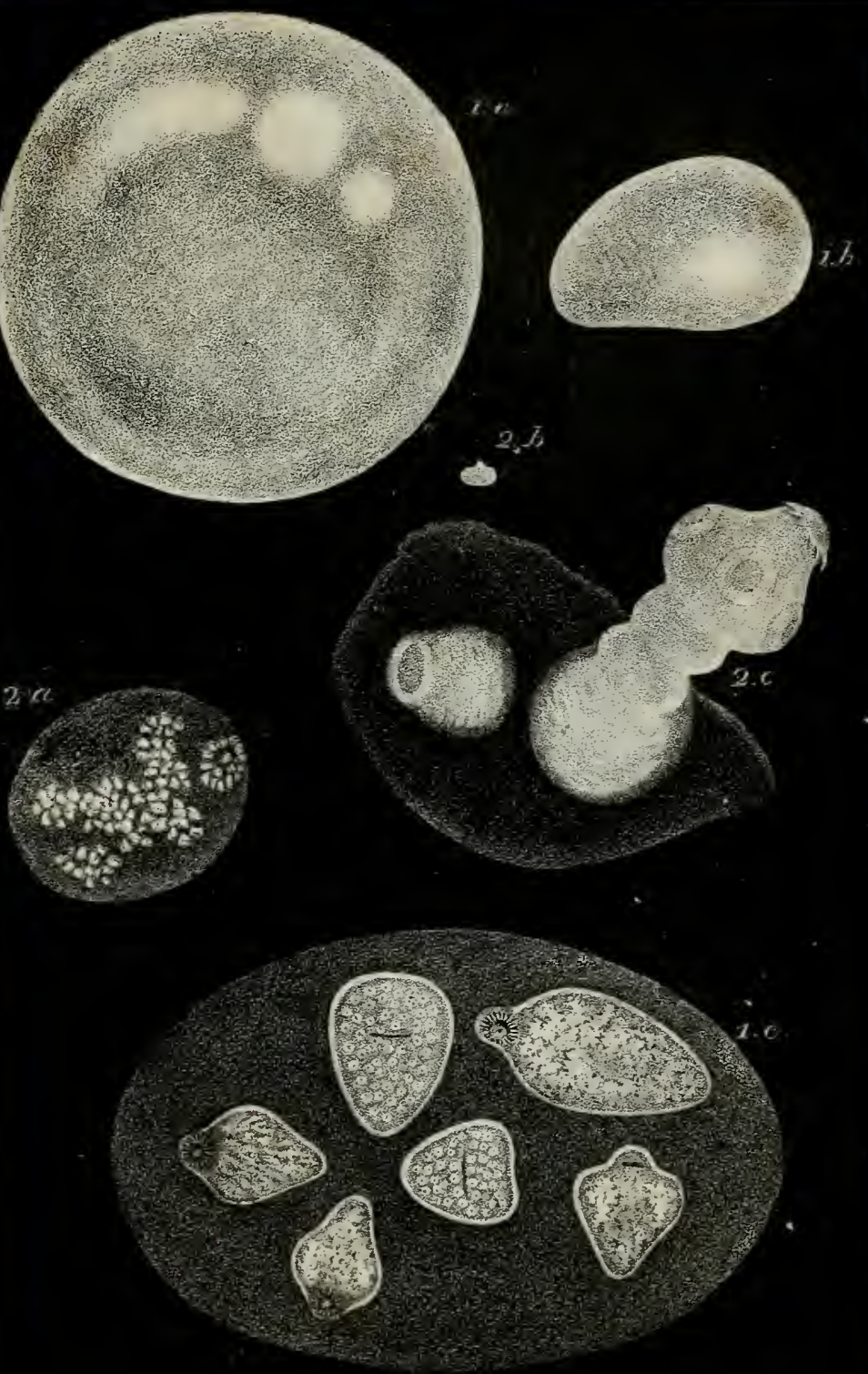



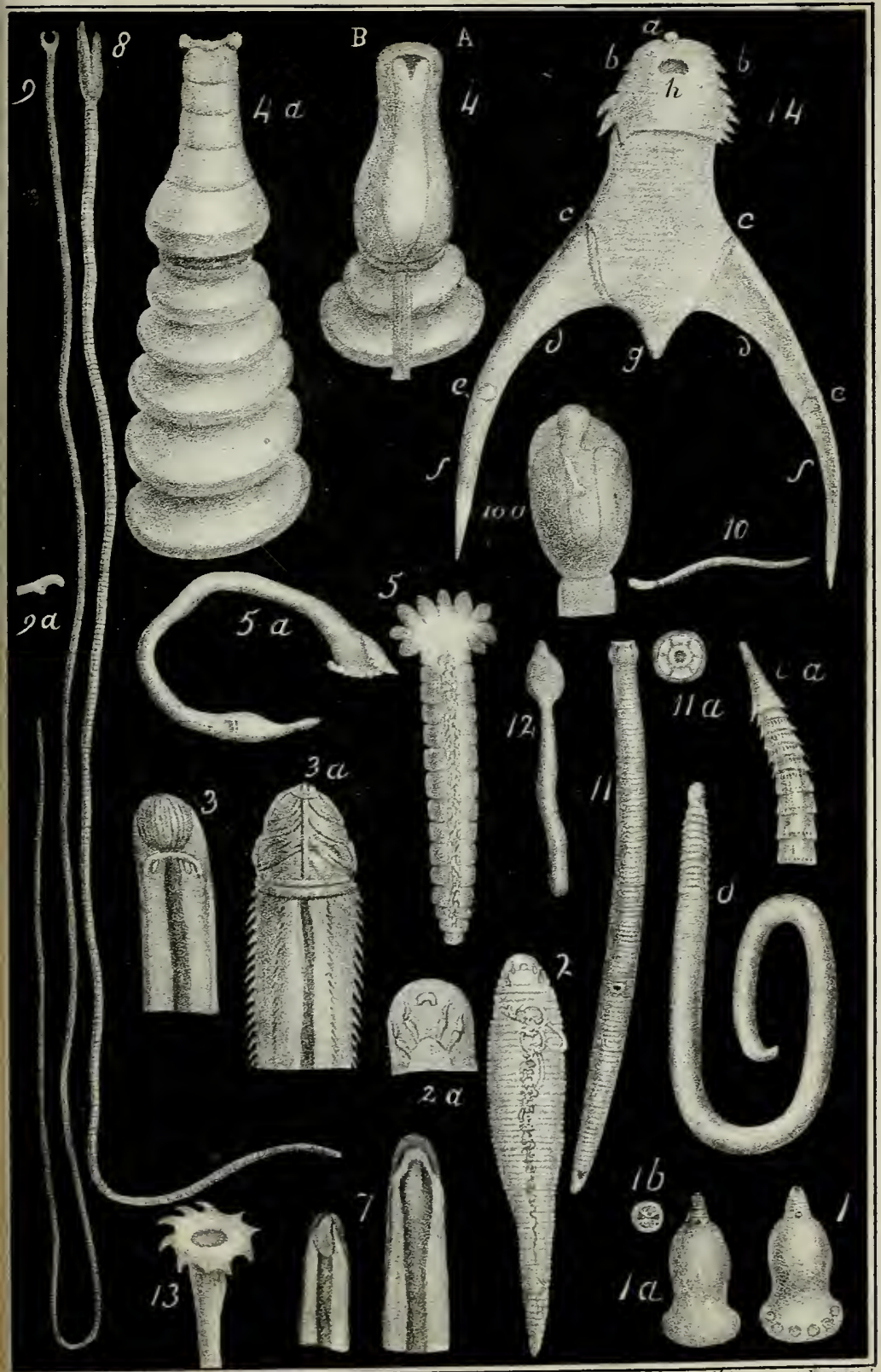



A P PEN $1 D \perp C L H$.

17. /I

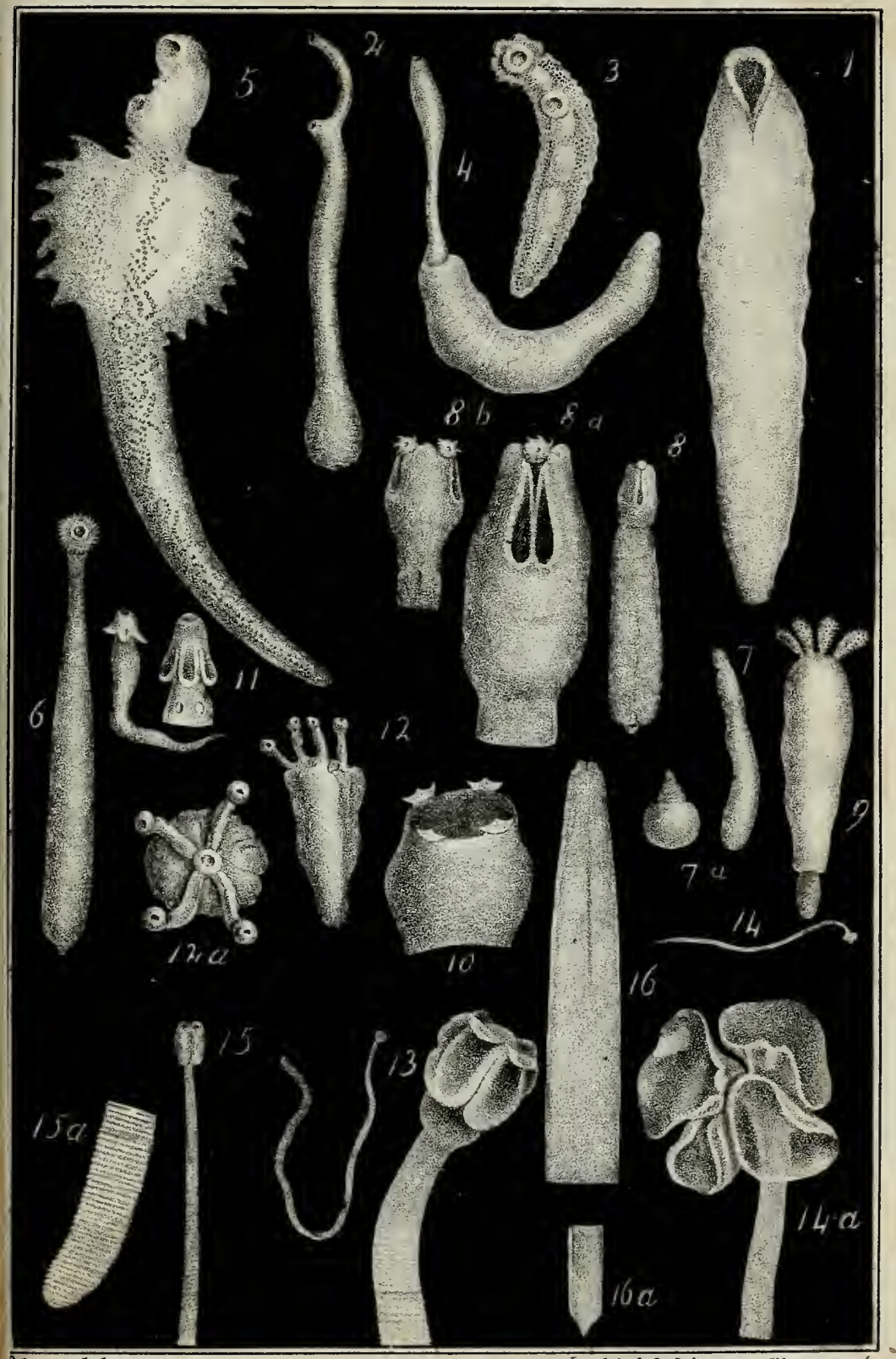

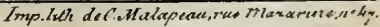




- $3:$ 



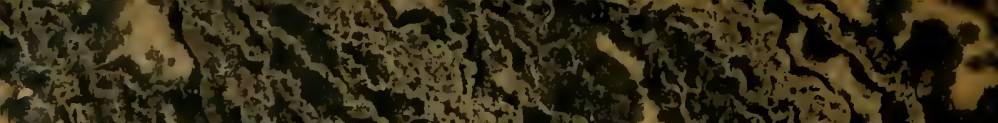

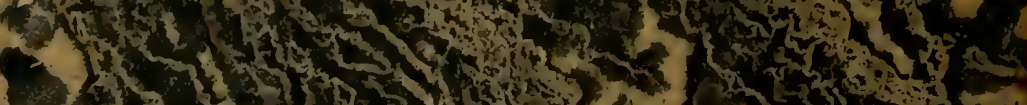

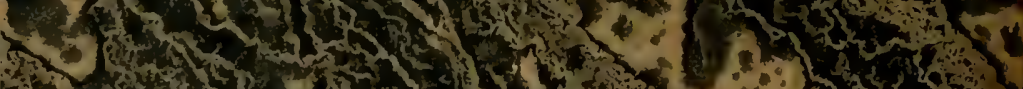

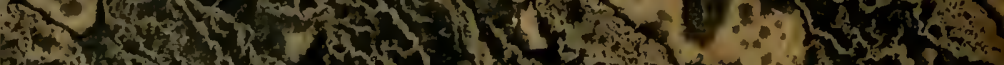

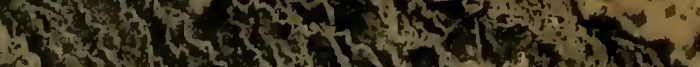

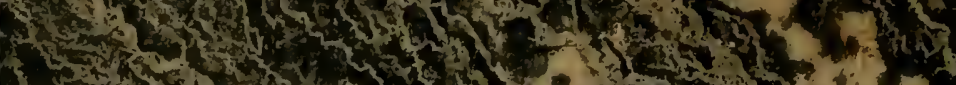

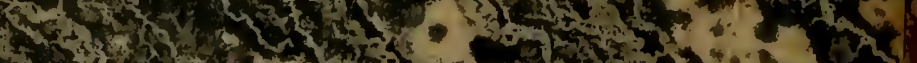

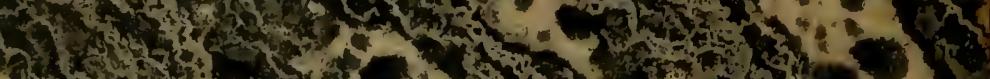

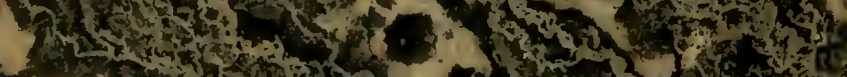

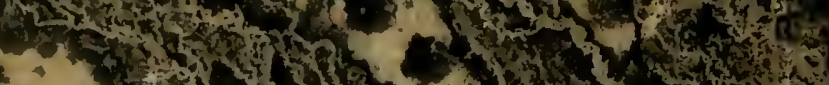

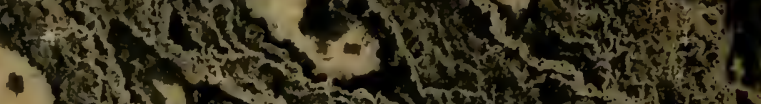

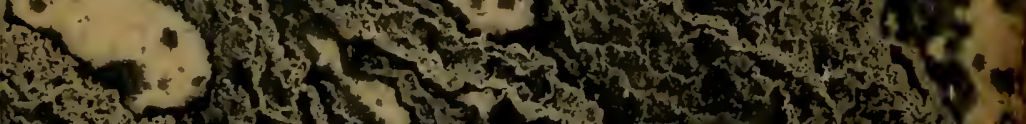

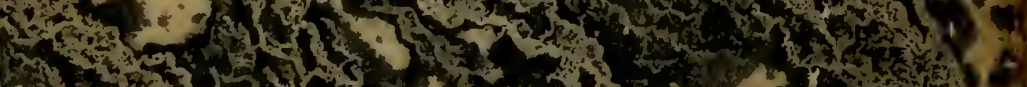

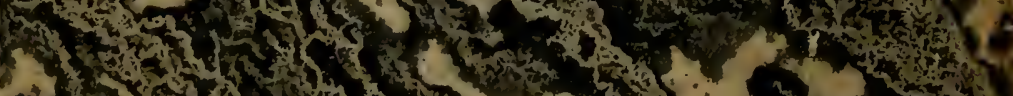
a sof

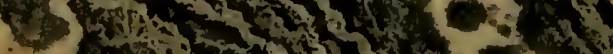

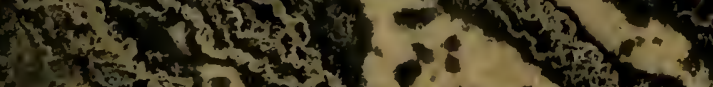

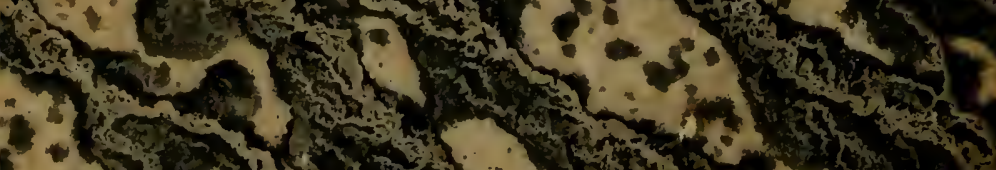

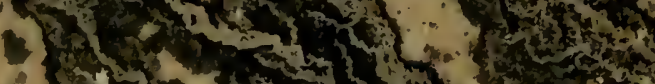

the

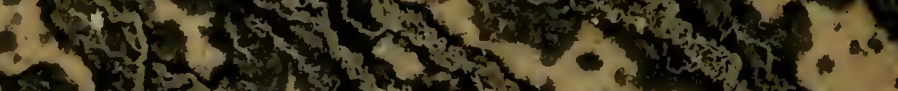

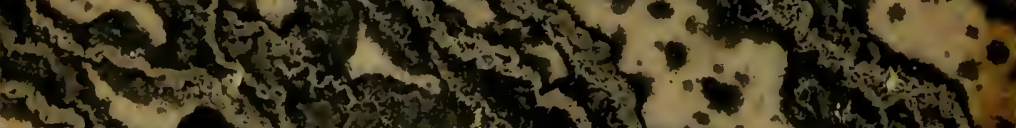
5 t

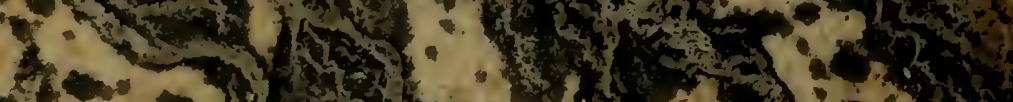

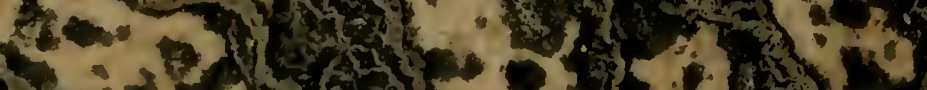

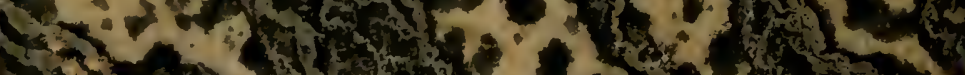

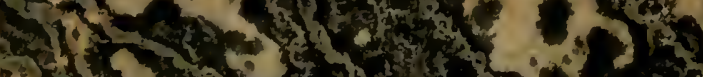

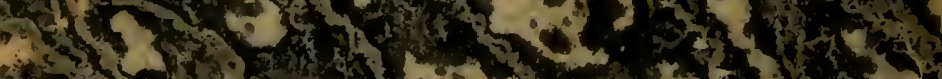

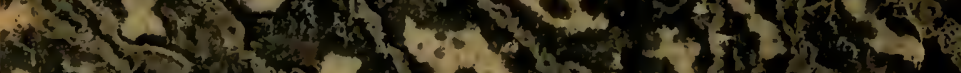

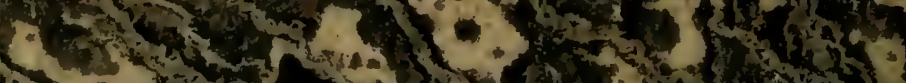

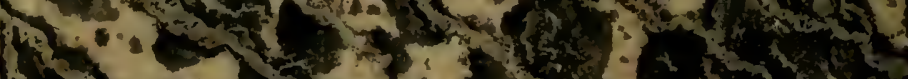

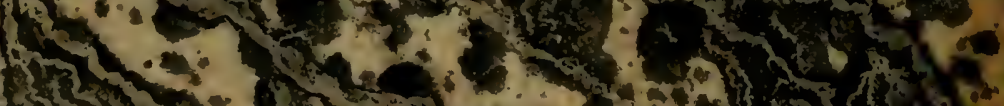

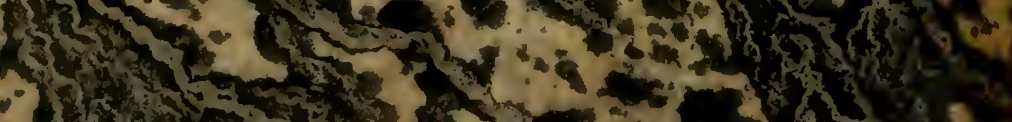

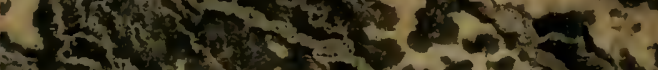
ats

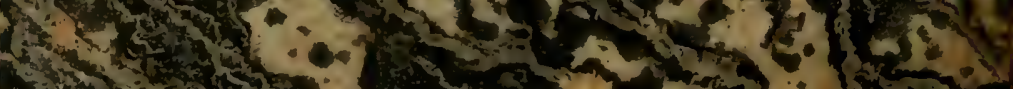

